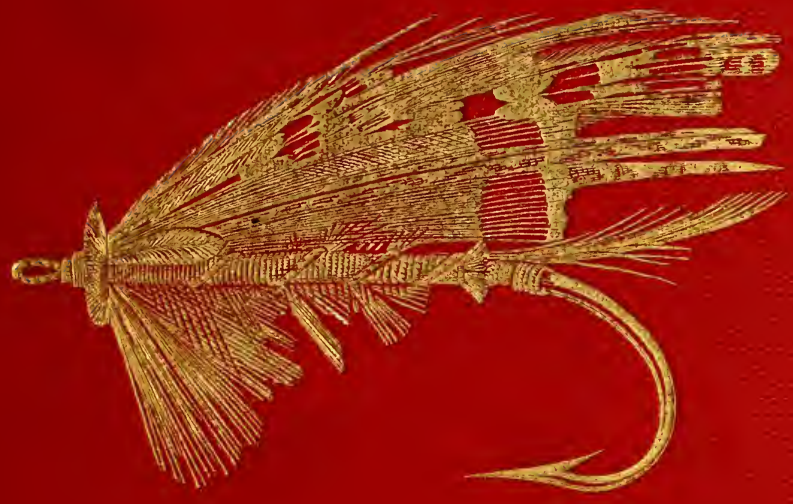





\section{SEP 1928}

Verlag von PAUL PAREY in Berlin SW., , o Hedemannstrasse

\section{Handbuch}

der

\section{Fischzucht und Fischerei.}

Unter Mitwirkung von

Dr. B. Benecke,

Professor in Königsberg i. Pr. und

E. Dallmer,

herausgegeben von

\section{Max von dem Borne,}

Rittergutsbesitzer auf Berneuchen.

Mit 581 in den Text gedruckten Abbildungen. Preis 20 M. Gebunden 22 M. 50 Pf.

Fischzucht und Fischerei nehmen, wie segensreich die Massnahmen vieler Behörden und die Wirksamkeit des Deutschen Fischerei-Vereins bislang auch schon gewesen sind, noch lange nicht die Stellung im Haushalte des Deutschen Reiches ein, welche ihnen gebührt.

Die deutschen Binnengewässer müssen zu einem grossen Teile neu mit Fischen bevölkert werden, und dazu gehört die weiteste Verbreitung von Kenntnissen in der Kunst der Fischzüchtung; die deutschen Meere mit ihrem unerschöpflichen Fischreichtum müssen in ganz anderer Weise ausgebeutet werden, wie bisher, und dazu gehört die weiteste Verbreitung von Kenntnissen in der Kunst des Fischfangens.

Das waren die Gesichtspunkte und Gründe, welchees wünschenswert erscheinen liessen, dass die Resultate der vielen wissenschaftlichen Untersuchungen und reichen praktischen Erfahrungen der beiden letzten Jahrzehnte, unter Heranziehung alles dessen, was die ausländische Litteratur über diesen Gegenstand bietet, nunmehr zusammengefasst würden in einem systematischen und ausführlichen, allgemein verständlichen Handbuch der Fischzucht und Fischerei.

Das Werk zerfällt in folgende vier Abteilungen:

Naturgeschichte und Leben der Fische (Benecke), Fischzucht (Borne), Seefischerei (Dallmer), Süsswasserfischerei (Borne).

$\mathrm{Zu}$ beziehen durch jede Buchhandlung. 


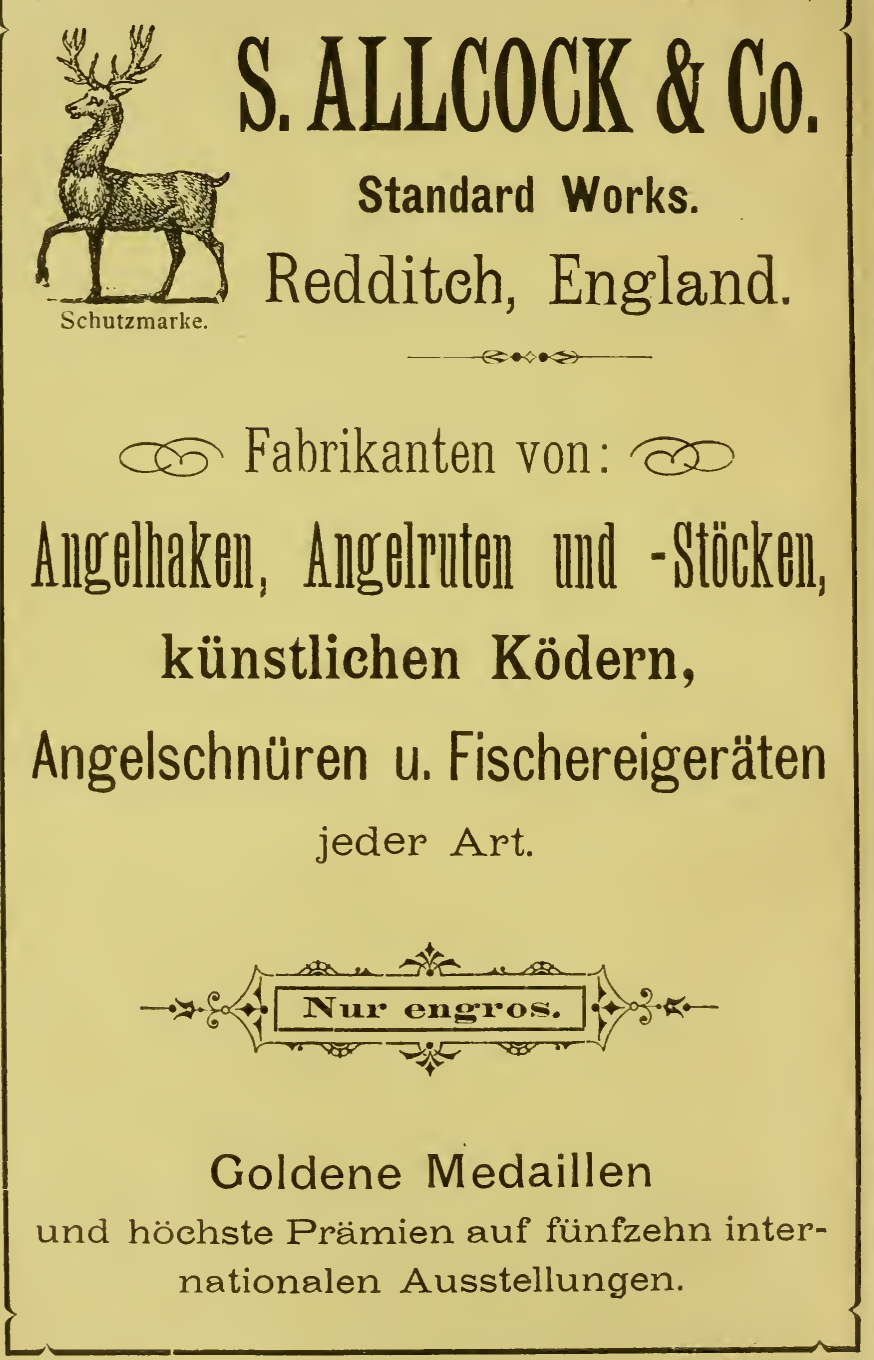




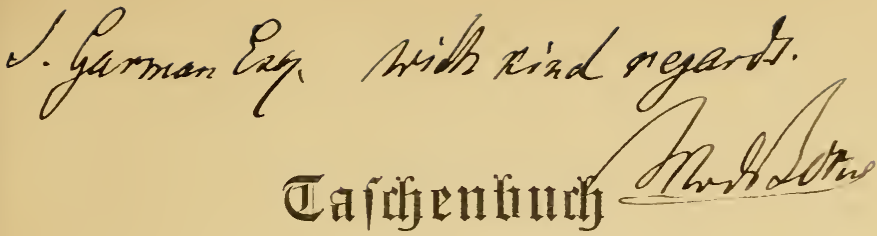

Ser

2ligelfifderei.

Don

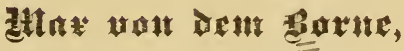

rittergutşbeïzer ani Berneutchen.

Drite, แmbarfeitete Puflate.

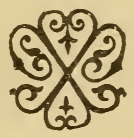

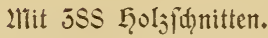

Barliu.

$\mathfrak{D} \in \mathfrak{r} \mathfrak{a} \mathfrak{g}$ von $\mathfrak{P} \mathfrak{a} \mathfrak{a} \mathfrak{p} \mathfrak{a} \mathfrak{r} \mathfrak{e}$.

Berlagbhanblung fur Qanbrirtiकaft, Bartenbau uno forfinefen.

SW., 10 Izeoemannjtrafze.

1892. 


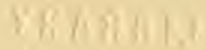

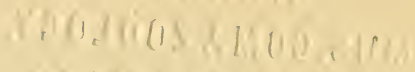

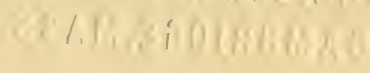




\section{Dormort.}

Dem Sport ber Angelfijcheret mirb in Deutjoland nidjt die $\mathfrak{A}$ upmerfiamfeit gejdyenft, weldye er als ein Mittel zur (Errifichung bes (Sieiftes und Rörpers verbient; er füfrt uns

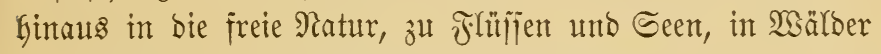
uno Wiejen bes (Siebirges und bes Flachlandes, unb an bas Meer.

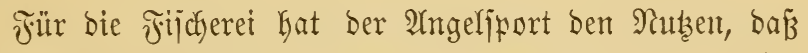
er Renntnis unb Riebe zur Sache in ben gebilbeten und ein= flüreidyen Sreijen verbreitet, unb bieje anregt, bie Jijcherei jut pflegen uno zu verbefjern.

Sd) Gabe midy bemüht, ben sejer mit alfem Eefannt zu

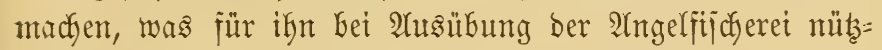
(id) jein fann; namentlicf) mit ben $\mathfrak{A}$ ngelgeräten, beren $\mathfrak{g e r}=$

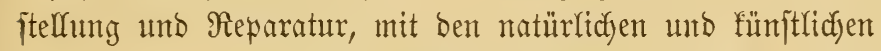

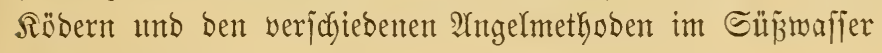
und in Meere.

Bejonbere 2 Uujmerfiamfeit jchenfte ich ben (silanzifiegen, weil fite bei uns nidyt bie Beadytung finden, beren fie wert jint. Wenn nady einem Jakrzehnt, wie ich hoffe, bas ameri= fanijçe Black Bass in unjeren (jewä̈jןern alfgemein verbreitet jein wiro, fo werben bie (silanzfliegen für ben âtgler nod) größere Bebeutung geminnen, als fie jedon jetst verbienen. 
Sab war bemilgt, alfes neue anto mertoolfe mitjuteilen, mas feit 1882, bem Erjoheinumggjafre ber vorigen Stuffage biejes Budbes, auj bent (5iebiete ber 2hugelfijdyerei bervor= getreten ift, utto es hat infolge beffen bie mene Aluflage eine erbeblicke Erweiterung erbalten.

Bejonders mertoolle Sallfe haben mir folgente Jeerren geleiftet, benen idy bafür verbintolidy it banfe.

Mer. Theodor 巨dyann in Ronon,

Jeer (5) hutat in Jranffurt an Main,

Mr. Eharles Farlow in Ronbon,

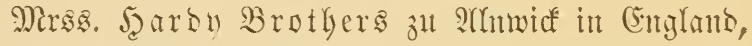

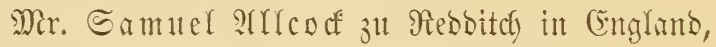

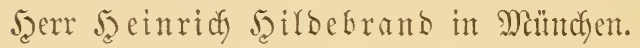

Berneuden, im Sommer 1892.

\section{Der Derfaffer.}




\section{Junhalt.}

(Eriter Teil. Die 2Utngelfijcherei im Sï̄̄wafier.

Eriter 2lbichnitt. 21ngelgeräte . . . . . . . . . . 1

1. Die 2̂ngelrute . . . . . . . . . . . 1

2. Die Millent . . . . . . . . . . . . 8

3. Der Pltgelhafett . . . . . . . . . . 11

4. Die $2 \mathfrak{A}$ gelichmur . . . . . . . . . . . 17

5. Die Snoten . . . . . . . . . . 22

6. Waache, Jinní, Leim . . . . . . . . . 32

7. Färben . . . . . . . . . . . 34

8. Flofie . . . . . . . . . . 35

9. Genfer . . . . . . . . . . 37

10. Berichiedente 2 ntgelgerätichaften. Sleidung . 38 3weiter 2Ubidnitt. Die SiöDer . . . . . . . . . . 43

I. Natïrliche Siöber . . . . . . . . . . . . . 44

1. 2̂Utgellöber . . . . . . . . . . . . . 44

2. (5rundëber . . . . . . . . . . . . 53

II. Sïnftliche Söber . . . . . . . . . . . . . 55

1. Süntitldce F̂tiegen unઠ Säfer . . . . . 55

A. Dašrsinden der fünjtlichen Frlegen und Säfer 57

Dag Mindent der Forelfenfliege . . . . 65

2Hoere Methode Forellenfliegen zu windent 70

B. Die fünitlichen Jorellenfliegen . . . . 78

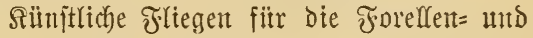

2lejchenfificherei . . . . . . . 81

Sïnftliche Säfer . . . . . . . . . 97

Das WBinden ber grop̉en (Slanzfliegen . 100

Bejdreibung einiger bejonders mirfiamer

Glanzfliegen . . . . . . . 113 
C. Seeforelfenfliegen . . . . . . 124

D. Mleerforellenfliegen . . . . . . . . 124

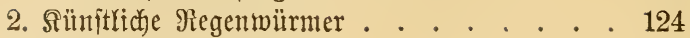

3. Sünftliche J゙leifchmaden . . . . . . 124

4. Sünftliche Söber für Spinnfijcherei . . . . 124

5. Sümittiche Röber für bas 5̧eben แnd Senfen 127 Dritter 2Hbichnitt. 2Angelmethoden . . . . . . . 131

1. Die Flof̧angel . . . . . . . . . . 136

2. Das feftliegende F̂ló̉ . . . . . . 146

3. Die Ģrumbangel ohne Frló̉ . . . . . . 146

4. Dą Bobenblei . . . . . . . . . 147

5. Die \$aternofterangel . . . . . . . . . 148

6. Dą Szeben und Senfen. . . . . . . . 150

7. Die Jif thenangel . . . . . . . . . . 150

7a. Die Epinnfifherei . . . . . . . . 151

7b. Die Irollangel . . . . . . . . . 169

7c. Die Sdnappangel mit lebentem Söber=

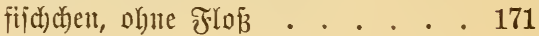

7d. Die Schnappangel mit lebendem Sïber= fifchden und dem Floß . . . . 173

7e. Die 巨chluctangel mit Yebentem Sïberfifd̆ 174

8. Die Fif cherei mit ber fünjtlichent Friege . . 180

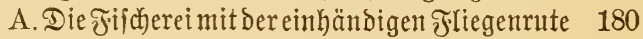

B. Die Fif fherei nit ber boppelhändigent

F) fiegenrute und mit Der Rachasurute. 182

9. Das 2Ingeln mit Der Friegenrute und natïr= lichen Söbern . . . . . . 187

10. Die $\mathfrak{B u f h b a n g e l e i}$ Dober Iippfifanerei $\quad . \quad . \quad 189$

11. Die Treibjhumr . . . . . . . . . 190

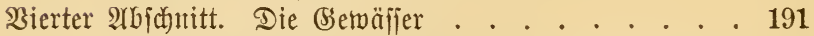

I. Die ßerteilung der Fiffuartent in Seen und Fritiffen 191

II. $\mathfrak{A}$ ngelitellen

III. Die MBaffertiefe . . . . . . . . . . . . 197

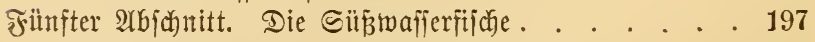

IYngelf́alender . . . . . . . . . . . . . . 197

Dą $\mathfrak{B e t t e r}$.

1. Die Frorelle . . . . . . . . . . 202 
2. Die Scefore!le . . . . . . . 208

3. Die $\mathfrak{Y}$ ejche. . . . . . . . . . . . . 209

4. Der \&achg. . . . . . . . . . . . . 212

5. Die Mieerforelfe . . . . . . . . . . . 214

6. Der Şuthen . . . . . . . . . . . 214

7. Ier Saibling . . . . . . . . . . . 215

8. Der Sitwarzbarich und Der Forellenbarid . 216

9. Der Etint. . . . . . . . . . . . 224

10. Der Blaufelchen. . . . . . . . . . . 225

11. Der Sillch . . . . . . . . . . . . . 225

12. Ier ๔đnäpei . . . . . . . . . . 226

13. Die groß̉e Maräne . . . . . . . 226

14. Die Döbel . . . . . . . . . . . 226

15. Der ら̧äjeling . . . . . . . . . . . . 228

16. Der lltelet . . . . . . . . . . . . 229

17. Der Echneidcr . . . . . . . . . . . 229

18. Der Mapfen . . . . . . . . . . . . 230

19. Ter $\mathfrak{A l a n d}$. . . . . . . . . . . 231

20. Ier Maififich) . . . . . . . . . . . . 231

21. Der Seecht . . . . . . . . . . 232

22. Der Barich . . . . . . . . . . . . 236

23. Der 3ander . . . . . . . . . . 238

24. Ier Siaulbarich . . . . . . . . . . 239

25. Der Bingel . . . . . . . . . . 240

26. Der ভtreber . . . . . . . . . . . 240

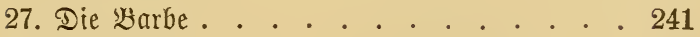

28. Die Plöge . . . . . . . . . . . . 243

29. Das Rotauge . . . . . . . . . . 246

30. Der Blei . . . . . . . . . . . . . 246

31. Die (̧üjter . . . . . . . . . 247

32. Ier Farpfen . . . . . . . . . . 248

33. Der Echlei . . . . . . . . . . . . 249

34. Der Briundling . . . . . . . . . 250

35. Die Maje . . . . . . . . . . . . 251

36. Die Flumber . . . . . . . . . . 252

37. Der $\mathfrak{A a l}$. . . . . . . . . . . . 253

38. Die Erike . . . . . . . . . 255 
39. Ier 巨tichling . . . . . . . . 25う

40. Die Sttappe . . . . . . . . 256

41. Die 3ärthe . . . . . . . . . 257

42. Der $\mathfrak{W e l}$. . . . . . . . . 258

3 weiter Teil. Die Angelfitherei int Mneere.

(5rjter 2rbichnitt. 21ngelmethoden und 2lngelgcräte . . 259

1. Tie Errundangel. . . . . . . . . . . 259

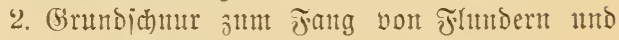
Iliejthent . . . . . . . . . 264

3 Die Jitherei mit ber Treibidum . . . . 264

4. Ias Jithen mit ber Edjleppangel . . . 266

5. Ias Jifichen mit Der 2(ngelrute . . . . 268

6. Ias Bobcublei . . . . . . . . . . . 269

7. Tie \$aternofter=?trgel . . . . . . . . 269

8. Regeantgelt . . . . . . . . . . 271

Die sintre . . . . . . . . . . . . 272

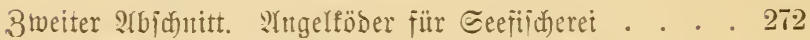

Dritter Afbiduttitt. Die Eeefijche unb Deren Fang mit Der 2lngel . . . . . . . . . . . . . 279

Antgelfalchber . . . . . . . . . . 279

Filiche. . . . . . . . . . 281

Sitteratur . . . . . . . . . . . 293 


\section{strterterts

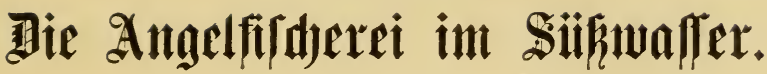

\section{Erfter 2lbiḑnitt. \\ $\mathfrak{d} \mathfrak{i} \mathfrak{g} \mathfrak{e} \mathfrak{l} \cdot \mathfrak{G} \mathfrak{e} \mathfrak{x} \mathfrak{a} \mathfrak{t} \mathfrak{e}$.}

(5s giebt Giegenden, wo es jumbierig ift, bie 2Angelgeräte ju faufen, unb es giebt \$erjonen, benen es Bergnügen madjt, ifre 2lpparate jelbjt anjufertigen, bie licber mit jelbjtäabrizierten 2rngeln fiifjen, wie mit gefauften. Sit ijt es audy notwendig, La

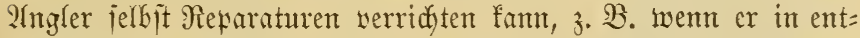
legenter (Begents, in einem einjamen Sirtshauje auf fidy jelbjt an= gemiejen ijt. Tie Befanntichaft mit ber Fabrifation ber (5ieräte

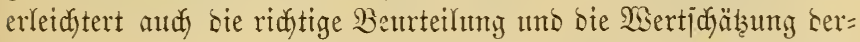
jelben beim (Einfauf. Deshalb jollte jeber Jreunto ber 2 ingelfijheret fidd) nit ber 2Injertigung ber 2Yngelgeräte befannt zu madjen judjen.

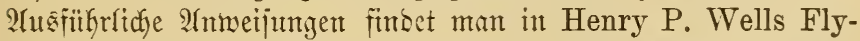
rods and Fly-tackle, London. Sampson Low, Marston, Searle and Rivington.

\section{Die 2Ingelrute.}

(B) wöhnlid fun bie Ângelnute in mehrere Teile jerlegt merben, um fie leidjter aufbemahren uto transportieren zu fönnen. Tie Serbinsung ber einzelnen Teile geidjeht meijtenteils in ber

v. ১. Borne, Ingeffifiderei. 3. 2uff. 


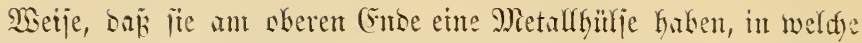
bas untere mit Metalf beidhlagene Enoe oes folgentoen Zeiles geitedt wirs.

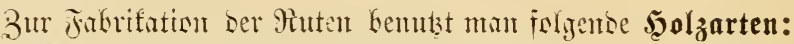

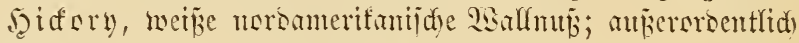
zäly uno sauterfaft, geneigt frumm jut wersen, wutro in Englans

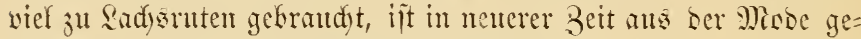
fommen; ju Eprisen nidjt gut jut gebranden. Epej. (Sem. 0,80.

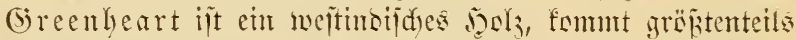

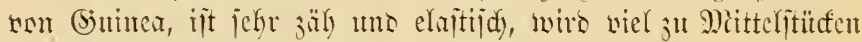
unts Equisen gefraudt. Epez. (5icm. 0,96-1,09.

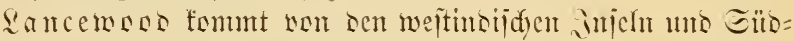

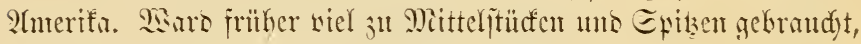
jetzt mentig rermentet. Elej. (Jien. 1,03.

Eijenthols ans Piorb=2lmerifin, won Ostrya Virginica. Tein= fajerig, iteif uno elajtijes; läjit jïh Yeidyt in lamge Eplitter teilen. Erej. Giew. 0,82.

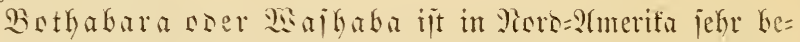

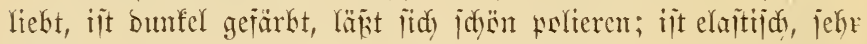

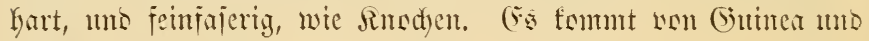
Gat 1,21 ifej. (jems.

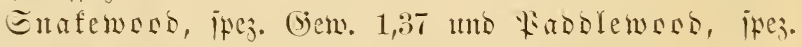

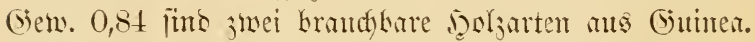

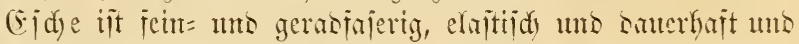
jefre gut ju Grifjenten. Epej. Sien. 0,78.

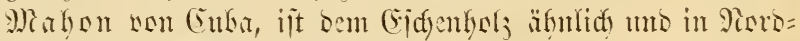
Panerifia beliebt. Epej. (Sin. 0,66.

Ias Sitinotjde Bantus wan Galcutta (Bambus Arundinacea) wiro 12-15 Mieter hod) uno bis $7^{1} / 2$ (Eentinter sidf; es ijt hobl unt hat fleme Rnoten, es liejert Das Material ju ben

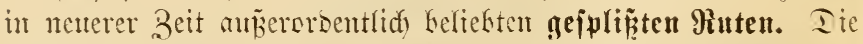

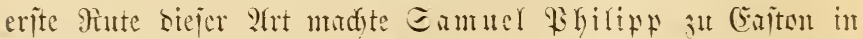

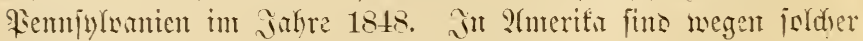

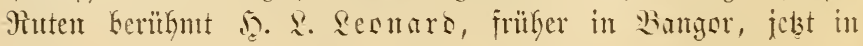




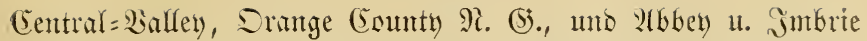
48 Maiben \&ante, Mem=Vort. Thif ber Internationalen Fijdyerei= 2tusitellung zu Sonbon 1883 erfielt bie Firma Jaarby Brothers,

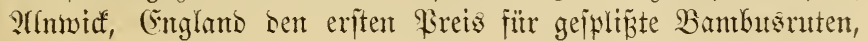

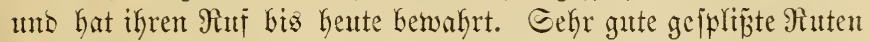

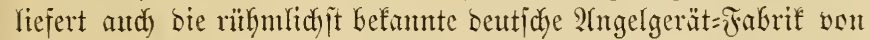

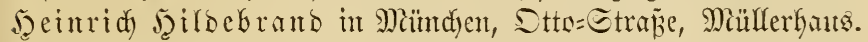
Das untere, biffite Cnoe des Bambus Yiejert bas befte Material, unto jeine Etärfe beruht in ber Cepibermis, weshalb von biejer möglidyjt viel in bie Mute gebradyt unb bas weiche Mart joviel wie möglidy entjernt wirt. Die Espibermis folf unverYetzt jein,

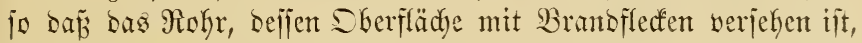

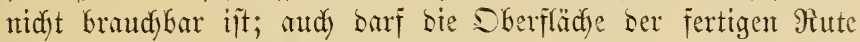
nidjt geglättet ober poliert werben. Die 6 Eplijien, aus melden

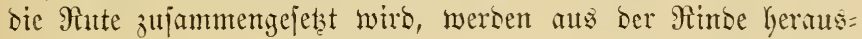
geidnitten, wie bies bie weipe Qinie in umjerer Fig. 1 a moentet.

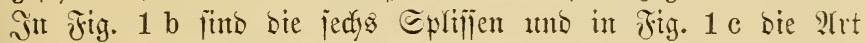
ifgrer 3ujammenfïgung veranjdaulid)t.

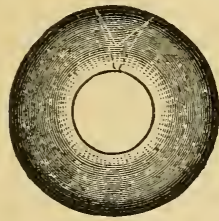

a

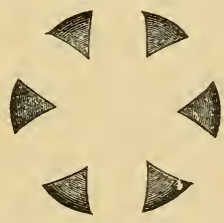

b

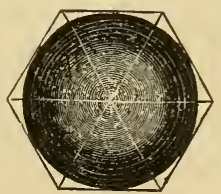

c

Fig. 1. Gejpliß̧te-ângelrute.

Der Reint, Dutrd meldyen bie Epliffen verkutben werben,

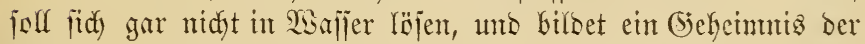
Fabrif, von jeiner Jaltbarfeit uno Danerfaftigfeit ift bie Siüte ber Mite wejentlidy bebingt.

Die oberen bïnteren Teile Der Auten werben ans jedjs Eplijien gemadd, Fig. 1 c, ebenjo ift es bei bem Griffentse ber

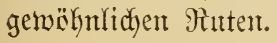


Bei ben bejten Ruten, bejonbers Len jegr laugen uno itarten, bejteken sie unteren Teile auв jowei Sagem won je 6 Eplifin,

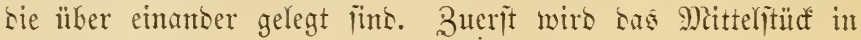
gewöhnlidjer Sieije aus 6 Sflijien jujanmengeleimt unb ge= trofnet. Dann fommt cine sweite Rage won 6 Eplifien barüber.

Cine jefr wejentriche Berbejerung in jaltbarfeit un 2 Surf=

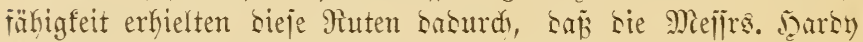
Brothers ine mit cinem Eentrum non Etafhl werjahen, weldyes iesem Gliebe ser Aute eingefügt ift. Ier Etahl ijt mie bie 1thrjebern gehürtet.

Ia Tonfingrofre ijt fajt garnidyt hofh, sümter als bas

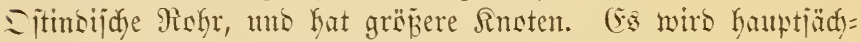

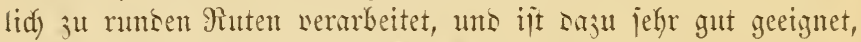

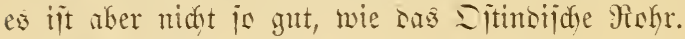

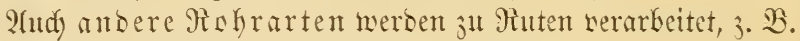

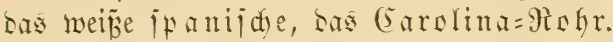

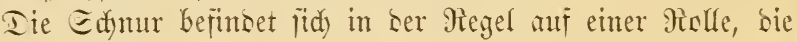
am unteren (Enbe ber Bute surd) einen werjdiebbaren Ring be= fejtigt mird; ïe wirb surd) eine $2(m j a f) l$ won Ringen gezogen,

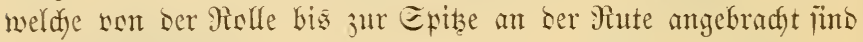
(ङ. Jig. 10). Tie Ringe itefen $14 m$ is näher ameinanser, je

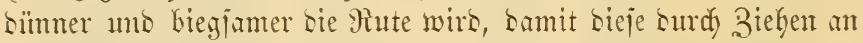
ber Echntr gleidgmänig gebogen wirs. Eie jimb entweser be= weglid, fie fömnen alfgeridftet uns umgelegt wersen, ober jie itehen feit uno anfredyt. Erjtere Form madyt bas Berfacen Der Pute bequemer, ift aber nidyt braudbar, wenn burd) bie Minge gemorfen werben joll (j. s.). (5s ijt beshalb ben aujredtitefensen Mingen ber Borjug ju geken. I ie cinfadjite form (i. Fig. 2) hat sen llebeljtant, Daj bie Edynur leidht Durd) bie Pinge gefangen wirs, wenn fie fid sarum jojlingt. Befjer jino folgente formen: Bell's Life Rings (Jig. 3). 2fmerifanijale Irahtringe (Jig. 4).

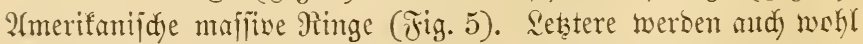

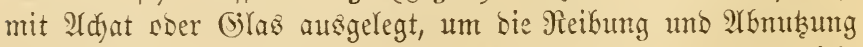
ser Edynur zu verminbern. Ier Enoring foll ebenfalls nidst 


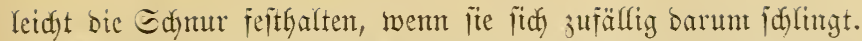

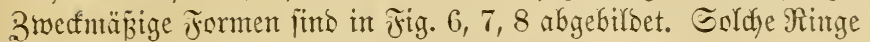

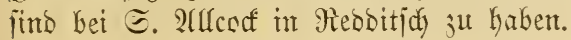

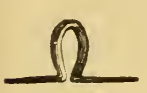

Fig. 2.

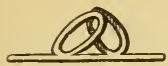

Fig. 4.

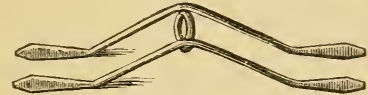

Æig. 3.

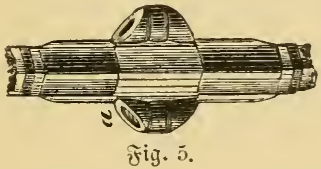

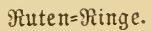
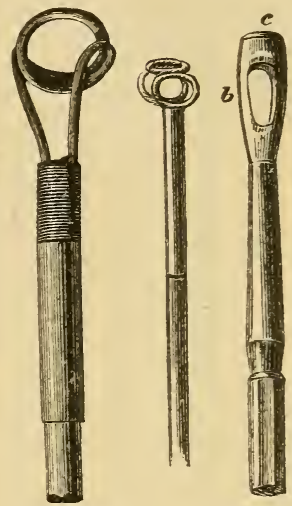

Fig. 6. Fig. 7. Fig. 8.

in ben lebten Gafhen fint bie Edyangenringe, Snake

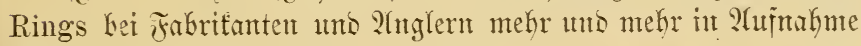
gefoumen, ïe jint jowoly ben beweg=

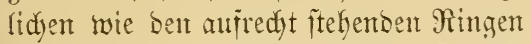
norjuziegen, weil ie haltbarer fint, uno iid) jefyr jower werbiegen lafjen uno wail

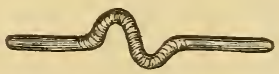

Fig. 9. Sd)langenring. bic Edymur fid) nidht baran verfängt.

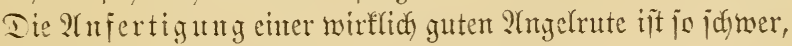

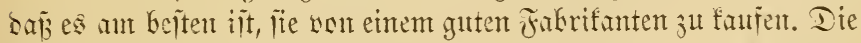
Epibe einter Foreffenfliegenrute itt taum io biaf, wie ber Siel einer

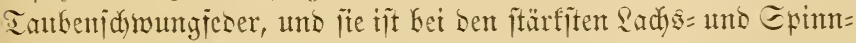
ruten faum io sidf, wie ser Riel ber Sdymungeder einer Gant. Der

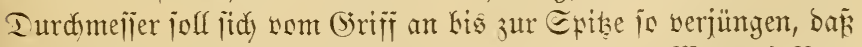

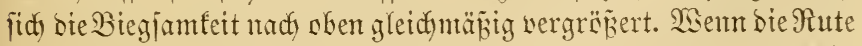

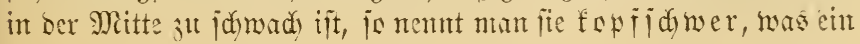


grozer Fegler ijt. Ter Grab ber Bieglanteit, Eraftijität

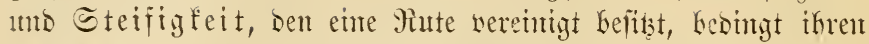

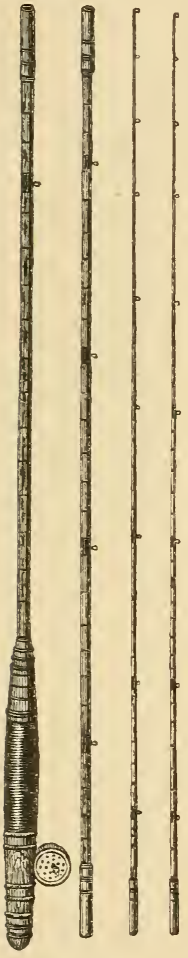

Fig. 10.

Tingerrute. Rert; fie jolf fid biegen, aber nidyt bredjen, wemt ein grojer jijch gehaft worden ijt. Diervon hängt

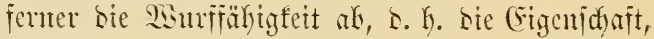

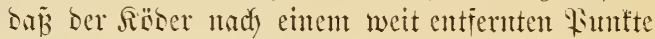
jidfer geworfen werben famm. Tie Mute jei nidyt

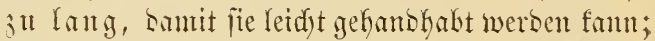
man wirft weiter uns fid herer mit einer funzen Pinte, sic bequent ift, wie mit cinter langent $u=$ Gequemen Mute. Eefyr michtig ijt jerner bas (b) = widyt ber Mute, je leidgter iic ijt, um jo bejier

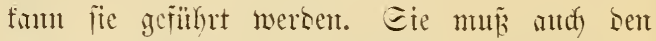
Edwerpunft an Der ridutigen Etelle haten. Bei sen chthänsigen Matent folf er ca. $1 / 3$ m ïber

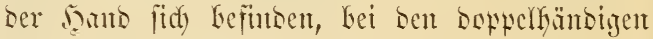
$1 / 3 \mathrm{~m}$ neiter anfwärts. (E) ijt fajt inmer bas

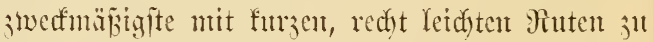
Fijden, tuto es mirb jelten notwendig jein, eine

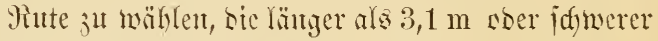
wie 500 bis $600 \mathrm{gr}$ ift.

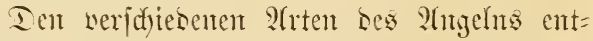

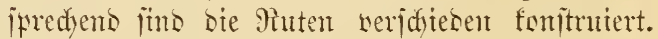

1. Tie einfändige Fliegenrute. Sidh Galte bie won Etemart entrighlene Mute für bie beite. Sie ift 3,1-3,3 m lang, umo nicht jul geidmeisig, jombern etwas iteif. Man fom sant weiter samit worfen, wie mit ciner längeren ju biegjamen Mute. Tie Rolfe befintset fich ant (Siriffente unter ber Etelle, wo bie Jant bes 2 (nglers anforit (Tig. 10).

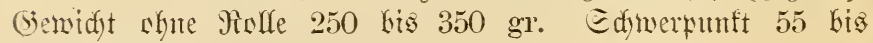
70 Eentinteter som Siriffentio entfont.

2. Tie boppergänoige Fliegenrute iteht zwijdyen ber

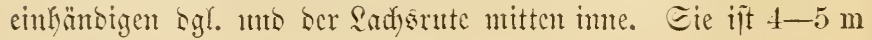




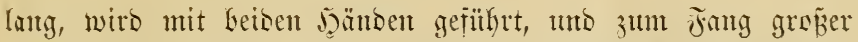

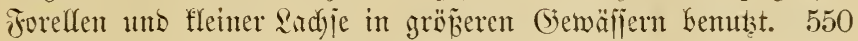

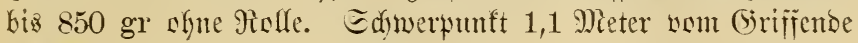
cutrernt.

3. Die Qad) rute 5,3-6,3 m lang. $1300-1400$ gr idyer whe Rolle, ber Edwerpunft $1,3 \mathrm{~m}$ wom (Sriffende.

4. Tie Nottingham= $\Re$ ute ijt $3,7 \mathrm{~m}$ lang uno hat auf=

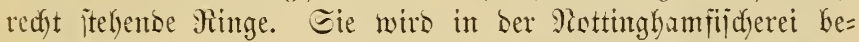
mutst, ijt mit ciner iefr leid)t brehbaren Molfe werjehen, unt ber siëber wirb in ber Megel won ber Molfe geworfen.

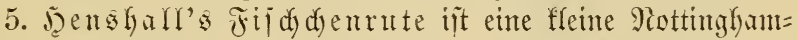
Mute, bie leidyt mit einer Santo gejührt werben famt. Sie iit $2 \frac{1}{2} \mathrm{~m}$ lang, an ber $\Re$ olfe ijt jie $25 \mathrm{~mm}$, an ber Epize $21 / 2 \mathrm{~mm}$

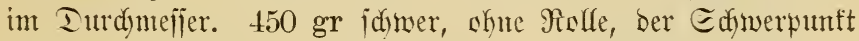
$0,6 \mathrm{~m}$ voun (jriffente. Die Rolle ijt $20-30 \mathrm{~cm}$ nom Griffende entfernt, jo baj bie Danto fid) unter ber Molle befindet, unb ber Dannen bie Berwegungen bor Rolle bequem regulieren fant.

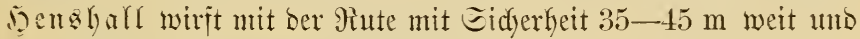
hat bamit bis 14 Wifo. idwere Dedfe gejangen. Cie fintm non

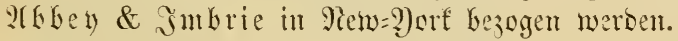

6. Iie Epinnute ift $3,1-3,3 \mathrm{~m}$ lang, reidyt uno elajtijd

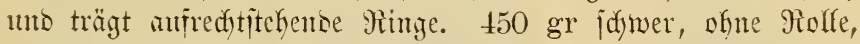
Der Edyerpuntt $1 \mathrm{~m}$ vom unteren Enoc.

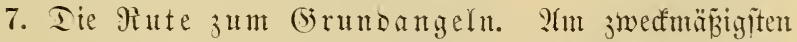
jind bajt bie Nottinghan=, Shenshall'jue und Epinnme. Beim Sijdjen wom lliper weroen bisweilent jebr lange Mtuten, - jogar bis $6^{1} / 4 \mathrm{~m}$ Qünge angemanot, Lie fïrzeren Mitten verbicnen aber

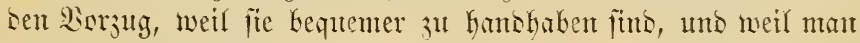
weiter bamit werfen fant.

Bon allen biejen Rutenaten bejibe id mupter= Gafte Gremplare bon ben beften Jabrifanten, bie es giebt; nad) Diejen Mujtern fertigt jeer Deinrid) Dirbe= brand in Miünden 2fugelruten, bie ebenjo gut jint, wie sie englijdent und anterifmijden unb bebentend billiger verfauft 


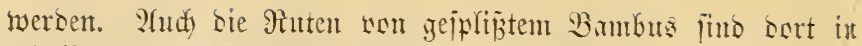
tabelfojer Bejuaffenteit zu haken.

\section{Die Rollen.}

Die Angelidymur ijt auf bie Rafle genvidelt, uno bieje ijt an ber Angelnute Durdy cinten werichiebbaren Ring befeitigt.

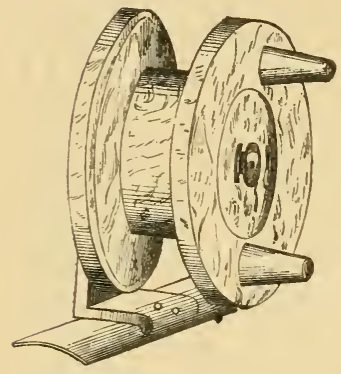

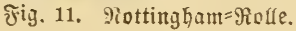

1. Tie Mottingham= Morle (Fig. 11) ift bie einfadyte. Eie iit bon Dolj uns oreht jidy mit gröpter Reidutig= feit, somit bei bem simf yon oer 丹olle Edyum abläut, wenu ber Röber, Jlofi ober Eenfer angigemorien werben, ofuc on joje Edum rorbansen ijt. Gine nenere Berbeflerung it einte Feberlemmung, sie abitellbar ijt. Sic joll Das Heberlanfat ber Rolle wer= Ginserm, wosurdy sic Edyutr in sier= mirumg gerït.

2. Metarrollen obue Federbemum (Jig. 12) haben ben

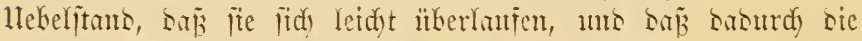

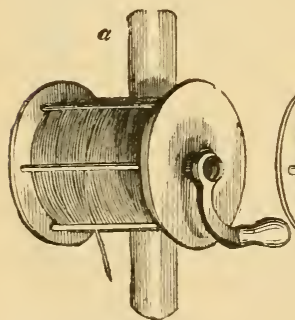

Fig. 12. Metalfrofre shne Feserhentung.

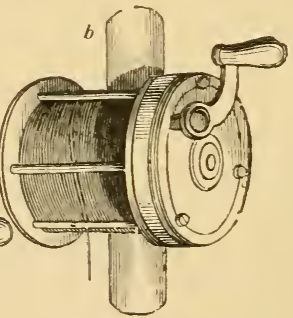

Fig. 13. Mietaflrolle mit Miultiplifator. Eduntr in Hmors: mung tommt. Sicje Bollen jüto nidut $\{u$ empiebleut.

3. Mietarluollen mit Febergem= mung (J゙ig. 14). Lie Fener nerfindert Die jul leidyte $11 \mathrm{~m}=$ irefung, sas lleber= lanfen und die Rier=

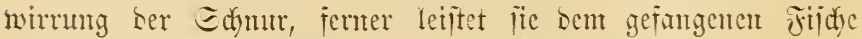
Siocritans, ment er Edymur son ber Bolle abjicht.

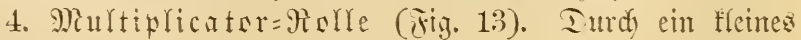


(S) triebe breft jidy bie Epindel breinal, menn bie Rurbel cinmal

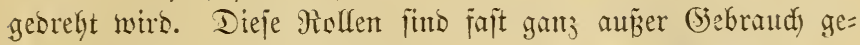
fonmen, weil fie leidgt in llnorb= nung fommen.

5. Mallod's, ,Sun and Planet" Molle iit cinc Multi= plifator $=\Re$ olle, wo fidt bie Epindel fünjmal oregt, ment ber (5rifif vier llmläufe madyt. SGre wejent=

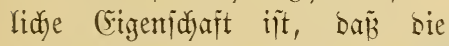
Spindel and utulaufen famt, went bie Eeitemplatten mit bem (briff feititehen, ber (Srifif breht fid ba= bei um jeine eigente $\mathfrak{A} d$ jje. Der geingene Fijd fant baher Edyutr

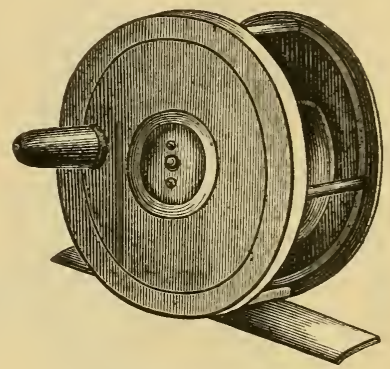

Fig. 14. Metalfrofle mit Feberhemmung.

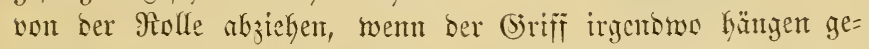
blieben ijt. Dies ijt nou sert bei Der Bontijiderei, uno wem

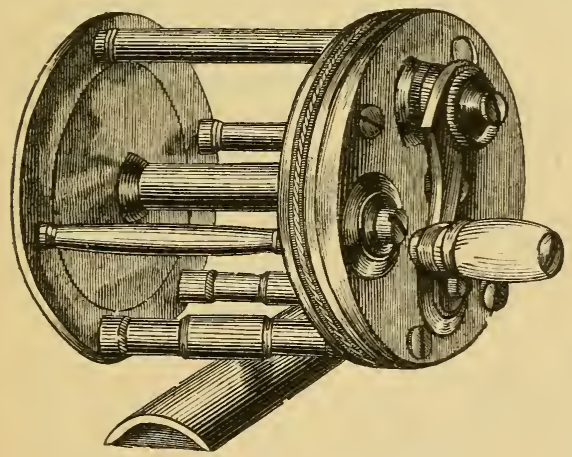

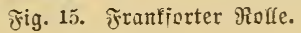

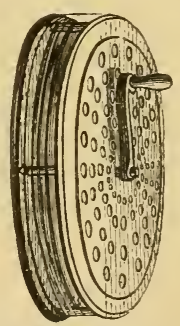

Fig. 16. Durd $=$ brodiene Rolfe.

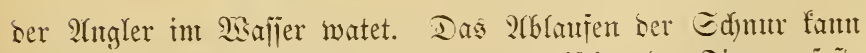
gekenmt werben, went man ben Srifi zwijhen ben Fingern feit= Gält uno bie llntbrefung um fïd jelbit Dadurd) aufgält. 
6. Die 2lmerifanijue Jranfiorter Borle (jig. 15) multi= priziert im sierkältuis 4 ju 1 . Eie hat eime Jeberkenmumg, bie

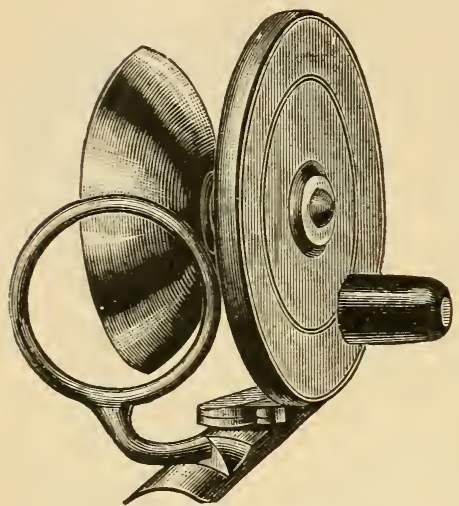

Fig. 17. Mallod's Mienterolfe. abgejtelft werben fam, und läuft auberorbentlidy Yeidyt tur. Eic ijt jefre jauber gear= beitet, utur wirb gefrautd, um bei sem ?tuge?n mit bem Fijdyen wout ber Mindfe jull werfen. Id habe viele ginfure ioldye Bolfen gebraudte, uno bin jebr mit senjelfen jut frieben. Eie jimb ein mejent= lidyer Beitmoteil ber Jens= falljen ?angel.

7. Iie 2 merifantide Durdorodene Molle (jig. 16) bat Den Bwedf, Das

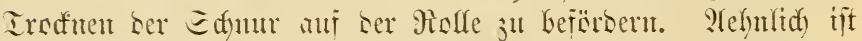
Jardine's Trout Reel fomptruicert, sie fïr bie Forellemfijdyerei

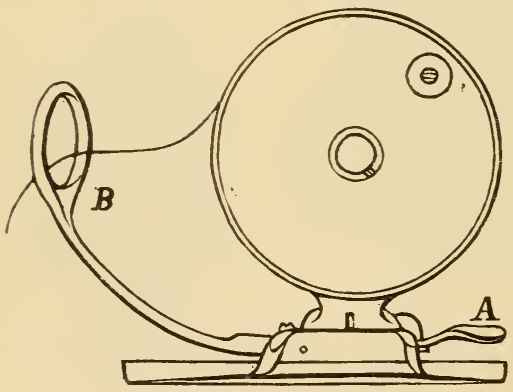

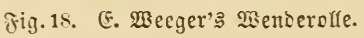
gebraudit mirs, uno jefre gerülynt wirt.

8. Iie 2isenbe= rolle (jig. 17) iit wout 2. I. Miallody in Fierth, Edyottlanto, erfunben uno bejondersis gut geeignet, um weite 2 simfe non ber Molle jut madyen.

Eefro gute 2 sembe= rollen, uad) ben Mujtern wou (5. 2leeger jertigt

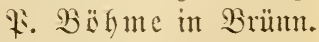
9. Follen yon Plumitutum jind jwar teuer, haben aber einige Sorjüge vor Midflen aus anterem Mietall worats. Wegent 
ifree grop̃en Reidytigfeit fömen jie erkeblidy grëß̈er gentadjt werben, unto man fann beshalb mit einter längerent Edynur fijdyen. Eime Forellen= rofle won $7 \mathrm{~cm}$ Iurd)= mefier wiegt $85 \mathrm{gr}$ unto eime?adfordfe won $10 \mathrm{~cm}$ Durdymelier wiegt 255 Gramm.

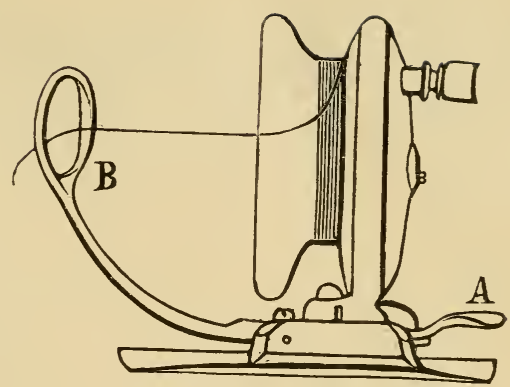

Fig. 19. E. Weeger $\mathfrak{\text { Wenterolle. }}$

\section{Der 2rngelhafen.}

Mian nennt bei bent 2rigelfafen a b bie Eptke, a ben Bart, b c ben Begen, c d ben Edyentel, d ben אapf (Jig. 20). Tie Jaten weroen ats bejtem Etahl gemadyt, und jo gehärtet, baj jie jefgr idswer brechen uno jidy nicht biegen lajien. Ia won biejer $B e=$

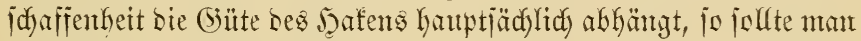

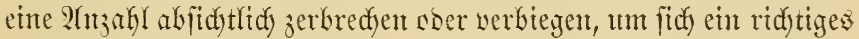

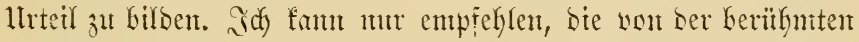

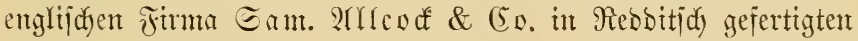
Daten, weldye jth ben bejten gefören, bie es giebt, bei biejer Friffutg als Multer ju nefymen, utb nit anderen jut vergleidjent. Daten, bie fid werbiegen lafjen, soer leidgt jerbrechen, jollte man nidyt gebraudjent. Der 2hrgelfafen jolf leidst eimbringen, unt Dant idwer wieber lasgemadyt werben fünten. (Sr bringt um

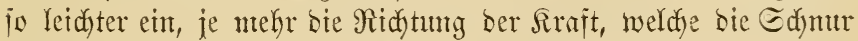
überträgt, ber Ridduturg Der Epitze fich nähert. Went wir bie Epibe a an einen barten biegentand anjeben unb sann an ber Edynur jieken, an weldyer ber Jaafen bejejtigt ijt, jo nimmt bie

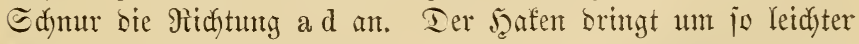
cin, je flenter ber isintel d a $f=\gamma^{\prime}$ ift. Den Sinfel $\gamma^{\prime}$ ganz

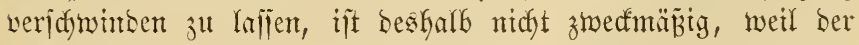

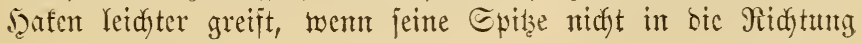


Der Rraft fällt. Man mennt beshalb $\gamma$ ben i, däoliden winfer

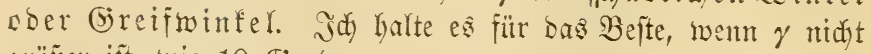
grëвеer î̃t, wic 10 Grab.

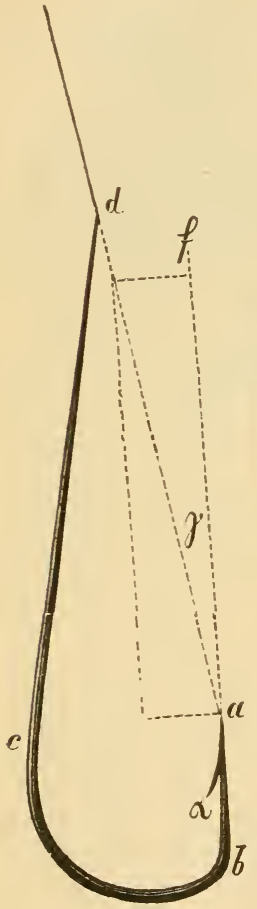

テig. 20.

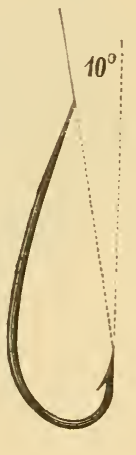

ฬig. 21.

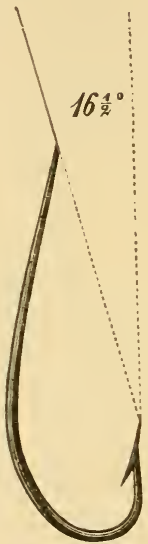

Sproat-bend. Tig. 22. Limerick.

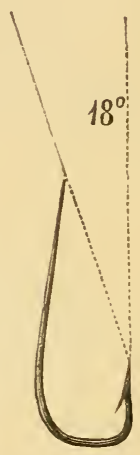

₹ig. 23.

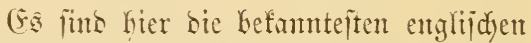
इafentormen mit ?ngabe ses idü̈rlidyen Minfels abgebilicet (jig. 21-28).

Der Sproat-bend (Fig. 21) Gat mach nteiner 2rnitht cine zu furze Spitze, Deshark

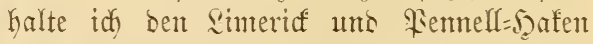
(Jig. 22, 23) ï̈r bie keiten. Ia j̈d für Situmfijdyerei ein Dafen mit runbem Bogen ant beiten eigut, is bat idy Derm Eam. 2racofi, emen round bend=\$aten mit langer Epise mo einem jđäblidben Binfel zu foultuteren, ber nidyt gröber wie 100 ît

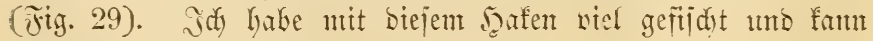
ibn beiten cmpjeblen. 
Mem Der Winfel $\gamma$ flein und bie Epitze lang ift, jo wirft Ler 3 ug an ber Edjur mefy bafin, baj ber Jaaten einbringt,

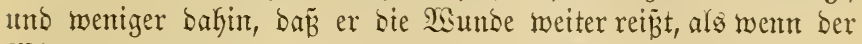
Winfel $\gamma$ grë̈éer ift. Sït nämlidy a bießugfrajt, jo ift nady bem Saķ von bem Faralfelegramm ber Rräfte a $\cos \gamma$ bie Rraft,

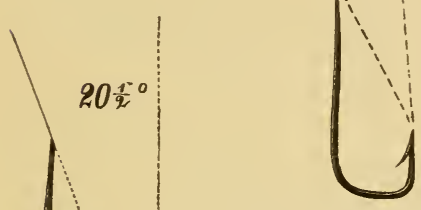

Jig. 25. Sneck-bend.
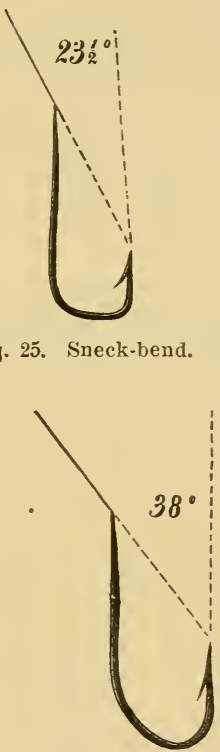

Fig. 27. Kendal.

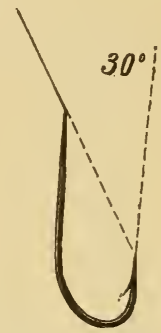

Fig. 26. Kirby.

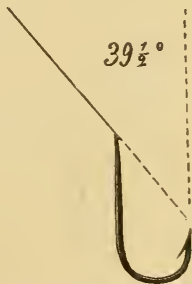

Fig. 28. Englijojer Brötzenkafen.

weldye ben Jafen einoringen läpt, und a sin $\gamma$ bie Rrait, weldje bejtrebt ijt, bie Wumbe aujajüyntocn (i. Jig. 20). Je jeiner ber Draft, itm io reidyter jumeibet er in bas Fleijd. Deshalb jitzt ein Jुafen um jo fejter, je jtärfer Der Iraft, je länger bie Epiże (i. Fig. 30) und je fleiner ber Sinfel $\gamma$ ijt. Die übrige Biejtalt bes Şafens ijt gleidgültig. In Fig. 31 entipridyt d a fe bem Fennell, 
$\mathrm{d}$ b f e bem Round-bend, $\mathrm{d}$ c fe bent Qimeriff. Da alle srei biejelbe Epitze unb benjelben joüblichen Winfel haben, io oringen jie alle

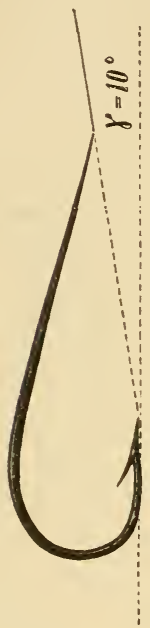

Jig. 29.

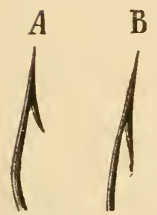

Fig. 30.

Fig. 32.

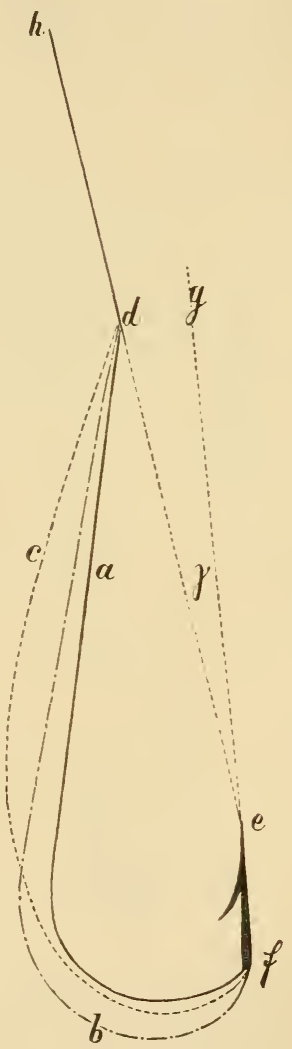

Fig. 31 . brei glcidy lecidyt ein, tuto hajten gleidy feit. llnt bie Befeiti= gutug ser હedutt am Taten jut erleidytern, fins mandedgaten am Ropi mit einem \$lätt= den, einer Seje ober citem Piabelifhr wer= jeketr.

Der Maifliegen= Gaten won Francis (i. Titi. 32) ijt jwedf=

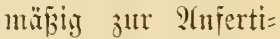
gutug fümtlidyer silic= gen, sie fdymmen follen, weil er jefr leidyt ijt.

Seit eunigen iar)= ren fonmen in (Eitg= lant, worjugsmeije bet ber Jijugerei mit fïmit= lidjen Fliegen, mehr umb mekr geäbrte 2ntgelfaten in (5e= brautd. Diejelben Gaten vor ben früber

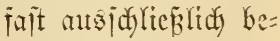
untrtert bic Borteile, saj jie Yeidyter uns

jenteller an Sutjaben bejejtigt werben fümen und baj bie Fliege

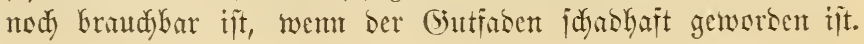


Das Sefr wiro entweber, ähnlid wie bei ber Mä̆huabel, Durdy ben Edyutel bes bafens geịlagen, soer baburdh hergeitellt, baj ber Edjentel ant (Ense umgebogen wirb.

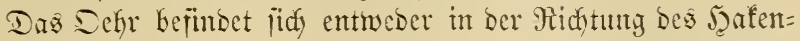
idjentels (jig. 33), ader es ijt nad) imten gebegen (Fig. 34,

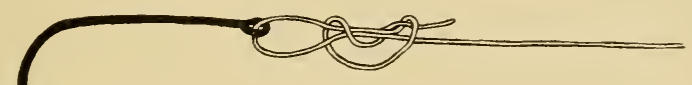

ริig. 33.

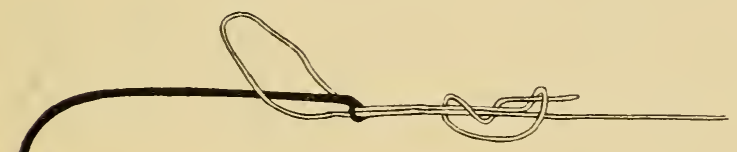

Fig. 31. Finnel's 5atent.

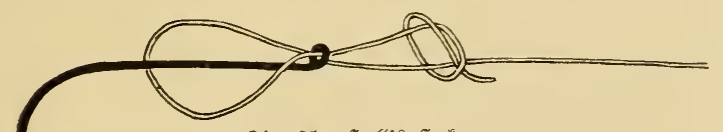

Fig. 3.. Satll': 5ูafen.

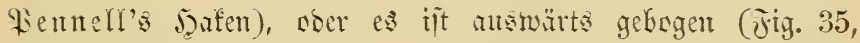
गुalla jaftert).

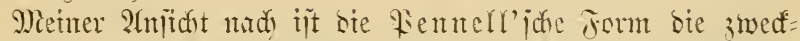

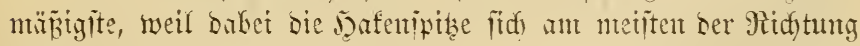
ber 2tngelidnut nähert, went lekstere angezogen wirb. Taburd

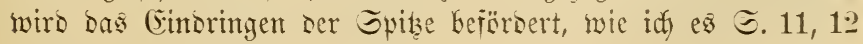
madigemiejen habe. 


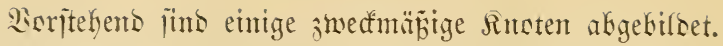

in Fig. 34 unt 35 ijt ber Jithen zweimal surd bas Sefr gejtedt und barauj surd einen einfadyen sber soppelten Sincten gejd)lojien; mit anteren 2 serten, es ijt juterît ein P(nferitid unto Laratif ein einjadier soer Sofpelter Inaten gemadyt. in Jig. 33 ift ber (butfaden mur eimmal surd) bas Sefr geitedt mo sann mit eintem Tappelfnoten rerjeken worben. Saenn bas Sehr io

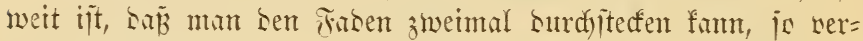
bient ber 2Anferitid wegen gröberer நaltbarfeit unb Eteiffeit ben Barzug. Iurdy Bujpizen bes Gutjasens mit Edyeere soer Mejier wirb es leiduter, ifn surdy bas Sehr jut jteden.

Eelgitredento mui der Dafen io flein jein, baj er in Maul

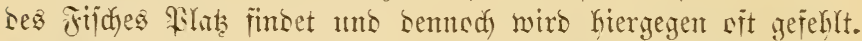

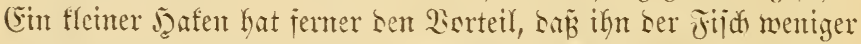
Yeidjt kemerft, bagegen ijt ein grejer saten bejier in jtambe, sen Fijd feituthaltent.

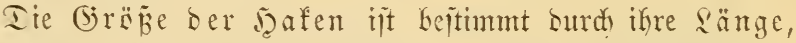
(Entienung bes Rapies d nom Rogen (b c Fig. 20) uno Breite (bie Entfermung ser Epitze a rom Echentel b c).

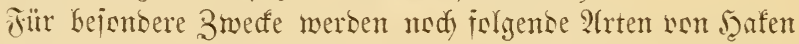
Gemuts:

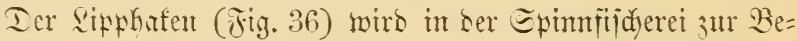
fejtigumy bes Rëserfijdyes gebraudyt.

Ier Iofpelfaten mb ber Iriangel io wie Fientells = Enshafen (Jig. 37) fincen efenfalls bei ber Epimangel Ber= wentung.

Breite ber Dafen, von ber Epibe jum Edyenfel ge= melien, fïr ben Fantig bon:

Forelle 3-5-7 mm. Tëfel $4-9 \mathrm{~mm}$.

Eeeforelle 1. Mecriarelle $4-9 \mathrm{~mm}$. Däjeling : $4 \mathrm{~mm}$. Yeja)e $3-4 \mathrm{~mm}$. llúfelei 2-3 mm. Dud ßapfen $4-9 \mathrm{~mm}$. Etint $5 \mathrm{~mm}$. Prïbe $4-5 \mathrm{~mm}$. 
Bhei $5-6 \mathrm{~mm}$.

(Siinter $5 \mathrm{~mm}$.

?llano $5 \mathrm{~mm}$.

(Situndling $4 \mathrm{~mm}$.

Barbe 5-10 mm.

Sarpien : $-7 \mathrm{~mm}$.
Ed)let $6 \mathrm{~mm}$.

Barid 5-8 mm.

Raulbaridy $5 \mathrm{~mm}$.

Jed t 7-23 mm.

Slal $5-7 \mathrm{~mm}$.

silutioer $5-6 \mathrm{~mm}$.

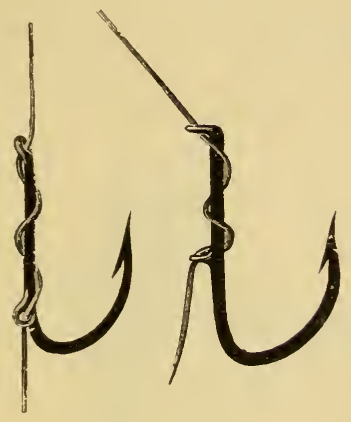

Jig. 36. Eipphaten.

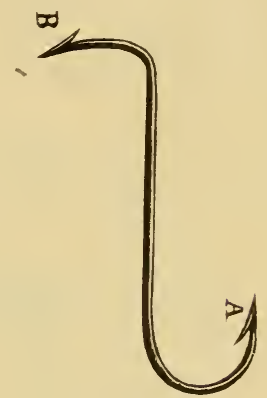

Fig. 37. Hennell's Enokaten.

\section{Die 2rngelínur.}

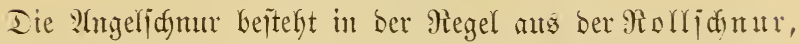

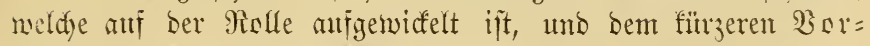
fad, an weldyent jidy Jaten, Eenfer, SBirbel it. j. m. befindent.

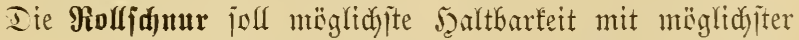
jeinteit verbinoen und feine Picigung haben, fid ju werbreken Lber ju werjürzen. afle bieje (Eigenjdyaften bejust bie aus 5-8 Eträhnen gef̃lodsterte jeibente Edymur int rolffommeniten Mañe. Turdy bas Firnifien wirb bie Edyut haltbarer und iteifer, ite gleitet aber nidyt jo leidyt burdy bie Rutemringe, wie sie ungefimiñte Edyutr. Man benutzt beshalb fïr bie 2tngel=

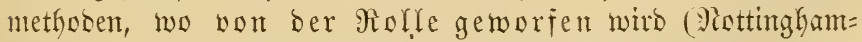

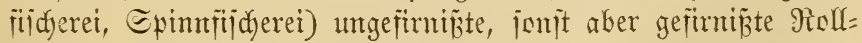

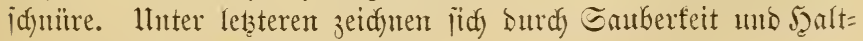

v. ๖. $\mathfrak{B}$ orne, 2rngerfifderei. 3. 2ufl. 
barfeit bie amerifantiden jogenumten emerald lines bejonocrs आn, bie aud water-proof braided silk lines gentunt werben.

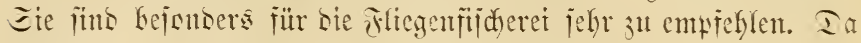
iid) mit ber ìliegentute cine etmas iteife Edyur beficr wirt, is

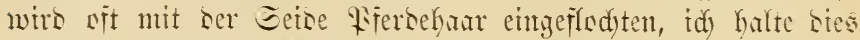

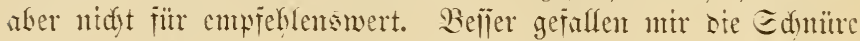

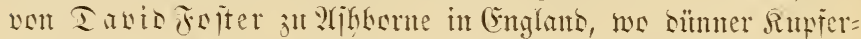

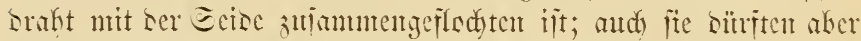
won ber emerald line übertreffen werben. Gute Éduntre jertigt (atd) S. Eto of in 14 m.

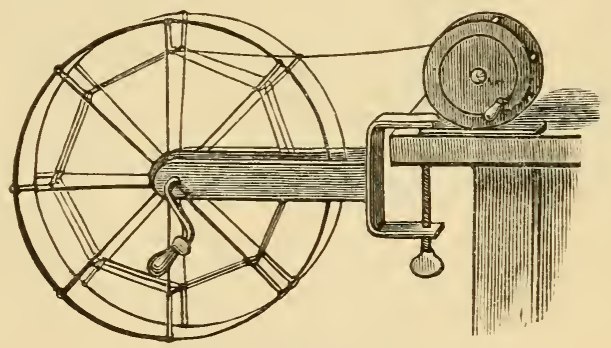

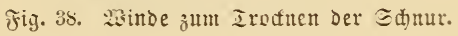

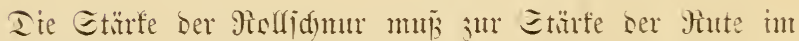

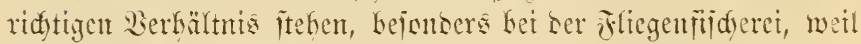
mant mur bann gut uns weit werien tant. (Es ijt am bejten, bic

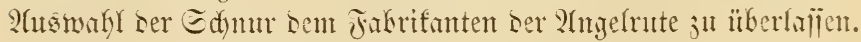

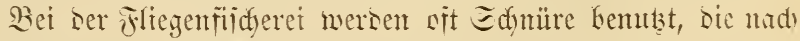
ben Enben 3 allmählidy bünner wersen, weil jie sann letditer

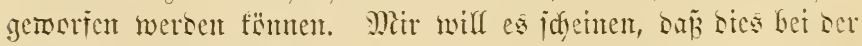

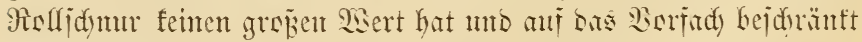
mersen fant.

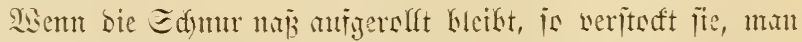

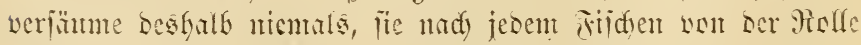

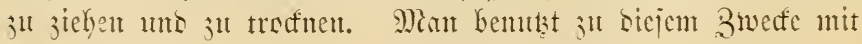




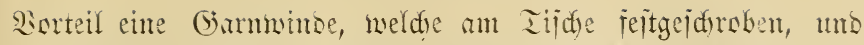

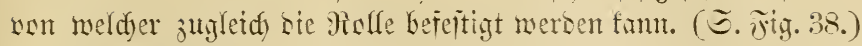

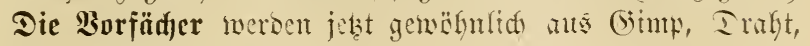
(jut woer Piferbefan gemadf.

Der (S) imp ift mit I ruht ïberiponnente Eecise, er miro zum Fangen wen Fildten be= mutst, bie int itutioe jüno, genobulidye Edymu ju 3 er=

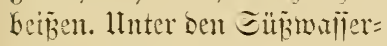
fijthen fom bies nur Der Sectht. Iic veridiebenen Etürfen, wie jie bie jefre re= unmmierte Fabrif ber Meeĩrs. Kenning, Sittle Britain, ¿onson, liefert, jün in jig. 39 abgebilbet. Mian betommt bort (bimp von jacer Keliebi= gon \&̇änge.

Rupfer= ober Mejiug= staft wirt in italien jefr riel zu Epimangeln fomtąt und ijt baju jefr jwedmä̈rig. I ie Traftrabrif wen trie= srids Rlebes in Piümberig fertigt gebrehte Edumire ats 9 bis 12 feinen Irïften, weldye ebenjo biegian, aber wiel Galtbarer fins alo bimts,

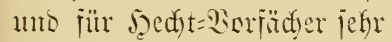
000 00

\section{0}
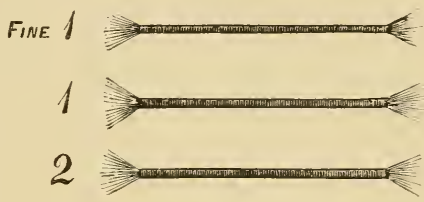

3

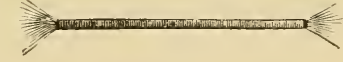

Fig. 39. Gimp.

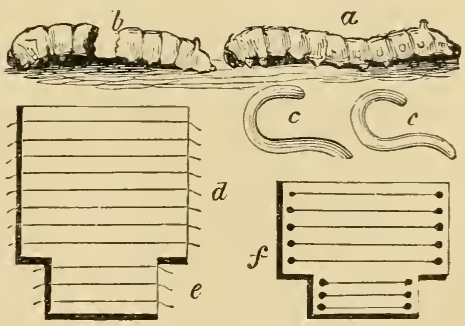

Fis. 40. Gutiasen=Bereitung.

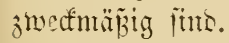

Ier (j)t, Ecisemmumbraft, Gut, Poil ofer Crin de Florence, Cava di Seta bejtely aus Derjelfen Eubitanz, aus weldyer bie Pante den Seibenjasen ipinnt; biejelbe wilo aber 
mu ju chten 0,3-0,9 m langen jaben ansgejogen, währens sie Manpe sarans mefrere $1000 \mathrm{~m}$ ifunt. Sein Etofi weremigt in

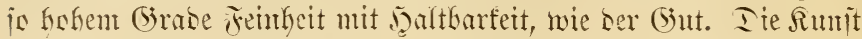

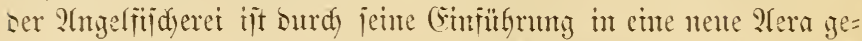

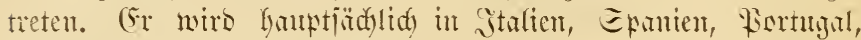

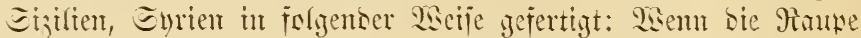

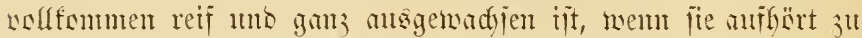

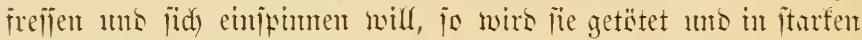

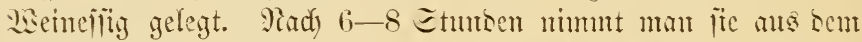

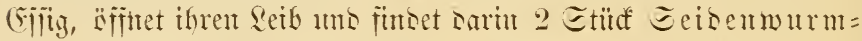
sarm won ungleidyer Gröbe (Fig. 40 a, b, c). Serjelbe wirs an beise (Ensen gejajt und angejngen. Siejen rohen (5)ut=jasen

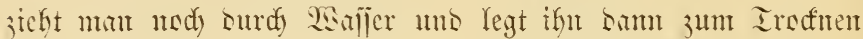
aus $(d, e, f)$. Ilu ifu von ser gelben jant ju bejreien uns ju

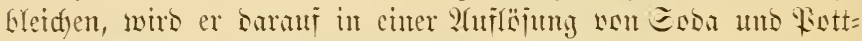

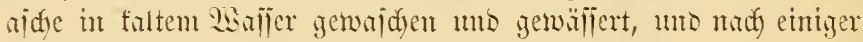

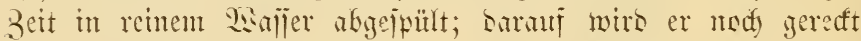
uno gebefut, inden man bas eine (5nbe jwijhen ocn Bäfnen Gält, Das mbere mit sen Fingern ergreift. Fnolid) werben sie

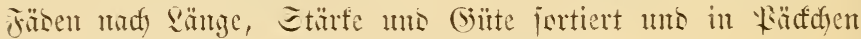
Lon je 100 gäsen in ben Jeanbel gebradt.

(Eine worjüglidye, billige Dezugsquelfe, sie surd) bie inter=

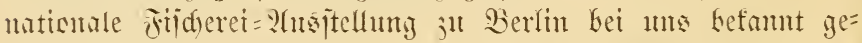

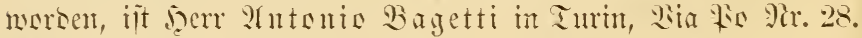
Eofr gute Bezugsumellen jins and ju Miurcia in Epanten, wo iid) mebrere Jiabritinten Gefinsen.

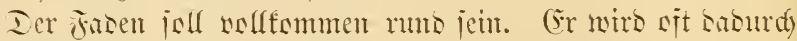

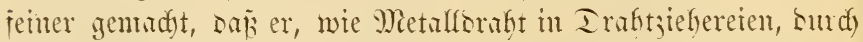
eine mit verjdjeben jeinen P̈̈d)en verjehene Metallflatte gejngen mirs, jeme jaltbarteit leiset aber jebr sasmds, mo ebento fenter

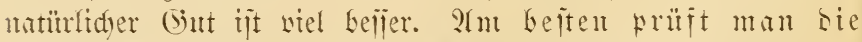

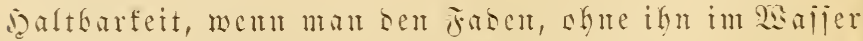

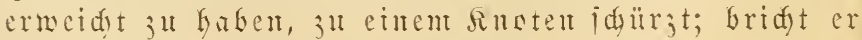
sabei nidit, io itit er gut. 


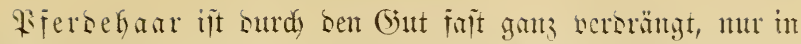

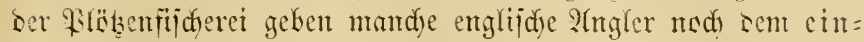
zelnen Rifordohan ben Borjug.

Ctarke Borfä̈ber werben aus mehreren Gutfaben ober aus

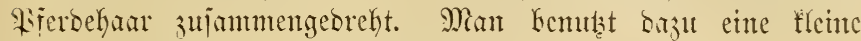
Epinnmajuine, weldye man in ben

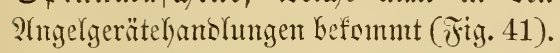
Tiejelbe wiro mit ber Edraube is in

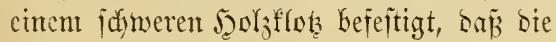
Surbel nady sben, bie brei Jaten nath unten geridytet fints. Tarmul bindet man bie Jaare in zowe coer brei gleidy itarfe Eträhnen зulammen, befejtigt bas cine Enbe jeber Eträ̈hne in ben Jaaten ber Exinmmajome, fnïpit bic anderen (Enden anjummen, uno bängt unten ein (biendidyt Gincin, uto zwar pro 18-20 \$iferdebanre Fig. 41. Epinmuajine. ein Pifun. Man breht mu bie Rurbel

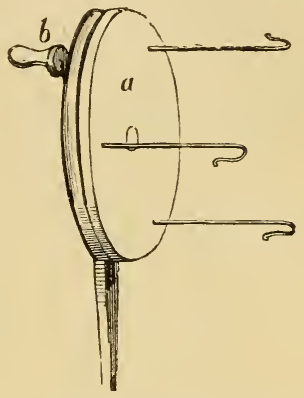

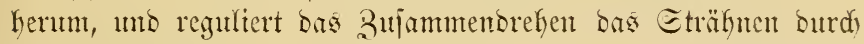
3wijdenthalten ber Finger ber linfen Jaanb. Ilm bie Eträfunen ganz julammenoreben ju fömen, hängt man worker in bie Jaten Det Majaje fleine beneglidye Saten aus Mejïngoraht. WSenn man mehrere Ednüre jujammenfnüpit, jo werben jie abwedjelnts

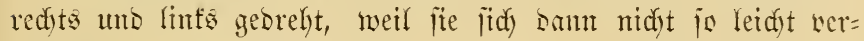
idjützent.

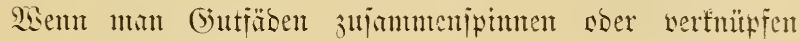

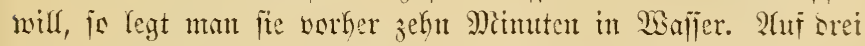
Fräben won ftarfem Ealntengut redyet man ein Gervidyt won

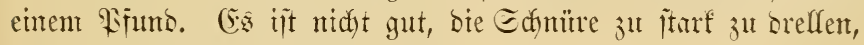
iie werben badurd wentiger haltbar.

(Das Sorfad) beiteft gewöhnlidi aus zwei Teilen, welde

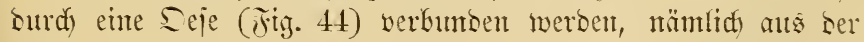
SEmfijnur oder bem Fuj (Casting Line, Sid Strap) uns ben Sorjdilag (Snooding), welder cinen ober meftrete 


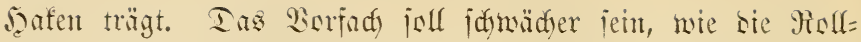

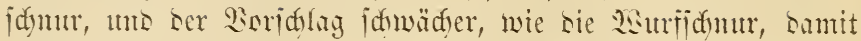
mëgliaft wentig werleren gcht, wemt bie Ecturur reipt.

\section{Sie Knoten.}

Ias Binten ber maiten Rnoten wirb mit Seritelfung einer Budt (jig. 42) ober sinter Edyleife (jig. 43) begnuten.

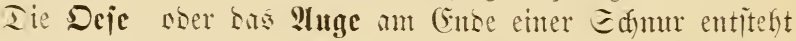

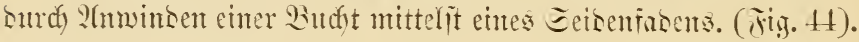

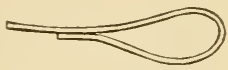

Fig. 42. Bud)t.

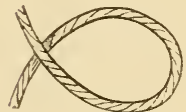

jig. 43. Edileije.

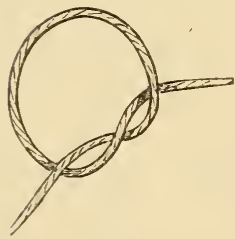

§ig. 16. Einjadjer sinoten.

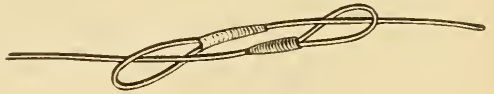

Tig. 4. Eeje.

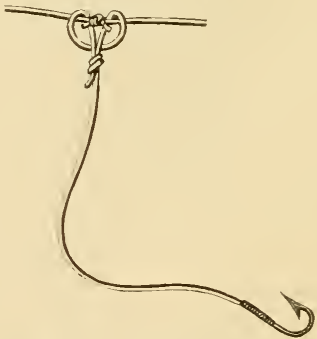

Fig. 45. Bejeitigung einer Ђängefliege am 3oriad.

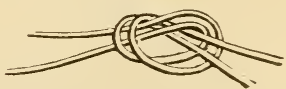

Fig. 47. 2acrbintong gweier Sdnüre surdi einfaben sinoten.

Iurdy Sejen fümncn jwei Edfnïte werbunden wersen (Fig. 4), insem man bie eine Edymt surdy bie Deje ber anderen Edumtr itedft.

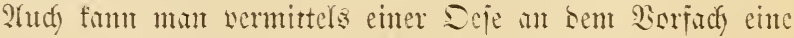


நängejdinge, woer an bem \&aternojter einen Dafen bejeitigen. (jig. 45 ).

Ier cinjadc Sinoteu ober Waifertunten soer Iteberband= fnoten (Fig. 46) ift eine gute Berbintsung jweier Edyntre, er

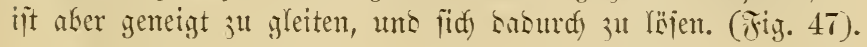

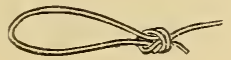

jig. 4..

Seie surdy einjadien sinoten gebunbert.

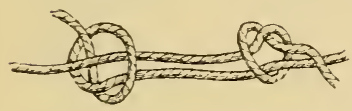

Fig. 50. Englijuer sinoten.

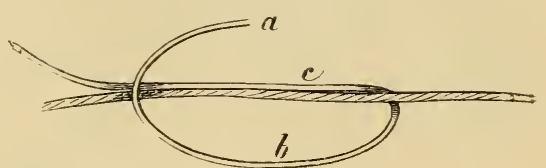

jig. 19. Binten des engliidyen șntens.

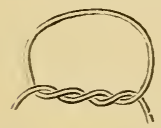

Tig. 52. Iopperter sinoten.

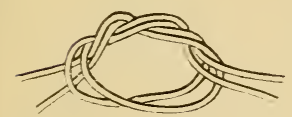

Jig. 53. Ioppelter sinoten.

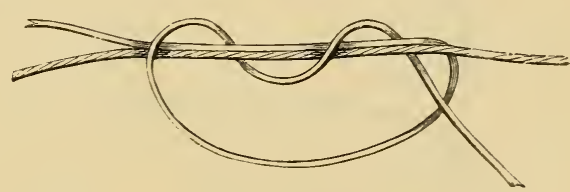

Fig. 54. Toppelter englifider snoten.
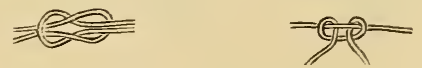

Fig. 55.

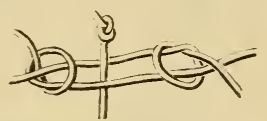

Jig. 51. Englijder sinote!t mit $\mathfrak{s u ̈ n g e f l i e g e . ~}$ 


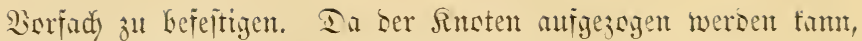
fo fann bie 万̧ängeffiege gewedjelt werben. (ङ. Jig. 51).

Der Doppelte finoteu soer Doppelte $\mathfrak{G a j i}$ erfnoten (îtig. 5:2) ift swar biffer, wie ser cinfache Rnoten, gleitet aker garnidst, ift

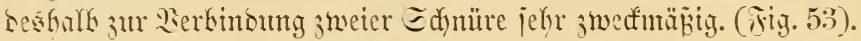

Ser Doppelte englijd) Sinoten nber Doppelte Fijdiferfnoten iit ähnlid bem einfadyen, Geiteht aher ans imei soppelten Busten,

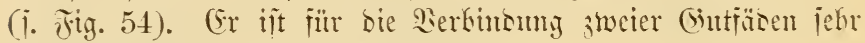
ăbefmäв̄iq, weil er niemalo gleitet.

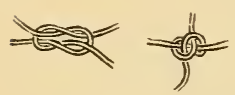

xig. 57. Fig. $5 x$. isalider sinoten.

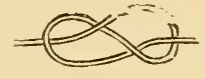

Fig. 59. r̂ämijđer sinoten.

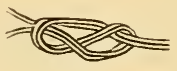

Tig. 60. toten.

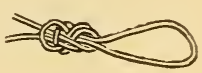

Fig. 61.

F̂ämijđer sutten.

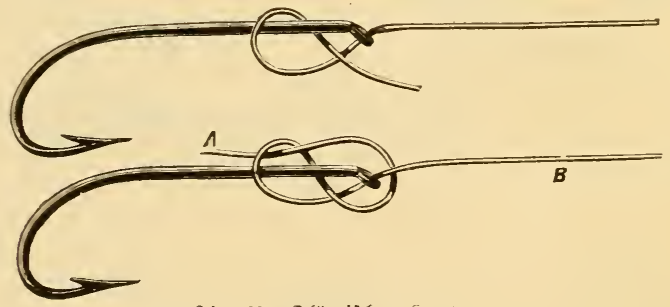

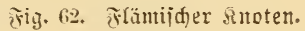

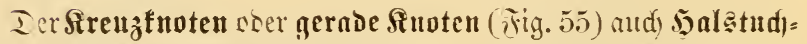

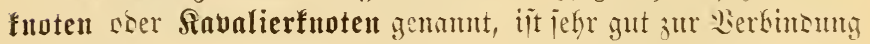

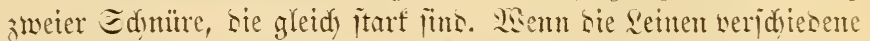
Etärfe faken, is nimmt ber Krengfnoten bie in Jig. 56 abgefilocte

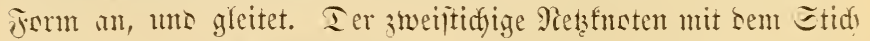
won sken ijt cin Rreujtheten.

Ior jaljuge soer Gropimutterfuoten (Jig. 5i) ijt nidjt gut,

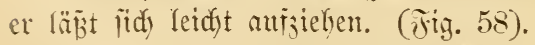

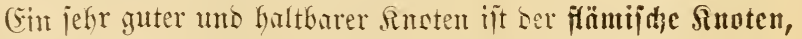

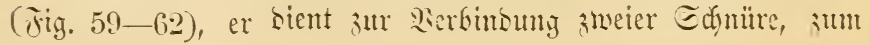


Soeritellen einer Deje an (Enbe einer Єdum, uns zur Berejtigung beş geöhrten 2trigelfatens.

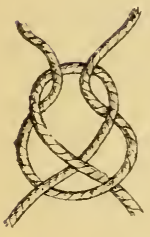

Æig. 63. Weberfnoten.
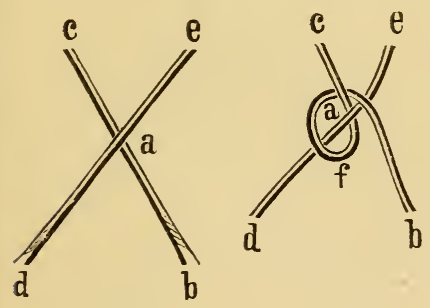

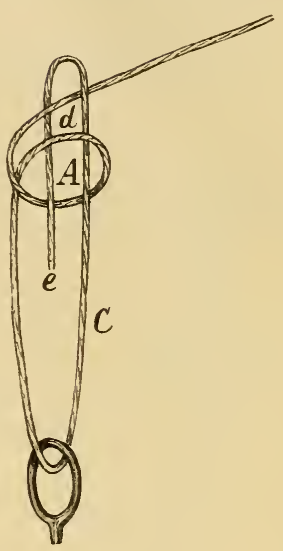

テjig. 65.

iseberfnoten.

Fig. 66. Weberfnoten.

Der $\mathfrak{B e b e r f n o t e n ~ ( F i g . ~ 6 3 , ~ 6 4 ) ~ i j t ~ D e r ~ S n o t e n , ~ w e l c h e r ~ b i e ~}$

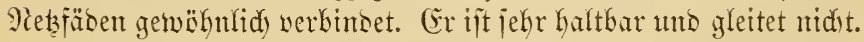
Fig. 65 zeigt bie Befeitigung eines Eeiles nou cintent ent=

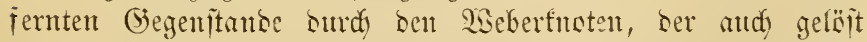
werben famm, went ber Gegenjtano unzugänglidy it, 3. B. Hei 5eadinatijer. 
Bei sent Etriffen won Vieţen wersen nen èngelegte Fäben ant bejten surdy ben

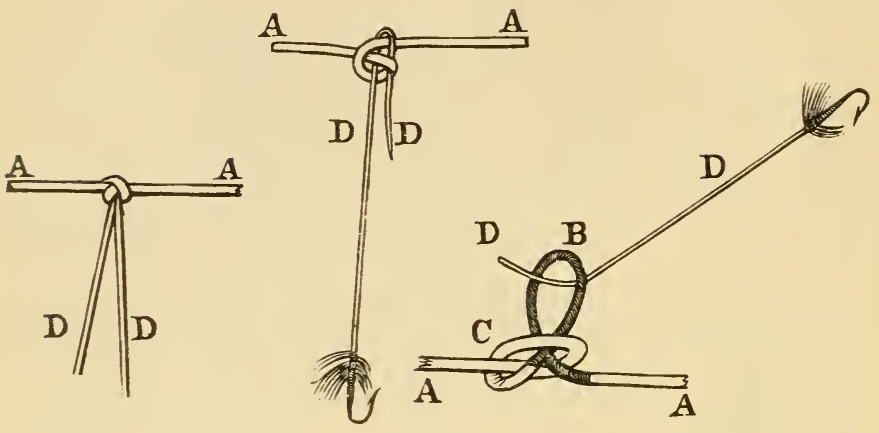

gig. 17. mieterfnote::

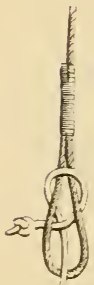

Titg. 68. meberfnoten.
Jig. 69. Geidleiiter sieberfnoten.

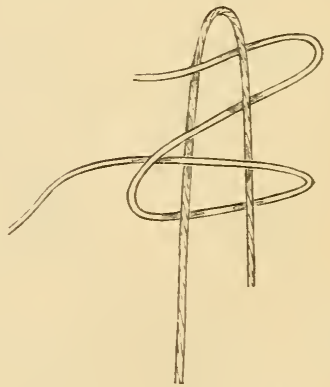

Fig. 70. (Beid)leifter iseberfnoten.

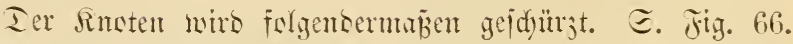
Buerit werben sie beisen Ecfnüre a b uno a d gefrenjt, Jig. 66. Inum bilset man mit sem unteren Teil ser Edumt a b sie Ed)leife af Jig. 66, som legt man bie Edjleife a f nady g him nern über, Jig. 66, unb jioht ben Finen e surch bie Edyleife $\mathrm{g} f$, 
Fig. 66; julebt fajt man bie brei Fäben d c e jujummen uno jieht ben Rusten mit jaben b jul.

Iurd) ben Weeberfnsten befejtigt man einte Dängefliege ant Dem Fliegentworfad, i. Fig. 67, coer einen פaten am Raaternojter, i. Jij. 68.

Iurd) ben geidleiften Weberfnoten, ber leidjt aut=

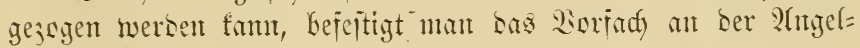
id)mur. Fig. 69, 70.
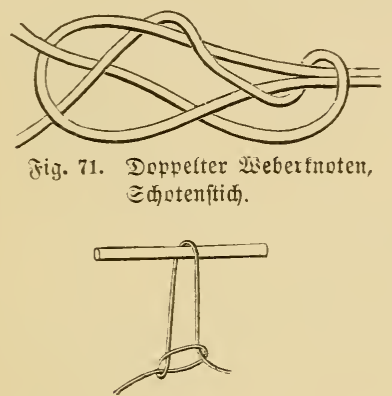

Fig. 73. Galeerenfnoten.

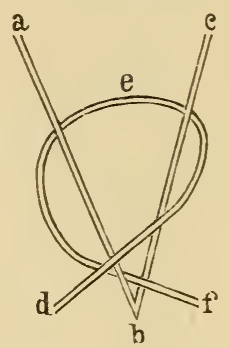

Fig. 72. Toppelter weber= froten, Sø̆otenitid.

Ier soppelte Weberfnoten sber Edjotenjtid) (Jig. 71) ift

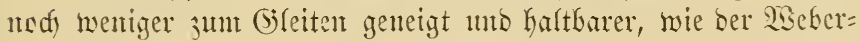
fntoten.

Mian madyt juerjt ben einfadyen seberfineten, Fig. 7.2, uno

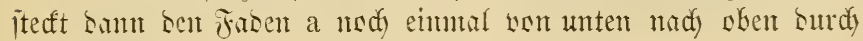
bie Edyleife e.

Ter Galeeren = noer Wärge = noex Edfinberfnoten bilset einte lanfentse Edyleife, bie zugejsgen werben fant; er wirb jur Serbintong zweier Edntitre bonutst. Fig. 73.

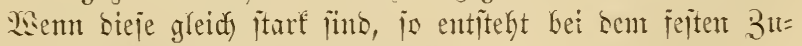
jiefent ein Seberfinoten. 厄. Jig. 67.

Mian fam vermittelit bes Galeerentustens ein Tau an einent jern liegenten Begenjtutse jo bejejtigen, baj bie Berbintung 
gelojit wersen famm, and) wem ber (begenitanto fiäter umjugäng= (id) ijt, 3. B. went ein Bont an eincm Ringe iejtgelegt ift, weld)er ipäterfin surdy bic Finth bebedt wirs. Fig. 74.

Der (jalecrenfneten wirs in jolgenser 些ije gemadut: Nian legt bie Edyur in cine Edyleife, uns zieht buth sieje sie Edmu in Jorm einer zmeiten Edileife hinourd).

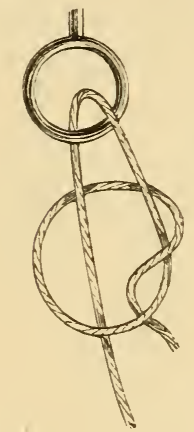

Fig. 74. Galecren= fnoten.

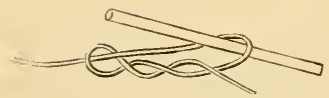

Fig. 77. 3immermannstnoten.

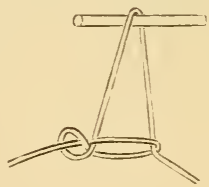

Fig̣. 75. Ioppelter Baleerenfnoten.

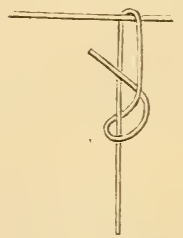

Fig. 7s. Syatber Bimmermanstnoten.

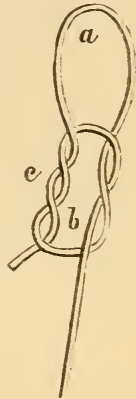

Vig. 76. Geidüryter

Ealeerentnoter.

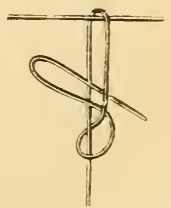

rig. 79. Gejuleijter halber 3immer= manistnoter.

Der Doppelte Balcerenfnoten, Fig. 75, wito is gemadit: Man legt bie Edumur in eine Ed)leife, breht bieje cimmil um iid) jelbit herum, uno jieht bann bie Edymur in Form ciner

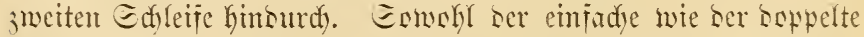
Galecrenfneten bilben gute Berbinoungen einer Sängeidituge mit bem Borfach, noer cutes Drafens mit sem Fiatemoiter. Der 
in jig. 76 afgebilisete finoten entitefit, menn man bie jweite Edfleife zweimal ourd) bie erite fintourdyieft.

Ier Bimmermannêfnoten cocr Dentide Sinoten sber bie Sajue (jig. 77) ijt eine iefr gute Serbinoung sümerer Edyüre

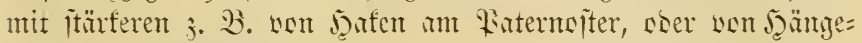
fliegen an Barfiadse.

Ier halbe Bimmermannefnoten, ungejdyeift (jig. 78) unb

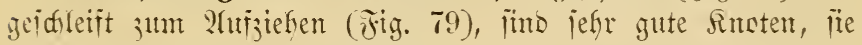
wersen gefrautd, um Dängefliegen an Borfad) sber Dafen am Baternoiter ju befeitigen.
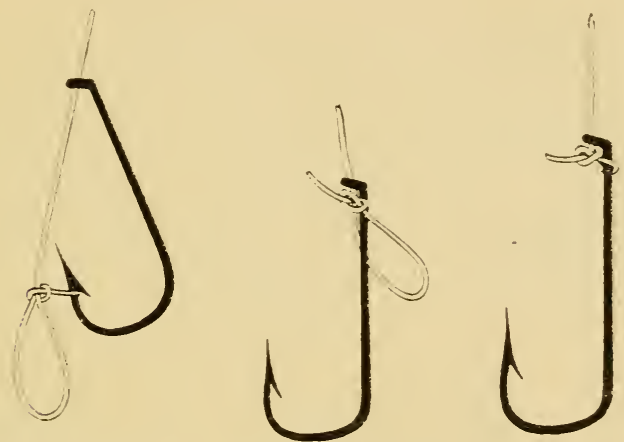

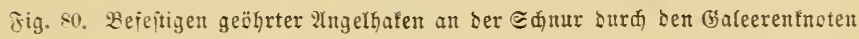

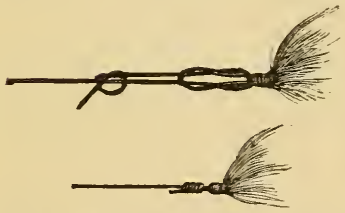

Fig. s1. Beiejtigen geöhrter $\mathfrak{A}$ ngel= bafen art ber Єđfnur.

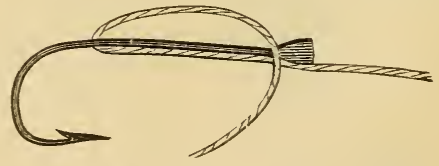

Fig. 82. Bejeitigen Der Wlättḑen=Sgafen alt ber Edumtr.

Beieftigung ber 2ugchaten an Der Edutr.

(b)ëhrte Angelfaten werben surdy ben Galeerenfusten an ber Edrum bejejtigt, wie es miere jig. 80, 81 barjtellt. 
Sie 2hgelfafen, welde mit Flättaen werjeben fün, wic folde in ber Fegel bei bom 2htgeln im Meere gefraud werben, befejtigt man is, wie wir es in jig. 82-84 abfilsen.

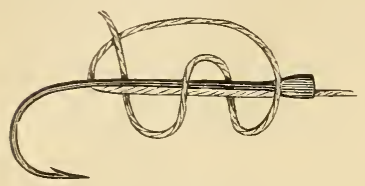

Nig. 83.

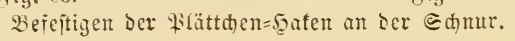

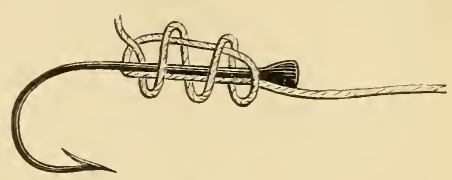

jig. 84.

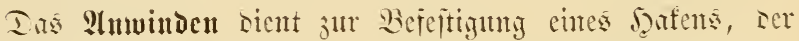
weser Sebr nody Shatte hat an Gitfinden, jum Befejtigen ser

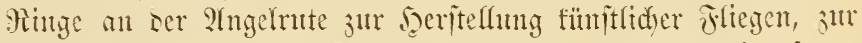
Reparatur zerbred)ener 2(ngelruten 1t. a. m. Der Eeisentiaben

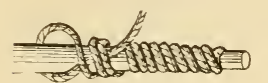

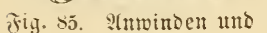
verborgener sinten.

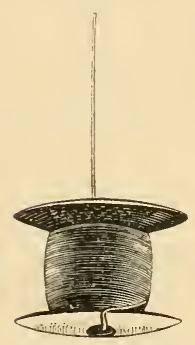

Fig. 86. *ntwintejente.

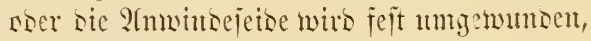
is saj jidy sie 2sinoungen berithren, aber nidit ilbersecten. vig. 85.

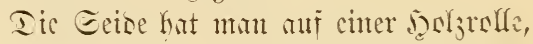
welde herabbängt, beint 2(nwinsen ben

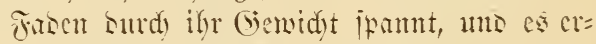
ermighlidut, sen Firoen madh Bebarf ju wer= längern. Fig. 86.

Ter berkergene Rnoten kilbet ben

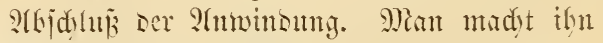
in werjofiesenter siseije.

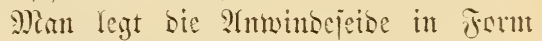

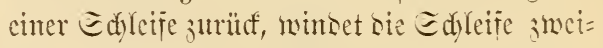
bis orcintal 1 m Den Deafen, zielyt fie fejt, 1 mo

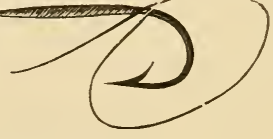

Fig. s7. Sierborgener snoten iduncibet bent Firoen ab. Fig. 87.

iit ser Gegen= itans is lanty, baj bie Edulcife nidut ïber= 


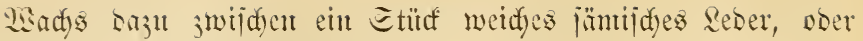
trägt es in flüifiger Form anf.

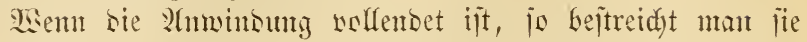
mit einem idneff trofutenten Firni

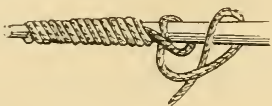

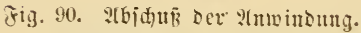

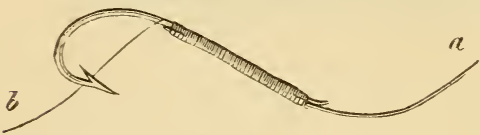

łig. 92.

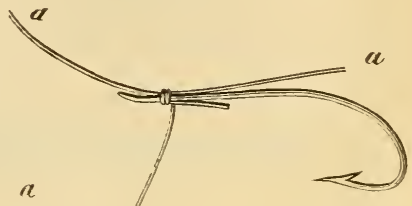

2tnwinten bes Şafent.

Fig. 91.

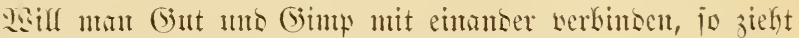
man io biel wou ber Drahtumbinsung bes (Bimps herunter, Daj man bie Esise umlegen uns an Den (5utiasen amwinton tam.

\section{Wadhs, firnifî, Leim.}

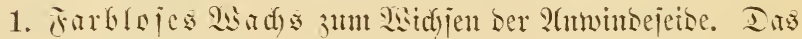

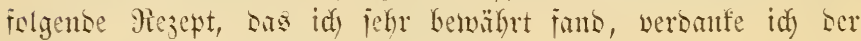
Mitteilumg cintes gewnisten gliegenfild)ers.

Colophonium Citrum wan (j)ke \& (É. wird ïber ge=

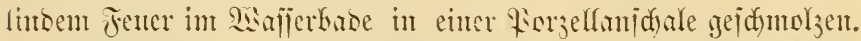

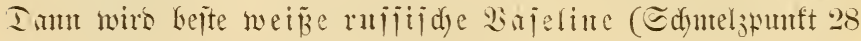

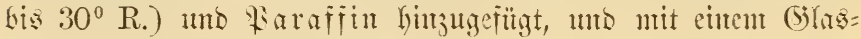
itake gut gemiid)t. WEenn bie Diijdung etwas erfaltet ift, io jebt man ein äterijdes Sel hinzu, Drs ans gleidjen Seilen

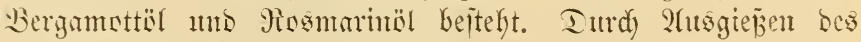

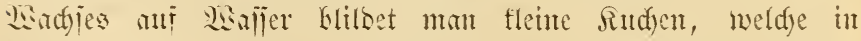

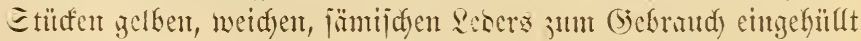
iwcrien. 
Ctala für sie särtegrabe ocs sades.

\begin{tabular}{|c|c|c|c|c|}
\hline & $\begin{array}{l}\text { Sidfo } \\
\text { phoutium }\end{array}$ & Bajeline & Baraffitut & $\begin{array}{c}\text { Hetherijides } \\
\text { Sel }\end{array}$ \\
\hline & 160 & $14 \quad-15,75$ & $6-6,75$ & 1,4 \\
\hline & 160 & $17,50-19,25$ & $7,5-8,25$ & 1,4 \\
\hline & 160 & $21 \quad-22,75$ & $9-9,75$ & 1,4 \\
\hline
\end{tabular}

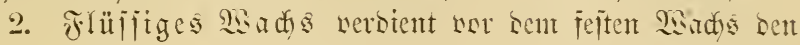

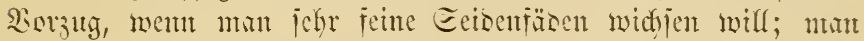
trügt es mit einem Giasitäbdyen anf bie Eecise auT.

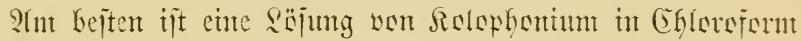
oder Yether.

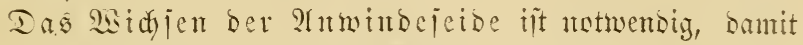
bie 2 sinsungen feit an einnor fleten.

3. Firnifje fütrerborgente Susten, tüntlide Jliegen, Anminoungen unb bgl. Tiejelben idfen jundf treftren.

a. Taspalthaf erit getroffitet, sam in (Ehleroform gelëft. (Dr. 2. Bettentorif).

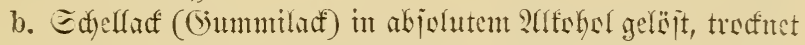
etmas Kangjant. (Dr. 21. Bettenowi.)

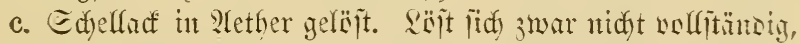

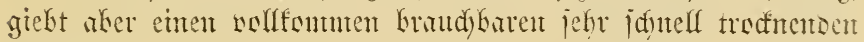
Firni亩.

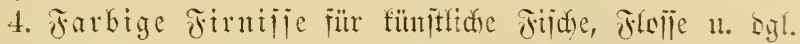

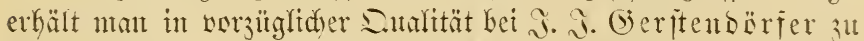
Fürth in Bayern, Blattmetulf=, Brofat= unt Bronjefarben= jabrit. Mian erkält golo, filber, gelb, wot, Glau, grün, Graum, fupfer tu wer:

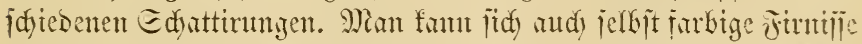

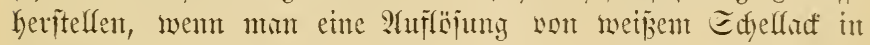

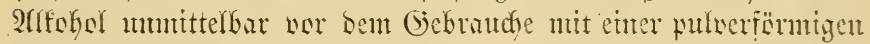
Farbe niicht.

Ias Firnifjen ser fertigen afumintutgen hat ben Buedf, dieje haltbar jul midhen.

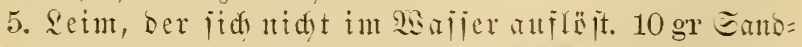

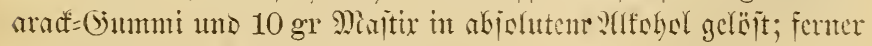

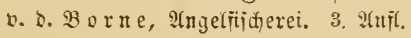




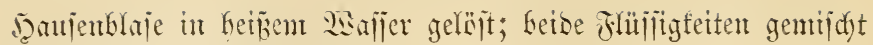
utb jofert heib augetragen.

\section{7. färben.}

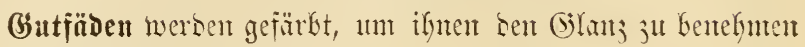
unto ite wentiger jidytbar ju madyen.

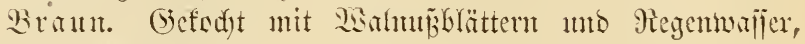
Rafiee noter Thee.

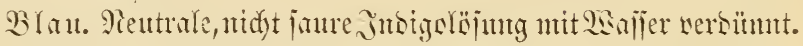

Brangran. Cinte Danto boll Blaubdy, $1 / 2$ Siter Begen= Wrijer, eint wentig Eosa mto Suffervitriol (jo grof wie einte Babure) werben getedyt, gut für(ft and wersüunte Tinte.

(S) rün. Fegenmajier lyatswarm $\left(32^{\circ} \mathrm{R}\right.$.) mit neutraler Jnsige=

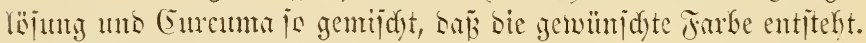

Jedern unb Ficlahanr. Ier Fliegenbinoer jollte jelkit im itumbe jein, jeine jesern unto Feljhate ju fürben, meil or jids

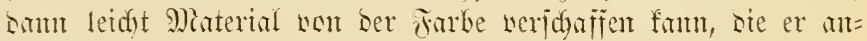

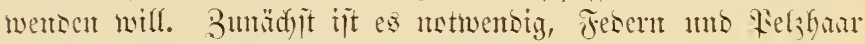
won Edyuts und jett wolfitünoig ju befreien. Iieje merben

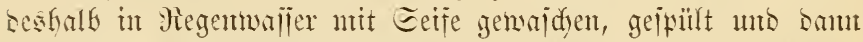
in 2 Siter Regentwaijer, ben ein halber Sheelëffel idwefeliane Shouterse jugejetst ijt, eine Etunte lang gefodyt unb bam ge=

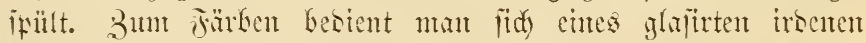

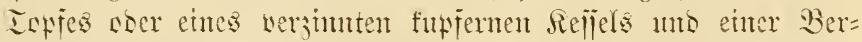

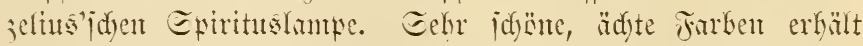

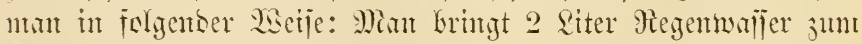

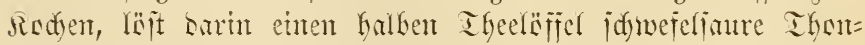
erbe uns bie Tarbe auf, ban thut man bie gerentigten Febern

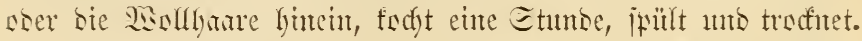
Bum (belbfärben nimmt man $1 / 2$ Theelöfel Flavin

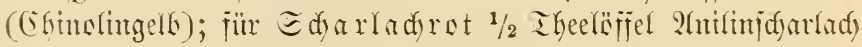

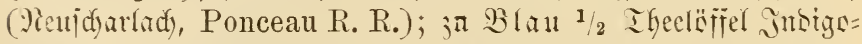

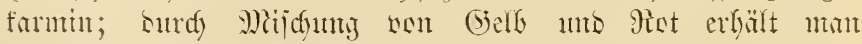

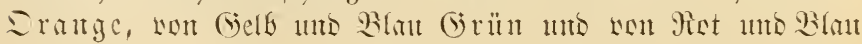




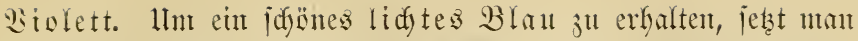

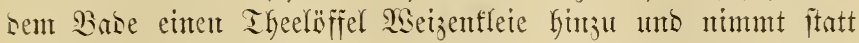

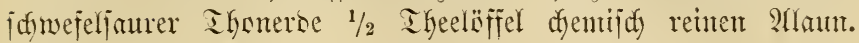

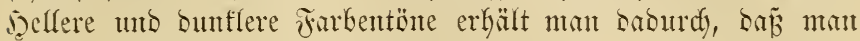
wentiger soer mefr Furbe bem Babe jujeł̧t.

Mgeitere Mitteilungen entgält bas Jantobud) ber Järberei wan Dr. Inton Epirf.

\section{8. floffe.}

Ias Jiñ ober der E(f)wimmer hat bent 3 wedf, sen $\Re$ ïber it einer bejtimmten Tieje jumbint= mento ju erkalten, uno surd) jeinte Bemegutug jut jeigen, went cin jiid ge=

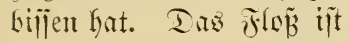
mul ba jweofmiñ̄ig, wo ser Röber ganz nahe ant Oinutbe itber cine weite Etrecfe fortipuminmen isll, roer wo ber Grunto

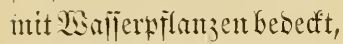
oser idylamming iit, unto bes: falb ser Räber brwon fern bleiben mun; ober wo mit lebentoen Röberfijd)en nad) Jedyten ober Barjdyen ge= aniglt wirs. Da bas jlo reidft bie Jijdye beun= rulfigt, namentlids im flacten Siajer uno an ber Sberfläche, io läp̆t man

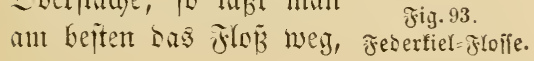

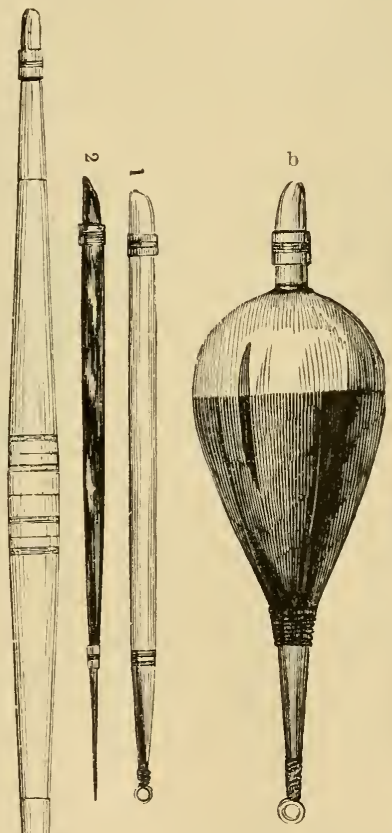

a

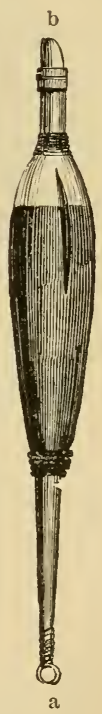

Jig. 94. sorf =\{rofife. 


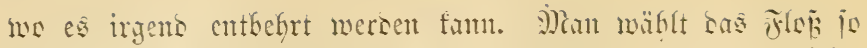

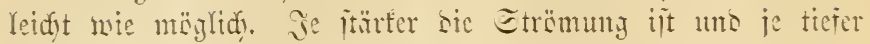

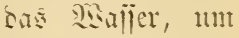
is grëser muj cs
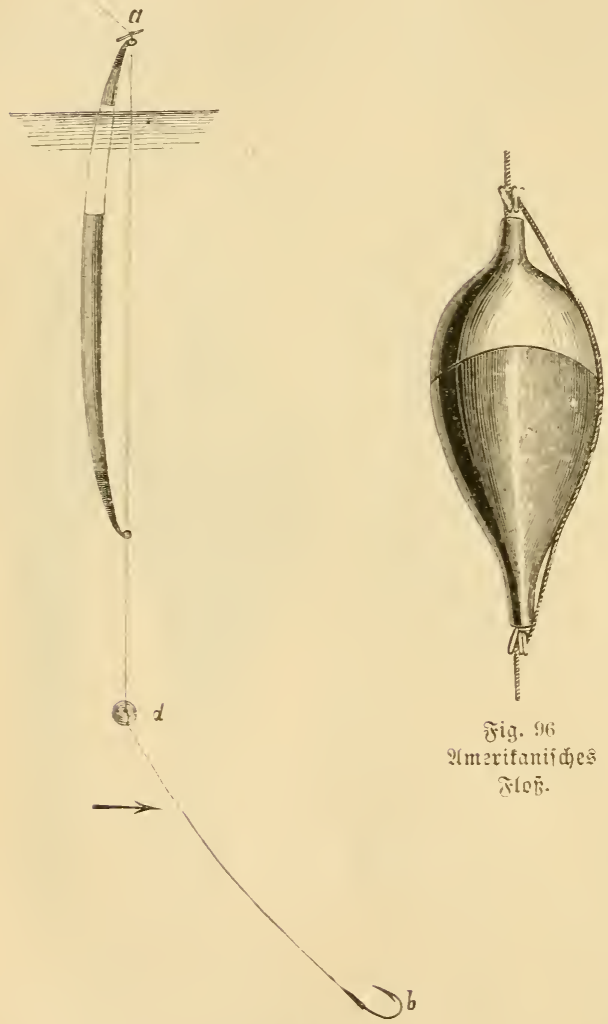

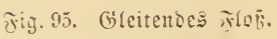

jeir; ekenjs jemit= siger sas Setter, rtm jo gröber jei

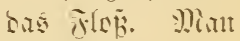
unterjudeiset:

Teserfielifolic non (5)̈nјe= Edymantielat (viti. 93, 1).

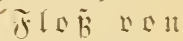
Etadialjamein= Goriten (Xig. 9:, $\because)$.

Ioffeltiel= fant (rig. 93, 3). (c) iोt aus mek)= reren Etüden won Teserifulen int= jammentigciest.

Batfolic (ïij. 91). ㄲan mäkle wentig ati= fallende kirben, tum bie vitiche is meniy wie ntëglid idenen all madfent. Berejtigung ses Tilaijes an set 2) ngeliditur:

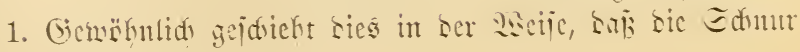




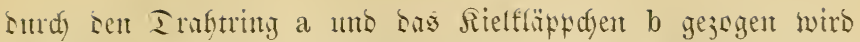

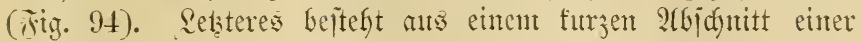
ieseripule.

2. Das gleitende Fró (Fig. 95) ijt an betbent (Ensent mit Iraftringen werjeken, burdh weldhe bie Edymtr gezogen itt.

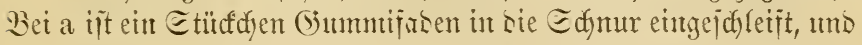

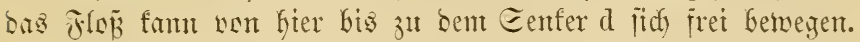

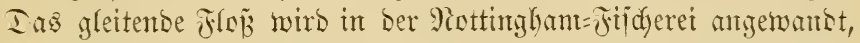

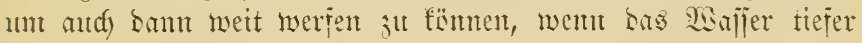
iit, wie bie 2lngelrute lanty ijt.

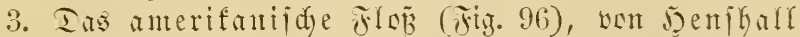
empioblen, iit an beiben (Snsen mit Epiralen aus itarfem Iraht werjefen und fam babutrd) leidyt überall an ber Edymur ange=

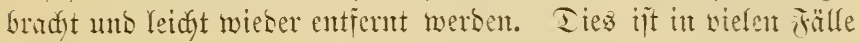
jehr zwectmiäig.

\section{Senker.}

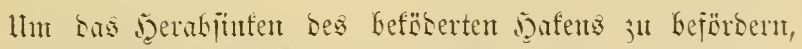
wirb sie Edyur oft mit Eenfent veriehen. Dierzu j̈̈to geeignet: Breifolie, bie man um bie Edynt mitfelt;

(bejpaltenes Edjot, bas man an biejelbe anflemmt;

Iurd) bohrte Bleigemidyte von fugelrunder ober länglidyer Form, bie mit einent Dooljpflocf bejeitigt werben;

Breigemidyte, bie an briben Enoen cine Iraftidyleife haben.

些irbel wou fintreidenter.

Eifnere, wo mant an einer Eeite bie Echnur, an ber miberen ben Dafen bejejtigt. Ier Senfer jei $25-30 \mathrm{~cm}$ wom Dafen entiernt. (Fine

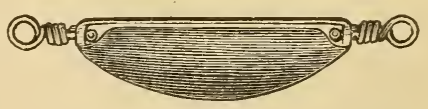

Jig. 97. Eenter.

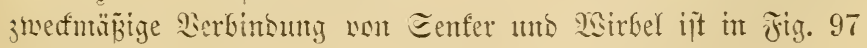
abgefiliset. 


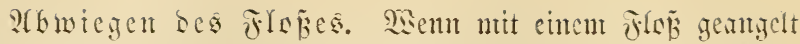

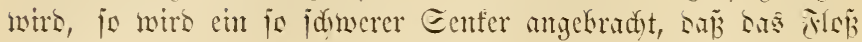

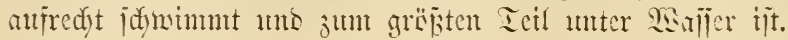

\section{Derfक̧iedene 2lngelgerätj̧̧aften. Kleidung.}

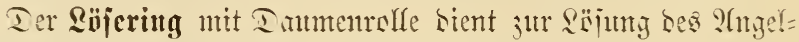

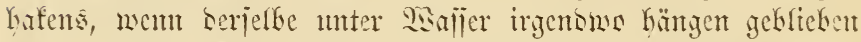

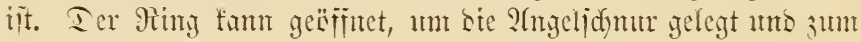

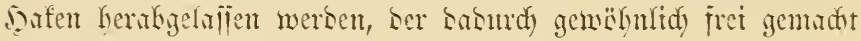
nirt. (ว̈ig. 9s).

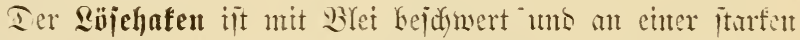

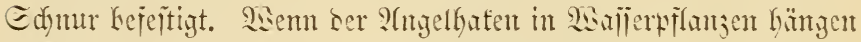

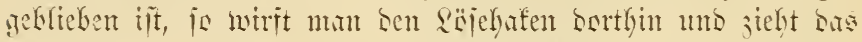

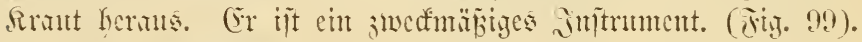

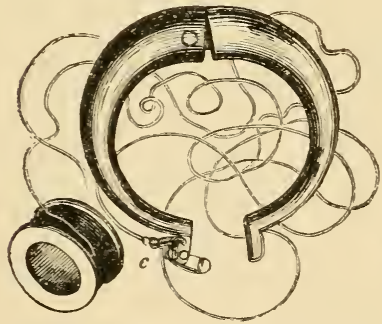

Fig 98. ¿äiering.

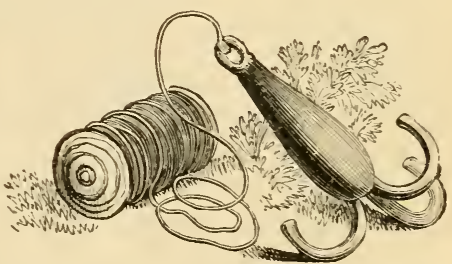

テrig. 99. S̈̈jehaten.

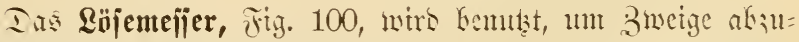

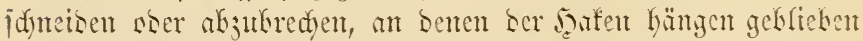

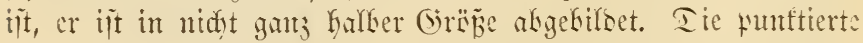

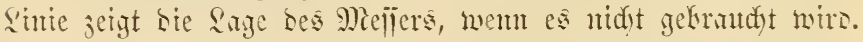

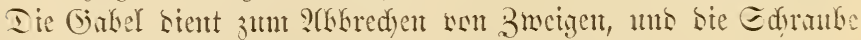

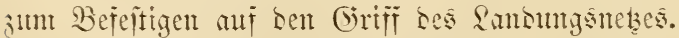

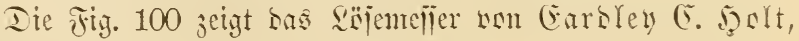
meldes (Eh. Farlom in Enton wertmit. 


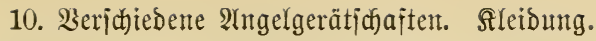

Die Rïberfanne, Fig. 101, sient jut 24ufbemahrung lebenser

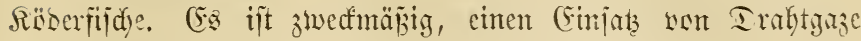
Ginemjutelfen, in weldyen bie Röberfijdye gejekt merben. Ias erleidytert bas beratsulymen ber Fijdye. Man fant biejelben

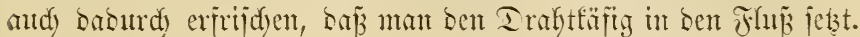

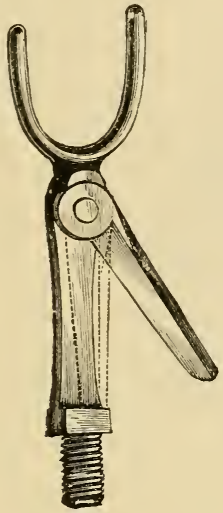

Fig. 100. ¿öjemefier.

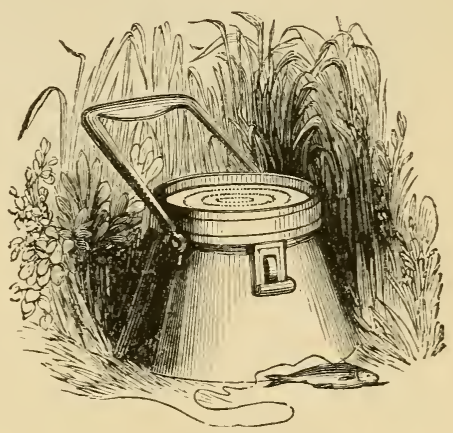

₹ig. 101. Sïberfanne.

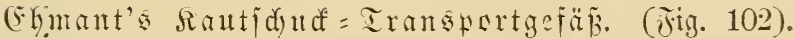
Sährent bes Jijens werben bie rebenden Tije darin ins

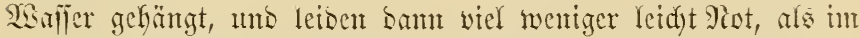

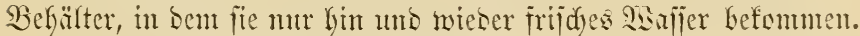

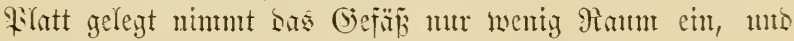
iit besfalk jefre trunspertabel.

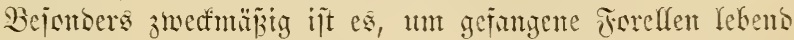
fut erkalten.

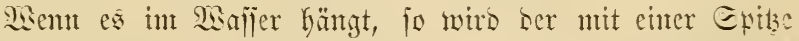
werjebene brifi in bas llfer geitedt unb sie Edymur, welde brs

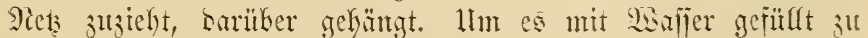
tragen, zieht man bas lek ein wentg auf uns ftecft ben Griff unter bie Eduntre, an melden bas (biä̈ hängt. 


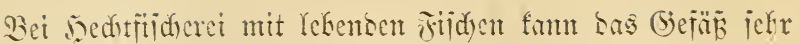

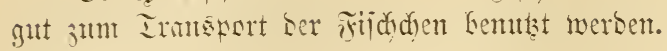

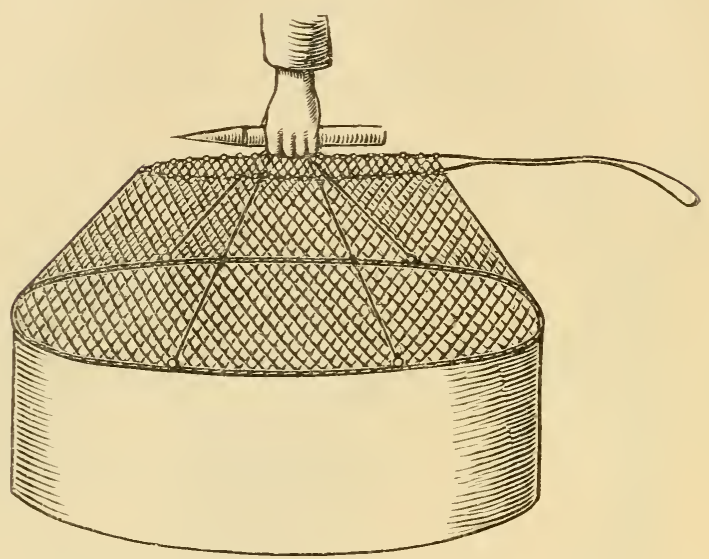

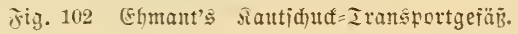

Bei Equmfitiderei anf Forelfen werben bie Geffriben in bem

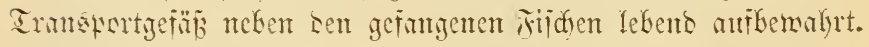

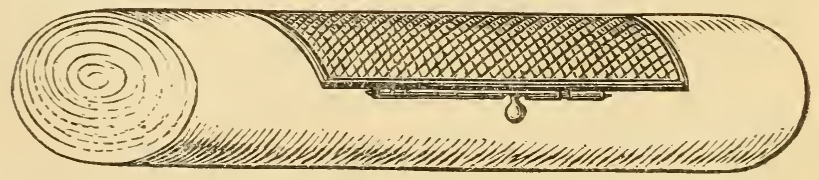

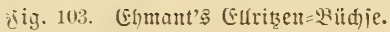

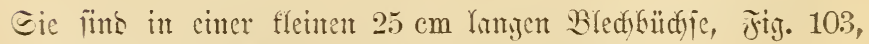

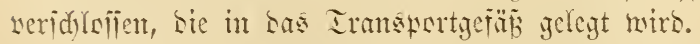

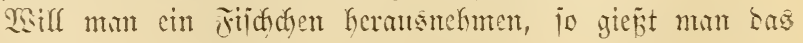

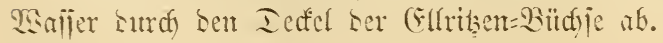


Sim Sienjennck, Jig. 104, fomt man Räberfiiche längere Beit an Peben erfalten, es wirb über Iraftringe geitricft, mit Bifet beichmert, uto faum jujammen=

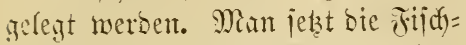
den Ginein, utb verjentt saถ Renjen=

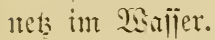

(Sime Büdjie, Jig. 105, jum

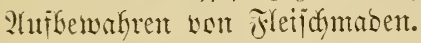

(Eine Bled)ooje, Jig. 106, um

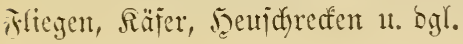
jth serwabren.

Bei I refumg bes Iecfelo seffint ïd) ein sodf, weldyes nutr einter

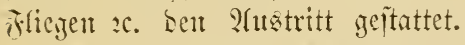

(ت̈in Reinwandbentel, Fig. 106, fïr. Regenwïnter.

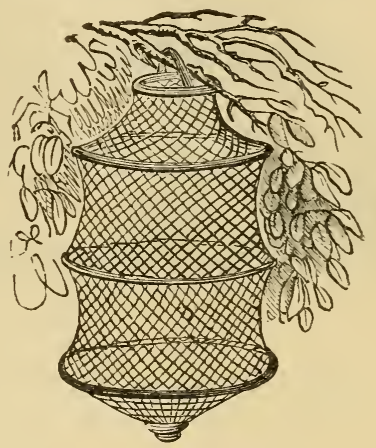

Fig. 104. Rentiennets.

Eine Feberwage, Jig. 107, jum \$siegen ber gefangenten Tiijue.

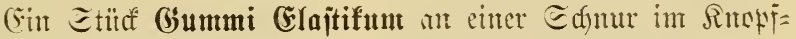
led getragen, jum (b) lätten bes gefräujelten Guttiobens.

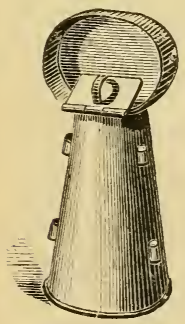

Fig. 105.

פiarentiufie.

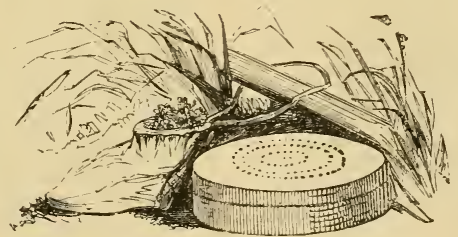

Fั!g. 106.

Jiegenooje unb warmbentel.

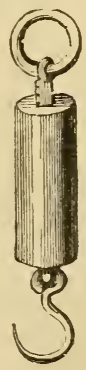

sig. 107.

Jiid)twage.

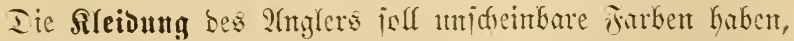

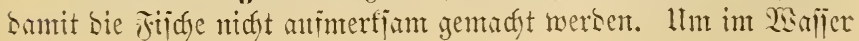




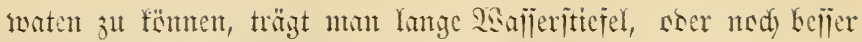
lange wajjersidute Etrümpfe soer Beintleider. 1leker letstere jicht

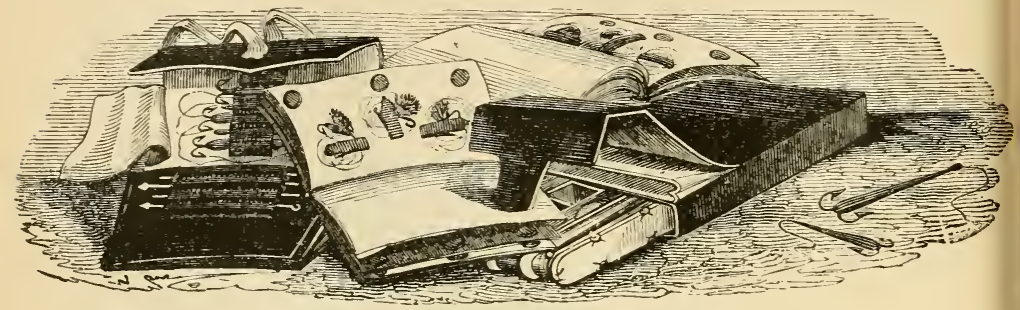

Fig 10s. Iajdenbüđer.

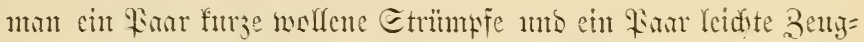

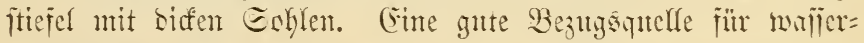
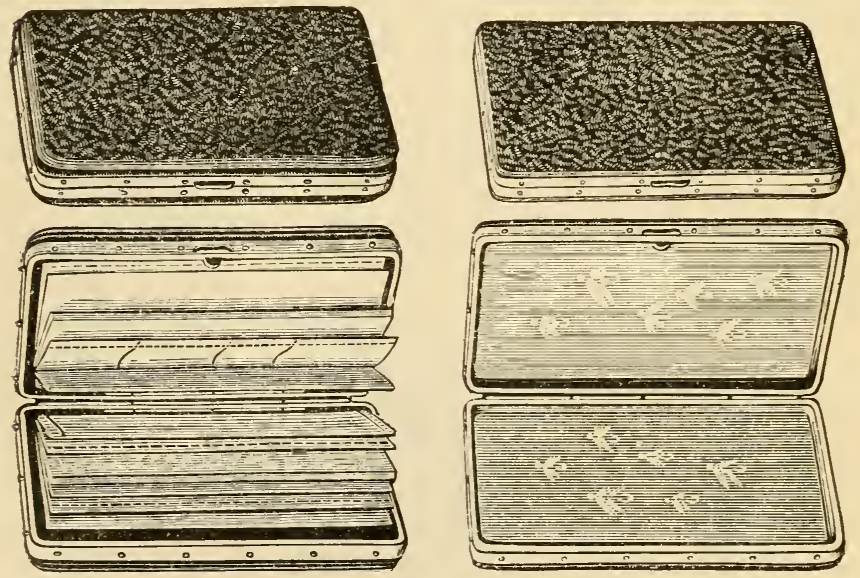

Fig. 109. Wh heatle y's Fliegentüber in Injenform. Fig. 110.

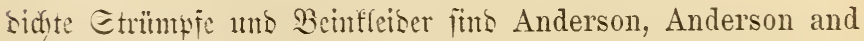
Anderson, 37 Queen Victoria-Street, City, E. C., London. 


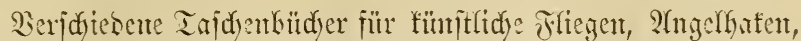
Angeljdütre, Guträsen, Surfädyer, Edjeren, Eenter, SËrbel 1. bergl. Jig. 108.

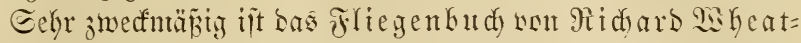
(eq) und Est. Manufacturers in Fancy Leather Goods 30 Hockley Street, Birmingham.

Daflelbe hat bie form ciner (Eigarren= Ioje, unb bie Fliezen werben barit garnidyt gebrïnt. Fig. 109, 110.

\section{כweiter $\mathfrak{A b}$ đanitt.}

\section{Die Söber.}

Man hat zwei 2raten won R̈̈ber, ben natürliden mo

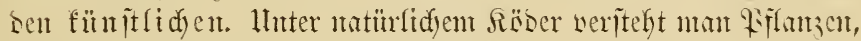

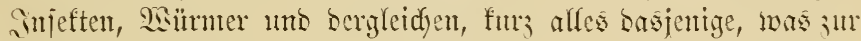

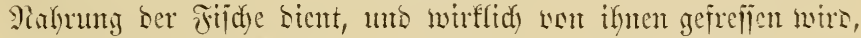

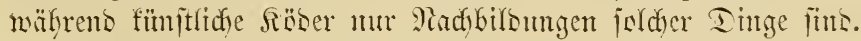

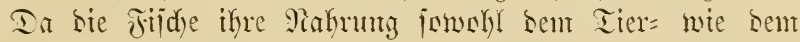
Fiflanjenteidye entuchmen, is giebt es, entipred)ent ber Mamnig= faltigfeit Keiber Meiche, einte fait unbegrentste Menge wat Sact= ipeijen für bie 2atgel, unb ber mit ber Rebentsmeije ber fijd) vertraute 2 ngigler wirb nidyt leidyt in Sierlegenfyeit fommten, wenn

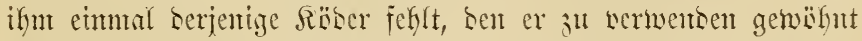

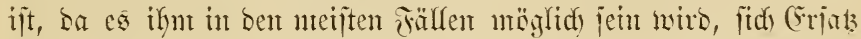
zu werjdaffen. En nimmt ber Forellenfifider, wenn jein Sourat

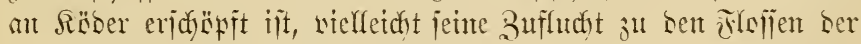
Gereits gejangenen Fijde, or angelt bantit wie mit ber tïmitlidyen

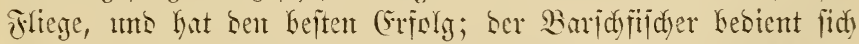
in ber Miot ber $\mathfrak{H}$ ugen ber gejangenten Fijde, ober bringt Etücfe

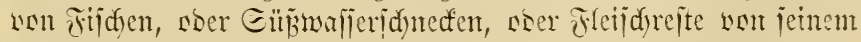
Trühytitud ant ben Soufen. 


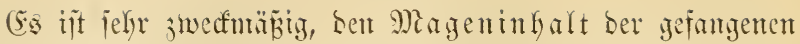
Fitide ju unterjuden, senn hierburd mirb man nit ber Seben: meije berjelben befannt un befäbigt, benjentgen Räber für bie

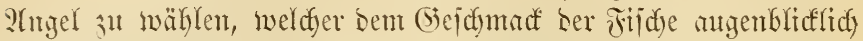
an meijten jujagt. Bei Forellen ijt bies gnuj bejonbers midytig,

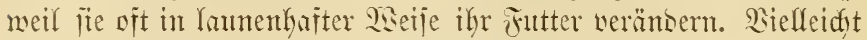
gelingt es cinmal bem stiegenfijeder nidyt, einen guten Fang 3 ul madyen; er unterjudt Den Magen einer gefangenen Forelfe, unto

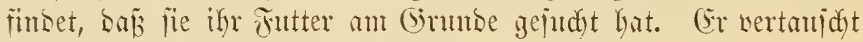

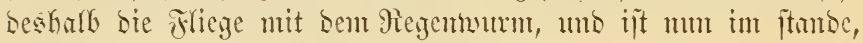
in furzer Beit jenten sijdfforb ju jüllen.

\section{Natürlidje Röber.}

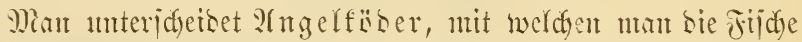

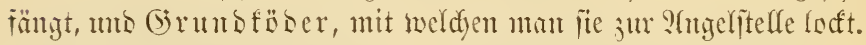

\section{2lutgelfëber.}

Ser Regenwurm ijt wobl ber an meiften gebrande sëber. Mian unterjofiset nefyrere 2frten.

1. Ter IGaumurm ît Ser gröbte unb bäufigite won alfen. Man fübet ibn überall in Garten uns Fels, bejombers an fendfen

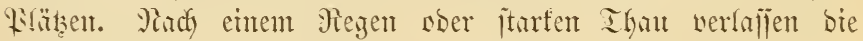

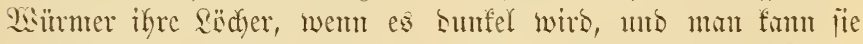
Emm bei Eatemenjedein in bent Garten ober auf bem Pfefer in

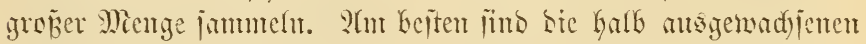

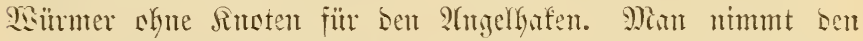

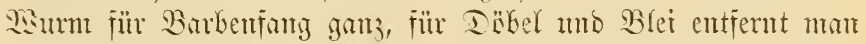

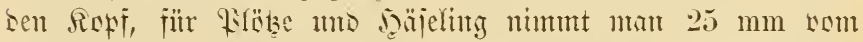
Edmanjensc. Dic gröjeren SGürnter mit Rnoten bentlist man jul Gruntrfïber.

2. Ier Motwurm ijt Heiner, wie Der worige, er lebt in Damfen modernber Blätter, ì werrottetem Tünger, ober unter

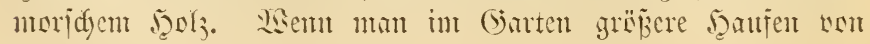




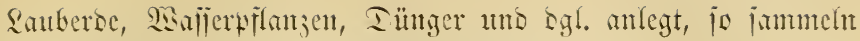

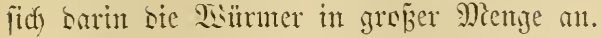

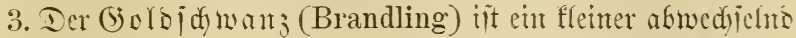
rot unb gelf geftreifter Fegenwum, ben man in verretteten

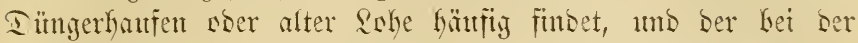
Berïfrung cinen gelfen, ffebrigen, wiberfidy ritedyenten Eait ab= fonbert. Er ijt ein jefre guter Rï̈ber.

Reinigen un 2 mfbenafren ber Regentürmer. Sum (bebrandy thut man bie Regentütmer in einen Iapf anf frijdes

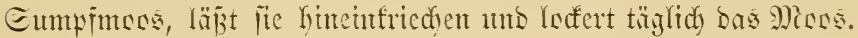
Son 3eit ju Beit entleert man Len Topf, wäjdyt ifn rein ats, thut frijdes Moos finetut und legt bas alte mit ben SSüntern Daranf. Iie gejunben siurmer friedyen in bas frijde Moos, bent Edymus, bie toten uns franfen Sisümer wirft man mit sent alten Micoje fort. Mian fam bie gïünter füttern, inbent man

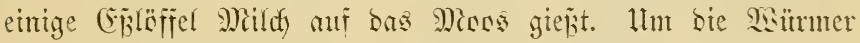

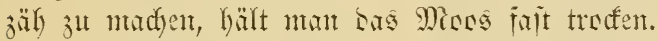

Fleifdnmaden Gilsen einen werjüglidyen Rëber, mamentlidt

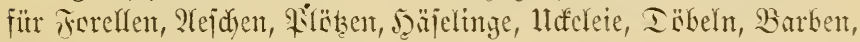

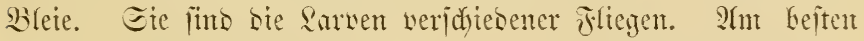
erkält man jie, went man cine Sefer wom Mint ober Edy a in

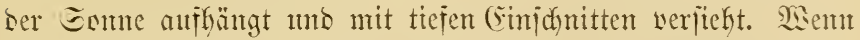
bie Fliegent ifre (Ëier abgejetzt haben, is legt man bie Seker in einen grofen Sopf, ber hanbhed mit jamarzer (bartenerbe gefüllt

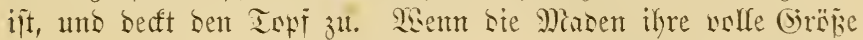

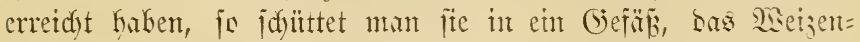
jdalenfleie enthält, bamtit fie fid) reinigen. Maron, weldie man mit Fijoten füttert, werben bejousers groj uno fins ein guter Rïber.

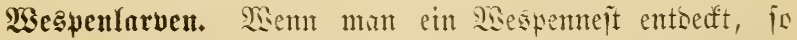
tötet man bie Bewolgner baburd), baj man ein mit (Syantaltumt=

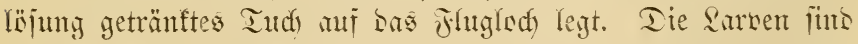

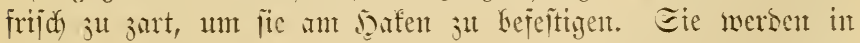

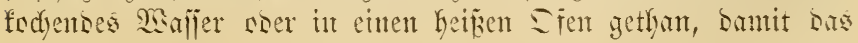




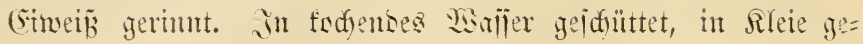

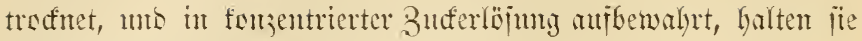
itid) juffrelonis gut.

Engerlinge แnd Räferlarven werben ähnlid wie \$Eespen= larwen Gebartiselt.

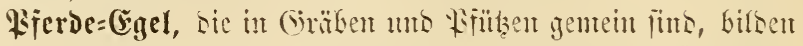

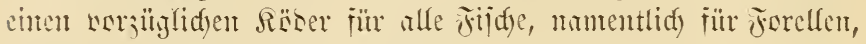

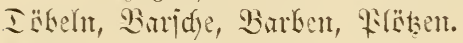

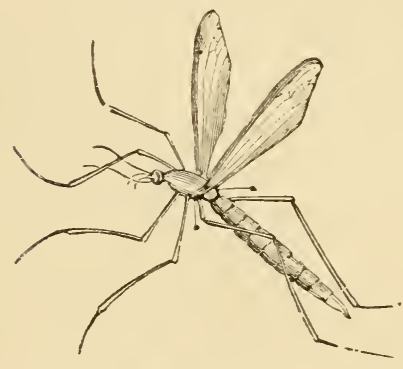

jig. 111.

Tipula rivosa.

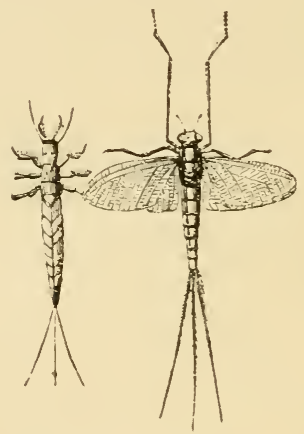

Fig. 112. Fig. 113. Miaifliege und :arve.

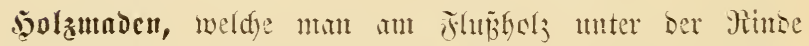
fünet, wersen bei Berlin mit Borlicbe junt Burjojung bentlat.

Madte Edneffen und Wajijerjuneden jüb gut, numentlich für jorellentintg.

Miehlwärmer füntuen leidyt in cintent mit Medh und Rleie

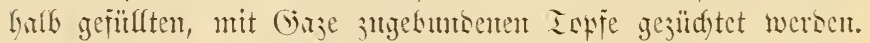
Eie werten gerïlynt für ben jomtg won jorellen.

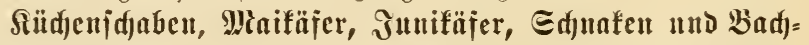
müufen, Fliegen für jorellen uto Ï̈beht. Jity. 111 ijt Tipula rivosa, englijd) Crane Fly, Harry Long-Legs, eit jefr guter

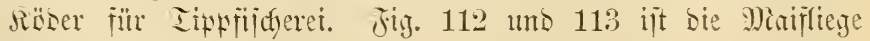
und iffe sirnve. 
פeupierddjen bilsen einen ber beiten Röber für Iäbeln, Reiden mo Ferelfen nem Juni bis Eeptember. Man fintet

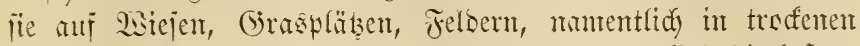

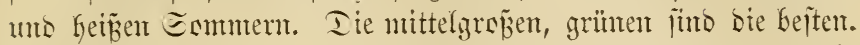
Man benahrt jie in bejonders baju eingertateten Büdjen, bie

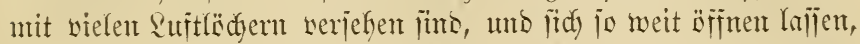
ba

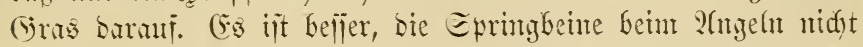
abjureiben. Mit einent Edymetterlingstejder fam man leidst eine Menge fungent.

\section{Die Larve Der Etcinfliege} (Perla bicoudata, Creeper) funbet man in \$sinter in

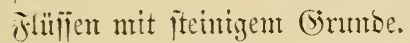
int 2 fpril erreidyt jie eine zänge non $20 \mathrm{~mm}$, unb hat cine büme, gelb umb braum geflecfte genut. Man fintoet fie im Fladgen ïe lüuft jefre idnell, mo ijt

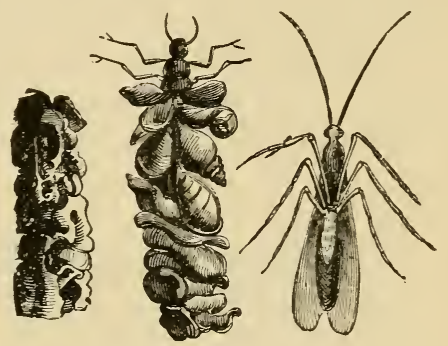

Fig. 114. 厄trofmum. midyt reidjt jut iangen. Int

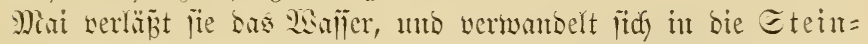
flicge. Eiejelbe ijt umgerähr $25 \mathrm{~mm}$ lang, iffe Flügcl liegen

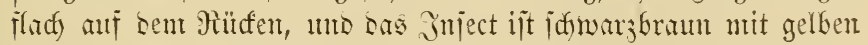
Flectent. Eomolgl bie Enve, wie bie Fitege nerbent viel junt

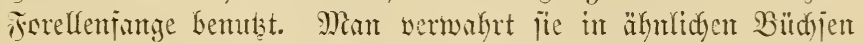
wie bic Denpferddent. E. Tig. 106. Die Sarven fünten in

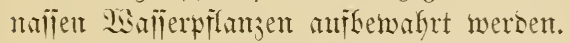

Ier Etrofwurut, Eproffe (Caddis, Cadbait) (Jig. 11.t) ijt bie Earve einter Fliegenart, ber Whryganeen, und Daburds

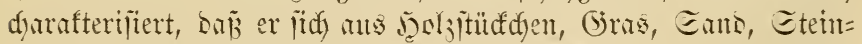
den, Majdyelidgaten u. i. w. ein Gefäuje batt, mit bent er im

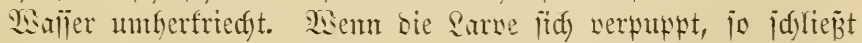
jie Sas (jefünje. Eie bilost einen jefr guten Röber für viele 


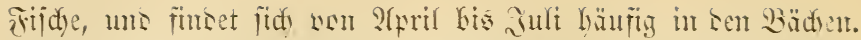
Mant jammelt ïe am bejten mit einent flemen mit Yangen Eticle

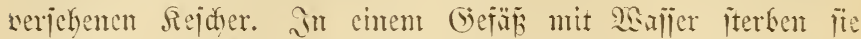
jebr kals; mat faun jie afer is lange am sefen erkalten, bis

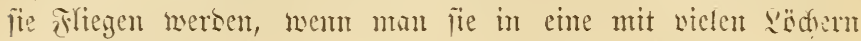
werjefrene Büdje thut, mo sieje in Den Bad jtelf. Sirr som (bibraud) thut mat ite in cinten Beutel, entmeser alfein, secr

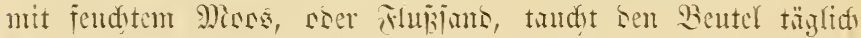

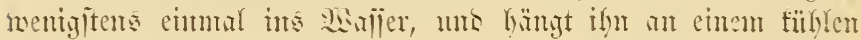

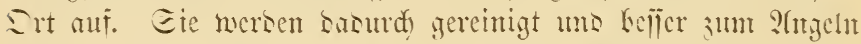
gecignet, als wemt ite gan frifol gejanmelt jims.

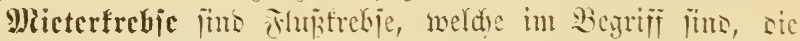

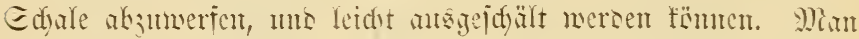

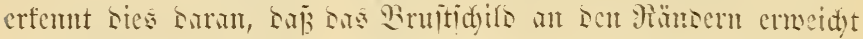

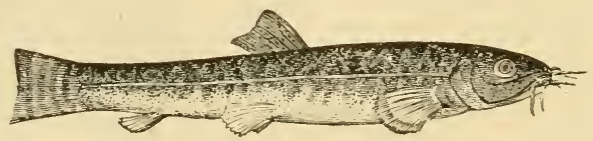

Fig. 115. Girünling.

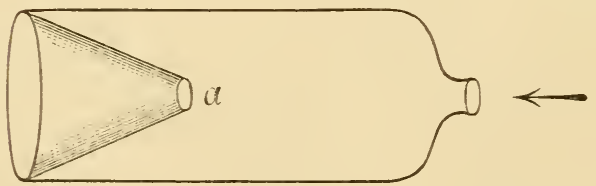

Fig. 116. Жenienflaide.

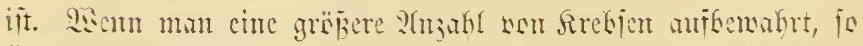

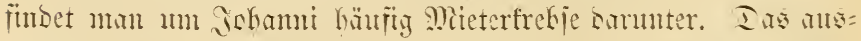

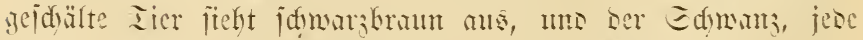

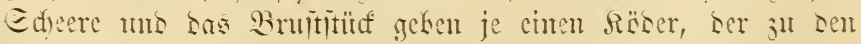

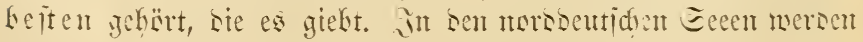

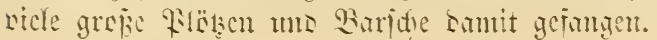




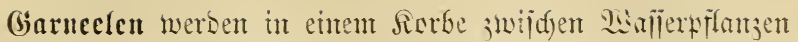

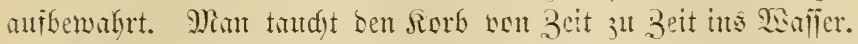

Steine Fröjde finto cint beliebter Räcer für ben fong went İ̈beln.

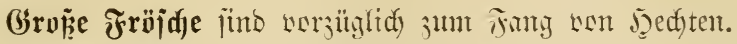

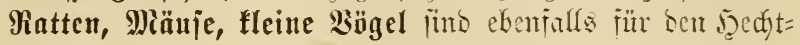
Fanty brandebar.

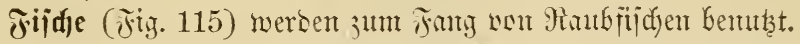

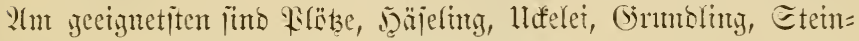
grumbel unb Celfrize nou $8-12 \mathrm{~cm}$ \&änge. Man fïngt bie

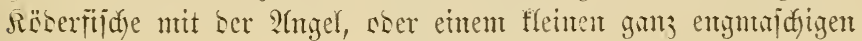
3itgntets.

Eine andere Fingmethode ijt bis mit ber Flajde, weldye bie in ber weritekenton Fig. 116 argebiloete Frm hat. Man

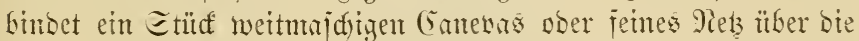
Wiënsum; nadsem man einige Brostrumen in Die jolade ge=

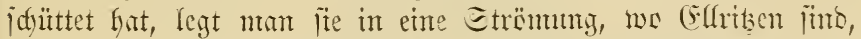

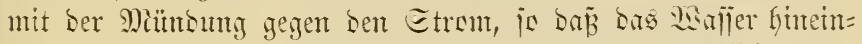

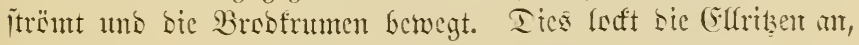

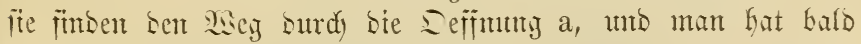

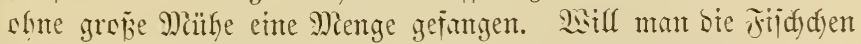

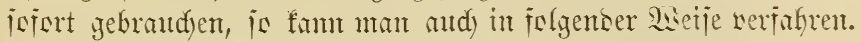

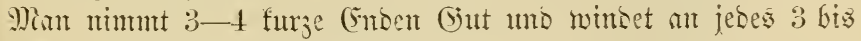
4 fleine Safen an uns binset jie mit eimem (jutfaben jujammen,

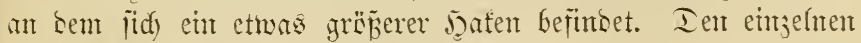
Dafen beföbcrt man nit einem Etïddyen Motmum uno Gängt bie

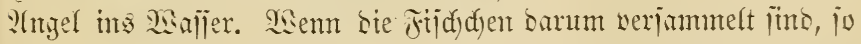
jicht man idnelf heraus, unb haft gewöhnlich 2 soer 3 auben ant Rërper. Ier Röber wirb gewëhnlidy wen ben grejen jijdent

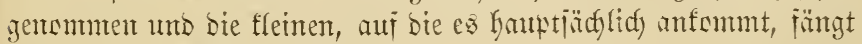
man an ben fleinen פaten.

Fïr bie Meije jollon bie Janggeräte leidyt trantsportabel jein.

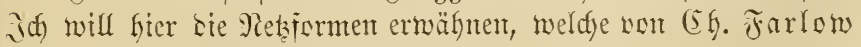
in Sontoon berfanft werber.

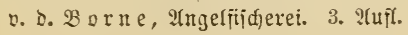




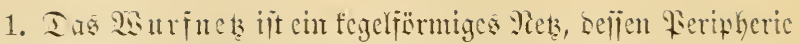

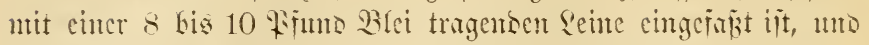

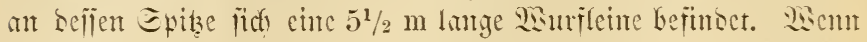

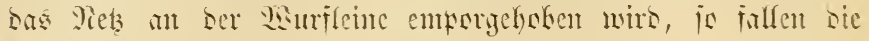

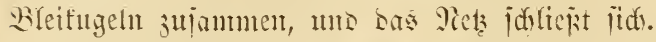

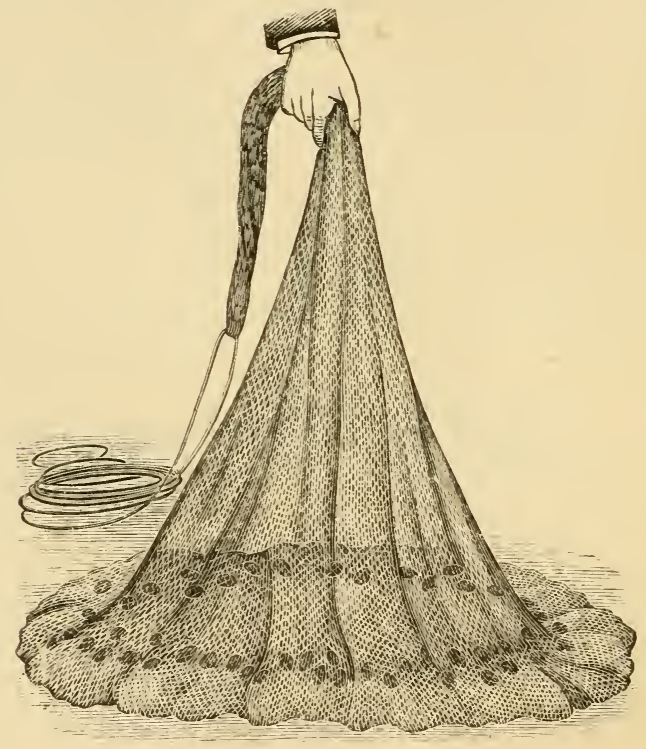

จig. 116. พืurinct.

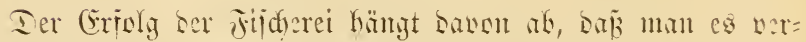

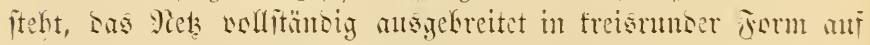

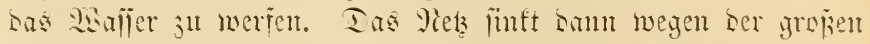

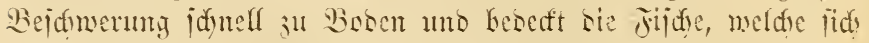

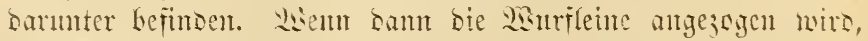
is rüfen bie Breigendide jujammen, uns bie jijde jommeln jid

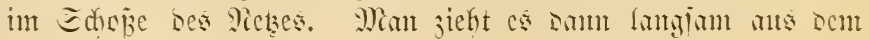




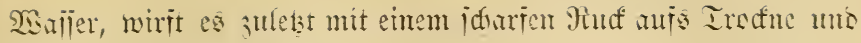
nimmt bie jijue herats.

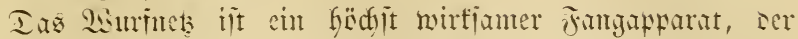

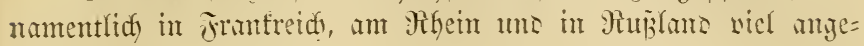
mentset niro.*)

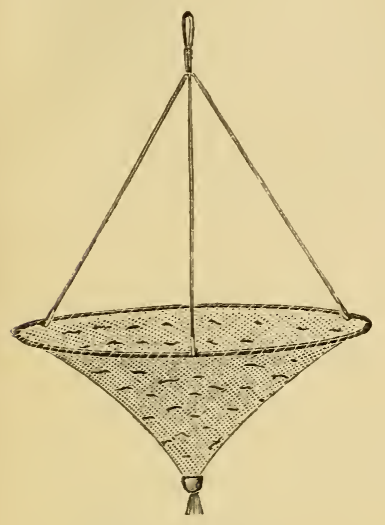

iig. 117. Eentnetz.

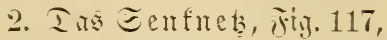
Gat einelt Iurdms jiler nou $1 / 2$ bis $1 \mathrm{~m}$, iit über einen eijernen ßeifen geipant, uth in ber Nitte surd cine Bleitugel bejowert. (5. mirs an cincr Etelfe in bas sitijer ge= legt, wo lid fleine Fijue authalten, mo nad) einigen Mimuten ifunelf

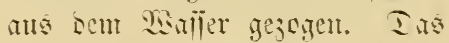
Mets ijt jehr gut jum jumigent won llafeleien, (Etfriben und flemen SEcifitilden geeigntet.

3. Iic 丹euje, Fig. 11s, ijt

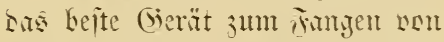

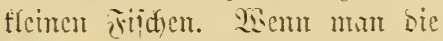

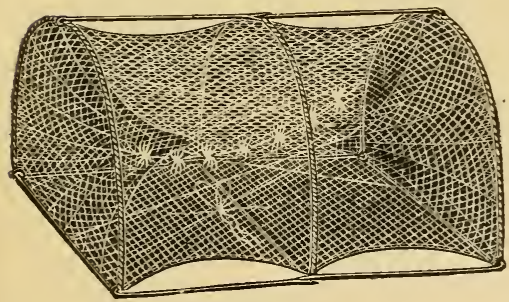

Jig. 118. Die Reaje.

Epamptïf: fortmimut, is fam man sic Reuje julimmentegent. Beint Eimlegen in Das : wirl fie surch binem= thut rom ein Par Eteinen beidimert Imt verientt. Mian itellt sic Hente an einer mit Edfili

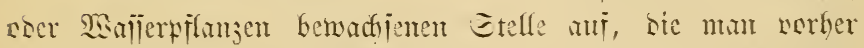

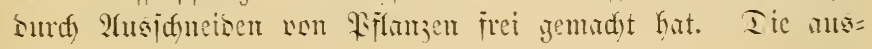

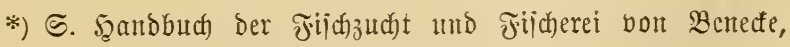
Daffmer uto v. D. SSorne, S. 608-614. 


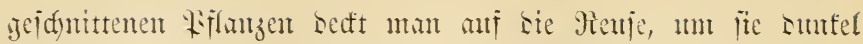
jll madjent.

Dian legt bie zun angenblicfliden (Gebranda bejtumnten

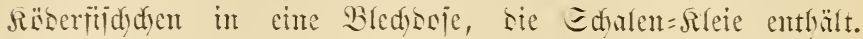

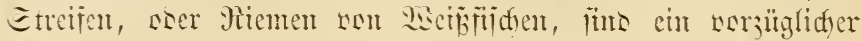

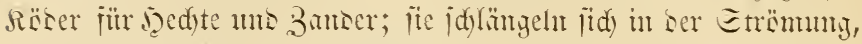

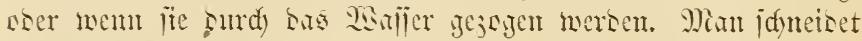

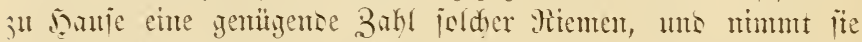

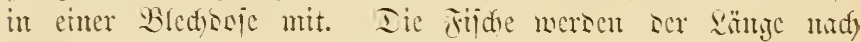

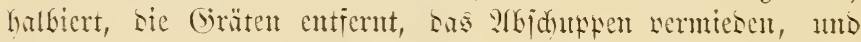

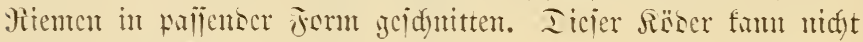
gemtig empishlen werben.

Soniervieren Der SïDerfijdge. SEerben Rïserfijde Had) wentigen Eajen gebrantedt, io brimigt ntan ite in tonjentrierte

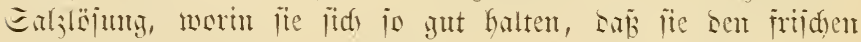
Tijdyen nidyt madytehen. SEill man je aber länger fonjervieren,

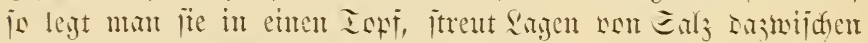

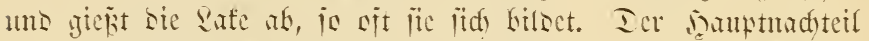
ser cingejaljenen Röserfijde bejteht sarin, saj lie ju Eteletten

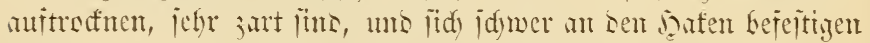
Lajien. Eoer man thut sie Jijdye in jdyadyen Exiritus.

Qlbjehaspelte Siofong Der Ecidenraupe werben an Sago Miagyicre als Rëser bentut.

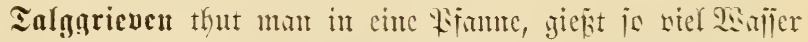

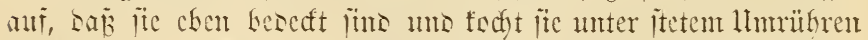
20 Mimuten, Sem jie erfalten, mui sie Malie jteif wersen,

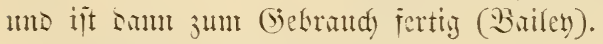

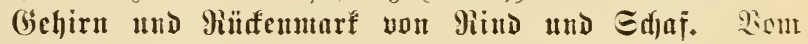
(jefirm enternt man sie Dont un reinigt es in Blut, bis es rein weif ijt. Som Bücfemmant entient man bie

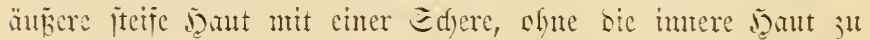

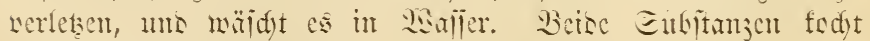
ntan 1 Mimute (Builen).

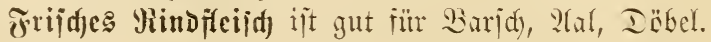


Gerifte, Weijen, Ferlgrnmpen werben geted)t, biş bic

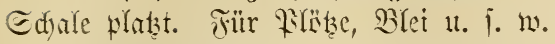

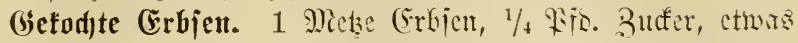

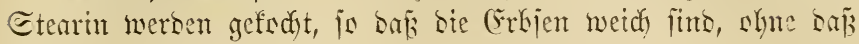

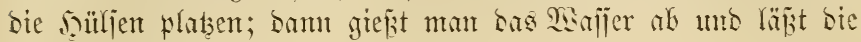
(Erbjen trodinen (Engel in (Eiijtrin).

Edjweizerfäje ift gut für Barben unt Iäbeln, er wirb in

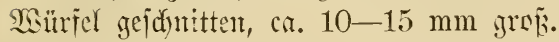

Siridsen füto gut für IBbeln. Man enterne ben Eticl, unto führt bear bafen ba eirt, we ber Etick geielien hat.

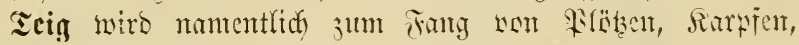

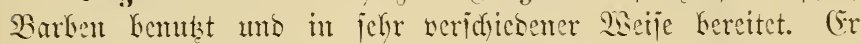

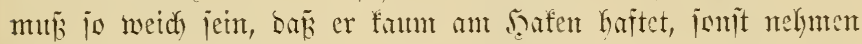
ify sie Fijde nidyt germ.

Brotteig. Man hülft bic Rrume won Metipgrot in cin

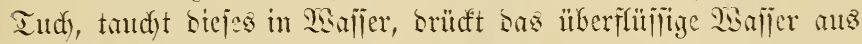

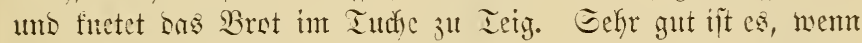

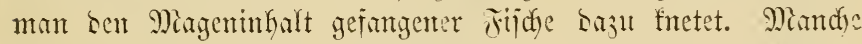

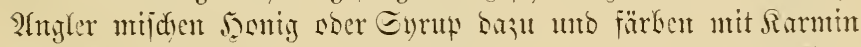

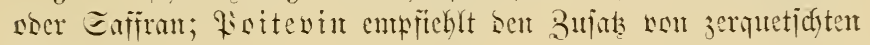
Sanftïrnern.

Räjetcig. Man jerreibt cinen zähen, alten Räje, thut

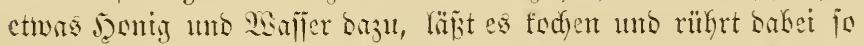
vicl Miekl bajl, baj man chnen weiden Ieig (etwa wie Enuer=

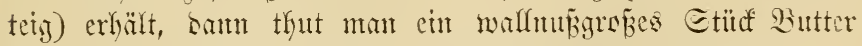
baju. Sorzüglid) für Rarpfen und Bleie (2rlter Fraftifus).

\section{Grun১pöser.}

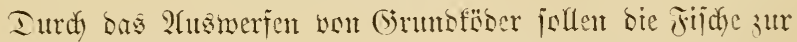

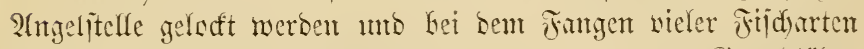

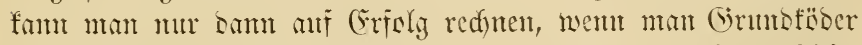
ammentoct. Ties gilt namentlich für Flöben, Barben, Blcie,

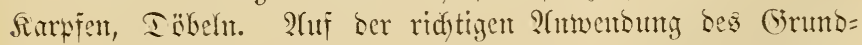

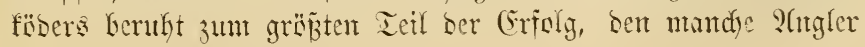


erjiclen. Mian bari aber and) nidyt wergefien, saj man bas $2(n=$

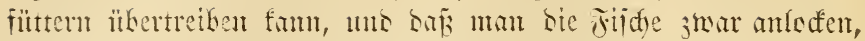

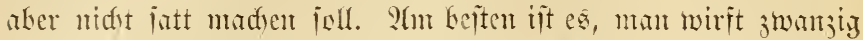

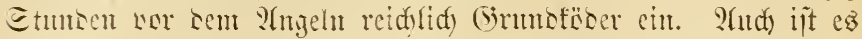

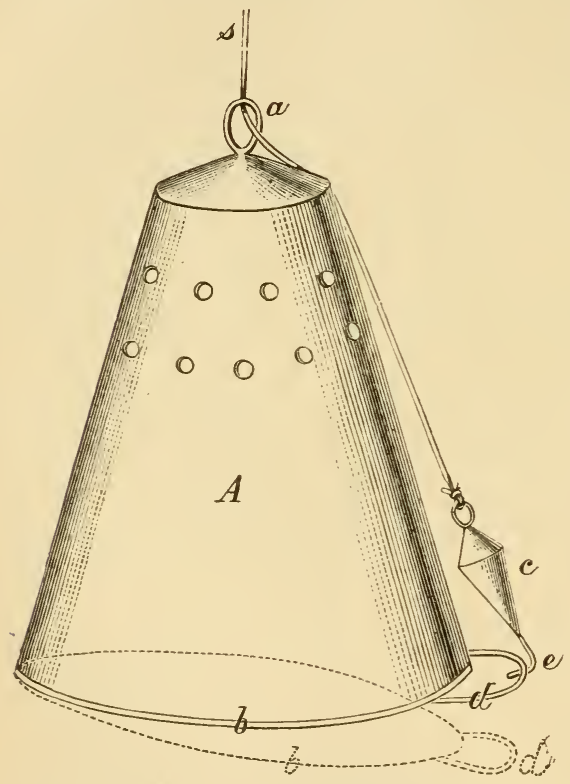

รig. 119. Grunstöscrküdje. gut, mefircre Etellen anjư̈ütern un jie abmedjelut jut be= Fiidyen, meil bie ixtidye, rie surdy ben Jamg an eimer Etelfe wer= idfeutd)t fints, bamt gern bie benadjerten anfitud)en. Dierfïr ijt Bater in Potting= ban unbedingt bie widntigite Sulte. Serjelbe wirft fït Den Barbenfangein: 1500 Enmü̈mer jwan= jig Ettursen wor sem 2tngehr; - ober 11 in tfeine 2ïürel ge= id)nittenen Räje 30 Etumben norfer. Girieven fint nidst ght, weil jie bie sitide jul jatt madyen. Mian muj genau willen, we ber Rëber ben (Stunt berïfyt, unb genau an biejer Etelle angeln.

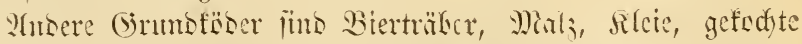

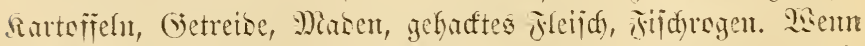
bie Etrömung jefr itarf, jo tuetet man sicje Eubjtanzen mit

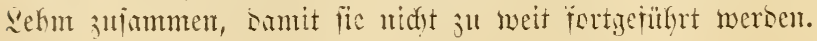

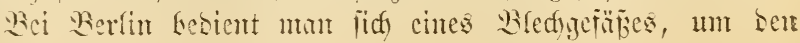


(Sinutsfïser ju werjenten umb ifn jujanmen ju balten. Ialjelbe

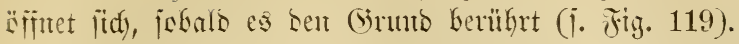

TEährend bes jijdens wirft num bon Beit ju Beit einige Etü̈fden won zeridfnittenen Regenwürmen, ober eintige Maben, soor syl. ein unb fijdyt gentan an ber Etelle, wo bieje Dinge ben

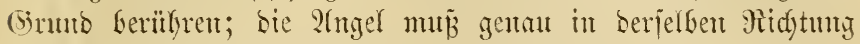
id)mumen, wie ber (5inutoföber. Setzteren wirft man immer an Serjelfen Etelfe ein linb wieberfolt bies jebesmal, went man

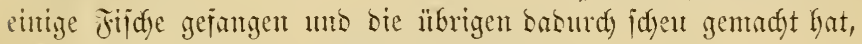
uto went bie Jijde autgören ju beijent.

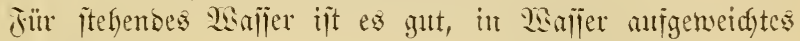
Bret eirjumerfert.

Fer Grumbföber des Capt. WSilliamiun ijt beritfmt. Dajer= mebl ober Sllete werben in einer fifante ïber Fenter gefräunt, mit Enrup ju Ieig geformt uno savon hajelmügrofe Rugeht eintigemorfen.

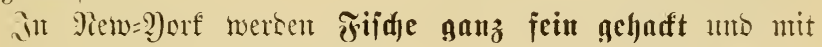

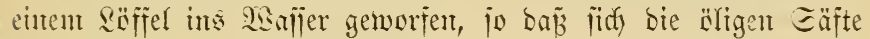
weit über bie Sberifäbe verbreiten. Ier Eecbaridy wirb Iasurdy ans weiter jerne berbeigeloct.

\section{Sïnjtlidye Sïber.}

\section{Künftliche fliegen un๖ Käfer.}

Man unteridyeiset jolgente 2lrten tünitlidyer Fliegen:

1. Taирепіliegen, Walmer, mit behantem Rörter, shne stïigel. jig. 120.

2. Eummente jiegen, jedel. jig. 121.

3. (5) flügelte jliegen. jit. 122.

4. 2tmeijenjliegen. jig. 12:3.

5. Räier. Jig. 124.

6. (3) anjiliege, \&adgrliege, Eeeforellenfliege. jig.125. 


\section{Eefle ber Glamifliege.}

1. Tag. Etift nder \$imme.

2. Butt. Edymanjtiorf.

3. Tail. Edfmant.

4. Joint. Gifies.

5. Hackle. Sïrpertyedrel.

6. Shoulder Hackle. Edulberfiediel, Bsins.

7. Wings. Flïgel.

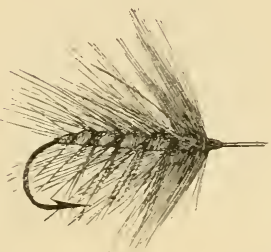

Fig. 120. Raunenfliege, Fafuter.

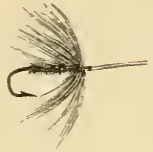

iviy. 121.

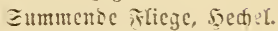

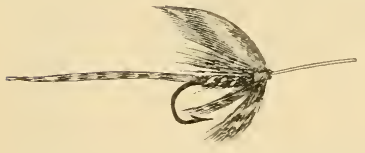

Fig. 122. Gợliţ̧elte rifiçe.

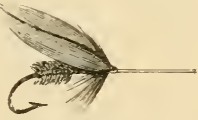

ז̃ig. 1:3.

:tmeipenfliege.

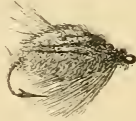

rig. 124. säier.

8. Toppings over. Ioffe.

9. Horns. நürner.

10. Cheeks. Sismign.

11. Head. Ririt.

12. Seje bes gafents.

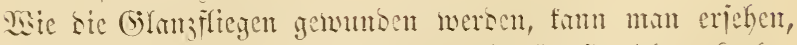

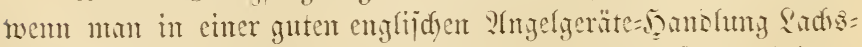

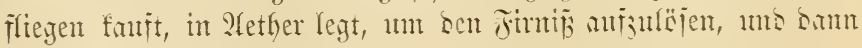
sie Fliege atscimanoer nimmt. 


\section{A. Das Winsen Ser fünftlid)en fliegen uns Lïffer.}

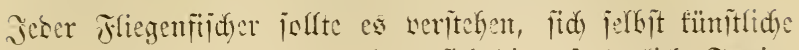
Fliegen $j^{\text {ll }}$ wimben. (Ein jeser fom jid bie erforberlide Fertig= feit leidyt unt in furjer 3eit surd einige llebung er= merben. (Es ift ein grojer Sortcil, won ben 2lngelgeräte= 5amslungen umate= frängig ôt jein, jid jeberzeit joltegen mad)en 3 lt fïnten,

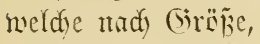
(5eitalt unto rarbe Sen Besürnitis ent= ifrectert, um jeser= zeit entretentoe Mä̈ngel ergänjen ju fümten, was jomit gar sịt gerabe sa nidut mëglide iit, wo man es am jodnery= lid)iten entifinset.

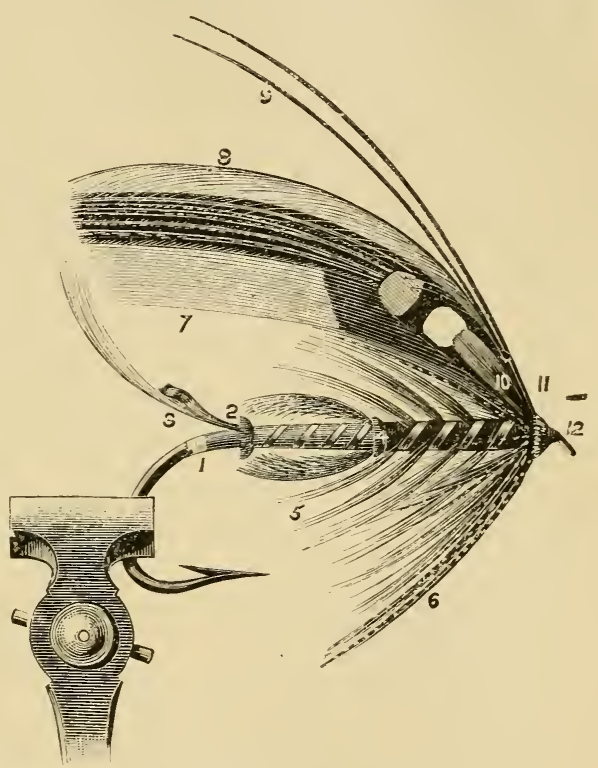

Fig. 125. Ģlantzfliege. Jock Scott.

Bei bem Fliegenwinsen werden folgende Merf = zeltge gebraucht:

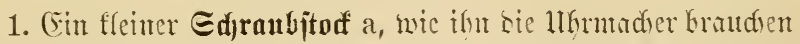
jum Jepthalten bes Sorfens (jig. 126). Man kefeitigt jein unteres

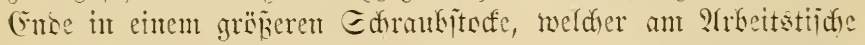

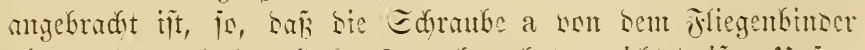
afgementet umb ser bafen b uad redits geriditet iit. Llujer:

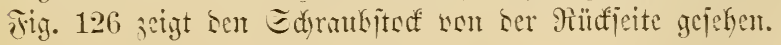


2. Federzangen (jig. 12ד) ster Pincetten jum jeithalten

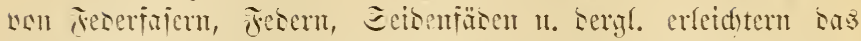

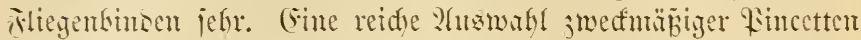
fintet man bei sen jofrifunten diturgijher imitnmente unter Don lnterbinoumgapincetten und Plrtcrienflemmen.

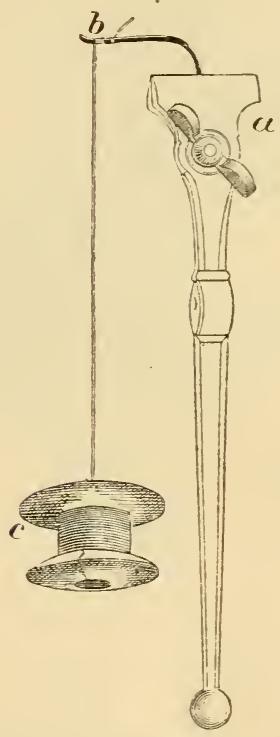

sìig. 126. Estraukited.

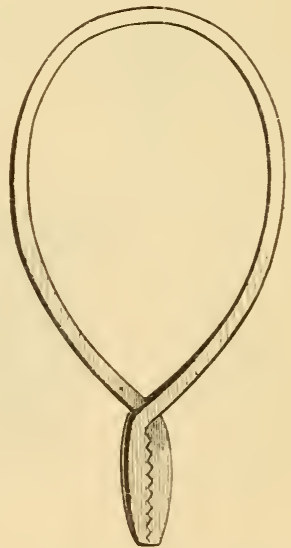

Jig. 127. Teserzange.

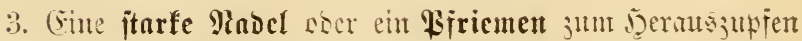
untergenumbener jescrfajern soer \&eljhane u. Dergl. und jum ¿äjen von Rnoten.

4. Ein Federmejier.

5. (Einige red)t idfuris, feint Edjeren. 
Matcrialien, aus welden bie Fliegen gefertigt werben.

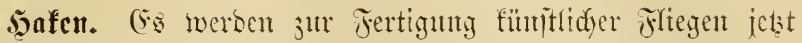
gemöhulidy geährte jatfen angewentet, weldye bie Borteile ge= wäfren, baj jie leidgter uno jdyneffer am (siutiasen bejeitigt werben fömten, unb baj bie jiliege und brandbar it, wenn ber (butraben juablyajt gemerbent ijt.

Die Anmindejeide jei jo fein uno haltbar, wie möglid). 2(nt bejten ijt bie für bicjen 3weaf won ben englijdyen 2fngelgeräte=

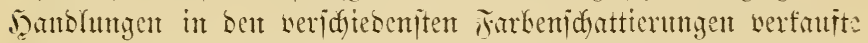
Eeibe, sie Pearsall's Gossamer Fly-silk heijt. Eie befinset

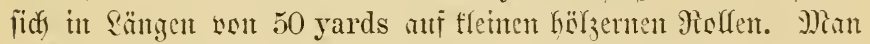

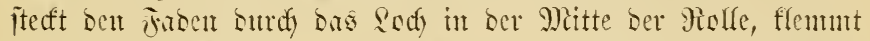

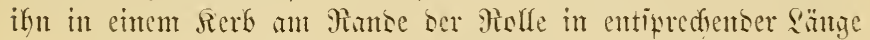

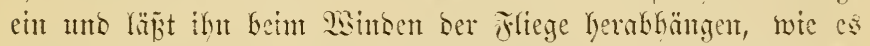
unjere jig. 126 zeigt. Ier Faben wirb is sutrd ans (jemidyt

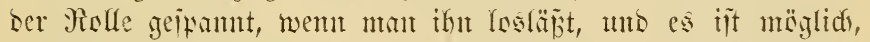

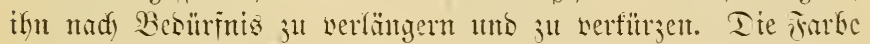

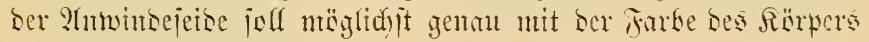
ser Fliege iibercinjtimmest.

Federn weridfebener 2hrt merbent siel jut Deritelfum ser

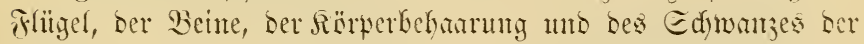
Fifiegen gebrandst. Dedelfebern memt mat biejenigen Febern, aus welden bie Bcine und bie Beharmen Des Rörpers hergeitellt tvitr. Mian faut kogelbälige gut unt billig in folgensent

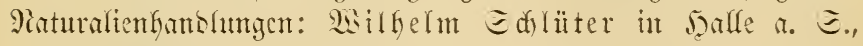

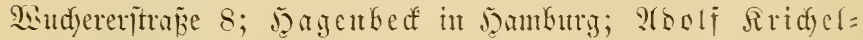
sorff in Berlin C. 42, Srantentrabe 135.

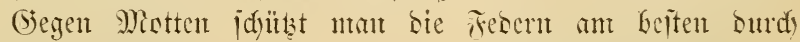
Siaththalintryitalle.

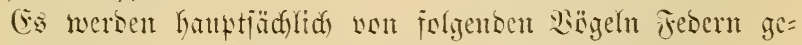

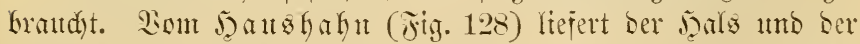

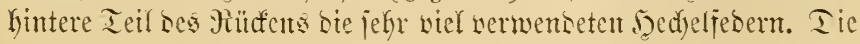




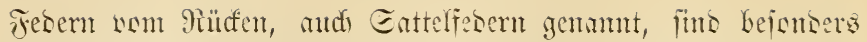
für grefie Fltegen bic beiten, weil fie länger, Kefjer geformt und glänjenter j̈ns, wentiger Flaumfebern baten, unt weil ser fiel feiner uno haltharer ift, wie bei ben Salsfebern. Mlan bratdet bie Fesern in sen werjofiebenten Farben: weij, gran, jumary, ret, gelf, - cinfurkig, gejtreift soer geflect.

furnace hackle hat einen jowargen Sëmgsitteifen in ser Mitte,

coch-y-bonddhu hackle bat in ber Mitte 1 mo $\mathrm{mm}$ Ratbe cinen jumarjen Sängsitreifon,

knee-cap hackle hat imci jomarie süngsitreifen an Mande,

cuckoo hackle hat sumfle S.ueritreifen.

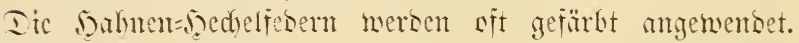

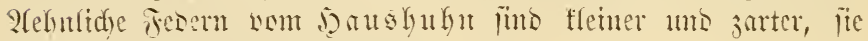

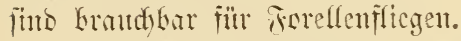

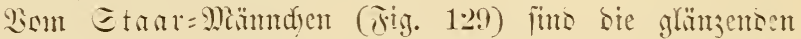

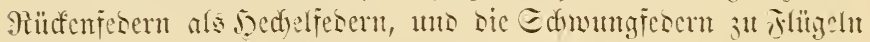
wat Forellenflicien viel gebramedt.

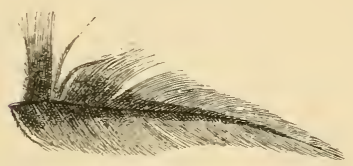

Fig. 128. Şedhelfeser vom 5̧ahn.

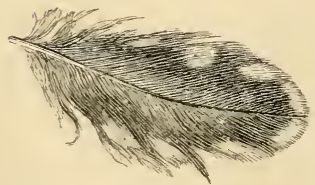

Fig. 130. Miorinelf $=$ Regenpicitix.

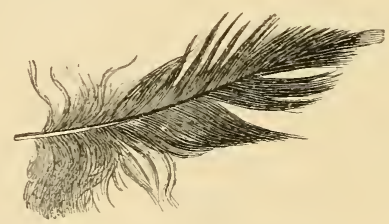

J̧ig. 12\%. Etuar=ßiüdenizber.

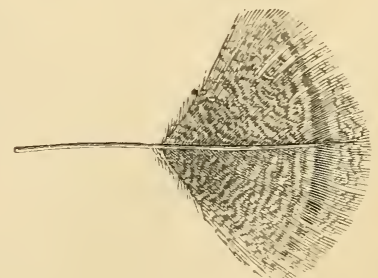

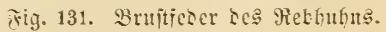


Pefnlid) Berwentury füben bie Febern won folgenden

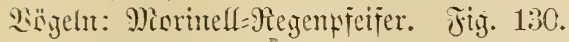

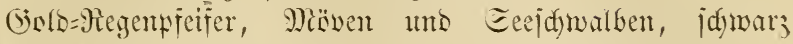

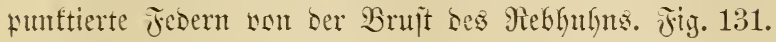

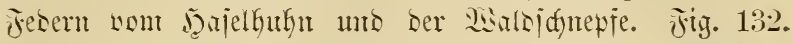

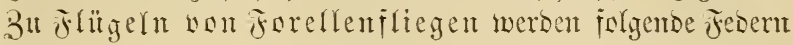
gebrindat:

Transparente Flügel werben burd) 2 fbjdytte aus Der Fafute Der Edyumigiedern bes jooljhäher, Etaar, ber Serdye noer aus

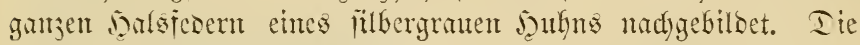

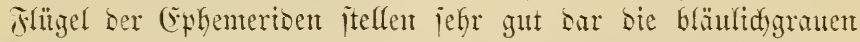
jebent unter bent shïgel bes Etar, Megenpieifer, Der Edynepie,

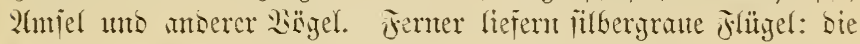
Jesern ber Eeejumalbe; bunfelgrau: Edjwungfeder von jeäfer

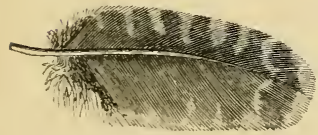

sig. 132. Fitügefieber Der

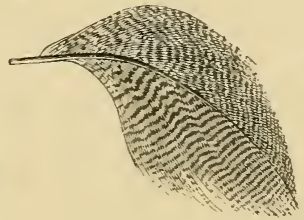

Fig. 133. Brujtieder bes wilsen Enterid)s.

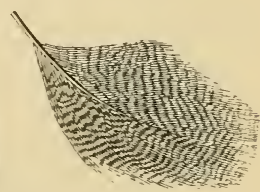

Fig. 134. Bruitieber Der sriffente.

nder Etarr; idmary: Edymungfeder ber idymarjen afmjel, bes

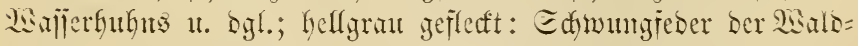
idunepre, des Hebhuhns, Der Jajanthente; Dunfelgrau gefleft:

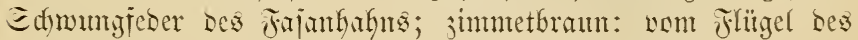
些adtelfönigs soer ber (Eule; braumret uno bunfelgrau gejtreift:

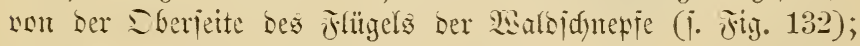
weí uno idyar; funftiert ober geitreift: von ber Brujt bes miloen (Enterid) (j. Jig. 133); eine ähnlid) Jeber von Siriderpel

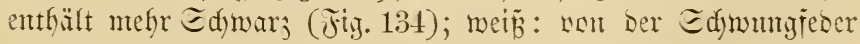
ber biats doer Iatbe. 
3u ben grojen Oblanjfiegen werben bie Eebern wer= juiescner anserer Bïgel gebraudyt.

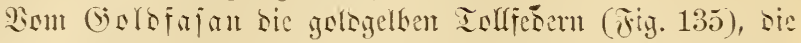

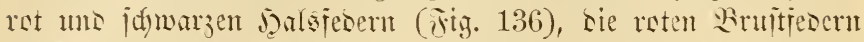

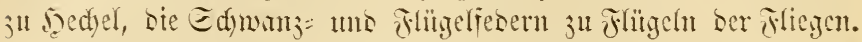

Fafageffeberm Der Prarauna, Sittace Ararama mis

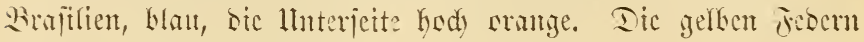

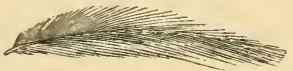

Fig. 135. Gotriajan=Infleber.

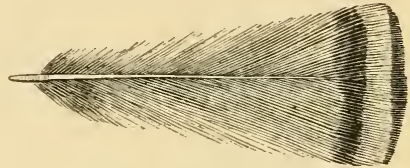

Fig. 136. Gntbiajan=\$atsieser.

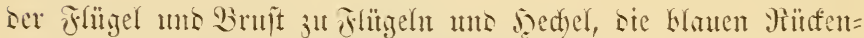

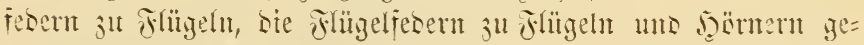
brattdyt. Blue Macaw.

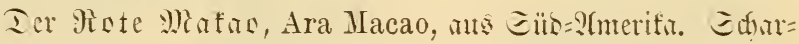

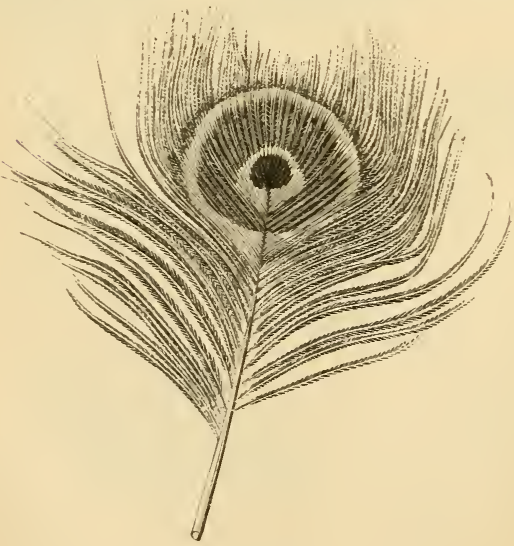

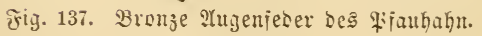
ladbrot, Miülen Gimmtel= Glau, Red Macaw.

Der Eoloaten= arara, Sittace militaris, ats Eits=? ?m:= rifa, grün mit reter Etim; Dinterrïfon, Edumingen uns Edwatzende finmt: Whut. Green Macaw. Ior $\mathfrak{A m a j o u e n =}$ papagei, Chrysotis amazonicus. jocll= grün, ₹̂lïgel rot. Green Parrot. 


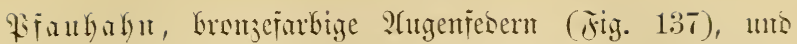

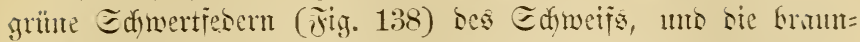
geflecten Febern ber Flïgel; bie Glauen Febern ber Bruit, umo

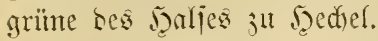

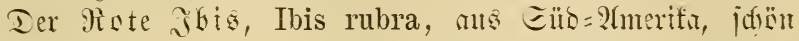
zimnoberrot.

Der Edjuppenfropiougel, nocr bie Jubijthe Rräte,

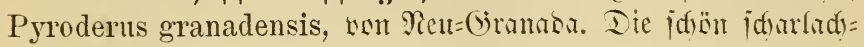

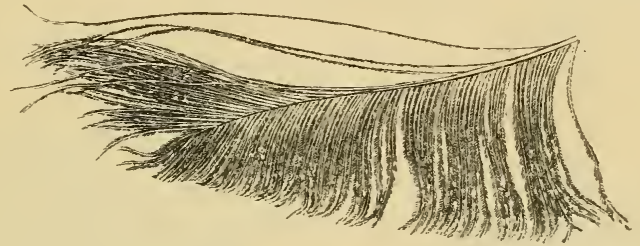

Fig. 13\%. Grüne @dwertieder des Wiaulahn.

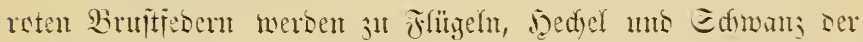
Fliegen riel gefrimudt. Indian Crow.

Der Sifefierfrejer, Iufm, Ramphastos Ariel, aus :Seit= Susten uno (buyan. Die gelben umb reten fobern an Brut

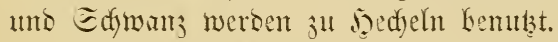

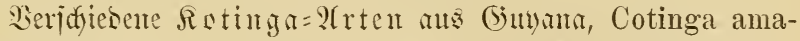
bilis, C. magnana, C. cincta, C. coerulea, liejern ifgën blane

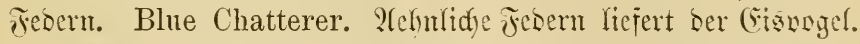

Ins Rlippenfufn, Rupicola crocea, aus (bunna, liejert

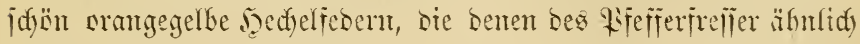
iins. Cock of the. Rock.

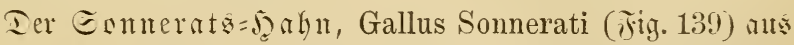

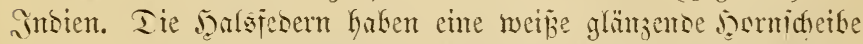

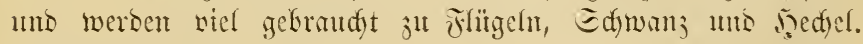
Jungle-Cock.

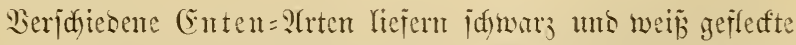

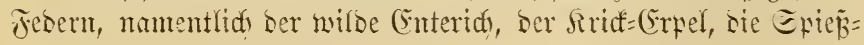


ente, Anas acuta, Pin-tail; bie Pifeifentente, Anas Penelope, IVidgeon.

Wou ber Brantente, Aix sponsa, Summer-duck, Wood-

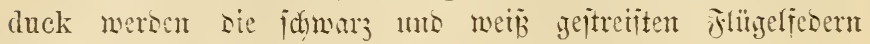
gebraudit.

Brown Mallard, Jig. 140, ijt sie branme, idswars geflecte jeser ber Bunt unter ben Siligeln bes wilsen (5uterichs.

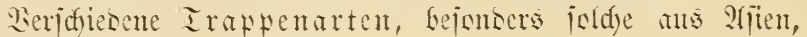
liefern brame, suntel geflecte und gejtrifte jesen, bie fegr siel

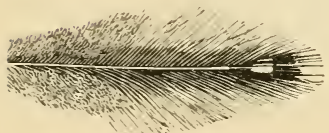

jig. 139. Şalsieser Des

eonneratshahn.

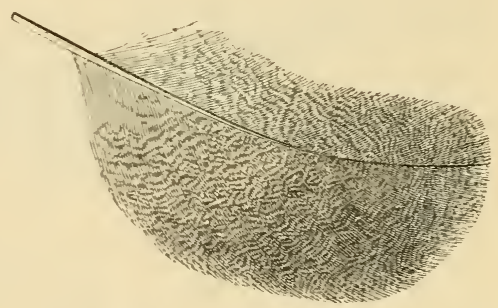

Jig. 140. Brown Mallard.

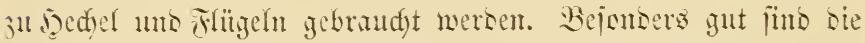
Fenern ses jorifin, Spheotides Bengalensis, Florican. Ier englijde Piante fïr bieje 2ingelarten ijt Bustard.

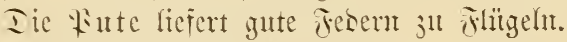

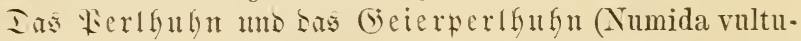
rina). Tie idnury uno weib geflecten Fescrn werben teils

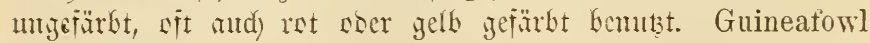
noer Gallina.

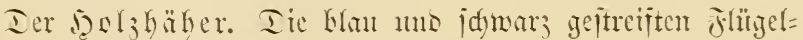
jesern werben eit als bedjel wermertet.

Eamanenfebern gelb, blan uno rot gefärbt.

Etraujen=Ed)

Bed te un linte Jebern. Jig. 141. Tiejelben Jebern vou ser redten und linten Eeite eines Bogels werfalten fid) ju

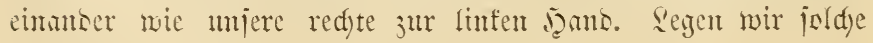




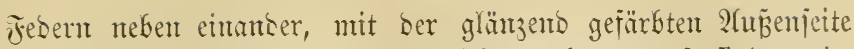
nach sben, bie matt gefärbte Sntmenjeite nad) unten, jo fünen wir,

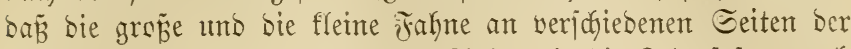
Riele ïţen, und baj jomohl bie Riele, wie bie Jeberfajern nady entgegengejestent Seiten gefrünmt jims.

Sient man eine redfte und eine linfe Feber mit bon Jimen=

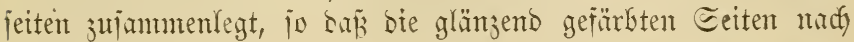
aup̃en geridgtet jims, io legen jidy sie Riele uns jajern aneinander, uno jizen inmmetrija) und mett. Regt man aber jwei redjte ober jwei linfe Jebern mit ben Jumenjeiten 3ujummen, jo freusen fid bie Riele und Feberiajern, unto pajjen nidgt auj eitander.

Man winbet jowohl ganze Febern, wie Tetle berjelben itets io an Dent Dafen an, Dan bie glänjenteren 2Ңūenjeiten mad) anjen ge= ridjtet ïns. Desfarlb ijt es notmentig, itets fïr bie redyte Geite redyte Jebern, unt für bie lunte Eeite linfe Jebern zu nefmen, bantit bie Jajern

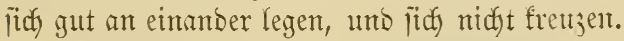

Pelzhanr, franje Edyweingmolle, Eee= humbshaar, Mishair, verjedieden gefärbt; jtm

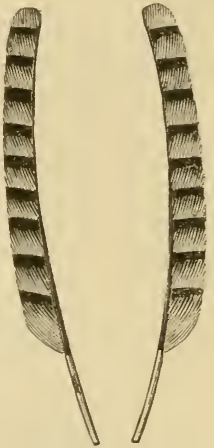

Fig. 1.11. Redjte unb rinte Flïgelfeber des Şolahähers. Rörper ber Fliegen.

Chenille, jatwarz, gelfe, rot.

Bsold = unD Silberfäben, wie fie ju ltniform= Etiffereicn vermenbet werben. Nan unterjatbet Iametta, bie aus band = förmigem Metallbledy bejteht, geipoutnene Fäben uno Edym; Metalforaft, ber aus überjponnener Eeibe bejteht, uno runb ober oval ijt.

\section{Daß Minben ber Jorellenfliege.}

1. Das Anwinden bes (5utiabens ant Dafen. Man

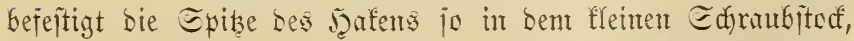

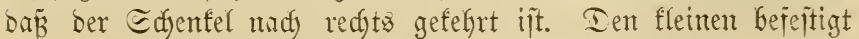

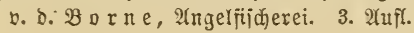


man in einem gröberen Edraubitof, ber am Arbeitotijue an= gobradyt iijt. Den Gutiaben winset man in ber bereits bejoriebenen

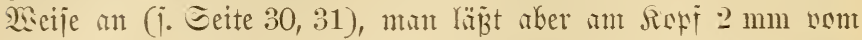

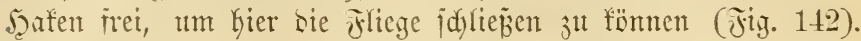

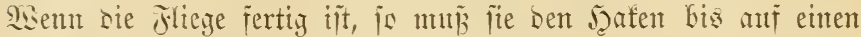
ganz fleinen Heit, ber fidbtbur bleibt, bebeden. BBenn Der Eeiben= faben angewumben ijt, fo wirb /er mit eincr Ed)leife (jig. 143)

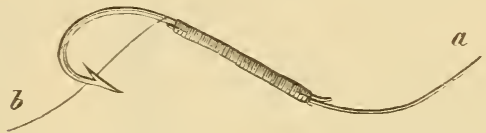

Fig. 142.

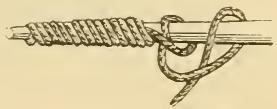

Fig. 143.

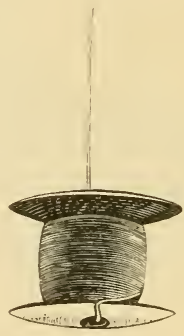

Fig. 111.

bejejtigt, und man läjt ifn, mit ber Feberzange ober mit ber fleinen jobljolle bejdwert, Gerabbängen (j. Jig. 144).

Bei ben weiteren $\mathfrak{A}$ rbeiten hält man ben Jafen, Die Tebern, Flodieise 11. Dgl. mit Daumen und Mittelfunger ber linfen Jano,

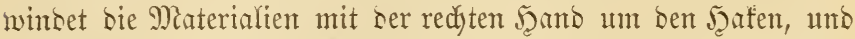

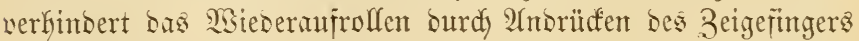
ber Yutfen janto an den Edyenfel des Jatens.

2. Winben be Rörpers. Went Der Rërper aus Jeber= fajern gemadyt werben infl, is legt man 2 ober mebrere Fajern an Den Bogen Des jatens, minbet jie mit ber Ceibe an (Jig. 145), bringt lebtere in weitläuftigen Sindungen bis babin, wo ber

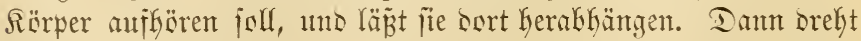
man bie Jeberfajern jujammen, wimbet jie un sen ฐaten, is Dak 
sie Wintungen jïf berübren, legt 2 -3 Mindungen ber Geibe

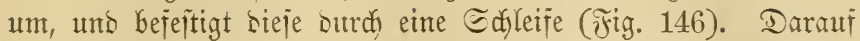

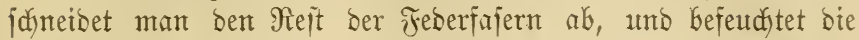

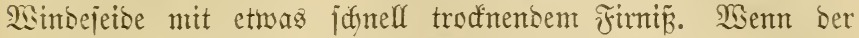
Sörper aus Flodjetbe ober Etidmolle gemadt merben jolf, is rerfährt man ganz ähulidy. Den Rörper aus Belzhaar madjt

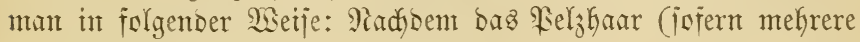

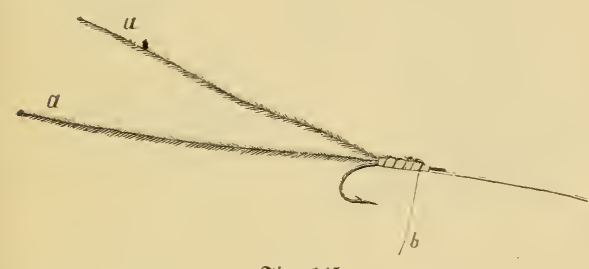

Fig. 145.

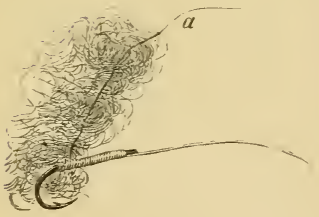

Fig. $14 \overline{7}$.

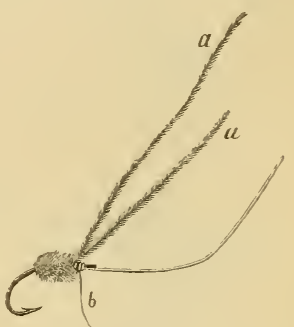

Fig. 146.

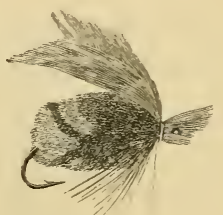

テig. 149.

2(rten genommen werben) gut gemijd tift, legt man es mit Daumen und Mittelfinger ber Yinfen 5anto um bie शrmindejeide,

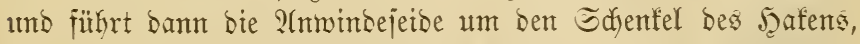

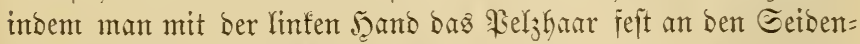
faben anjieft (Fig. 147). Sit bas 5aar furz, jo legt man bavon bei jeber Sindung neu ein. Sit es lang, wie z. $\mathfrak{B}$. Edyeinsmolle, io winbet man es entwenter mit ber linten 
Janto um ben Jaten, ehe man netes Felahaar einlegt, coer man legt bei jeber Windung neu ein, uno beidneibet ben Rörper (i. Jig. 148) nadher mit ber Edhere. Iurdy (Einlegen ver= idfiebenfarbigen Reljhanres fann man bem Rörper abmedjelnto Farben geben, 3. B. jumary uno gelb, wie in jig. 149 uno fo Den Rärper einer நummel nadybilsen.

3. Piadfatmug ber Beine. Dies geidjeht gewähnlidy

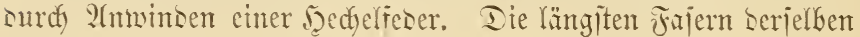

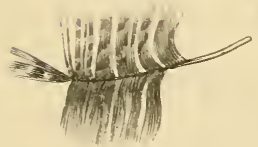

Fig. 150.

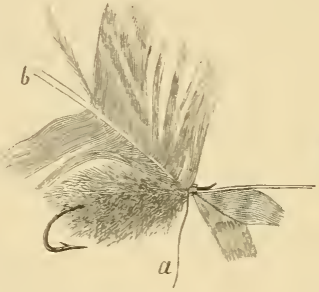

Fig. 151.

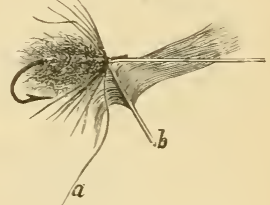

స̂ig. 152.

inflen ungefäfr fo lang jein, wie ber jafenjuenfel. Wan itreidyt bie jeberiajern io juriuf, wie es jig. 150 jeigt, unt bejejtigt bie Epibe ber Feber b Surd ein parr Sintungen bes Jabens a (Jig. 151). Daraui fajt man sen Riel b mit einer jweiten Jeserjange, tnto mintet bie Feber um ben Jafen, inbem man bei jeber Simoung bie Jeberfajern mit ber linten 5and jutüt

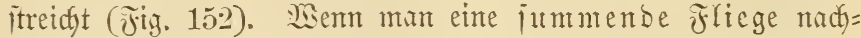
afmen will, io wirb bie Feber $6-8$ Mal ober sifter umgetwumben,

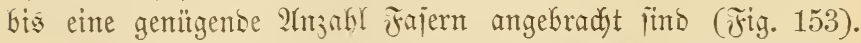
Man wintet barauf sen Riel ber Feser mit

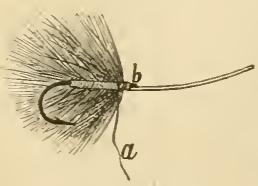

సig. 153. bem Faben an ben Jaten an, madyt cine

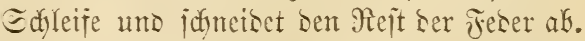
Iarauf wirb bie frliege burdy mefrere 2 sin= Dungen und Edjleifen, bie man mit ber Seibe madjt, rollentot, leţtere mit Jimin bejeudutet und afgeidynitten. Der Jafen iit 
mun bis zum Ropf fajt ganz beoceft. Sienn man eine geflüg gelte Friege nadyen will, fo legt man bie sectelfeber nur zmei= bis oreimal um ben 5ृafen, um nidyt jut viele Beine barjultellen,

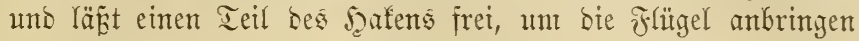

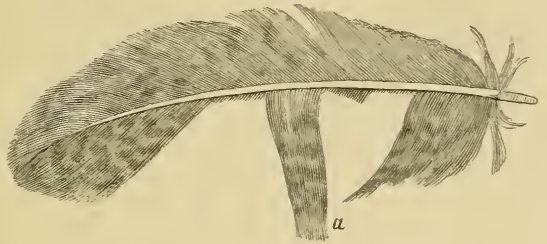

ริig. 154.

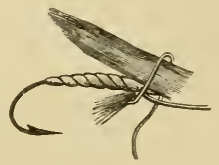

Fig. 155.

zu tönnen. Man bejejtigt bie Feber in ber bereits bejdriebenen Seije und janneibet bas Heberfliujizge ab.

4. Das Antwinben ber Flügel. Diejelfen werben ge=

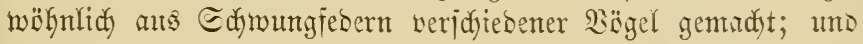

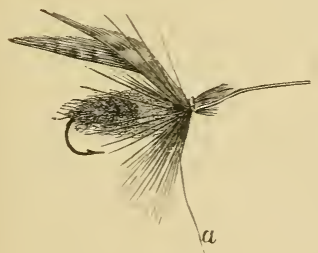

Æig. 156.

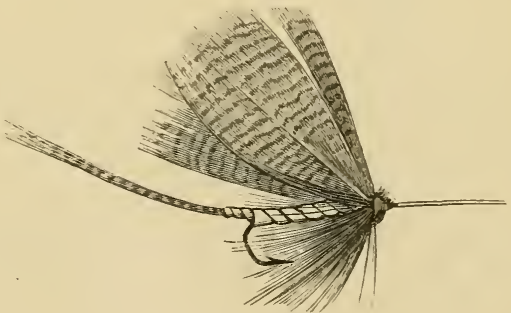

₹ig. 157.

zwar juneibet man ben redyten uno linfen Flïgel ber Ffiege aus zwei forrejpondierenden rechten und linfen Edymungfebern bes Bogels, bamit bie Flügel ber Ffiege inmmetrijdy unb nett gejtellt merben fönnen. Ulnjere Fig. 151 joll vermidjaulichen, in weldyer

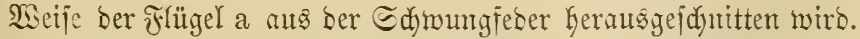
Die Flügel ber Fliege follen ungefäbr jo lang jein, wie ber 


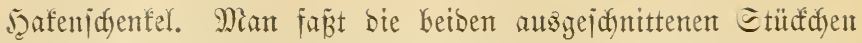
Der Feberfafnen feit zuwijden Daumen uno Mittelfünger ser

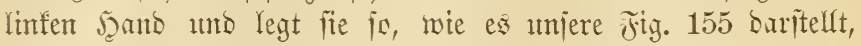

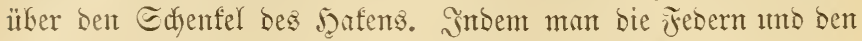

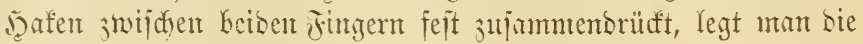

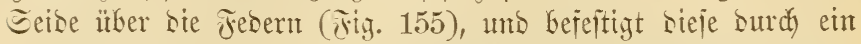

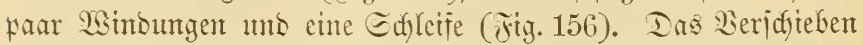

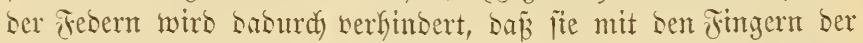

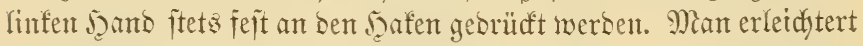

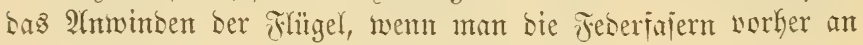

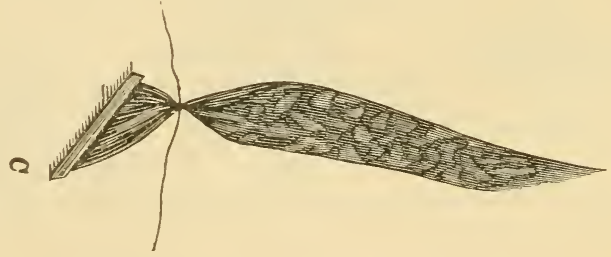

Fig. 158.

Der betreffenten Etelle surdy einen Faben zujammenjanitrt.

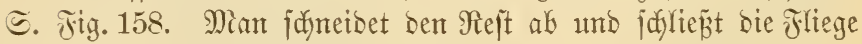
in ber bereits bejuriebenen Siseije.

In ähnlidber Meije verfährt man, wenn bie Frügel aus żmet ganzen Feberdien beiteben, mie es $j$. B. Kei Der Maifliege noer ber Francis ser Fall ijt (Fig. 157 und Fig. 183).

Anbere Methode Forellenfliegen ă winden.*)

Die folgende Methose wirb hauptiädifich angemendet, $九$ m idymimmente Fliegen zu mintent.

1. Befeitigen Der QHminbejeibe aut פafet. Machbem ber Safen in Dem Edyraubitodfe Kefeitigt unb bie 2rnwinbejeibe gut

*) Floating Flies and How to Dress Them by F. M. Halford. London 1886. 


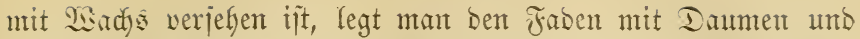

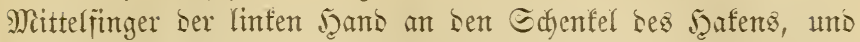

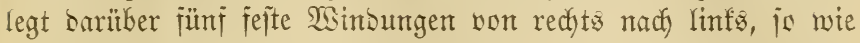

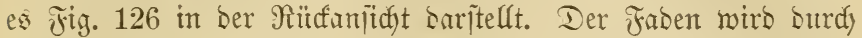

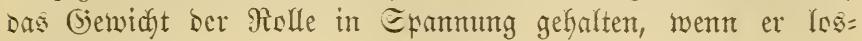
gelaijen wirb.

2. Dą Anwinden Der Sedjelfeder. Piadjeem man bie

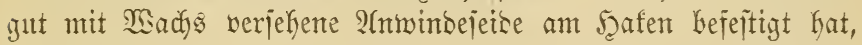

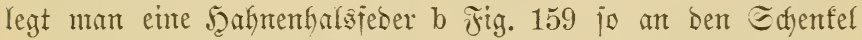

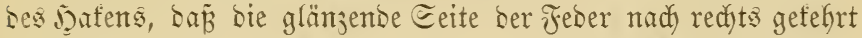
ijt, und mindet ben Sitel ber Feber c am Echenfel mittelit ber Eeibe d an. Tarani nummt man bie Epize ber Jechelfeder b in bie Rinjette und wintet jie jo un ben Edyenfel, baj bie

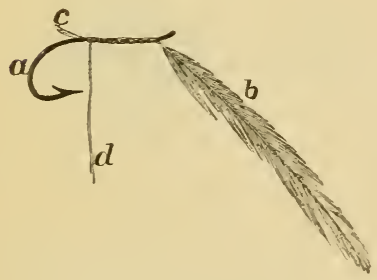

テ̛ig. 159.

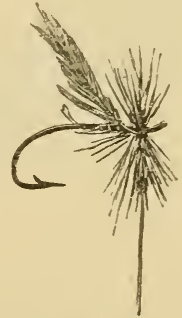

Fig. 160.

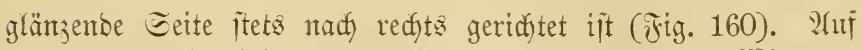
ber untern Eeite bringt man babei bie Singette bei jeber Winoung möglidjit weit nady reçts vormärts, weil bie Fajern bant viel belier gejtellt werben. Wenn vier bis fün Sindungen umgelegt finto, jo fübrt man sen Eetbenfaben ütber bie Sechelfeber in mefreren Windungen mady bem vorberen (Enbe bes Satens und

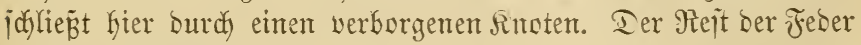
wirb abgeidynitten.

Senn man eine Fliege mit ganz Gefaartem Rörper winben will, jo jüfrt man bie நechelfeber nom Sicpi = bis zum Edymany= 
ente über ben ganjen Rïrper ber Fliege (j. Fig. 161), mobei bie Wintumgen am Soff näber an emander gelegt werben, wie anf ben Rïrper. Darau fülgrt man bie Ceibe in geitrectiten

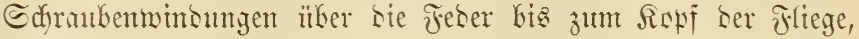
fdyliep̃t fier mit einem werbargenen finsten mo idyncibet ben Rejt ber Feber ak. Iurdy bas lleterminten bes Eetbentiaben

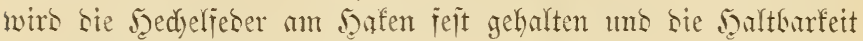
ber Fliege boseuteno erkibt.

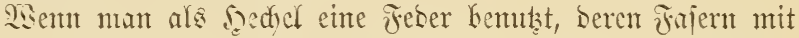
Flaum befletbet jims, uns sie baburdy an einanter haiten, is

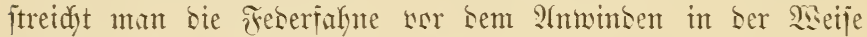
zurücf, wie es unjere Fig. 162 zeigt. Dies gilt 3. B. won Bnutt= febern bes Regentipeifers uno ähnlidyen jesern.

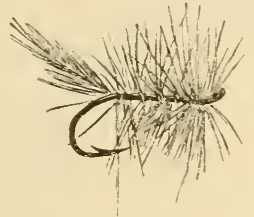

Fig. 161.

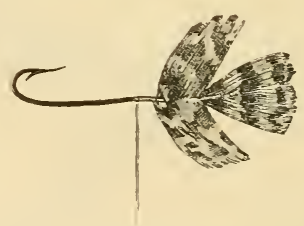

ซig. 162 .

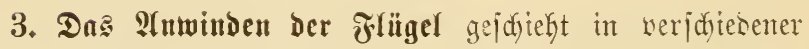
Sisetje:

A. Man fanteiset ans zwei forrejpensierenden Edyung= febern eines redyten uno linfen Fitigels won ben Feberfahnen

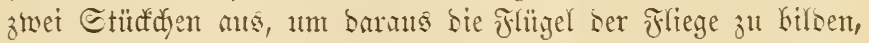

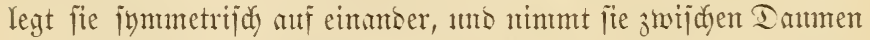

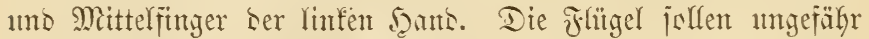
fo lang werten, wie ber Edyenfel bes batens ift. Darauf Gringt ntan bie Flügel ba auf Den Scafenidyentel, wo fie angemumben werben fiflen, srïtft bie beisen Finger, weldye sie Flügel Galten, fejt zujammen und an ben Edhenfel, fringt bie 21mminbefetbe

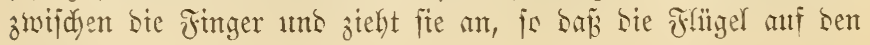




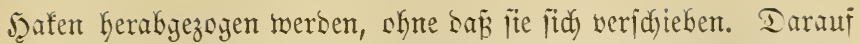
legt man bie Eeibe nod) bret= bis riermal an berfelben Etelle feft un und entfernt erit bann bie Finger ber linfen ந̧ano, um zu jeken, wie bie Speration gelungen ift. Die Etellung ber Flügel muj jetzt jo jein, wie es unjere Fig. 163 zeigt.

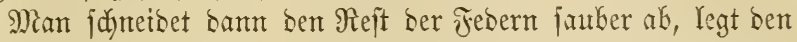
Faben nod) ein paarmal wor und finter ben Flïgeln um utho id)ließ̈t burdy einen verborgenen Sinoten.

B. Nin nebmen an, baf bie Flitgel aus zwei ganjen Febern gemadjt werben follen. Man nimmt biefelben in ber bejurtebenen

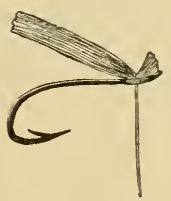

Fig. 163.

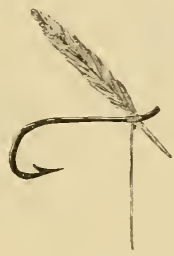

ซิig. 164.

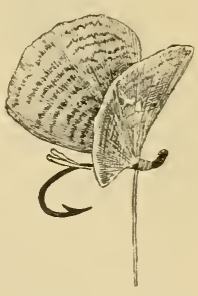

Fig. 165.

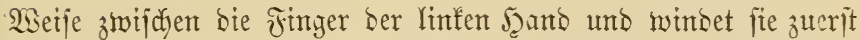
fo an, wie es unjere Fig. 164 darftelft. Dann legt man bie beisen Riele juritud an ben Jafen und winbet fie fo an, wie es unjere Jig. 165 zeigt.

C. Eehr gut ijt bie folgende Methode, mobet bie Flügel jefr. feit jitzen uno jebe beliebige Stellung erhalten fïnnen. Buerit

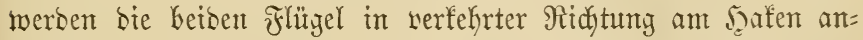
gemunben (Fig. 166); barauf trennt man bie bciben Flügel mit bem \$ritemen und minbet alsbann bie Eeibe abmedjelno und bem Besürfnis entipredyen bald vor und bald finter ben Flïgeln um ben F̧afen; ferner legt man fie abwedjento unto fre euz= weije zmijdien ben Flügeln burdb), eimnal vor bent redften und finter bem linfen Flïgel, und bann umgefefrt, Ginter bem red)ten 
uno nov bem linfent Flügel burdy. Tie Seise umidylungt alfo

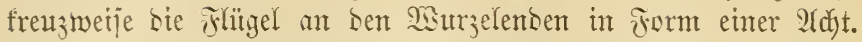
Je nachbem man bie Eeibe roun roer hinten an bie Flügel an= brïct, fann man bie Etelfung berjelben veräıtoem; man fann ifinen leidyt eine iteile nber fladje Eteffung getien. Tie Eeibe sarf aber mur an ben Kisurjelenden, nidyt an ben Fahnen ber jlïgel angelegt werben, weil jonjt sie Jeberiajern in llumoromung gebradyt werben.

Buleb̧t werbent bie Rejtenten ber Flügel jo bejdyitten, wie es bie fig. 167 jeigt.

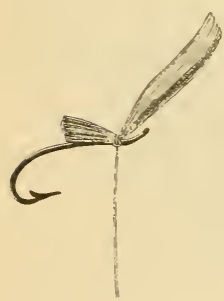

§ig. 166.

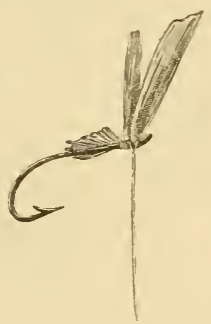

Jig. 167.

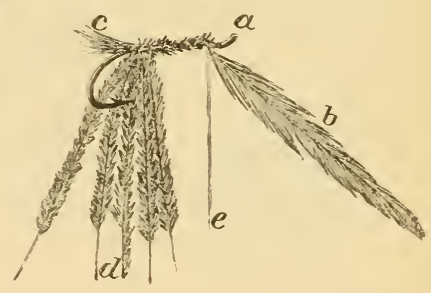

Fig. 168.

\section{Dą $\mathfrak{A}$ mwinden Des fïrpers und Die Bolfendung Der}

Fliege. Man unteridyetbet: geflügelte uno ungeflügelte filiegen,

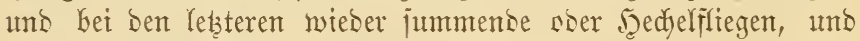

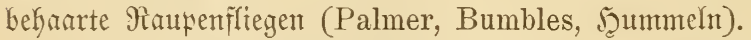

A. Ias Man winbet bie Jedjelfelber b Fig. 168 is am Jaten an, wie wir es obent beidjreben Gaben. Darauf merben vier voer fünf Fajern d won eiter Edywanjeber bes Rijaubahnes (ober anderes Material, aus bem ser Rörper ber Jitige gebilset werben joll) am நafent angemunben, ber Sdjwanz $c$ ber Fliege wirb ange= bradt, unb bie Simbejeibe nady bem Rapiente ber Fliege e ge=

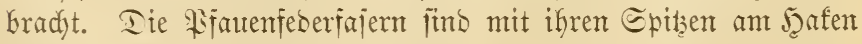
befeitigt; ite wersen an sen unteren (Enben in einer Feberzange 


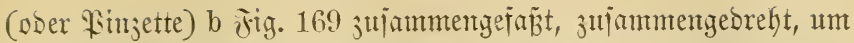
Den Jafen wom Edymanzende zum Ropfende portichreitento um=

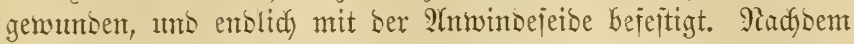

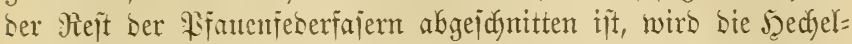

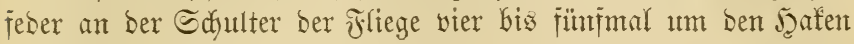
gemunber. Tant wirb bie Geibe won finten nadf vorn über bie Däfelfeber gewumben unb bie Flicge natge am Sefre burch eimen verborgenen Sincten geidblofien. 厄. Fig. 170.

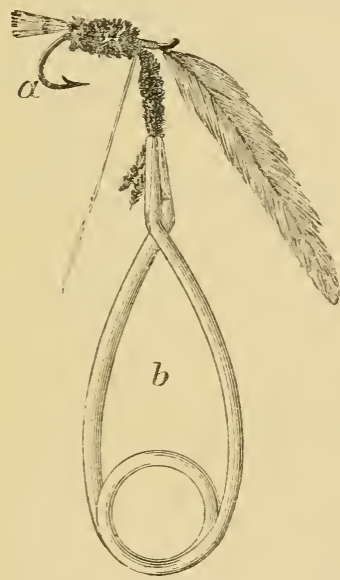

jig. 169.

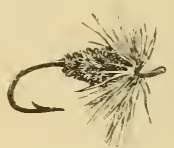

Fig. 170 .

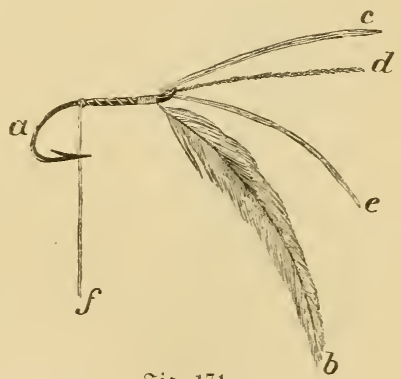

Fig. 171.

B. Das Minden einer Mapenfliege mit befarten Rörper (Palmer, Bumble, joumntel). 2Tm Ropfende merben 3ugleidy Flodfeibe c Fig. 171, Golbjaben e, bie Ffautenjumert= feberfajer $d$ und bie J̧edyelfeber $b$ angenumben. Die 2 Unwinbe=

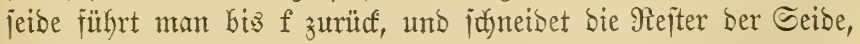
Febern und Des Goblofabens ab. Dann mindet man bie Flocfjetbe um ben Jafenidjenfel, bejejtigt jie bei $f$, barauf folgt in weiten 
Edyraubenlinien ber Goldfaben, bie Fifauenfeberfajer unb bie

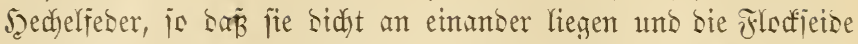

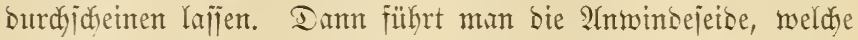
biejelke Farke hat wie bie Flaffeise, über bie Fliege vom Edymanz=

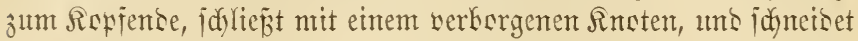
sie Refter won Febern, Setibe unb Golbiaben ab. Iie Fitiege hat bie Form unjerer Fig. 172.

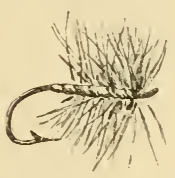

Fig. 172.

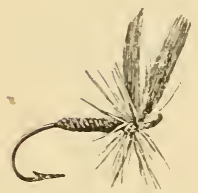

ซig. 17 .

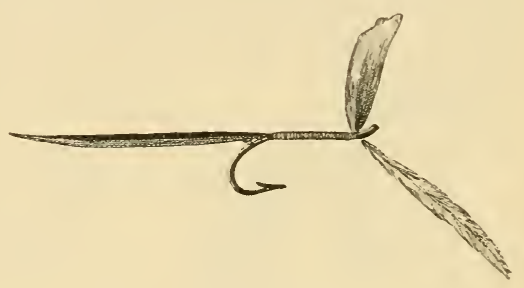

₹ig. 173.

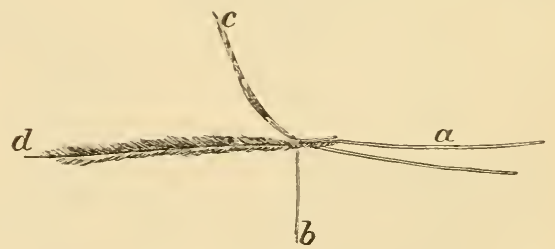

Æig. 175 .

C. Geflügelte Fliegen. Man miniet ant Ropiente bie Flügel unb bie Jechelfeber an, und am Edumanzente bie Feber= fajern (ober jonjtiges Material), ans welden ber Sïrper gebilset werben jolf (Jig. 173). Iie 2lmwindejeide bringt man bis bidgt Ginter bie Flïgel. Darauf formiert man ben Rörper, inbem man bie Feberfajern bis sid)t Ginter bie Flügel um ben Jaten= jdenfel wintet. Man fann bas Brutidyilo nadjbilden, wenn man bas Rërpermaterial in ber weiter sfen bejdrtekenen SEcije

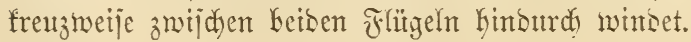


Darmuf wirb bie Jెedyelfeber Ginter ben Flïgeln umgenunbent, mobei man bei jeber Windung bie Pincette auf ser ltnterjeite möglidyit weit redfts nady worne bringt. Enolidy legt man bie Irminbejeibe in mefreren 2 sindungen itber bie jechelfeber und

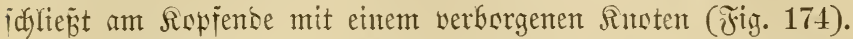

D. Fliegen, beren Rörper von bem Jafen getrent jins. Der Rïrper wirb um eine Edyeinsborite in gemöbnlidyer Weije genumben unb bann an bem Jafen bejejtigt. Man biegt bie Edymeinsborjte a Fig. 175 in ber Mitte $\mathrm{tm}$, fo baj fie sappelt zu liegent fommt. Dann befejtigt man bie 2Anwindejeibe bei $\mathrm{b}$ an Edyanzente uno winbet bort bas Material d für ben Rörper unb ben Edyanz c an.

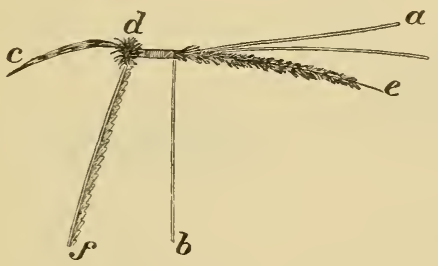

Jig. 176.

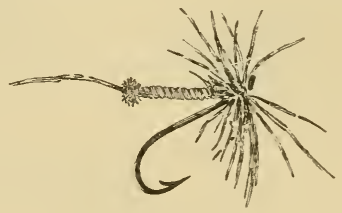

Fig. 177.

Imeijenfliege. Wir nefmen als Beifpiel bas Winben einer 2 Imeifenfliege unb jwar fer unter bem Namen Horrocks befannten Fliege. Der હdjwanz c Fig. 176 bejteft aus Graunen Rutenfebern, ber Edfronzfnotend aus ein paar Wintungen cinter Riautenidneif=Feberfajer. Der Rörper nirb burd ben Feberfiel $f$

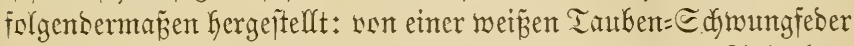
murbe bie Fafne abgeitreift und bie Fajern nafe am Riel abge= idynitten. Diejer Riel f nitro barauf in ber bejariebenten Weije um bie Borite gemidfert und jo ber Rörper ber Frliege gebilbet. Man jetst barauj ben Rörper rittlings zwijden ben beiben Beritenenden a auf ben Angelfafen und winbet ifn mit ber Seibe b feit an. Die Berbindungsiftelle ummintot man mit bem 


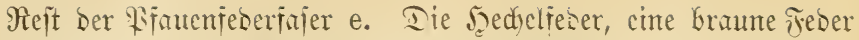
vom Sïrper einer Fite, wiro in gewïhnlider Sieije angebradyt und bie frliege hat num bie Giejtalt unjerer Fig. 17 .

\section{B. Die fünfltiden forellenfliegen}

find jum Teil Rachbiloungen won Injeften, bie fich bäufig am Wafjer finten unb wen ben jifdyen gefreflen werben. Tie bäufig= ften fint folgende:

\section{Coleoptera, Sị̈̂r.}

1. Staphylinidae, Rumbecfer, Quedius haemarrhous. Peacock-fly, Little Chap (ङ. Nir. 9 unjeres Berzeichniljes fïmptlidjer Filiegent.)

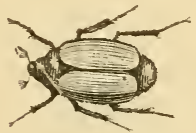

Fig. 178. Эunifüier.

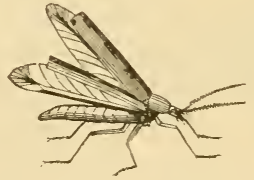

Tig. 179. Fern Fly.

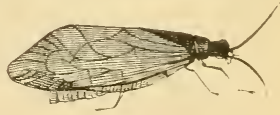

Jig. 180. Alderfly.

2. Scarabaeidae, Silatthornfiffer. Amphimalla solstitialis, Junifüfer (Jig. 178). Coch-y-bondhu. (ङ. Pir. 7 Desิ Sjerjeidutitifes.)

3. Telephoridae, SEeidfk̈̈ter. Telephorus lividus, cit srangerster Räfer, Fern-fly. (F̈ig. 101.)

\section{Hymenoptera, şattfilitgler.}

1. 'Tenthredo viridis, grüne Blattweșp. Yellow Sally. (2ir. 71 bes Sicrzectuntifes.)

2. Formicidae, 2Imeijen. (Ox.121-123 beș Berzcidnifies.)

\section{Neuroptera, Niekrifiigler.}

Erite ll ntcroronung: Flattflügler, Planipennia.

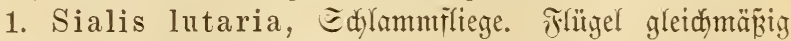

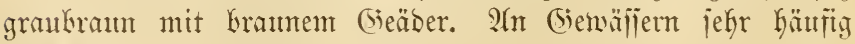

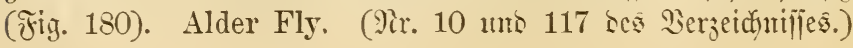


3weite llnterorsung: Trichoptera, Belzflügler. Phyrganidae, Jrïhlingsffliegent.

1. Limnophilus striatus, Grannom. (अir. 51 bes Berzeidynifies.)
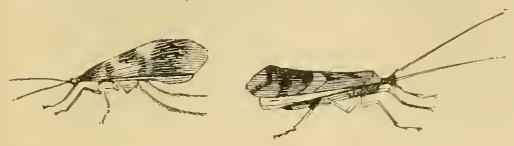

Fig. 181. Santofliege. Fig. 182. Bimmetffiege.

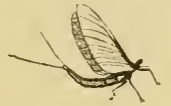

Fig. 184.

Pale Evening Dun.

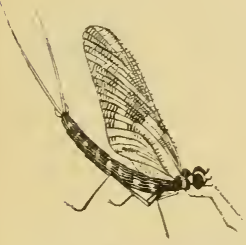

ケig. 186. March Brown.

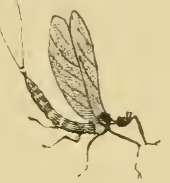

Fig. 185. Red spinner.

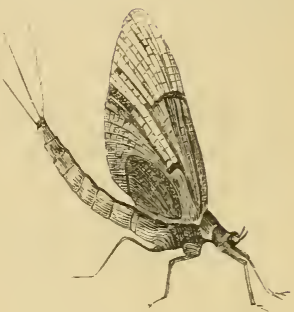

Fig. 183. Maifliege.

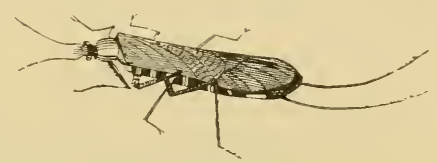

テig. 187. Steinfliege.

2. Limnophilus flavus. Sandfly, Ganbfliege. (Fig. 181. Nir. 61 umjeres Berzeidynifjes.)

3. Limnophilus stigmaticus. Cinnamon Fly, Zintmet=

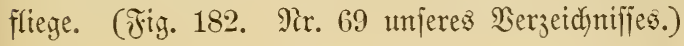

\section{Orthoptera, Beradfiiglex.}

\section{A. Ephemeridae, (Eintagofliegen.}

1. Ephemera vulgata, Maifliege (গ̇ig. 183), bas unvoll= fonmen entmicfelte Injeft. Green Drake. (Nr. 100 bes $\mathfrak{B}$ er= zeid)nilles.) - Das volfitündig entwidelte Imieft Gray Drake. ( Mir. 101 des Serzeidynifies.) 
2. Clo ë on pumila, Pale Evening Dun. (9ir.67. Fitg. 184.)

3. Cloëon auliciformis. lltursigebilket, Sky-blue. (Nir. 46 m 99.)

4. Clo ë on d ip tera. Ilnentwicfelt Ironblue Dun (Nr. 14). 2rusgebilset Jenny Spinner. ( Nir. 28, 35.)

5. Potamantus rufescens (jig. 185). Hnumgebildet Blue Dun. (Nar. 43 unjeres Nerzeidnifics.) Das austgebilbete Sinjeft ijt Red Spinner. (ङ. 2ir. 88.)

6. Baëtis longicauda (Jig. 186). Unnuggebilibet MarchBrown (i. Mir. 59, 60). Ifusgebildet Great Red Spinner.

7. Baëtis flava. Lnumsgebilict Yellow Dun. (Mr. 45 uto 98.)

8. Baëtis obscura. July Dun. (Vir. 4.)

9. Baëtis fluminum. August Dun. ( $(2 r .109$.

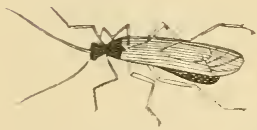

Tig. 188. Red Fly.

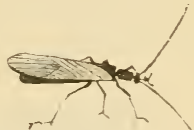

Nig. 189.

Willow Fly.

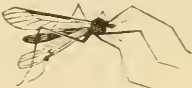

F̌ig. 190.

Gravel Bed.

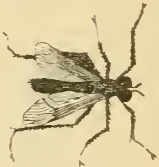

Fig. 191. Hawthorn Fly.

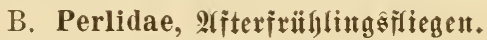

1. Perla bicaudata. Stone-fly. Etemfliege (Jig. 187. Pr. วิ bes :

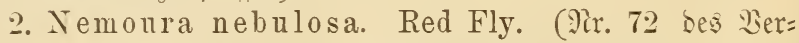
jeidjuijes. Jig. 188.)

3. Nemoura fuliginosa. Willow Fly. (jig. 189. 9ir. 41 bes sergeidyuifies.)

\section{Lepidoptera, Edjutterlinge.}

Berjojiesente Mistten. (Mir. 27, 76, 86.)

\section{Diptera, 3weiffïgler.}

Tipulidae, Étrafer.

1. Anisomera obscura, Gravel Bed. (Frig. 190. Mir. 38 Les :ierjeidunifies.) 
2. Bibio Marci, ந̧aarmüffe, Hawthorn Fly. (J̃ig. 191, Nr. 25 Des Berjeidjnifies.)

Muscidae, eigentlide Fliegen.

Scatophaga stercoraria, Imtgrfiege, Cowdung Fly. (9r. 83, 92.)

Leptis scolopacea. Edynerienfliege, Oakfly. (Mr. 105 Des Serzeidnifīes.)

\section{Empidae, Ianjiliegen.}

1. Ramphom yia aethiops, Edynabelfiltege, Black Gnat, Tailey Tail, Black Spider (Stewart) žu ungeheuren Miengen

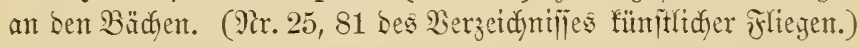

\section{Künjtliche fliegen für bie forellen= uns 2lejकृenfficherei.}

Die Bafyl ber füntlidyen Fltegen, weldye in ber Frrellen= unto Pejdenfijderei gebraudjt merben, ijt bei weitem nidjt is

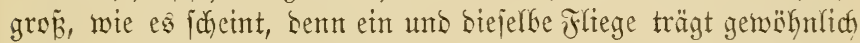
mekrere Nanten. Sid babe fier bie Fliegen nady ber Farbe ber Rörper gecromet.

\section{Sinfaltis =lleberid t.}

(I ie Bahlen beziefen fidy anj bie forthujenbe 9iumerierung.) भloam=Fliegen 39, 63, 64, 70, Black Gnat 25. $78,79,81,84,94,95,96,103$. Black Midge 25. Alder Fly 10, 11 7 .

Alexandra 119. Amber Fly 75. Ash Fly 105. Ashy Dun 29. August Dun 109. Autumn Fly 6. Bank Fly 84. Barm Fly 68. Big Dun 94. Black Ant 121.

Black Palmer 26 . Black Spider 81. Blue Bottle 23.

Blue Dun 43.

Blue Midge 32, 37.

Bracken-Clock 7 .

Braune Miotte 76 .

Brown-bent 90.

Brazil Fly 72.

Brown Drake 59.

Brown Moth 76.

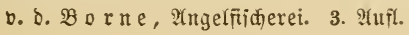


Brown Palmer 80.

Brown Skipjack 52.

Brown Spinner \&.2.

Buff Dun 103.

Bumbles of Derbyshire 124

Caddis Fly 66.

Cadow 100.

Cannon Fly 105.

Caperer 77.

Cinnamon Fly 69.

Coachman 4.

Coch-y-bondhu 7.

Cocktail 43, 100.

Cowdung 83, 92.

Crossing Brown 79.

Cuckoo Dun 57.

Dabby 55.

Dark Blue Dun 44.

Dark Bloa 112.

Dotterel Hackle ti.

Downhill Fly 105.

Down-looker 105.

Early Dark Dun 33, 43, 56.

Eden Fly 64.

Edmead 115.

Emerald Fly 16.

Erlenfliege 10, 117.

Evening Bloa 28.

Tamfrautfliege 101.

February Red 7‥

Fern Fly 101.

Tetueriflege 8.

Fire Fly 8.

Flat Yellow 71.
Francis Fly 11.

Frog Hopper 90.

Furnace Hackle 104.

(S) (iiifwurm 3.

Golden-eyed Gauze Wing 19.

Golden Palmer Hackle 14.

Golden Plover Hackle 18.

Good General Palmer 91.

Govermor 6.

Grannom 51.

Gravel Bed 38.

Grey Drake 101.

Grey Housewife 53.

Grey Spider 30.

Great Dark Drone 31.

Green Drake 100.

Green Midge 17.

Greentail 51.

Greenwell's Glory 2.

Grouse Hackle 89.

(S)rün ㄲöcfe 17.

Hammond's Adopted 52.

Hares Ear 49.

Hares Ear and Yellow 43.

Hares Fleck 43.

Harry-Long -Legs 55.

Hawthorn Fly 25.

Hazel Fly 7.

Ђeujdjrecte, tümitl. 22.

Hofflands Fancy 111.

Horrocks 123.

House Fly 23, 25.

Saction=fliege 112 .

Jenny Spinner 28, 35. 
Indian Yellow 70.

Iron-Blue 35.

Iron-Blue Dun 28.

July Dun 44.

Jumper 90.

Suntifäศอ 7.

Sutfmittfliege 83, 92.

Sutidyer 4.

Light Dun 95.

Little Chap 9.

Little Dark Bloa 35.

Little Dark Spinner 116.

Little Gosling 97.

Little Iron-blue Dun 35.

Little Pale Blue Dun 41.

Little Sky-blue 24.

Little White Spinner 28.

Little Yellow May Dun 62.

Local March Brown 78.

Mackerel Governor 6.

Maiffliege 58, 62, 100 .

Narch Brown 59, 60.

Harlow Buzz 7.

May Fly 58, 62, 100.

Mealy Cream Moth 86 .

Verlin 44.

Mündfenter Grassfüpfer 22.

Maltby 74 .

Needle Brown 48.

Oak Fly 105.

Old Beson 41.

Old Joan 72.

Olive Fly 20.

Orange-brown 105.
Orange Dun 103.

Orange Fly 107.

Orange-headed Dun 39.

Pale Evening Dun 67.

Partridge Hackle 54, 93.

Peacock Fly 5.

Peacock Palmer Hackle 12.

Pearl Drake 28.

Quill Gnat 31.

அaupen 2, 26, 114.

Red Ant 121.

Red Fly 72.

Red Hackle 1.

Red Ibis 120.

Red Palmer 2, 114.

Red Spider 88.

Red Spinner 109, 113.

Red Tag 3, 15 .

Robin Hood 36.

Sailor 102.

Sanded Dun 61.

Sand Fly 61.

Sand Gnat 38, 49.

Edymeinffliege 23.

Edyneisers Jeenjarede 22.

Edfneprenfifiege 105.

Sedge Fly 65.

Shade Fly 23.

Shamrock Fly 41 .

Shell Fly 51.

Shorn Fly 1, 3, 7, 13.

Skip Jack 77.

Sky Blue 46, 99.

Small Black Caterpiller 25. 
Soldier 102.

Soldier Palmer 114.

Spanish Needle 48.

Spider Fly 38, 49, 124.

Spring on Dotterel Dum 96.

Eteinffiege 58.

Stemartfliegen $30,50,81,87,88$. Stewart's Winged Fly 50.

Stone Fly 58.

Stream Fly 40.

Tailey Tail 81.

Wasp Fly 108.

Watchett 35.

Water Cricket 105.

siseisenfliege 41.
Nהcije Mrotte 27.

Sipe 108.

Whirling Blue Dun 85.

Whirling Dun 42, 56.

White Moth 27.

Wickham's Fancy 118.

Willow Fly 41.

Withy Fly 41.

Woodcock Fly 105.

Woodcock and Yellow 87.

Wrentail 73, 90.

Yellow Dun $45,98$.

Yellow Sally 71.

Bimmetrfiege 69.

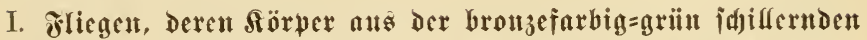

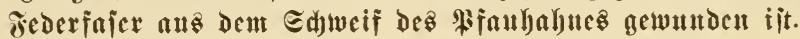

1. Edulterbä̈felfeber, rote Dahnenfulsfeder. (Fig. 152, こ.61.) Tie Jiege heijt Red Hackle; Shorn Fly.

2. Rampenfliege. Die rete Dorfunthalsfeber ijt ïher sen ganjen Rërfer genuntion. Red Palmer.

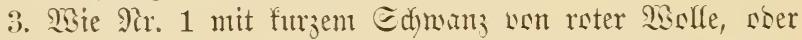
ciner flemen jdurfadyreten Jeser. Shorn Fly, Red Tag, (s)iith)= iwurm.

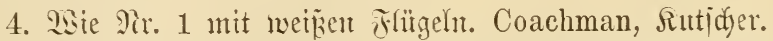

5. Sice $2 x, 1$ mit Flügeln ans einer bunflen Edywumgeber bes Ctrar noer jolzhäher. Peacock Fly.

6. Sdymatnoten won srauge Flacfjeide. Rörper gold gerippt, ment bie Fliege groí ijt, fontt wie bei $9 x$. 1. Baine

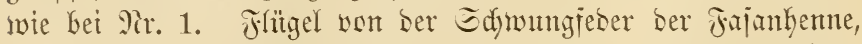
nber einer granten Edymanjeber bes ßebfufn, sder einer weifen fdymar; putuftierten Feber von ber Brujt bes wilden Enterich) (Gray Drake F(ïgel). Governor, Autumn Fly, Mackerel Governor. 
7. Eabulterfechel, eine Ejenjeber (eine rote jafnentalsjeber mit jafwarzem Rern). Bisweilen ifit ber Rörper mit roter Floof = jeibe gerippt, ober bie bronzene Pifanenjeberfajer ift mit jafwarzer Etrauffeberfajer gemifdyt. Coch-y-bondhu, Marlow Buzz, Shorn Fly, Hazel Fly, Bracken-Clock, Junifäfer.

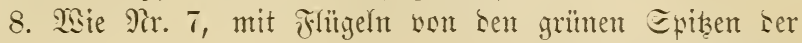
Edmertiebern aus bem Edyweif Les Rinutahns. Fire Fly, Fenteritiege.

9. Edyulterfectelfeber, grau. Little Chap.

10. Edyulterbedyeljecer, idywarz ober rojtbrau; Flïgel braun und jdwars gefledte Edyungieber bes Fajantafnes. Alder Fly; Errlenfiliege.

11. Rörper mit fupierroter Floffieibe ge= rippt; Beine und Flügel won mittelgrauen Jenlsfebern bes Juthns. Francis Fly. Jig. 192.

12. Rörper mit Eilberfuben gerippt; Sirperbecter unto Edyulterbechel won einer

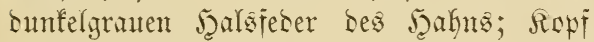
won jdyarladjrotem Misfair. Peacock Palmer

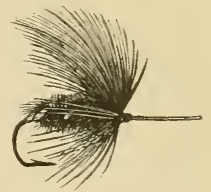

Fig. 192. Hackle.

13. Rurzer Edywantz von orange Madle; Edyulterbedyel Eunfelgrane J̧afuentyalsafeder. Shorn Fly.

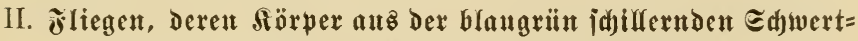

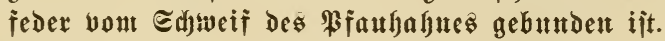

14. Rörper mit (joldofaben geript; Rörper= uno Edylter= Kedyel won eimer rotbraument Feser. Golden Palmer Hackle.

15. Rutrzer Edywanz won jdyarladyroter Wiolfe ober einer

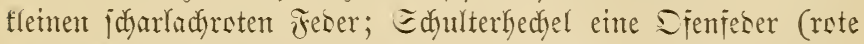

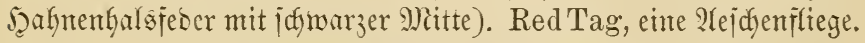

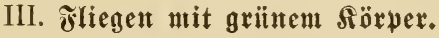

16. Rörper von imaragogrümer Filocjecioc; Edynanz graue Jeberijern; Beine bunfelrot; Flügel won ber Edymungfeder ber \&ercje. Emerald Fly. 
17. Rörper apjelgrüne Floffeise; Sdulterbedel lidut jither= grau, junmens; gant; fleine F̂liege. Green Midge, grüne Nï̈cfe.

18. Rärper gelfgrüne Flodfetbe; Beine Aüdfenfeder Des (5) old = Megentfeifer, juntments. Golden Plover Hackle.

19. Rörper lidjt gelfgrïne Flocfjetbe; Beine lidytgrau; Flïgel son einer surdjidfeinentsen lidftgrün gefürbten Jeber. Goldeneyed Gauze Wing.

20. Rörper sunfel olinengrünes Mohair; ïlberner Edywan:= tnoten; Cdymanz graue Brujtfeber nom (Enterid); Flügel Edymung= feber bes Etrar. Olive Fly.

21. Rörper suntel slivengrüne Flodieise, mit jemem Bols= faben geript; Beine eime Sienfeber; Flügel won ber Eatwung= feber ber sialsidmefie. Greenwell's Glory.

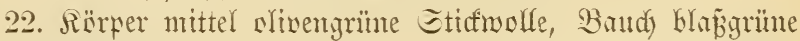
Etidfmolle, beibe über ein Etüfdyen Jiskly gemumben, geript mit srange Eeibenjaben; Beine uno Fühler Friern won ber Edyung= feber bes blauen 2 trarama=Papageis, flügel non ben Edywumg= feberm bes Glaten Trarama=Papages, sben graugrün, unten

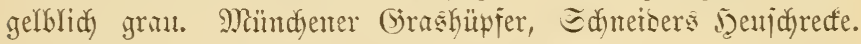

\section{Sliegen mit blatem Sörper.}

23. Rörper lidytblaue jocfieide, braun gerippt, aud mobl

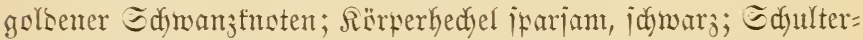

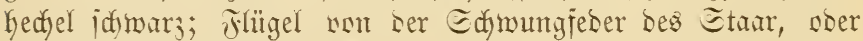
funmens. Ränge bes ฐ̃afen $12 \mathrm{~mm}$; Blue Bottle, House Fly, Shade Fly, Edymeiñfliege.

24. Rörper fimmelblaue Flodieide; Edyulterhedyel jdyarz; Flügel won ber Edynungieber bes Etrar; gantz flein. Little Skyblue.

\section{Fliegen ntit idjwarjem $\mathfrak{A o ̈ r p e r . ~}$}

25. Rërper von jdwarjer Jeberfajer bes Etrauß; Bente

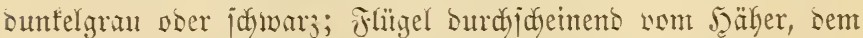
Staar ober ser Serdye. (jroje Fliegen: Hawthorn Fly. Mittel= groß̈e Fliegen: House Fly. Rleine Fliegen: Black Gnat, Black Midge, Small Black Caterpiller. 
26. Rörper von idwarzer Etran̈feberiajer, aud mohl mit bronzejarbiger Bjautenjeberfajer gemijat, gerippt mit Golo ober Eilberfaben; Rërper = uno Edjulterkedyel jdmarz ooer brimmet. Eime ßaupenfiliege. Black Palmer.

\section{Flicgent mit weip̌ent Sörper.}

27. Rörper won weiper Wolle, Mohair ober Pelzhar, bicf;

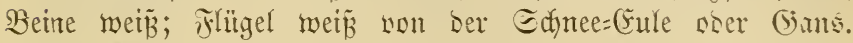
White Moth, weize Miotte, Madytflicge.

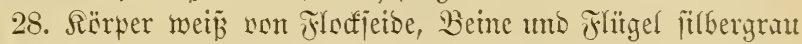

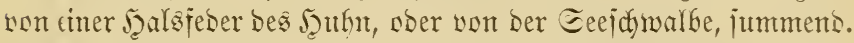
Jaater $8 \mathrm{~mm}$ lang. Jenny Spinner, Evening Bloa, Little White Spinner, Pearl Drake. Die Fliege entiteft mis ber Iron Blue Dun.

\section{F̧iegen mit gratent STöruer.}

29. Sïrper filbergraue Flocfjeide; Beine lidgtgran; Flügcl lidyt, Durajidjeineno. 5aafen $10 \mathrm{~mm}$ lang. Ashy Dun.

30. Rörper griu; Beine unt Flügel won einer Feber unter

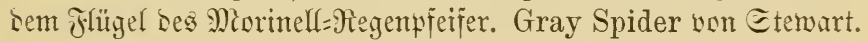

31. Rörper vom Riel einer Edyungfeder Des Ctaar; Beine suntelgrau ober brammot; Flügel helle Etar = Edymungicoer. \&änge $12 \mathrm{~mm}$. Quill Gnat.

32. Rörper lidyt jujefergraue Floficibe ober Relzhanr; Edulterhedyel filtbergraut jummend; ganj flein. Blue Mlidge.

33. Rörper non Naulwurfogar; Beine grau; Flügel Strar= idjmutgfeder; Edywanz grau. Early Dark Dun.

34. Rïrper Maulmurishaar mit jamarzer Etrauj̃ederiajer gerippt; Beine grau, jumment. Great Dark Drone.

35. Rörper Naulmurishaar; Edyanz gelbgrau; Beine gelb= grau oder ingwergelb; Flitgel sunfel bleigrau. ¿änge $10 \mathrm{~mm}$. Little Ironblue Dun, Ironblue, Watchett, Little Dark Bloa. Bermandelt ïch in ben Jenny Spinner.

36. Rörper Maulmurighaar; Ropf von rotem Mohair; Beine blutrot; Flügel graıt von ber Brujt ses (Enterid)s. Robin Hood. 
37. Rërther Heigraute Ěeibe; Beine gran; Flïgel bräunlidó

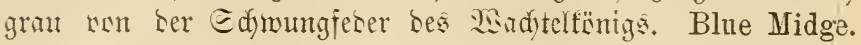
38. Rörper bleigraue Flodjeise; Beine jdwarz; Flügel grau

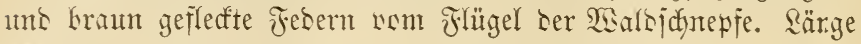
$14 \mathrm{~mm}$. Spider Fly, Gravel Bed, Sand Gnat.

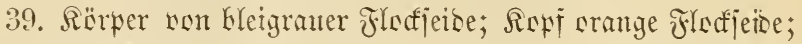
Beine ichlüfijelblumenge!b; słlügel mittelgran. Orangeheaded Dun non 2rroam.

40. Rörper bunfel bleigrane Flodjeise; Beine bräuntiḑ; Flügel wie Gei 9ir. 38. Stream Fly.

\section{Fliegen mit gelbgrautem siörper.}

41. Rörper won Miatlwurfshanr, mit gelber Seibe geript; Echulterkedyel sunfelgran mit fupjerretem Echein, jummens. ¿änge $10-13 \mathrm{~mm}$. Willow Fly, Shamrock Fly, Little Paleblue Dun, Old Beson, Withy Fly, SEeiden=Fliege.

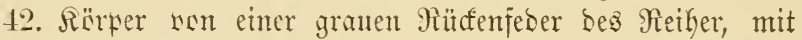
gelber Eetse geript; Beine sunfelbrangrau sber flutret; Flügel suntle Edyuntgieder bes Etant. Whirling Dun.

43. Rürper ans gelkem und granem Pelzhan gemijcht; Edjwanj transparent grau; Beine Gellyran; Flitgel transparent yrau rom Etrar noer நäher. Blue Dun, Cock Tail, Early Dark Dun, Hare's Ear and Yellow. Hare's Fleck.

41. Sörwer wie hei Ler wortgen Fliege; Edywanz Sunfelgrau; Beine suntelgrau; Flügel sunfle Edymungeber ses Etaar, gelb gefärkt. July Dun, Darkblue Dun, Merlin.

45. Sïrner wie bei ber Blue Dun, aber mit mehr gelb;

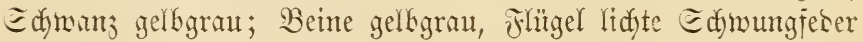
bes Etarar. Yellow Dun.

46. Sïrper lidft, wie bei Blue Dun; 巨dymanz uno Beine blägelb; Jlügel lidjtgrau non ber Eeejchmalbe gelb gefärbt. Skyblue.

47. Rörper non gramem Feljhar, bas nuі gelbe Ceibe ge=

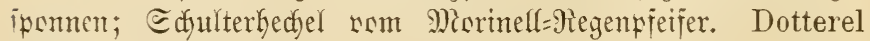
Hackle. 
48. Rörper won bem graugelben Siel einer Drofjelfeber; Beine grau; Ilnterflügel lidyte Edymugfeser bes @taar, Sber= flügel janarze Edymungfeber Łer 2tmjel, sänge $12 \mathrm{~mm}$. Needle Brown, Spanish Needle.

\section{IX. Şliegen, Deren Sörperīarbe eitte Miīhung von grat, gelb und braun iit.}

49. Rärper ron Dumfler Şajendhrwolle; Edymanz braune, idfwarzpunftierte Feber nou ber Brujt bes (Enteridy; Beine an ber

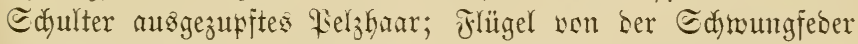
ter 2 Salofdinepie. Hare's Ear, Spider Fly, Sand Gnat.

50. Rörper wie bei ber vorkergełenten Fliege; Beine juwarz; Flügel surchjdyeinent von einer Edywungieder ber \&erdye. Stewart's Winged Fly.

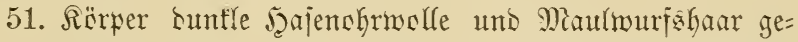
mifat, Edwangfnoten grïne Flodfeibe, Bippen braune Eeibe; Beine blä́ngwergelb; Flügel won ber Edyungfeber ber Fajanten= henne. \&änge $12 \mathrm{~mm}$. Green-tail, Grannom, Shell Fly.

52. Rörper wie bei Der worbergehenden; Beine grau; Flügel wie bei গir.51. Brown Skipjack, Hammond's Adopted.

53. Rörper Jajenthrwolle mit lidtbraunem Makair gemijat; Edjwanz graue Brujtfeber ses (Enterid); Beine graue Feser vom

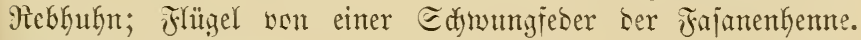
Gray Housewife.

5t. Rörper helle und Dunfle Dajenofrwolle gemijut; Beine braungeflefft wont 円ebhuhn, junments. Partridge Hackle.

55. Rörper lichte Dajenthrmolle mit wentg gelbem Miofair gemijot, gelf gerippt; Beine braumrot; Flügel Fajantemen= こdyungieber. Harry Long-legs; Dabby.

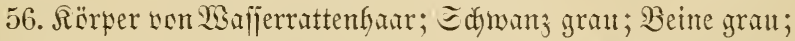
Flügel Echnungfeder bes Etrar. Early Dark Dun, WhirlingDun.

57. Rörper Wajferrattenfaar mit gelfen Misfair gemijat;

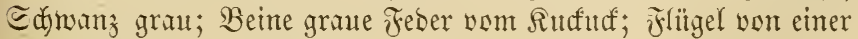
Edyungfeber ser Fajangenne. Cuckoo Dun. 


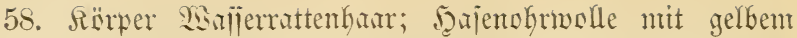
Mohair gemijd, finten mehr gelb wie vorne; Sdywan brum=

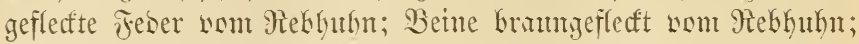
Flügel non ber Edyumgfeber einer Jajantutheme; Dönter Bart= haar eines Ranindyen. $23 \mathrm{~mm}$ lang. Stone Fly, May Fly, Eteminliege.

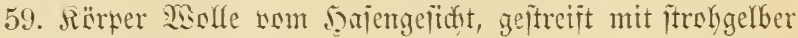

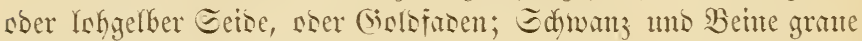

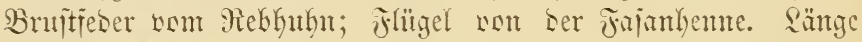
$23 \mathrm{~mm}$. March Brown, Brown Drake, bie mämnlidje Fliege.

60. Nite bie worige, mor ijt im Rërper gelbes Miohair bei= gemijht. Das Weibchen ber March Brown.

61. Rörper Molfe your Dajengefidit uns gelbent Mebatr ge= mijht; Beine lesergelb; Ilnterfitigel Etanrichnumgieder, Sber= flügel Edumungieser von 2.ad)telfënig. Ränge $15 \mathrm{~mm}$. Sand Fly, Sanded Dun.

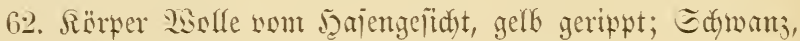

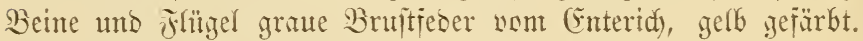
Little Yellow May Dun.

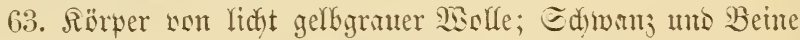
rebergelb; jlügel won ber Edynumgieber bes Etam. Summer Dun vou Aldam.

64. Rörter wie bei ber workergehenden; Sdywan; itrohgelb;

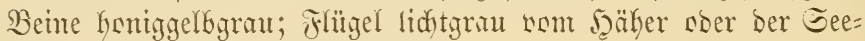
idnualbe. Eden Fly won Aldam.

65. Rërper lidjt lebergelb mit Giolofaden gerippt; Rörper= uno Edulterbedsel bla Sberflügel 2Badtelfänigidnumgieser. Sedge Fly.

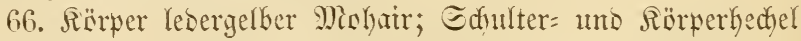

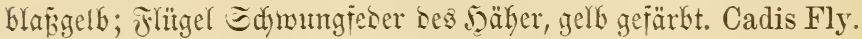

67. Rïrper jd)mutzig lebergelbes Poljham, gelb gerippt; Sdyman; hellgran; Beine hellgrau ober honiggelbgrau, Jlügel vom Etiar, gelb gefürbt. $10 \mathrm{~mm}$ lang. Pale Evening Dun. 68. Rörper licht crêmejarbiges uno lichtbraumes Welzhaar ge= 


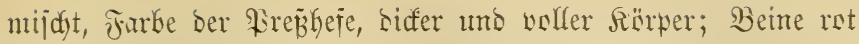
ober ingmergelb; Jlügel sunfel vom Jajanenflïgel soer ber bratuten Eule. 14-16 mm lang. Barm Fly.

69. Rörper wie bie vorkergehente, ein wentig sunfler; Beinte

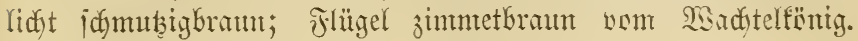
$15 \mathrm{~mm}$ Yautg. Cinnamon Fly, Bimmetfiltege.

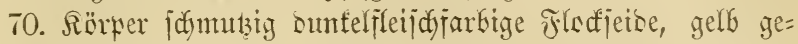
rippt; Beine lidjt lebergelb; Flügel einfarbig mittelgran. Indian Yellow von Aldam.

71. Rörper blä̈gelfbraun ober ftrobgelb mit lidjtgrmuent Edyein, braun voer apfelgrün gerippt, Beine blä zitronengelb woer gelbgrau; Jlügel helfe Edymungfeder bes Jä̈her; gelbgrïn gefärbt. Ränge $15 \mathrm{~mm}$. Yellow Sally, Flat Yellow.

\section{F̧liegen mit grantoten $\mathfrak{s}$ rëper.}

72. Sërper Ginten jounubig Gorbeanmetes Mohair, worn ntit

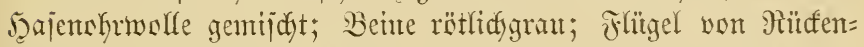
febern ber Pifuhenne. $15 \mathrm{~mm}$ lang. Red Fly, February Red, Brazil Fly, Old Joan.

\section{Fliegen mit brautent $\mathfrak{s}$ örper.}

73. Sörper jimntetbrautes Minfair, gologerippt; Cdulter= hedjel eine braungeflecte Feber bes Bauntinnigs. $7 \mathrm{~mm}$ lang. Wren Tail.

74. Rätwer wie bei bent Wrentail; Sdwand braut= punftierte Brulffeder bes (Enterid; Beine Dunfelret; Flïgel von einer Waldjonepjenjdwungfeder. Maltby.

75. Rörper wie bei ber worigen, haurig; Rörper=und Edutter= hedjelfeber ambrafarbig; Flügel rote uno grane Feberfajern aus

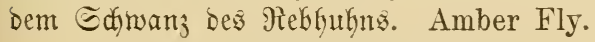

76. Rörper wie bei ber worigen nder wou labgelbem Felz= Gaar; Beine ingmergelb; Interfiügel crêtnegelf wa ber Cutule, Dberflügel gratte Bruftieser vom (Enterich. Brown Moth, brimte Mette. 
77. Rörper rojtbrames Mofair; Beine lidytrot; Flïgel von ber Ednumgfeser ber Jajantente. Sünge 1t-16 mm. Caperer, Skip Jack.

78. Rörper mafagonikraumes Weljhar wom Dhr Les fudy; Beine braune funfticrte Feser bom Bauntënig; Flügel graue

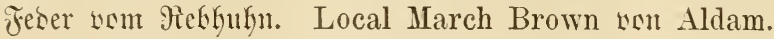

79. Rärper wie bei ber workergehenten, aber lidyter; Beine

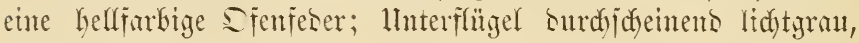
Sberflügel jansig fräunlid). Crossing Brown wen Aldam.

80. Rërper Graumes Fieljhan; Rërper= uno Edjulterbedyel Graum aber retbraum. Brown Palmer.

81. Rörper won einer Granten Futenfeber ober Granter Eetbe;

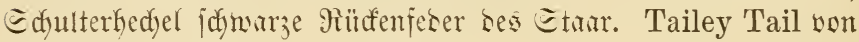
Aldam, Black Spider won Stewart.

82. Rörper lidatbrame Eeise, gulsgeript; Edymanz und Beine lidft retforaun; Jlïgel lidgt fräuntlidy grau. Brown Spinner.

\section{Fliegen mit braungelbem $\mathfrak{A o ̈ r p e r . ~}$}

83. Rërper haarig, lobgelbes unto srantge Reljhaar gemijdyt; Beine ingwergelb; gringel braun rom Nindytelfüntg. Cow-Dung.

84. Rërper weridyolfene ormige Eeise mit rotent (Eid)gorn= fyar; Beine unt Jÿ̈gel wie bei ber workergekenton. Bank Fly non Aldam.

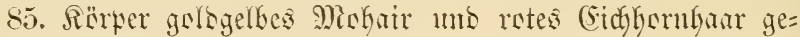
mijat, mit gelber Eeise geripts; Edymanz unt Beine blaj ingmer= gelb; Flügel sunfle Edywungferer bes Etaar. $11 \mathrm{~mm}$ lang. Whirling Blue Dun.

86. Rärper Glä́ crêmegelbes Relzhar; Beine ingmergelb; Unterflitgel Gellgelb, Sberflïgel sunfelgelk, beibe sou ber Sule. Mealy Cream Moth.

\section{Fliegen mit gelbem $\mathfrak{S o ̈ r} \mathfrak{p e r}$.}

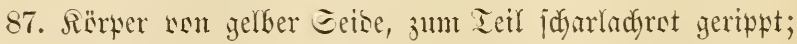
Flügel vou eimer Marbidnnepfen=Edynungfeder. Woodcock and Yellow von Stewart. 
88. Rörper wie bei ser norkergekenden; Beine rotbraun wom SEadjtelfünig, jummens. Red Spider von Stewart.

89. Rörper wie bei ser workergehenten; golsener Edywanz=

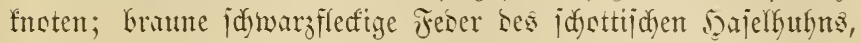
jumments. Grouse Hackle.

90. Fïrper ingwergelbe ober golsgelfe Flodieide; Edylter= Kect) ol brambeflectte Fober som Zaumfünig. $7 \mathrm{~mm}$ lang. Wren Tail, Frog Hopper, Brown Bent, Jumper.

91. Rërper goldgelbes Mokair, Rërper= unt Edultertyedyel Dienfeber; Flügel bunfle Edyungfeber wom நä̆fer coer Etaar. Good General Palmer.

92. Rörter won zitrenengelbem Mofair; Beine ingmergelb; Flitgel braum vom :Badtelfönig. Cowdung mady Ephemera.

93. Sïrter zitronengelke Eeioe; golbenter Edyanjthaten; Edjulterkediel graue Jeser wom Rebhuth, jumment. Partridge Hackle.

94. Rörper won julitilelbfumengelber Flodieibe; Beinte etmas bunfler gefärbt; Flügel Yidgtgrau won ser Ceejdyalke. Bigg Dun von Aldam.

95. Rörper ganj lidyte jdulïïelbhumengelbe Seibe; Edyulter= Gedel won ber Seejdyalbe, jummens. Light Dun von Aldam.

96. Rörper won lidyt grïnlidgelber Jlodjeide; Beine nom Morimell Negenpieifer, jumment. Spring Dotterel Dun won Aldam.

97. Rërper won ber Farbe Ser gelben (5änşdyen, grünlid)= gelbe Floffectbe nder Mishair; Beine zimmetbraun; Flügel non einer Edumungeber ber \&erdfe. Little Gosling.

98. Rörper won einem Fesertiel, ber Glapitrobgelb Gis sliven= grünlidjgelb gerärbt iit; Beine ftrobgelb; Tlïgel son einer Staar= Edymungeser; Edyman; itrofgelf. Yellow Dun.

99. Rörper itrohgelb; Edjwanz lebergelk; Beine honiggelb; Flügel won ber Eeefdroalbe; fleine Fliege. Skyblue.

100. Rärper itrofgelb Gis fräunlidigelb; Edyantz von einer Sdinumgieser ser Fafankeme; Flügel grate Brutteser wom 


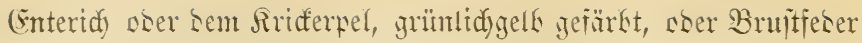
Ser Aix Sponsa, weldye unger̈̈rbt gensmment wirb; Beine lidjt lebergelf fis lidyt rlivengelfgrïn, $25 \mathrm{~mm}$ lanty. Green Drake, May Fly, Cock Tail, Cadow, Maiffliege.

101. Rörper wie fei ser vorigen; Edyman unt Beite graue Brutjeser wom (snterid); Flügel won serjelben Jeber. Gray Drake, entiteft aus ber wrigenanten Fiege.

102. Rörper srange Jitodjeise soer Kanfe, gologeript; Beme feuerrat; Flitgel wou einter Edyumgieber ber jajumbente soer bes Rebruthes noer bes Etanr, $15 \mathrm{~mm}$ lang. Fern Fly, Soldier, Sailor.

103. Rörter orange Eetibe, an ser Edulter suntfer werbent; Beine mittelgrau, jumments. Orange Dun rber Buff Dun sout Aldam.

104. Rörper orange; Beine won einter Efenjeber; Flïgel Edyungieber ber Irsijel. Furnace Hackle.

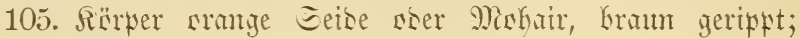
Sïrperfectel braumet, tutrz gejdoren; Beine Sienfeber soer fonig=

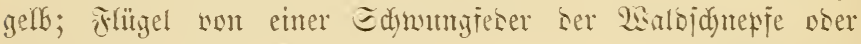
Jajantente, \&änge 18 mm. Oak Fly. Ash Fly, Cannon Fly, Down Looker, Downhill, Orange Brown, Woodcock Fly, edunepientilicje.

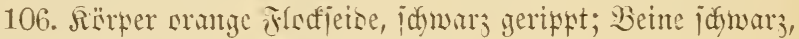
jumments, $8 \mathrm{~mm}$ linty. Water Cricket.

107. Rärper uts ßippen wie Kei Der varbergehenton; Beinte Dienfeser; Flitgel suntle Etaar = Edymungfeser, $10 \mathrm{~mm}$ lang. Orange Fly.

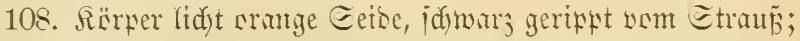

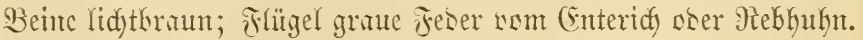
Wasp Fly. Misospe.

\section{Fliegen mit braunrotent förper.}

109. Rörper rotbraum, aus Siolle vem Dajengejüt, gelb geript; Edwan; awei Ranindfentbarthare; Beine rotbram; Flügel son einer Sdymungieber ber Fajanfenne. August Dun. 
110. Rörper Gelf brimurot; Edfmanz uns Beite rot; Flügel

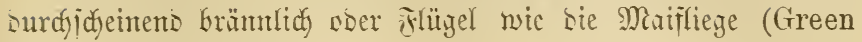
Drake). Red Spinner.

111. Rörper wie bet ber workergehenden; Beine rot; Jithgel

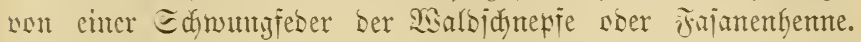
Hofland's Fancy.

112. Rörper nou bunfelfraumroter Eeibe; Beine unt @dynanz won ciner idwarzen Dafnentalsfeber; Flïgel won einer bunflen Feser von ber immenjeite bes Jlügels bes wajijerfufns. Dark Bloa won Jackson.

\section{Fliegen mit rotem förper.}

113. Rörper won einem rotgen̈rbten jeserfiel ober roter Escibe, gologeript; Beine unt Edyronz rot; Flügel Gellgraue Dalsfeber Des Detuths. Red Spinner.

114. Rörper idg̈n rot, grlsgerippt; Rörper und Edyulter= bedjel blutrot. Red Palmer, Soldier Palmer.

115. Wise Red Spinner; Flïgel graue Bruftieber bes (Enteridy. Edmead.

116. Rörper maulbeerjarbige Eeibe; Echmany uno Beine purpurrot; Frlïgel won einer Edymungfeber des હtrar. Little Dark Spinner.

117. Rörper bou Sunfelforbeanrrotem unb fupferrotem Mahair gemijat; Cdymanz, Ḑörner uno Beinte bunfel rotbraum; Ilnterfilügel Staaridymugieser, Sberflügel rotbraume Feber wom sadetelfüuig ooer Rebguth. Alder Fly nady Ephemera.

\section{Fliegen mit goldiger oDer filberner Sörperiante.}

118. Rörper von Golb=Rametta; Rërper= uns Schulterfyedyel

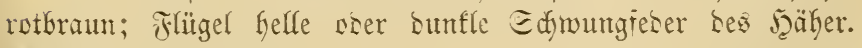
Wickham's Fancy.

\section{A. Gejlügelte Alexandra.}

Rörper won Silber= arer Golb = \&ametta, roer =Faben; 


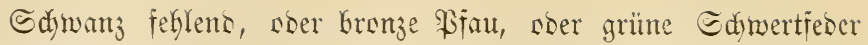

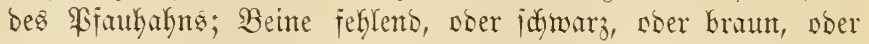
bronze Ffau, coer grüne Edywertfeber; Flügel jumment von

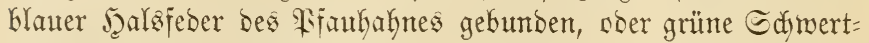
feber noer Kronze Sifu, ober ein gemijdyter Flïgel ans sifuten= ¡dweif=Feberfaiern, roten, grïmen, braumen Jeberfajern, aud mit Febern nom J⿹als bes Eommerats=5ahnes.

B. Ilngeflïgelte Alexandra, Räferform.

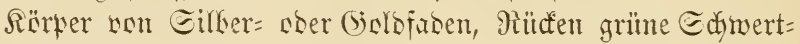

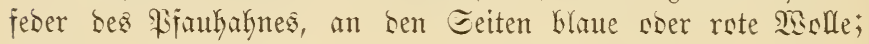
Edymanz Edyertieber= noer kromze Fifmenfeberfajern.

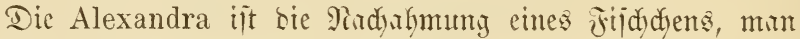
fiicht mit werjunfener foliege, beshalb wirb ser Rörper aud mobl mit Blei bejdywert.

\section{Red Ibis (2(merifanijde $\mathfrak{F}$ ficge).}

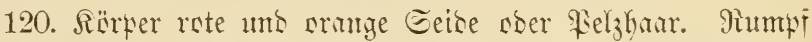

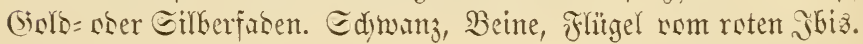

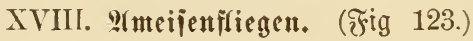

121. B lack Ant. (Bowlker, Ephemera, Francis.) Sïrrper idymarzbraune Eeide, Edywanjtnoten jđwarz wom Etraup.

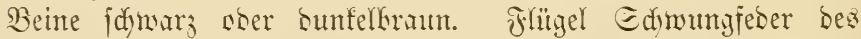
Ctaras noer Jeäher.

122. Red Ant, Little woer Large Red Ant, Small Common Ant. (Bowlker, Ronalds, Ephemera, Francis, Aldam.) Sïrper fupierrete Eeibe, Edymanjfnoten brenje \$rau. Beine rot. Flügel Edinumgfeder nom Etaar noer

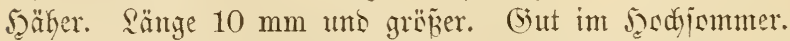

123. Horrocks (Jig. 193) won John Horrocks. Rörper vom abgejtreiften Bart einer meipen Taubenfeber, bie Fajern turz gejdyoren; an ber Edjulter uno am Edyanz ein paar

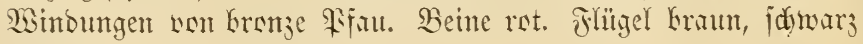




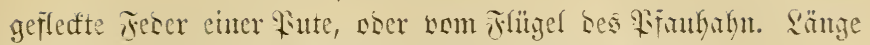
$25 \mathrm{~mm}$. (Fine in Icutid)lano siel gebrande Fliege.

\section{XIX. מgedłclĭliegen, junmende Fliegen, Mï̌fen, Spider Flies.}

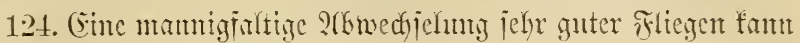

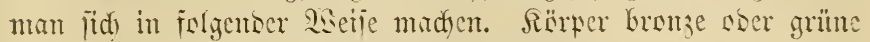

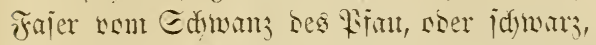
hellbram, bumfelforam, gelf, rot, non Ëeise,

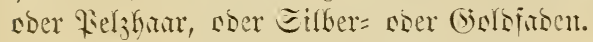
Beine und Fitigel non Febern ber Befrilime, bem Pitüfen bes Etanr, Iedfebern wom

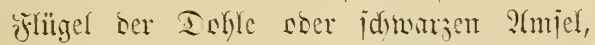
vom Rücfen Des Marutelfreigentififers, Dem Rïlden roer Der Brult bes Goloregen= beifers, won ber Sber= ober Unterjeite bes Jig. 193. Horroeks.

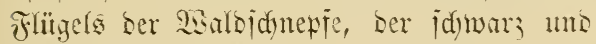

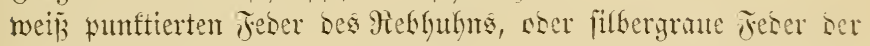
Seejdmalbe: alle jumment gemunoen.

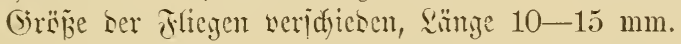

\section{125. Bumbles of Derbyshire, \$ummelı,}

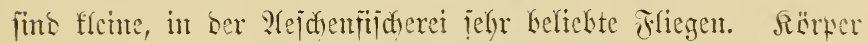

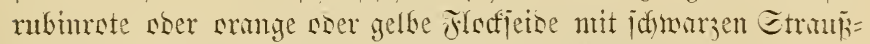

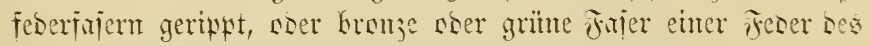

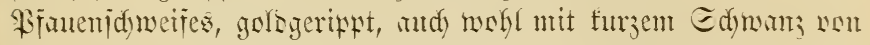
roter Eeibe aber einer flemen jourladureten Feber. Beine Gellgrane

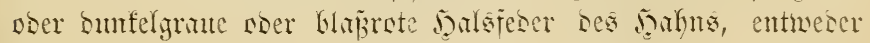
ïber ben ganjen fïrper ober mu an ser Edulter. Filuge jummtento gebuntsen. Sïnge 10-11 $\mathrm{mm}$.

\section{Sünjtlidye Rärer}

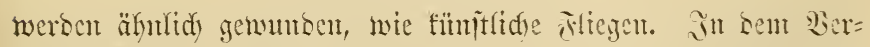

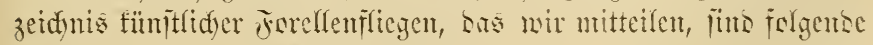
Pachbifoumigen von Rärent enthalten:

๖. ๖. Borne, Ingelfitiderei. 3. 2uff. 
7. Coch-y-bondhu, Birdyfïfer.

5. Peacock Fly: Little Chap.

101. Fern Fly.

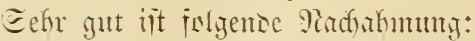

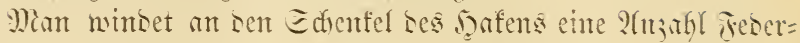

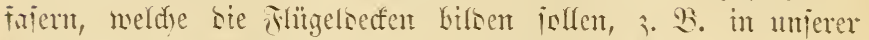

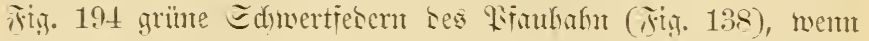

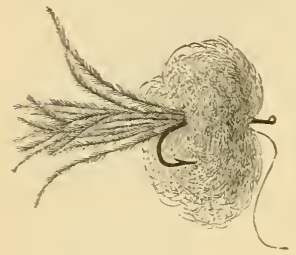

Fig. 194. mir einen Rojenfäfer nadybifon wolfen. Iarmif wintot man in ser kejdriebenten Secije frimute Edweinsmolle an uno be= idnutset bieje is, ban lie ber Rërperform

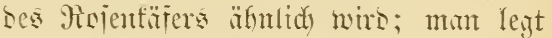
Dann bie Edfmertfesern über Den Mïhtent uno wintset fie ant ber Edulter an, unt sie Thïgelsedfen зu filbent. 1tm bie Edyulter fomnten srei simsumien einer framen

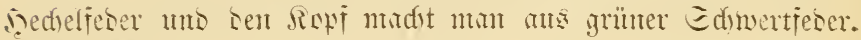
(jig. 12t).

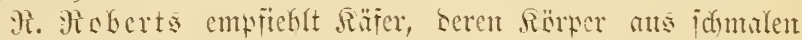

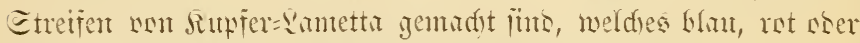

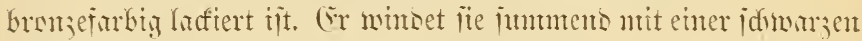

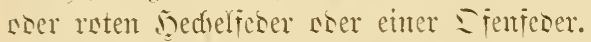

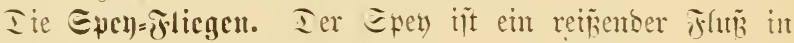

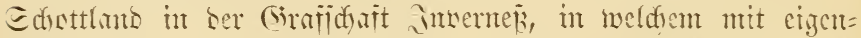

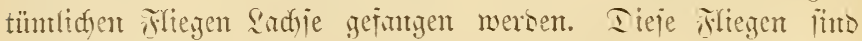

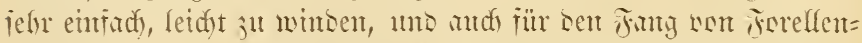

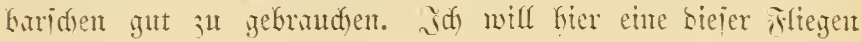
gentu bejareiben.

Purple King. Ier நafen ît $45 \mathrm{~mm}$ lang. Die Dechel=

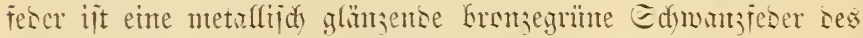

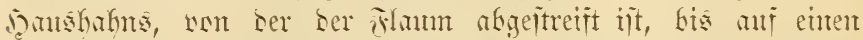

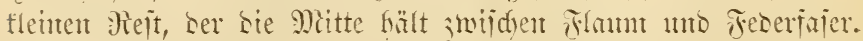
Ier befieberte Ieil ber is sorbereiteten nedrelfeber ijt $120 \mathrm{~mm}$ lamis. Jig. 195. 


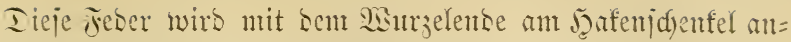
gemutsen, unb bic Jajern jurïhgeitrid)en. Fig. 195 unten.

Bugleidy mit ber jeber werben ein banbförmiger uns eir gejpennener Eilberiaben an Jatenidhenfel angemunben.

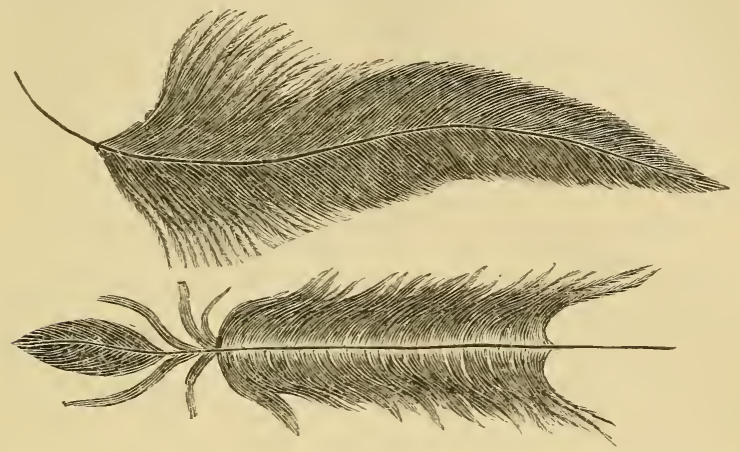

Fig. 195.

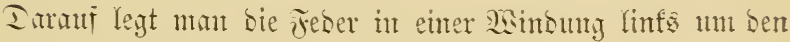
Dafen, bic heisen Eirberfäben in einer Simbutg nady redyto unb

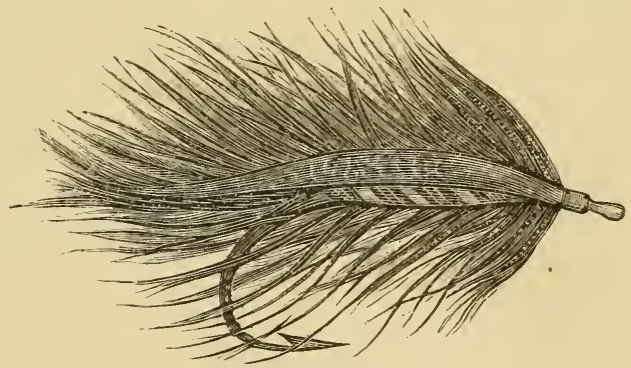

Fig. 196. Epen=Ffiege.

filfut sie Ëllberiäben freujweije ̈̈ber bie Feber. in berjelben. Weije

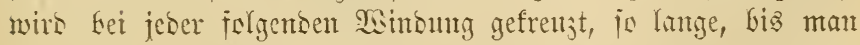
bic Deje bes Dafens erreidyt hat. Daranj ziegt man mit einter 
Mabel bie Feberfajern Kerwar, welde wan ben Eilforfïbon besedt fins. Ite Flege kat bant bie Gejtalt, wie in Fig. 196.

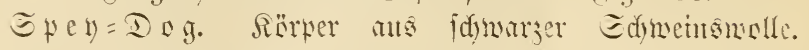

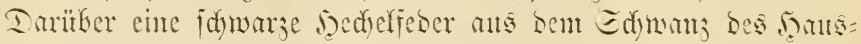

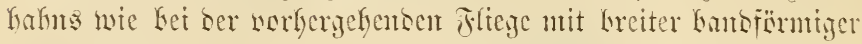

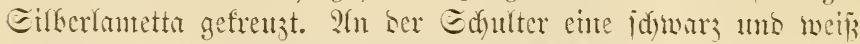
geflectte Dechelfeber wou ber Bruit ber Sriffente; Flïgel Eran=

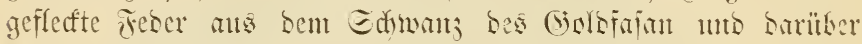

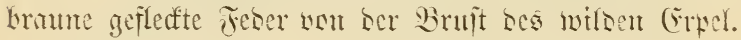

\section{Das Minden ber groben Gilanjflicgen.}

Die kejte 2huleitung jum winsen ser Granjfliegen gicht

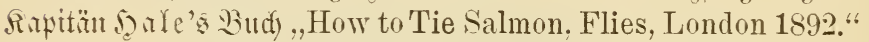

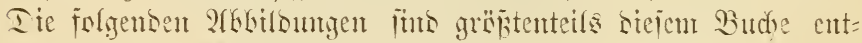
Yehnt, wir banten bent Dern Berleger Mir. M. 2). Mariton werbinslidy, saj er bie satederigate geitattet hat.

1. Ier Ettit woer bie Fimue, Tag, beitcht in ber Regel

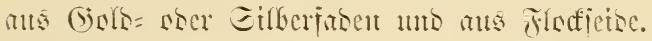

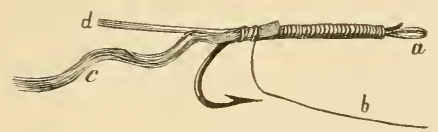

Æig. 197.

Badysem ber Dafen in bent Edrrabutode befeptigt iit, winteet mat juerit sen (Sals= foce Silberfirben d mo baratif cintige Mitfinter soment enternt

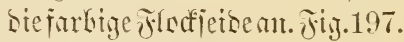

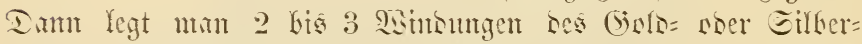

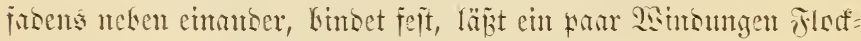

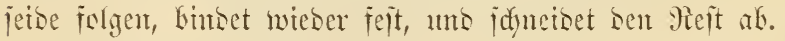

Ier Edjwanz, Tail, ijt in ser Regel eine fleme grlogelbe Ioll=

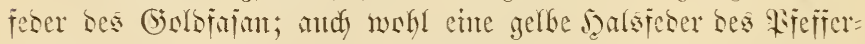

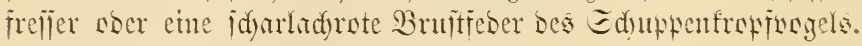
Inju merben bismetlen chitige fajern folgenter febern gefïgt:

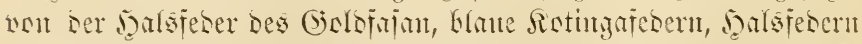

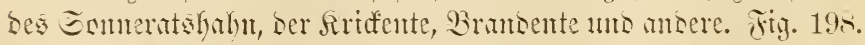

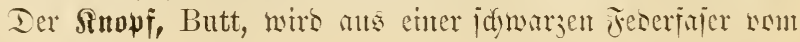




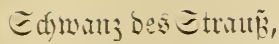
aber einer fromje = farbigen Pinutgafu= Ed) weiffeber genult= Don. ivig. 199. Tig.200.

Ias Mejultat ser. bisherigen ?rbeititellt Titig. 200 bar. Ter Baten joll anmittel= bar am Snafi unbe= Secft geblieben jein; bie 2tmmindejeibe füngt bei a heraf. rig. 201.

Ierfïrper, Body, jull am fisfifer stiege etmas itärfer jein, wie

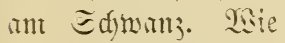

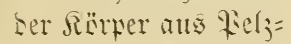
fout gemed)t wirs, Gabe idf bet bem Bintoen ber Farelfen= fliegen beidyrieben. E. 67. Minn legt bas Donr mit Inumen un Mittelfinger ber linfen நand unt bie Wnwintejetbe, und jüfrt sieje sorrmi um sen Edjentel Des joufens, indent mun Eas Reflyar feit an bie 2trmintejeibe an= zieft, tmb mady $\mathfrak{B} e=$

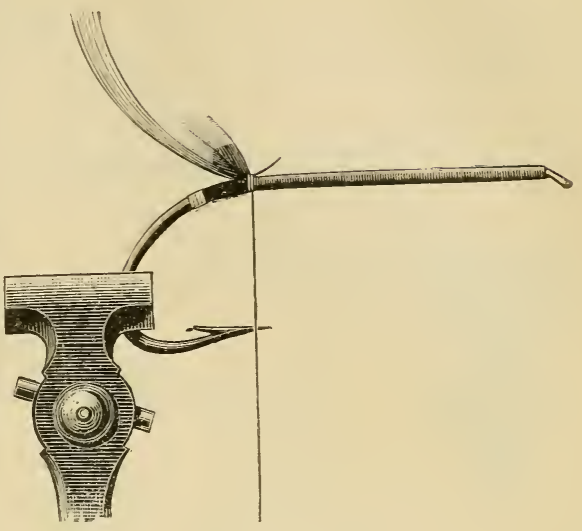

รig. 198.

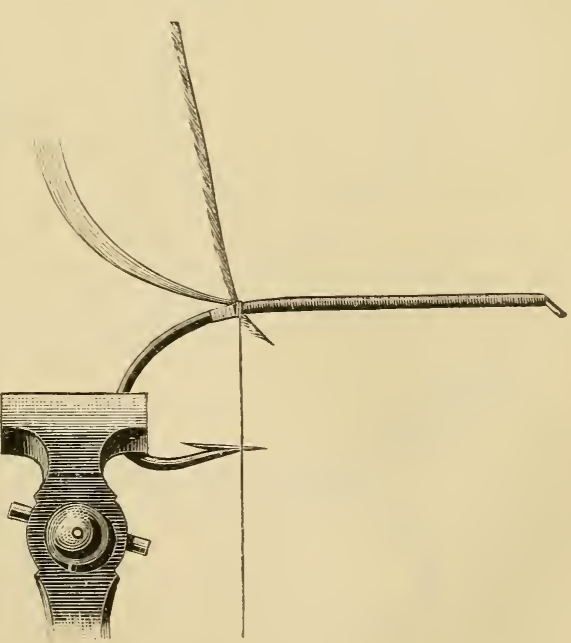

Fig. 199. 


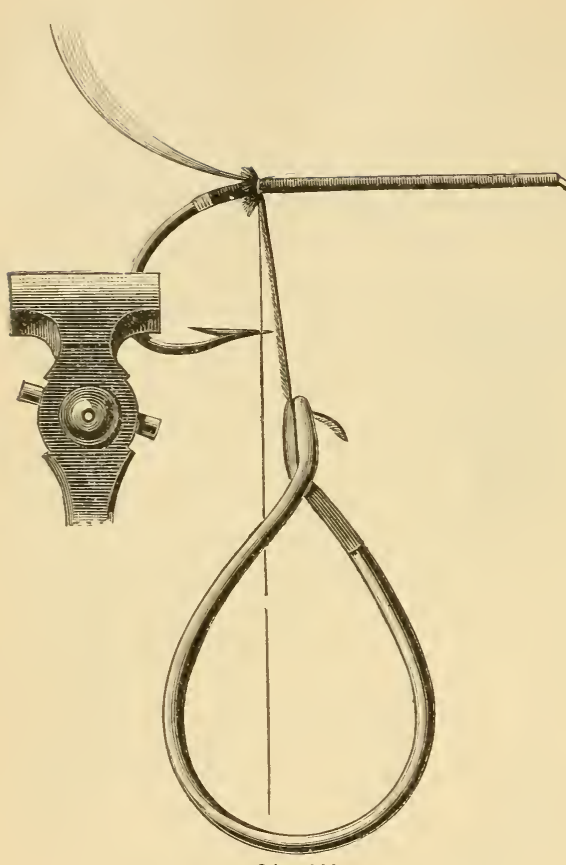

รijg. 200.

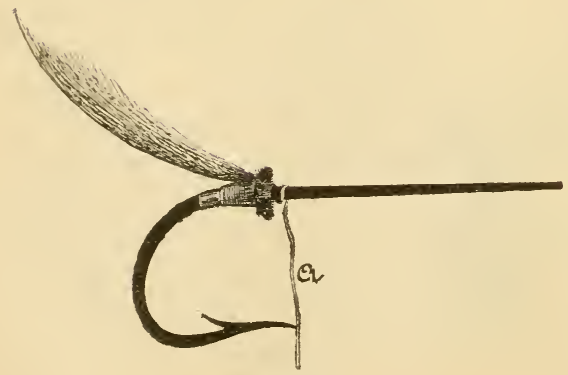

ซิig. 201.

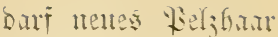
anlegt. 2/nt maijten mersen Edyments: molle, Eechundsham nocr Misbrix maje= wertbet, bie gell, wrange, rot, violet, blat oser idinar; ge= fürbt juns; sit entbïlt cin Sïrfer mebrere jorben. (jemöbulion mixd smäber ent Gors = rocr Eilber: firben, atmo sit muth eite sedielfercr gc= wunten.

Iic nedrelfeser wirs in ciniger (5nt= formung bom

Edumantmofi mmac= fäbr sa angemunten, wo bie jmeite 2sin= sumy bes Gold = noer Eilbertabens b: giunt. Fity. 202, 20:3. ภํrper สแ Eilber = nex (jold = Lamettr. Ginter Dent Sincten mirs bie samettr,

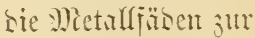
Seriphum, ms sic

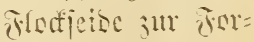
micrmyscsRërper 
anyemumben. Insis
Enoe ser Lametti nire worferipiţuno idyräy jutgejuntten; heror fie anyemum= Een wirt, formiert man ben Sïrfer mit Der Freffecioe ing = fältig, glüttetifnn mit ciner jesermelier= ffinge, uno tränft ifn mit Firnik.

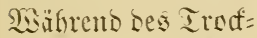

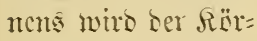
fer mit ber Feber: mellertfinge ge= glättet. joty. 204.

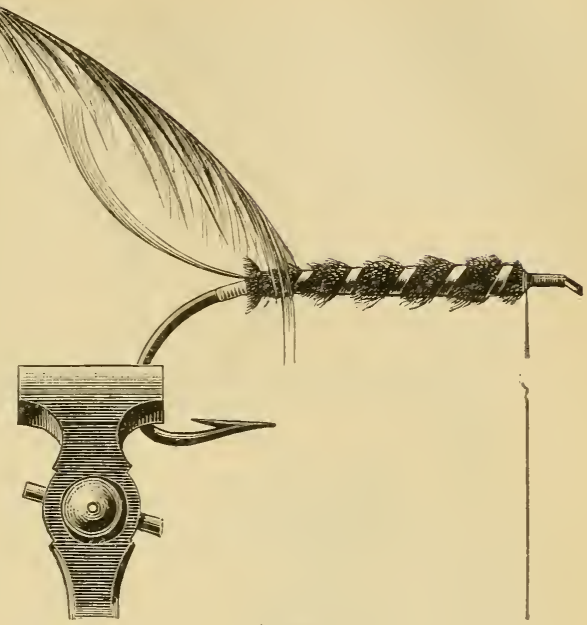

ฟ̌ig. 202.

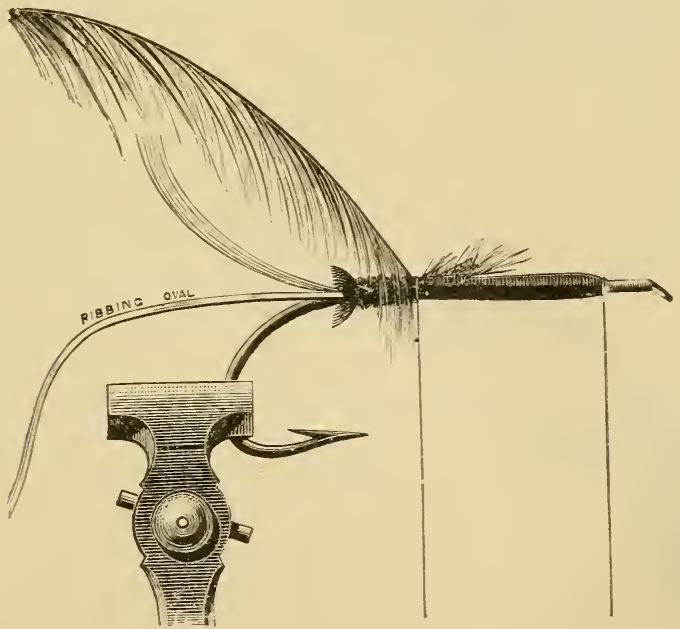

ริiy. 203. 


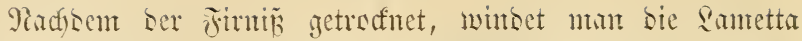

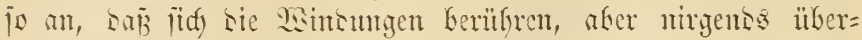

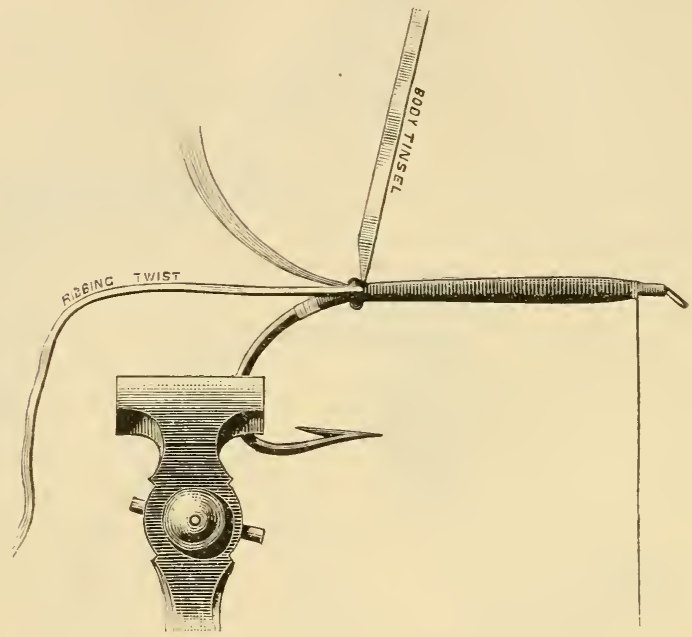

Fig. 204.

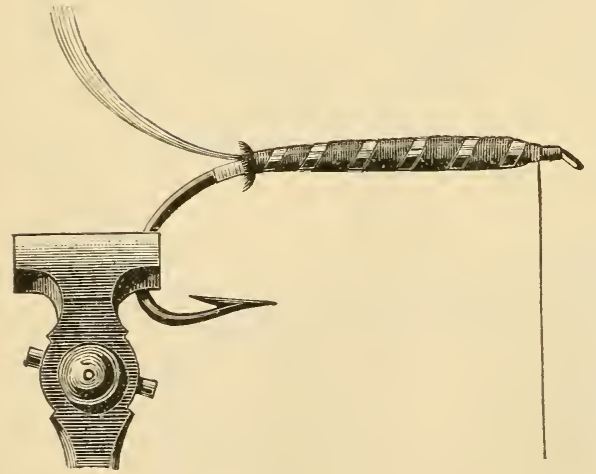

Jig. 205.
Decfent. Iann minoet man sie?antetta mit ber 2luminseleibe jeit, idfueibet ben Reit ab, glättet bent 2(bidynitt mit bem Fingernigel, und fïgt ein farr fernere 然insungen ber 2 (1) windeceise finju. Iann läjt man bic Beripkung und bie jeedfelfeber folgen. 
(Ein Rärper ans Flodjeibe wirb ähnlid) gemadyt, wie ber ans :amettr; fower man bie Eecibe anmintoct, welde bie

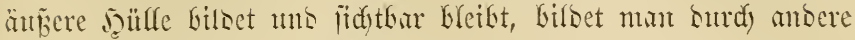
Tincferse wen beliebiger jarbe ente llnterlage, ant bie llneben=

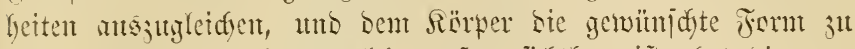

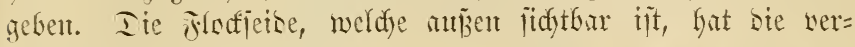
juicoeniten, gemifhn= lidf) redyt glänjento Firbert, namentlids gelb, ret, flan soer idjwiry. Iarïber fonment genöbulidd) Ripten ณแล Smuttr, Metulfiä́den unto einte bectelfeber. (jig.205.)

Iie jed el feber wirb is angerwunten, wie wir es bei ben jorellenfliegen be= idrieben bitben. Iie beijtefiende jig. 206 jetigt, wie ser Riel mid) Scut Plumintent ber Feber feit ge= bunben mirs.

Wenn ser Riel Der Jedyelfeber io bief

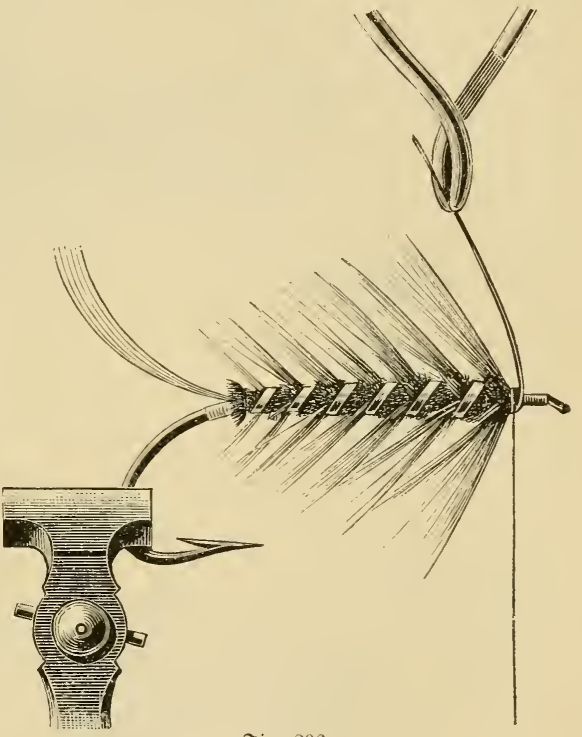

Fig. 206.

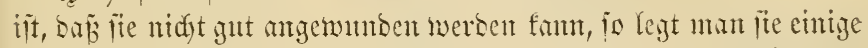
Beit in 2 saner, ftreift bie Feberfafute ab, unto nimbet mu bieje att. SEenn bie Rërperfechelfeber angelegt ift, fo folgt bie Sdfulter= Gedjelfeber. Fig. 207, 208.

IEem ber Siel ber Eedulterfechelfeber jafr biaf ift, wie

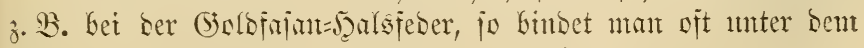
Jafen ein Bündel Feberfajern an. ङ. Jig. 209. 


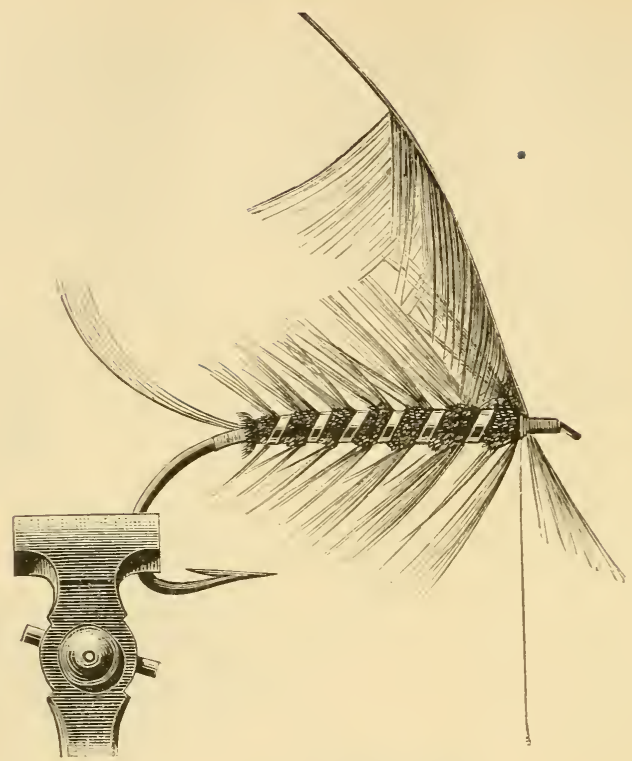

Tig. $20 \%$.

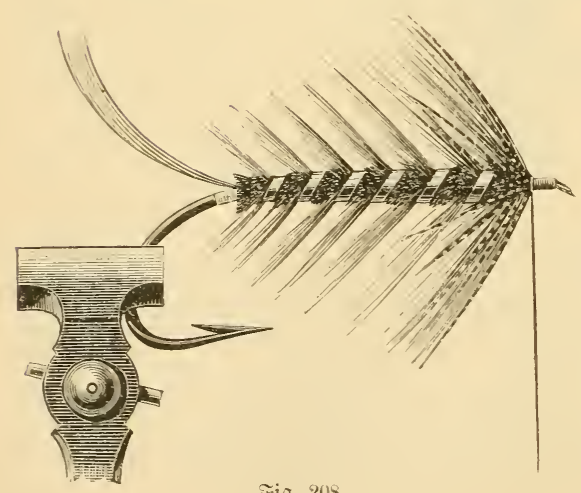

รig. 208.
Befiederte Ä̈r= fer. Ier Järfer ber (S) anifliegen wird oit mitatt mit amtgemu= Denen. Soedfelfebern mit ganjen Febern Ketlei= ret. Man mendet Daju

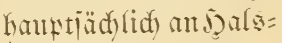
federn Des Rifeffer= Freilier, Bruttiederm Ses Eduppentrept= wegels (Indian Crow), Ghue Brutfedernvem 
Sintinga (Blue Chatterer), metallijó glämjende Febern vout

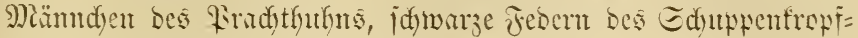

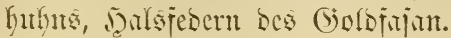

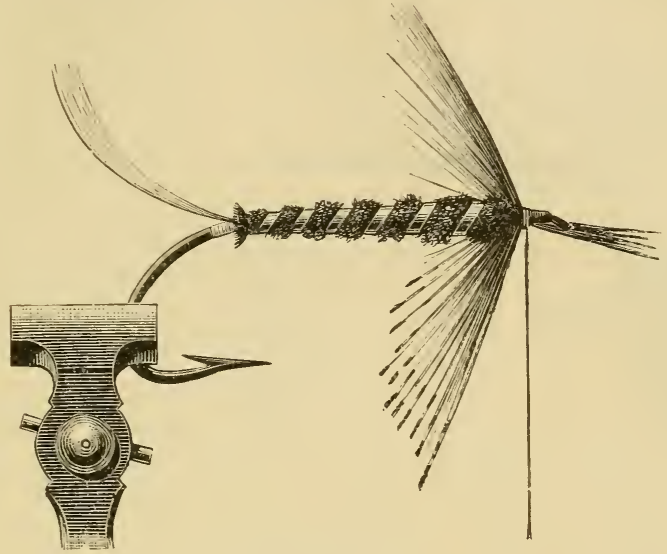

ชิiฺ. 209.

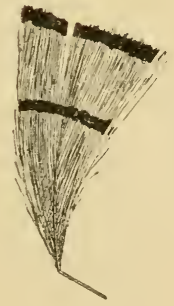

Fig. 210.

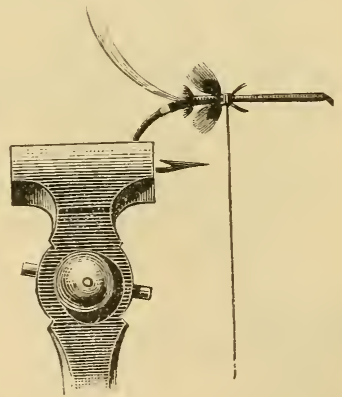

ซig. 211.

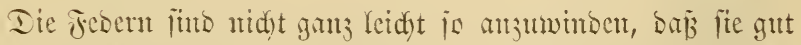

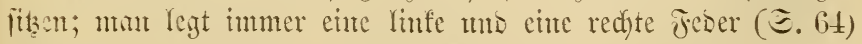

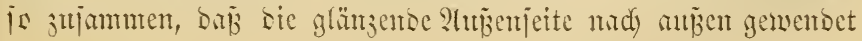


ijt, Sen Siel ser jesern Lrüfft man nit sem Beigefinger gegen

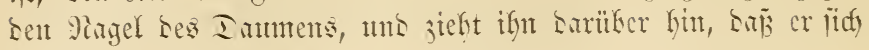
trïmmt. Jig. 210, 216.

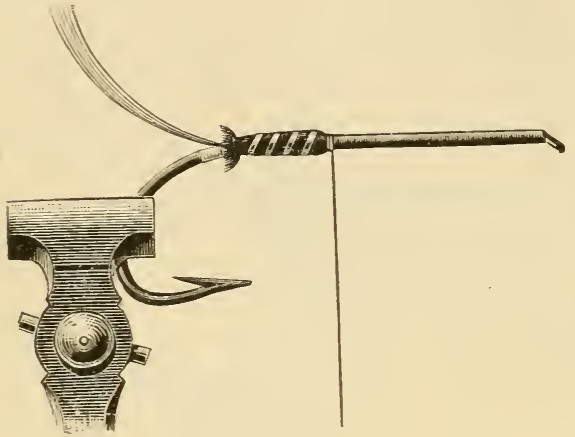

Fig. 212.

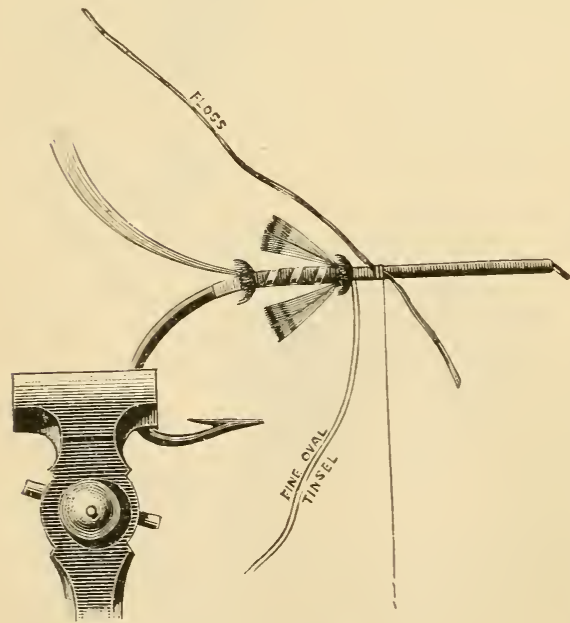

Fig. 213.

Ite Sïrter berge= glicserten irlicgen merten oft mit ganjen icsern befleiset.

(Fingeglieberter Rörtuer miro folgen= sermajient gefunsen: Yirdibem bas erite (5ilies gejertigt iit (vity. 212), wiro einte redite uno linte Feser ïter, un ein ähnlides Fanr mter sem iofen angemutsen. तitg.213. Tie folyentort Gilicber minset mant it serielfen 然eilic, uto nimment babee für jeses folgente bified ctmas grëв̈ere jesern. jig. 214.

Girs sit mirb and Ginter jesem (itliebe ser jofiege cinte Dedrelferer tum ben Daten genumben. Jig. 215.

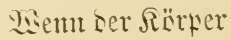
wollentet, unb bie Decdelieser an ber Eifulter angebracht 


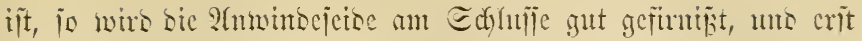
weiter gearbeitet, wenn ber Fimin nolftommen trefen scmerson

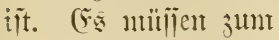

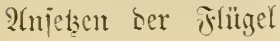
unto jut Bifoung bes Rofies wenigitens ned) $5 \mathrm{~mm}$ wem 5̧afenidyenfel preijein, wem ber fïrter woll= eltoet iit.

Iie হrïgel, Wings, Eer (B) anjifliegen twer= ben jum Teil ats ganjen Febern eser ant Ecilcu won feber= fafuen, rocr rus Bündeln nen jeder= frijern gemunsen.

Fïr Flitgel ans gamjentedent werben Gruptiädylidy folgente Jesern nermentet: gelbe Fibern wou ber unteren Ceite bes Flügels bes folnten Trarmun = Fipageis, blate Febern nan bemielben Bagel, nte= tallijd) glänzentoe jebern bes Firadit= Guthes, Soulstragen= fesern bes Gelofajan=

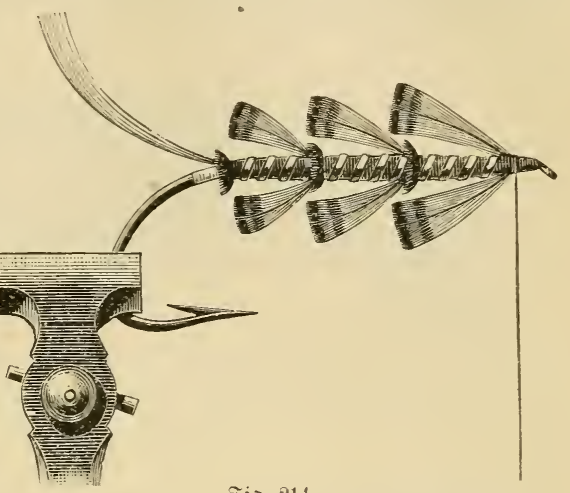

rig. 214.

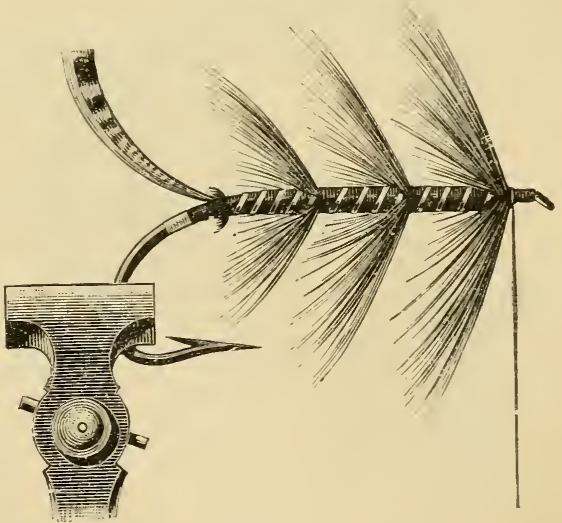

รig. 215.

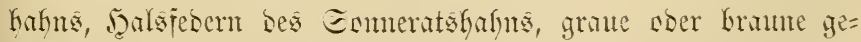
flecte Feocrn, ober cine grö̈̈ere Bahl Fenem won ber Tolle bes 


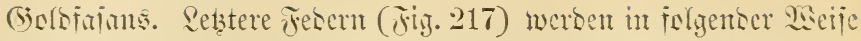
angenumben: eine Iollfeber, jefn sitnsungen ber Ëeibe; eine

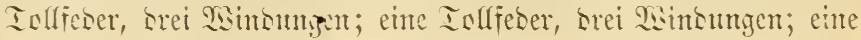

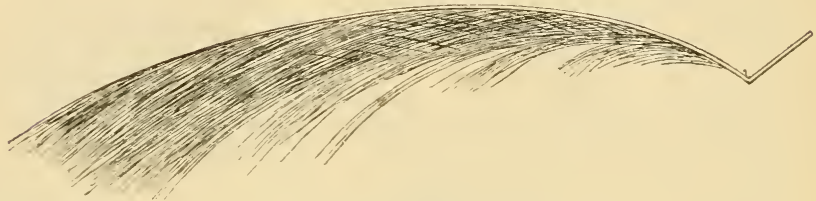

Fig. 216.

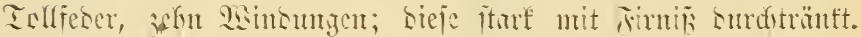

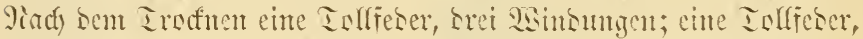

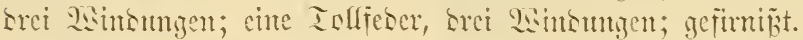

Ier Riel ser (Side =

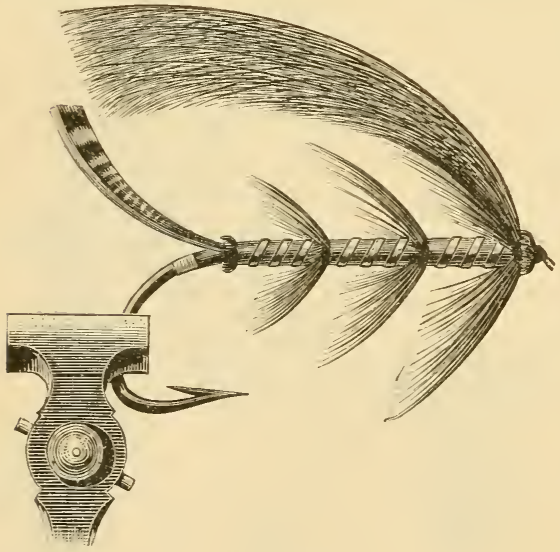

Fig. 217.

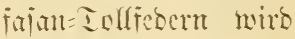
wer sem ?tminton ba= Surd) gefrïmmt, ¿ã cr gegen bic Edyäri: Des Immtemtagels ge= sritcfét uno sarüter fin= gezogen wird (i. Jig. $216)$, bie Feber fitht bann clegant, mit jier= Cidfer Rrïmmutng añ sem Datenidientel.

int itarf bewegtem 然affer werbienen bie Elitigel aus gamjen Terern in Ler Fiegel Dert Ranzty, weil ite mentiger leidyt surd bie Etrïnumy in ltnersmung getradyt meroen,

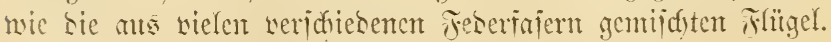
Mian legt inmer redste uns linfe jesern an sie redyte unt 
mns linfe Eecite ber Flliege an; Samit bie Riele fid) gut an ben

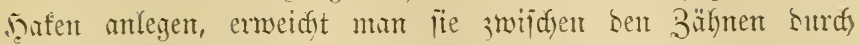
Beipen un Biehent.

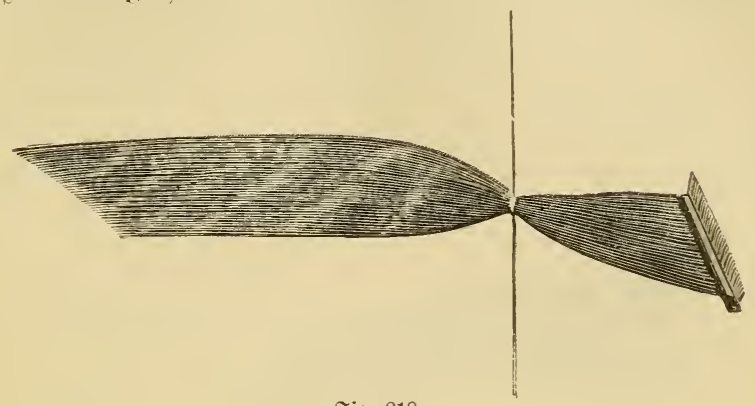

Fig. 21s.

Flügel aus Teilen von Feberfahnen werben in ähnlidjer 2aije anyemunden, wie bei Forellenticienen. Fig. 218, 219.

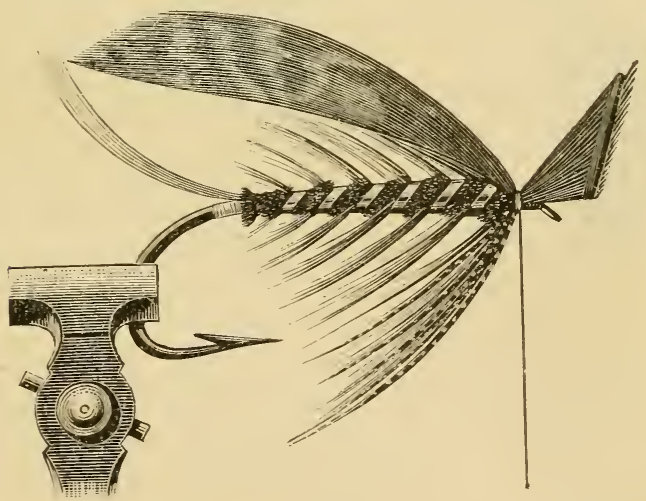

Fitig. 219.

Bujammengejebste Flügel, Built Wings. Sit fitellt

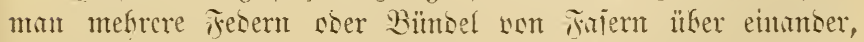
uno unteridyeiset sann linterflitger mo Sberflitgel. 


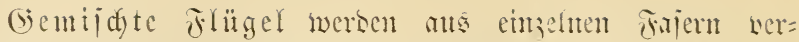

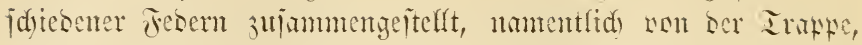

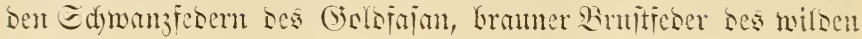

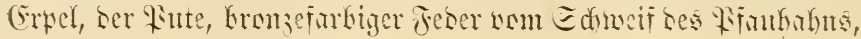

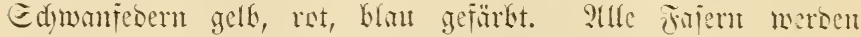
getrent uto is gemijdst, baj bie Ensen jujummentalien un

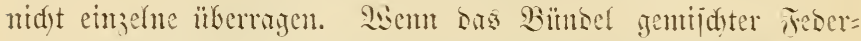

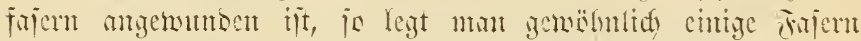
wot einer antoren Feser baritber. Jig. 218.

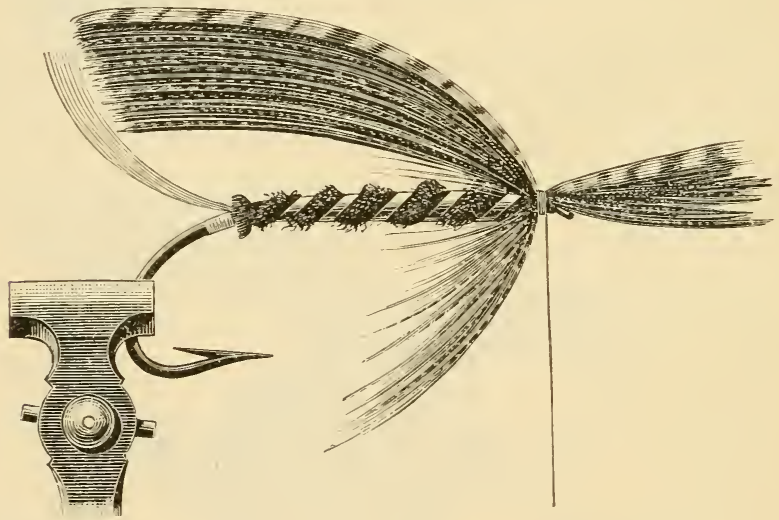

$\pi i_{j} .220$.

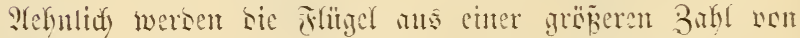

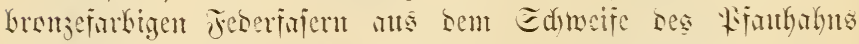

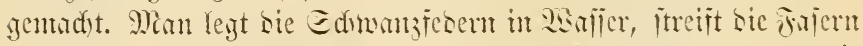
ab, uns wählt baju cinte redte uns eine linte Édimeiffeser, Damit jid) bic Fajern red)t glatt mo nett an cinanter antegen.

I ic Tolle, Topping (Jig. 125.8.), beitebt aus cuter coce cit

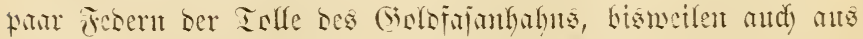

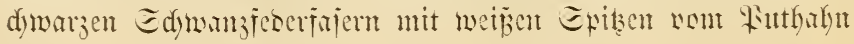


ober aus braungeflecten Feberfajen von ber Brult bes wilben (Enteridy (Brown Mallard).

Die Iolfe mird unch ben Flägeln angemunten, bie seinoutig gut mit Fintin burdatränft uno getrocfuet.

Die $\mathfrak{B a n g e n , ~ S i d e s , ~ C h e e k s ~ ( J i t g . ~ 1 2 5 . ~ 1 0 ) , ~ w e r b e n ~ n a d ) ~ b e r ~}$ Inlfe angenumben unb befteken aus folgenton Jebern: blaute Jebern wom Rotinga, wentiger jän füt Cisvogelfebern, rote

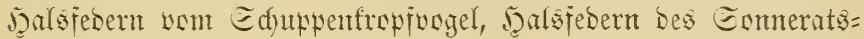

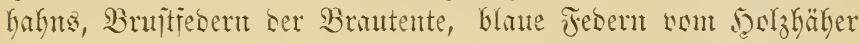
4. a. m. Die Mangen werbent mit jeds sitnoungen befeftigt, Dann wird gefintipt.

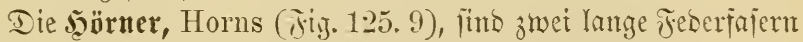

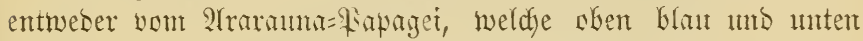

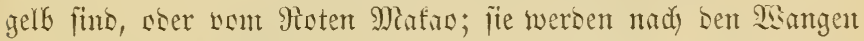
angewumben. Iantit jie jidy gut an bie Fitïgel anlegen, werben jie gefrïmmt, inbent man fie itber bie Edyärfe bes Daumen=

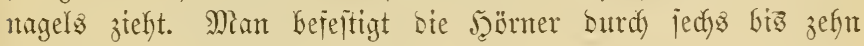

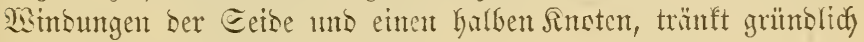
mit Timin unb läjt jie trocfuen.

Der Siopĩ, Head (Fig. 125. 11), madyt bent Edylub; er wirb hergeltelft ans einem jeinent Saben jumarzer Ettufmolle ober enter jumarzen Fiajer einter Etranbenfeber ober einer bronje=

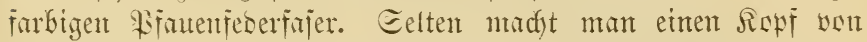

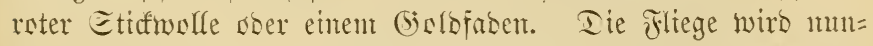
mefr mit brei \$stnoungen ber Eetoe, bent verborgenen Suten

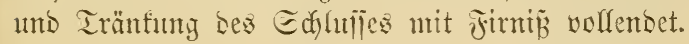

Befdreibung einiger bejonbers wirfamer (3) lanziliegen.

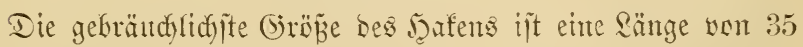
bis $50 \mathrm{~mm}$ uno eitu Breite won 15 bis $20 \mathrm{~mm}$.

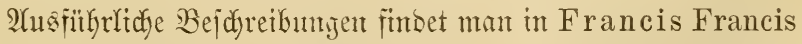
a Book on Angling; - in Rarpitïn Jaale, How to Tie Salmon

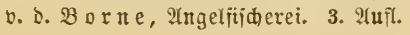


Flies. - 2. (j. Reljon veriffentlidyte 1889 in Land and Water

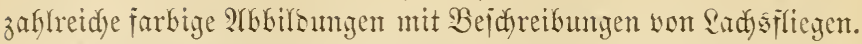

Die Elgannot=Fliege, Fig. 221, wirb auf bem gleidumantigen

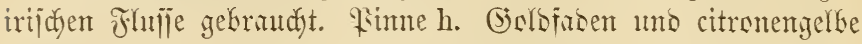

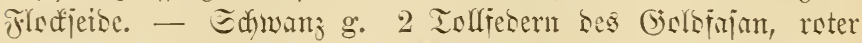

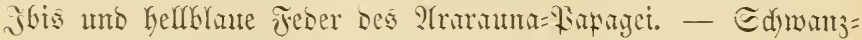

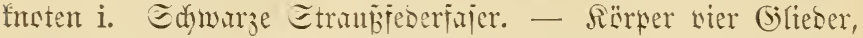

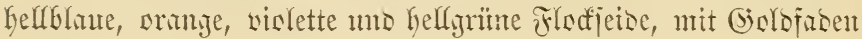

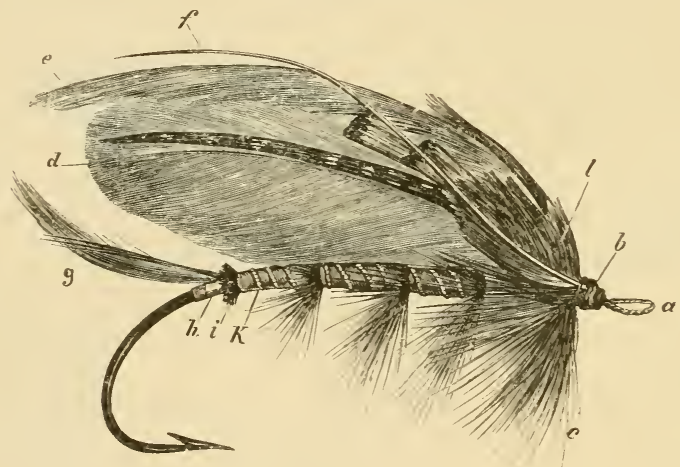

Tig. 221. Ehamnon.

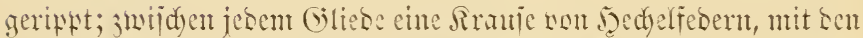

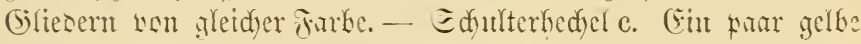

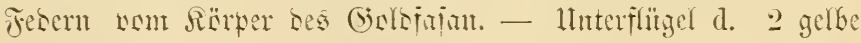
Traramaflitgelfesern mit jumarjer Mitte, Feberfajern wem 2frgus fajm, bgl. wen ber Dalsiferer bes Golsiajm. - Eberfïgel e. Golsfajantelfesern uno halb is lange Feber vem Moten Mafis. -

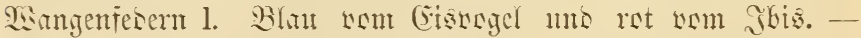

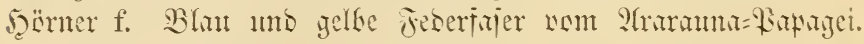
- Sisti b. jumarj.

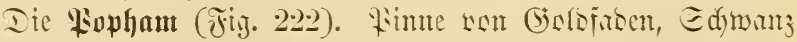

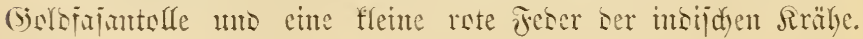


Sd)wanjfnoten jumarz nom Etraun. Rörper in orei Sticbern, unten gelbe, in ber Mitte blaue, oben orange Flecfeibe, mit Gols= faben gerippt; bajwijotsen je ein Sinopi von brenge Binuenjeberfajern unt 3-4 roten Febern bes Sduppen= fropfrogels, jo lung wie bic Şlieber bes Rörpers. Flügel gemijed: Braum uns jowar; gejtreifte Feberfijer vom Edymanj des Goldiajam, braute, idwarg punttierte Feber ber Trappe, bes Rriff= crpel, werjujeben gejürote

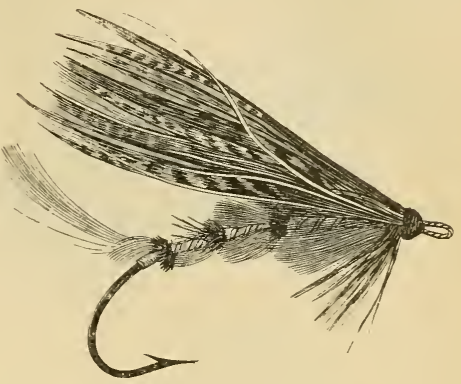

Fig. 222. Fับpham. Teberiajern wom Edyman.

Black and Teal. Ed)warz uno Rrifente (Jig. 223); eine ber bejten Fliegen, bie es giebt, für \&adjle uno gref́ze Forelfen

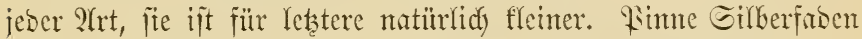

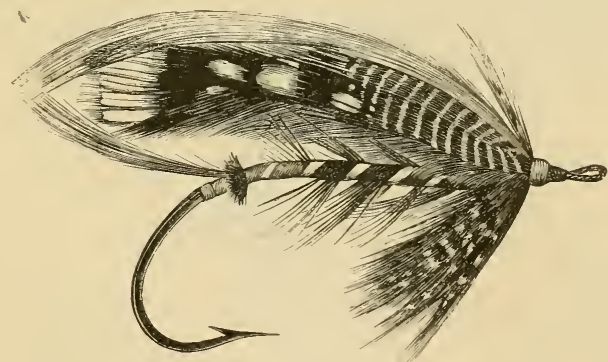

Fig. 223. Black and Teal.

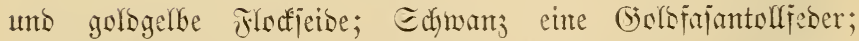

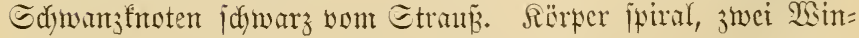

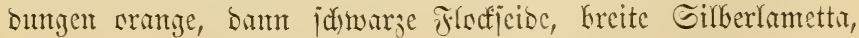




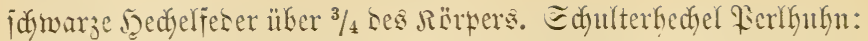

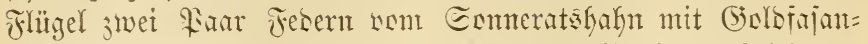

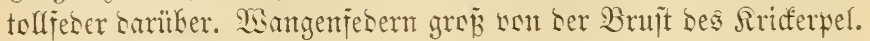

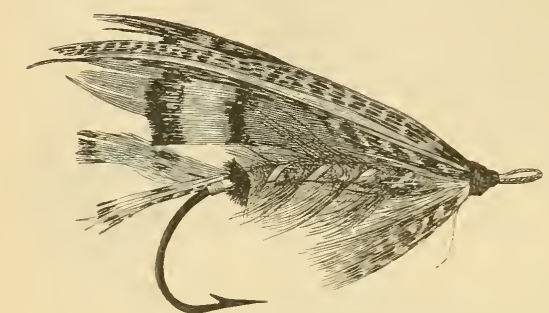

Fig. 224. Sđlädter.

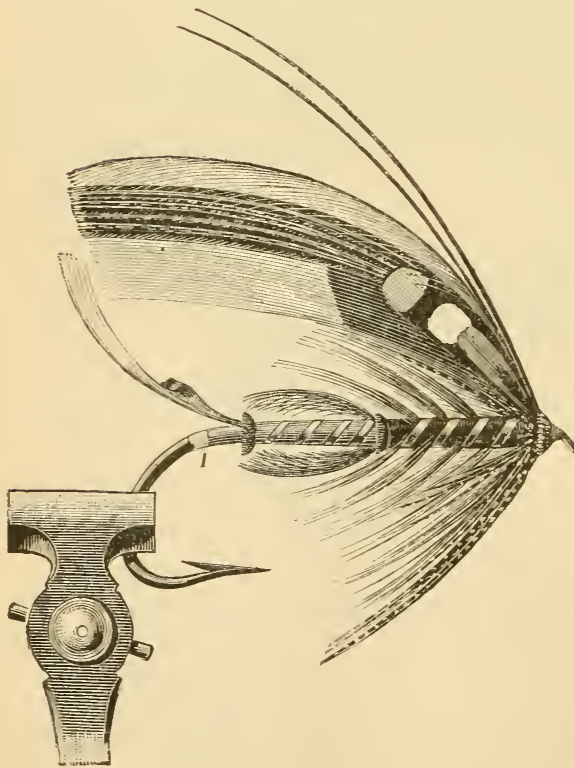

Tig. 225. Jock Scott
Rapi (Salbitiden.

\section{Der Edłlär(jter,} Butcher (jig. 22t). Finne Gidsfisen und oramige ivfocfjeise. Edmans ajern einer Doulafeber bes Gols= fixion; Edymanz= turster idyarze Stramijeder. Sërper

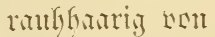
Ed)meinswolle, ipiralförmig, in sier Proteifument, abuect)= jelus licy tblau uno ret, geript nit (jols= lamettis. Edyulter= hedtel forscanuret. Iluterfiügel Bruit= jeber nom Golbiajur. Sberfinigel gemijat; grau wont Enterid), braum, idjwarjgcflect nonder Irappe, Rinu= Gafnerflügel, blau uno gelb geF̈̈rbte Edyman= jeberjijern.

Jock Scott. (ritig. 225). शinne Eilber= forsen unto kellgelte Jirafieioe. 


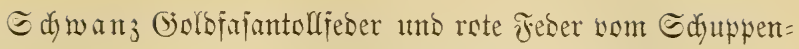
fropinogel.

Sunten idnarge Ctraujpeberfijern.

sïrper in 2 gleidylanjen Giliebern.

Errites Gilico Kellgelbe Fladieide mit peiner Eilberlametti

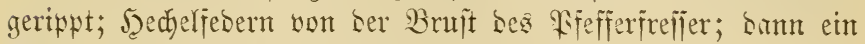
Snoten von jobwarzen Etraubigderiajern.

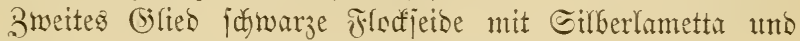
Eilberfaden gerippt, und idwarzer Dedyalfeber.

Edulterhedelfeder vom Ferlfuth.

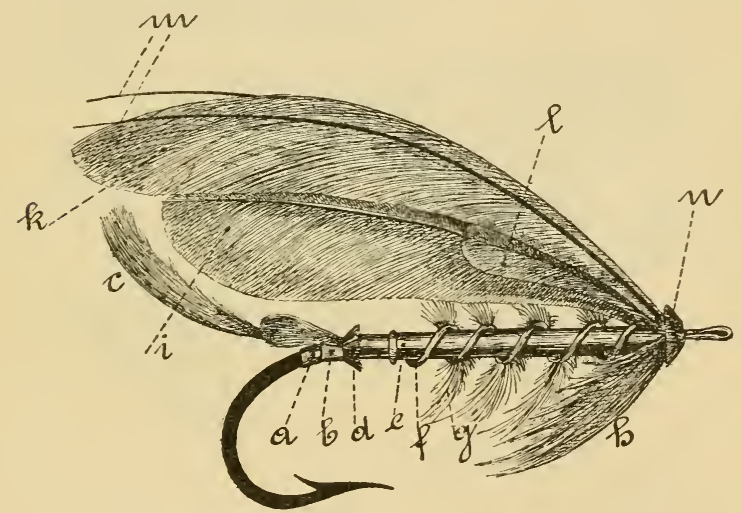

Fig. 226. May Queen, Mai=§önigin.

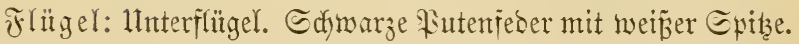

Sberflügel gemijdyt aus Jajern won ber Trappe, grauem

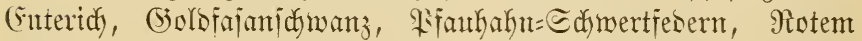
Mafao, blat umb gelb gejärbten Ed)wanenfebern; barüber ein Bündel Fajern von grauent (Enteridy unt Iollebern vom (bolbjajan.

Wangen vom Rotinga uno Sonneratshafn.

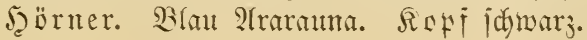


May Queen, Mani=Sänigin (ふig. 226). Pinne Eilber=

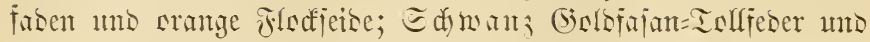

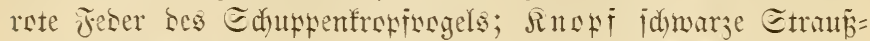

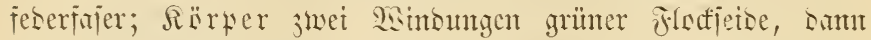

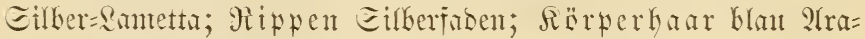
rama; Refle gelb ?trarama; Jlügel roter jbis gelb gerärbt;

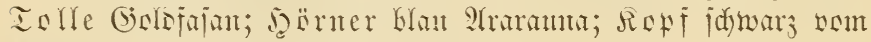
Etrinu.

Stevenson (jig. 227). Finne Cilberiason mb kellblaute

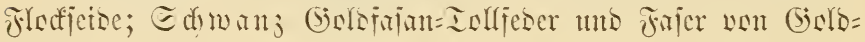

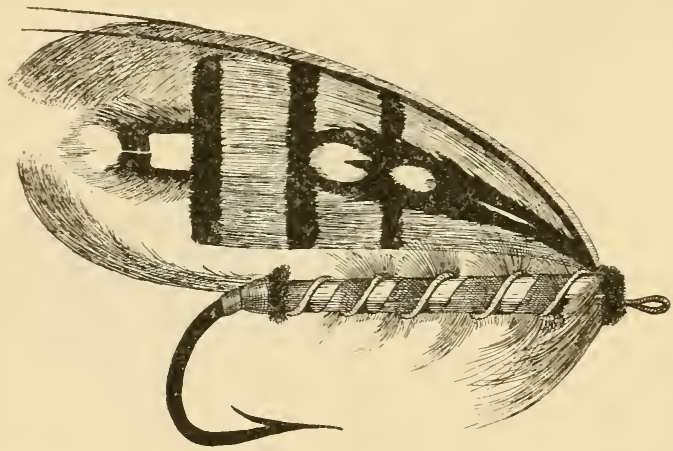

Fig. 227. Steveuson.

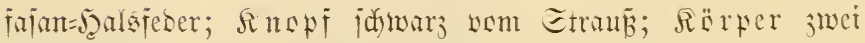

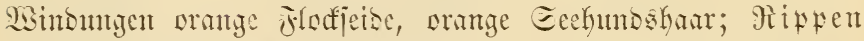

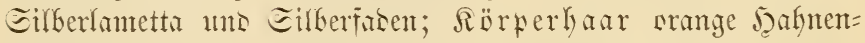

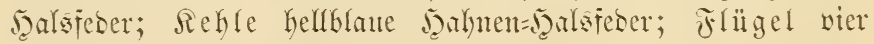

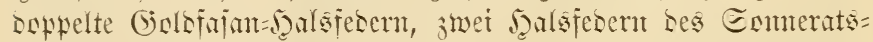

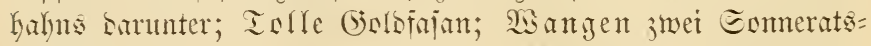

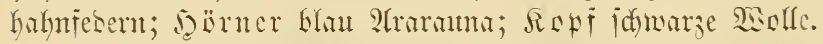

Infallible, Die Unjehlbare (Jiy. 228). Finne Eilber=

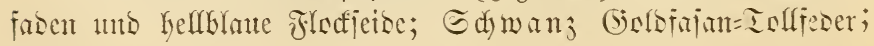




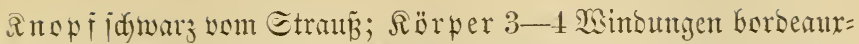
roter Floffeibe, Dann Dunfelgelbe Flodfeibe; Rippen breite

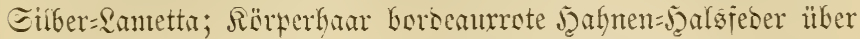

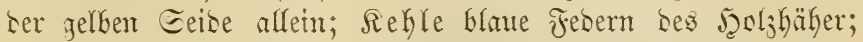
Flügel unten zwei (jolbrajan=ந̃alsjebern, barïber ein gemijater

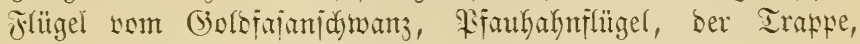
braumen (Enterid), Glau 2trarauna; Tolle Golbjajan; WSangen

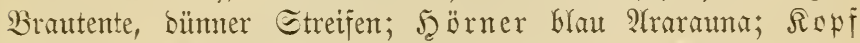
idwarz som Etrauj.

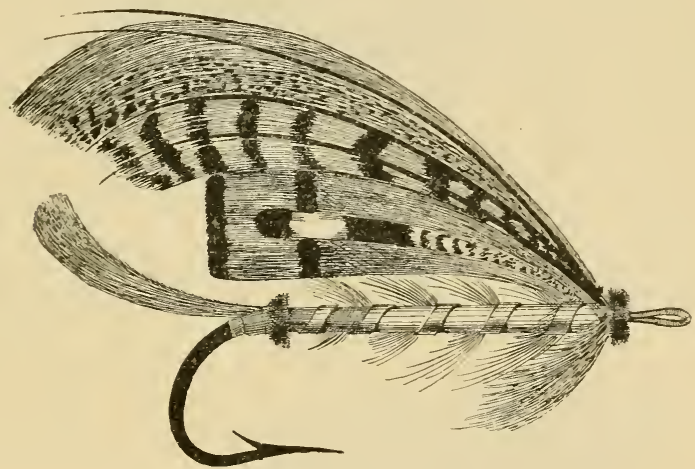

Fig. 228. Infallible, die unjehlbare.

Golden Butterfly, (5oldidjmetterling (Fig. 229). Binne Eilberiaben unb hellklate Flodjeibe; Edyanz eine Golbfajan= Iolfeber; Rnopi idwar; Ctraub; Rörper lidbtblaue Flodjeide; in füñ Arbteiluntgen geteilt, oben uno unten mit (Solofajan=

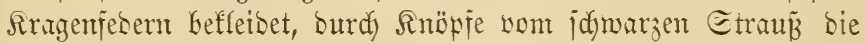

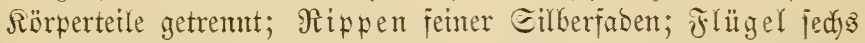
Golbfajan=Solfedern; Sö̈rner Glau Ararauta; Ropf idyarz Etraun.

Dawson (Fig. 230). Binne Silberiaden uno gelbe Flodjeibe; Sdyanz Golbfajan=Iollfeber unb blau Rotinga; 


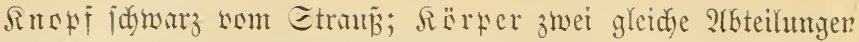
won Eilber=?ametta mit Eilberfaben geriptst mit je zwei rotun

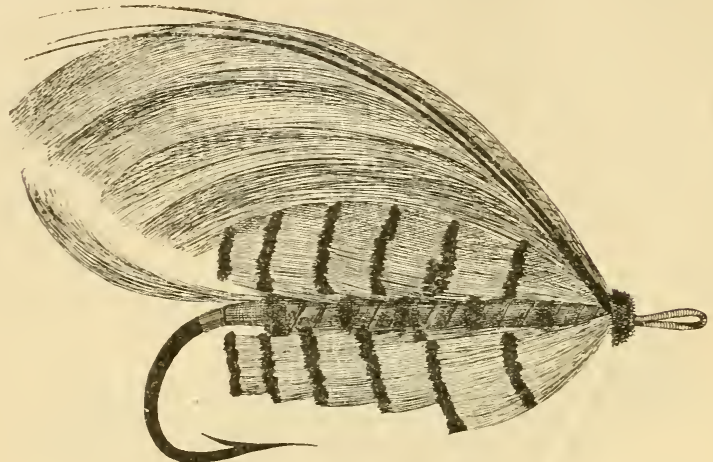

Fig. 229. Golden Butterfly, Golbidmetterling.

Jebern bes Edfupenfropiongels (Indian Crow) theide getrennt Durd) einen Snoten non janarzer Etranjieberiajer; Dals rote

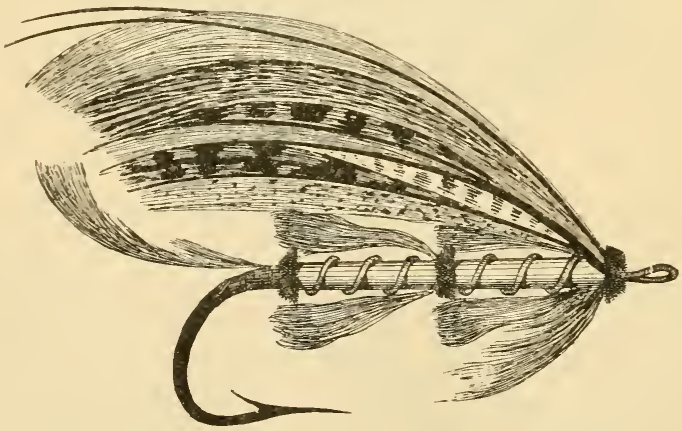

Fig. 230. Dawsou.

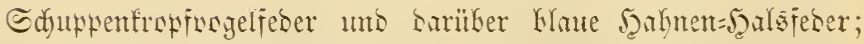

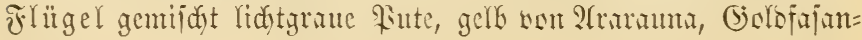




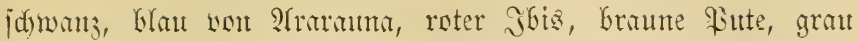
(Enteridh, braun (Enterid); Tolle von Golsfajan; Dörner blau

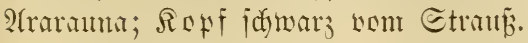

Gitana (Fig. 231). Fintue Eilberiaben umb Glaute Flocfeibe; Cdunanj Golbrajan=Tolfeder unb Hau Rotinga;

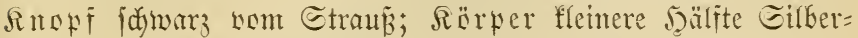
Ramettr, gerippt mit Silberfaben, barïber zwei rote Jebern bes Edyupenfropfwogels, bant ein Rnopf won idwwarzer Etranj=

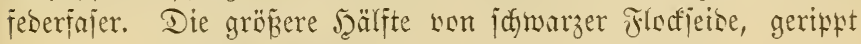

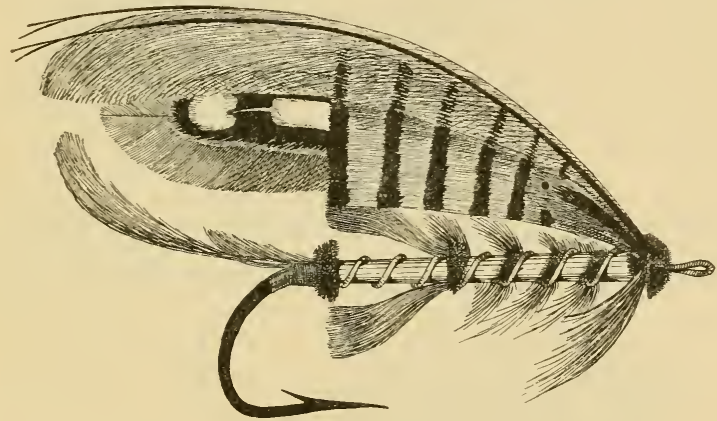

テig. 231. Gitana.

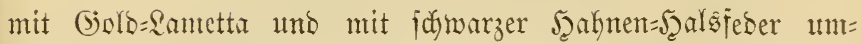

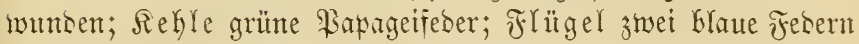

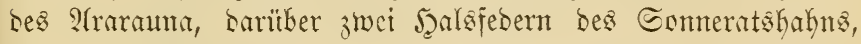

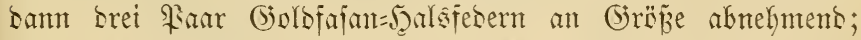
Iolle Golsfajar; Sangen blan Rotinga; Jä̈rner je zhei

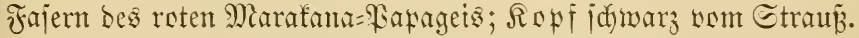

Dandy, Der Etukzer (Fig. 232). Finne Eilberfaben und gelbe Flodfeibe; Єdyanz Goldfajan=Tolffeber, Fajern einer Brautentenfeder, Glau Rotinga; $\Omega$ örper Silber=?ametta, barauf= folgent lidutblate Flodjeibe; Mippen Eilberfaben; Rörper= 


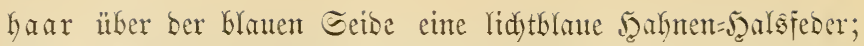

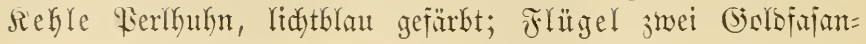

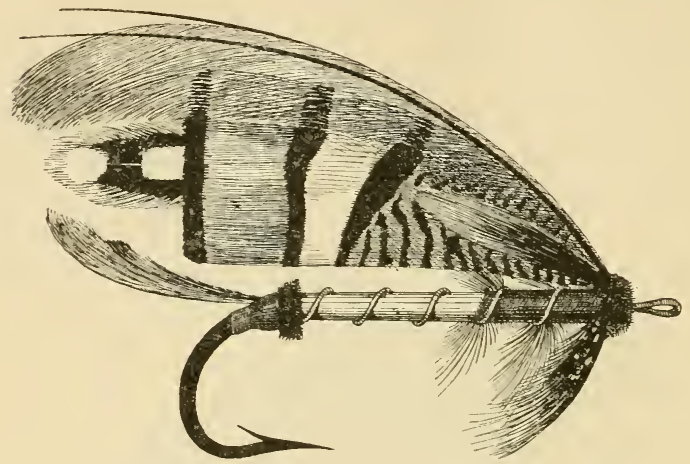

Fig. 232. Dandy, Stuţer.

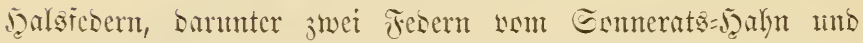
Darïber zwei Febern ber Bratente; Iolfe mefrere Goldfajan=

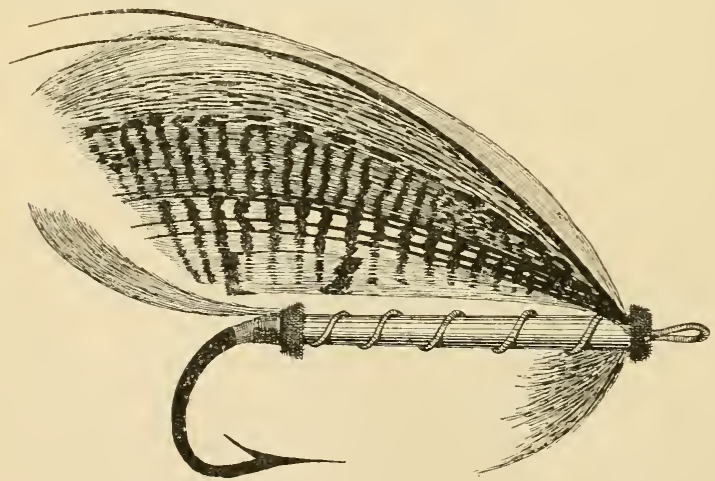

Fig. 233. Silver Doctor, Silker= Doctor.

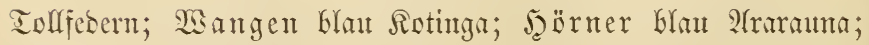
Ropf jumarze rialle. 
Silver Doctor, Eilber=Doctor (Fig. 233). Pinne Sirberfaben unb gelbe Flodfeibe; Edymanz Gorbajan=Tolfeber; Snopf idnarladyrote Wolle; Rörper Gilber=Rametta; Rippen

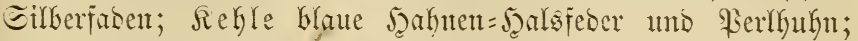

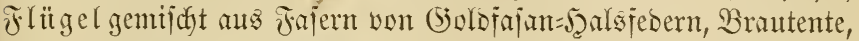
Epibente, Gorbjajanjwanj, gelb und blat gefärbte Edyman=

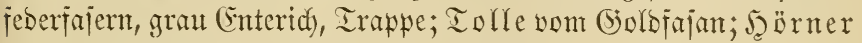
Glau Atrarauna; Ropf jdyarladyrote Wadfe.

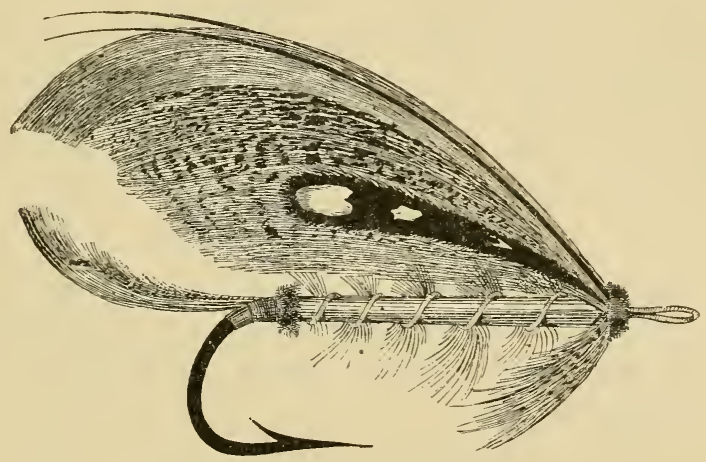

Fig. 234. Silver Gray, હilbergrau.

Silver Gray, Silbergraue Fliege (Fig. 234). Fimne

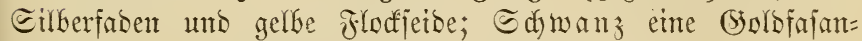
Tolfeber und Feberfajern non ber Brautente; Rnopf fdwarz

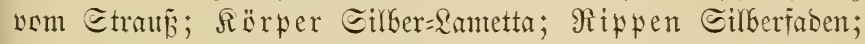
Rörperhaar graue Jahnentalsfeber mit jamarzer Mitte; Rehle graue Enteridfeber; Flügel gemijdyt von Fajern ber Goldajan=5alsfeber, bes Golbfajanjomanzes, Trappe, Edyman gelb gefärbt, Werlhufn, blau 2trarauna, gran (Enterid): Iolle vom Golbfajan; J̋̈̈rner Glau 2trarauna; Wangen 5̧alsfeder Des Sonneratihahits; Ropf jumarze Molle. 


\section{Eeeforellenfliegen.}

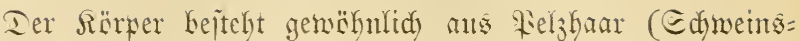
molle, noer Eeehumbifinar) noer ans Flocjeide. Iie Farben fint

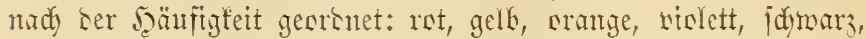
blan, grün; bisweilen ijt Ler Rärper mefrifarbig; oft wieber mit (jols = noer Eilkerfiben geript. Tie Beine funt gewähnlid ebenjo gefürbt, wie ber Sürfer, cóer man bentlit eine Sienjeber (ङ. 60), noer fraume, jamarzpunftierte Jebern, 3. B. nom Sajelfufn noer ser 3mergtrappe. Iie Flïgel fints nom (Enteridy,

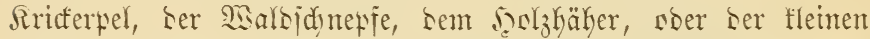

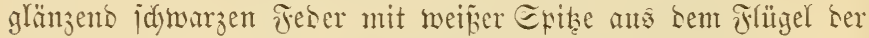
wilisen (Ente. - Man Grandyt aud) Farellenfliegen, Gejonbers March Brown, Blue umb Yellow Dun. Ier Jeaten ijt 5 bis 6 min breit.

\section{2lieerforellenfliegen.}

Mian fimbet eine reidye 2 Hasmahl in bem wortrefflidyen Budje won Francis: A Book on Angling. 2(m beiten fint groje See=

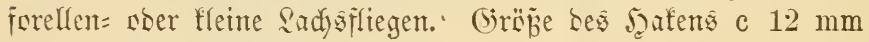
breit $141025 \mathrm{~mm}$ lang.

\section{Küunftliḑe Regenwürmer}

jefre maturgetrent, uach Baron Idyerfajoy non E. 2rryco of in Rebsitjch gefertigt, etwas zart und jumierig jut handhaben, werben an Etemarts sisumtworiad bejeitigt.

\section{Zünitliche fleif̧maben}

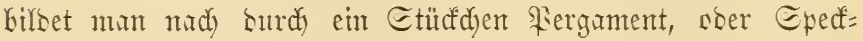

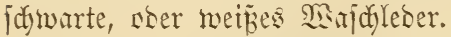

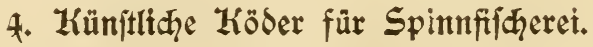

Man fijdyt mit fünitlidyen Exinmenn entweber mit ber Epinnmute ebenjo wie mit natïrlichen Röbern - cober man Kemut fie bei ber Edyleppangelei binter einent fafremben Baste. 
In erîterem jalfe werbienes bie natürliçen, in Keł̧terent bie fünitficfen $\Omega$ s̈ber ben 2 orżug.

Int allyenteinen find bie fïmitlidyen $\Omega$ öber bie bejten, weldye am beiten ipiment.

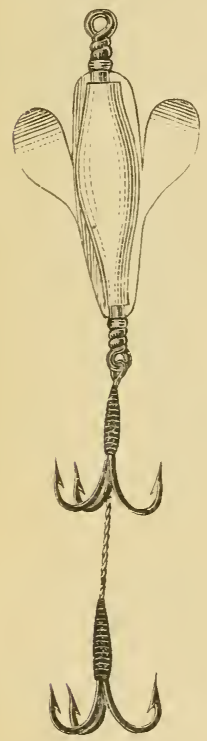

Sig. 235 .

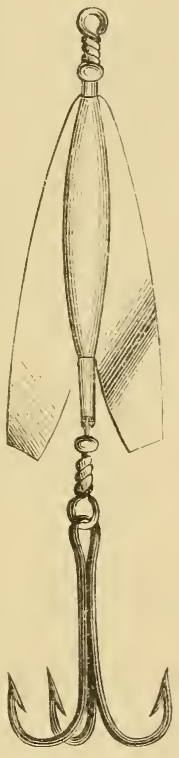

Fig. 236.

Plano-comnex Minnon. Silver Spinner.

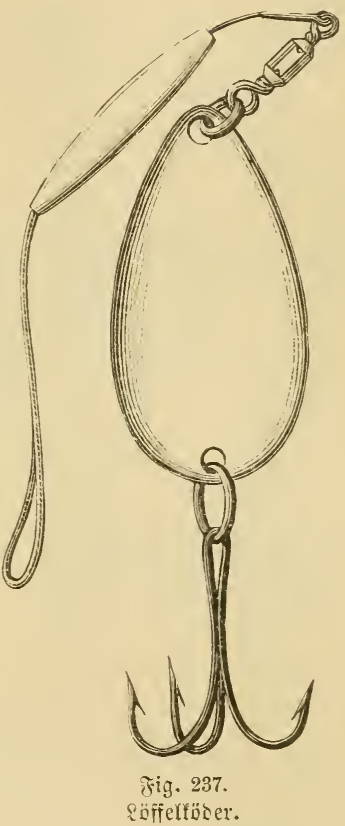

soifeltöber.

Plano-convex Minnow (jitig. 235) uns Silver Spinner (Jig. 236), namentlidy fïr Ed)leptangelei.

Fud bie Röfielföber (Jig. 237) jins jefr zu empfehlen.

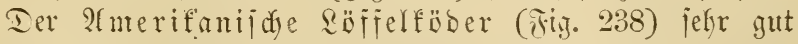
uno giebt einten lendutentoen, wedjelnbent Edjein, ber ifn jefr anjiekents fït bie Raubfitide madyt.

Ier Blinfer (Jig. 239), weldyer bei Etraljumb in ben 


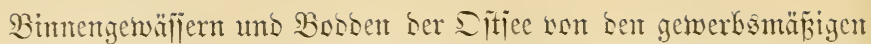

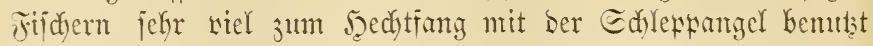
wirb, ijt wegen jeiner (Eimfackeit,

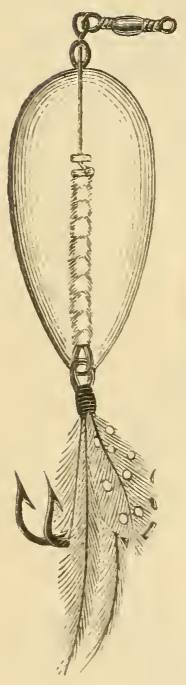

Fig. 238. Imerifanijd)er ¿äffelföber.

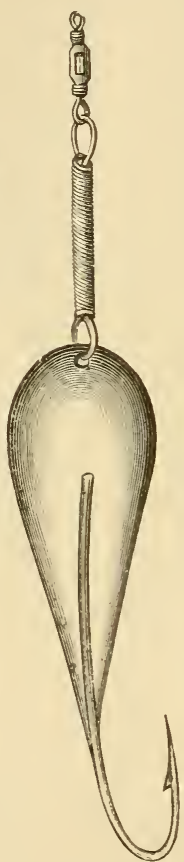

Fi่g. 239. Brinfer. tmo jeines vorjüglidyen Eximens einer ber beiten finmfliden Epinner wou affen. (Er erzengt ähnlidy, mie ber amerifanijue ?ëfölföber, cinen wed) Yenditensen Edhein, uns ijt nertrefï= (id) für ben Fang won ग̃ed), Barid 11. Dergl. Seinrid) Deiloebramb in 2lïndien jertigt anf mein $6 \mathrm{~s}=$ judyen joldye fioser, sie mit Silatina Flattiert fint, unt intmer glänjent bleiben; er madyt jie in allen Grizĩen, ben eliffel won 15 bis $150 \mathrm{~mm}$ Iang, Den நafen wergimut, is saj er and int Ealjwafjer ge= braudit werton form.

Sünftlide Epinuer für bic Jriegenrute.

jarlow Hog-backed Fly

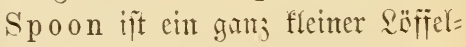
fëber, ber mit ber fliegenrute ge= soorfen werben famr; er ijt jefre gut für sen Fang bon Forellen, Forellen= barjiden, Bariden unto Miatrelen. Der Gejpeniterifid, Fhantont=fijd den, Phantom

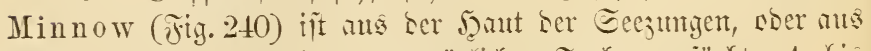
Seise gefertigt, tus in ben natïrfidyen Jarben geförbt; 4 bis $15 \mathrm{~cm}$ lang. (Er ijt jo leidyt, Laj er mit Ler Fliegenrute geworfen

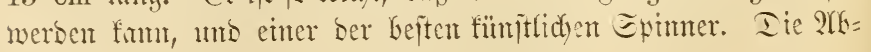

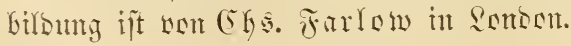


Das Feberfielfijdden (Jig. 241) ijt cit anderer febr beliebter uno jehr Yeidyter fïtiftlicher Röber, ber mit ber Fftegen= rute gewarfen werben fann; es ift jefre wirfian in flarent fladyem 2inilier.

Epinnende fünttlide Flicgen, bie mit ber Fliegenrute

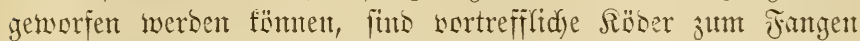
von Sedfen, Forellen, Foreffenbariden, Bariden n. Dgl.

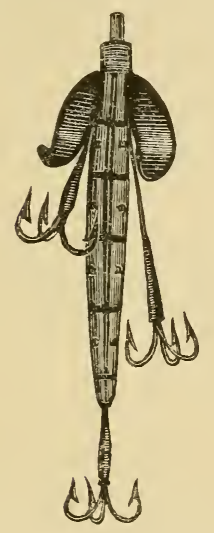

Fig. 241. Feberfie(ïid) den.

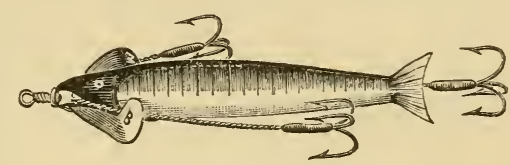

Fig. 240. Geipentertitidan.

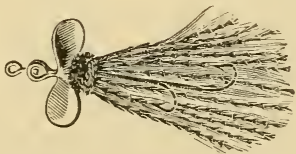

Fig. 242. Epinnenbe łinntlidje frliege.

Die Fig. 242 zeigt bie Spinning Alexandra won Farlow;

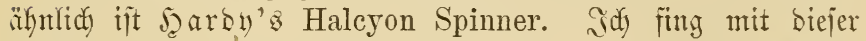

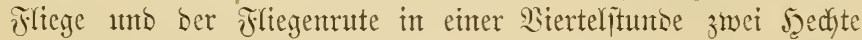

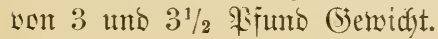

\section{Künttlicḩe Köber für das Geben und Senken.}

Dieje $\Omega$ äber finto jo jtart mit Blei bejdyert, baj je jednell

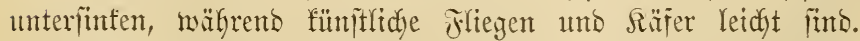

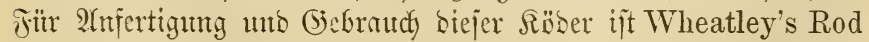
and Line bie Somptquelfe. 
1. Roflraupe (Cabbage Grub). (Ein Etüdficn geglïften Meijugbraft legt man zujammen, und breft es in eine Edjleife

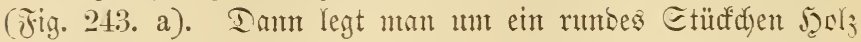
einen Fapiercylinser mo flebt ifn an ber Eeite mit Eiegellad 3ujammen. In biejen (Sylinder jteft man ben Mejïngbraft uns

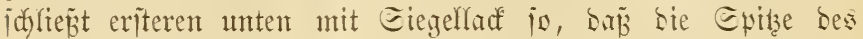
Meiningorafts $15 \mathrm{~mm}$ unten noriteht. Iarauf unflülft man ben

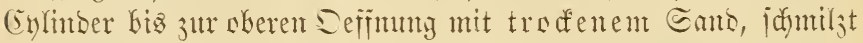

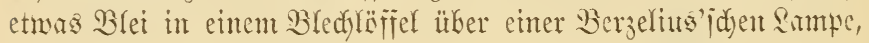

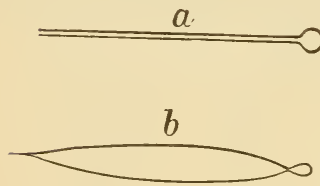

Fig. 243.

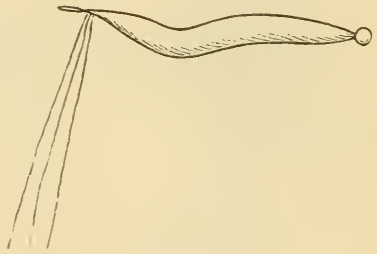

Fig. 244.

und giefit ben Fapiercylintor woll Blei. Ier Eanto smi nidat

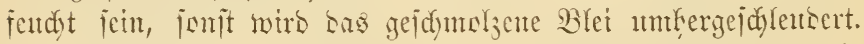
Mit Mejer, Feile uno Folieritahl gieft man bem Ganjen bie (5ejtalt von Fig. 243. b, uno kiegt bas Blei, wie es in Fig. 241

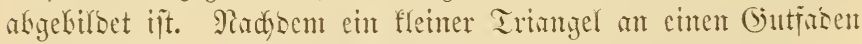

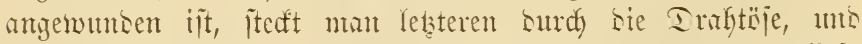
binbet ifn jejt an. Ier freibleibende Teil bes jirbens jull io lang jein, baj, wenn man sen Triangel nad) bem Edymanj ber

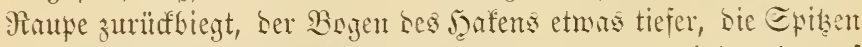
etwas häher wie ber Edwans jeken. Man wintet bamif unten am Meifingoraht jwei Fiben won nidyt jut Sunfler grïner

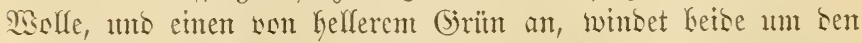
Meifingoraht nädjit ben Blei um (Fig. 241), umb biegt ben

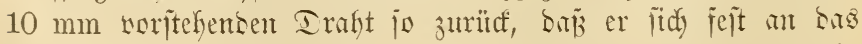
Blei anlegt. Man wintot Lam bic Sadfe um bas Blei bis 
zun Rofi, uns bintet fie an ber Deje fejt. Bugleidf woirb eine Fajer cinter Rijuent soer Etranjenfeber, ober ein Stühdyen

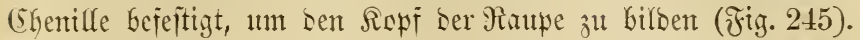

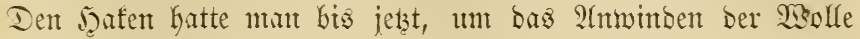
nidit ju rinbern, von Rörper abgebogen; num legt man ben

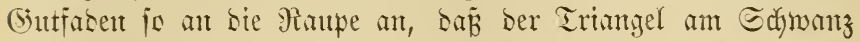
liegt unt befejtigt ben (butfaben in biejer Etelfung Durd) cinige Ssindungen ber Eeibe. Num winbet man mit ber Feberfajer voer

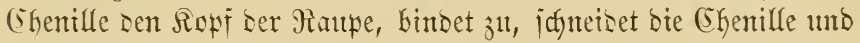

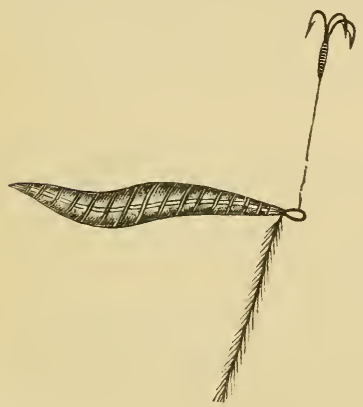

Fig. 245.

Siohlraupe.

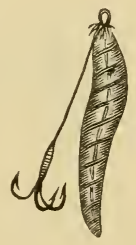

Fig. 246. Siohlraupe.

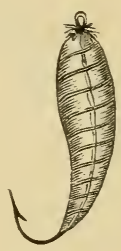

Fig. 247. Grashüpfer.

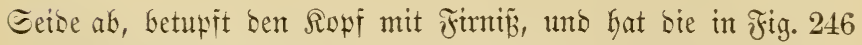
abgefilbete ingenamte Roflraupe, einen ber Geiten Röber, bie e⿳亠丷厂 giebt.

2. Der Grashüpfer (Grasshopper) ijt ein Dem worker= gefenten äfnltider Röber, unb wirb vieffeidyt nted mefr in ber Yejdenfiidyerei gebraudyt, obgleidy er ifym an Wirfianteit etwas

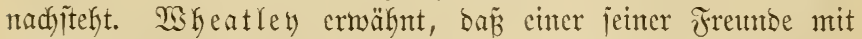
biejem Rëber in brei Tagen (wovon an einem nur brei 巨tunben

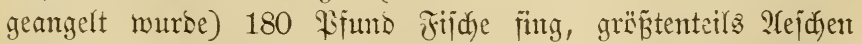
uno einige Forellen. Der Grashüpfer ift in Fig. 247 abgebiloet. Er wiro folgenbermañen angefertigt: Man nimme einen etwas

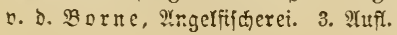


größ̈eren Jafen wie bei ber Rohlrampe, formt wie vorker aus

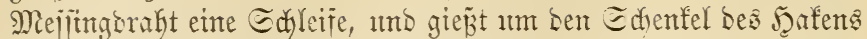
uno bie Draffichleife Blei, weldes man mit Meijer, Jeile und Polieritabl is formt, wie es in Fig. 247 abgebilset ift. Dann

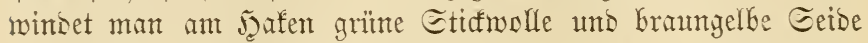
an, winbet bie Molle um bas Blei biş jum Rofie. Dam legt man an jebe Seite bes Rërpers ein idymales Etüdfden Etroh, unt winbet bie gelbe Eetse in Efiralminsungen baritker. Den Ropf bes (Srashüpier madyt man ebenis, wie ben ber Koblratipe.

3. (Sin anberer guter $\Omega$ ïber ijt bie in Fig. 248 abgebilisete

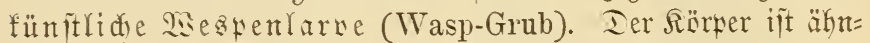
lidh wic bei ser Roblraute, aus weijer Chentlle gemumben.

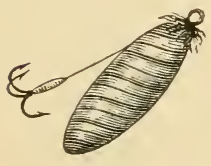

Fig. 248. mespentarve.

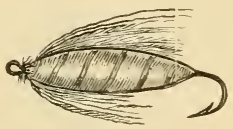

Fig. 219. Miaifliegenlarve.

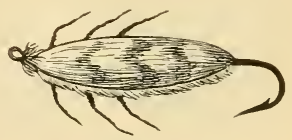

Fig. 250 .

Eteinfliegenlarbe.

In Fij. 249 iijt eine Piadjbiloung Der Sarve ber Mai= fliege (Green Drake Grub) afgebilset, furz beror the Iaz

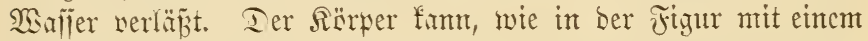
Jaffen, nder, wie bei ber Rofinme mit einem Triangel bemelyrt wersen, und iit, wie bie frïheren, mit Blei bejdwert. (Ex ijt mit idmutziggelber Ghenilfe befleibet uno mit brauner Eeibe gerippt. Die Fligel fint biejelben, wie kei bem Green Drake, uns liegent an Rörper an. Intüber wirs eine ingwergelbe Jectelfeber gemumsen, um sic Beine nadyubilsen. 2tudy bies ift ein jehr guter Sïser, namentfich f̈̈r Forelfent.

5. Sünitlidye Ereeper (Creeper, Crab, Cad-Bait) (Fig. 250). Die (Ereeper jind bie Sarven ber Eteinfliege und cin jehr

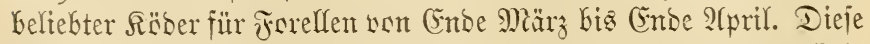

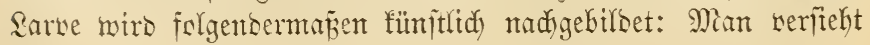




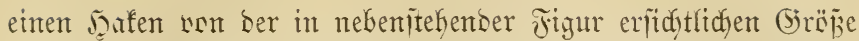
mit Blet, uno giebt lebsterem eine flache (jejtalt. I am veriährt man ähnlich, wie bei ber Darjellung fümitlicher Räjer. Das

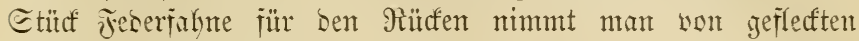

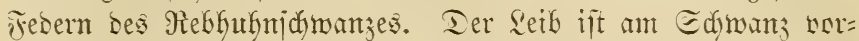
herridsent gelf, und weiter sberfalb gelb= uno bratm gemijht. Er wirb mit gelber Seibe gerippt. Die Beinte fint man aus biden Fäson slivengrïner Ceibe ntaden, mant bindet ïe an ben saffen, nadbem er mit Blet werjeben iit uno ehe etmas anderes gemadyt wirb.

\section{Dritter 2lb/hnitt.}

\section{$\mathfrak{A} \mathfrak{i} \mathfrak{g} \mathfrak{e} \mathfrak{l} \mathfrak{m} \mathfrak{e} \mathfrak{t} \mathfrak{j}$ o d $\mathfrak{e} \mathbb{H}$.}

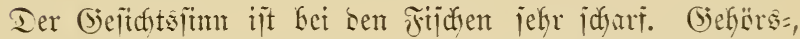

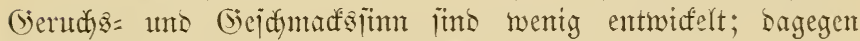

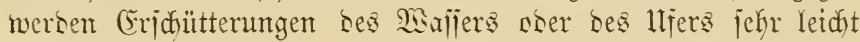
won ifnen bemerft. Wier es werjeft, in flarem 2 sajier ju fijdsen, shne won ben Fijdjen wafgrgentwmen jtt werben, hat bie grëpite

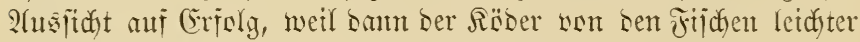

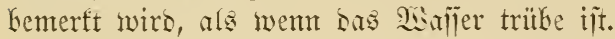

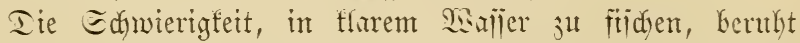

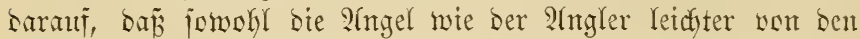

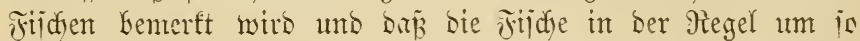
jucuer jüns, je älter uns größ̈er je jün; ber Borteil, ben bieje Frijberei gemährt, went obige Edywierigfeiten übermutnden fino, iit, baj bie Fijdye Den Röber viel Yeidter wahntehmen.

ltm Las 2lngelgerät mëglidyit ju verbergen, madyt man es jo fein wie möglid) uno vermeiset alle glänjenton, aui= fallenten Tarben.

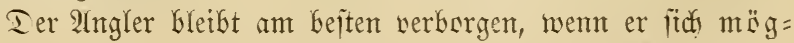


lidjit entiernt wen Ien Jifden fält unb wenn er in nidit ju raid) fliçentem 些affer ftromauf fifdt.

Ifle Jijde itehen mit bem ふ઼ofi gegen bie Etrëmung ge= ridhtet, weil jie mur is ifre Etellumg behaupten fünnen; jie jefon

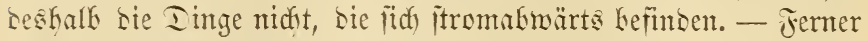
wiro ein Fiid, weldyer ben Räber ergriffen, leidjter gefaft, went er itromabmärts angefanen wirs, weil ifm ber Darfen ins Maul

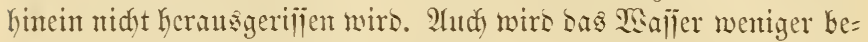
unrubigt, weil alle Jithe itromab fliefen, wemt ie gehaft jinto, fie fonmen baher in 2 sajper, weldes bereits abgefiidt ijt. Prlersings ijt sas Tijden jtrmauf jdwerer, es giebt aber viel grëв̈ere 2 (uskente, numentlid) viel grë̈ere fiid)e, went man bie Edymierigfeiten bejiegen famm.

Ins jijden itromab ijt in jtorf itrïmensem, in tiejem

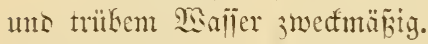

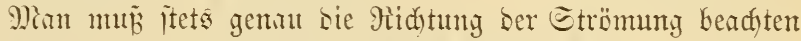

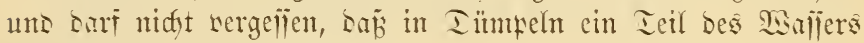
rüıfläıf̈̈ge Etrëmung hat.

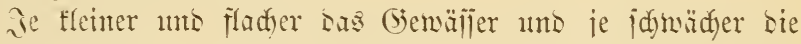

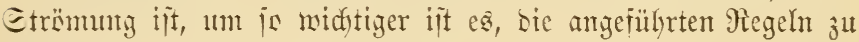
beachten.

Infauen nent man sas idnelle Tnjiehen ber Prngelidnur,

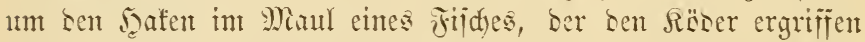
Gat, jut befeitigen. Miandye jitide pflegen ben natïrlid)en Röber

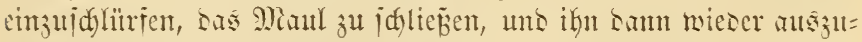
ifecen; bei ifnen mub iofort angehauen werben. ?tnsere ergreifen sen Rëser langian uno serjidtig, uno entidyliejen jid erit mit ber Beit, ifn ins Diaul ju nefmen; hier ift jofortiges P(nhauen ein

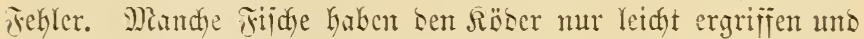
idyimmen Lamit eine Etrefe fort, währent antere bie $\mathfrak{A n g e l}=$ ichmer faum bewegen uno bof Den Röber ganz ins Miaul genemmen

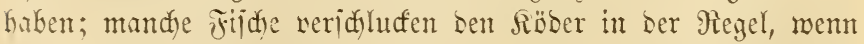

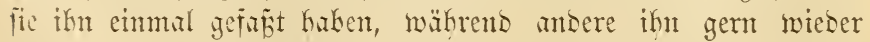

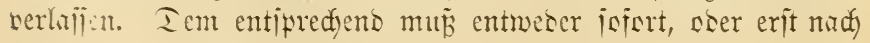


einiger Beit angehauen werben. Exfertiges 2tnhauen ift zmedt=

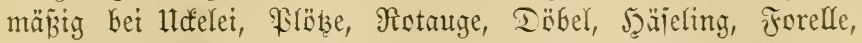
2leidje, Eecjorelle, Miecrisrelle. Man martet einige Beit, che man

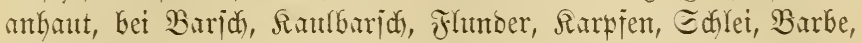
Blei, 2hand, 2lal, \&ady.

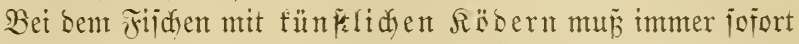
angehruen wersen, weil ber Fijd bie Täujdung bemertt, jowie er ben Röber ergrifien hat, unb biejen besfalf jofort mieber aus= ipeit; bie Sdyutr mun itets jtraif gefaltent werben, um jofort an= hauen zlt fümen.

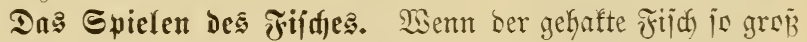

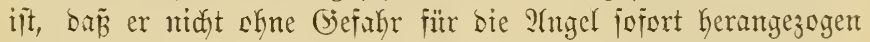
werben fant, fo wirb or vorker ermilbet. Dies gejdieft in ber Seije, oaj man ifn bei jeinen Bemegungen umunterbrechen be=

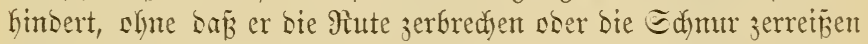
fann. Senn es notwenbig ijt, läjt man ifn mehr Edyur von ber

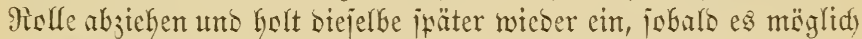

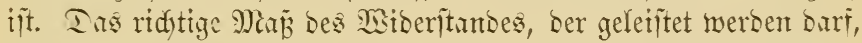
mui man burd) llebung fennen Yernen. Man ijt bann initande,

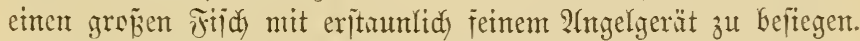

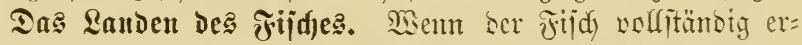

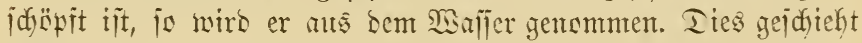
entweber mit ber 21ngel, coer ber Jants, ober bem Entoutygnets ober bem Lanbungshafen.

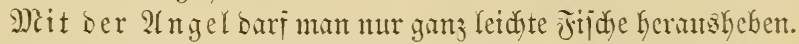

Mit ber Jaand hebt man einen wollitändig ermübeten jijd heraus, inbem man ifn jeit finter sen Riemen fajt. (Es er=

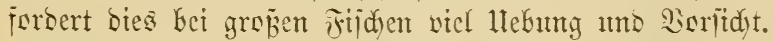

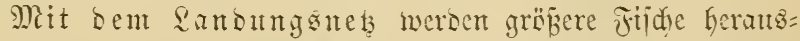

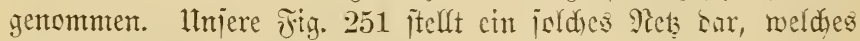
jujammengelegt werden fant.

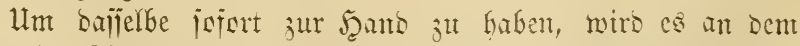

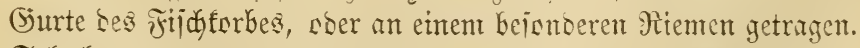
Sehr bequem uno zmedmü̈̈ig ift oas ,Slip" Landing-Net, meldyes 
Carter \& Peek 137 St. John Street Road, London werfaufen. Der (brifi ijt iejt mit sem Mes werbunben, nidyt angejdyrabt,

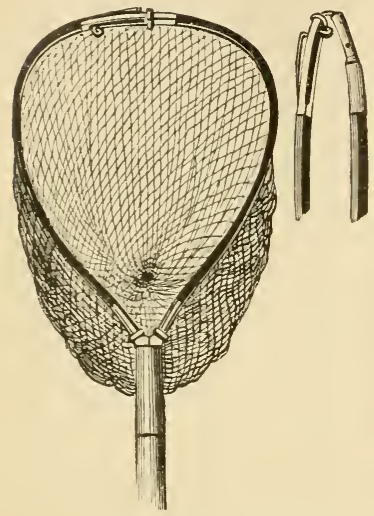

Fig. 251. \&anoungsnetz. fann alje nicjt loje merben, es miro mit eintem Riemen über ber Edyulter getragen, uno läğt fich leidft uno be= quem fanshaben. (Jig. 299.)

Ter sanbugithaten, ijt in frëmentem 然ajper umb Kei grofen Fithen ficherer wie bas Ranoungsinetz; or hat bie Form eines grofen ?higel= Gaten, uns jein Grifi beiteht

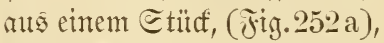
rober er fam wie ein Fermohr jujammentgejdoben werben. (Jig. 252b.) Die Entize Des Sorfens muj io sermafyt jein,

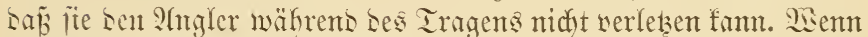
ser jitch ermitset ît, is näkert man sen Ransungshafen won ber

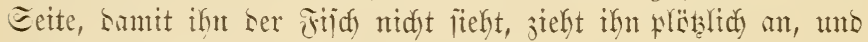
fringt sen jiid io juntell wie mëglidy ats sem :

Der Ђafenlöjer (Jig. 253) whit angewendet, um ben Fijd)

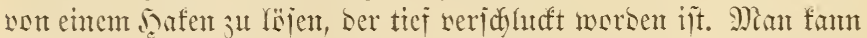
ifn mit einer Sanbiäge aus itarfent Mejïngstaht, soer einem Etüd Gartent $S_{e} \mathfrak{l}_{3}$, wer Dem Griff einer Bafnbürite anfertigen. Dan

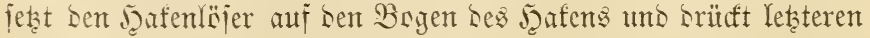
herants. 
Die Jaedidjere. Ias Röjen bes Jedyt won bem Jafen eriorbert Burfidyt, meil bie idjarjen Bähne leidgt bie Finger arg verlez̧en. Um bas Maul jo lange offen z̧u halten, bis bie jaten gelöjt jind, bebient man jich einer Art won Schere, bie feit geitellt werben fann. (The ,Jardine" Gag, Pike Scissors).

Ier Fifdforb, Fig. 25t, bient zum Iragen ber gefungenen gijdje.

Iamit er beim Siehen und Saten im Wajjer jejt jitzt, uno jidy nidyt werjdyiebt ober gar überjdlägt, trägt man ifn sfft an eimem (s)urte über Der linten Edyulter, Der mit einem (5)urte um ben Seib verbunben iit, jig. 255, io baj ber rechte 2 (rm vollftändig frei bleibt. Man tfut Giras in ben Rorb, bamit er midst burd) Den Edjleim ber Fijdye verumeinigt wirb. Sm Decfel befindet jid) eit Sod) zum (simptecten ber gejangenen Fijdye.

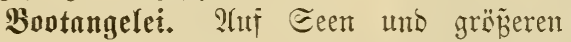
Flüijen iit oft bie $\mathfrak{A}$ ngelei vom Boote viel mirt= jamer, als bie vom llfer, beshalb junb bie trans= pertaffen Bonte Der Bendfung Des 2Anglers mert.

Die Berton Boat Company (Limited), 150 Holborn Viaduct, London jertigt zujammen= Yegbare Boote, bie won 2 Männern getragen werben fümen, bie $2,2 \mathrm{~m}$ lang und $1 \mathrm{~m}$ breit futro uno mur 8 sitrl. foiten.

Cording's Collapsing Fishing Boat, สนติ

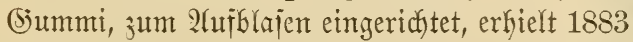

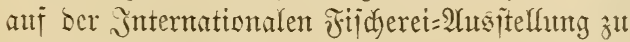
Sonow einen Preis.

Das Corasle, Fig. 256, non wix Gierbei abbilden, iit angeblich bas älteite $\mathfrak{B}$ oot
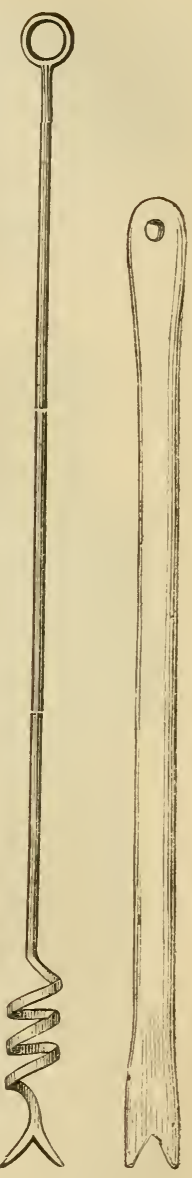

Fig. 253 。 Şafenlöjer. 


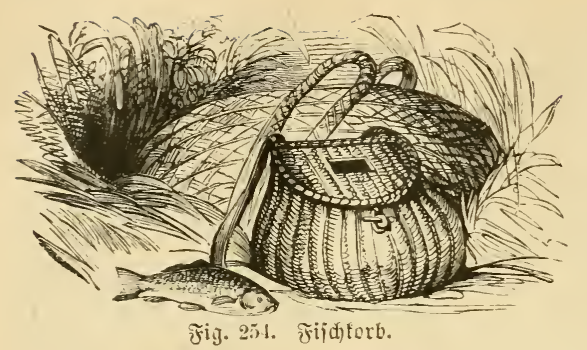

Englants. Es maro bereits 1188 ermähnt, als (Erjbijadof Bald = min einten Rreuzzug predigte, unto wiro nod) Keute auf bon Flitifan in Sales riel zut Picts= uno 2tngel= fiict)erei benutit. (5:

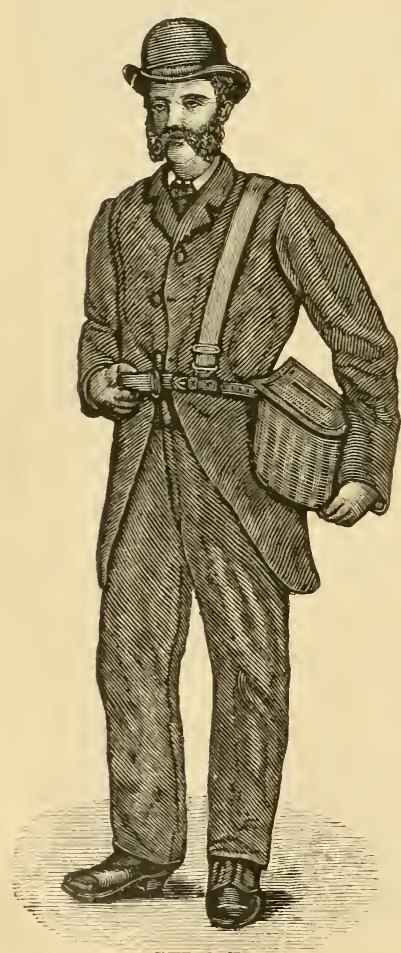

Fig. 255. Fijdfort. gehërt jur Bemtbung vielellebung, weil es jodmer ju rutsern ijt mo Yeidut umijd)lägt.

Daŝ (Soracle ijt ein Rerb won (Sjd)en= noer Secibentutenge= fledit, ber mit gepidyter uno ge= itrictener Qeimmato imen und minen betfeiset ijt. (E) ijt $1,25 \mathrm{~m}$ lang unt $1 \mathrm{~m}$ freit.

(5. wirs an cinem Giurt ïber. ber Edyulter getragen, ber an bem Sibbrette angebradyt ift. Das fleine Puber wirs bei bent Eriggen quer über ben Rilden gelegt. (5) iit and) mit einem ?nfer werjeken.

\section{Die flofangel.}

In Tloj kat ben 3wect, ben Rïber it einer beitimmten 2Sallerticje 3u trigen, uno ifn in

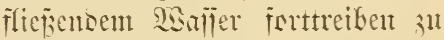

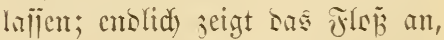
wem cint vilid angebifien hat. In fladjent uto flarem seafier

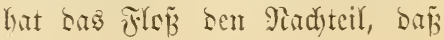




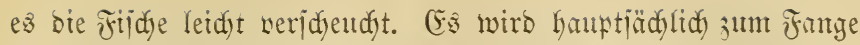

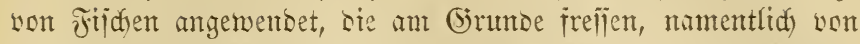

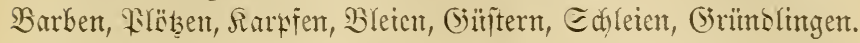

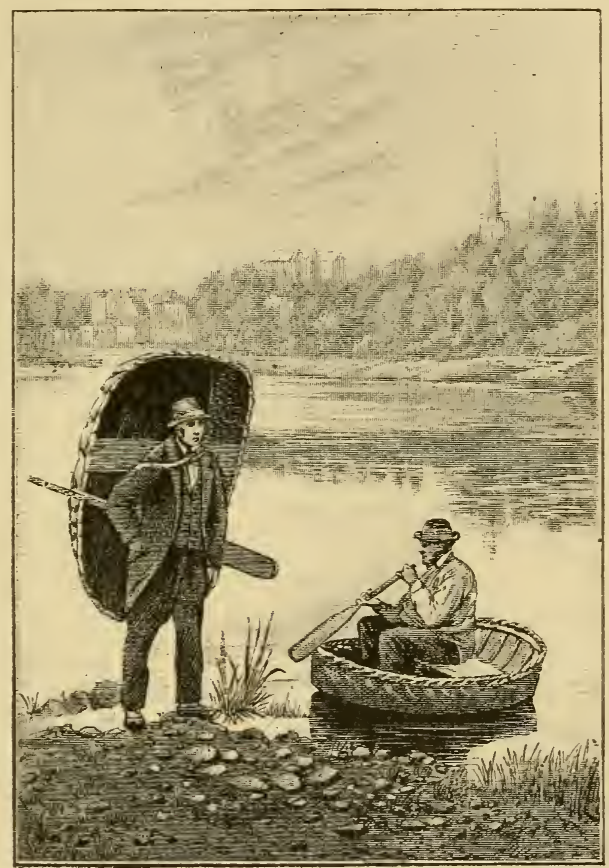

ฆig. 256. Coracle.

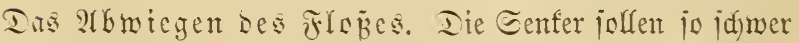

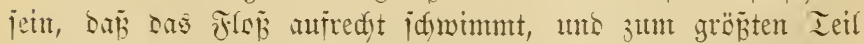
unter Saljer ijt. Je weiter es verienft ijt, un fo empfintolider

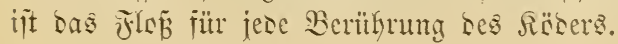




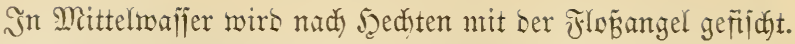

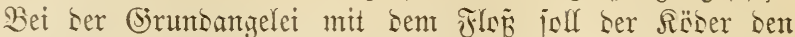
(Strunb berülgren, soer fïh wenige Sentimeter barüber befünen. Man min̈t seşalb juerit bie Tieje ses Mafjers mit einem Eentblei, weldyes an bem Jaten bejejtigt wirb. Fig. 257 a jeigt
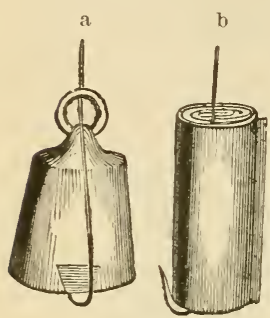

Fig. 257. હentblei. cin Eentblei, weldes unten mit Rerf ver= jehen ijt, in meldyen ber Salen eingeitedt wirs. Fig. $257 \mathrm{~b}$ itellt einen Etreifen Bleiblecty Iar, weldyer um Ien jafen ge= widelt wird.

Mant besient jidn am bejten ser Rottingham=2lngel. Die Angelmute it ganty leidyt, 3 bis $3,7 \mathrm{~m}$ lang, mit aupredyt jtehenben Mingen, sie Molle sreht jidy

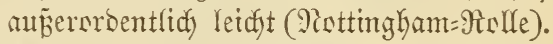
Ite Rollidymur ijt won Geibe geflechten und ungefinnipt; fie wiegt bei $100 \mathrm{~m}$ Sänge fïr \$edtrang 23 bis

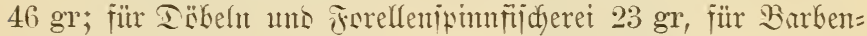
fang mit Brsenblei $20 \mathrm{gr}$, für Barbeñang mit J゙ln bangel $12 \mathrm{gr}$,

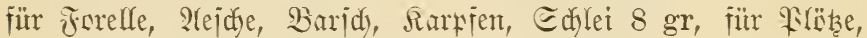

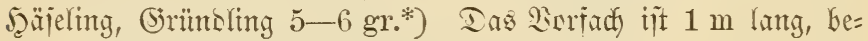

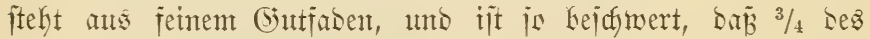

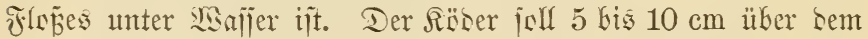

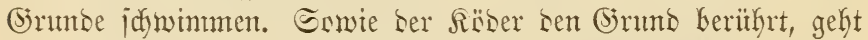

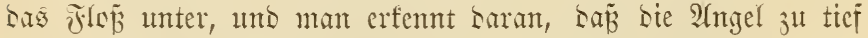
geitellt ijt. Die Sobnum inll leidyt gejpannt gebalten werben, bamit ser fïber surd bie Etrïmung wrauggetrieben wird.

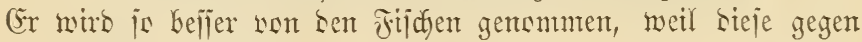
bie Ctrömung Jront gemacht Gaben, unt ben Röber eher erbliden, wie bic 巨afyur. ๔. Jig. 258.

*) IBilliam $\mathfrak{B}$ ailey. The Angler's Instructor. Nottingham. Die wichtigite Suelfe für bie Prottingham= Jitichere. 
Bejejtigung Des äbers an bem Nngelfaten.

1. Der einfadje $\mathfrak{g a f e n .}$

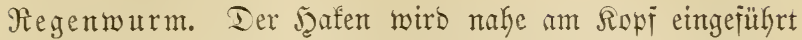

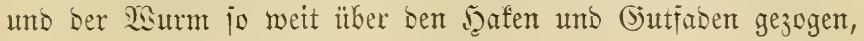

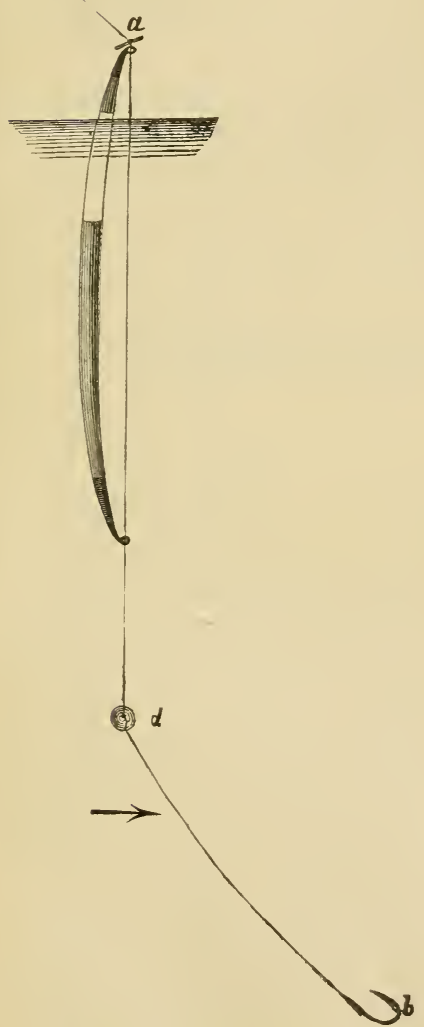

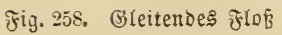

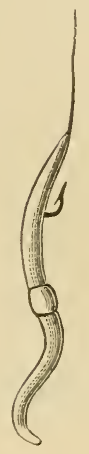

Fig. 259.

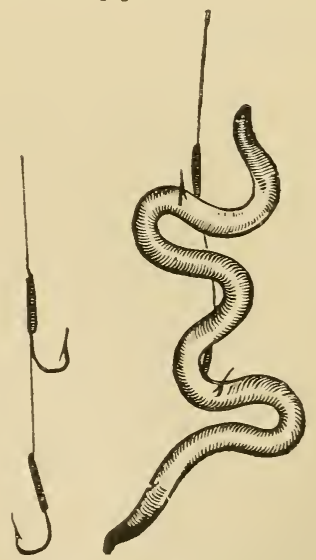

Fig. 260. Stewart's Murm=:oriad). 


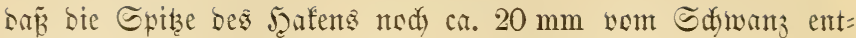
iernt ît.

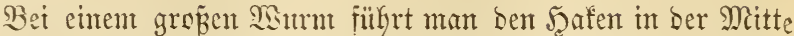

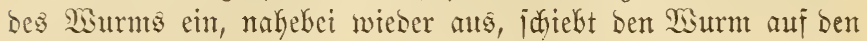
Gutfaben Ginauf, führt ben Şaten am Ropj nochmals in ben

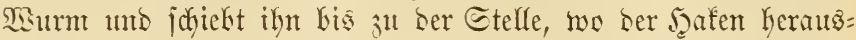
gebradjt uvar, baum fajtebt man ben Teil bes Sarmts, ber auf bem (Sutfaben ift, Kerab (Walton).

(j) Gejeitige ben Sisurn gemëhnlich jo, wie Fig. 259 zeigt, wobei idy cinen fleinen $4-6 \mathrm{~mm}$ breiten Jaten armente. Jit)

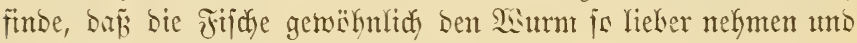
ben Ђafen woniger leidyt bemerten.

Farlom wintet an ben Saten eine ober zmei Edyeins: boriten an, um ben sium feitjufalten, unb Defien Ferafgleiten

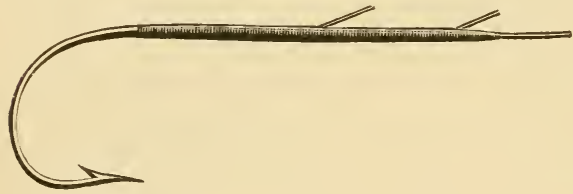

Fig. 261. Murmoriach mit Boriten. jut verfindorn. Fig. 261.

Maben. 然enn man mit 3 bis 4 Plaben angelu will, is nimut man cinen 4-5 $\mathrm{mm}$ breiten Dafen. Man fübrt bie Epitze einige Millimeter nom biden Enoe in eine Mabe cin uno am bünnen (Ente heraus; ebenjs veriährt man mit einer zweiten uns britten Mlabe und bebecft baum mit einer vierten bie Eptze Des Datents. Will man zwei Maben Graudien, io nimmt man einen $4 \mathrm{~mm}$ breiten 5 afen, itecft bie crite

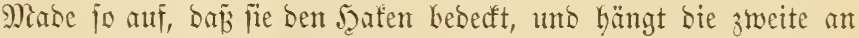
Sic Epitze. Mabl bie bejte Methroe ijt bie folgente: Man nimmt einen 3-4 mm breiten 5aten und itidyt jeine Exibe am bidfen Enbe eiter Mabe ein werrig unter ber Saut burd, io baj ber gamje Salfen unbebeft bleibt. Man fann fo aud 2 soer mehrere Maben miteden, bie bann am Jaffen hüngen, wie ein Bunb Edjlitifeln am Minge. Der Sanutorteil biejer Miethode bejteft barin, baj ber Räber länger am Reben bleibt unb burd) jeine 
Bemegungen bie Fijdje in Gokem Grabe anlodt. Man muź für Maben einen Jafen aus feinerem Iraht nefmen, wie er jomit zocedmäp̄ig jein würbe.

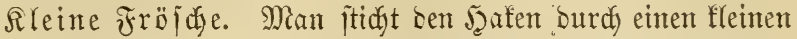

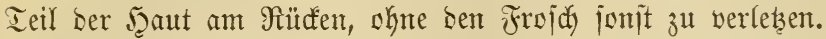

Mespenlarven. Man ninnt einen $3 \mathrm{~mm}$ breiten Jृaten

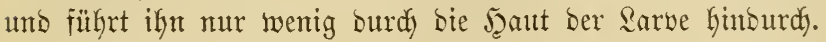

2. Etewart'sorfady für Megennürmer ijt als 2 biz 4 Şafen won $4-5 \mathrm{~mm}$ Breite gejertigt (i. Fig. 260). TSie ber

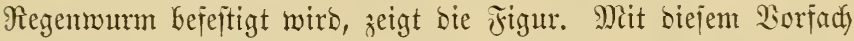
fing Etemart ntefy Forellen, wie mit bem eimfadyen \$atent. Dem entipridyt nteine (Erfahrutg mit ber äfnlidyen, aber nod einfacheren 2Anorbmung (i. Fig. 269).

F̌ig. 262. Relle.

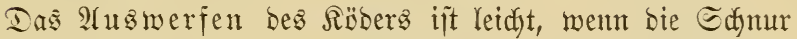
nidyt wejentfidy länger iit, wie bie Rute, es erforbert aber um

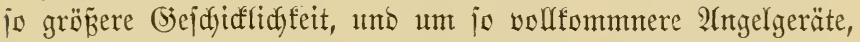
je mefgr bie \&änge ber Edjnur bie \&änge ber 2lngelnute über= trifit. Wenn bas Majier tiejer, wie bie Angelrute lang ijt, jo bebient man jid bes gleitenten Flojles, jig. 258, weldjes jid jwijhen bem Eenfer d unt cinem an ber Edynur bejeitigten Etüdfden Siummifaben frei betwegt. Wenn man auswerfen will, jo rulgt bas Floz auf bem Eenfer d, und ment man ausgemorfen hat, io jieht ber Röber uno ber Senfer bie Sdynur Durdy bie beiben

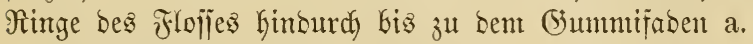

Bei Berlin benuţt man bie Relle (Jij. 262), um beim Angeln in jefr tiefem $\mathfrak{B a f j e r}$ bie Flofangel auşumerfen. Sie ift von $\sqrt[5]{ } \mathrm{ol}_{3}, 50 \mathrm{~cm} \mathrm{lang}, 2^{1}{ }_{2} \mathrm{~cm}$ breit und mit einer $2 \mathrm{~cm}$

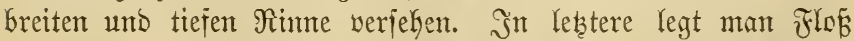

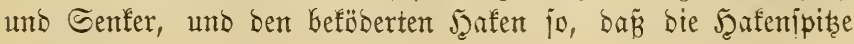


ganz rorn liegt. Iann crgreift man sie Relle mit ser redyten Sant, Gebt fie Garizental über bie redyte Echulter, uno mirft mit bem Unterarm. Bei einiger Utebung lernt man balo meit

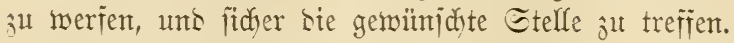

Der gegabelte Etod, mit meldyem in Englans beim Jechtfitidyen unt bei bem Angeln im Neere rom lifer gemorien

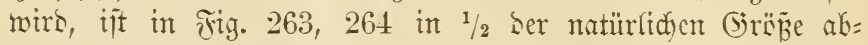
gekildet. Fig. 263 fteflt eine (5abel non Etahl sar, beren Etiel

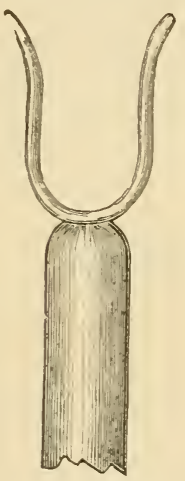

Fig. 263.

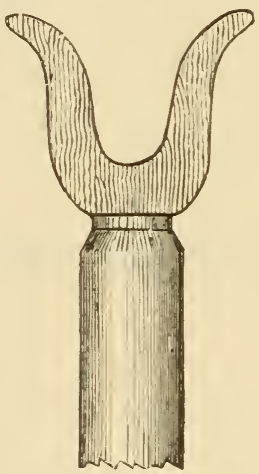

Æig. 264.
Gegabelter @tod.

won 万olj und $1,4 \mathrm{~m}$ lang iit. Man wendet aud (biabeln an, bei benen eine Binfe fürzer ït, als sie anbere. Fig. 264 itt Mr. Eamber's hablyerme (5a = Fel, mit ser er auj sem 2rnglerturnier in Sononn $18 S 1$ wari, fite ift ron Jocly uno flads. Ier Etiel ijt Bambus uno $2,3 \mathrm{~m}$ lang. - Beim Merien legt man sie Edonum in sin= sungen an sie Erse, legt bie Rute aus Ser Juns,

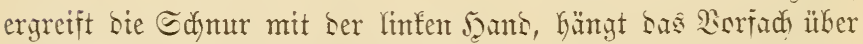
bie (sabel, uns wirft in ähnlider Metie, wie mit ber Nottingham=

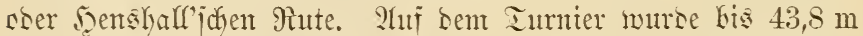
weit gemorien. Iie Mitteilungen wersante idy ber biute meines Jreundes Mar. I. Sdrwann in Sontont.

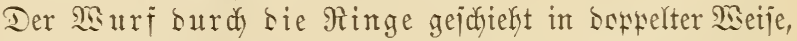
inbem ntan entweber io viel Edum sorber non ber Holle ab= zieft, als ausgemorien merben joll, noer insem mat son ber

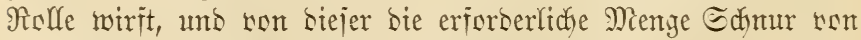

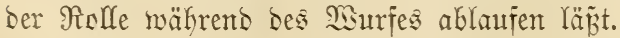

Menn man bie Edyur nor bem Murf oon ber ßofle ab= 
zieft, is legt man ïe entweber in Mingen yor bie Füñe, sober mun jummelt fie in ber Yinfen Jaand auf. Den Röber zieft

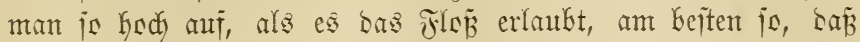
mod) 1 bis $1 / 2 \mathrm{~m}$ Edum non Ior Efitze ber Edumtr berab=

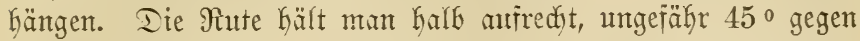
ben Jaoriznt geneigt. Innt bringt man burd eiten Edyung ber Aute bas Sorifad in pendelnse Bemegung, und wirit mit einem fräftigen Sdyunge nady oben nadh ber gemünjuten

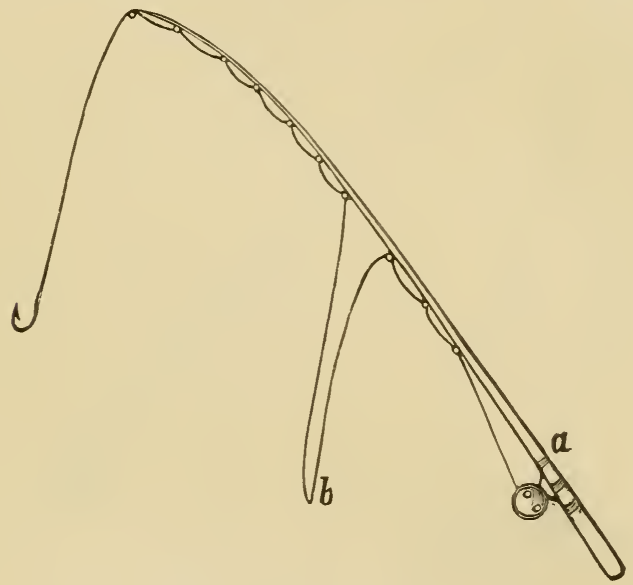

Fig. 265. Wint Durd bie Ringe.

Richtıng. Der Räber und Las Fla fr fliegen bant in einem Brogen nady sben bem Biele zu, und jiefent bie abgemidfelte

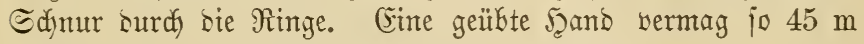
meit zu werfen, uno Das Biel fither jut treffen.

Man fam ben Wurf mit anfigezsgener Edjur aud in folgender 些eije auŝühren.*)

*) J. W. Martin The Nottingham Style of Floatfishing and Spinning. 


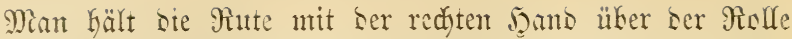
Fei a Jig. 265, uns jicht mit ser linfen jant sie erforberlidje

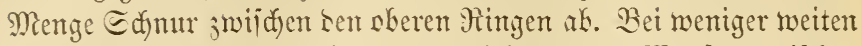

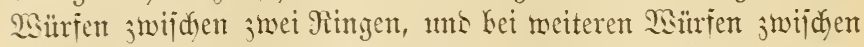

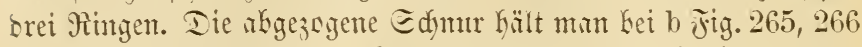
mit ber linfen Deanto fejt. Iann fühtt man sen Sisur aus, uno

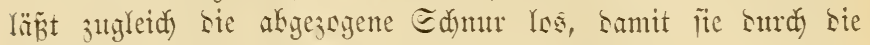
Ringe gleitet, uno einen weiten siuri ermigglidyt.

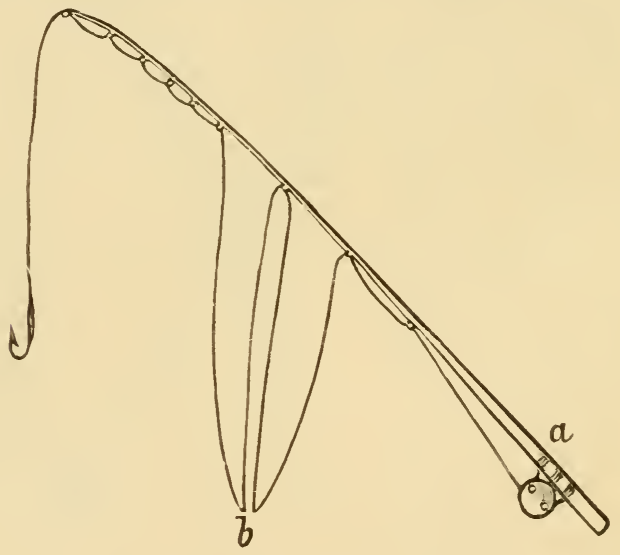

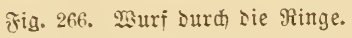

Ter Murf won Ser Rottinghamrolle ijt nidyt leidyt, uns fann mur surdy längere ltefung crlent werben. Der Röber

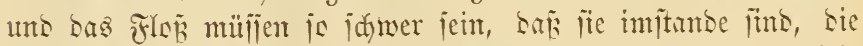

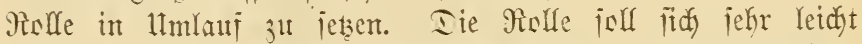
sreken, unb beshalb wirb ifre Spinsel Güufitg mit Sel gejdmiert.

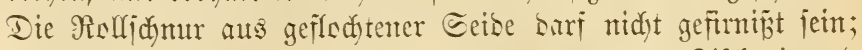

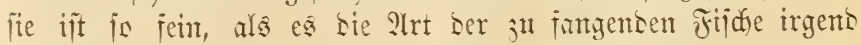
geitattet. Iie Rolle ijt io weit mit Edyutr gejüllt, wie es

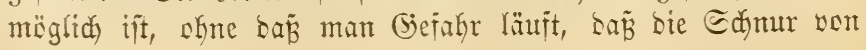




\section{Die Frlö̉angel.}

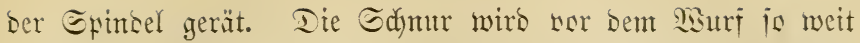

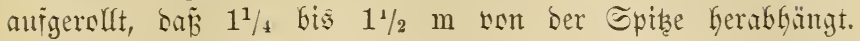
Ionn wirt ser Marf io ausgefülyrt, wie wir es norker be=

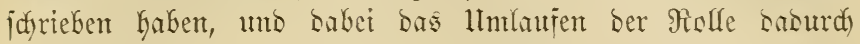

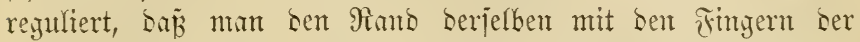
linfen Damo berilfyrt. Bei bem

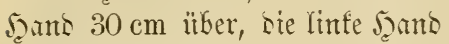

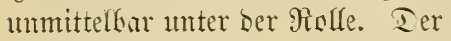
Beigefinger ber linfen Jumb gält Fithlung mit bem rotierenten Teile

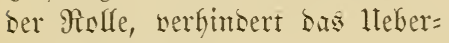
laufen berjelben uno fält je an, bencr eben ber fïser bas silfer berïthrt.

Bei rem Epielen eines̀ ge= Gaften Jijdes befintet jidy ber fleme Finger ber linfen jamo an

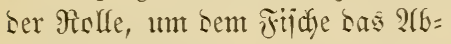
ziehen ber Ėdjur zü erjefweren.

Der Saur won ber Molfe iit riel leidyter, woun man eine Senoerslle bentht, bie won bem Edatten Miallod eriunten uno wan (5. Seeger in Brün ver= foijert worben ijt. Є. 10 เms Fig. 267.

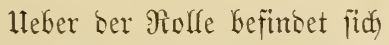
ein metulfener Seitring, ourd) meldyen bie Edanur läut; bie

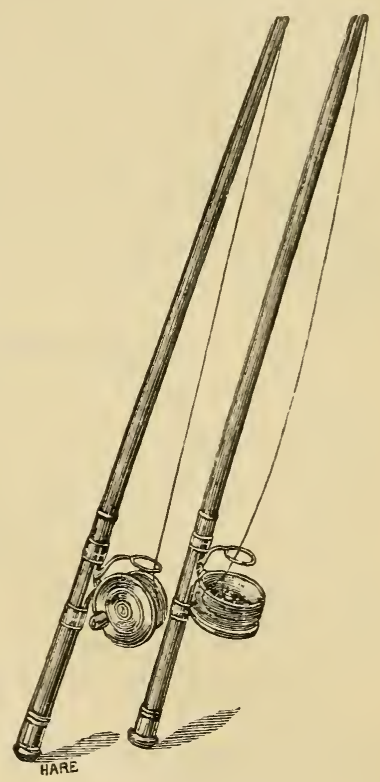

テrig. 267. Wenterolle.

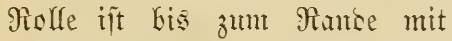

Cdymur gejülft. Wem gemorfent merben jolf, io wentet man

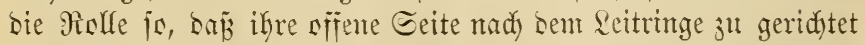
ijt. Man Gält bann bie Edyun mit ber linfen Jann nber mit

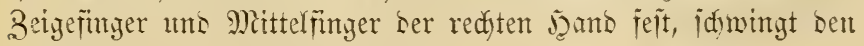
v. ๖. Borne, Ingelfitiderei. 3. 2qufl. 


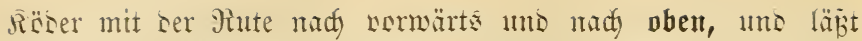
jugleidy sie Edymur los. Dieje lüuft bam mit ber gräbiaten

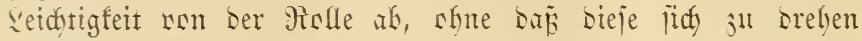

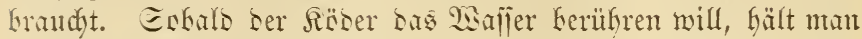
sie Edyur feit, santit jie nidyt weiter aflünft, sreht bie Holle in iffre

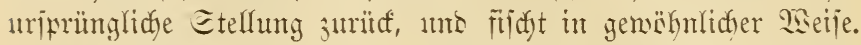

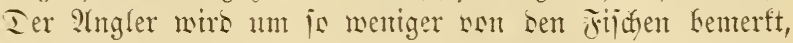
je weiter er won benjelfen entfernt ift. Iesfalb genährt bie Disttinghant=2(ngel einent groben Barteil, weil man mit serjelben

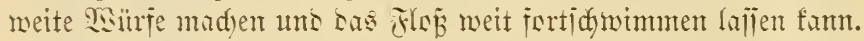

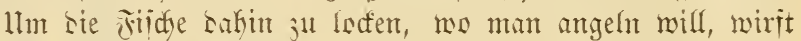
mant an mefreren Etellen (Sirunstäber ants, uno wedyjelt sie Whatitellen io sit, als sie Fithe authëren ju keipen. Benor man fortgeft, wirft man bon neuem Grumbfiscer aus, um sie jiidje wieser anjulocten.

\section{Das feftliegende flok}

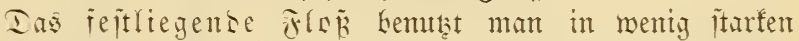
Etrümungen, roer in itehenbem 2 sajler, um an einer Keĩtmunten Etelfe, we man wor längerer Beit Grunstëser singemerien hat,

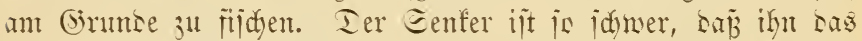

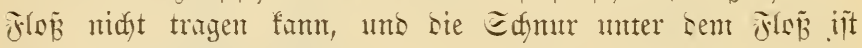
$1 / 2 \mathrm{~m}$ länger als bas Saljer tief ijt. Bei Berlm wirb bieje

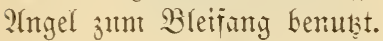

\section{Die Grunbangel ohne flof.}

Sie (i) runs angel of me Flo

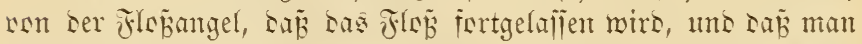
Den Bí Des Fijhes nidyt an ber Beroegung bes flofles pieft,

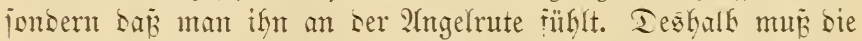
Sdym immer itrafi gefalten werben, weil man jonit nicht fïlblen famt, wem cin Jijh anbeipt. Tie 2lugel hat ben Borteil vor

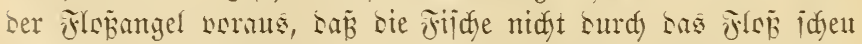

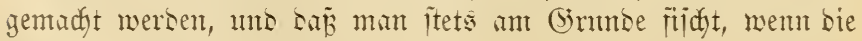
Echnur genïgento bejdwert iit. 


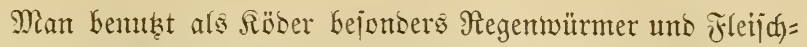
maben, und fängt jefr gut au bieje Weije Barjdye, Blöbzen, Blei,

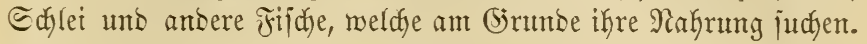

\section{Das Bobenblei.}

Ias Bobentelei, Fig. 268, ijt eine Grunbangel mit jumerem Senfer, Der ant Grumbe jejt liegen bleibt. Daljelbe ijt flachs

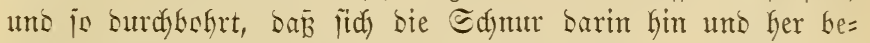
megen fann. Llm bas (b)leiten ber Edyur anf einen beittimmten Haum ju bejuränfen, bejejtigt man an ber Sdynur an beisen Ceiten Des Bodenbleies je ein Sdyrotforn.

Der Räber jolf nidft weiter wie $30 \mathrm{~cm}$ vom $B$ obenblet ent= fernt jein, meil man jonit ben Biz Des Fijdes nidyt mefr jo Dentlidy fühlt. Tie Echnur wird itrafi gehalten, bamit man jofort burd) Das (jeïitgl bemerft, wenn ein Fitid ben Röber nimmt.

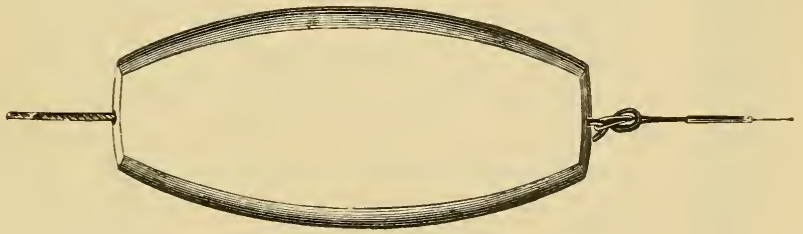

Fig. 268. Bobentlei.

Farlow in Znnson verfautt Bobenbleie, bie leidyt angebracht und abgenommen werben fönmen, shne bie Angelidymr ausein= ander zu refmen.

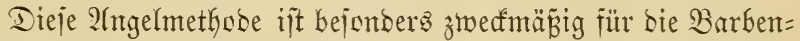
fiid)erei in itarfen Etrömungen.

In jefr tiejem, jtarf itrömendem Wajier fijht man sit mit grö̈em (Erjolge won einem weranterten Rafn mit bem Bobenblet uno einer ganz furzen, mur 30 bis $40 \mathrm{~cm}$ langen 2lngelnute, Fig. 269,

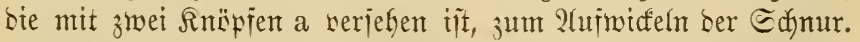

Iieje $\mathfrak{A}$ ngel mit' jefr siel in ben weiten Mündungen ber Ströme in bas Meer bemuşt. 


\section{Die Paternopterangel.}

Die Piatemoiterangel ( (5rundangel ofne Flo

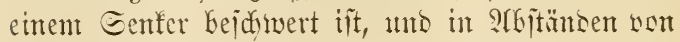
$30 \mathrm{~cm}$ uns mefr Jäben won (5ut noer (5imtp trägt, an benten bie Denfen kejejtigt jïts.

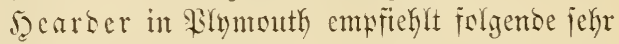

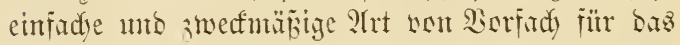
Piatemojter. Betze (Enden eitrer $35-10 \mathrm{~cm}$ langen Edyntr won biut ober (Simp weriegt man mit einer Edyleife a unto c, und legt banm bas Entoe a is $u$ m, saj cine stitte Ealleife b gebiloet wirb.

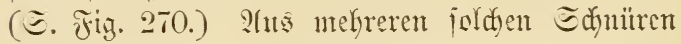

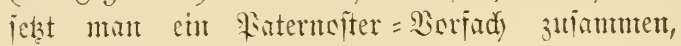
utsem man lie basurd berbinbet, baj man bie Edyleife b ber cinten Edyum in ber Edhleife c ber anberen Edyur cinfängt. Mam fam io bas Soriady Yeidyt beliebig werlüngern. In ber Regel

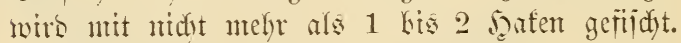
Mian fant and bei frantigem (biunte, von bem man fern bleiben will, coer bei jefr tiefen

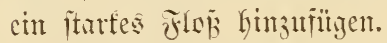

Fïr sen Baridjanty ijt bie Fatemejterangel

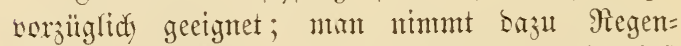
wütmer als Röber; bei sem Etintfange wirb fait

Fig. 269. Ingelrute für Die Fifderei mit Dem Bodenblei.

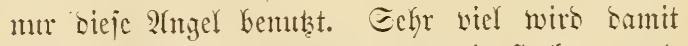

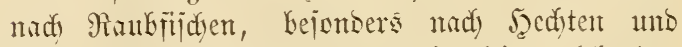
Banbern geangelt, uno baju mit Heinen lebenton Jijujen betöbert.

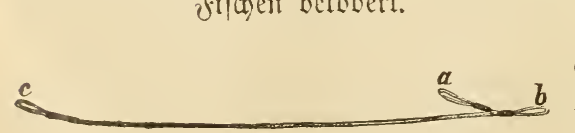

Fig. 270. Searocr'` \$aternoiter=21ngel.

Mañ fuălt Geim ?ntgeln sic Edyur itraij gejpannt, oamit man fühlt, wenn cin 
Fija) anteipt, uno haut nad) cinigen Eefunten an. Das Blei läb̦t mat $3 \mathfrak{u}$ Boben finfen, bringt es nad) eintger Beit an eime antere Etelfe und fijdt io affmäflidy bas ganze Wafler ab.

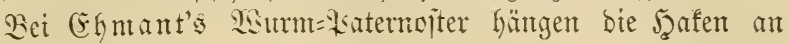
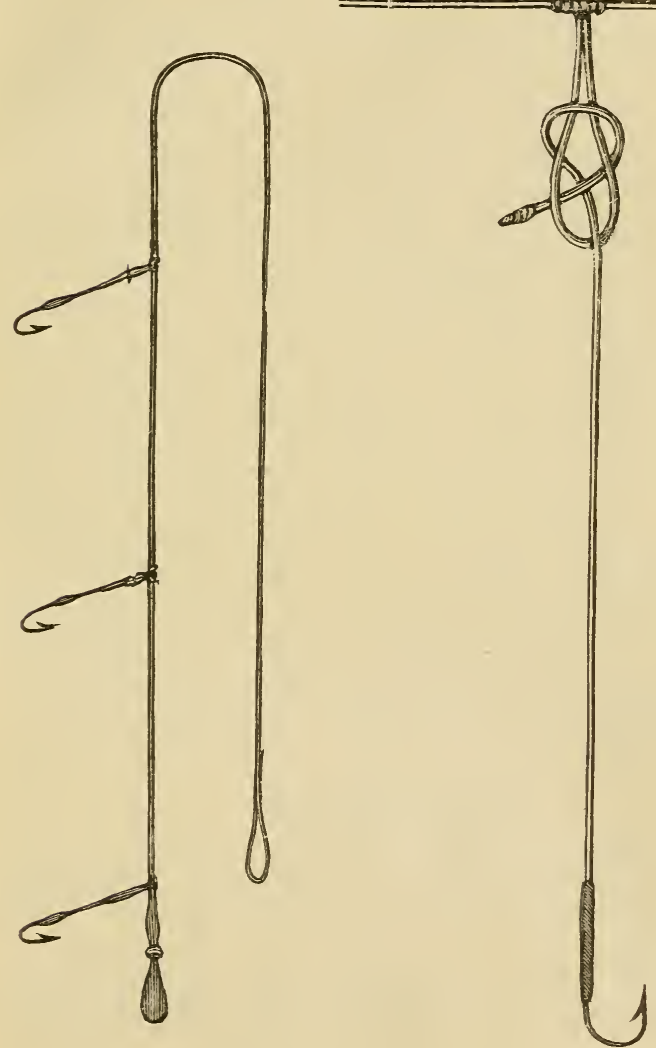

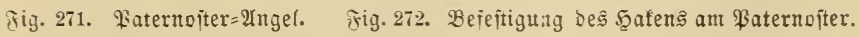


einem, und bas Blei an einem anderen Gut=Borfach. Beide Sorfächer fimb oben sereinigt, uno unten burdy einen bünnen, $12 \mathrm{~cm}$ langen Draft auseinanbergehalten, bant fie fidh nidut wericylingen.

\section{Das Geben uns Senfen.}

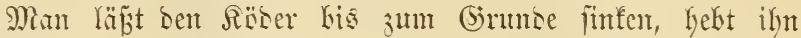
mieber bis zur Sberflädye, uno kält ifn io in fortwährenter Benvegutg, won eimer Etefle zutr anberent fortidyreiteno, fis maut alfmählidy das ganze Sajjer abgefijdyt hat. Man fijdyt entweber unit ciner Prottingham=21ngel, noer mit einer Fliegentute. \$ienn

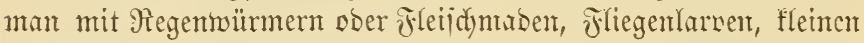

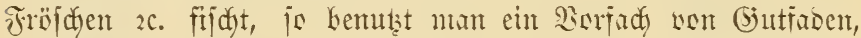

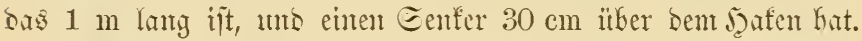

Eehr wirfjam funo für bicje afrt wn Fijcherei bie mit Blei

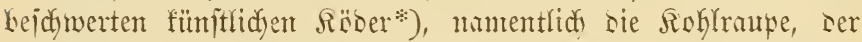

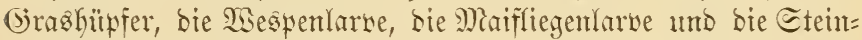
fliegenlarve, bie wir Geite 127-131 bejuricten kaben. Die

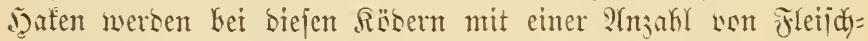
maben kekängt, und Sann wirb, wie sken bejdrieken, Kebent unt jentento gefifdyt. Manchmal wirs aud) ein mefrere (5enti= meter langes feberfielfilon angebradjt, um leidut beurteifen ju tömten, wenn Der Röber ben (Sruno berïhrt. Die Edyur hält man jets gejpannt, bantit, ntan jeben Biß̄ jofort jüblen fam.

Fïr ben Rejdenfang ijt Das Jeben und Eenfen ganj bejon= berz wirfiam.

\section{Die fifhḑenangel.}

Mit bent Fijdyen wersen Maubfitide, namentlid Sechte,

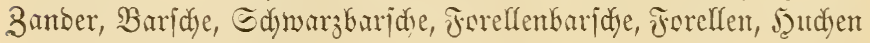
u. . . w. gejaugen.

Man bentht entweber einen Yebentent roer einten toten (coer fümitlichen) Röberfijdy uno unteridfeiset bei beiben Âten won $\Re$ öser

*) H. Wheatley. The Rod and Line. London 1840. 


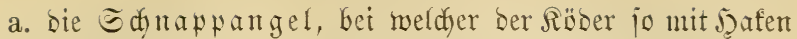

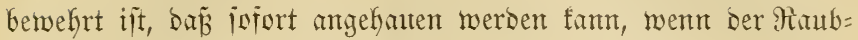

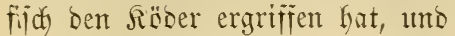

b. bie Edyludfangel, bei meldyer bie jatfen bidyt am

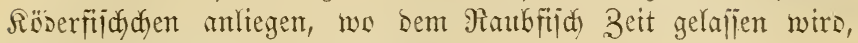

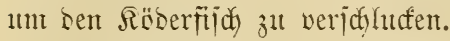

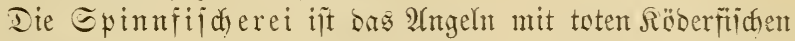
uno ber Edynappangel.

Iie Irollfijderei ift bas ângeln mit toten Röberfijicten unt ber Edylutangel.

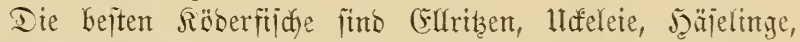

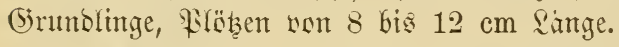

\section{7a. Die Spinmfifherei.}

Man nennt Spinnfijderei bas 2 ngeln mit totem ober fünit=

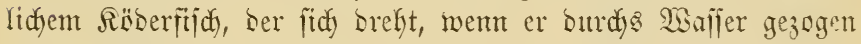

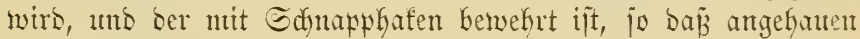
werben fann, fowie ein Ratbfijich Den Röber ergriffen hat. MRan füngt Damit Jechte, Joudyen, Echmarzbaridje, Foreffenbaridie, Baridye, Zanber, Forelfen und andere Maubfijdye.

Die Epinnrute ift $3-3 \frac{1}{2} \mathrm{~m}$ lang uno redit Yeidyt. Mian

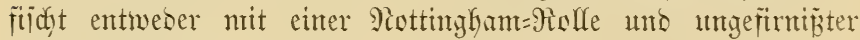
Eeisenidyur, ober mit eiter senderolle.

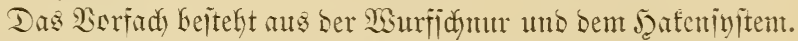

Iic 2 surficnur (Jig. 273) ift nit ntelgreren sistbeln, (Fig. 274,275 ) verjehen, bamit jie ourdy bie I rehungen bes Räber. nidyt veridflungen uno veridjürat wirs.

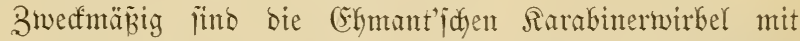

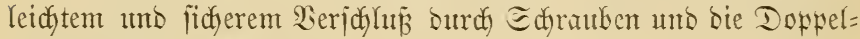
twirbel, (Fig. 27t, 275).

Man unteridyetoet folgende Arten von Sitrbeln, jowohl von Etahl als won Meifing gejertigt:

geid)loilene, Budfel=, Patent=, Rarabinerbaten=, Rarabinter= idrauben= uno Epiral=sirbel. 


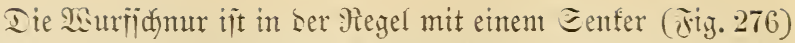

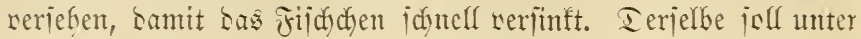

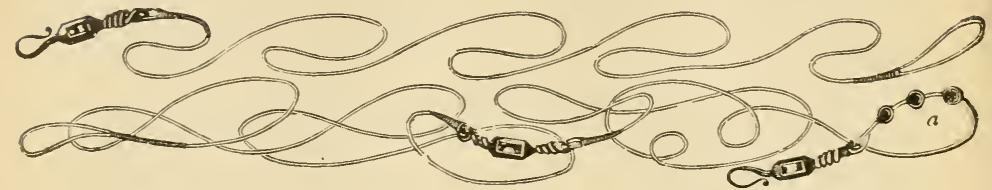

Fig. 273. Sisuridunur mit Şafentirbeln an heiben Ensen

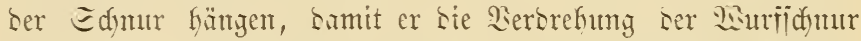
rerfintert; er it and sit nod) mit einem rber jwei sirbeln

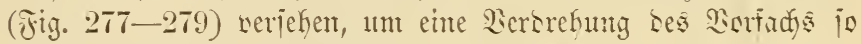
niel als mïglidy ju verhüten; ïe infl nidyt länger jein, als ein

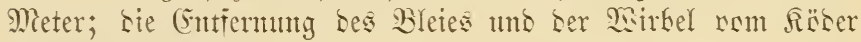
jei 50 bis $60 \mathrm{~cm}$.

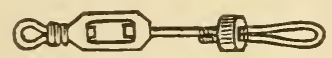

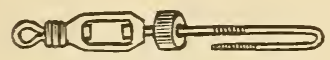

Fig. 274. Rarahinermirbel mit Sdraube.

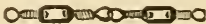

Fig. 275. Inpfelmirber, beise geidrlolien.

In ftarfer Etrönntug ober tiefent

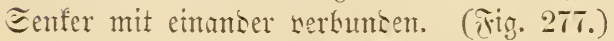

Dafenintem. Tas Eximen, bas keipt bie Irefung Les

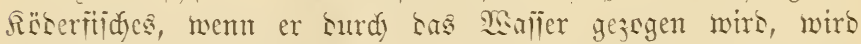

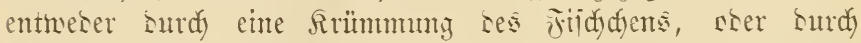
Edyraubenflügel kemirft, welde am Ropie seljelten angebrad)t

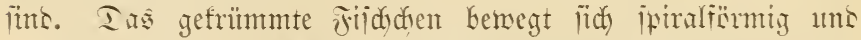
ppringens, mit sen Edfratfenflitgeln sreht es fids unt jeine

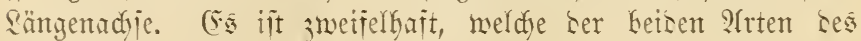

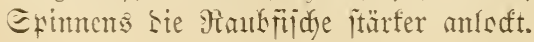


7a. Die Epinnfijderei.

153
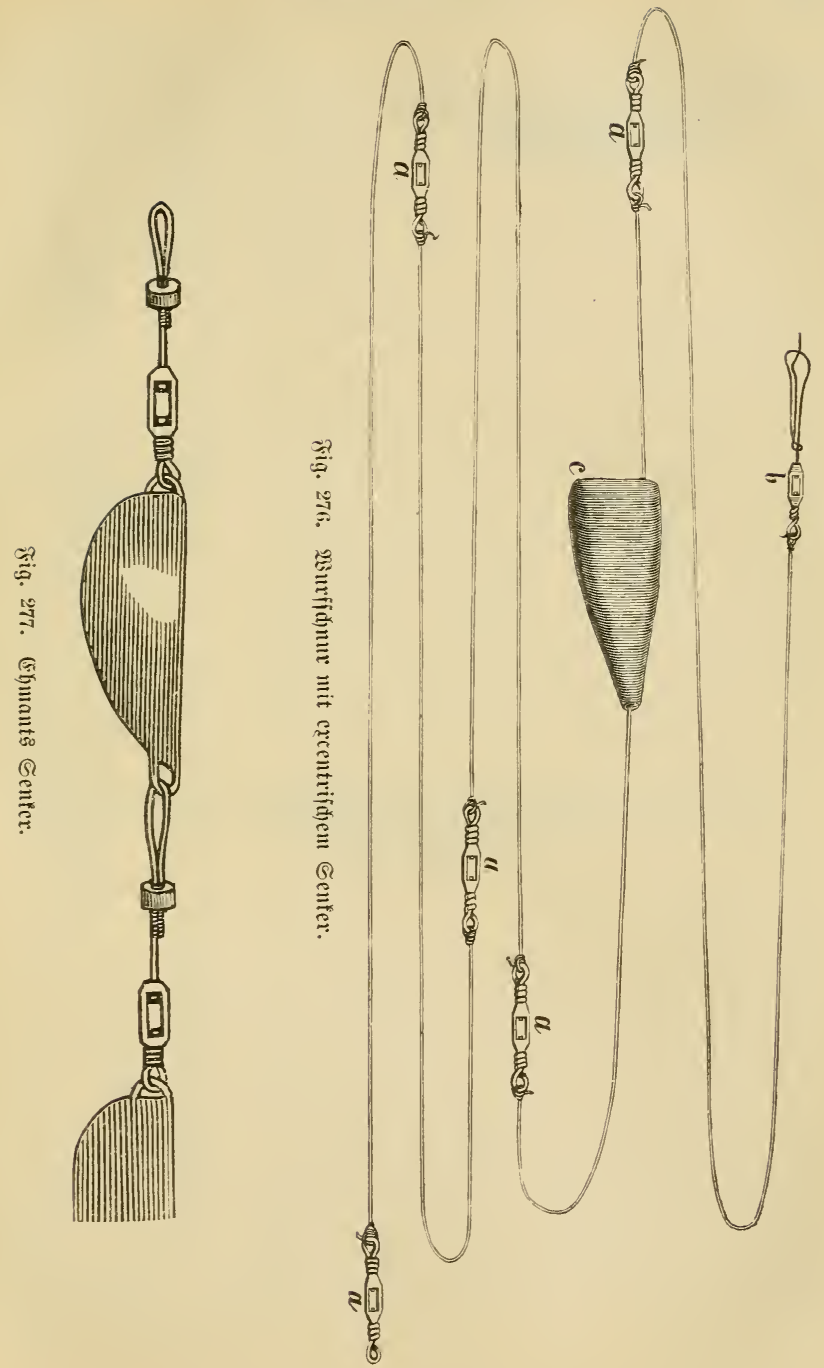


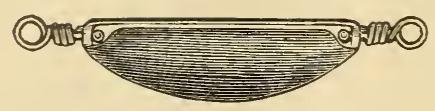

Fig 278 .

Im alfyemeinen fangen bie Sateninjteme mit menigen gropen Daten beller, als bie mit vielen fleinen פaten wer= jehenen Eyiteme. Fïr sent Jechtiang werben bie Jaten an (jimts, noer nody belier

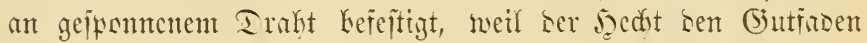
zerbeižen fann.

J3an ats einent eingigen bafen uto itcht an Sirtifamfeit tirum einem anseren Enjtent nad. Sisalton bejdreibt jeine Epinn=

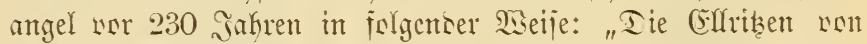
mittlerer Gräbe und weijer Tarbe find bie bejten Röber. Ias Fijdyen ioll io an ben Syafen geitedt werben, baj es fid streht,

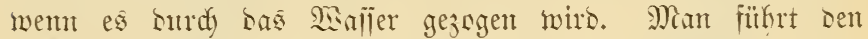

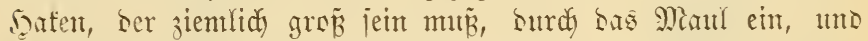

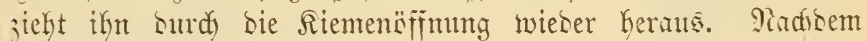
man bie Edyur 5 kis $8 \mathrm{~cm}$ Kerworgezogen hat, füfrt man sen

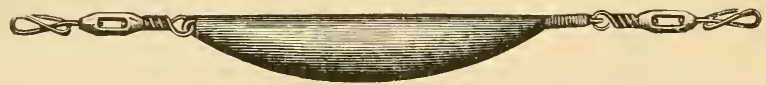

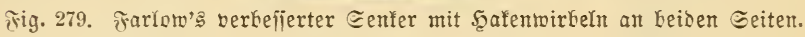

Jaffen nod eimmal burdy Das Miaul uno bringt ifgn am Ectwan; Geraus. Dann bintet man Dafen uns Echmanz redst jauter mit einem weip̌en Faben zujammen, wodurd bas Fijdyen un

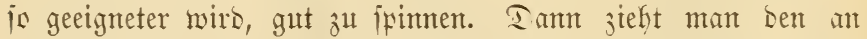
Der Riemenöfïmung eine Edyleife bilbenten Teil Der Edyur itrafi

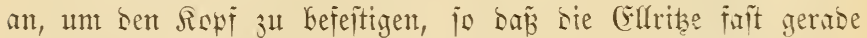
gejtredft anf bent Jarfen fitz̧t. Sit bies geideken, is jicht mant fie surdy bas : nidyt jajnell breft, is menset man ben Edwmanz ein wenig nadh reduts noer Yinfs, bis jie fidh flinf breht, benn wenn fie sies 


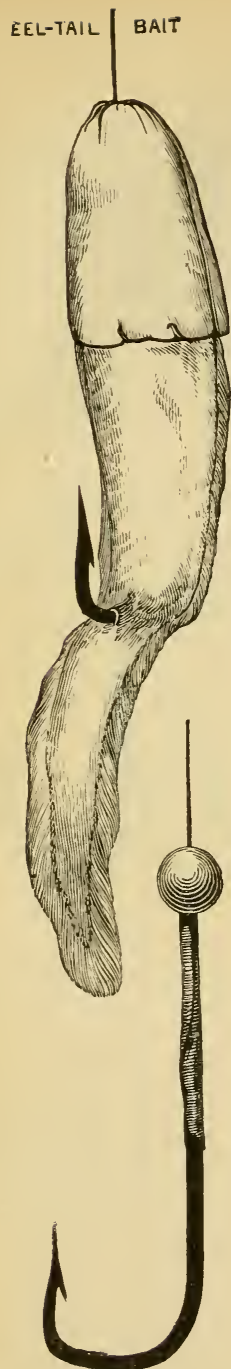

AND TACKLE

Fig. 280. Pentell's Aalidinanz=̊öber.

nidyt thut, fo läuft man Gejahr, nidyts zu fangen. Denn man milife, Daj fie fich midyt 3u iduell brehen fann. Scat man feine Elltize, jo ijt aud ein fleiner Steinbeizer, coer ein Stidyling ober ein anberer fleiner Fijdy jefr gut; aud fann manfie eimjalzen unb baburdy 3-4 Iage ober länger brauchbar erfaltem." Bennell's 2laljdyanzföber (Ftg.280) a beiteht, wie $23 a l t o n ' s$ Spinmorfad, aus einem einzigen Saten, und ift bem gleidy= namigen $\Re$ öber jehr ähulids, ber in ber See= fijd)erei benutst wirb. Er jpinnt jefre gut, ift jebr bauerhaft, wirb won Jeedenten utmo Eachjen gern genommen, uno ift audy für bie Ed)leppangelei geeignet. (5s ijt gut, ben 2Har 2 bis 3 Madjen wor bem (Siebraudy in Salz zu Yegen, bamit bie Şaut bläuliç umb zäh wirb, - und ifn bann 10 bis 15 Etunden wor bem (Sebrattd zu mäffern.

Man Gemuląt 2 lale von $30-35 \mathrm{~cm}$ Qünge.

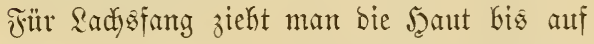
$10 \mathrm{~cm}$, vom Edjwanz gemefien ab, juneibet fier bas Fleifd ab und $5 \mathrm{~cm}$ bavon entfernt aud bie Jamut. Fig. 280 zeigt, wie Kieraus ber Röber gemadyt wiro. Für ben jecht= fang madyt man ben $\Omega$ öber $15-16 \mathrm{~cm}$ lang.

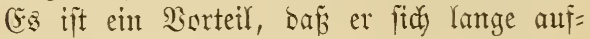
bewahren läp̃t. IInter ben fonjervierten Söberfiiden ift er wohl ber bejte.

Das Edyottijue Jafeninjtem, rig.281, weldyes in Edyottlano jehr viel zum jorelfen= fanty benubzt wirb, bejteht aus einem grojen freisförmig gebogenen Enofaten uno einem Sipphaten. 
Man füfrt ben grofen Enofaten in bas Maul bes Röser= riidjes ein, am Rüdfigrat entlang uno nahe am Edymanz ans; julekt wirs ber Sipphafen burdy bie Ripfen bes Jijdyens non when nady unten gejtedt. Bet ber Jorelfenfijdyerei wirb ser Sipk= bafen ieit an ben Gutiaben angemumben, bein jectijang wirb sit ein gleitenter Sipklyafen angemanot (Jig. 282), um bas

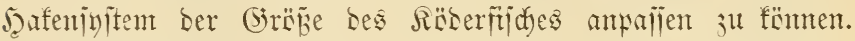
Ite 2indungen ber Edyur um Den Dafenidjenfel madyt man

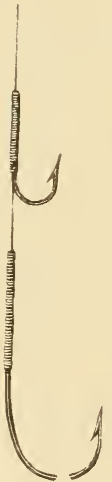

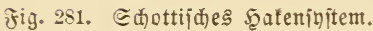

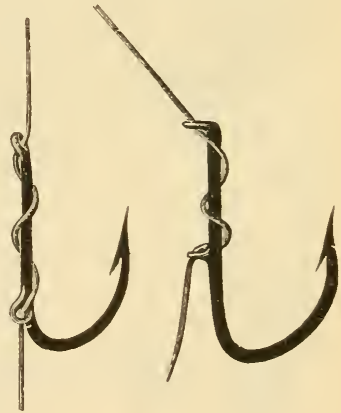

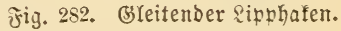

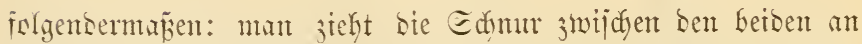

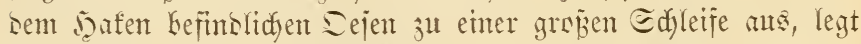
ben unteren Teil ber Ed)leife um ben Dafertidenfel, jtedt bas Ente ser Edynu surd) bie Edyleife umb jieht bie Edyleife ein.

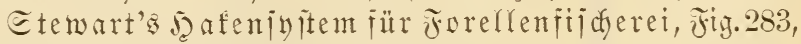
ijt sas Edyottijue bafenjutem mit einem fliegenben Triangel, ber fid) einige (Sentimeter finter bem Räserfijdyen befintot.

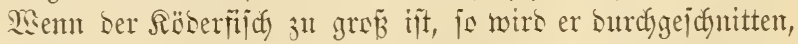
unb mur mit bem Edymanje gefijdyt. Ier fliegende Triangel, Jig. 28t, fann an jebem Dafeninjtem angebracht werben, wenn 
bie Edyum, an ber er befeftigt ijt, an (Ende mit einer Ed)leife verjeken ijt.

Der fliegende Triangel joll jidy bei ber Forellenfifiderei Ginter,

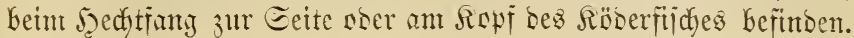

v. D. Borne's Satenjutem Pir. 1 (Fig. 285). (Sin jehr einjaches Jatenjyitem faum man jidy in folgenter Weije leidyt
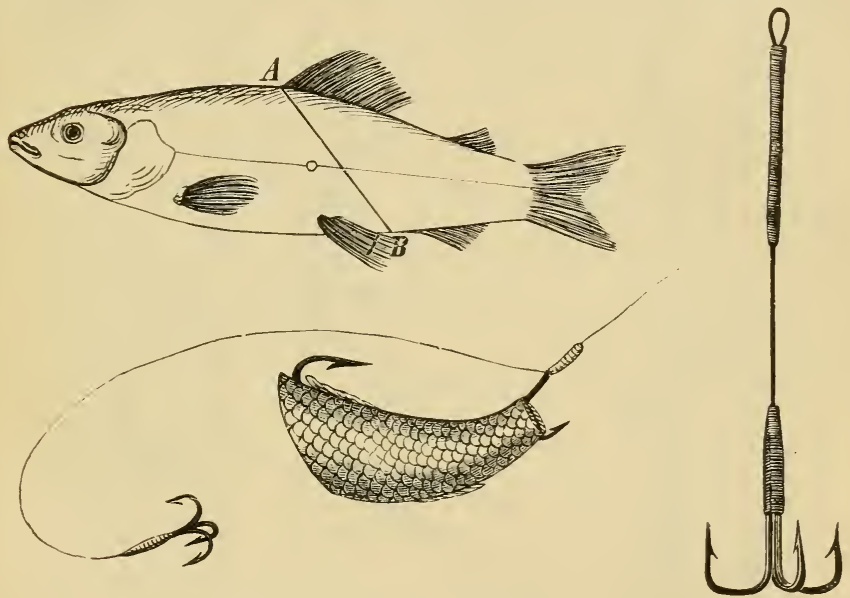

Fig. 283. Etemart's @yjtem.

Fig. 284.

Friegender

Iriangel.

felbit anjertigen. Man glïgt iturfen Meifingoraht, um ifu meich unt biegjam zu machen unb lötet an eintent Enbe eine Deje, an bem ambcren Enbe einen grojen Triangel an. Den Drafyt füfret man butdy 2Ifter und Maul bes Röberfijdyens, und biegt biejes zugleidy mit bom Mefiungbraht, burd llmlegen

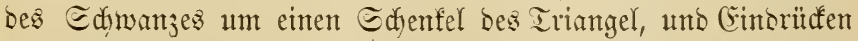

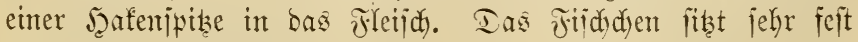
unt ppinnt ausgezeidynet. 
Mian fann jidd io and ein Eyjtem mit zmei Iriangeln an= fertigen uns, went man will, einten fliegenben Iriangel anbängen.

21 ber Surfidmur wiro bas Eyjtem mit einem Rarabiner= Gaten=sinbel befertigt.

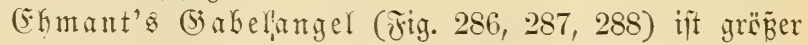

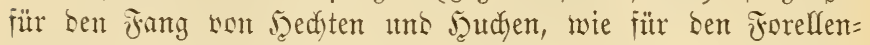
fang. Die langen Gabelyinfen werben io in bie Ceite bes
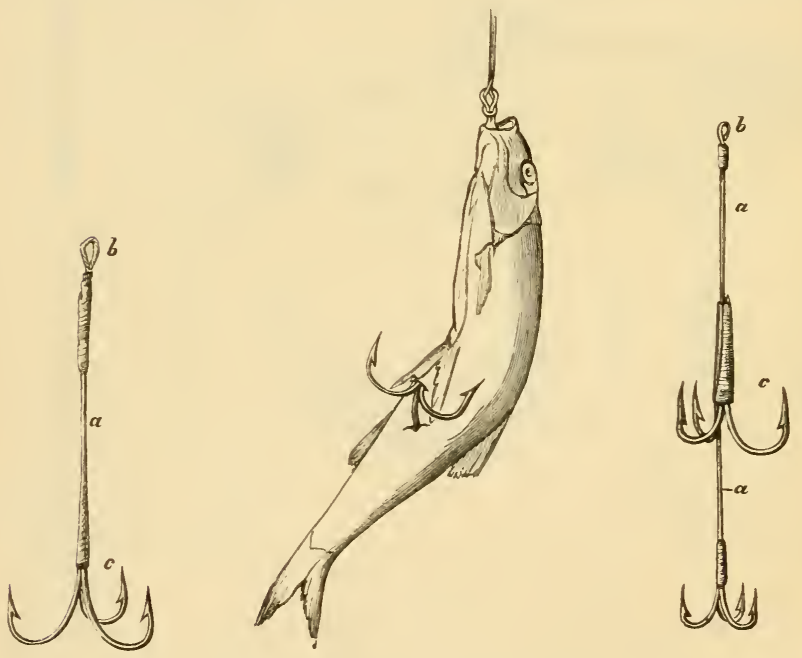

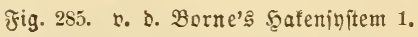

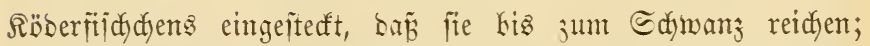
bann werben bie futrzen Binfen nady ber Ridytung zum Ropi cingeitectt, ber Ripphafen surdy bie Sippen geitoden, unb burd

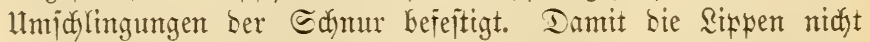
jerreipen, wirb eine Slammer (Jig. 287) 3u J̋̈̆lfe genommen. Den Sipphafen füfrt man burch bas Sefr ber Rlammer,

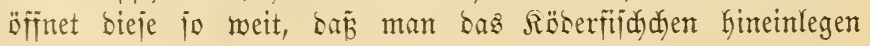


fann, uno srït sant bie Rlammer Ginter ben Siemen in bas Säberfijd) cint.

(Siemëhnlid) iit ser Senfer in einiger (Entiernung von bem

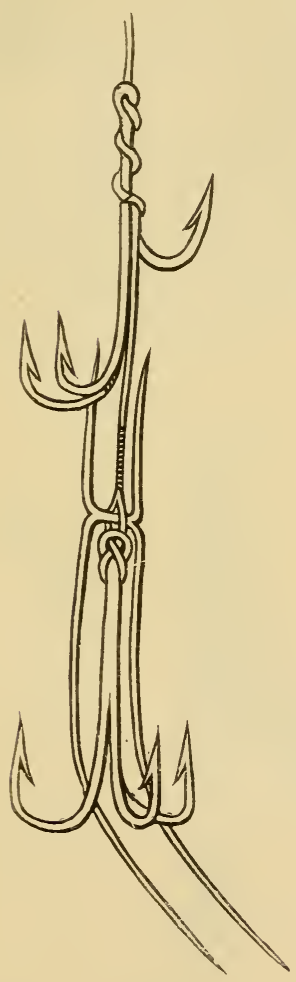

Fig 286. Ffhmant's Brabelangel iür ફ̧edftiang.

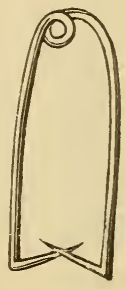

Fig. 287. (5hmant's Яlammer.

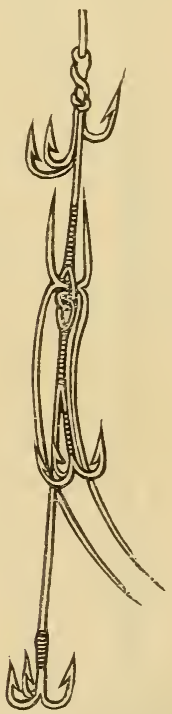

Fig. 288. CFhmant's B̧abelanget für forelleniang. 
Räberfijdiden an Borfad) Kefeitigt; bet ber Fijderet in fleinen Flitilen aber, wo nidut weit gemorien wirb, ijt bas Balei
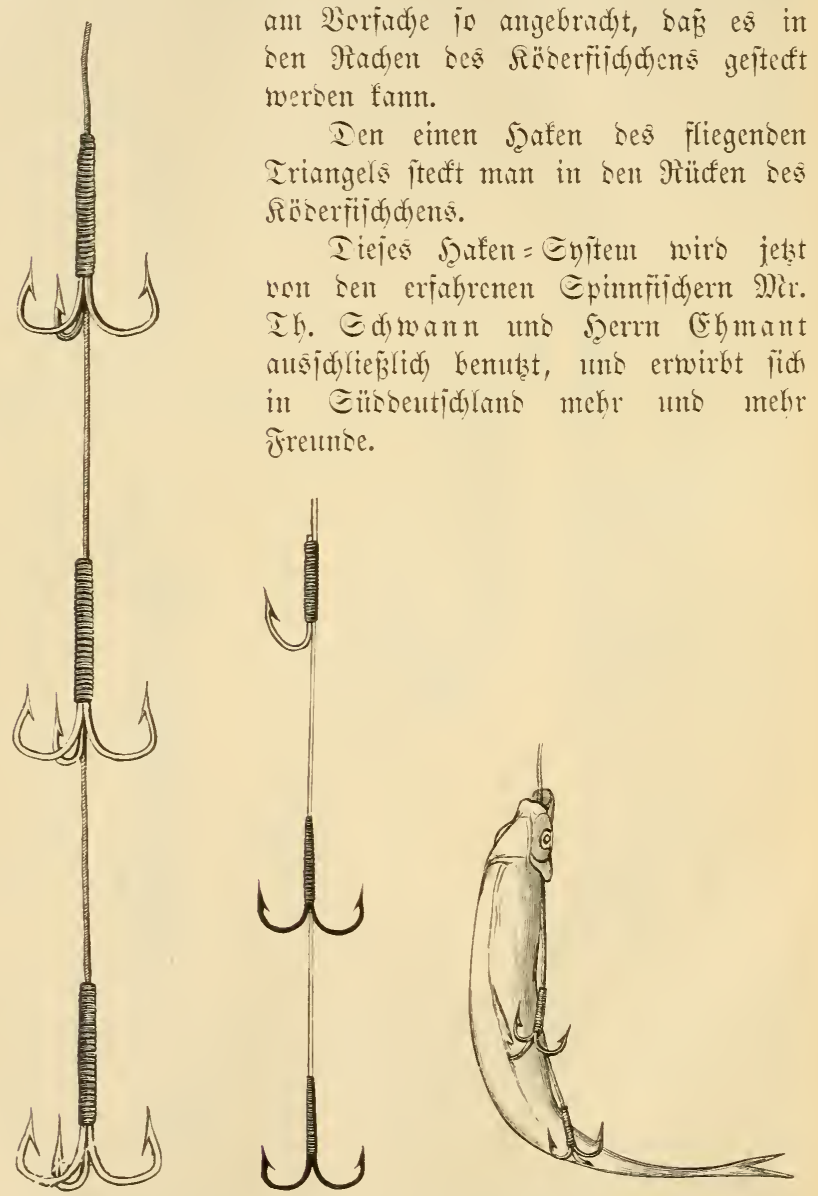

Fig. 289. Rottingham=5eafeninftem. 


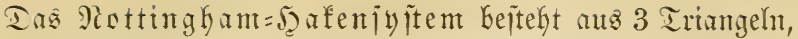
coer 2 Triangeln utro 1 Sipphafen, ober aus 2 Doppelfafen unt 1 Sipphafen (Fig. 289). Eetzere Form hat ben Borteil, oñ jie bequem in ein Iajdentudy gethan merben fann. - Man füfrt bie Ed)ur in bie Riemenöfinung ein und aus sem Maule aus. Den Sipphafen führt man ebenfalls burdy bie Riemenöfinmung in

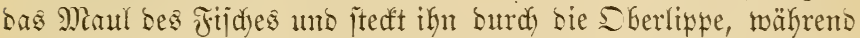

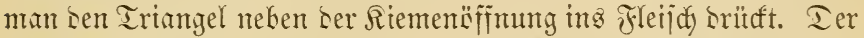
mittlere Iringel wiro Ginter ber Rücfentrlefie eingesrülft uno giebt bem Röber bie Rrïnmung. Ter Endtriangel wirb ïber ben Ritfen ge= legt uno am Edymanj eingebrüctit. Wenn sas Fijdyen ridftig bejeitigt ijt, to ipimt es ansyezeichnet.

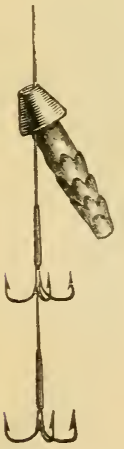

Fig. 290. Tee= Daferipitem.

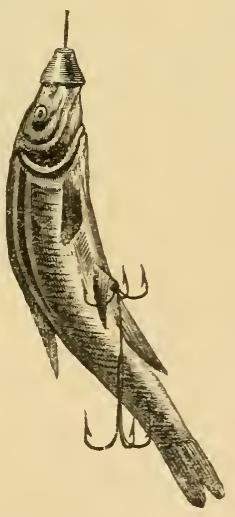

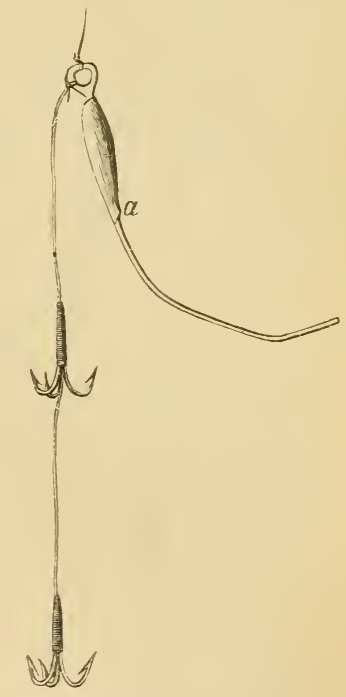

Fig. 291. v. D. Borne'马 Saleniniten 2.

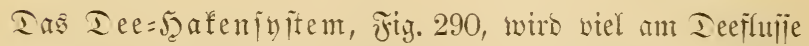
in AGerbenifire gebraudyt, uno ijt jebr entrieblenswert. Man

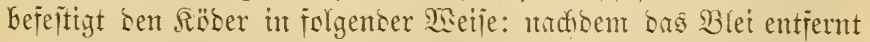

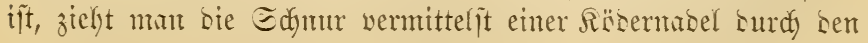

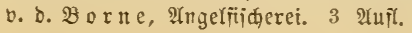


Sifter ein uns burd bas Maul aus, bann ftreift man bas Blei mieser auf bie Edynur, idjeft es io tief mie möglidy in sas

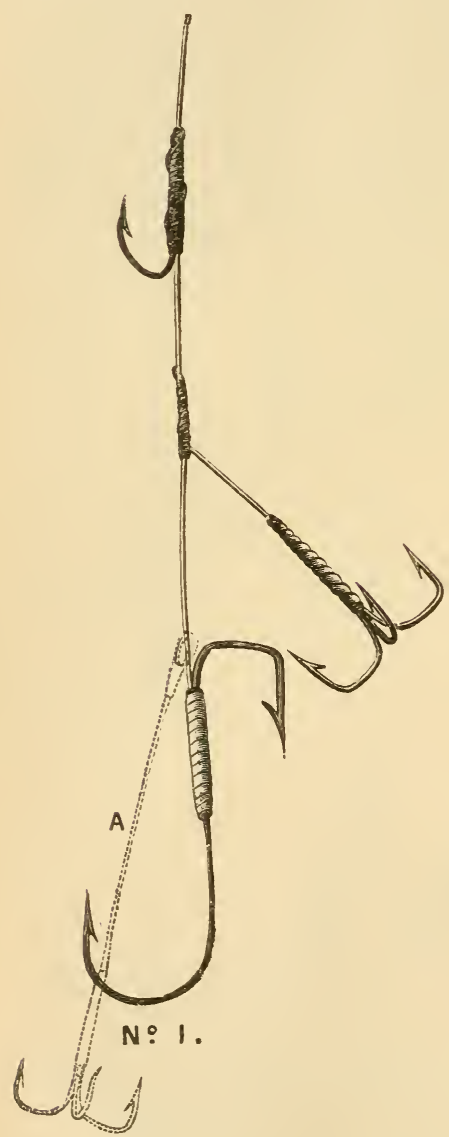

Fig. 292.

Fennell's Sateninftem.

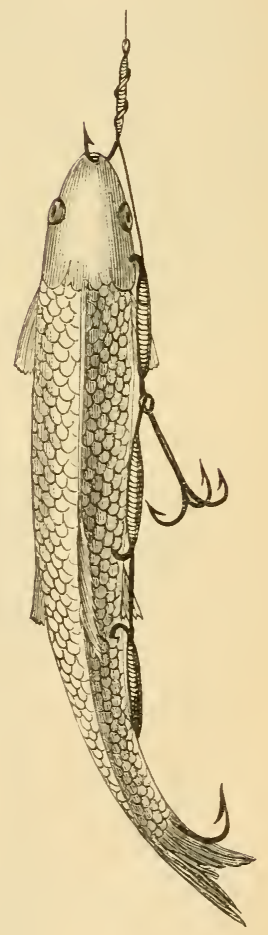

Fig. 293. 
Mant bes Röbers fincin, unb frïnmt ben Räberfifd, wie es Fig. 290 anbentet.

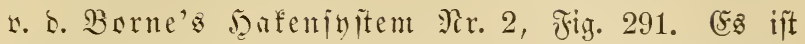

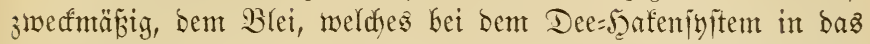
Maul bes Röberiijhes gejdjofen wirb, un einen jtarfen Meifing= braft zu gieñen, Der bis jum Edymanje Deś Röberfitides reidyt. Das Fifdyen form bum mit bem Drahte jutgleidy gebogen werben, cine Röber= misel iit nidjt notmenbig.

Rennerlog nafen= it) item, Jig. 292-295. Iic Rrümmung Des Rëberfild)es wiro Durdy einen Fientullidyen Enofafen bemirft.

In newerer Beit hat Pennell biejent (Enchafen einte anbere Form gegeben. Fig. 294.

Ier Röber wirb it folgenoer Sieije Gejeitigt, i. Jig. 293: man itidyt ben

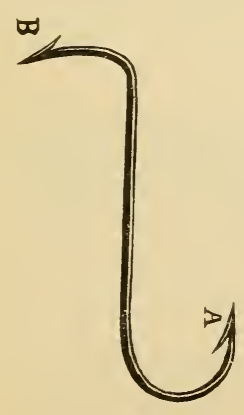

รig. 294. Eñohafen.

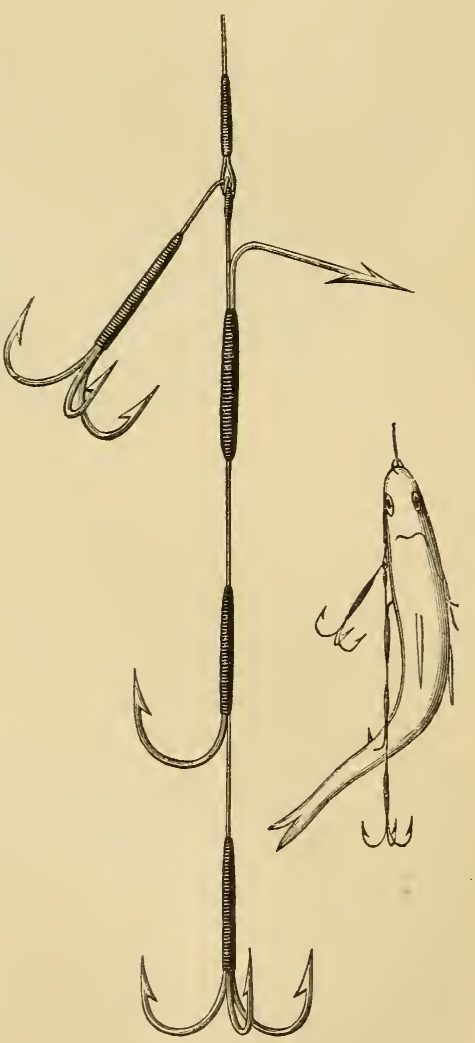

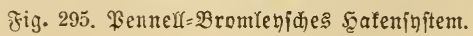


Enskafen nake an Edwanj unter ber Saut surdy und an ber

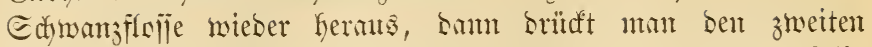
Edjenfel Des (Enohafen in sen Röberfitich ein, jo baj bericlbe sie zum Epimnen erforterliche Rrïmmung crfält. Enolich be= fejtigt man ben Ripkbafen. Bei grezen Söberfijdyen bringt man

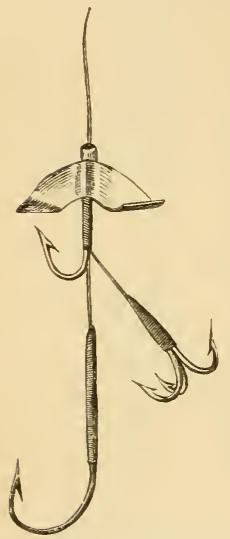

Fig. 296. Francis' Safen $=$ Stftem. einen fliegenten Iriangel an, wie in Fig. 292 angereutet int.

Eine wejentlidie Berbejierung biejes

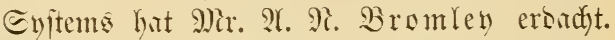

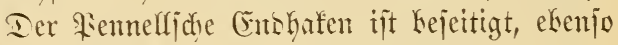
ber Sipphafen. Der Simp miro nuter bem

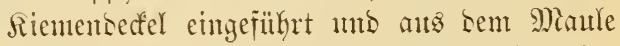

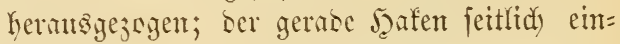

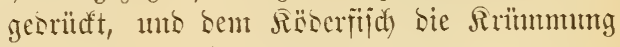
gegchen, wie Fig. 295 antsentet.

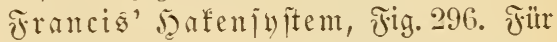
Frrellentijderei uns bie Edhleptangel entpfiehlt

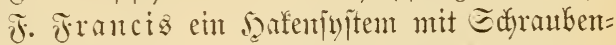
flïgeln, an bem bas Fijdydyen ungetrünmt be= feitigt wirs. Ter Sipflyaten wirb butdy ben Sapi uns ber (Enbhaten burdy ben Leib bes Rïserfijdyes gejtect.

Wm. Bartleet \& Sons, London EC, 23 Gresham Street

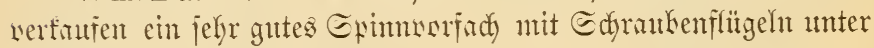
bent Pianen Archer Spinner.

Der Wuri mit Der Epinunuger wotro efenjo anşgëüfrt,

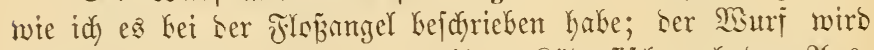

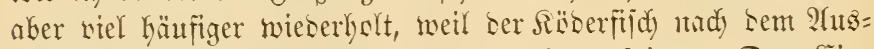

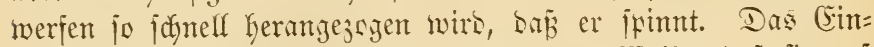
zieken ber Edymur gejejiegt entweber in ber Meije, baj jie auf

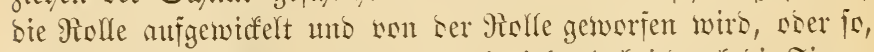

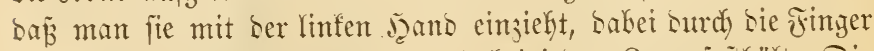
ber rechten \$anto gleiten läjpt, unto kei jebem Buge fejthält. Die abgezsgene @dynur lä̈̈t mant entweser ju Bosen filfen, ober 
man iommtelt fie in ber linfen 5ुanto auf. Durd) cintige Hebung

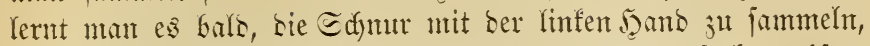

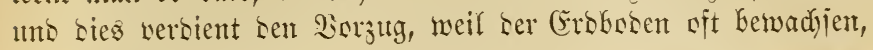

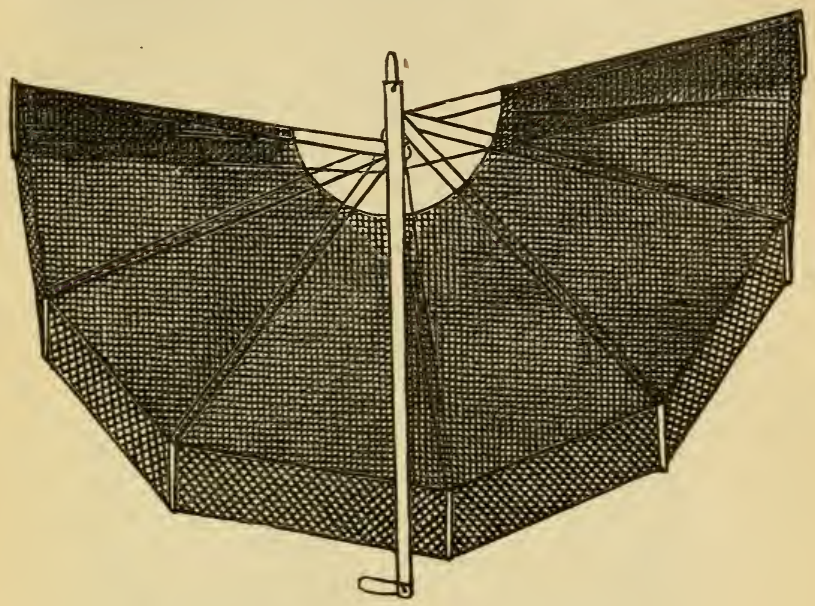

Fig. 297. E乌mant's Fäder, aufgefpannt.

ober mit Steinen besedt ift, wodurdy bie @dyutr in 1tnorbmung

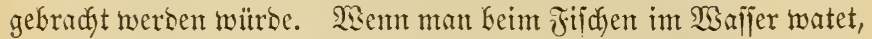
fo fann nan bie abgezegene Edymur ebenfalls nidyt fallen laffen.

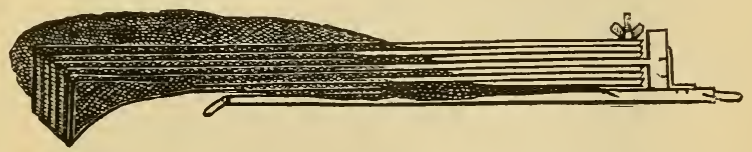

Fig. 298. E乌mant' Fäđjer, zulammengetlappt.

Der Fädber won (5hmant (Fig. 297, 298) wiro benubt,

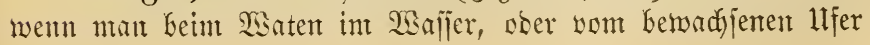




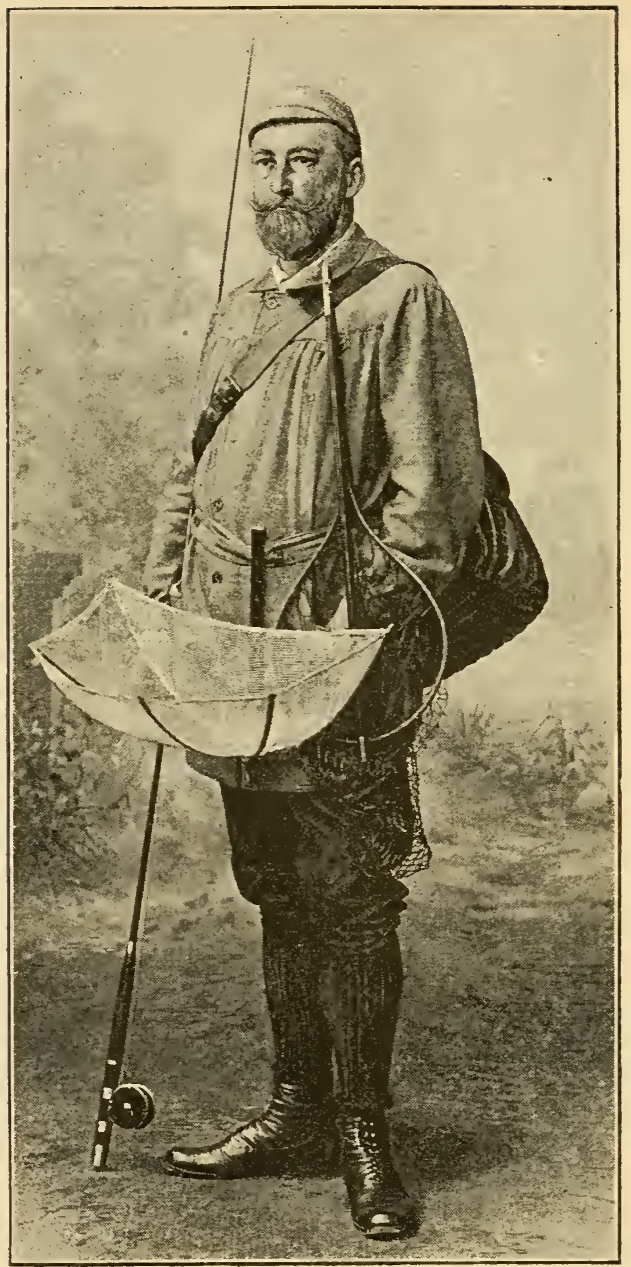

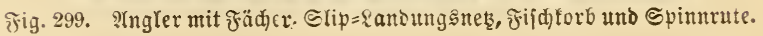


aus weite Würie madyen will. Aujgeipant unt ieitgejdraubt bilbet er eimen Rorb, ber an einem (3)ürtel ober am linten 50 jen=

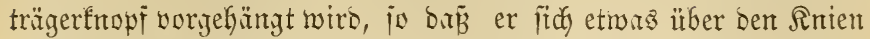
befindet, uno feine ber beim Filifen nötigen Bemegungen Ginbert, Fig. 299. Man läp̈t beim Crimjiehen bie Sdynur barauf

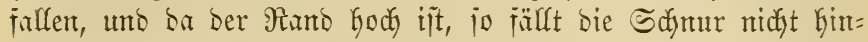

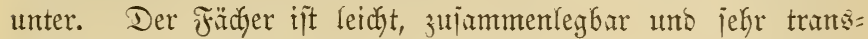

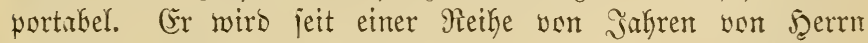

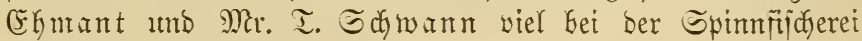
berutbt.

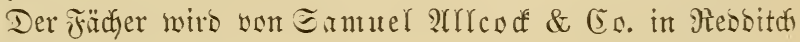
angejertigt und nerfinut.

Die Fijdjerei mit Der Spinnangel ijt ebenjo intereliant, wie bie Fliegenfijiderei, weil ber (Errolg ebenjo jehr yon ber (5ejejicflidffeit bes 2fnglers abhänyt, bie fidd burdy llebung jort=

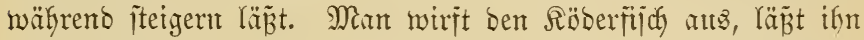

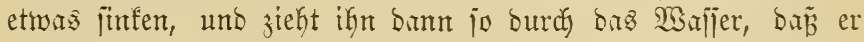
gut ipinnt. Dabei mui bie Sdynur itets itrafi jein, bamit man

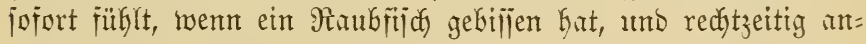
Gauen fant. Wenn ber Röberfiị ausgemorjen, uno bis jur gemïnjhten Tieje gejunten ijt, jo jieft man ifn entweder ruct=

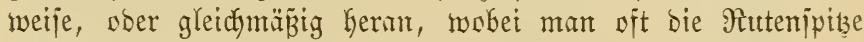

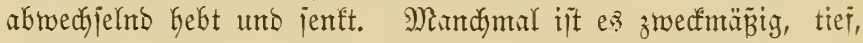

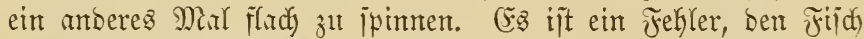

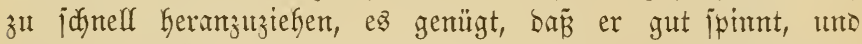

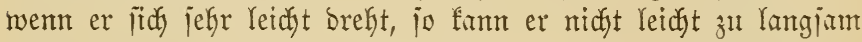
Kerangezogen werben. Desfalb bejdyert man bas Borjach nidjt

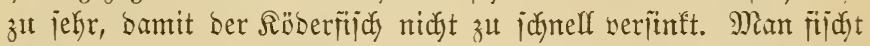
immer zuerit in ber Päke, unb bann erjt meiter entfernt, um bie Fijde nidyt vor ber Beit zlt veridjendfen!

Natürliche Röberfijale fint in allgementen viel wirt= jumer, wie fïmptlidye Spinmer, you letsteren finto bie Qöffelföber uno bas (Siejpenjterfijdyen am bejten.

Die Edjleppnngelci (Darrijidjerei, Edwebeangelei) beiteht 
Earin, ¿aj man finter einem geruberten ober jegelnsen Boot eine lange mit Blei bejdmerte Sdymur nadjidleppt, an ber iid ein matürlicker ober fünjtlicher Räber befintset. Dieje Ingel=

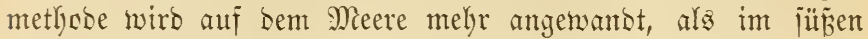
Saffer, weshalb wir anf bas werweifen, was Gierüber sub rubr. Eeefijdyeret gejagt ift. Für bie Süfwalierfijderei ijt bie Edylepp=

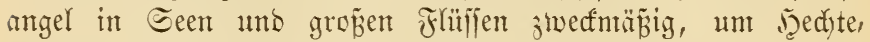
Baridye unt Eeejorellen zu fangen. Man nummt bakei bie

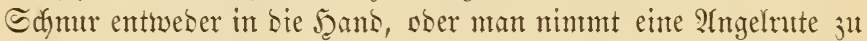

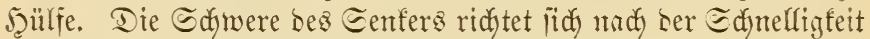
ser Fahrt unb ber Majijertieje, in ber gefijdt wercen joll. Man wentet in ber Megel fünjtlidye Epinner an, uno unter biejen jimb

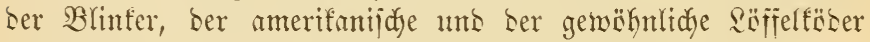
sie bejten, weil fie antagezeidynet ipinnen, uno einen eigentüm= lidyen wedjelnoen Edyein geben, ber sie Fijdye itarf antodt.

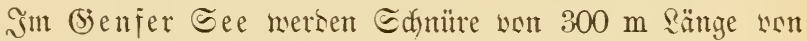
geflodtener Seibe für ben Seeforelleniang gentmmen, unb es wirb zu gleidyer Beit flad) uno tief geangelt. Für bie fladje 2tugel jint bie Eenfer 30-100 gr jumer, bas Borfach hat jwei Sitrbel, bejtegt ans boppeltem (Sutfaben und ift $9 \frac{1}{2} \mathrm{~m}$ lang; für bie tiefgehente 2lngel ift ber Eenfer 170-400 gr jchmer,

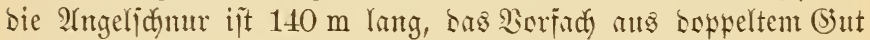

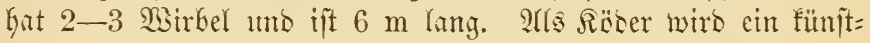
Yidfer Spinmer genomment. 2tm (b)enjer See brandft man auds im 2lpril umb Mai natürlidye Röberfijdse. Fär Eeejorelfen wiro audy oit baz Borfach mit fïnjtlidyen Fliegen werjeken.

Die Sdyleppangel für J̧edyt hat ein Borfady von (Simp, nder befier geipontentem Draht, utb als Räber ein Fijd hen wher

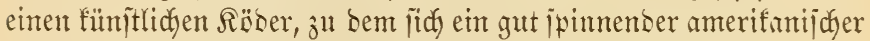
Ëffelffiber ober ein Blitfer bejonders eigntet.

Die Sdyleppangel für Barjh hat ein Borfach von eit= fadjem iturfent Salmen=(Sit, uno einen ganz flemen fünjtlidyen

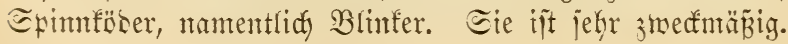




\section{7b. Die Crollangel,}

5. f. Edyludangel mit totem Röberifid.

Tiele 2ngelntethode Gait zuerit Nabbes*) 1682 bejdrieken, uns biejelbe ijt jeitbem im im reeientliden unveränbert geblieben. Eie it wou allen bie beite, $1 \mathrm{~m}$ ein itarf ntit Rraut

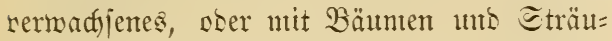
doern itarf bejestes Walfer, wo bie Erinn= angel nidyt angementet werben fanm, abzu=

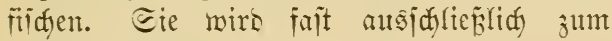
Jedutrange benubst.

2ugelgerät. Man benubst Siejelbe Mute, Polle unb Edymur, wie bei ber Efinn= angel. Das ₹oriacy entbält einen Nirbel, ift aus (Simp ober geiponnenem Draht gemadyt, unb Gödyitens $1 / 3$ io lang, wie bie 21ngelrute, neil innit nidyt gut geworien werben fomm. Ier Soppelfafen, ber Gier angemant mirs, ijt mit Blei Gejdyert (Fig. 300).

Man fann bieje Safen in jefr einfadjer Saeije jelkit mit Breigenoidyt und Irahtzije werjefien. Man madyt aus geglühtem Melifing= Iraft bie Seje, und winbet ije mit gants feinent Mefiumbraht an ten Ioppelfafen. Iann formt ntan ïber einent runten Etücf= doen Goly einen Rapiercylinter, weldyen man an ser Eeite mit Eiegellacf jujanmenflebt; itecft sen mit ber Irahtidyleife verbunbenen Edentel ses Ioppelfafens in sen Tiater=

*) Rev. Rob. Nobbes The Complete Angler. London 1682, lefrt Den Fang des Sgechts mit der Irollangel io, wie er nod) heute ausggeführt toiro.

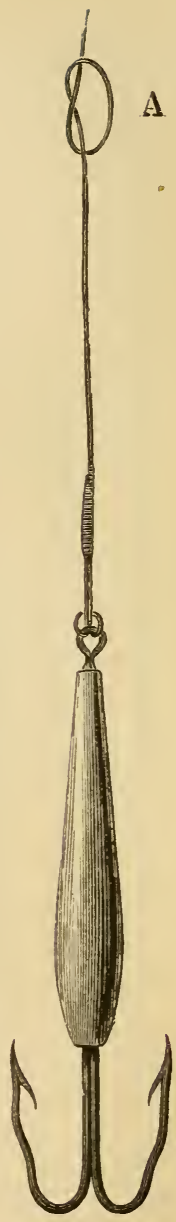

Fig. 300 . Iroflangel. 
Angelmethoden.

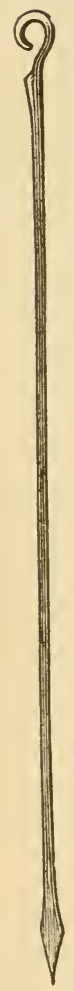

Fig. 301. Söbernabel.

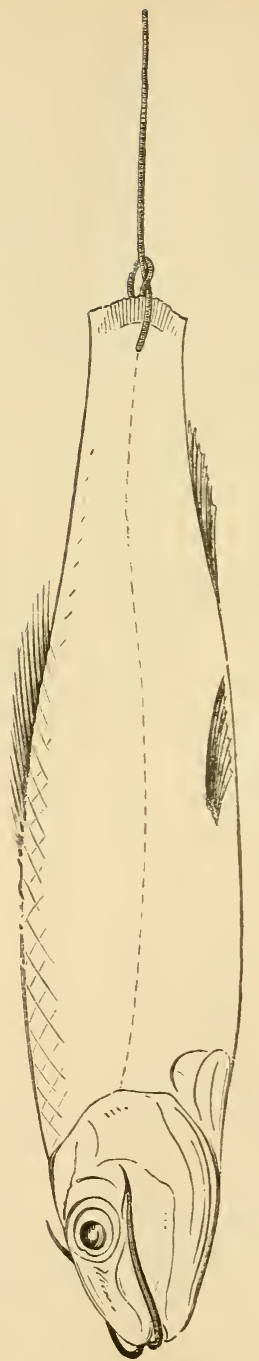

cylutber, und verjalieñt biejen unten mit etmas Ciegellad, is

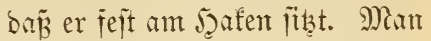
jeflt bas Sianje jo tiej in trodfenen Gand, Dä bie Bapier: Gülje bis an die obere Def̈nmmy ımidylojien ijt, jumilyt in einent Bledylöffel über einer Berzeltus: joben Sampe etwas Blei, lins giept Dantit ben Bapiercylinder

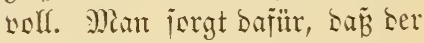
Eand ganj trocfen if̈, Lamit bas gejdymolzene Blei nidyt umber= gejdyleusert wirs. Nady bem (5r= falten bes Bleies wirs das Papter entfernt, und bem Blei mit Melier, Jeile umb Rolieritahl bie gewünjute Sieitalt gegeben.

Man beieitigt Den Röberfijo in folgenter Meije am 5aten: Mlin Gängt die Edyunr, ant meldyer fich ber Doppelyafen befinbet, in eine Röbernabel (i. Fig. 301), fiilyrt bieje in bas Maul ses Fijotjes ein, und ant Sdywans ans, zieht jie jo weit

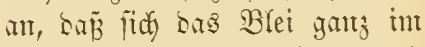
Gnnern bes Jijdyes berinbet, uno

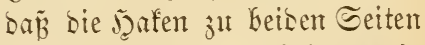

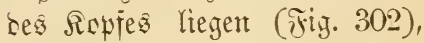
ïe nü̈jien ein wentig bom Ropi bes Röbers abitehen, samit ïe greifen, wenn ber Jecht ธa Jijachen neriduludt hat.

Fig. 302. Iroflangel. 
Fijden mit ber Troflangel. Man wirft ben Röber an

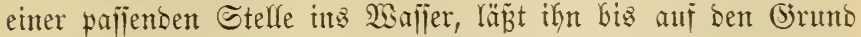
finten, zieft etwas Edyut ein, inbem man ben $\mathbb{R}$ öber bis zur

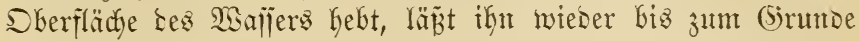
funten, unb fijđt auf bieje Weije alfmählich bas ganze Wajjer ab. Enbals ein bedjt ben Räberfijd ergreift, giebt man ifut freien Epielraum, bamit er laufen fann, wohjut er will, uno giebt ifm

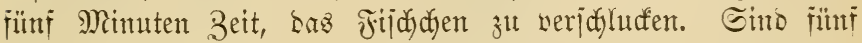
Mimuten verfloilen, is jieht man bie Ecturn itrafi uno haut ant.

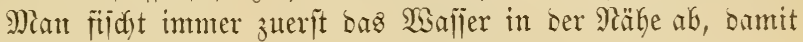

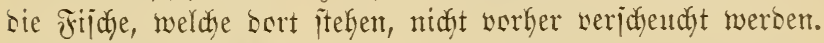

\section{7c. Die Sֲnnappangel ntit lebendem Köberfifhchen, olnue flof.}

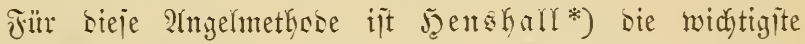
Suefle. Das anjelgerät habe idy wat 2(bbey \& Jutbrie,

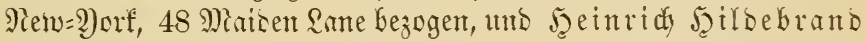
zur Renntuisnafme gegebent, jo baj es an beiben Suellen gefaut werben fann. Tie 2fngeltute iit ganj Yeidyt, $2^{1 / 2} \mathrm{~m}$ Yang uno

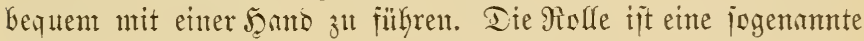

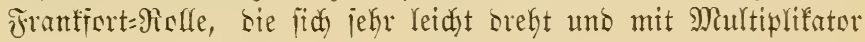

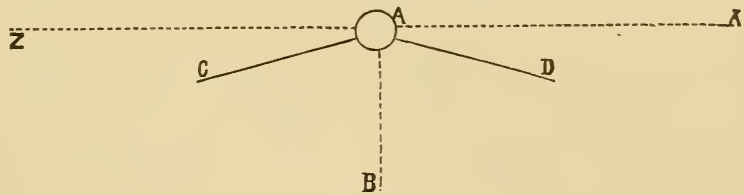

Fig. 303.

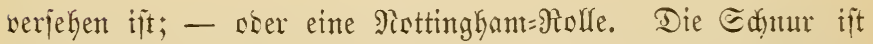

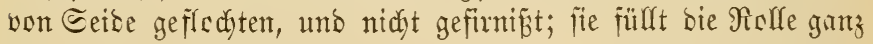
aus, bamit ber Durdmelier berjelben bie ridytige Srö̈be hat. Das Sorfach Kat einen Sirbel, ift aus (Sut gefmüpt und mit einem $\mathfrak{5}$ afen verjefen, ber $10-15 \mathrm{~mm}$ breit ift. Weenn ez er=

*) Hen shall. Book of The Black Bass. Cincinnati 1891. 
forberficy mirs, is idjaltet man einen fleinen Eenfer ein. Das

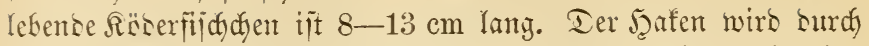
bie Unterlippe uns ein Pajenleds, - oser Kei flement Fijdyen surd) Las Maul uns eine ?agenfïhle gefüfrt.

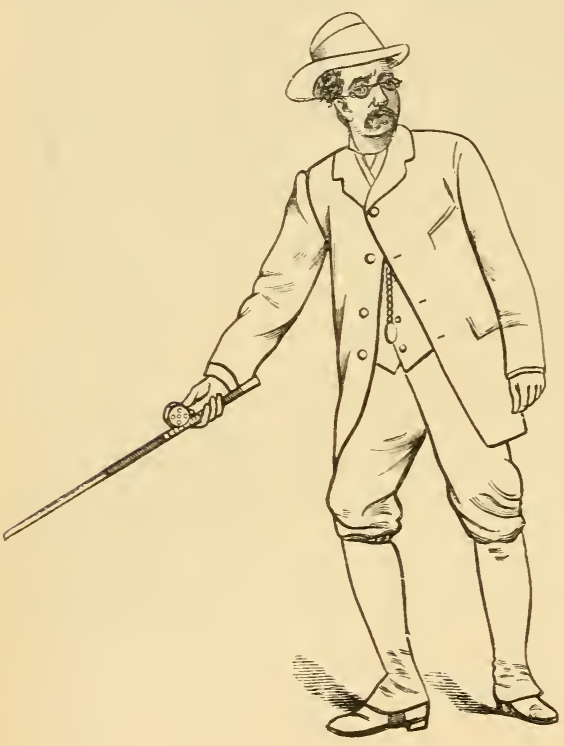

Fig. 304.

2ur bem Dafen itt eine lebende (E) Uritise keieitigt. Iie ङdyum wird is weit aujge= rollt, baj ser Mirbel bie Mutenipize be= rïfrt. Iie 2 rrt, wie

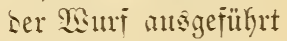
wirb, iit an 2rbbiloun= gen erläutert, weldje sem Denifallidien Butde entlehnt fint. Itnjere Fig. 303 gieft ein Bilto aus ser Bingelperjpective: A ijt

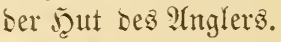
$\mathrm{AB}, \mathrm{AC}, \mathrm{AD}$ bie Prute, und $\mathrm{X}$ ber Fiunte, wohin ge= morjent werben joll. Iie Piute miro mit Ser redyten $\mathfrak{5}$ and mittelfar unter ber Prolle gefalten, uns ser Iaumen befinbet jids an ber Efule, um sas 2hblaufen ter Edyur za beherridjen. Die Etellung bes Panglers ijt io, wie es unjere Fig. 304 barjtellt; bas Fijdyen befintet jidy sidjt ïter sem Boben, Las (biejidyt ijt nady bem Runfte gementet, wohin geworien merben joll, bie Pute hat

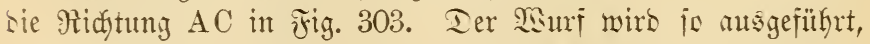

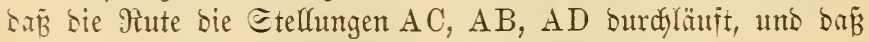
zulest ser 2Ingler is iteft, wie es Fig. 305 jeigt. Ier Surf iit 
nad) fben geridytet, insem bie redyte Janto iait eine gerabe Sinie

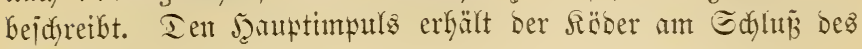
Surfes.

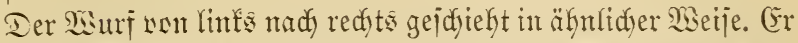
ift in Fig. 306 und 307 abgebilset, uno wirb Gäufiger gemnd wt, wie

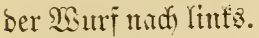
Der Röber fliegt, wie aus Jig. 303 zu erjeken iit, nidjt in ber Aitdytutis, welche bie Pitte jut= Yetzt eimimmt, jont = bern ein wenig nady Gintert, beshalb muib ber sinfel DAX nidyt fleiner wie $30^{\circ}$ werben.

Die Bemeging Der Aislle wirt burdy ben redfen Iaumen regultert, was sutrdy fleip̈ige llebung er= lemt werben muis. Cobald ber fïber bas Majler Gerïfrt, wirb bie Palle an= gekalten.

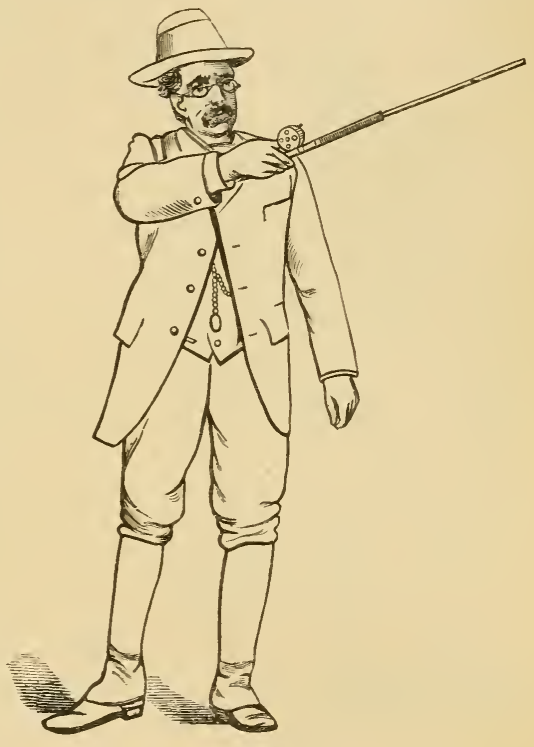

Fig. 305 .

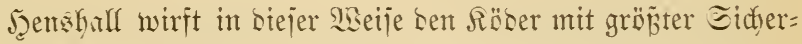
heit $36-45 \mathrm{~m}$ weit.

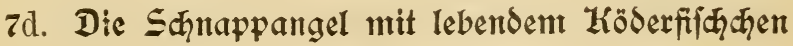 und bem flot:}

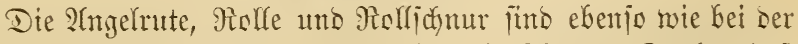

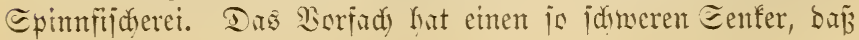




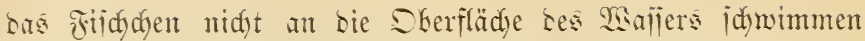
fann, umb ein itartes Rorffló. Fig. 94a, 96.

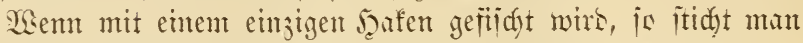

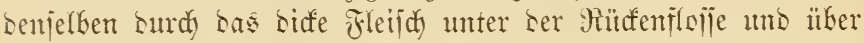
bem Mituffirat. (Fig. 308. ङ. 175).

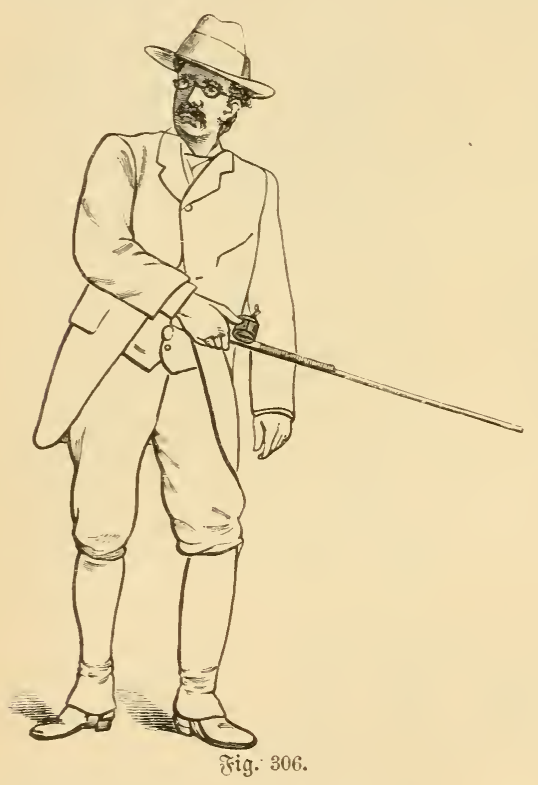

Iis itg. 309 jeigt eit anteres Doufeniyltem uns sie 9 (rt, wie sas ふ̊̈̈ьerfifdyen baran kefeitigt wirs.

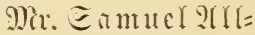
coff jull Feoritidy in (Englans werfauft unter ser Bejeidyunt Pir. 2674 ein Doufenfyjtem, Fig. 310, 311. Ter gleitente Iriangel mirb an Rüufen bes RöLer= iijoles kejeitigt. Ter jogentannte Biffersufe= Triangel B mit Iem 3urïtfgebogenen ந̧aten wirb in ser ?äke bes Ritementedelo unter ber Doant bes Jijdyen be= feitigt, uns ser fliegente

Iriangel C hängt loje herak.

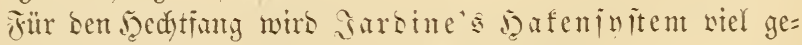

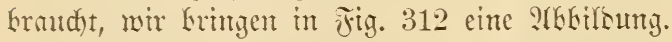

\section{7e. Die Sđhludfangel mit lebentem Köberfif}

Das Rorfach heiteht aus (Simf, boer geifontenem Draft ron $20 \mathrm{~cm}$ Sänge unઠ einem Doffelhaten. Hm Len Röberfif

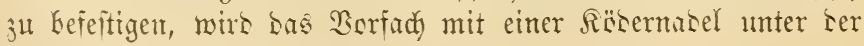




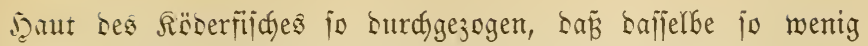
wie mëglid) verlekst wirs, unb lange am Seben bleibt.

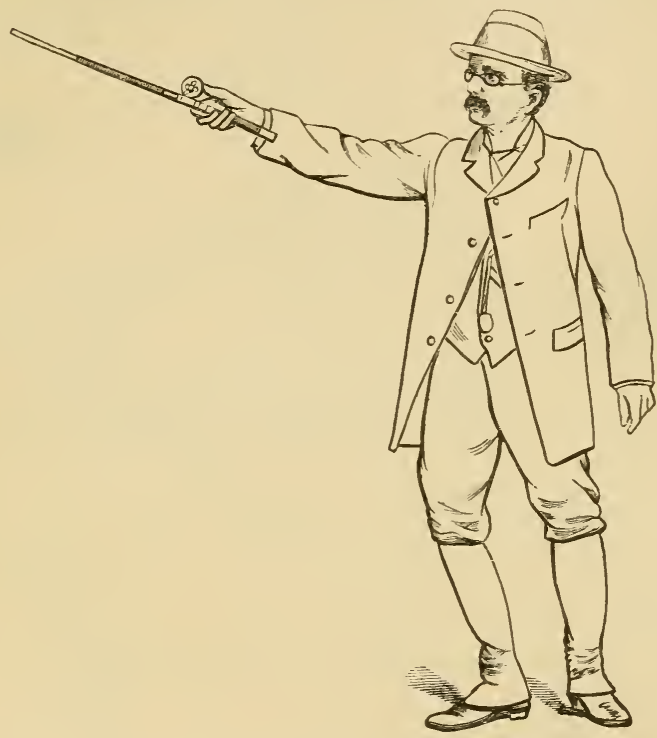

Fig. 307.

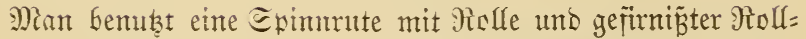
iđjnur, ein jtarfes Fla †̈iñ Minuten Beit зum Edfudfen, wenr ein $\Re a u b=$ fiic) gebijien hat. Dieje

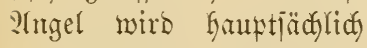

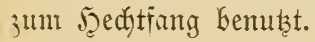

Dic $\mathfrak{S e d}$ tpuppe ift eine Ed)ludfangel mit lekentem

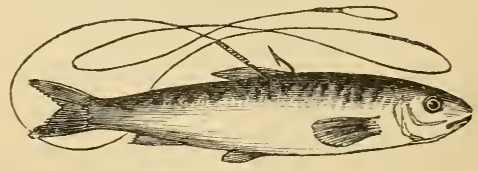

Fig. 308. ङdnappangel.

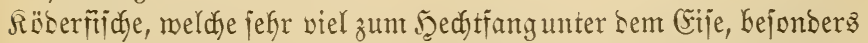

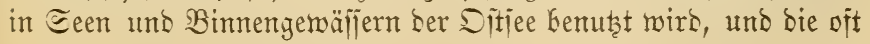




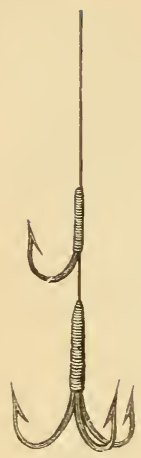

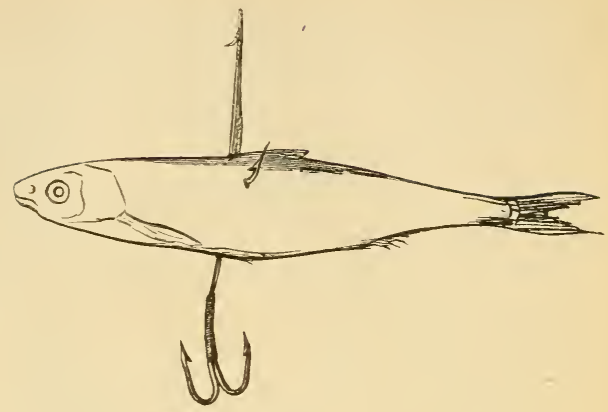

テrig. 309. Sdnappangel.

reidye Beute liejert. Die Puppe ijt ein Eylinter bon bartem Jasly, ber $12 \mathrm{~cm}$ hed iit, mo $4^{1 / 2} \mathrm{~cm}$ Durdmmeller hat. $11 \mathrm{~m}$ bie \$uppe wirb eine 8 fis $10 \mathrm{~m}$ lange iturfe Plngelichmur ge= midfelt, uno an bieje bas Borfach mit bem Röberfichdien kejeitigt. Man Kaut baraui an Gielegen won Rokr, Shilf aber Binjen Sëder in bas CEis, widfelt jo viel

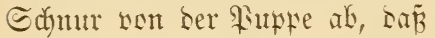

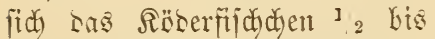
$3 / 4 \mathrm{~m}$ unter bem Sije befintet, uno ftellt bie \$ruppe neben sem Sodje auf bas Cis. Heber sas Sod legtmaneinen gabelförmigen Sneig um bie Ednur, weldyer bie \$uppe zurücthält, wenn ein Sed)t ben Röberfitid ergriffen buat, uno Damit fortictwimmet, unt ifn

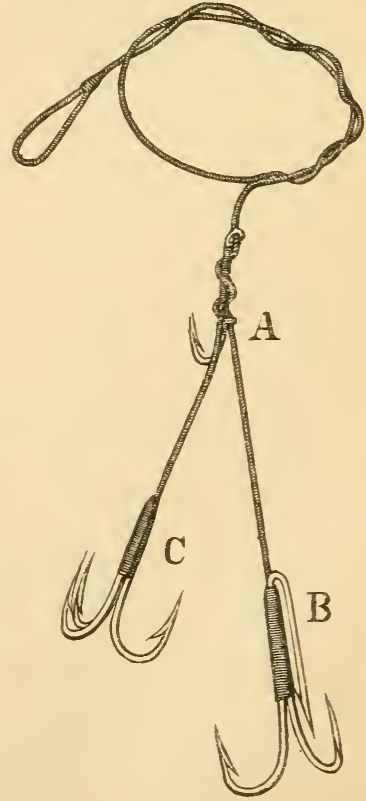

Fig. 310. Shmappangel. 


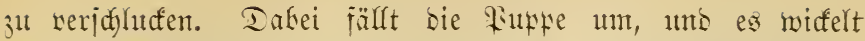

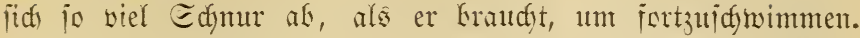

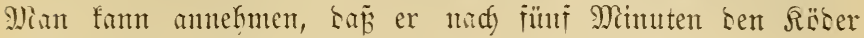
Leridylufft hat, uno gerangen ijt, io $\delta a \tilde{B}$ er aus Lem Wanjer

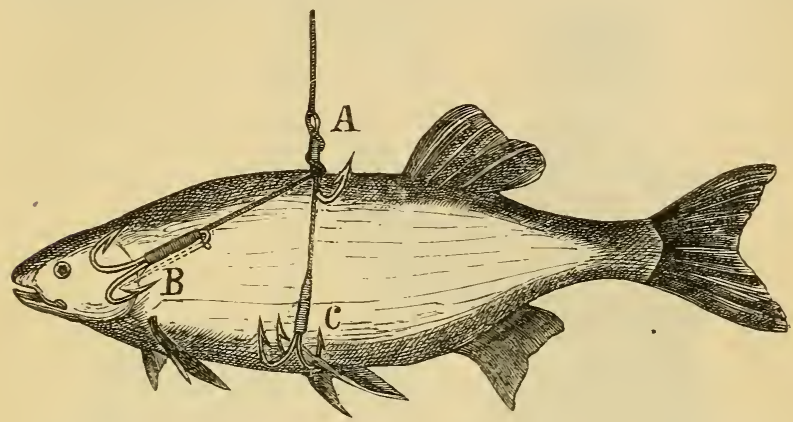

Fig. 311. Sdnappangel.

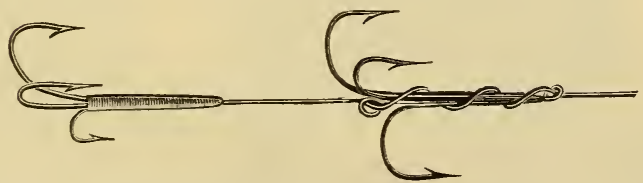

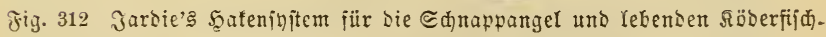

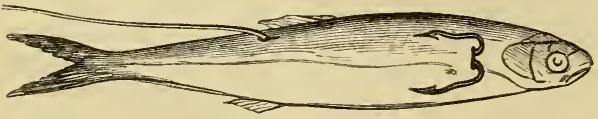

Fig. 313. Shlutangel.

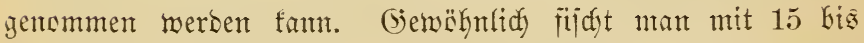

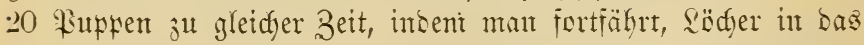
Cis zu hauen, uno immer bie Fupfen heransminnt, uno vorn wieber einlegt.

Für bie Angelfiiderei unter Lem (Eije ijt ber (Befraudy bes v. ๖. Borne, Ingelfiiderei. 3. 2uff. 
Tijejerjditten zmedmäвig, meldyer auf ber Intemationalen

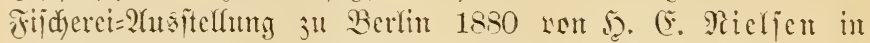
Chriftiania anseiteflt mar. SEir jeben in Fig. 314, wic ser

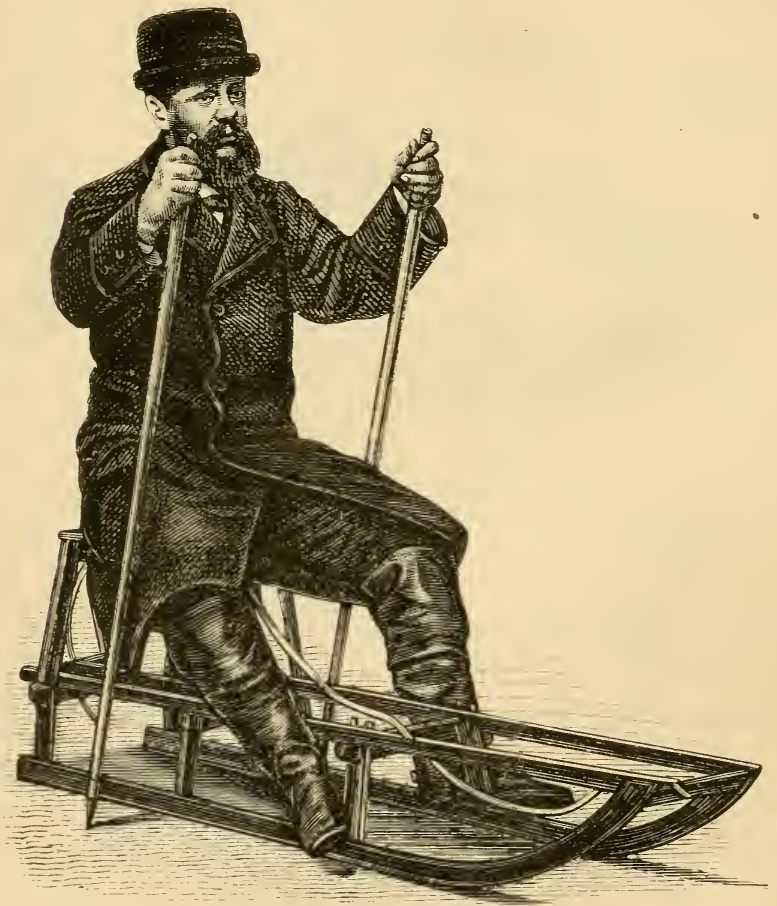

Fig. 314. Fijderidilitten.

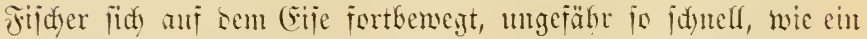
Wiferbejditten. Dis Eegel, Fig. 315, meldyes auth jur Fort=

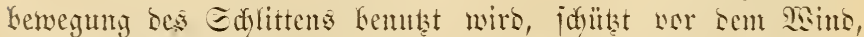
went bie 2lugelitelfe erreidfit ijt. Die Edymiel bient zur Ent= 
7e. Dic Schluffangel mit lebendem Söberfijh.

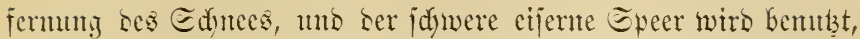

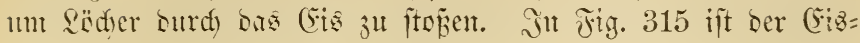

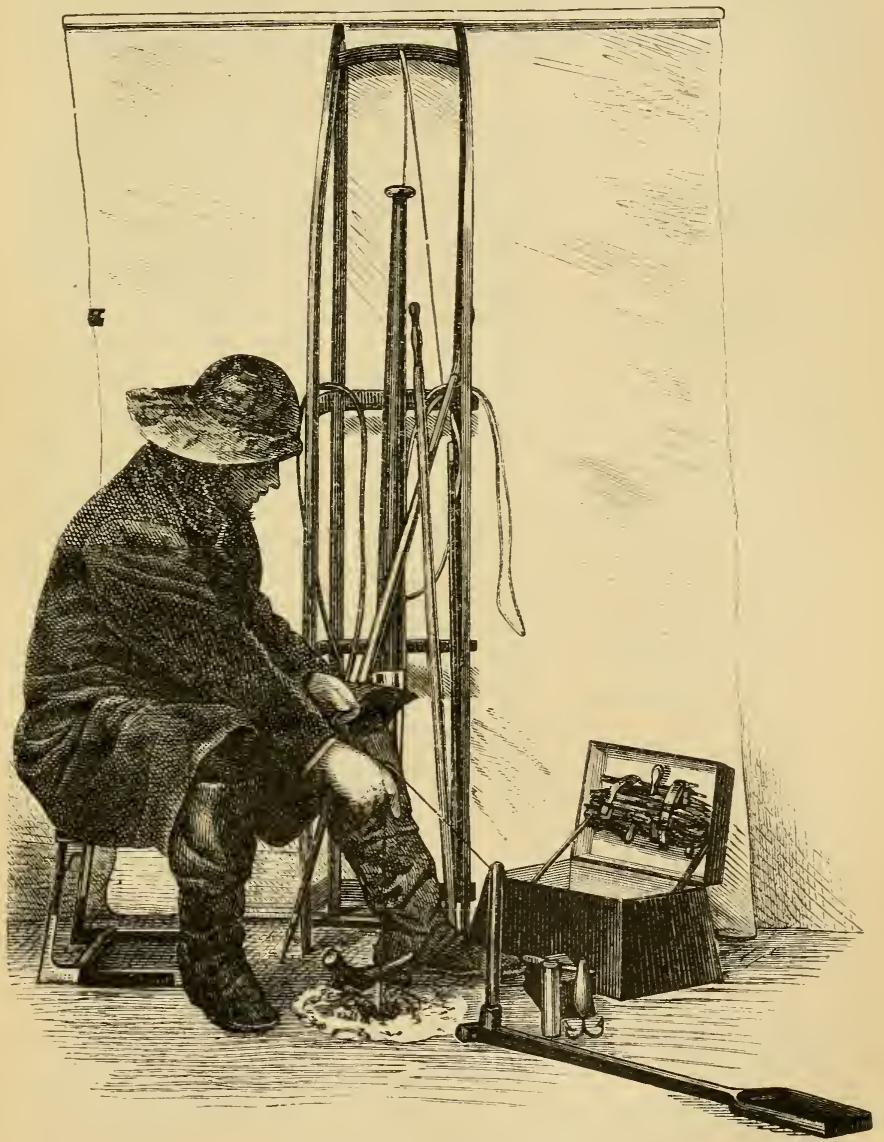

Fig. 315. Fiiderid)litten. 


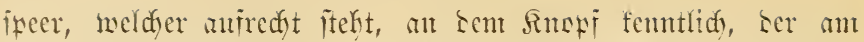

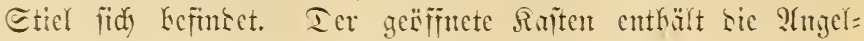

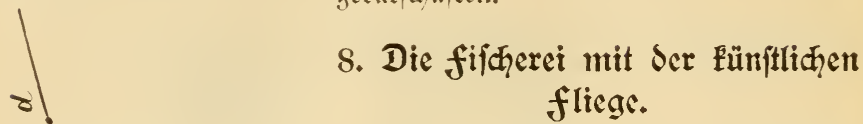
gerïtijuaften.

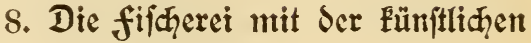

Tie Fificterei mit ser fümitliden Fliege iit eine ser wirfiamiten uns be= Yiektejten Angelmethosen. Man unter= idjeiset bie Fitidyerei mit ber einkänsigen uns bie mit ser soffelfünsigen Fliegen= rutc.

A. Die Jijucrei mit ber ciu= hänoigen Jiegenrute.

Die Rute jei 3 bis $3,2 \mathrm{~m}$ lang, jefr

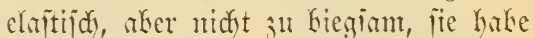

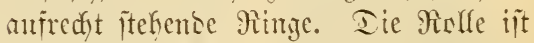
she Multiplifater mit Fererkemmung

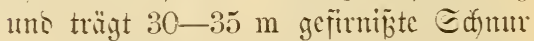
won gefleditener Ecise. Ias Sorfadt iit nou (5)ut, $1 \frac{1}{2}$ bis $3 \mathrm{~m}$ lanto, 1 mo wirs nad) Ier Exithe ju feinter, es trï̈gt eine (ensfliege a, umb gemöhnlid) cinte Gis stec நüngefliegen b, c, Jig. 316.

Ier 28 ur feiteht in einer offpelten

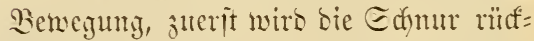

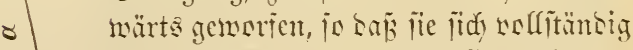
gernes itrecft, sam wirt fie nad now= wät ge genorien, is sab fie fid wiederum gerise itrect, bener sie Enoffiege bas

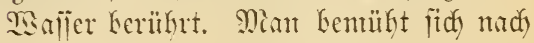
Fig. 316. Fliegen=?orfad.

\section{裁}




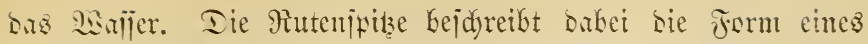

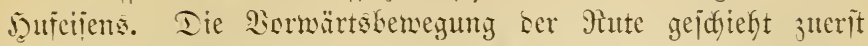

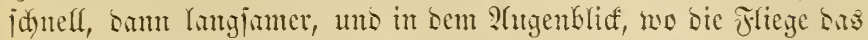

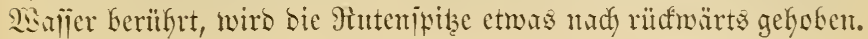
Man bült bie Pute is fteil wie mïglidf, umo jentt jie nidut mehr als $45^{\circ}$.

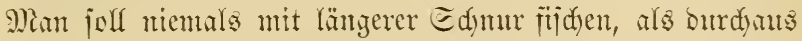

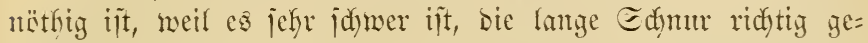

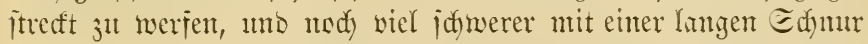

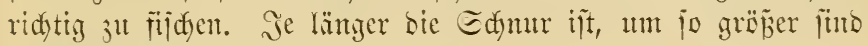
bie Edynierigfeiten.

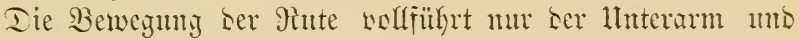

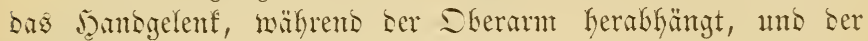

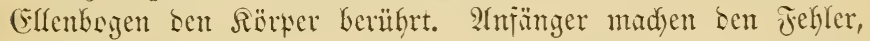
baj jie sie Mute ju jefr jenten, uto ju viel Rrajt auf ben werwentent. Je jeifer bie Rute bleibt, umb je geringer bie wer=

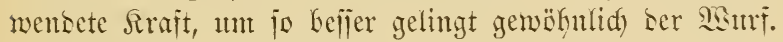

Sem ber erite Simf gemadyt ift, jo wirb bei ben folgenoen

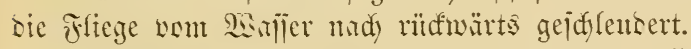

(Ein $12 \mathrm{~m}$ weiter sinf ijt in ber Megel volffommen ats

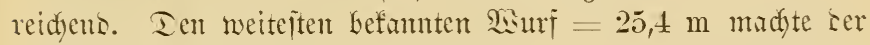

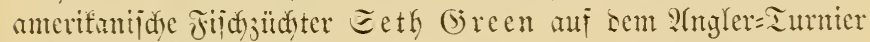
in Pew=? orf 1880 .

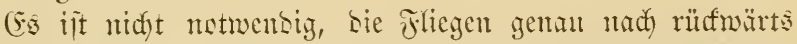

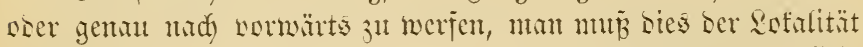

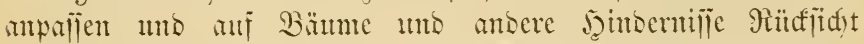

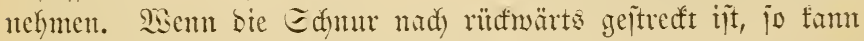
mant mady jeser beliebigen Ridytung werjen uno bismeiten ijt es bejier, jie nidft mady finten, jonsem nady sben sber jeitwäts $\hat{\jmath}^{\mathrm{U}}$ itrectert.

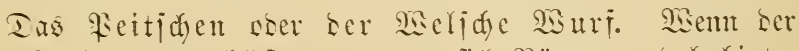
2Sinb ftarf entgegen blät, soer ment fid) Bäunte u. Sgl. Ginter

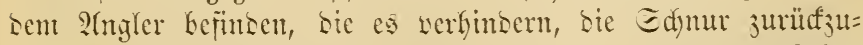

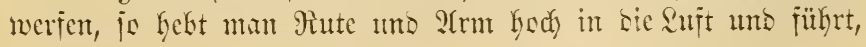




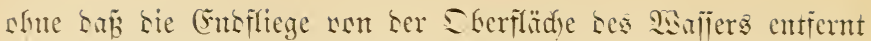

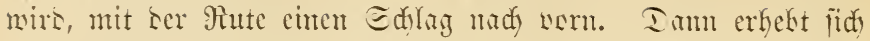

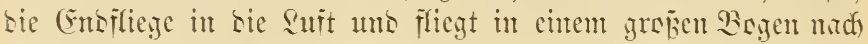
ser gewilmidyten Etelle.

B. Die Fijherei mit ber boffelhänoigen Jliegen= rute uno mit ber sadyrute

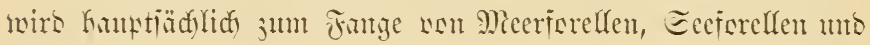
Sadjen geilft. I ie soffelfünsige sliegentute ijt 4 fis $5 \mathrm{~m}$ แn bie Sadystute 5,3 bis $6,3 \mathrm{~m}$ lanty.

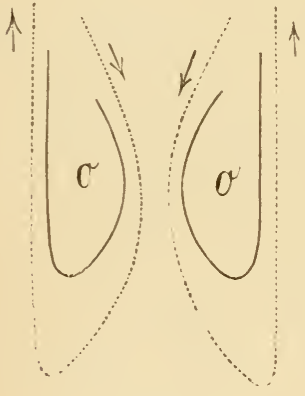

Fig. 317.

Ier 2 stur wits in ganj TEeije antsgerührt wie mit ser ein= Gänsigen ìliegentute, sie Bute mirs afer iffer Echwere weyen mit fieisen Dänsen geritfert, intsent jidy bie redite Dant über unb sie linfe unter ber Balle Kefturet. in Fig. 317 kejeidutut 0 bie Etelle, wo ser ?higler jeft, bie ants:

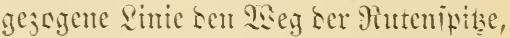

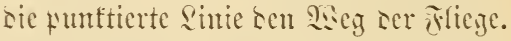
2ieciter wie 20 - $25 \mathrm{~m}$ wiro ielten ge= worien, $30 \mathrm{~m}$ it cin langer sisur uno $37 \mathrm{~m}$ รie äujerite erreidyte Grenje.

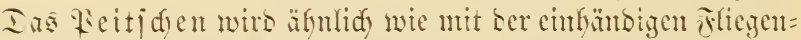

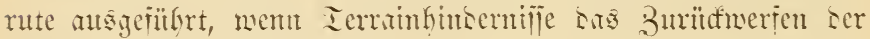
Edyur unms̈glidy madien.

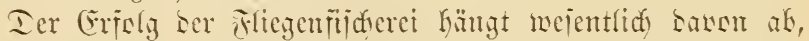

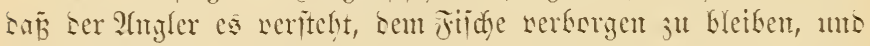

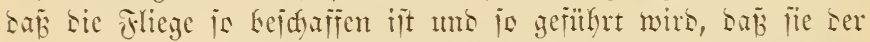
Fitid) fïr ein Yebenses falb idl

1. Sie Gitalt, jarke utb Grïbe ser ittege jmedent= iprectento jein;

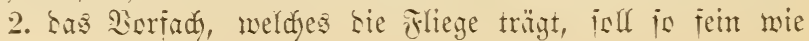




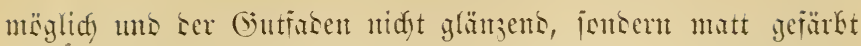
ich, Eantit ifn ser Jijh nidyt leidft bemerft;

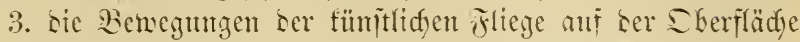

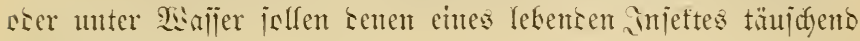
älunlid) jein.

(5) wirb in werjuchener

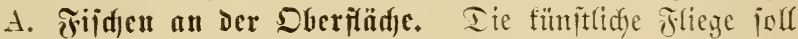

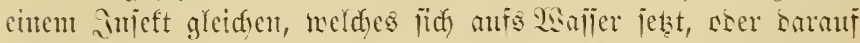

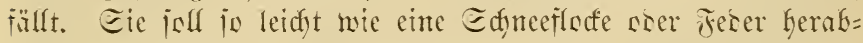
fallen uns nidft berfinten. Mian unterideicet bier ferner bie Titicherei mit Ler troffenen uns ber mafjen siliege.

Bei ser sijdjerei mit ser troffenen Fliege mirb biejelke nad) jebent Simf mefreremale in Breijen surdy bie Suit unther

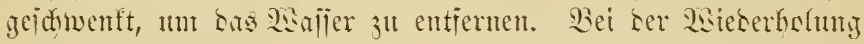

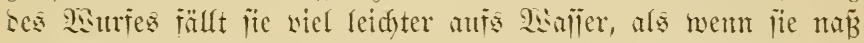
ijt. Die stiege ind in gewunten jein, baj jie jdyimmt, uno

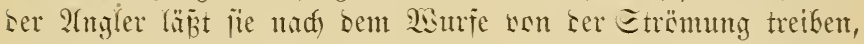

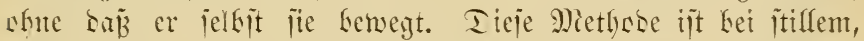
Felfem Setter uno bei rubigent, flarem Wafler kejonsers mirfjan

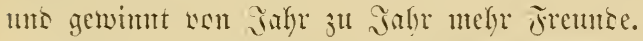

Siei Ier Fijdjerei mit ser แafien Fliege wersen sie Sïure whte meiteres wicberfolt. Es geidjieht bies zmectmäpigermeije bei windigem, trïbem, regnerifdem Sietter uns anf femegtem trïbem 2sajier.

B. Fijden unter Der sberfiläde oder mit Der verjunfenen

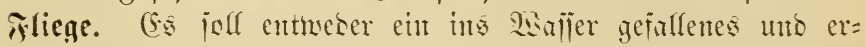

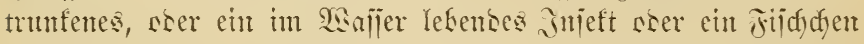

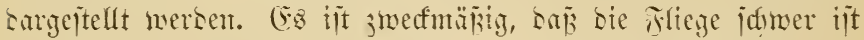
uns reidyt rerïntt, weshalb jie sit mit Blei feidwert wirs. Man wirit itromab, soer quer über sen Etront, soer auj eine jtebente 然aljerfläd)e, läpt bie Jliege mefr sier weniger, sit bis jur

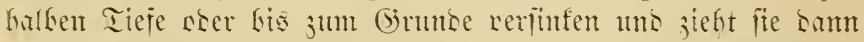

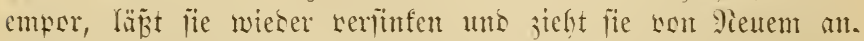
Mian nent bieje Methobe, wenn jie mit itarf kejomerten, fdunell 


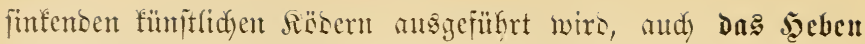

\section{uto Ecufen.}

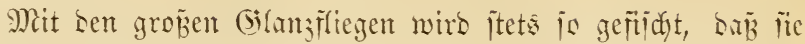

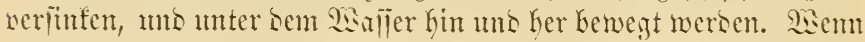

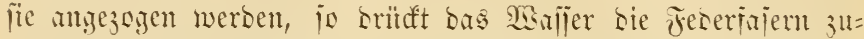

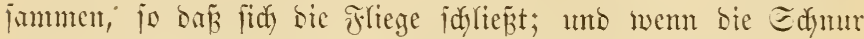

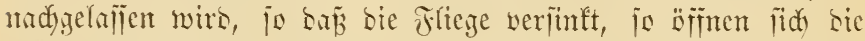
Feserfajern, und bie ganje Pradyt ber Farken wirs fidthar. I ie

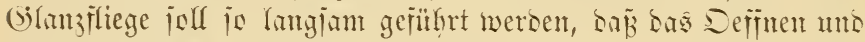
Edyliejen wirtlidy erjolgt, weil ber Fijdy bie Fliege gemöbulidy

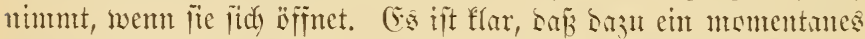
Edylaffwerben ber Edyur erforberlidy ijt. Deshalb jenft man

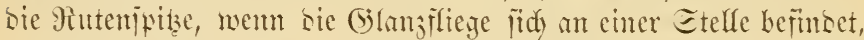
we man einen Fijd sermuthet. In itrümensem sisaljer wirit man quer ïfer ben Etrom, und läß̈t sie Fliege herumtreiben.

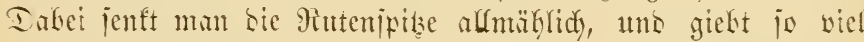

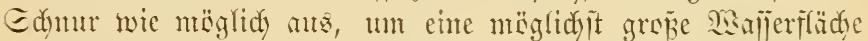

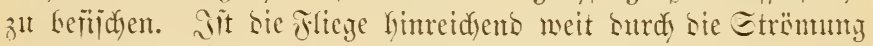

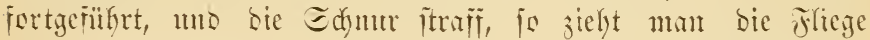

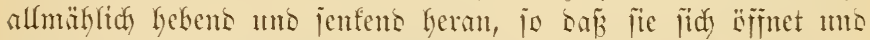

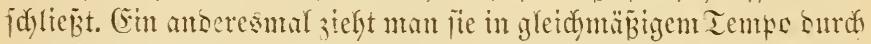

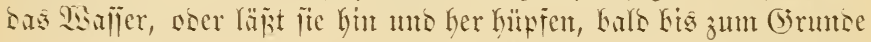

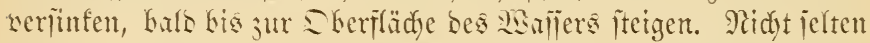

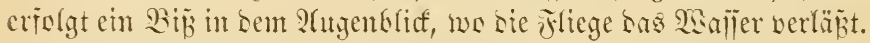
Derr Dr. 2. Bettentorf in Bont, ein jefr gemanoter mb criabrener Fliegenfildyer, teilt iolgente Methose ber Fliegen= fiid)erei mit, bie jebr wirfjom, namentlid) für ben 2(ejd)enfang,

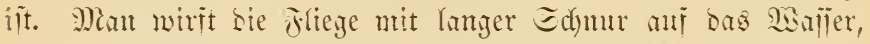

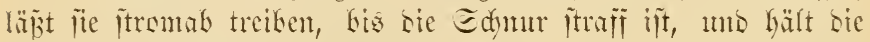
Triege sam mbig an serielken Etclle, wobei fie melyr ofer

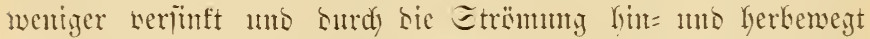
swirs. Nad chingen Minuten geht man einen Edyritt weiter

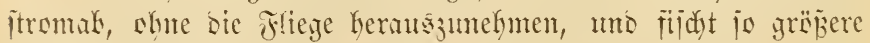

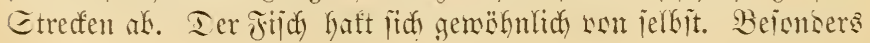


empiehlenswert ijt bieje Mlethobe in fleinen Bädhen unto Etrom=

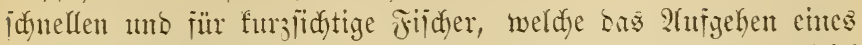

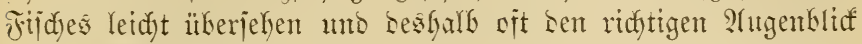

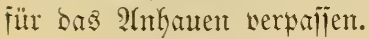

Tienn bie Tijdye bie Fliegen an ber Sberfläd)e nidyt nefmen

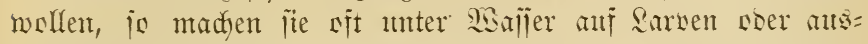

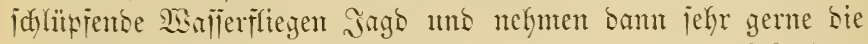
rerjuntene Fliege. Ferner ijt bieje Methobe ganz bejnoers empieblenowert für sie Befiid)ung wn tiejen rufigen Dümpeln, non Mïhlobermajier, Ieidyen uno Eeen. Ier amerifantidye Edwar $z=$ un forellenbarid) nehmen sie werjunfene Fltege

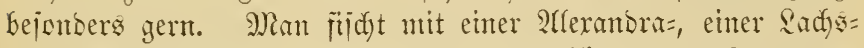
ober Eeeforelfenfliege, einter biffen Mä̈rzfliege ober Governor,

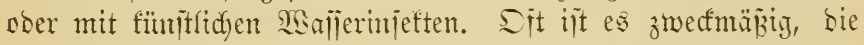
Fliege basurd zu bejdweren, baj man am Ropi berjelben eiu geipaltenes Edyotforn am Gutfaben anfmeift. Fenter erfïft co vit bie sirfiamfeit ber serjunfenen Fliege besentens, went eine bide Fleijdmabe an sen Jafen gebüngt wirb. Raltes, minbiges,

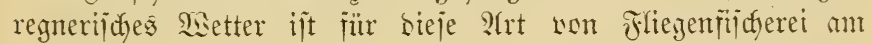
gümitigiten.

Man wirft in Flüfjen umb Bädyen bie Fliege entweber fromaui, voer mant fijdst itromab.

Das Etromanifiidgen ift mu in rufiger Etrömung möglid,

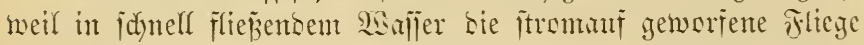
io junell bent jijdyer entgegentgejüfyt wiro, baj er nidyt int itanse ijt, bie Edyur itrafi ju erfalten. Ties ift aber not= wensig, um jojort antianen ju fönnen, went eint Fifd) nad) ber Friege augeht. Ias Etronmufijidyen hat Len Borteil, Daj es

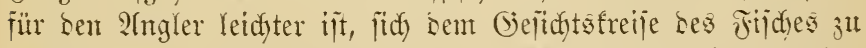

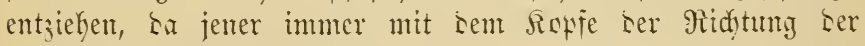
Strömung 3 thgewentiset ijt.

SEenn man gemorfen hat, io hebt man bie Shgelrute, ionie

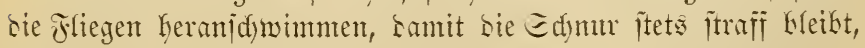
Die Filiege jolf mur surd) bic Eträmutạ, nidit surd ben 


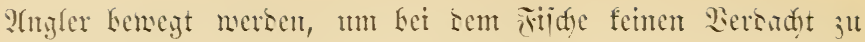
erregent.

Bei sem Etromabiidaen mirft man momëglid quter über

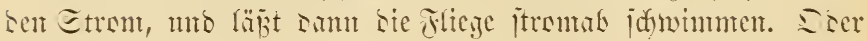

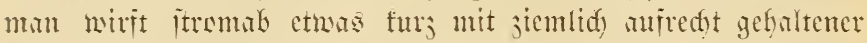

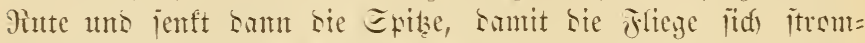

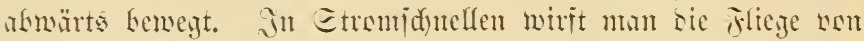

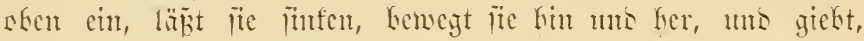

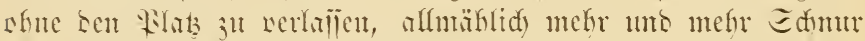

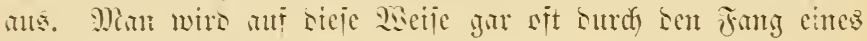
guter Fitides erfreut.

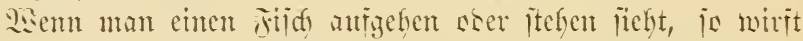

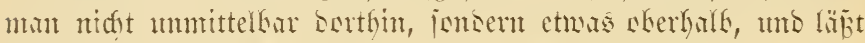

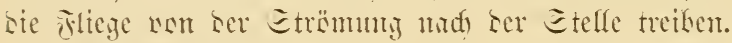

Ser Edyatte Etemart, einer ser gemanstejten Fliegen=

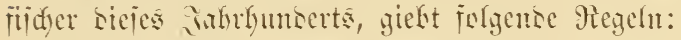

Cïnen tiejen, rubigen Pämpel isllte man mur Gefididen,

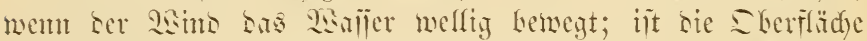

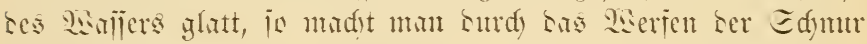

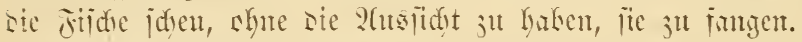

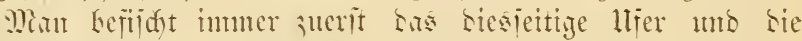
nädite llngegens, uns mirit erit ifäter meiter uno nach bem

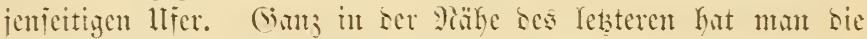

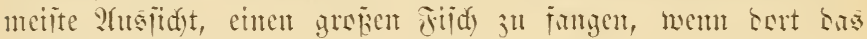
㷛rijer tiej iit.

Man whit itremant, uno läpt bie oflege, madecm fie sas

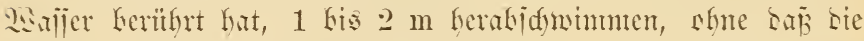

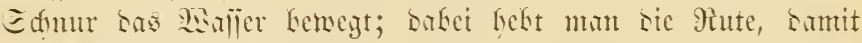

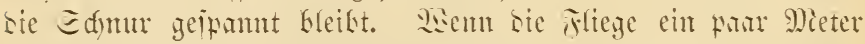

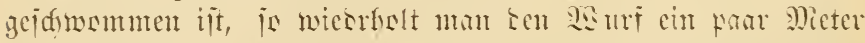

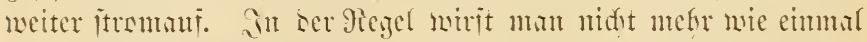

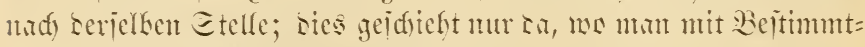
beit cinen guten itija crmartet.

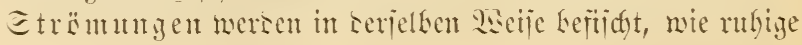




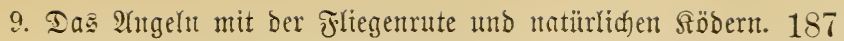

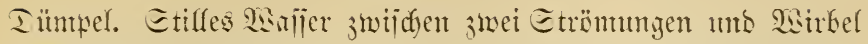

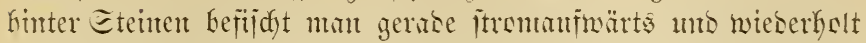

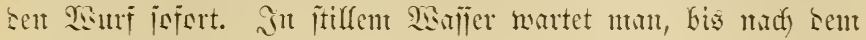

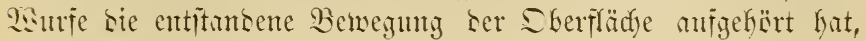
jeft sam bie stiege langlan heran und wirft bant bon nenem.

Die Evinüijderei mit ber sllegenrute uns ganjleidgten fünjt=

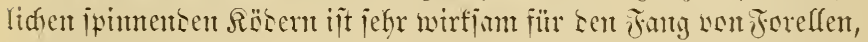

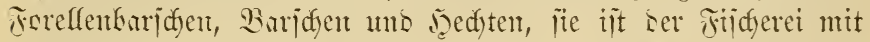

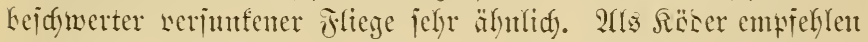
ïd) (sejpenterfijd den, Feserfielfijdden, Spinning Alexandra unt Halcyon Spinner, fleite suffelföser, wie Forlow's HogBacked Fly-Spoon; ser Bafy =Epinter.

\section{Das 2lngeln mit Der fliegenrute un' natürlichen Köbern.}

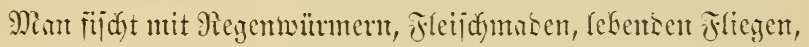

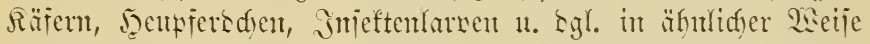
wie mit ser füntlidten sliege. Ier Räser wirb in folgenter 2ieije am jaten bejejtigt:

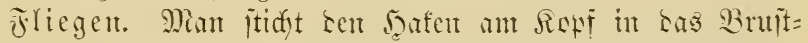

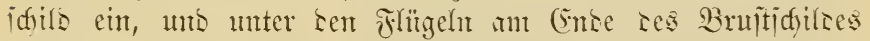

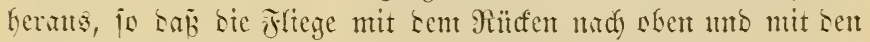
Beinten กสd) unten kängt.

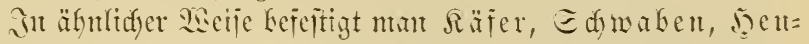
piersden unb bie Earwen ser Eteinfliege, (creeper).

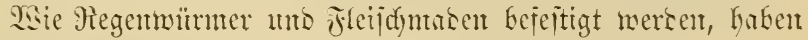

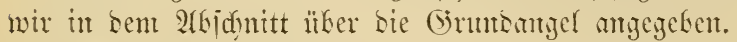

Etemart's Sorfad) für Etemiliegentarben. (Es werten jwei 6 bis $7 \mathrm{~mm}$ breite jaten an einem Gutforen mit gelber Eeise angemuncen, io baj ifre Exiben $15 \mathrm{~mm}$ nou cimntoer entfernt fins. Man ninmt eine Sarwe, mo fidid ifm sen unteren

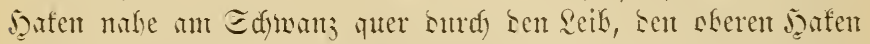

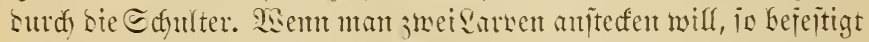

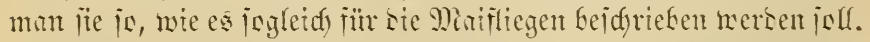
Etemart's Borfad für Miaiflegen. Iies Borfads 


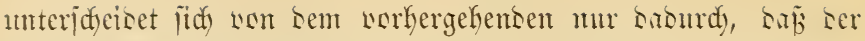

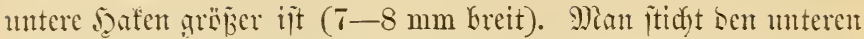
groben Saten in bie Datte einer Marifliege cin, bringt ifn an

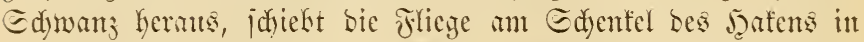

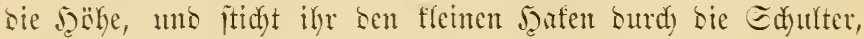

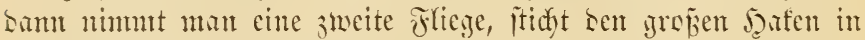

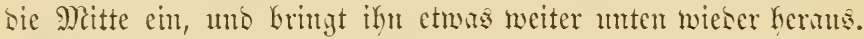

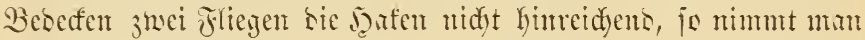
mod) cine oritte biuzu.

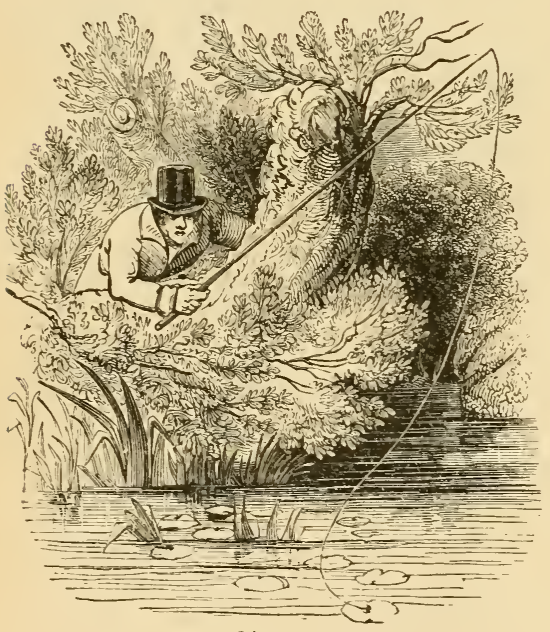

Fig. 318.

Man mun der Burt= beit bes Röbers wegen worpidstiger werfen, In mit serjelbe utdyt wom saten abfliçst. Ieshalb bemtist man entmeser eme lamize, reid)te, soppelfänsige otiegenrute ofer eine leid)te Mesttinglyan= 2tngel und wixit Surd) bie Minge. Die Mic= those ift jebr worfjam uno jum Fantuge wieler jijde, bejonbers ser Jorellen, श्ajedsen, Dëbeln, Sä̈jelinge,

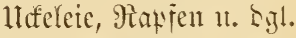
geeignet. Maan fijdyt in ser Begel ganj obne Eenter; anşge=

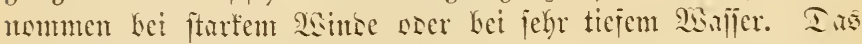
Fijden itromanf ijt fier nody wodytiger wie bei sent 2hugeln mit ber fümptlidyen Fliege, weil man nidyt jo weit mit sen zarteren

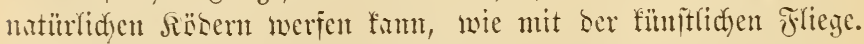

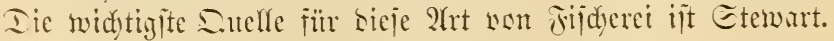

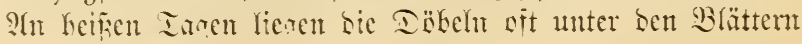




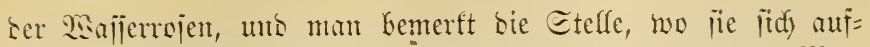

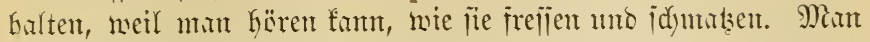

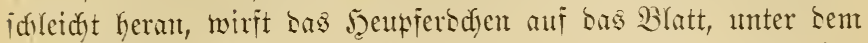
cin Fijey liegt, jieht es bis jum Mante ses Blattes unto ins

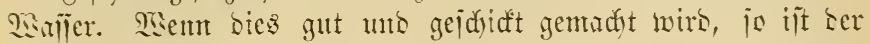
Fitich jidber gejangen; sent er iit jwar trïge, aber jebr gefräßjig,

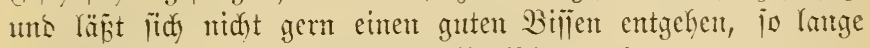
jein $\mathfrak{P}$ rgiwekn nidyt rege gemadyt ijt (Jig. 318).

\section{Die Bujhangelei ober Tippfifderei \\ (テ: 319 )}

witro bauptiäd)lidy jumt Finty nou Iibeln uns Freflen mit natürfichen Rësern, namentlidyleben=

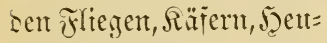

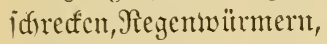
fleiten Frïidyen, ange= mantot. Tie Injeften be= ieitigt man io an bem Taten, ba is manbenjelfen ant Ropf eint=, uno hinter sem Brutitiditse herans: führt, samit ber Röber mit sem Rüdfen nady

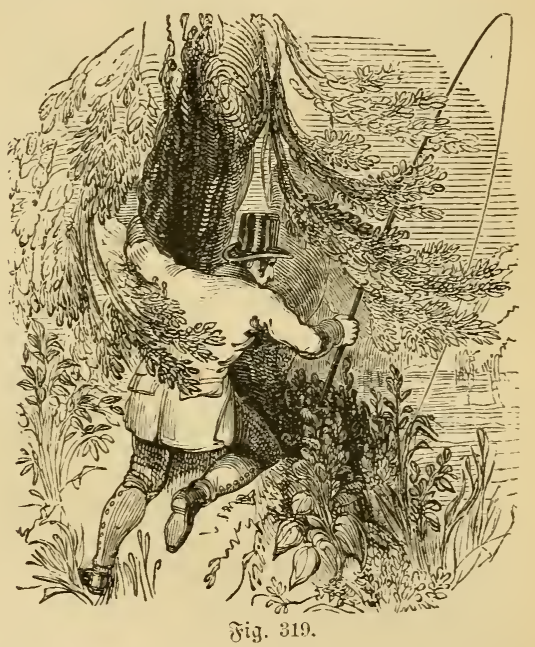

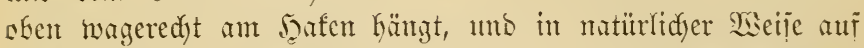

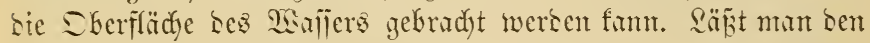
Sïser nidyt une ben Ropf eines rufig itehenten Fijdes, jontern

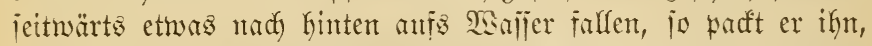

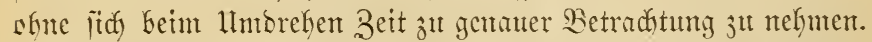
T ies ijt eine surdy (Erfinfung jefr Gemährte Metfobe. Ier Rïser

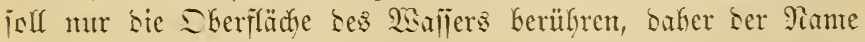

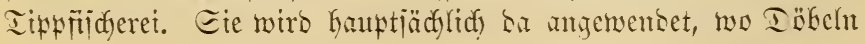




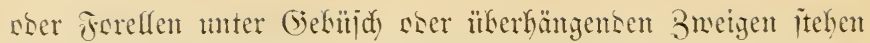

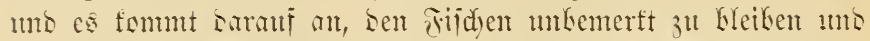

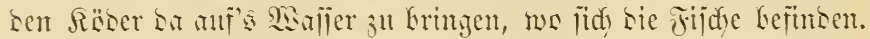
Tie Jamptamelfen fïr sieje jijderei fims: Izaak Walton, North Country Angler uns Francis Francis. Iie theite Beit itt, is lange wie wames Enmmerwetter berridt uno bie bejten Etunten

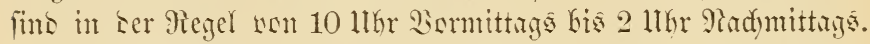

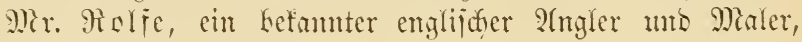

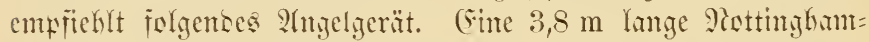
Bute mit Bisttinghan= Balfe uns ungefirnibter geflechtener jeibener

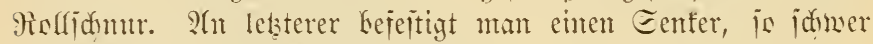
wie eine Büdejentugel unb barm ummittelbar ben Boridglag mit

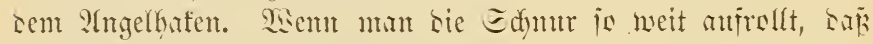

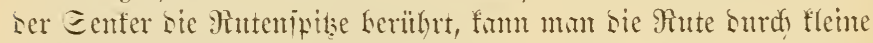

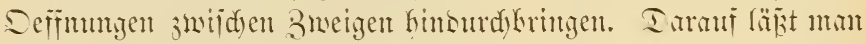

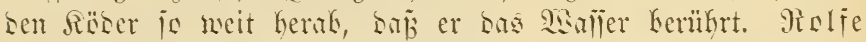

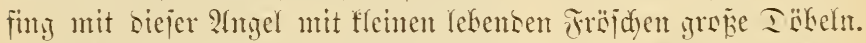

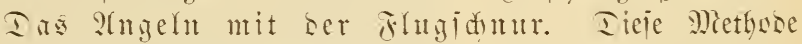
wirs fejonsers zum Fangen won Forellen in 2fmmensum gefract. Mant hat cine lange leidnte Hute, womtïglid) $5^{1 / 2}-6 \mathrm{~m}$ lang; cin

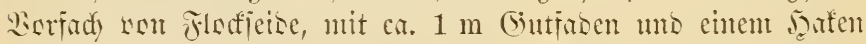
werjeken. Mian kejejtigt saran lebente Fliegen, wie fie am Bady

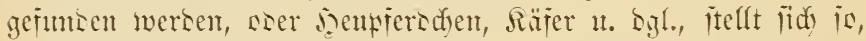
Б八в man sen

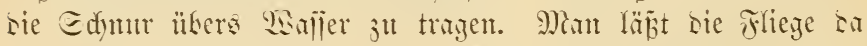

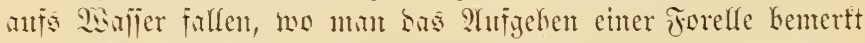

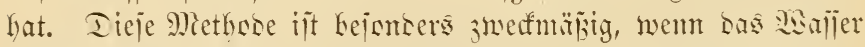
wenig berwegt ijt, weil sam sas Fijdyen mit ser tïmptlidjen Fliege dimerer itt, als bei mellig̈er Sterflädye.

\section{Die Treib/dynur.}

Iieje :ngelnnethose, meldye bei ser Eeefijherei siel antgewentet

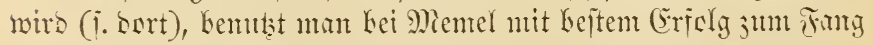

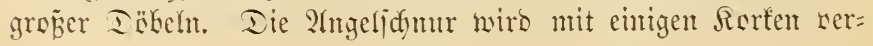


I. Dic Berteifung ber Fiidartent in 巨een แก Jiilïen. 191

jehen, samit jie jefwimmt, uns mit Yefenten injeften gefïsert.

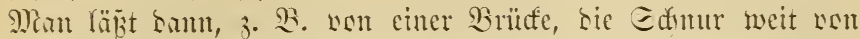
ser Etrümung jorttriben un bleibt au sieje siseije aujerbulb

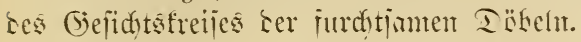

\section{Dierter 2lbfänitt.}

\section{$\mathfrak{D i \mathfrak { e }} \mathfrak{G} \mathfrak{e} \mathfrak{w} \mathfrak{a} \iint \mathfrak{e r}$.}

\section{Die Dertheilung der fifdarten in Geen und flüffen.}

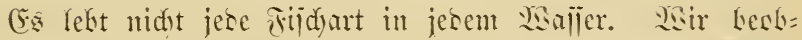

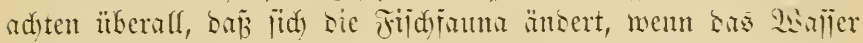
cine ansere Bejdafienkeit amimmt, ielkit anj furjen Etrecten,

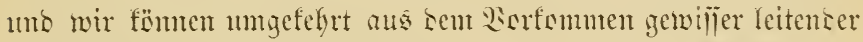

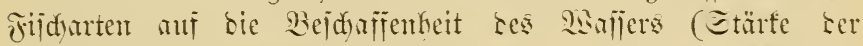

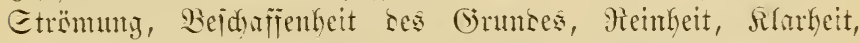

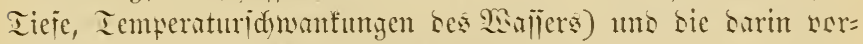
frmmenten anberen irijd)urten Edylülje madyent.

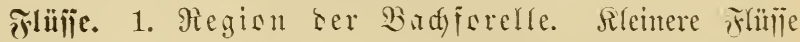

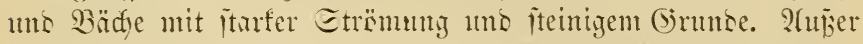

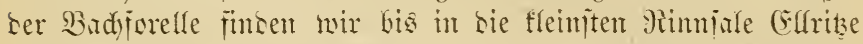
(Phoxinus laevis), Mählfaffe (Cottus gobio) ums Ectumerle (Cobitis barbatula). Miadjent ber Bad maljerreidjer gemorsen, treten neben ber Forelle juerit Iïbeh (Squalius cephalus) uno Pinjen (Chondrostoma nasus) auj, bom erjdeinen Fijde ter 2(ejden= uts jpäter ter Barben=9iegisn.

2. Region ser 2(ejde. Gräbere Bäd)e uno fleine rïlle

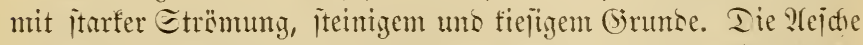

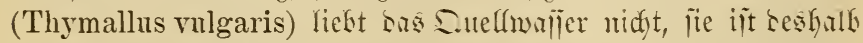

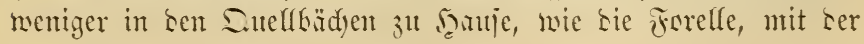


jie jum Teil juinmmenlebt, jum Ieil reidgt jie in bie Barbent= Begisn fintein, unt jwar neiter, wie bie Forelfen. Im Innu=

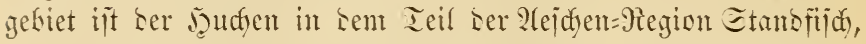

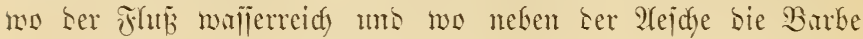

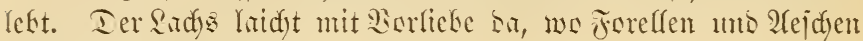
juinmen werfommen.

3. Region ber Barte. Größ̃ere Flïlje uno Etröme mit

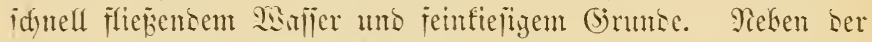
Barbe tommen wor: Iöbel, Nare, Raffen (Aspius rapax), 3ärthe (Abramis vimba), Edneirer (Alburnus bipunctatus), Däieling (Squatius lensiscus), (Sründling (Gobio fluviatilis)

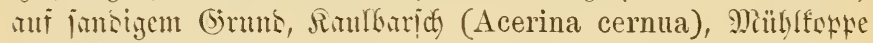
uns edumerle.

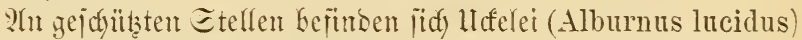

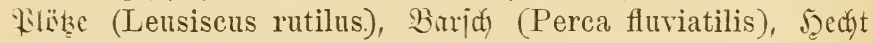
(Esox lucius), Rirricu (Cyprinus carpio), Burber (Lucioperca sandra), S.urffe (Lota vulgaris).

4. Region bes Blei. Sanglime Etrömung, junbiger unb

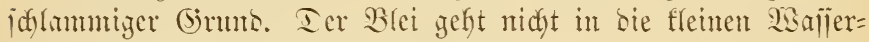
läufe Ginein, wir redunen biejelben bennedy zur Blei=3iegion, wenn ser 2 saljerlanf träge uno ber Grumb meidy itt; wir finsen

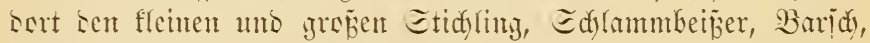

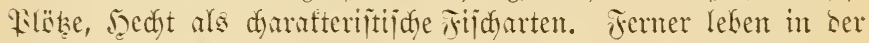

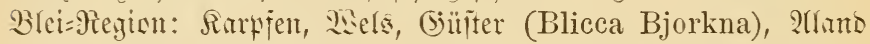
(Idus melanotus), Pistruge (Scardinius erythrophthalmus), Bitterling (Rhodeus amarus); jemer Binter, Barjo, Raul=

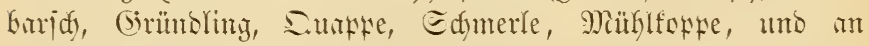
gecigneten Etelfen Barbe. Ier Bunser ijt fier redyt eigentlidy

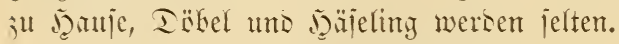

5. Farauje no Edyet leben in ser Bleiregion in itehenten 然aljer.

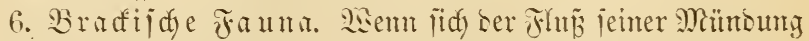

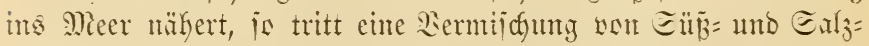
maijerfijdyen ein, insem mundye Eeefijdse, namtentlidy Jlunbern, in 


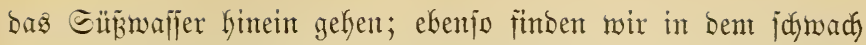
jalzigen Wajier ber Sitjee, wo es wentiger wie 1 pert. Ealz

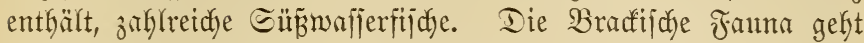
ungefähr jo weit in bem Flujje Ginanf, mie Gbbe uno Flut; in biejent Teile bes Flufjes fint Raulbarjd, 2Ial, Flunter, \&adyz, Etïr, Ednäpel, Maifijd, Finte, Wanbermaräne, Neunauge bejonders fräufig.

Ecen. 1. Badforelfen finben wir in Eeen, bie mit Forelfentäbjen in Serbindung itehen, fie gehent in ben Eeen ber Irlpen ant Gödyiten Ginaũ.

2. Seeforellen leben it äfnlidyen, nber gröperen Eeen

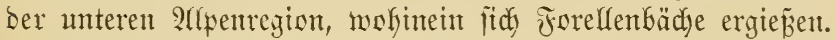

3. Saiblinge vellangen Maffer, bas nidyt wärmer wie $14^{\circ}$ R. wirs. Cie fommen in झrpenjeen bi $1900 \mathrm{~m}$ ïtber sent Meere vor.

4. Maränen, Felden, Renfen Yeben in tiefen Seen bes Fladjlandes unb ber 2llpen.

5. Der Etint fintet lidy in Seen bes Fludylanbes, in flarem Majier auf Eteingruns.

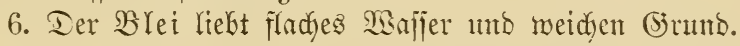

7. Die Raraujde left in flement ähnlich beidgaffenen Safferbecten, wie fie ber Blei liebt; namentlidy ba, wo anbere Fijdyarten in itrengen Sintern Yeidyt eriftiden.

\section{2lingel=Stellen.}

Die Jijde mählen iffen Etansort am liebjten ba, mo fie sen meijten Edyut wor ifren Feinten unt bas meifte Futter finsen. Bei Fijchen, bie einjiebleriidy Yeben, wie bedyt uno Forelfe, fütbet man bie itürfiten an ben.günitigiten Ctelfen. 280

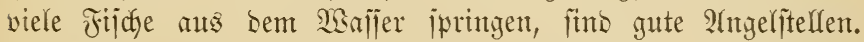
In fleinen $\mathfrak{B a ̈ d j e n ~ f a n n ~ c i n ~ F r e m b e r ~ Y e i c h t e r ~ b i e ~ g u t e n ~} 2$ (ngel= jtelfen erfennen, als in groben Flitfien, an johweriten ift bies in Eeen. In unbefonntem Terrain ift sie Führung ortzfunbiger Seute jefr worteilfaft, oft notwendig.

v. ๖. 2 o $\mathfrak{n}$ e, Ingelfitiderei. 3. 9tufl. 


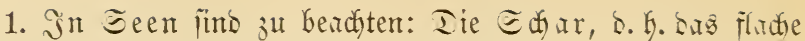
Borlano. Die weize Eatar reidyt jo weit, mie man waten fann, bie jawarze 巨dar won ba bis jum Iiefraller. Ien

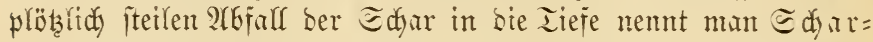
berg. Wo ein Echarberg lanbzungenartig in bas Tieimaljer

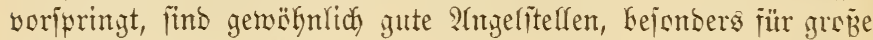

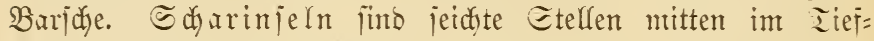

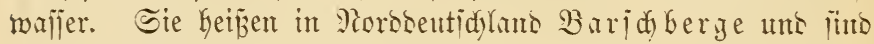
bie bejten Etclfen für ben Fang groß̉er Barjhe, \$rlöben, Pist= augen, (jü̈ttern u. a. m. In mandjen Eeen haben bie Barid = berge nur $1^{1} / 4-1^{3} / 4 \mathrm{~m}$ Sajjer, in anteren jüb bie bejten $91 / 2$ bis $19 \mathrm{~m}$ unter Wajler (Ëweriner Ceee). Sie haben jeiten, (iteinigen, junbigen soer Yettigent) (Stumo, und fint manchmal mit Mafjerpflanzen besedt, mandimal fahl. Eie jino rings unn Tieprafier umgeten. Man angelt an einem iteilen 2abfang

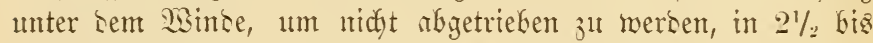
$7 \frac{1}{2}$ bis $12 \frac{1}{2} \mathrm{~m}$ tiefem $2 \mathrm{~s} a j$ er, in Epätherbit jelbit bis $25 \mathrm{~m}$ tiej.

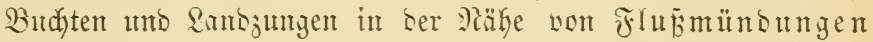

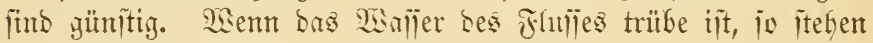

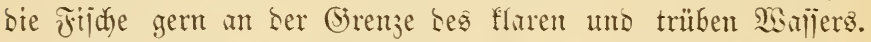
Nienn ber Eee wellig kemegt ijt, is entiteben Edyum= ftreifen paralfel bem 11 fer, bie won gropen Jijden gejudt werten, weil fie siel ertränfte Injeften entGalten.

Tie (5) elege (5. h. mit Edyilf, Robr und anteren über sas

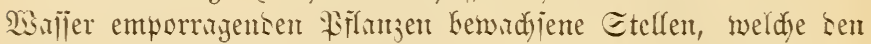

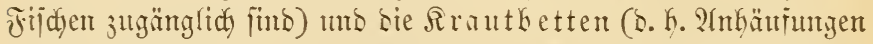

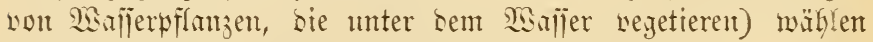
Soedfte unt Eeeforellen gern jut ifrent Etmognurtier.

II. In Flüjien un b $B$ äden wählen bie veridiebenen

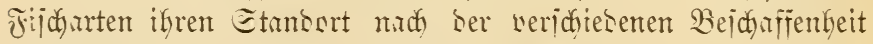

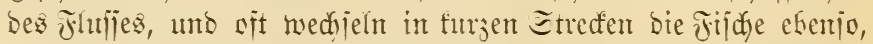
svie fidd bie Ratur bes Baches werüncert. Die Beichaffenteit bes

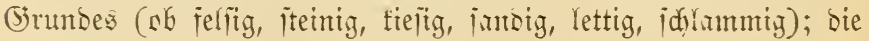
Etärfe ber Etrënumg (sb mit idyüımenber, mogenter, welliger, 
gefräujelter soer glatter Sberflüd)e); bie Temperaturidyantungen

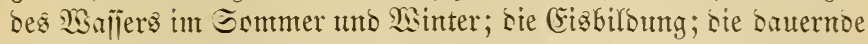
noer periobijche Alarkeit ober Irïbung; bie Tieje ses Wanilers; belien periobijdye 2Injchwellungen; bas Borkansenjein sber Fehlen von (Silegen uno Rrautbetten uno won Edylupiminfeln Keeinfluilen

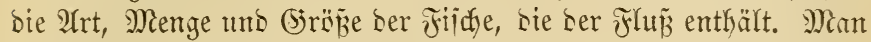

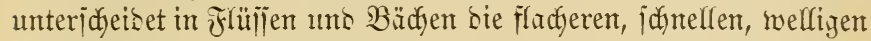
Etrömıngen (stream) uno die rufigeren, tiejeren Düntpel (Rolf, Strubel, Runfel, (Strumpen, pool). Die Stromrinne ijt ber tiejite Teil ber Strïmum, weldyer bei niebrigen Waljer=

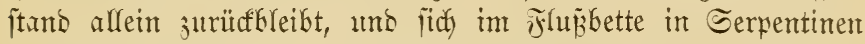
von einem llfer jum andern hinïberjieft. Bei Berlin heip̈t bie

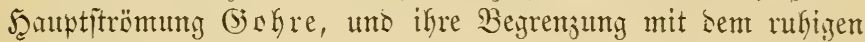
Nebenwafjer Edyar. Die Bleie jiehen in ber (jobre umber, uno

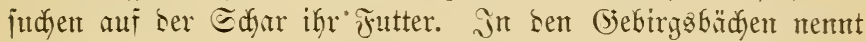
man bie Eteffen, wo Das

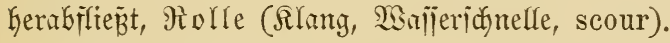

Die Dümpel fint in ben Biegungen ber Etrönung (wo am concaven llfer bie Etrömung redftläufing uno idyeller, an

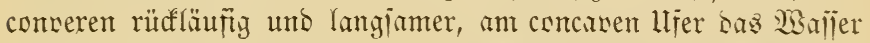

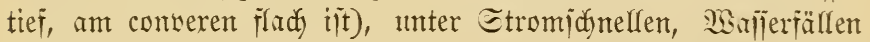
unto Mefren. In Sjebirgobädjen ijt sit in Dünteln bie concrve, tiefe llferjeite jeljig, bie convere, flache Eeite janbig. Die Dümpel

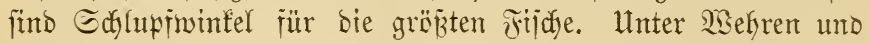
Wajjerfälfen ichię̧en bie Fiiche gern in bas joüumende und

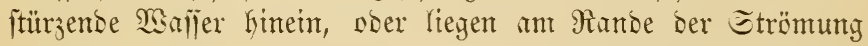

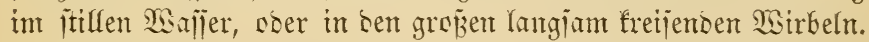

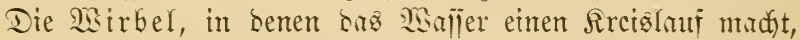
befinten jidy in sen Dümpeln, bie oft einen einzigen gropen Wirbel

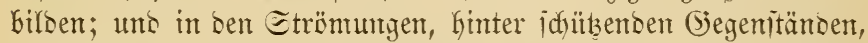
wie Bufnen, sorjpringenden Felien, 然zelitëcfen u. Dgl. J̄ebes Kerabidnimmente Teildyen verweilt treijento eintige 3eit in bett

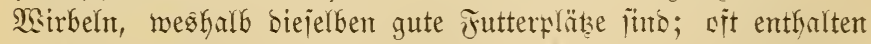
fie Edrantantäujungen, bie jïh srehen. 
Fermer füro güntige Etelfen bie Mänsungen won $\ u=$ früjfen; Banmmurzeln; über bas Wafjer häugenbe

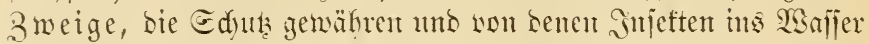
fallen; fteil abrallente unb hoble ufer; Bollwerte, bie sas

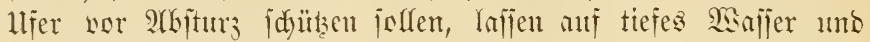
gute Seritecfe idyliefen; unter boblen Berbretterungen unb Matermerf an Mefren un Mäblarden, an Brüfen=

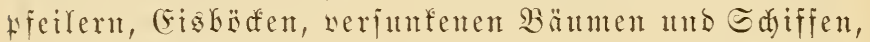

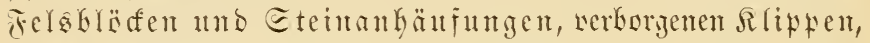
nu Rratbetten wählen bie grofen Jijche ifren Etant. Sinn Sistytigfeit find alle Etellen, wo nidyt mit Pieşen gefijdyt werben

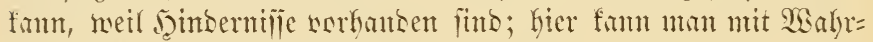
idfeinlidfteit anf einen guten fiang red)uen.

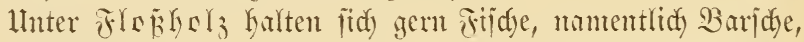

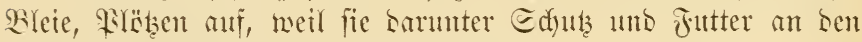
unter ber Borfe Yefenten Miaben finden.

Iie 2utwäfjer (Bredfen, sisajjerladyen), bie mit hent Flujje

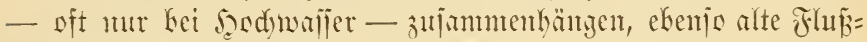

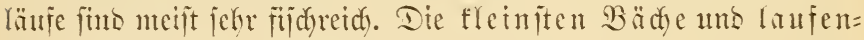
sen (bräben baben gemöbutidy an eirjelnen Etellen groje Jijdse. Fliifie, bie jefr anjumellen, uns bam ganj flein werten,

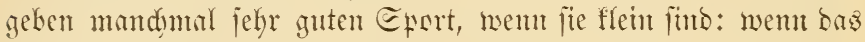

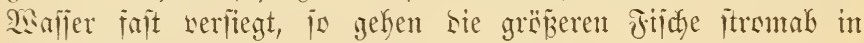
tieferes sisaljer, uno fteigen ket Sondymafier wieser auf.

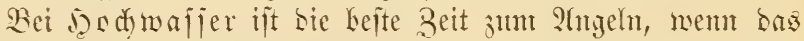

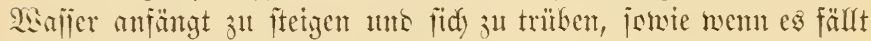
unt jidc) fait geflärt hat.

Man kerbad)tet in Sliifjen Käufig Etreifen un 2(nkäufungen

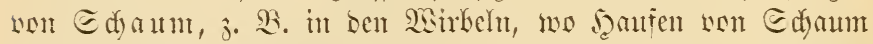
rotieren. Da ber Edhanm jtets viele crtränfte Injeften enthält, fo lecft er bie Fifde an, namentlidy bic Forellen.

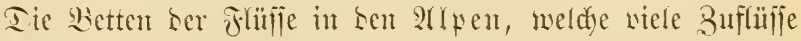

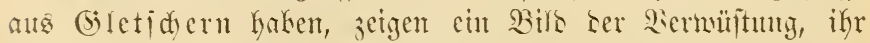

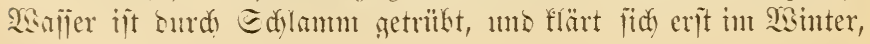




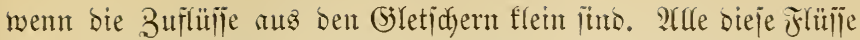
find arm an Fijden. SBent jie einent See burdjitrönt haben, fo treten jie wolfommen flar barats hervor mo jüb num befje: beichaffen.

Die Bädye, weldye aus Ralfiteingebirgen fonmen, itehen

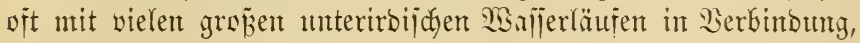

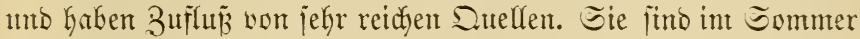
falt, im \$inter warm, unb borzüglich für Forellen geeigntet,

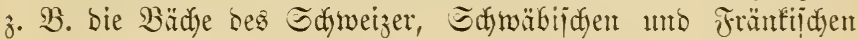

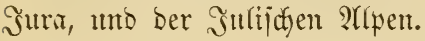

\section{Die Waffertiefe,}

in weldjer gefijdyt werben muris, ijt für bie verjojiebenten Fijd)= arten nidjt biejelbe. Man finbet am Grunde: Blöke, Blei, Illand, Rarpfer, Sajlei, Grünbling, Barbe, 2tal, Flunder, Barjd; - in tiefem Mittelwajier: Baridy uno Sarrize; in mittlerer Iiefe: Barja, Döbel, Seedt, Sä̈eling; - an

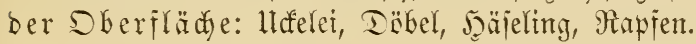

\section{fünfter 2lbfđąitt.}

\section{Die Sulfwalferfildje.}

\section{Angelffalender.}

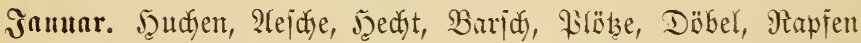
beipen gut. - In ben Rorbbeutichen Seen werben unter bem Cije an ben (5ielegen Jeedte gejangen; ebenjo in ber Sitjee.

Februar. Eaidzeit. Mitte Des Monatz füngt ber Jedyt ant zit laictjent. 
Fangzeit. Für ben Denchen bie beite Beit. Ier Zach nimmt bie f. Jiliege.

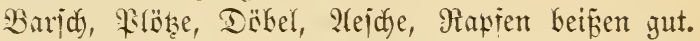

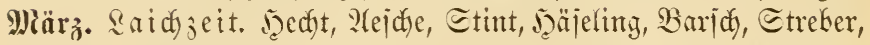
Fangzeit. Ier sadys jteigt gut nady ser f. Fliege. ebenjo bie Jorelle an warmen Tagen. Iäbeln beipen.

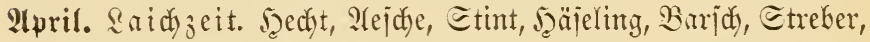

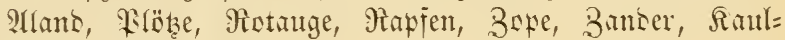
baxid, Bingel, 5̧udyen.

Fangjeit. Ier Rady jteigt gut.

Iie Frelle iteigt an mammen Tagen nady ser Fliege.

Ier Pllutb geht in bie Aflwälfer, um ju laidyen, unb beibt nady bem sardyen an kejten im ganjen Safre. Iökeln beízen.

Mini. \&aidjzeit. Jeedt, Barjd, Rlant, Plöke, Motange, Mapfen, Bope, Bunber, Katlkarjd, Bingel, Rarbe, Blei, Dökel,

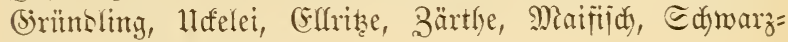
barich, Torellentarid.

Fanjzeit. Tie Forelle: im märmeren Ieil bes Mai beginnt bie bejte Eaijon für bie Fisegenfijdyerei uno sas 2rngeln mit Megenwurm.

Der Sady iteigt jefy gut nady ser f. Flieg̃e.

Aland mie im afprif.

In ben Porbbeutjyen Sandjeen fümen in nidyt jefr

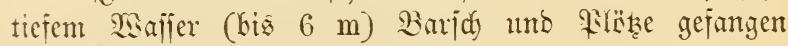
werben, went bie Saidyeit noritber ift.

Juni. Saidjeit. Barke, Blei, Däkel, Grïndling, Bärthe, Rarpfent, Etidyling.

Fangzeit. Für bie Forelle bauert bie bejte Beit

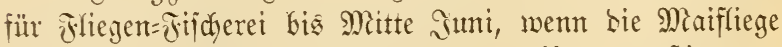

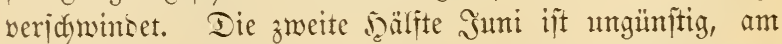

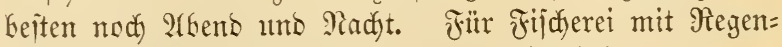
mum unb lebenten Injeften ijt bie keite Beit. 


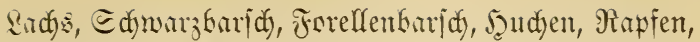
Töbel, șäpeling, llctelei nehmen bie Fliege gut; Döbeln wersen mit Ririden gefangen.

Iie Eaijon für bie Strumsangel beginnt; namentlict) in ben Piorbbentidjen Ranbjeen für Barjd) unb Prlöke (man antgelt nicht jefr tief, bis $6 \mathrm{~m}$ ); ckenio an ben Molen

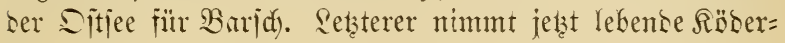
friche bejonbers gern.

Allano beip̄t gut bis Mitte Simi,

Etint bei ït in ganzen Minnat.

כuli. Raidjzeit. Rarpien, Etichling, Mels, Edylei.

Fangzeit. Für Forelle ift bie Eaijon für Fliegen= jifderei ekenjo bejdaffen, mie Enbe Juni; aud bie beite

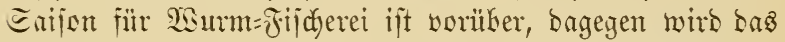
Fijodyen jeţt am Keiten genommen. Man madjt in Berg=

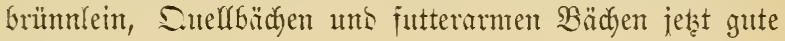
Beute an Forelfen.

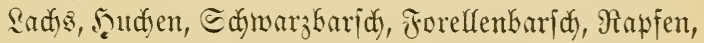

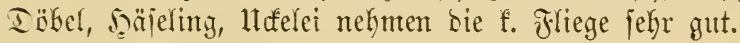

Dëbeln werben mit Rirjden gefanjen.

(Srünbling uno Barbe Geiz̄en gut. Für Blei beginnt

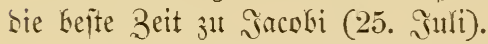

In ben Morbbentichen Eeen ijt bie bejte Beit zum

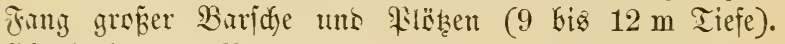
(5benfo für ben Burjofang an ben Molen ber Ditfee.

Ter Etint beiṕt gut.

Die Eaijon für Len Fal beginnt.

Auguit. Fangzeit. Iie Forefle nimmt bie f. Fliege etmas

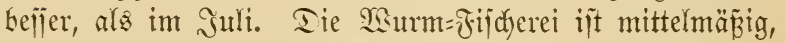
fït bie Fifdyenngel bie beite Beit.

Die 2lejhe. Beginn ber Eaijon.

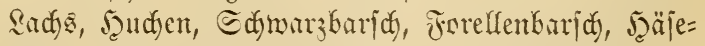
ling, İ̈bel, Ildelei, Rapfen nefymen bie f. Fliege gut. 
Der Błet. Die bejte Beit bauert bisิ Bartolomä: (24. 2fugut).

2lal, Barbe, Griunbling, Rarpien, Banter, Etint beip̄en gut.

Für bie 2Angel=jijderei in bent tiejen Porbbentiden

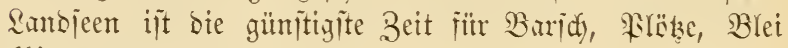
(bis 9 unt $12 \mathrm{~m}$ Tiefe).

Fïr Secht beginnt sie Eaijon.

September. Saidzeit. Ladj, Meeriorelle, Rildy.

Fangzeit. Die Saijon für \&adis ijt vorüber. Die Forelle nimmt jwar bie f. Fliege wieser jehr gut, iit aber

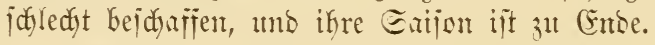

Southen, Sdywarbarid, Forelfenbaridy, Säjeling,

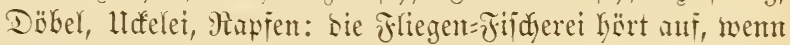
bie Padytiroijte eintreten uns bie Fliegen veridyinten.

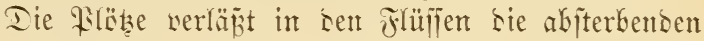
Rrantbetten, geht in bas tieje Sanjer, uns ifre Caijon hat brmit in ben jłinjen begennen.

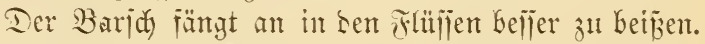

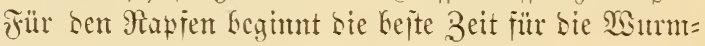
Jijacterei.

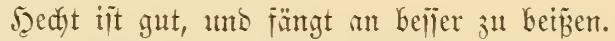

Barbe und Bander: bie Geite Beit.

Iejace, Srü̈bling, Blet, Rarpien unto Etint beī̄en gut.

2llanb tritt Crnse Eeptember nodyuals majientaft in bie 2 Ytwäjier uno beijit gut.

Int ben Mersbeutichen Eanbjeen ijt bie bejte Beit,

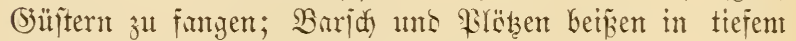
23alier gut (bis 15 unb $18 \mathrm{~m}$ tiei).

Detober. Saidjzeit. Sady, Meeriorelfe, Rildd, Forelle, Gee= forelle, Saibling, Sdintipel.

Fangjeit. Barben beip̈en jefr gut (namentlid) an 


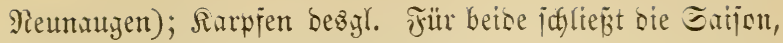
jobald bie eriten ftarfen Padatiojite eintreten.

Jaedit und Banber: bie beite Beit.

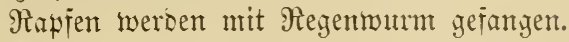

Peide, Edymarzbarid, Forellenbarjd, Barid, Plötze, Dëber, 2Aal, Blei, ভtint beizen gut.

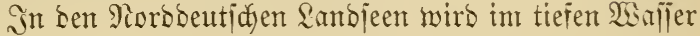
(biz 15 und $18 \mathrm{~m}$ ) ofit ein guter Jang gentadjt.

Movember. Raidjzeit. Ead), Meeriorelfe, Rildy, Forelle, Eee= forelle, Eaibling, Ednäpel, Nabü= Maräne, Suappe.

Fangzeit. Die beite Beit für Jutchen, Jeed)t, \&lötze.

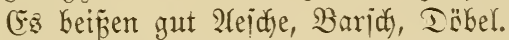

In ben Porbseutjon Ranbjeen fun in tiejem Wijer

(bis 15 und $18 \mathrm{~m}$ ) nod reidje Beute gemadjt merbent.

Dezenber. Raidzeit. Forelfe, Ceejorelfe, Nabü= Maränte, Duappe.

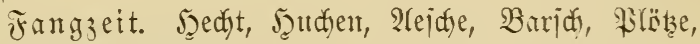

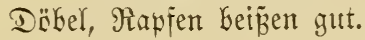

In ben Norbseutichen Eeen iit bie beite Beit, unter

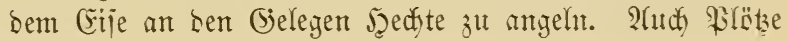
werben unter bem (Fiije gejangen.

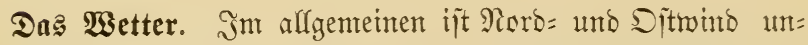

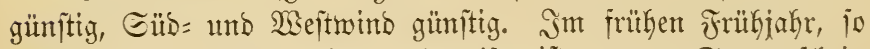
lange es bes Pladjts friert und reift, ift warnter Sonmenidein

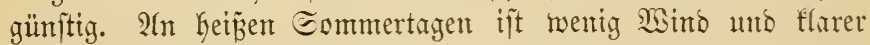

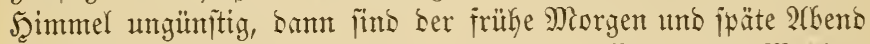

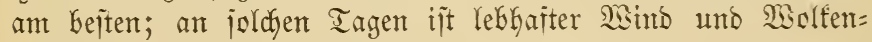
id)atten vorteilfajt. Bei jehr jawülem Wetter, mo man viele

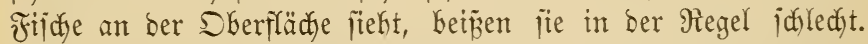
Dann ift oft in sem füflen Majier tiejer \&anbjeen bie gïnjtigite

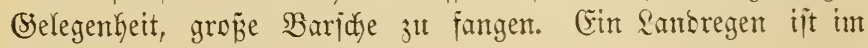
Sommer gemöbnlidy vorteilfaft. 


\section{Die forelle (Trutta Fario).}

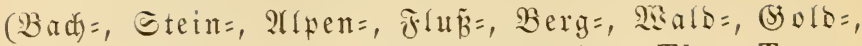

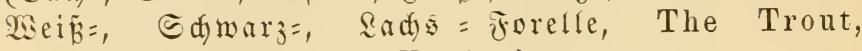
La Truite.)

Die Forelle füllt fidd im Eeptember mit Said uno jollte won

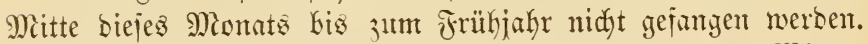

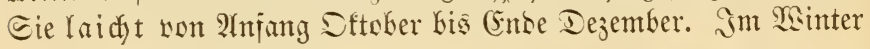

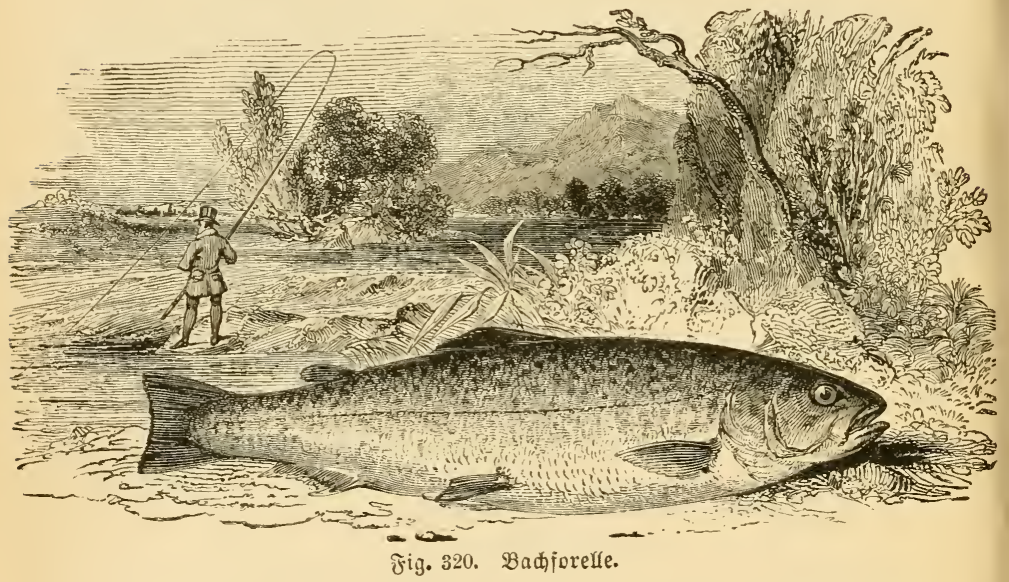

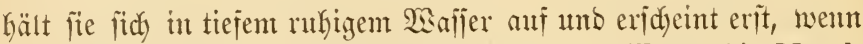

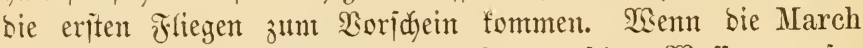
Brown (Baetis longicauda) 8-10 Tage auf bem $\mathfrak{x a j i j e r ~ g e m e j e n ~}$

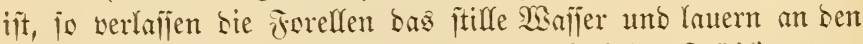
Ränbern ber Dümpel auf Beute. WSenn bie falten Frtühfingstage

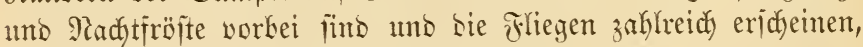
To werben bie Forelfen judnell frä̈tig, fudten itärfere Etrönungen auf unb fangen allmählidy an, fid im filuije z̆ verteilen. (Enbe Mai eridjeint bie Eteinfliege (Stone-Fly, Perla bicaudata) umb etwaş fpäter bie Miaifliege (Green Drake, Ephemera vulgaris). 
Tie jorellen jinto nun mohlgenährt, judjen jtarte Etrömungen aif umb geben auf bie Rollen.

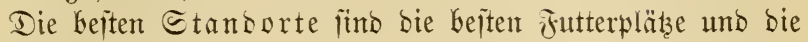
geidühtzejten Etellen. Sie merben won ben grëßten Forellen bejeb̧t. Iies jind namentlidy bie $13 i r b e l$ an ber Eeite ber Etrömungen und zwijhen Bufuen; bie $\subseteq$ d)amitreifen, bie immer eine Mienge ertränfte injeften enthalten; tiejes Saljer unter über=

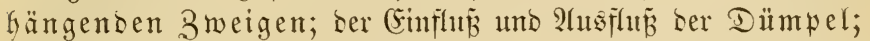
grope Steine, (Srasbulten, Baummurzeln, verjunfenes

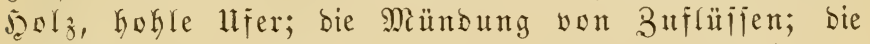
I itmpel unter Wefren, wo man ben Räber bis in baz

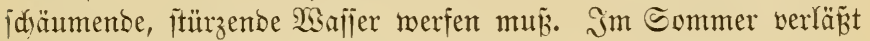
tie Forelle bes $M i a d$ ts gern bie Düntpel unb jdyimmt in bem benadbarten Dümpel und auf ben Rollen umber.

Seenn im Eommer nady anfaltender Ditme ber Bad jefr wajierarm mirb, jo gehen bie grof̈en Forellen jomeit ftromab, bis fie tieferes Majfer finsen. Sobald bann joodmajier ein= tritt, iteigen jie wieber ftroman und zwar dit in ein faar Tagen mehrere Meilen. Bei J̧odyaffer verlafien bie Forelfen bie itarfen Etrïmungen, jobalb bas 2 ajjer jteigt unb fid trïbt umb jammeln

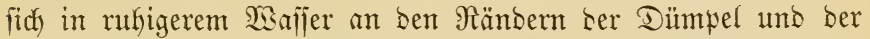
Etrömungen. Die gröbiten sijde ftehen ba, wo bas Wajfer anfängt, tiej zu merben.

Die Frezitunbe (time of the rise), wo bie Forelfen majienhaft aufgeben uno ipringen, ijt bei ber Fliegenfiidyerei

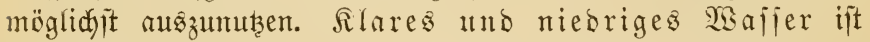
ein Borteil, wenn man es weriteht, bem Jijd werborgen zu Gleiben. $\mathfrak{S o l f}$ enidyatten ïno im Sommer günjtig, Esnnenj ijt an falten orriffingstagen günjtig, im warmen Esmmer un= günitig.

\section{Der Ĵorelleniang mit Der fünitliden Şliege.}

Man unterjdeibet zwei neridjebene Eyjteme ser Fliegen= fiicherei. 
a. Das englijde Sritem emprtehlt Fltegen, welche

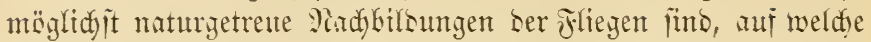
bie Foreflen eben jago madyen. (Gs mersen viele verjutesene Fliegen angemantst.

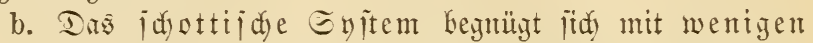

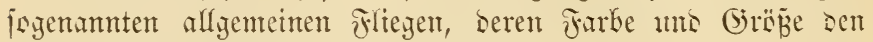
llmitänoen entipred)eno gemählt wits:

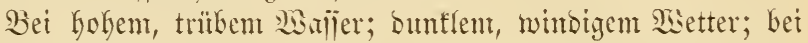
gut beifenden jijden; in Früfjafr bis jun Serjominden ser Mariflegen nimmt man gró̈e viliegen;

bei niesrigem, flarem sisalier; bet hellem, uthigem WBetter, in Eommer; bei trïgen Fijuen: fleine Fliegen;

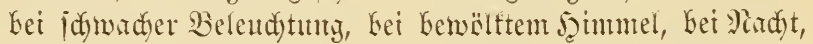
bei trübem, bobem sifler mählt man belle auffallenbe Farben;

bei grelfer Beleudjtung, Eomtenidyen, flarem, niebrigent Sinjer; bunfle, urideinbare jarben.

SBir faben ant Eeite $81-97$ eine 3ujummenitellumy oer rensumierteiten tümitliden Forelfenfliegen gegebent.

Ifm beiten fün in alfigemeinen March Brown, bie Blue unt Yellow Duns, bic Alder Fly umb Stewart's Black Spider. ixt ben Mionaten georonet, fimb Folgende Fliegen im alfyemetnen an beiten:

Gis Ense Tprif und Tnfang Mai Blue Dun, Narch Brown, Red Spinner, Cow-Dung, Coch-y-Bondhu, Yellow Dun, Iron Blue, Black Gnat, Sand Fly, Gravel-Bed, Grannom.

im Mat: Sie sorigen zum ₹eil, fermer: Stone Fly, Pale Evening Dnu, Alder, Little Blue,

im Simn: Green unt Gray Drake, Alder Fly, sie Dun's uno Spinner, Coch-y-Bondhu, Fern Fly,

im İtli: Red umo Black Ant, White un๖ Brown Moth, im 2lutguit: August Dun, Cinnamon, Willow Fly.

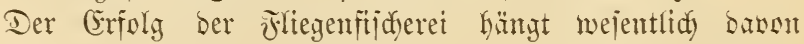

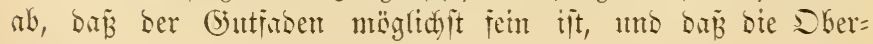




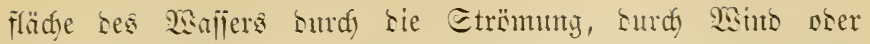
Megen gefrüulelt iit. Tie beite Eaijon begimt 8-10 Tage nach sem (sridyeinen her March Brown unto entet mit bem :er= jabinten ber Marfflege Mitte Junt; Geintsets ber wärmere Teil bes Mai und 2fniang Iunt iit am beiter. Int früfen orrüh= jafri, io lange sie Madft froitig, jutro inunige Morgen, von 8-12 1thr, ans bie 2thente ant bejten. Ralter Sitmint ijt jeţt

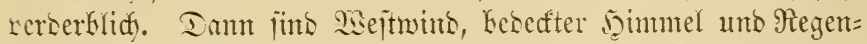

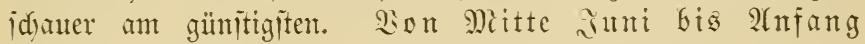

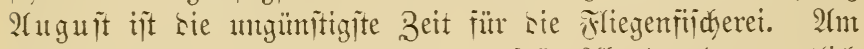
beitent fins jebst ser frühe Morgen anto jpäte 2lkento ano namentlid

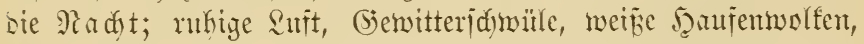
trüber Dimmel shne 2 sinto füto umgüntig; helfe winsige Tage jinto mittelmäp̈ig; bet Eonnenjedent bentbe man bie Beit, wo Isolfenjartten vorüberjieben; wolfige Iage uno smoregen fint ant keitert. In bicier Beit wirb bie Siliege beljer gentomment, wenn ntan Len Dafent mit einent fleinen roten 2 sum ober einer Miabe verifeft. Im 2 ugujt ninmt sie Forelle bie jliege wieder belier uns ser Eeptember ijt fait ebenis gut, wie ber 2tnjang ser Enijen, mur jinto bie Fijdye idyledyter.

\section{Der Joreffenfang mit Dent Pegenwurmt.}

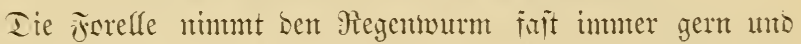

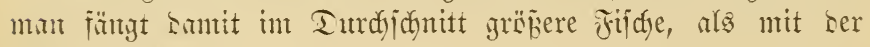
fïnitlidjent jorlege.

Man angelt entmeser itrontani mit einer langen Fliegentute uns entem fleinten $5-8 \mathrm{~cm} \mathrm{Vangen,} \mathrm{toten} 2 \mathrm{sum}$, ober fromab

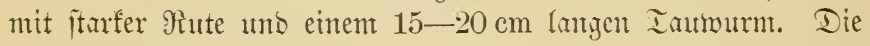

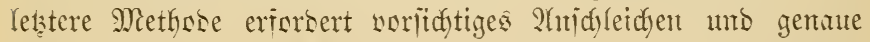

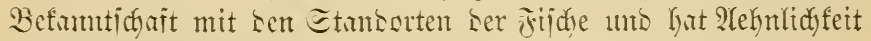
nit ser Bujdantgelet.

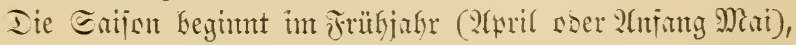
went bie Raudfidmalbe idmirrt unto "wie wie“ idyreit.

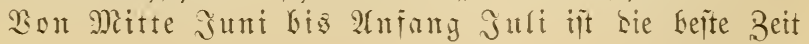




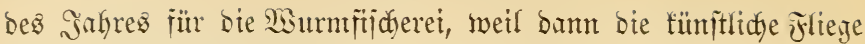
nicht gut gettommen wirs.

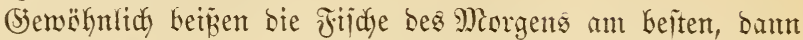
folgt eine Sauje wo $1 / 2$ Etunde, bann beizen tie wieder etwas

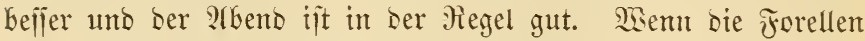

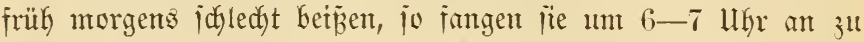
frefien. In ben Dümpeln ift bei Iage wentig zu ntadjen, wemt

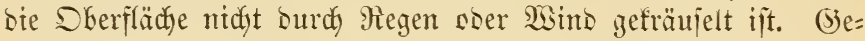
mitterlujt ijt nidyt ungünjtig. Wenn an einem trüben Sage na (d)= mittags bie Eonne burdjbridjt, jo pflegen bie jitide gut zu beijen, ebenjo bei eimem milsen Aeger. Sehr gut ijt ein trofener Mrorgen mit wentig Iau; bei fintem Inu ober Mebel warte man, bi: bie Sonne ben Tau werflitdytigt hat.

Bei $\mathfrak{5} 0$ dwajier nehmen bie Forelfen bie füntltidye Fliege nicht gut, jobalo bas 1 arjer trïbe wirb. Dagegen ift bei feigen=

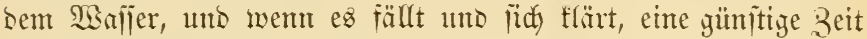
für bie Sisurmfijderei. Da onn mur wenige Etellen jün, mo man bie fitide jut juthen hat (i. o.), jo ijt es zmedtutäpig, nidyt zu idnelf ben Etantoort 3 w wedjeln. SEenn nady langer Dürre

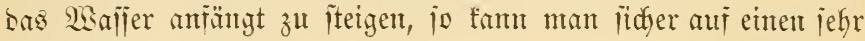
guten Fang rechnen.

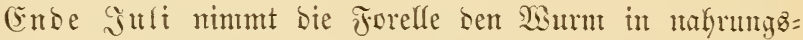
reidjen Bächen nidat mefyr jo gut, weśhalb man bam bie fleiten

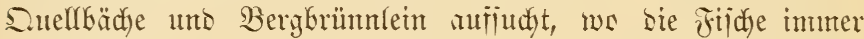
fungrig ïmo.

\section{Ser Forelfeñ̄ang mit Aäĩern.}

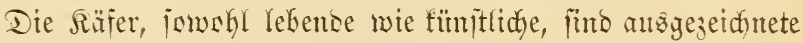

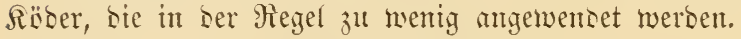

\section{Der Forelleniang mit senpierdeden, lebenden Fliegen und Sliegenlarven.}

Die Sarve ber Eteinfliege (Creeper) ift im 2htiang Mar ein

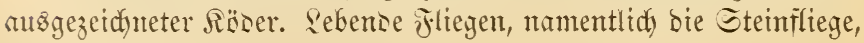




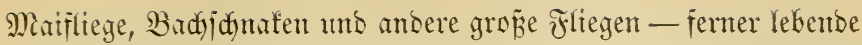
Soeufiers djen fund jefr witfame Röber. Man fifdt entweber mit einer Yangen leidyten Fliegenmte, ober mit ber Flugidnur. In niebrigem flarem Wajier fängt man bie meijten Forelfen in ben

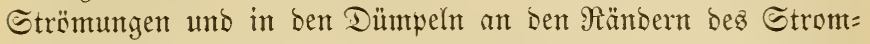

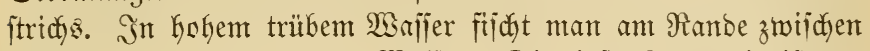
rufigem uno itröntendem Wajier. Die bejte Tageszeit ift ber

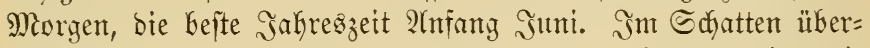
Kängender Bweige, two bie gräßten Forelfen iteken, wirb mit Sorteil bie Bujdangelei angewanbt.

\section{Der Frorellenfantg mit Der Spinnangel}

ijt Geionbers zum Fang ber gräß̈ten, an- ben Raub gemöhnten Forelfen geeignet. Der beite Röber itt eine gut jpinnente, $4-5 \mathrm{~cm}$ lange, glänzende Corfrike.

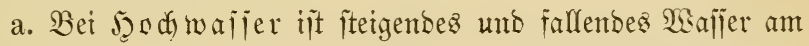
beiten. Im Mai ijt ber Sormittrg, im Suni uno Suli bei

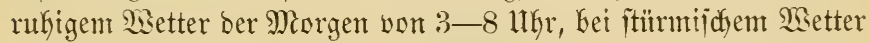
ber ganze Iag gut. Bei ber Sgähe ber Flut iit mentg zu madjen, bamt ijt eint gelegentlidjer Keller Sommenblid won Borteil.

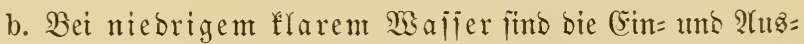
flüfie ber Dümpel, bie Dümpel unter SGehren und Wafjerfälfen,

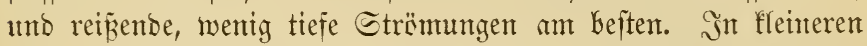

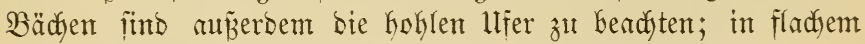
Manjer fijdt man ohne Senfer. Die gümitigite Jahreszeit ift ber Sonmer. Die günitigite Tageszeit it bei flarem SBetter

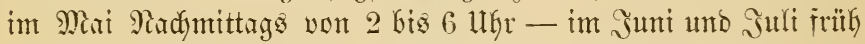
morgents uno ipät abento bis Mittentadyt. Irübes SBetter ift

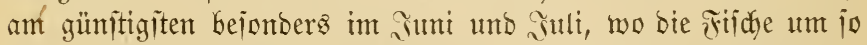
bejier beizen, je trüber ber Sag ift. In warmen Sommer= näd) ten ijt bie callribe ein verloctenter Räber, namentlidy in

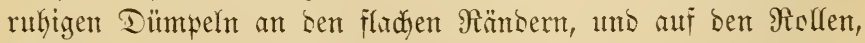
wo sant bie groỉen Forelfen unheridy wärmen. 


\section{Dex Sorcllenfang Durd) Şeben แth Eenfen}

ijt in tiejen rufigen Dümpeln, in Eeen, im Stermaijer non Mïflen jwectmäpig. Man fneift an ben Ropf einer fünjtlidjen Triege ein Edyrotforn, ser man fijd) mit ben in immern mit Blei bejdyerten fïnjtidyen Räsern (Grasshopper, Cabbage Grub,

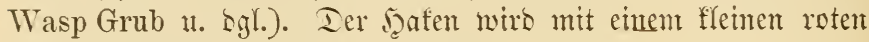

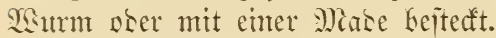

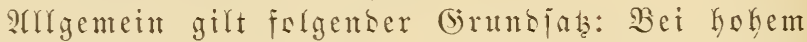
trübem sajijer ijt es leidyter zu jijden, wer es aber weriteht, bei flarem niebrigem asajer mit. feiner Edunt ftrmauj zuangeln, ser wirb bie retchite Beute madjen.

\section{Die Secforelle. Trutta Lacustris.}

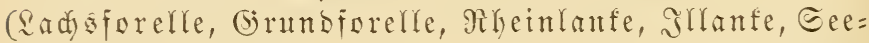
ferdye, Jerbitlads, Bahl= ober Ealfijd, Edylls, Edyebeforelle, Maiforelle, Eilberlads, Carpine. The Great Lake-Trout. La Truite Saumonée.)
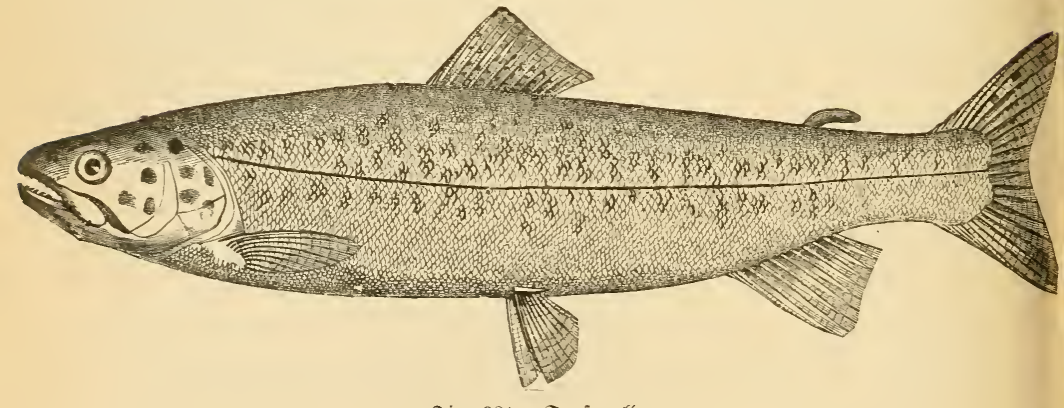

Fig. 321. Eeciorelle.

Tie Seefrrelle Yebt in grofien Sanojeen, namentfidy ber Iryen, uno fteigt im Jeerbit in bie Flizffe, unt zu laidfen.

lim fie zu angeln ift $\mathrm{cs}$ am beften, einen ortôtunbigen fö̈frer zu nefynten, Ler bie beften Etelfen fent. Man findet sen Fijdy 


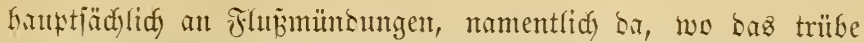

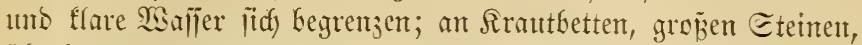
überfängenden 3tweigen, bei $1 \frac{1}{4}$ —3 m 2 Saljertieje; bei winbigent

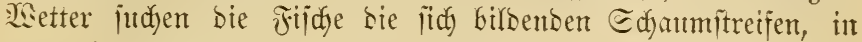
Senen jie viel erträntte Inieften füton.

2luf ben englijden Eeen filidst man int Mai bei norment

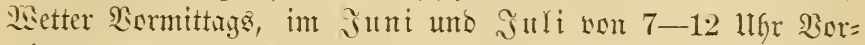

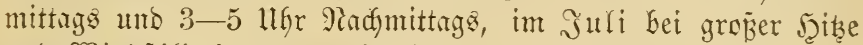
uno Minditilfe in ben jwei erjten Morgenjtundent.

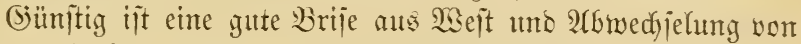
Megen und Eommenjhein. Für bie Fliegen=Fijderei iit Eomnen= ichein, für bie Echleppangel Dunfles ftürmifiches खsetter am bejten.

Man angelt ant beiten wom Rafyn. Iie Fliegenangel wirb auf ben englijajen Eeen mefr angemant, als in ben 2llpen. Iie 5aten, an weldje bie Fliegen gewumben werben, fint 5 biz $9 \mathrm{~mm}$ breit. Die Jarben ber Fliegen werben nady benjelbent 丹egeln gewähit, wie jum Fang won Badjorelfen.

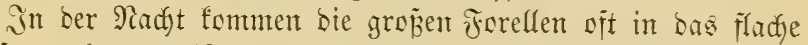
Sinjer nake ant llfer uno jüto bann gut mit oer Fliegenangel 3tl jantgetl.

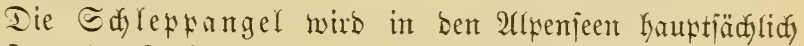
jun Fang ber Eeeforellen angemanot. Man fijdyt mit natïrlidyent

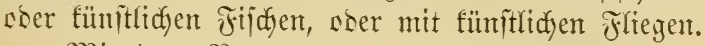

Mit Dem Regentwurm angelt man an ben 3uflüjen von

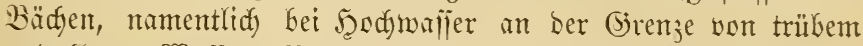

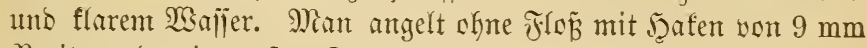
Breite mo mit groỉen Iaumürmern.

\section{Die 2ref̧̧e. Thymallus Vulgaris.}

(Epringer, Mailing, Epalt, Etalling, Jaarr, Etrom= maräne. The Grayling, L'Ombre.)

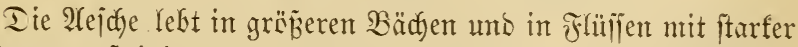
Etrömung, iteinigent und fiefigem (Srunbe, zum Ieil mit ber Forelfe, jum Teif mit ber Barbe julammen, liebt bie Sultebädje

ท. ๖. $\mathfrak{B}$ o rne, Ingelfifiderei. 3. 2ufl. 
nidyt, uno fommt mur weiter unterfalf wor. Sie ift in Deutich= rano in jehr vielen grïlïen vorkanben uno ojt jehr hänfig. Sie

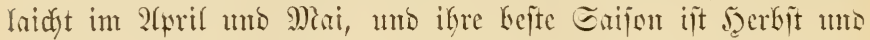

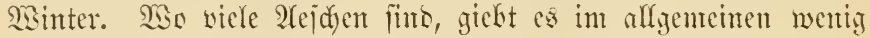
Forelfen uno ungefegrt, weil fie fid gegenjeitig auf ben Said)=

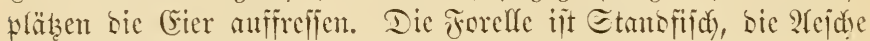

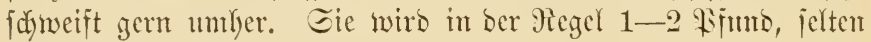
3-4 Rifuro fduwer.

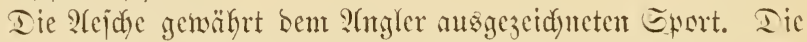
Enijon beginnt Enbe Mai uno bautert bis jum Frithjufr, bic

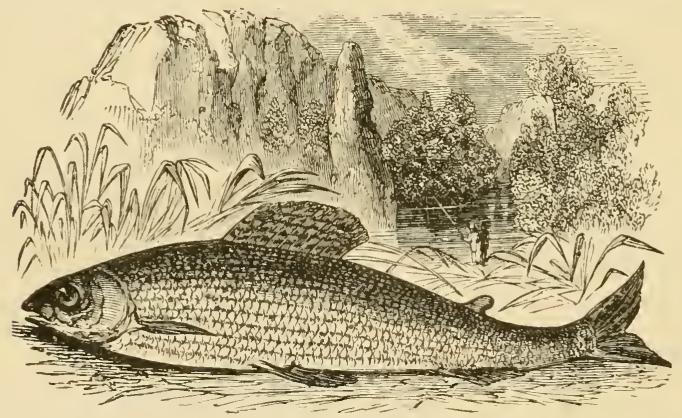

テig. 322. 2(ejđ)e.

bejte Beit ift nom Sftober bis in ben 2 inter, atd bei Jreit= wetter, namentfidy in ben Mlittrgsitumben.

Die beiten 2 Angelifeffen fino $1 \frac{1}{4}-1^{1} / 2 \mathrm{~m}$ tieje Ctrönungen, wo man bie meijten Fijdye an sberen Enbe funbet. Niebriger

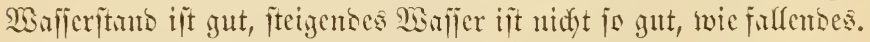

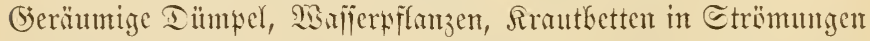

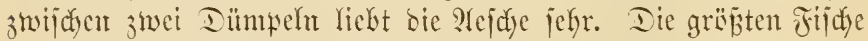

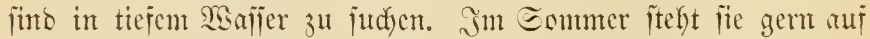

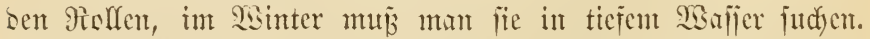
Angelfüber. Ereper, Eproden, Murou, Mespenlarsen, Fliegen, Jenterodyen, Megenmürnter. 


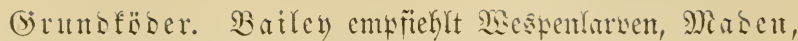
zeridynttene Regentwünter als jefr wirfjam.

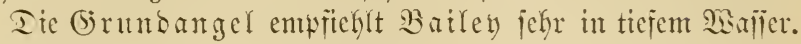

Fliegenangel. Die fïmitldye Fliege it ausigezeidyutet für

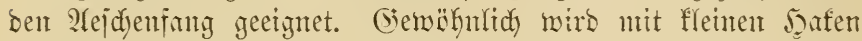
gefijot, uno man erfïgt bie NBirfung, menn mant cine Miabe baran kängt. Sute Fliegen fino bie Bumbles, Fliegen mit Rörper wan brenje Sifatmifederfajer, Black uto Red Ant, Iron Blue, Yellow

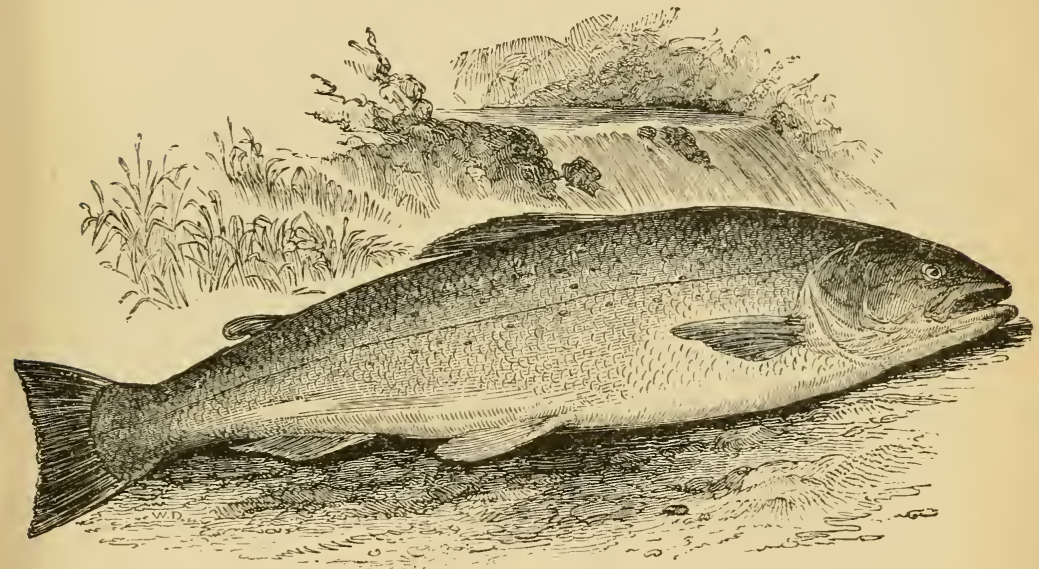

Fig. 323. உađis.

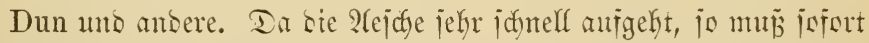
angebanen werben, uto ba jie wentiger ideu als bic Jorelfe ijt,

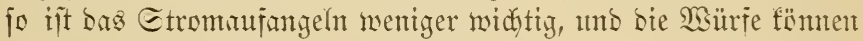

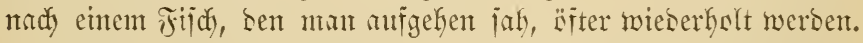
Rad) 9)ejie ijt jolgentes bas "(Behemmis ser Rejuen= fijderei": Man fijcht mit jeinjtent Gutjaben, wirft bie Fliege anj tieje jtille Iümpel Yeidyt wie eine Edyneeflode, tmo läb̆t jie ca. $25 \mathrm{~cm}$ finfen. Das 2lujgeben erfemt man m einer Rrüujelung 
ber Skerfä̈due. Socr man watet unto wirft bie Jliege unter üherfängendes (jebüjd.

Das நeben uns Eenfen mit Maben ijt eime anberorbent=

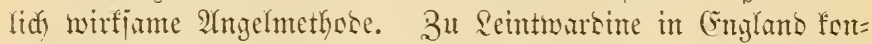
jerviert man bie Masen io lange wie möglidy in Len Sinter Ginein, umb ftedt $6-7$ Marben an einen ber fümitliden mit sislei

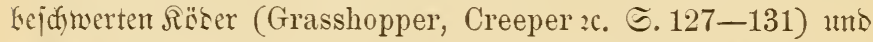

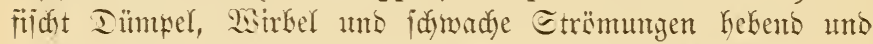

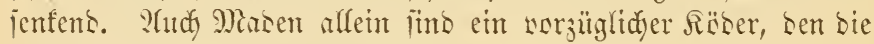
Teidye alfem anteren worzicht.

\section{*. Der $\mathfrak{S a d y s . ~ T r u t t a ~ S a l a r . ~}$}

(ङalm, The Salmon, Le Saumon.)

Ier Sady laidyt in ben Fritilen, jeine Brut lebt bort 1 bis

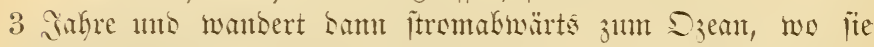

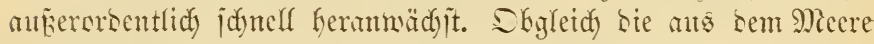

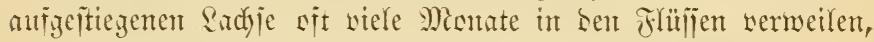
io idyeinten jie body mäfrent biejer Beit feine Pabrung jut nefmen, uns mu won ifrent lleferfluz an Fleijd und Fett 3 u jefren, ben jie mitfringen, sesm man funbet ben Magen jtets leer.

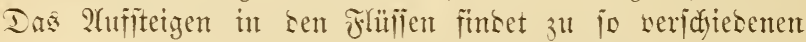

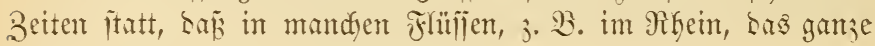
Gabr finturdy Radje gefangen werben.

Tie Saidfoeit fällt in bie Mnonate Eeptember, Sftober, Mowentiter.

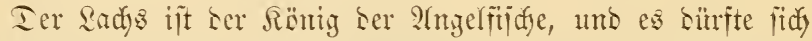
in Ieutjdyland bier und ba Gelegenbeit finten, biejen Eport

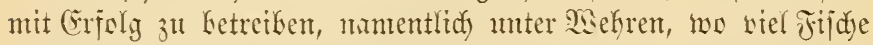
gejangen werben, wie kei நameln in ser Mejer, am Etoljen=

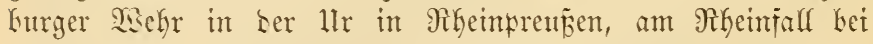

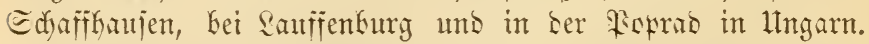

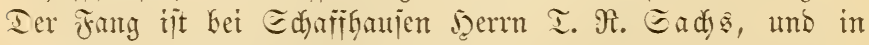

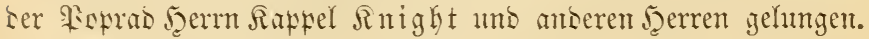

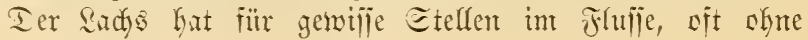




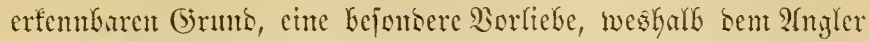
ein ortshtumbiger Füfrer unentbefrlidy ijt. Bejonders gern liegt

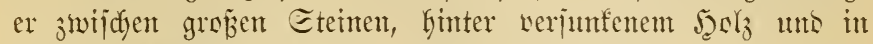
rufigen Iümpeln, unter sichren uno șanjerfällen, bic er über= ipringen will.

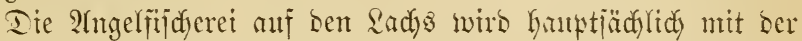
Fliegenangel betrieben. Thäerbem wirb mit (Erfolg bie 厄pinn= angel unb bas jeben uno Eenfen angetwambt. Bu ber Yekzteren

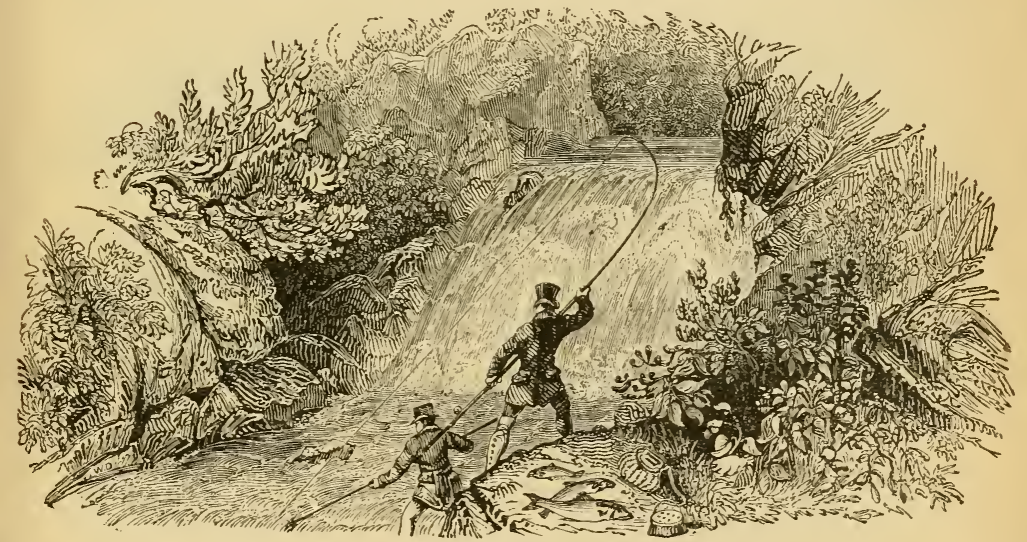

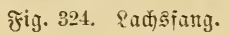

Methobe bejtedt man einen Sadjafifen won $12-15 \mathrm{~mm}$ Breite

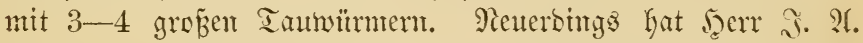

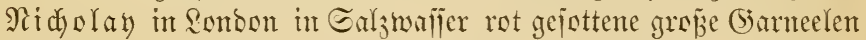
(Prawn) mit gutem Erfolge bemulat. Die Oarmeele owirb mit einer fleinen Röbernabel an Jafen bejejtigt, mit Ecibe jejt= gebunben, und $1 / 2 \mathrm{~m}$ über ben Röber eine groje B̉eifugel (ca. $3 / 4 \mathrm{~cm}$ Durdymejier) angebridyt. Sit itarfer Etrömung nimmt

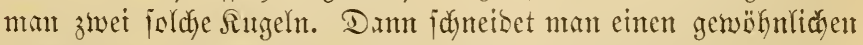

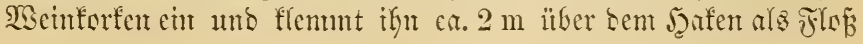


is an sie Edfutr, bañ er affällt, weme er beint Santen cines Ruchs Ginberfidy wirt.

5. Die Mieerforelle. Trutta Trutta.

(Eadsiforefle, The Salmon Trout, Bull Trout,

White Trout, La Truite de Mer.)

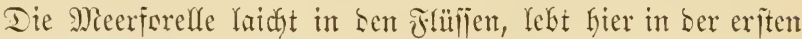

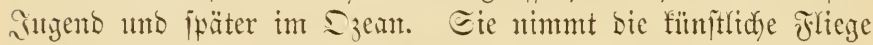

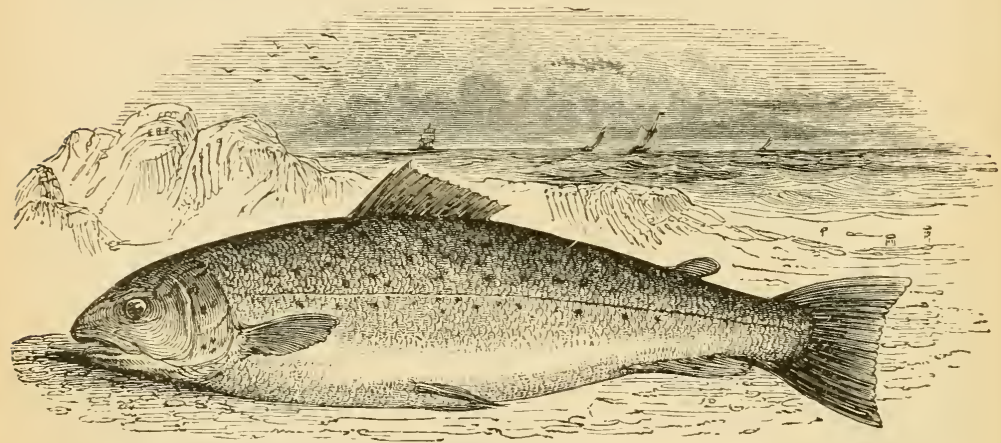

Fig. 325. Micerionelle.

fefre begierig, wirt and mit ber Eximnangel gejangen unt ift für Len ?trgel=

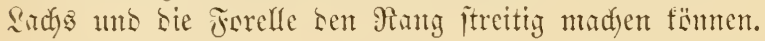

Rein Fitid) jteigt io breift nad) ser fintritliden Friege, wie

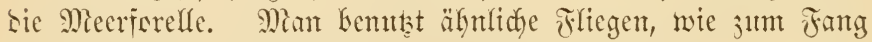

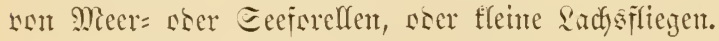

\section{Der Guđhen. Salmo Hucho.}

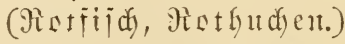

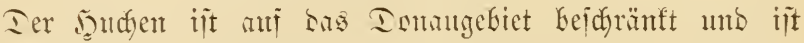

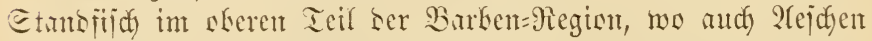

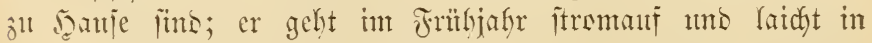

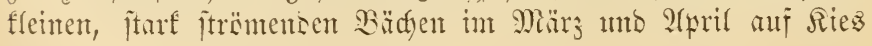




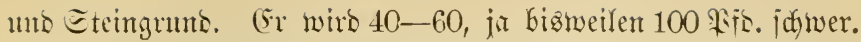

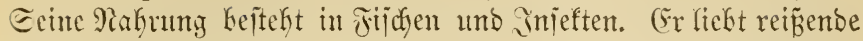
Etrëmungen uno Seritedfe finter Feljent, Eteinen uno verjunfentent Dody. Tieje 2fusbudtungen bes lliers, wo ser Borb unterjpült

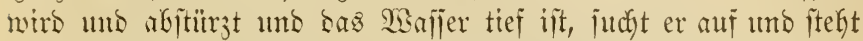
sort oft bidft am llfer, woer in jtarfen Etrïmunisen, wo fie in

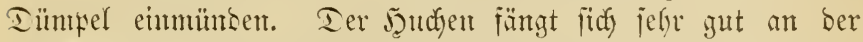
2hngel, er beiñt bas ganje Juhr; bie Keite Eaijon ijt ber 2 Sinter, wou Sftober, went es Nadjts reift, bis (Ense Fefruar, jo lange ans Saffer flein und flar ijt.

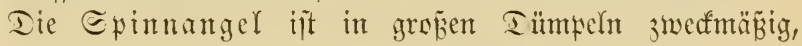
numentlidy int Jetelt: 00 R. unto jelbit 2-30 Rälte finto bie Eciten Temperaturent für bieje itidjerei.

Iie Fliegenangel mit tlenten Radysfliegent ift mur wom juni fis jerbjt wirfjam.

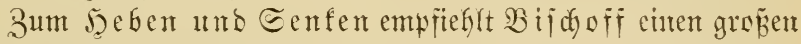
Inppelfafen ober Triangel, an Lent ein Bünsel Iaumürmer befejtigt ijt. Man famm fier jidyer ermarten, Daj jeber நuthent sen Röber nimmt, ber ifn erblict.

S.uellen: Bifdofif.

\section{Der Saibling. Salmo salvelinus.}

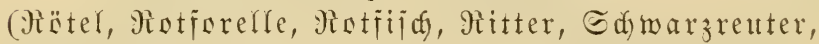
The Char, L'Ombre-Chevalier.)

Ier Eaibling lebt in Gebirgsieen ber 2lpen und (Englants, sit in grof̈er Tiefe, aus ber er im Dftober bis Iezentber mijiteigt, um jul laidfen. (Er ijt ein gejelfiger Jijdy. Bismeilen fängt man ifn an fer fünitlichen Fliege, soer mit eintem Jijddyen

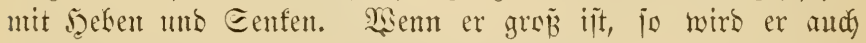
2ailofing Eaibling genannt unb man füngt ifn bant mit einer Wrt non Fatemojter=21ngel. Eie bejteht aus einter wohl $200 \mathrm{~m}$ langen edumtr, an weldyer alle $6-8 \mathrm{~m}$ ein $10 \mathrm{~cm}$ langes totes

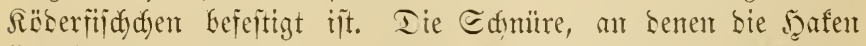
fid) befinden, fint $30 \mathrm{~cm}$ lang. Iie Jamtptleme, weldye an 


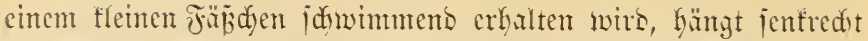

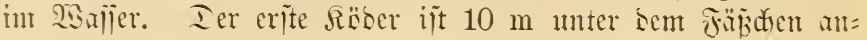
getradyt.

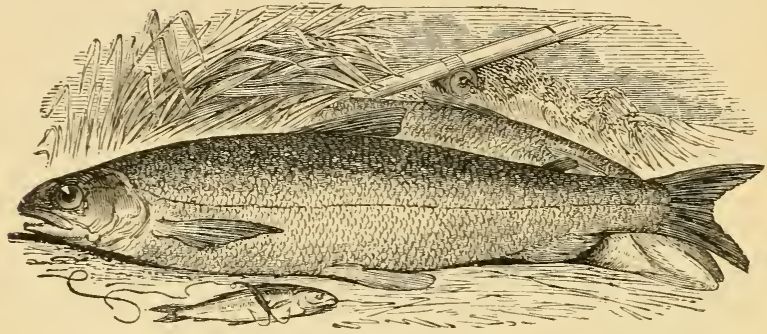

Fig. 326. Saifling.

8. Der Sđhwarjbaridh und der forellenbaridh, Black Bass.

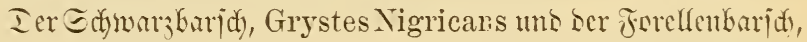

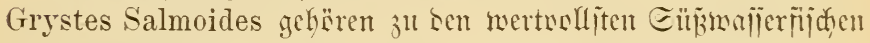

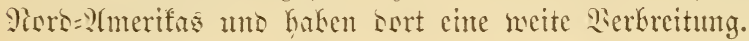

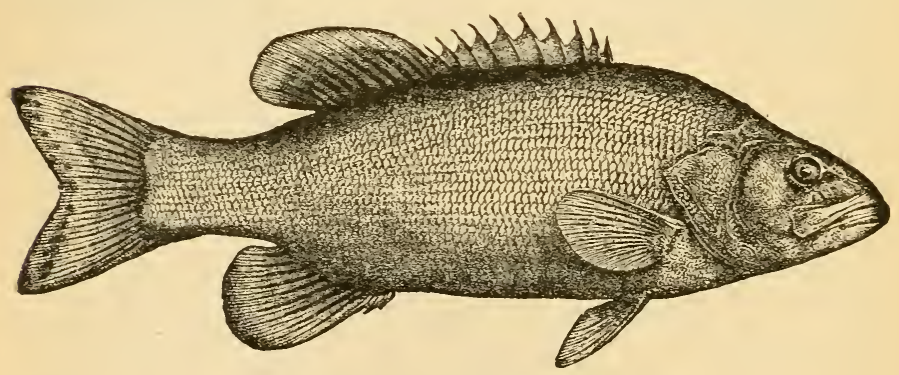

Fig. 327. Der હdinargbarid.

Sie funs int Gebiet bes Et. Qamrence= Etromes unt ber

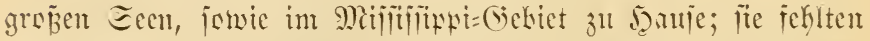
urjprünglidy in sen Flüilen, weldye jüolidy won Et. Eamuence in

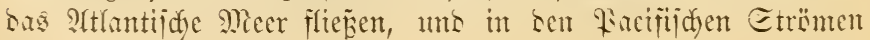


im Meiten bes Feljengebirges, weldye in ben Etillen Szean pidy ergiez̈en. Sim Morben fommen fie vor in ben nörblidjen Buflüijen ber grojen Eeen, un im Ë̈ben in sen Etrömen uno Budyten ant Merifaniiden Meerbujen uto int Rio Grande.

Der Edywarbarjd ift mefr int Rorsen, ber Forellentaridy mebr im Eitben jull Jamie, fie fonmten aber aud auf weiten

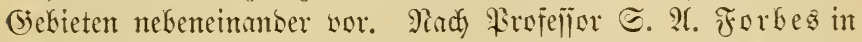
Illinois fonment ber Gdynarzbarid nod im (bebiet bes Wabash River im Etante Illinois vor, feflt aber meiter jüblidy. Ier

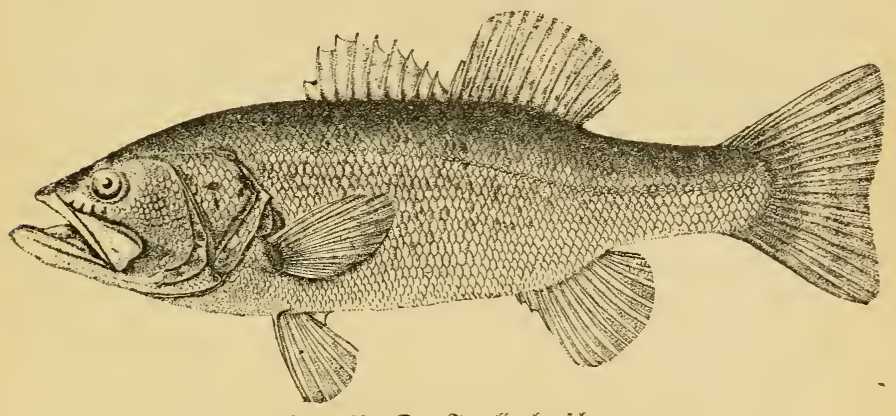

Fig. 328. Ier Forellentarid.

Forellenbarich gebeigt in ben warmen Gimälïern ber Eitojtaten vortreffrlidy, uno ijt bis zum Sntario= Eee verbreitet.

Shre Pabrung bejteht in Tieren affer 2frt, namentlidy Intujorien, Wïtrntern, Miujueln, Edhedfen, Rrebstieren, Injeften, Sistierfäjern uto Sarven, Fröjden, Frojd)larven uno Fijdyen. Sdh fint in Commer 1887 in bent Magen zmeijömmeriger

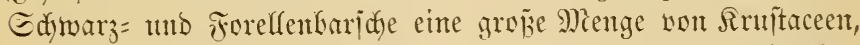
mantentlich Daphnien, Sujeften, Salïerfïfem un Edyneden. Barjue, bie jtets retdylidy mit Futterfijden werjorgt waren, zeigten bei meitem nidft ein io idntelfes Madystunt, wie bie,

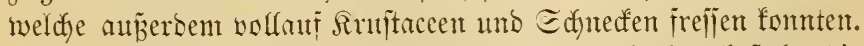
Mady Dr. (5. Eterling in Clewelmo, Shio, bejteht bie 


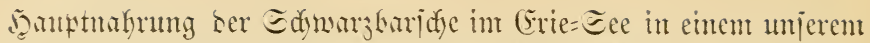
Ilffelei werwansten Fijdye, ser Silver Sided Minnow (Alburnus Nitidus), ber fis $7 \mathrm{~cm}$ lang wirs uno wie ber Eams an Mieere in jufferier Mienge worfommt.

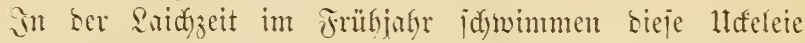
Ecite an Eeite $1-2 \mathrm{~m}$ breit uns ticf in ummeterforstenen Bügen, bie oft an einem Pintfe bes llfers 3-4 Inge lomg

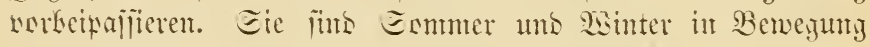
unt in alfen Mionaten gleid) bäufig. Eie leben won flemen,

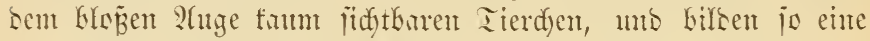

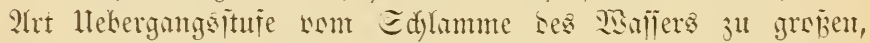

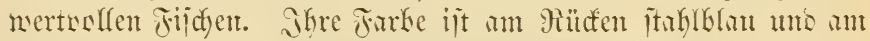

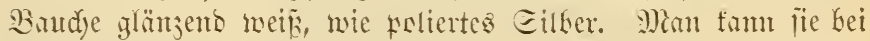
flarem 2 sifjer nod in 10-12 m zieje erfennen, went jie ifre Eeite ser Esume iuferren.

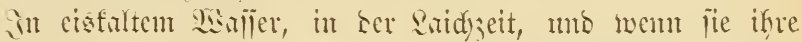

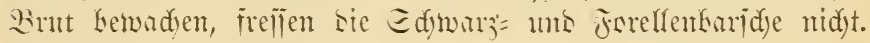

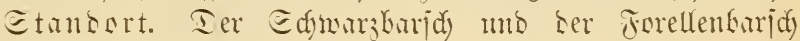
fonmen an vielen Exten juilmmen vor, ïe bemobnen jowohl Eeen, wic Jitilie.

in Eeen befinsen jie jidy im simter im ticjen 2 sajer, uns rerjaffen in Sethargic, wem bas seajler eistalt woirs; im

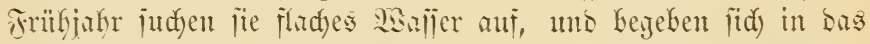
nämite

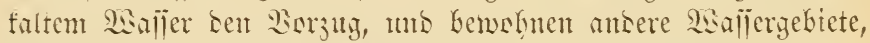
wie ser Black Bass. Iicjer iteft gern am Mante fer Edyar, wo Ier Grumb in bie Siefe iteil abjüllt, jerner an Rlipten, Eteinen uns Sirntbetten, uno wo es viel fleme jijdje giebt.

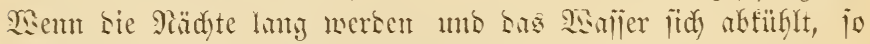
begeten pidy bie Baride in bie Tieje.

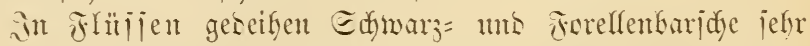

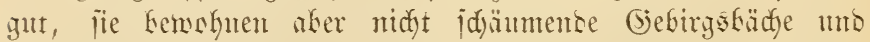
Enellbädse, weldye im Enmmer falt jins, unt wo bie Bad $=$ forefle an beften geseift. In fleinen Jitifïn won $10 \mathrm{~m}$ Breite 
unto geringer Iiefe giebt es oft viele Barjdye, unt fie werben Eort 2-4 Sifuto jumer, jie gebeifen aber an bejten in grop̃en Etrönten, in ber Brrten= unb Bletregion. Eie lieben eine

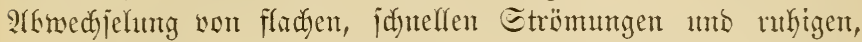
tiejen Tüntpeln. 2H(B Etanoort wählen fie gern tiejes, fitlles

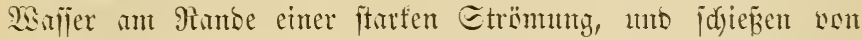
sout in ben Etrom auf worïberjdwimmenbes Futter. Sierjunfenes

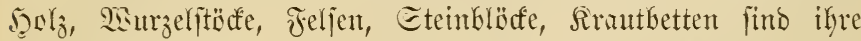
Sieritecfe. Im Sinter fint fie in tiejen, rufigen Iümpeln, ober in Eeen, weldye mit bem Flujie in Berbindung iteben. Pady

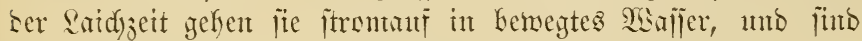

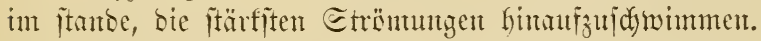

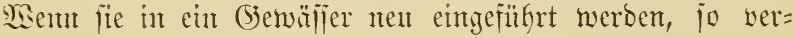

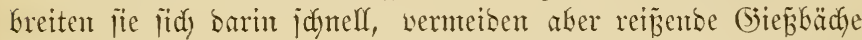
unt falte Suelffïijle. Im Susquehanna River fint bie Barjhe

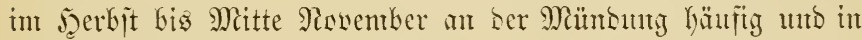
grofen Exemplaren, in Eommer find fie bort jelten zut finton. Ter Forelfenborid fideint wentiger weite 19 antberungen ju unter= nefmen, wie ser Edymarjbarjd. 4-6 Madjen var ber Raidjeit werlaffen bie Barjae bie SBinterquatiere und gehen in ben

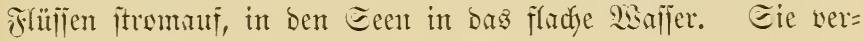
freiten jidh wiel joneller in einem fluffe itronanfmärts, wie itrontabwärtz von ser Etelle, wo jie antggejekzt numben; aber

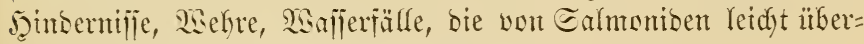
wutsen merben, verntögen fie nidyt zu ̈̈berjofreiten.

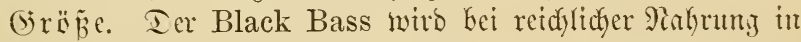
6 Mionaten 5 bis $14 \mathrm{~cm}$, in 18 Monaten 20 bis $30 \mathrm{~cm}$ lamg, unb fann jpäter jäfrlid) 1 Fifun idywerer merben, bis er aus =

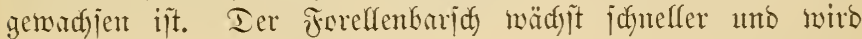

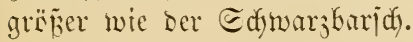

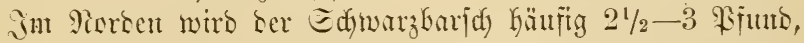

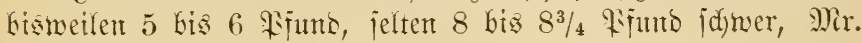

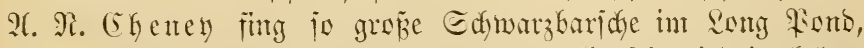

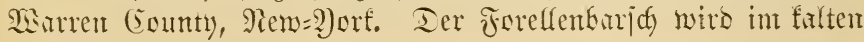




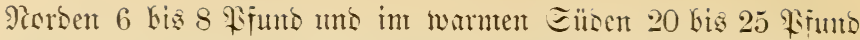

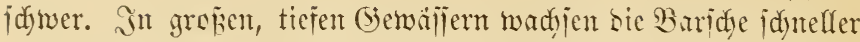

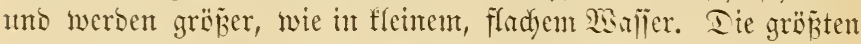
Fifche finto jefr fett, trïge uno wenig famtrfluitig, beut bejten

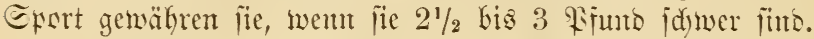

Eiuführung in Ieutidyland. Im Tebruar 1883 jumbte

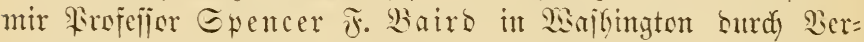
mittelung won Mr. Fred Mather in Mem=2)ort 7 Edymaz=

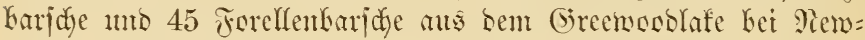
Yorf. Sie fumen zwar alle Yebeno fier an, infolge ser langen Reije jtarben aber in furzer Beit bie meijten, unto idy bebielt nur 3 Edymarjbarje mo 10 Forelfentaride. Jetst habe idy sawon

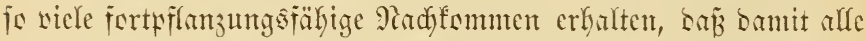

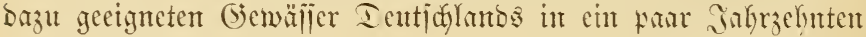
bewälfert werben fönnen.

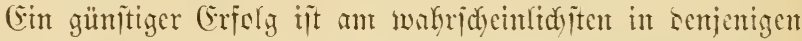

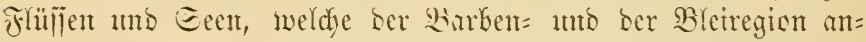
geförent uto bie fich in Eommer bis $15^{\circ} \mathrm{R}$. unt bariber er=

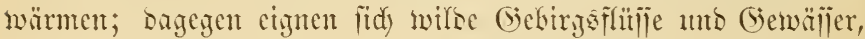
bic im Eonmer fult jüs, nidyt für Black Bass.

Rein mberer Fijd übertriff̈t ben Black Bass an Rübntheit

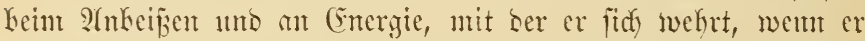
gefjaft ijt. (Er hat bie pieiliduntelfe Bemeguty ber Forelle, sie

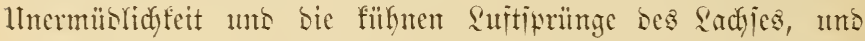

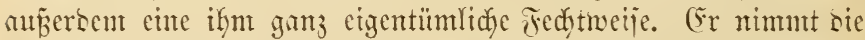

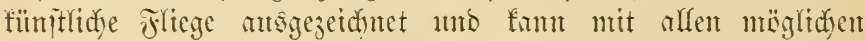
Prten sou matürlichen utb füntlidyen Röbern gefangen wersen.

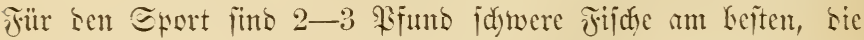

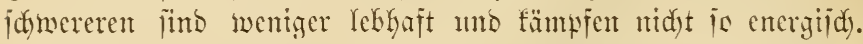
Int Flïijen ijt ber Efart in alfgenteinen befier wie in Eeen.

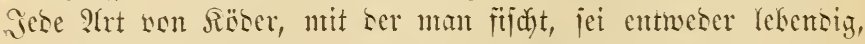
soer man bewege ifyt jo, saj er lebento zu jeit idjeint.

Sk fïr ben Epert ber Edjmatjharidy den Borzug werbient,

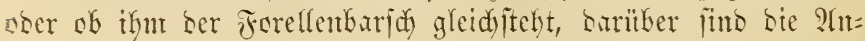




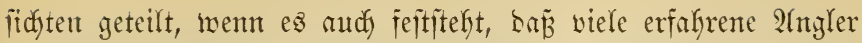
bem Edjwarjbarid bie Walme reidjen, io juätzen body anbere Fijejer von allgemein anerfannter 2Tutorität (jensharl u. a. m.) ben Forelfentarids gleid) hod).

Da man in 2 merifa Gerbadytet, bañ bie Forelfe burd) bie fort=

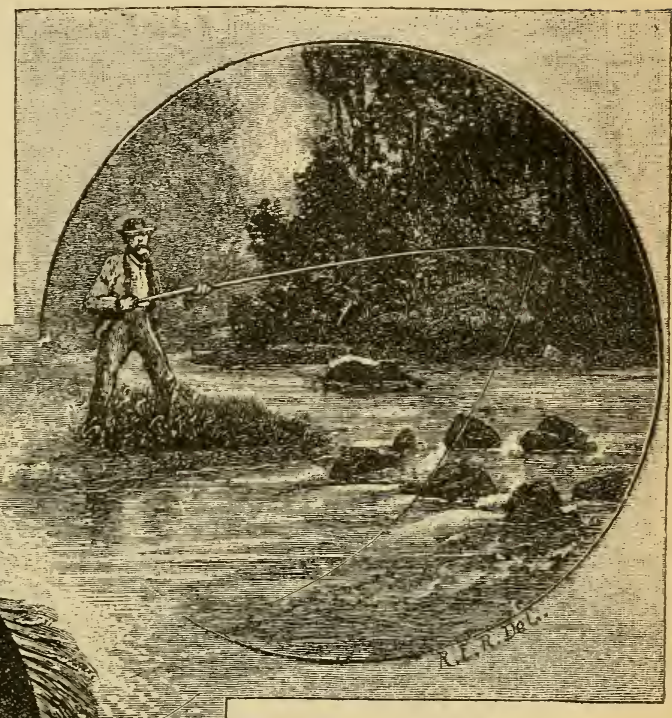

jadreitenbe Sultur ntefr und mehr verorängt mitro, währento bagegen ber Bass aufifalleno wenig empfindlidy ift, jo glaubt man, baj leţterer in ber 3ufunit als Eportfiid Fig. 329. Fang bes Forellenkarimes. Lie eritte Stellecintefment mirs. Wiele jint ber $\mathfrak{2} \mathfrak{n}=$ jidyt, baß̉ ifm bieje હtelle juon heute gebüfrt. Die Black Bass nefmen faft jesen nat ürliden ober fün it=

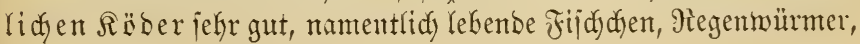




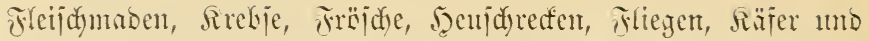

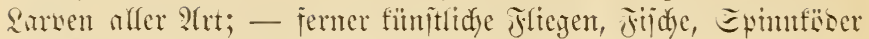
uno Rïser junt Detren anto Eentent.

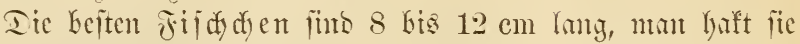
surdy sie 1tuterlippe, umb fout erit an, wenn man Len jweiten Pitucf Les Black Bass füblt.

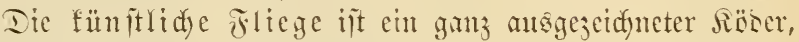

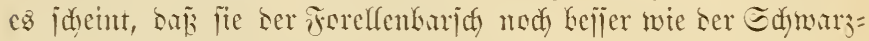

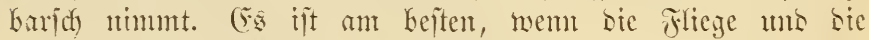

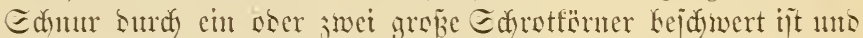

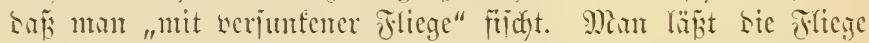

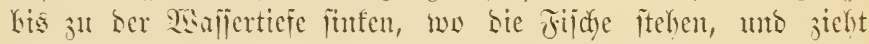

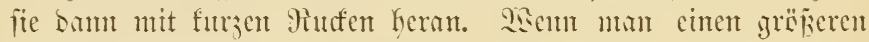

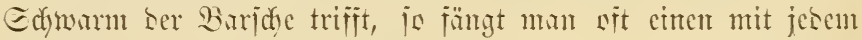
פismic.

Tie Fliege jei 25 bis $35 \mathrm{~mm}$ lang mo hate, wie bie eng=

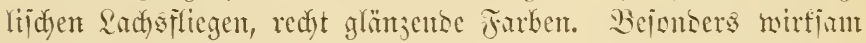
jülo gelf, rot, jatwar; tmo wein.

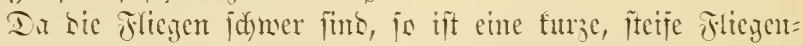
rute erforberlidy, id empifhle worjugsweije bie 3 bis $3 \frac{1}{2} \mathrm{~m}$ lange Etemartiche Fliegentute, wie jie bei Deiuridy Difocbramo

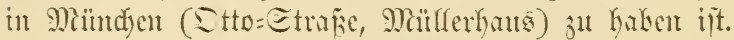

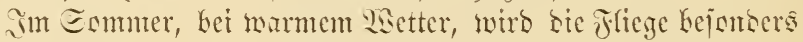
gut genontmen.

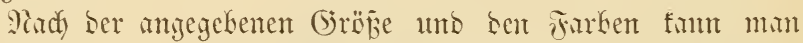

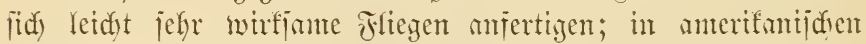
Büdjern unb Beitjuriften ift cine grofie Mienige werjobicsenter Muiter Geidyrieben uto empishlen.

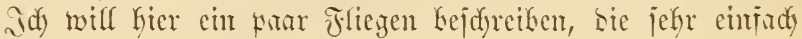
finto uns bie fich mir ars gant bejonters mirfiam erwiejen baben.

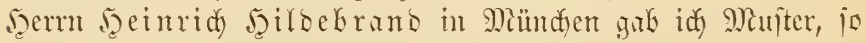

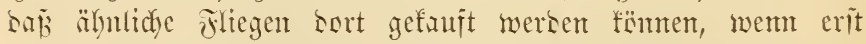
umjere seutjenen riüije mit Black Bass bevëlfert jein werben. Pad) amerifmijden (Erfahrutgen fömen mit sies mady 10 bis 
12 Jafren erwarten. Imm wirb bei uns ber Fliegenfilider wortreffidyen Eport ba finsen, wo er jebt nidyt jul haben ijt, in unjeren grojen Frïjien uro Ecen.

1. Die Bob=irlicge (Fig. 330) ift weiter nidyts, wie ein an bem 2tmgel= hafen angemutsenes Bünsel itrohgelben Mablairs. Sidh afymte io einen iche frimitiven tümptlidyen Röser nadh, mit weldyent in sen amerifmijdyen Cüb= ftuater, Gejonoers in Florisa, Soupiann mo Teras, mit grojem (Evfolg nad) Foreflenbariden gefild wo wirt. Ier bort

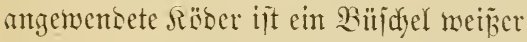

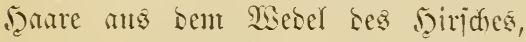
bejeitigt an cincm Irimgel; sft werben weib̈e Febern uno etreifen juarladyoten Iudfes finjugefügt. Mant fijdyt int ber Regel won einem langiant fahrenben Boste.

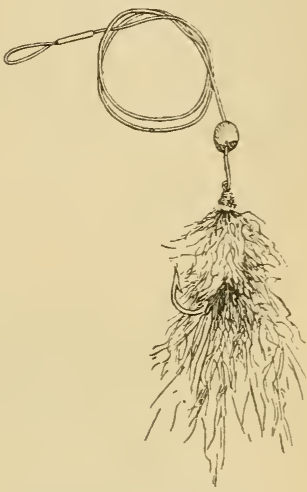

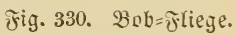

2. (Ein red)t rauber Fintuter (Jig. 331 uns 332) ans gefürbter Edimeinstwalle ober Eeehunbshan, unten itrohgelb,

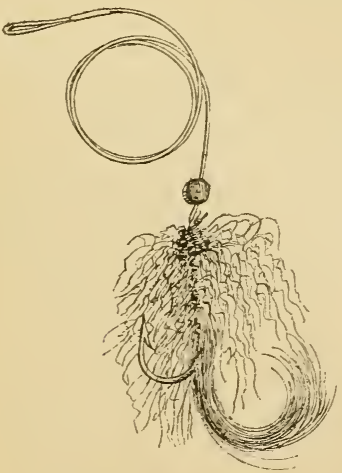

Fig. 331. Fialmer.

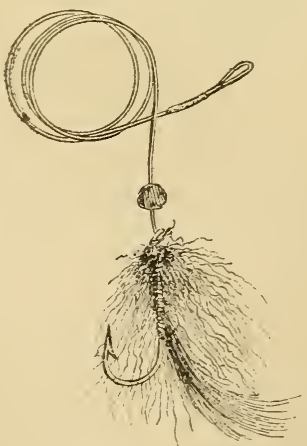

Fig. 332. Hialuer. 
an Lev Edulter jdarladyet, aud mobl mit Gold = ober Cilber= fasen geripts; ser Edywan; ats itrofgelbent Mobair oser won

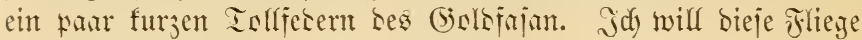
die Befr memen.

Ginte Jebern für Bass-Jliegen Yiejern: weipe Zaube, Gold =

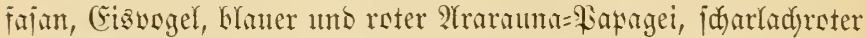

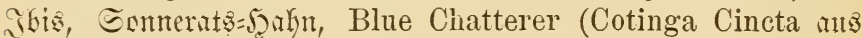
Brit. (Sututer); Indian Crow (Pyrodorus Scutatus); Cock of the Rock (Rupicola Peruviana); Rifefferfrejier (Tucan, Ramphastos Ariel) und andere B̈̈gel mit bunten Tesern.

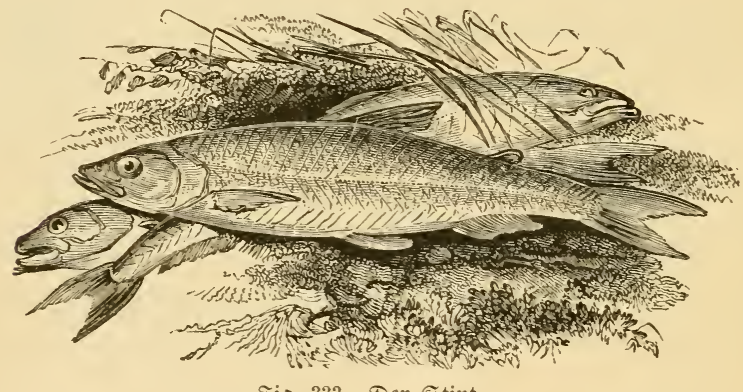

Fig. 333. Ier @tint.

9. Der Stint. Osmerus eperlanus.

(EpierYing, The Smelt, L'Eperlan.)

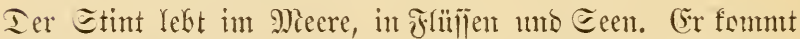

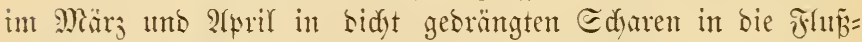

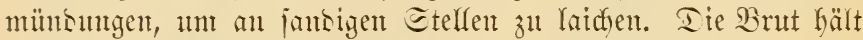

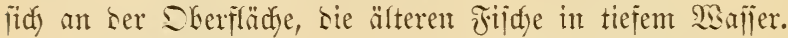

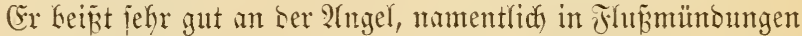
uno in Sanfjen, won Suni biz Piowember. Jut Eommer ijt ber 干rüke Morgen uno ipäte 2fbent bie beite Beit. Man fängt ifn erit $2-2 \frac{1}{2}$ in unter ber Sberfläche.

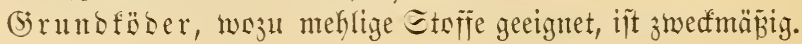




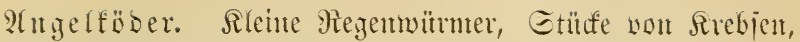

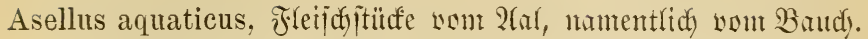

Der Dafen jei $5 \mathrm{~mm}$ breit. Man angelt nut einem Fater=

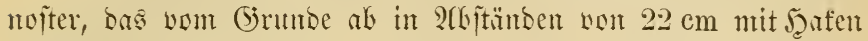
werjeken iit. Da man ben Biz ber Fijhe nidy füglt, jo muis in Interbalfen wou einigen Eefunoen leidgt angehanen werben.

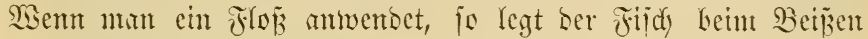

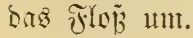

\section{Der Blaufelđ̧en. Coregonus Wartmanni.}

(Gimeine Renfe.)

Ter Blutieldyen lebt meift in ben gröperen Eeen ber nörs=

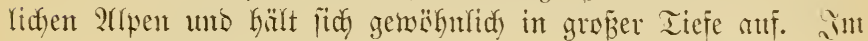
Bitricher Eee wirb er mit eimer eigen= tïntlidyen 21ugel gefantyen, bie Degner genant wirs. Thi einer Janorolle (jig. 33t) befinbet ïdy eine lange Fiferbeharidym, bie bis mif ben (jintur bes Cees reidyt umb an beren Ende ein Breigenvidyt angebradyt iit. Das Sorfach ijt jefr lang, bejteft aus (Sutjaben unto trägt 20 Jaafen won $6 \mathrm{~mm}$ Breite. 2Im Bogen ber

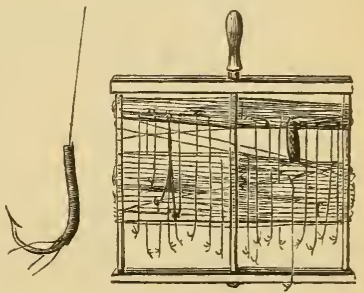

Fig. 331. ફ̧egner.

Jaafen befinton fich furze Riferbehante, bie ben cinzigen Sëber bitben. Bein Ângeln wiro bie Edynu langian beweyt, was bie Felden zunt Afnbeiz̄en veranlajit.

\section{Der Zíth. Coregonus hiemalis.}

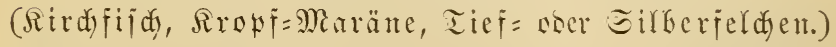

(5r. fonment im Bobenjee unb 2(ntmerjee sor tmb folf in reţterem mit ber 2 tngel gejangen werben.

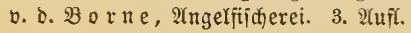




\section{Der Sð̧näpel. Coregonus Oxyrhynchus.}

(Dïttelmann, Igielemann, S.bneịen.)

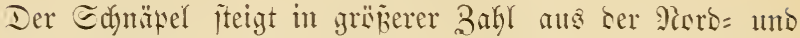

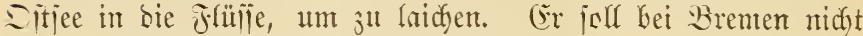
jelten mit ber Afngel gejangen merben.

\section{Die grof̧e Maräne. Coregonus Maraena.}

(M) a ü = Maräne.)

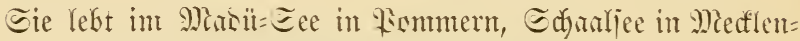

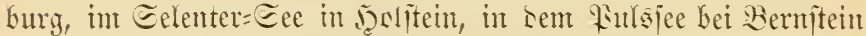
und einigen Eeen bei Bimbrum. Eie wirb im Marü=Eee an

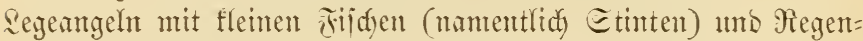
mütmetn gefingen.

\section{Die Döbel. Squalius Cephalus.}

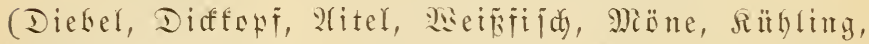

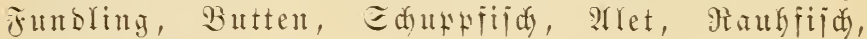

Iubling, Bratfifd, The Chub, La Chevenne.)

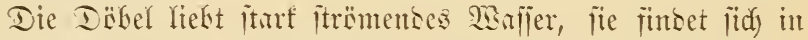

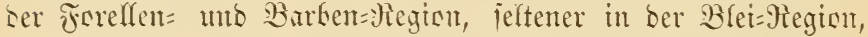

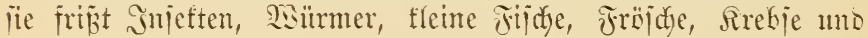

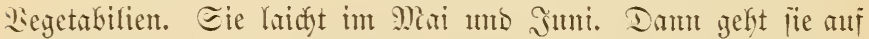
Inntiefen uno Etromidynelfen unb bie Enten ser Dïmpel. Sm

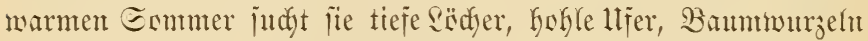

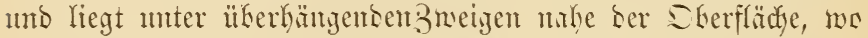

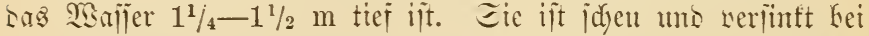
jebem Edyatten, ber fie trifft, iteigt aber furb wober empor. Im

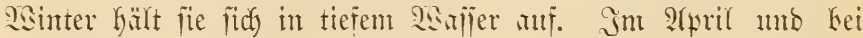

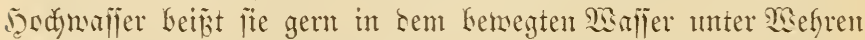
uno Mühlgerinten an Sammümern. Eefr gut ijt ber Binter

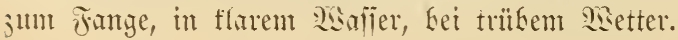




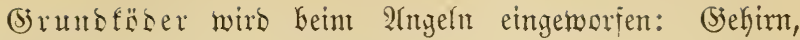
(j)rienert.

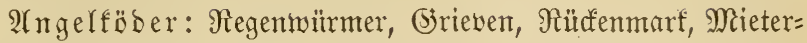
frebje, Räje, Maben, Räfer, MEeŝpenlarven, Jeujadrecten, fleine Fröjdye, frijdjes Sbjt.

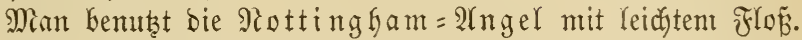

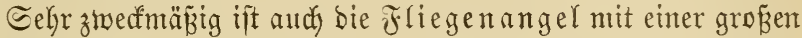
fïmitldyen Fliege, namentlidf, wenn eine Mabe ober ein Etüdthen

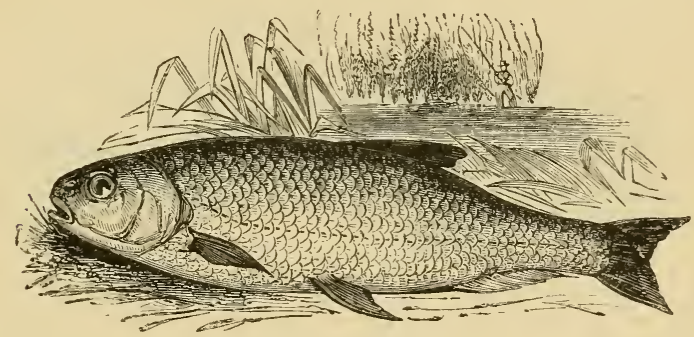

Fig. 335. Döbel.

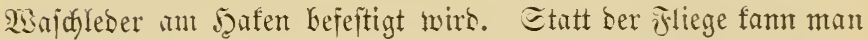

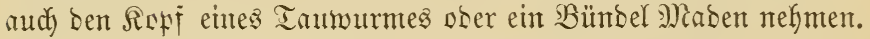

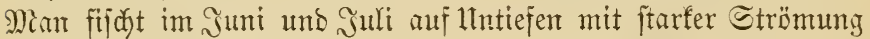
von 8-12 1thr Bormittrgs und nady 4 1thr Nadymittags, und in Conmer unter überbängenden Bweigen. Die Buj dyangelei ijt im Eommer unter Baumzmeigen uno (jebrijich jefr z'wedt=

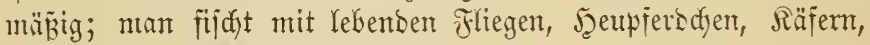
Mistten, fleinen Fröjchen u. Dgl.

Sn Mientel fiidst mum mit ber Ireibịnur. Sine ca. $30 \mathrm{~m}$

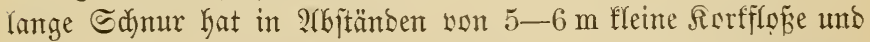
wirb von ber Strömung meit fortgeiüfrt. Man fijdt mit Räfern, Fliegen, J̃eupferddyen u. Dgl. uns fängt jo bie gröbten Döbeln.

Sueflen: Sizaaf Malton, Enlter, Bailery, Francis. 


\section{Der Gäfeling. Squalius Leuciscus.}

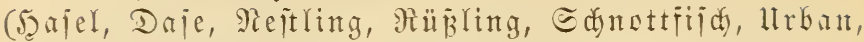
Springer, Märzling, (j)rätling, The Dace, La Vendoise.)

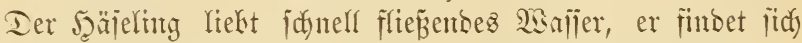

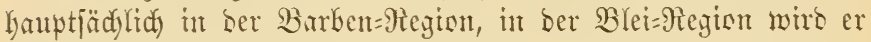

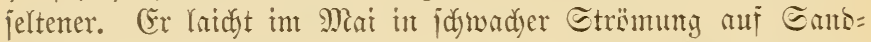
grunb. Int Ermmer kält er fid in itarfer Etrëmumy, int

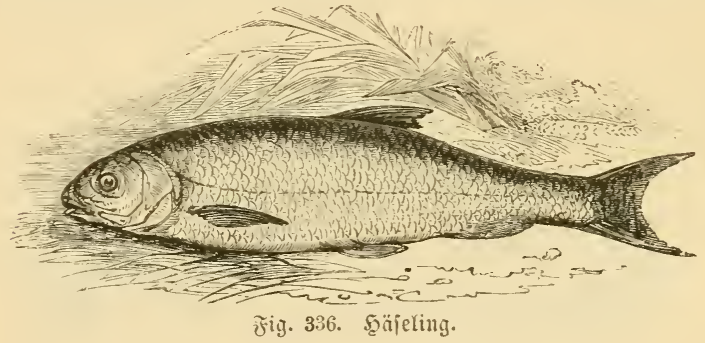

Sinter in rubigerem ca. $1 \mathrm{~m}$ tiejen Safjer auf Eant= woer Ricsิyrumb auf.

Saijou: Sou Juli bis Sftwher, er beipt aud in Winter.

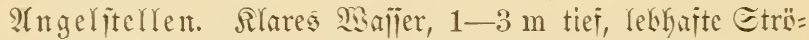

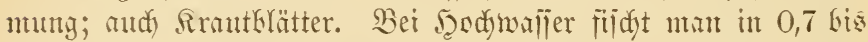

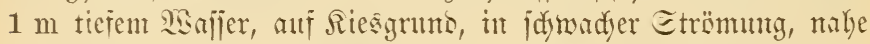
am llier.

(5) ruts öber. 2ard Bailey 5-600 Inumïmer 20 Stum= ben war sent :Hugeln. Darauf fijd man mit ser Pottinghant=2lngel wahe an Grunbe unt mirit won Beit zu Beit ein paar zerjeduttente

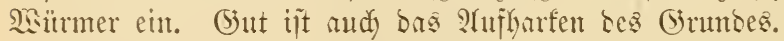

Mant fijdyt mit ber Nottingham=Flo

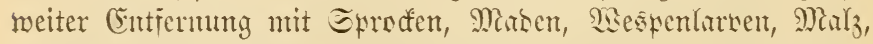

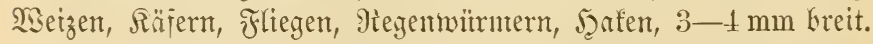

(Er ijt wout Mitte Sult kis in ben 2luguit jegr geeignet jüt bie Fliegenfijderei. Man nummt ganz fleme füntlidye Friegen. 
Pad) wirfjamer find bieje, wenn man ben Şafen nit eitrer Mabe werjityt. (ङs muг idnell angebauten werben.

\section{Der Udéelei. Alburnus Lucidus.}

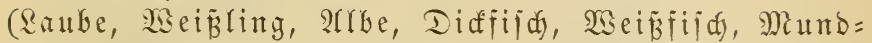
fijd, Edneiberling, Marienfijd, Bliede, Maiblefe, Sduppenfijd, sangeli, The Bleak, L'Albette).

Ier Itfelei lebt in Fliijien unb Ceen in ber Blei=ßegion ge= jeflig und iit jefr Yebhaft. Saidyeit 2lpril bis Juni. Bei warmem

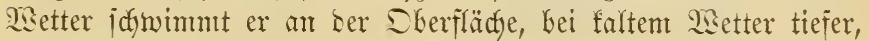

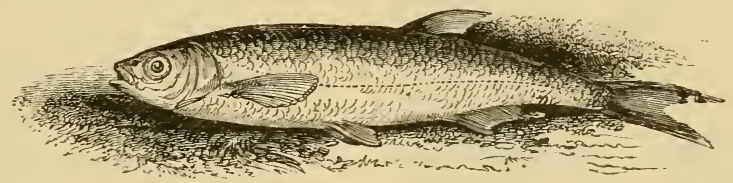

Fig. 337. Udf́clei.

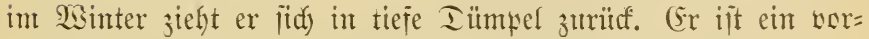
trefflidjer Räberfijid. Flo

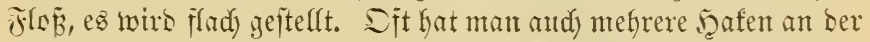

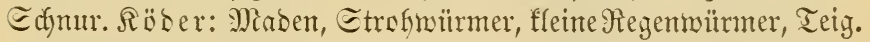

2(nfüttern fann mant, intem man etwas fatwinmende Sleie ins פanjer wirft.

Man mú idnelf ankauent.

Die Fliegenangel ijt jefr wirfjam unb eine wortreffliche Hebung. Man fïht mit ganz fleinen Jliegen unb fyängt eine Mabe an ben நafen.

Suellen: Ealter, Blaine.

\section{Der Sdhneider. Alburnus Bipunctatus.}

(Flecte, Etreifling, Gierbe, Mranbblecte, Maiblecte,

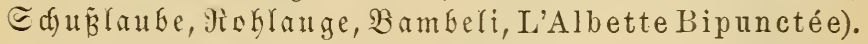

Der ๔dyneiber, Fig. 338, left in ber Barbenregion unb hat in

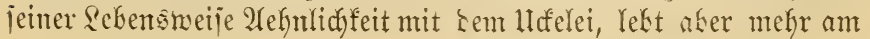


(3runbe wie biejer. Raidyzcit 2 pril und Mai. Fong ähnlidy wie ber bez lldéfeYei.

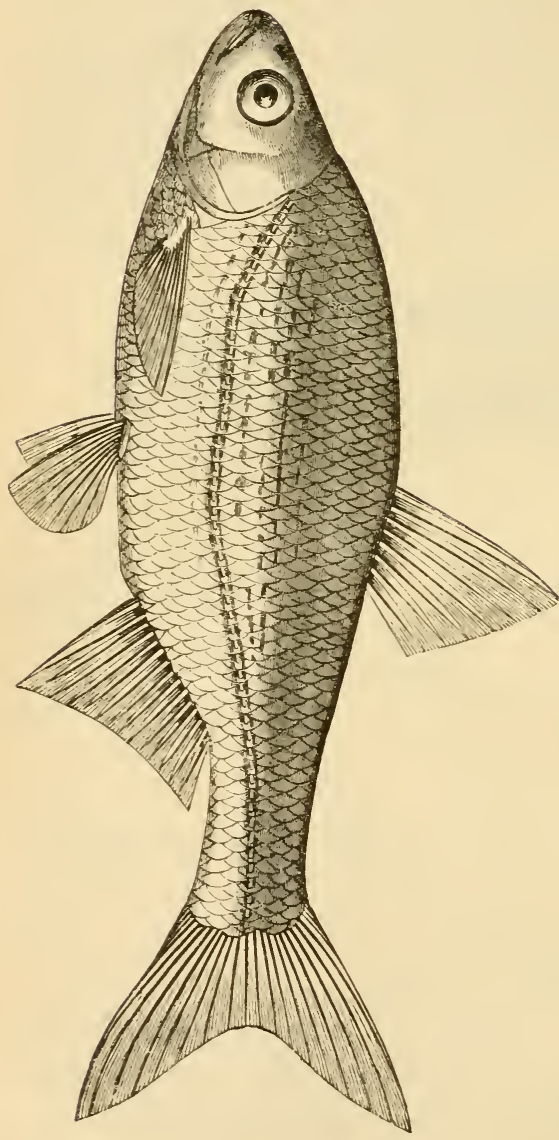

Fitg. 338. ๔dneiber.
18. Der Rapfen, Sdhied. Aspius Rapax. (3allat, Mülfe, Mäuјебeizer, L'Aspe).

Der Mapjen lebt in ben grëperen Ecen unb Flülïen Mittcleurcpa's, 3. B. Ser Inna, Socr,

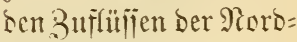
uno Sitjee, 2ttterjec, Ehiemjee, ben מ̃afjen, bejonser bäufig uno

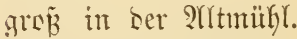
(Er jeflt in sejer und Mhein. Er gefourt ber Barbenregion an. Die Eardyzeit ijt in ?rpril uno Mai insen Flïijen. (Sr erreidyt ein (Sicwidst won $6 \mathrm{~kg}$, lieft jtarfe Ctrëmungen uno $\mathfrak{S i r =}$ bel, ftefit gern an

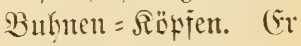
frijt Begetabilien, Sïumer uno fleine Fijdie, Kejonters fleine ulfeleic, פä̈jelinge unt sergleidjen.

Der Fang mit ber 


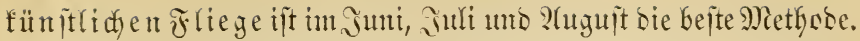

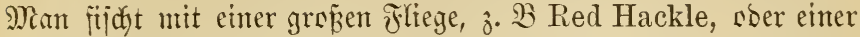

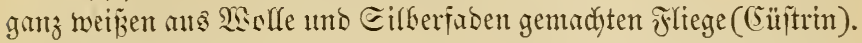
Mrit fleinen Fifdyen, aud mit eiment Silver Spinner läjt fid ber Rapfen jehr gut fangen.

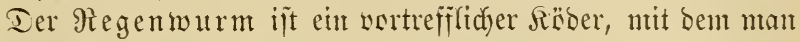
sen Fija) jelbjt in winter fangen fann, wenn man ben Dümpel

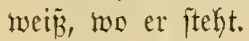

\section{Der 2rand. Idus Melanotus.}

(Drie, Merfing, Göje, Molutarpien, Jaatforf, Diof =

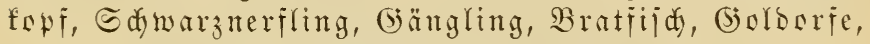
The Ide, l'Ide).

Ier 2lland lebt gejellig, und jdymumt meijt an ber Sber= fläche. (5r liebt jtarfe Etrümungen, WBefre, janbige Ilntiejen, und laidyt im 2qpril und Mai. Bu biejer Beit jieht er maflen= Kaft aus itarfen Strömungen in rubigeres 2 saljer, 3. B. Gei Cüftrin, Sranienburg, Eefle. Eine ähnlidye Santerung finbet sit Enbe Eeptember itatt; in biejen Beiten wirb er in Maijen unit ber 2 (ngel gefangen.

Man bemulzt eine Fro

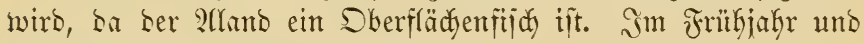
Serbjt wiro am Borumbe geangelt.

(5) rubföber: (Erbjen, Fegenmümer $2 c$.

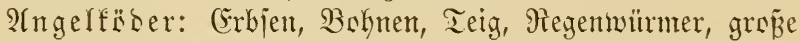

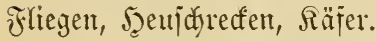

Ier Pllano beig̈t langjam, umo man barf nidyt zu fdyelf anthauten.

\section{Der 21aifif́, Alosa Vulgaris.}

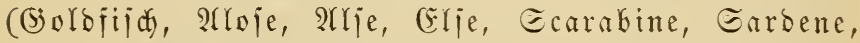
Plgone, (Eeppino, The Shad, L'Alose.)

Der Marifijd fteigt aus ber Morojee uno bem Mittelmeer in bie fritilie, um zu laidjen, utto wirb in ben italienijden 2lrpenjeen häufig mit ber Fliegenungel gejangen. 


\section{Der Gecht. Esox Lucius. \\ The Pike, Le Brochet.}

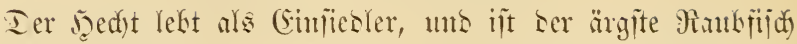

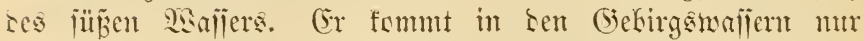

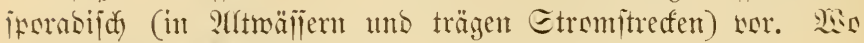
er bie Derridaft gemimnt, sa werjaminsen bie Forelfen mo

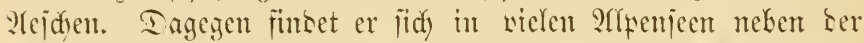
Eeeforelfe. (Er laidyt non Mlitte Februm bis Ene ?pril. (5r

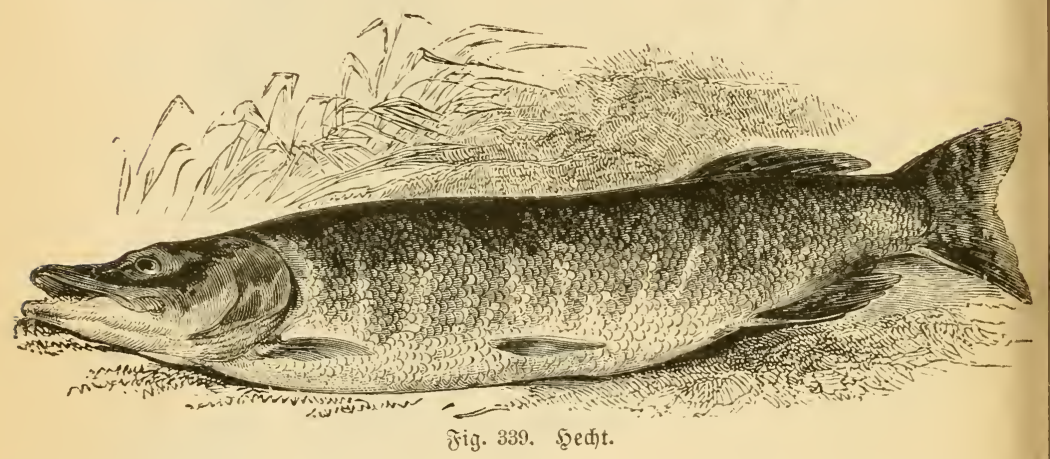

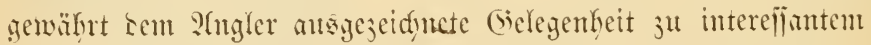
Eport.

Die Eaijon Les Dedrtanges beginnt in Eeen im Jumi mo

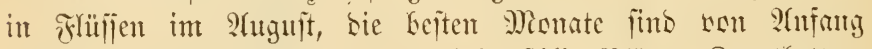

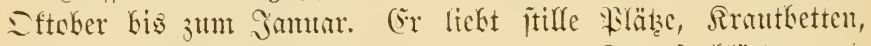

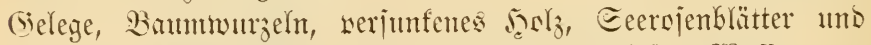

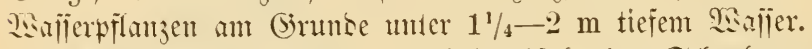

In Eeen theft er gern an teil abjallenten Edyarbergen in

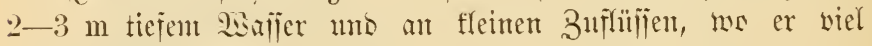
fleine gitdo funset.

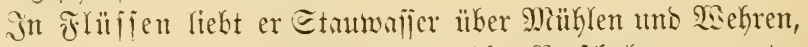

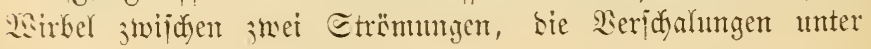


ben Mählen uno Welyren, tiefe Dümpel, frautreidyes, nidyt tiefes,

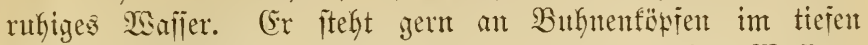

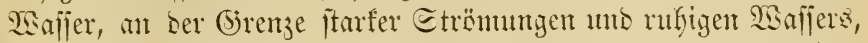
ant liebiten aberfarb ber Bufnen. Im Bereidy ber Gejeiten

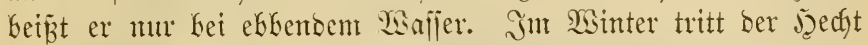
gern in sie Belege, er ift in jerbjt mo 2 sinter am meijten geneigt, an bie Ângel zu Geipen.

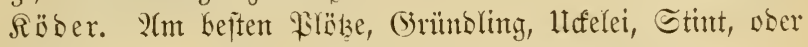

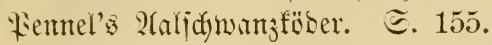

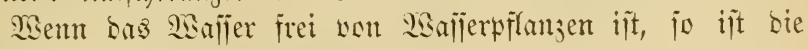
Epinnangel jefr empfehlenswert, bie mir C. 151-167 ats = fülyrlidy bejdyrieben Gabent. Ins tate gijdyden wirb jo an ber 2tngel befejtigt, baj es fidy orelyt, went es burd) bas wajier gezogent

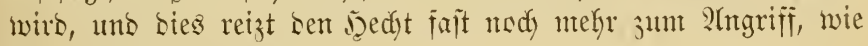
cin Yebentoes Jijdyen.

Für frautreidjes Siller bebient man jïd ber Trollangel

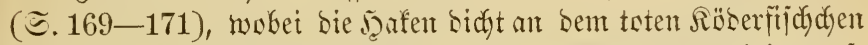
anliegen. Dieje 2tngelmetfore ijt jefr mirfjam, unb bringt cit

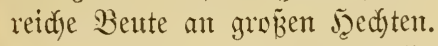

In Eeen, größ̈eren Flüfjen uno in Den Bimtengewäfjern ber Sitjee miro ntit gref̈en (Erfolize mit ber Sdyleppangel (Darre) won einem fafrenten Boote gefijdyt. 2(m bejten benubzt ntan

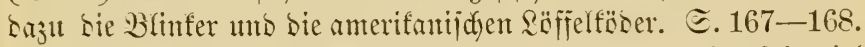

Mit ber $\widetilde{S}$ bludangel uno Yebenben Räbern wirb jefr viel unb ericlgreidy nady Sechten geangelt. (ङ. 174-180.)

Mir. Alfreb Sarbine gilt in (Sitglanto für ben gejdidftejten

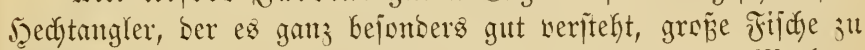
fangen. Jah will beakalb bie won ifm angemande Methore Gier bejdyreiben. Mar. Jarbine angelt hauptjächlidy mit Eer

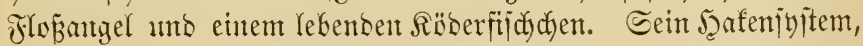
von bem wir eine 2tbbilbung bringen unt ein (Exemplar an

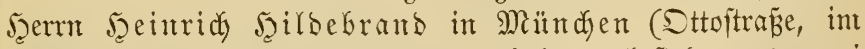
Mülferbauje) als Mujter gejanst haben, beiteht aus zwei Triangeln, weldye an (Simp won Supferornht angebunten fint. 
Der Enstringel kefteht ans zwei grofen Denfen, bie 10 bis $15 \mathrm{~mm}$ freit, 25-35 m lany füb uns einem fleinen 5aten, ber

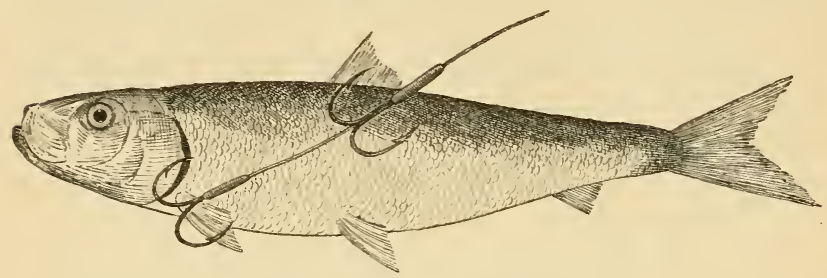

Fig. 310. Sarbine's Eqnappangel.

$4 \mathrm{~mm}$ Greit uns $10 \mathrm{~mm}$ lang ijt; Ser mittlere Trimyel Gepteht

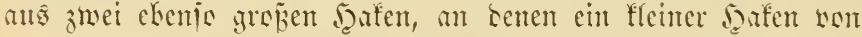

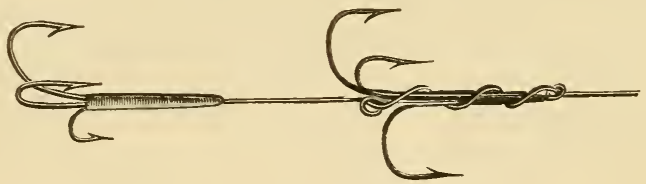

Fig. 341. Jarbine's Sfinappangel.

$7 \mathrm{~mm}$ Breite uno $10 \mathrm{~mm}$ Sänge angelötet ijt. Ter 26jitano

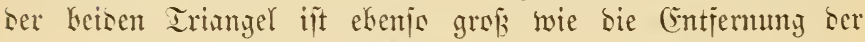

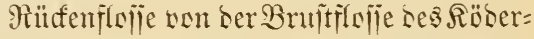

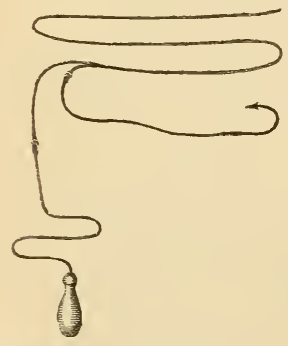

ชig. 342. Faternofter. fildes, 5. K. $45-65 \mathrm{~mm}$. Der (End= triangel wird au ber Bajiz ser Brupt= flofie, ber Mitteltriangel an ser Bajts

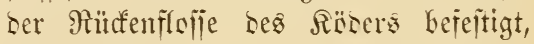
in baj biejer is roentig wie möglidy wer=

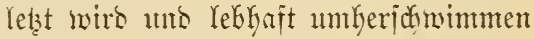
fann.

Da bie grofien Dedyte jefr aujmert= fam und idfeu fint, fo wirb bie angel mit grëzter Sorfidgt eingenorfen umb 
ber 2fngler judyt jidy jo gut wie möglidy ju verbergen, er fijdt zllerit bas Maffer nathe bei uno erit nadjher bas in weiterer

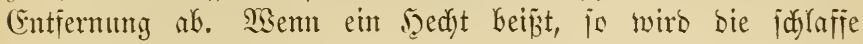
Edyur eingezogen unb barau nad etwa 5 Eefinton angebauen.

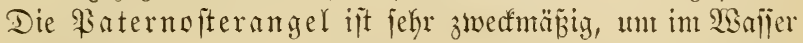

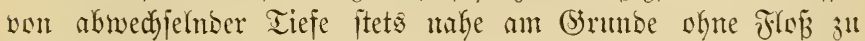
fijd)en. Die Rute ift lünger (bis 4,2 m) und weniger fteif wie bei ber Flofangel. Das Sorfady ift $1-1 \frac{1}{2} \mathrm{~m}$ lang und bejteft, wie kei ber Flojangel, aus grünlid)gran gefärbten, ftärfiten, ein= fadyen ober geflodytenen (Sutjäben. Das Bleigemidyt ift an einem 0,4 m langen (Stutjaben befeitigt, ber jeiner ift wie bas Borfad) uno in ber (Endjdyleife Des Borfades eingehängt ift (Jig. 342). Der Saten iit 7-10 $\mathrm{mm}$ breit und an $25 \mathrm{~cm}$ langem (Stiml

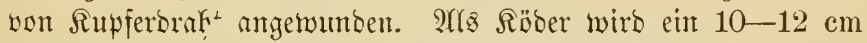

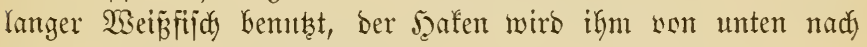
sben burdy beibe sippen gefïthrt.

Bisweilen, namentfidy wenn bas Wafijer warm ijt, uno went viele fleine Fijdye vorhandent fint, ergreift ber Jeedyt ben Röber=

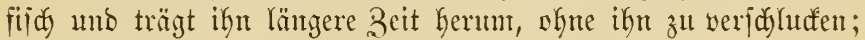
Dann befindet fid ber Saafen nidyt in jeinem Maule unb trifift ifn beshalb nidt, wem angehauen wirb. Wonn fid bies ëfter wieberfolt, io fülyrt man ben J̧afen entweber burdy bie Bafts

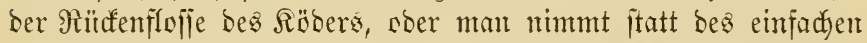

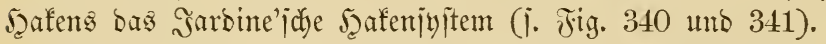

Man fijdyt mit bem Saternoiter bidyt an Bretterbettungen

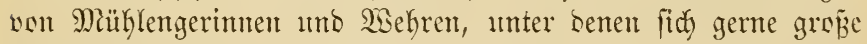
Sedyte verbergen; - an überfängenben 11 jern, - an Belegen

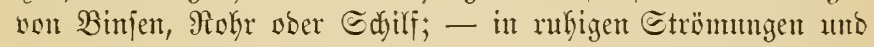
tiejen Dümpeln, wo rïdfäufige Etrömung iit. Im Jeerbit, wemu bas Rraut am (Srumbe nod) nidjt nfgeitorben ijt, fijdyt man is,

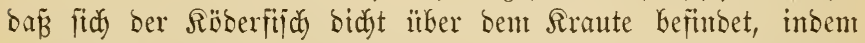
man bie Entjermung zwijdjen Bleigewidyt und Şafen bement= iprectyend wählt. Beim Fijdyen wirft man bie 2tngel ein, läßjt fie furze Beit an einer Etelfe ruhen mo bringt fie bam 
alfutählid) woำ einten Plab̧e jum anteren, inbem mant jie in

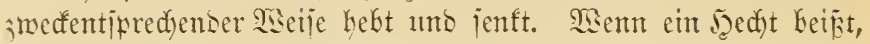
io Gaut man mad) ca. 5 Eefunden an.

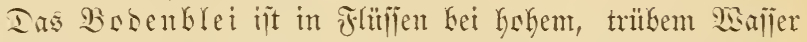

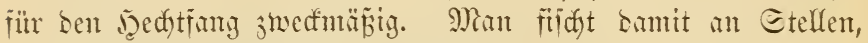

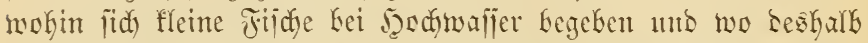
and oft igedyte itehen. Das Bleigemidyt ift 20-30 gr idfwer uns $50 \mathrm{~cm}$ won Räseritich entiernt; lețterer ijt an Jarbine's Dafeninjem kefeitigt.

Ier Fung mit ber Jed tifiege. Bisneilen nimmt ber

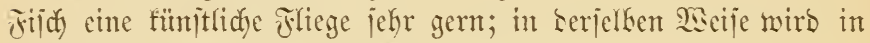
Diorbamerifi audy ber Söfïelföber bemulzt.

\section{Der Barfd. Perca Fluviatilis.}

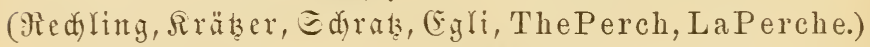

Der Barja Yeft wa Fijden, jujeften unb sïmtern, er fintot fidy in ber Barbenregion an gejdütbiten Etellen, nut ijt

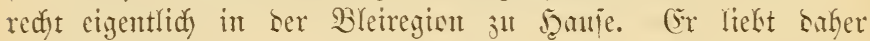
rufiges Salfer, unb wählt nidft itarfe Etrömungen ju einem

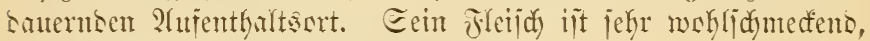
umb iteft sem ber Forelfe wenig Mady.

In Flïjien lieft rer Barje Dümpel unter SEefren, wo er

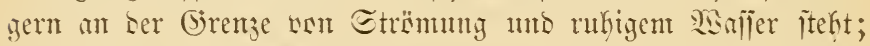

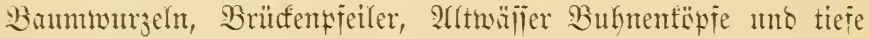

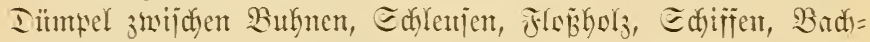
mïnoungen, Eteinankäufungen ant (jomtoe.

In Eeen jins Edyarkerge, sie lansjungentrtig worpringen unt Barjakerge sie kejten (i. Fngelitellen).

Ier Barja wiro jefr siel surdy Jeben uno Eenfen mit einem Binnfitidyen gefangen.

in ber Sitjee, wo ber Burja juffreich unt in grejent (Exemplaren werfommt, liebt er fteinigen, jansigen, frontigen

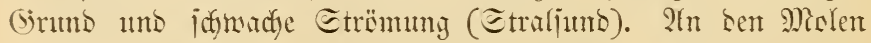

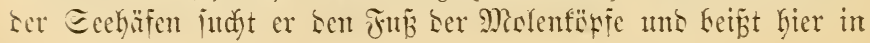




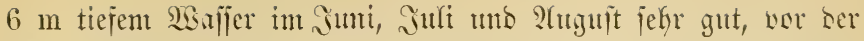
Flut, wenn bie Strënumg nuggeht, nidyt nad) ber Fitht bet cin= gekendem Etrome.

Eaidjzeit. Mitte März bis Ento Suni.

In Frïjien ijt ber Barjed int Frïgjakr in wenig tiejen Etrömungen uno beipt am bejten von 7-11 Mhr 3orntittngs

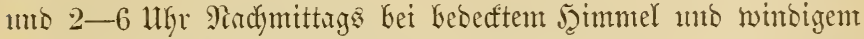
Wetter. Wo Ebbe uno Flut wirfen, ift beim Eteigen bes 2Baffers bie keite Beit. Int $\mathfrak{g} 0$ djommer geht er in tiefes

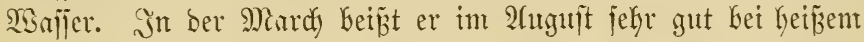

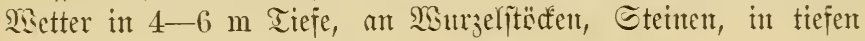

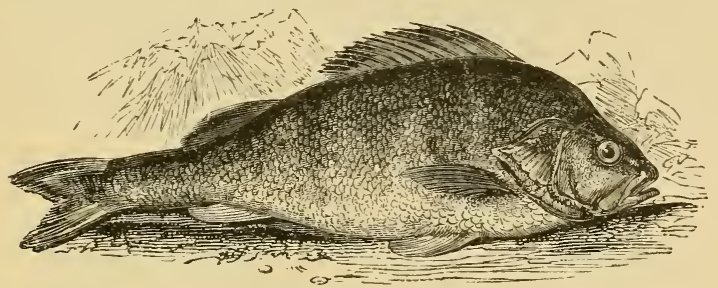

Fig. 343. Baridj.

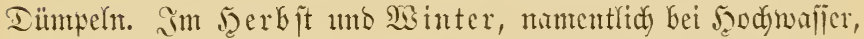

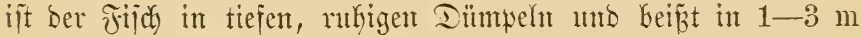

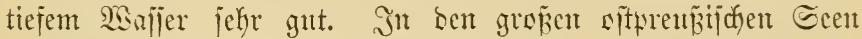

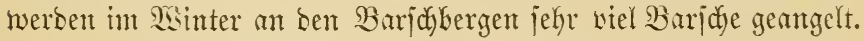

In Morbbeutiden Sanbjeen ift bie bejte Enijon won

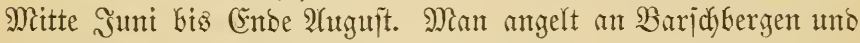
wortretenden Edyarbergen (i. Angelitelfen).

(5) runbföber. 20 Etumben vor bem 2tngeln wirft man 3-400 Iaumïrmer ein, bie in $30 \mathrm{~mm}$ range Stüdfe zerfdynttent fint. (2Bailey).

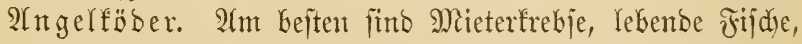

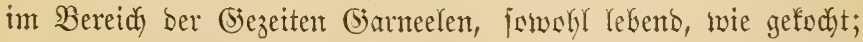

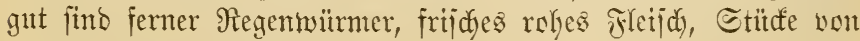
Fifchen, Maben. 


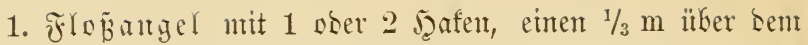
Girunbe, ben anteren in halber Tiefe.

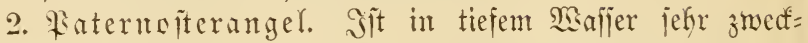
mäв̈ig. Man fildyt mit $2-3$ Jafen, und föbert mit rebenten Fijdyen unb anseren Röbern. Bei frautigent Sirunbe nimmt

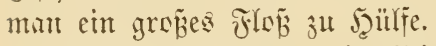

3. Epinnangel mit fleinen Jijddyen, ober fünthlidyen Efimtern.

4. Edyleptangel mit fleinen fümitlidyen Epintern liejert sit reidye Beute an grojen Barjoen, bejonsers in Eeen.

5. Fijdyenangel mit tlemen lebensen jijdyen ift im Mlai uno Juni jefr gut.

6. 5eben und Genfen mit Mieterfrebjen neer Tau= mürmern ift jefre gut in itümijidem sietter uns in fliejentom sisfier, Gejonbers in tiejen Dünteln mo Mirbeln.

\section{Der อ̄ander. Lucioperca Sandra. (ङ (ૃill, Plmaul, The Pike-Perch).}

Ier Zunter fehit in siejer= mo Atheingebiet. (Fr liebt tiefes fliez̃entes ser Tiefe auf. Sn Flüljen ijt er an unteren Ente ber itarten Eträmungen, woer hinter grejen Jeljen, noer in ben Etrom= iduncllen, ba mo jidy bas sisafjer über Feljen bridyt, zu juctyen. (E. fribt Fijhe uno Injeften, wie ber Barid.

Man angelt entweber mit ciner Flojangel uno Fijdyen,

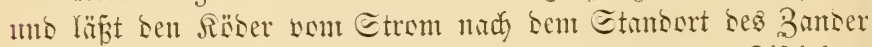
treiken - ober mit bent Piaternoiter uno ntefreren Fijdyen, uns wiryt sie 2fugel nahe wherhalb ser Etelle ein, no man sen Bander vernutet. Bijajoff empfieflt ein won Gräten befreites

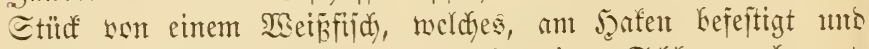
Durdy sarjer gezogen, jidy wie eine Edylange berwegt.

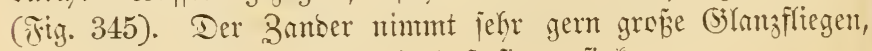
sie mit Blet bejdwert jins, jo saj jie werjinten. 
Minu angelt mit Flo

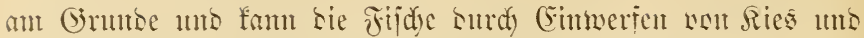

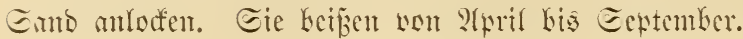

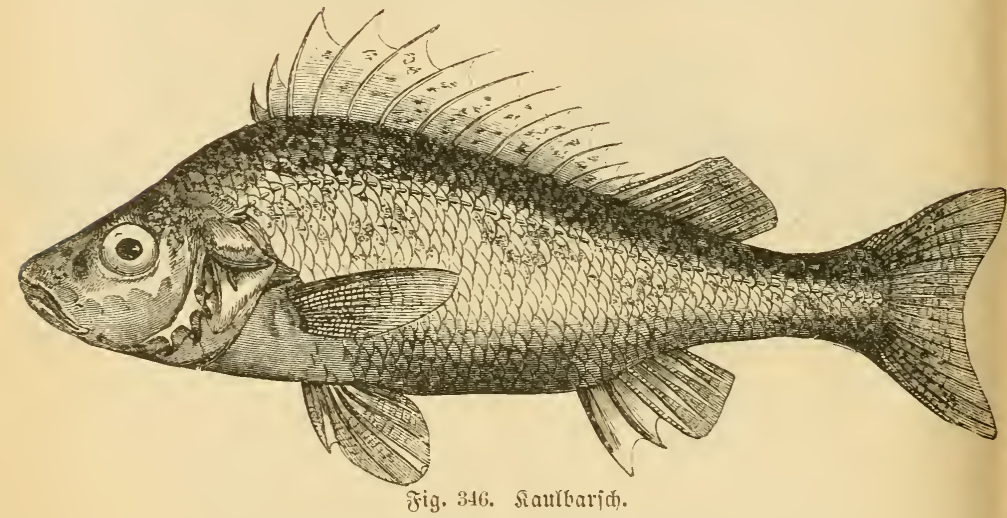

\section{Der อ̄ingel, Aspro Zingel,}

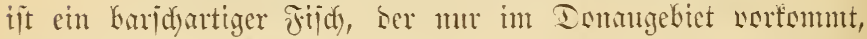

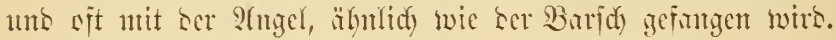

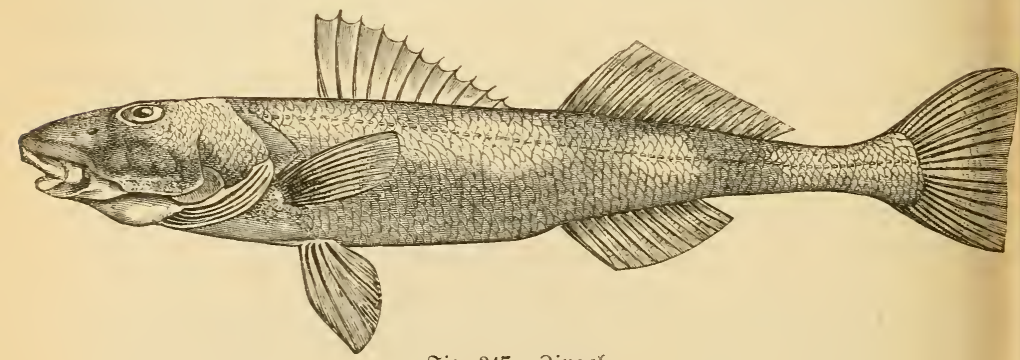

Fig. 347 . 3ingel.

26. Der Streber, Aspro Streber,

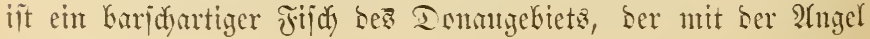
geraugen mits. 
27. Die 23arbe. Barbus Fluviatilis.

(Barbine, Barme, The Barbel, Le Barbeau.)

Die Barbe lebt in größeren Fliijen uto in Etrëmen, in

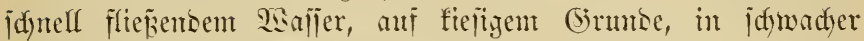

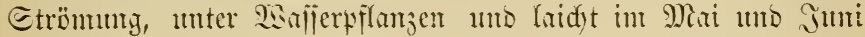

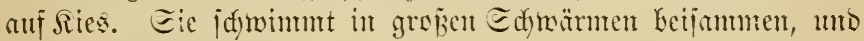

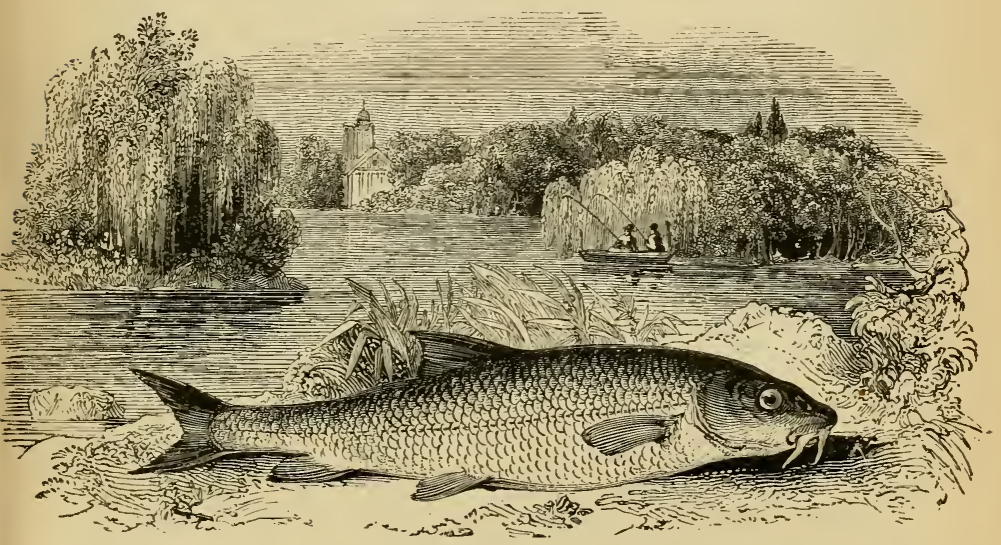

Fig. 31\%. Barbe.

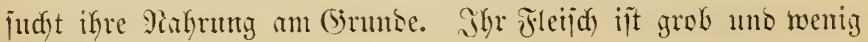
gejd)ätżt.

Fï ben 2 ngler gemährt jie ausgejeidneten Eport. Die widytigite Suefle iijt Briley wor! Nottingham, ber Len Fang Let Barbe mit Rorliebe betrieb.

Eaijon. 1 bia 2 2iscden mad ber Enidyzeit fränfelt bie Barbe, bonn geht jie in bie itärfiten Etrönumgen, ım fid

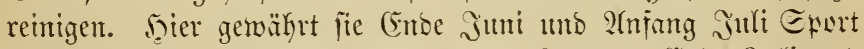

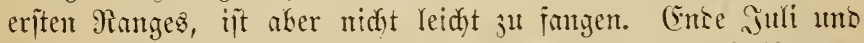
Anfang Plugult begiefst jie fid in tiefes Sanjer, unb bleibt bort bas ganze Jahr, mit 2hŝnahme won helfen, Keifien Tagen, an v. ๖. $B$ orne, Angelfitiderei. 3. 2ruft. 
Senen fie fladye Etrëmungen aufiludyt. PJit Sem (Eintritt von

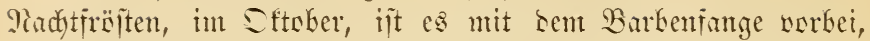

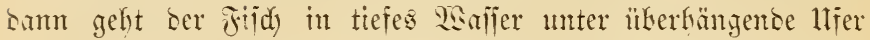
uno gejdürte Etellen, in jein sinterquartier, serfällt in Sethargie, und wirb erit int Frïblath nieser munter. Sm sie Barbe nidft.

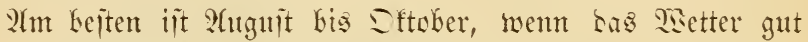
unb bas Sajer flar iit. Bis Mitte Mai ijt gute Beit, went bas Tsetter marm, uno bas 2 anjer niebrig uno flar ijt.

Ingelitellen. Der Gruno jei möglidyit Gorizontal, frei son Etcinen, Rrant, Jaols H. Sgl.

Bei flarem \$:alfer, wo man bie meijten Barben fanjen fann,

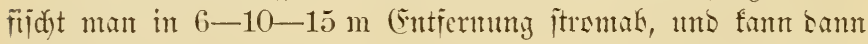
iehr guten Eport haben. Bei hohem, trïbem wajier iteht sie Burbe in $1^{1 / 4}-1 / 2 \mathrm{~m}$ Tieje auf Riesgrumb bidyt üker tiejent 2safjer uno itarfer Etrïmung.

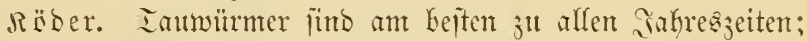

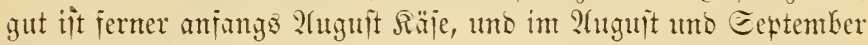
Grieven. Eproden fino wor ber Eaidjeit uno 2 -3 黑odyent nadyber gut, in mandien Flïfien füto aud Maren gut.

(5)runbfäber. 20 Etmisen wor bem 2tngeln twerbent 1500 Saumümter cingemorfen; voer 30 Etumben worber cin Siter

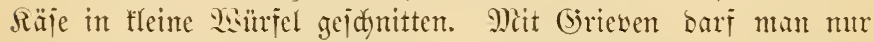
jebr verjichtig anjüttern, weil jie jefrr leidyt bie Fijđye überiättigen.

Flo unb mit möglicbit feiner Echmur ijt in allgemeinen am beiten, bejonbers bei ntebrigem, flarem Sisajier.

Ins Bosenblei ijt bei hohem, trïbem sajijer am beiten. Man wirit furz wer Beginn Des Jijdens 50 jeridunittene Inu= mürmer cin uno angelt $4-5 \mathrm{~m}$ entiernt.

Räjefijderei. Iie kejte Beit ijt anjangs 2lugujt in tiefent, rufigem

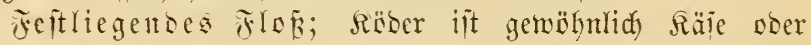
Biricwert. 


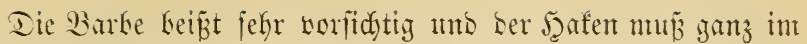
Röser werjtedt jeit. Se nadjem bie gijde mehr ober meniger idfett fins, nimmt man $5-6$ ober $7-8$ ober $9-10 \mathrm{~mm}$ breite Dafen won jtarfem Iraht. Die bejte Tiefe ijt bei flarem Saffer $1^{1 / 2}-3 \mathrm{~m}$, bei tribent 2 sajjer unter $1^{1 / 2} \mathrm{~m}$.

\section{Die Pröłe. Leuciscus Rutilus.}

(Motruge, Breier, Mottern, Motalter, Furn, Cdinalen, Rocfern, The Roach, La Rosse.)

Die \$röbe lebt in Eeen unb Flüfen in ber Bleiregion unb iit ein gejelfiger Jijh. Sie laidyt im April und Mai. Snt

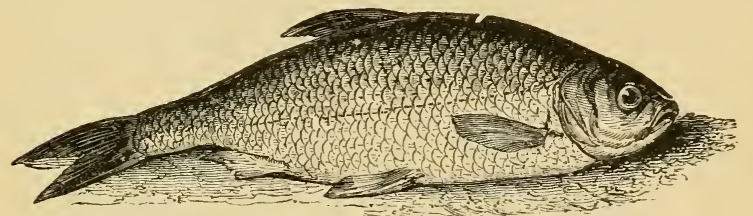

Fig. 349. Flötze.

Eonmer hält lie fid gem in Sirnutbetten anf Untiefen, wo fie

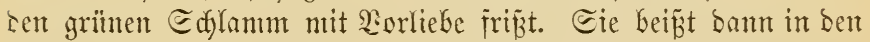
Sritifen nidyt gut, bie bejte Beit iit 2-3 Etunten nady Eonnen= angang, vber bei Tage int idäumenten Sbajjer unter Webren

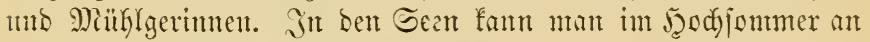
Ien Edjur= mo Barjdbergen in $4 \frac{1}{2}-5 \frac{1}{2} \mathrm{~m}$ tiefem $\mathfrak{B}$ afjer mit Mieterfrebjen grö́e Blöken in Menge fangen. Im September, wenn bie Safferpflanzen abjerben, judyt ber Fijd tiefes Waffer auf und jekt beginnt in ben Früijen bie bejte Enijon für

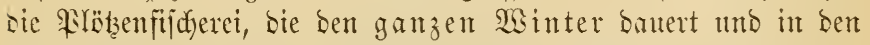

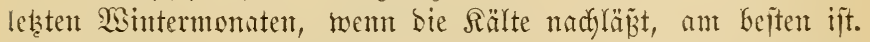
LEo Flophlolz aus bem Wajjer gezogen wurbe, ftehen im Winter

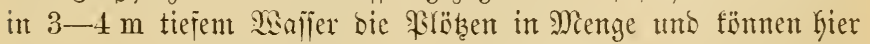
iefre gut auds unter bem Eije gejangen werben. Sism Edynee Len Boben bedect, ober wäbreno eines Edyneefalls iit günftige 
3eit, went aber bei Tanmotter viel હednecmajier im Fulje ijt,

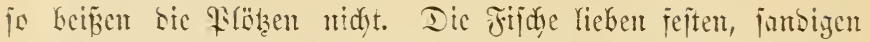

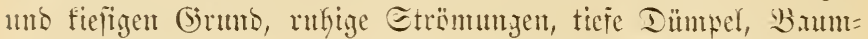

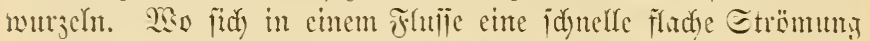

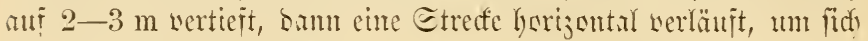
Dam wieber ju verfladyen, ba ijt eine gute PIngelitelle, namentlid wo bas Pnjteigen begiunt uno bas Futter zujammengetricben wirt.

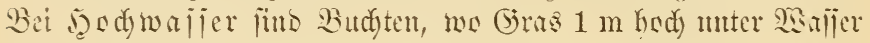
iteht, ju kendytert.

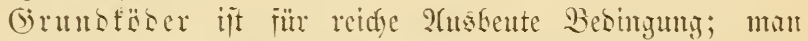

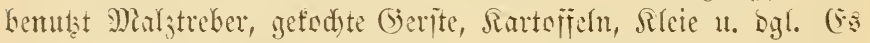

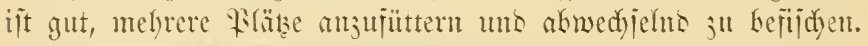

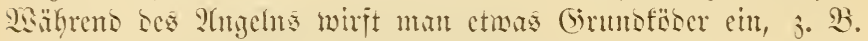

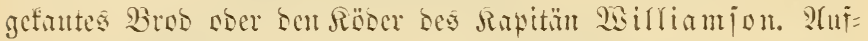

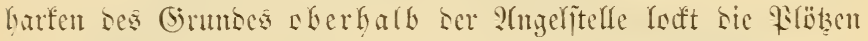

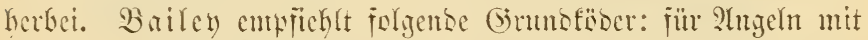

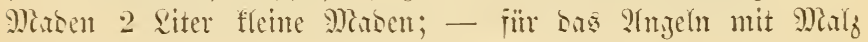

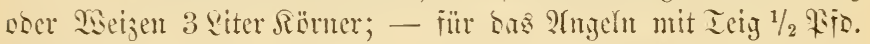

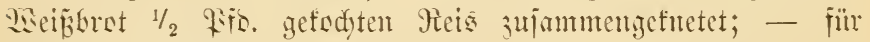

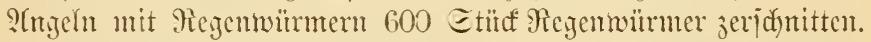

Mageltäser. Man jiidut mit Ieig, Maben, Mcieterfrebien, Regenwümern, gefodytem 2:eizen, Gerite, Erbjen, mit Enger=

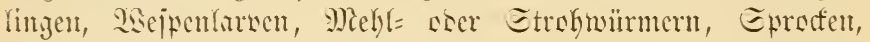
grümen 2 Ilgen.

Der Safen ijt $4-5 \mathrm{~mm}$ breit. Das Sioriad ijt jo jeit wie möglidy, vit ans einen Biferdebar beitehens.

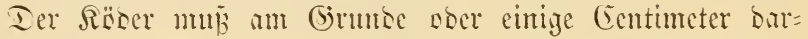
über jein.

Die Flo

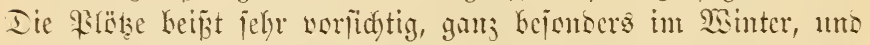

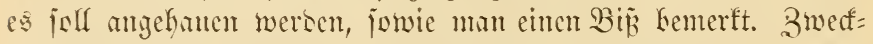

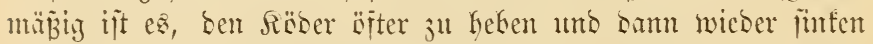

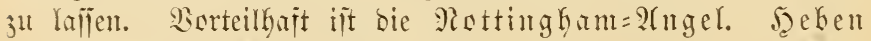

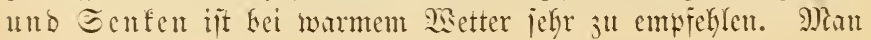




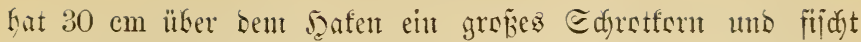

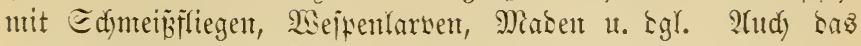
Bobenblei ober fejtliegende Fló ijt jwedmä̈ig.

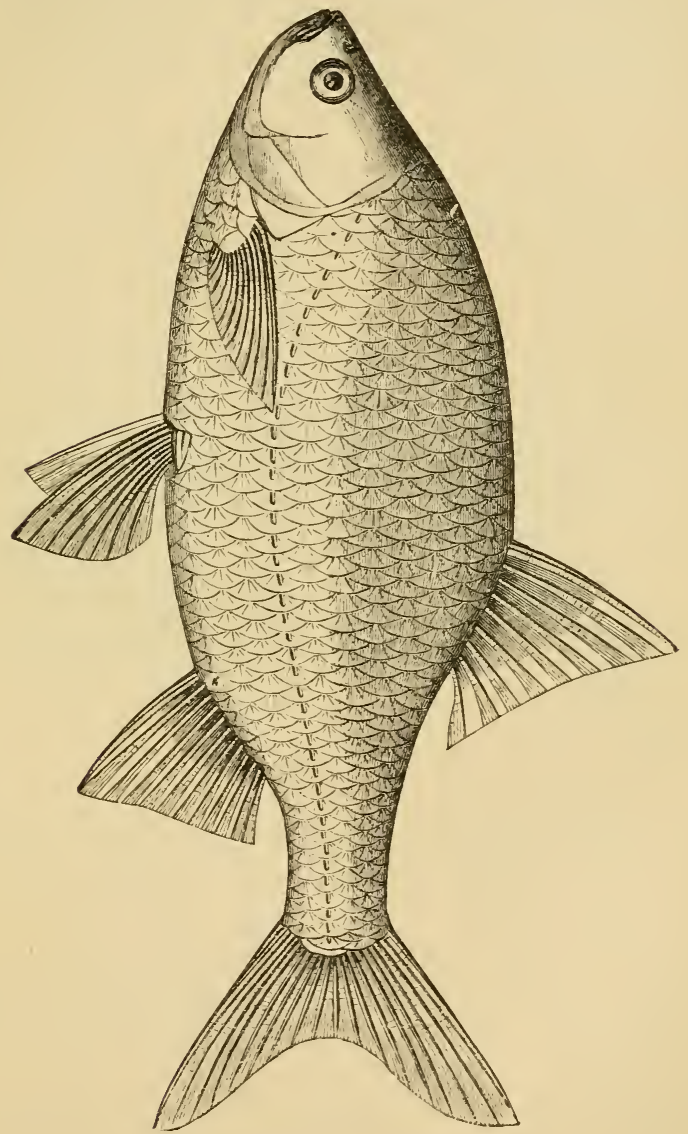

Fig. 350. Rotauge. 
29. Das Rotauge. Scardinius Erythrophthalmus.

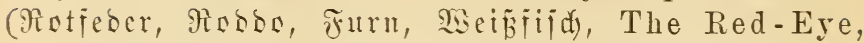

The Rudd, Le Gardon Rouge, Le Rotengle.)

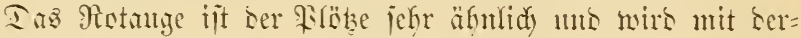

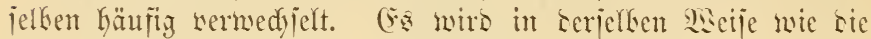
Pläkze geinngert.

\section{Der Blei. Abramis Brama.}

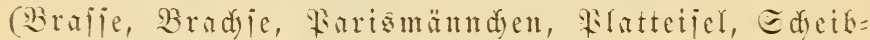
fleinzen, Euntifd, seid, Ricid, The Bream, La Brême.)

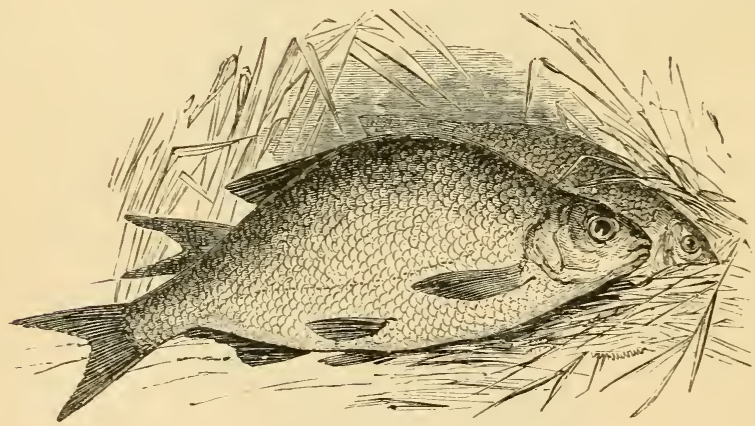

Fig. 351. Bici.

Ior Blei lebt gejellig in flitijen unto Eeen, liekt tiejes,

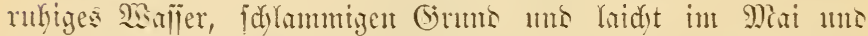

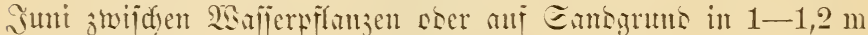
tiefen 经的er.

Eaijon. 2fm kejten ijt bie Beit non Sirentit (25. Silli) Kig Bartholomäi (24. 2Augut); gut fins aud Ceptember uns

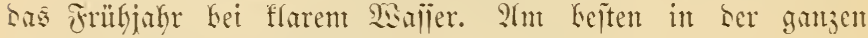
Eaijen iit flares, niesriges

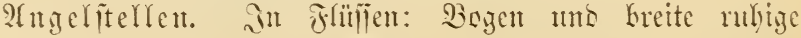

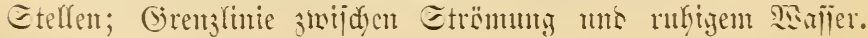


Sn Eeen: Der 2 tbgang bez Edarbergez neben tiejem Walier: momẗglid) $3-8 \mathrm{~m}$ Tieje.

Dodyajier. Der Blei verläpt bie Dümpel und geht in janfte Etrïmungen von 1-1,2 $\mathrm{m}$ Tieje. Sim Frïhjahr fam man

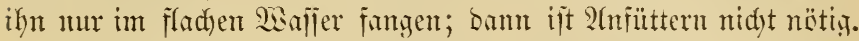

(5)runbfüber. 1500 Tammïmer 20 Etmoen vor ben

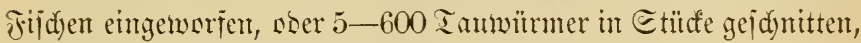
nder Brieven, noer Rëtfenmart.

Angelföber. 2(n beiten iit ber Gdyanz eines Tauwumb,

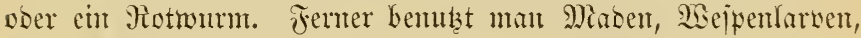
Seig, Erbjen, Bubnen, Rurtofïeln.

(D) afen 5-6 $\mathrm{mm}$ breit.

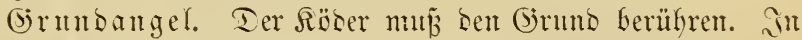
Fliefentom 2Eaffer fijcht man mit ber Piottimgham $=21$ ngel in

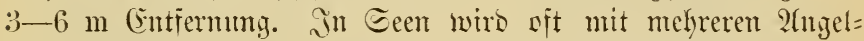

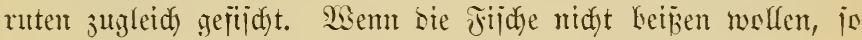
fijd)t man nidst weit geutug entfernt, oser nidst ba, wo ber

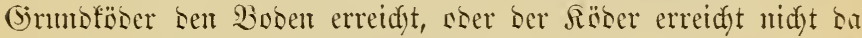
Den Grumb, wo bie Jifide itehen. Sor Begim bes Jijdens wirit man 20 Sanwütmer ein, bie in $2-3 \mathrm{~cm}$ lange Etüde gejdutten jüb uno wieberholt bies, wenn $3-4$ Fijdye gejangen find. Sit bas SBajler tiefer, wie bie Rute lang ift, fo nimmt

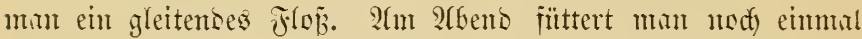
ftarf an und fam beu folgenten Tag anf ben befter Fanty redinen.

Ier Bfei beip̈t langiam und er wird micht fojort angefanten. Sueffen: Jjant Walton, Bailey, Ealter.

\section{Die Ğüfter. Blikka Björkna.}

(3ieber, Breitfifd, Blicfe, Botieder, Srattifid, Slieter, salbbrabien, Bobelpleinzen, Breinzen, Edyeiber, Bletten, The White Bream, La Bordelière.)

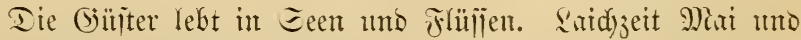

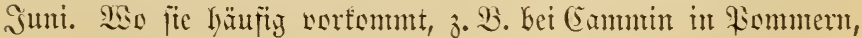




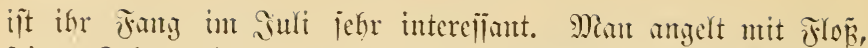
Feinter Edyur, in 6-61/4 $\mathrm{m}$ Tieje. Ffngelföber: mürfer Scig nus Rartoffelut unt alter geriebener Ecmmel, Regenwürmer, Miaben. Sint Minpinter Ese wirb jie im Eeptember in $91 / 2-19 \mathrm{~m}$ tiefent

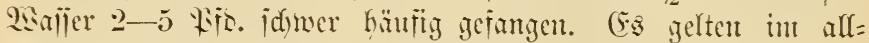
semeinen bicjelben Regehn, wic für ben Bleijanty.

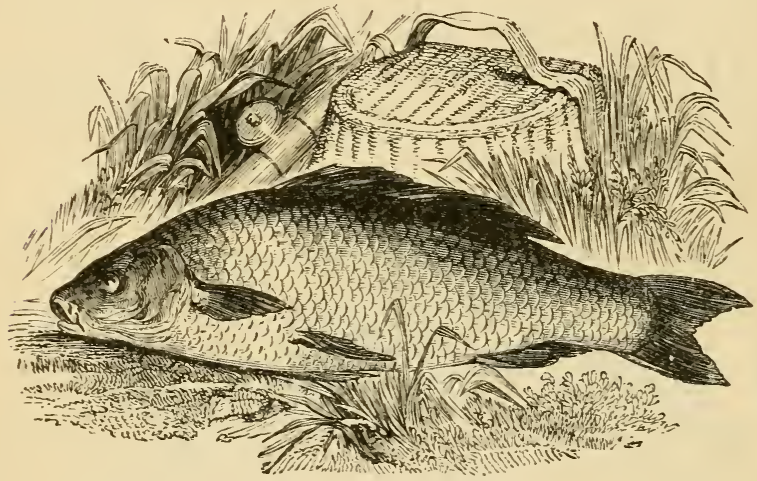

รig. 35.. sarpien.

52. Der Karpfen. Cyprinus Carpio.

(The Common Carp, La Carpe.)

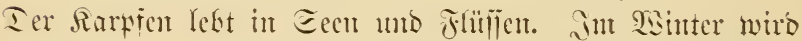

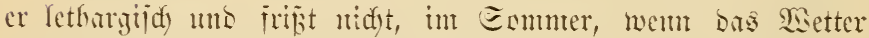

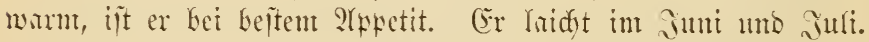

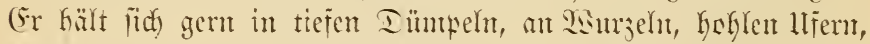

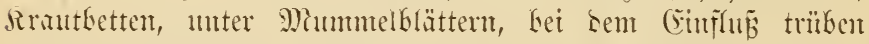

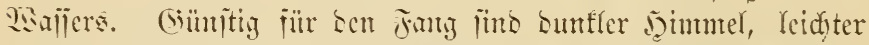

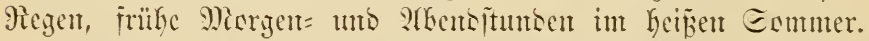

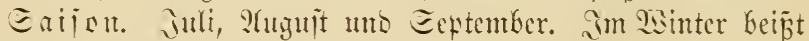
ser ijijd nidyt.

Grutbfäber. 700-1000 Inumürmer, in 2-3 Etüfe

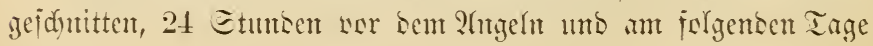




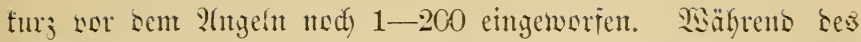

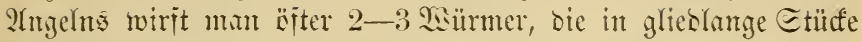
jerjanitten funs, ins sajier. (Es ijt gut, an mefreten Eteffen anjuïüttern.

Rugelfïber. Edyanz eines Iaumums ober Fotwurms.

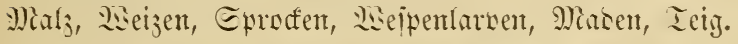

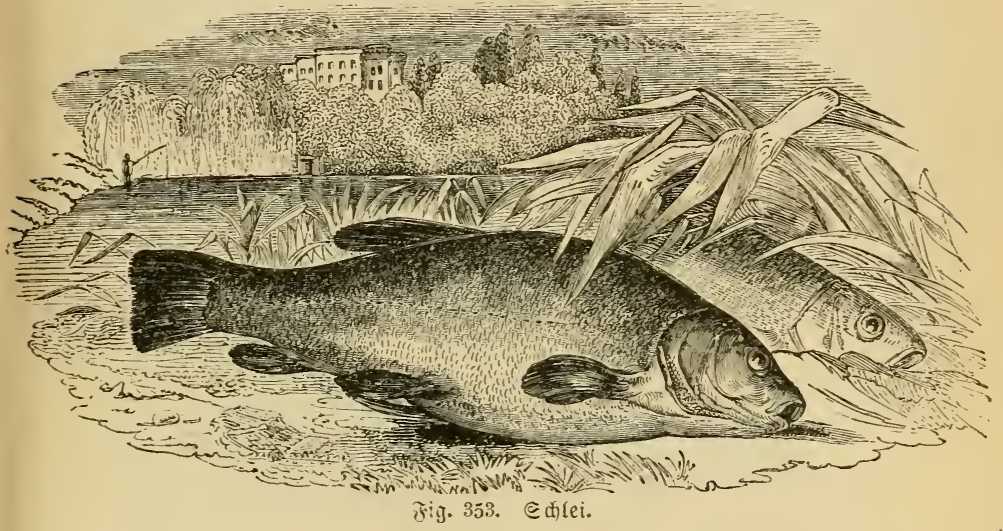

Man fijde mit ber Misttingham = 1 ngel, ber Röber liege

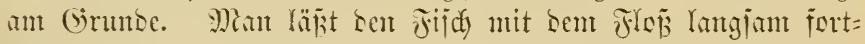
idfwimmen, ehe mon anthint; bie 2higelitelle mur wenigitens

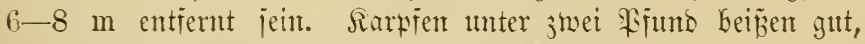

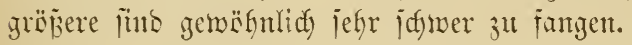

\section{Der Sđ̆let. Tinca Vulgaris.}

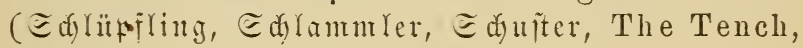

La Tanche.)

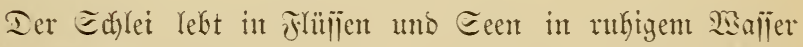
mit id)lammigen Srunde; er liebt Rrautbetten, bejonbers fleine sifente Etelfen im Rraut. In ben Ecen jteft er bei Keipem 2ietter an ben Gelegen. Die Eardyzeit ijt Jutl. 
Bismeilen beipt ser Edylei gut, gemöhnlidy iit bieß aber midyt ber Jall.

Eaijon. Juli bis Detober, bismeilen beipt cr and im Jrübjakr bei jünem, warmen Saetter.

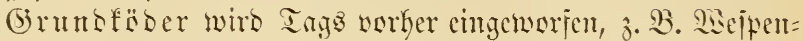
larven sber Regentwürmer.

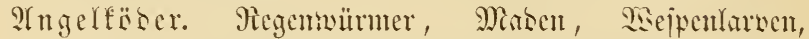
Eproffen, Mnicterfebje, Teig. 2lngel wie beint Rarpfen. Dor

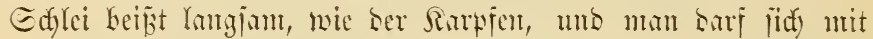

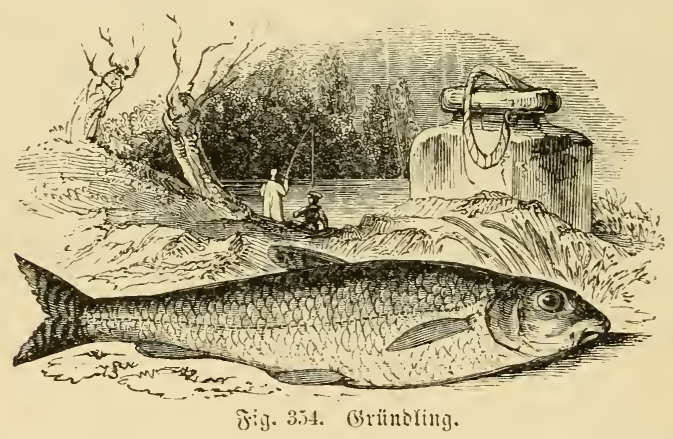

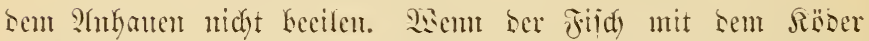
ipielt, io jielgt man ifm senjelfen einige Centmeter fort, was ify verleitet, endlicf) zu beijen.

\section{5ł. Der Gründling. Gobio Fluviatilis.}

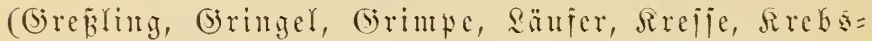
fifd), secterfij(y), The Gudgeon, Le Gonjon.)

Der Grïnoling lebt gejellig, liebt flares, nidut jtarf jtrömen=

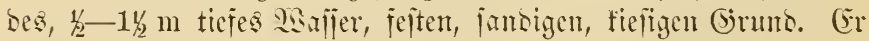

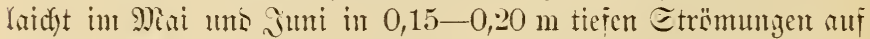
jeinem Rites.

Enijou. Suli bis Ceptember ijt am bejter; or beipt bas 


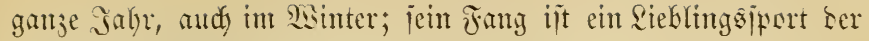
englijdyen Damen.

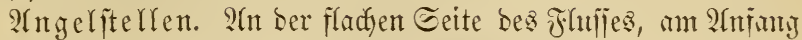
nder Enoc tiefen 2iallers, ftarfe Etrömung, 0,60-1,20 m Tieje, Riesgrunt.

(5) runbföber. ca. 20 fleine Maben einwerfen; PUffarfen

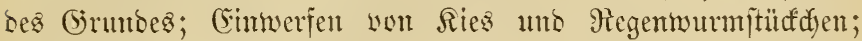

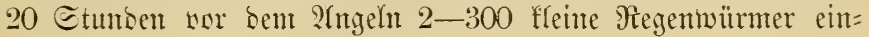
werfen.

Pngelföber. S(m bejten fint Mlaben und Regentütmer; Maben am 2hnjung ber Saijon, Megenmürnter bie ganje übrige Beit. Man nefme inmer beibe Röber mit, um zu probieren, weldyer an befter ijt.

Flobangel, fleites Flok, Jaten $5 \mathrm{~mm}$ breit; ber Röber liegt am (Srunbe. Maan muß idyell anhauen. Suellen: Baileb), Y)eile, Griter.

\section{Die Zaafe. Chondrostoma Nasus L.}

(Munditid, Mafrele, Edwarzbady, Bapfen, Buppe,

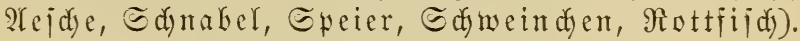

Dic Naje lebt in ber 2 (eidjen= nno Barbenregion, fie feblt in

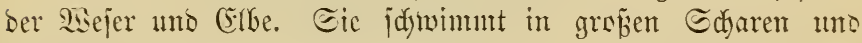
fteigt zur \&aidzeit (2rpril uno Mai) sft in ungekeurer Mienge int fleinere Filiije.

Mady Dr. Bettentorf wirb fie in ber 1turgegend won Bom in folgenber SEeije mit ber $\mathfrak{A} n g e l$ gefangent: $\mathfrak{A}$ nfüttern ijt not=

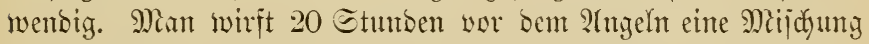

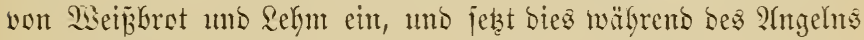
fort. Sorfad) won feincm (b)ut, $3 \mathrm{~m}$ lanty, braum gefärbt, gant3 empfindlider Edynimmer; jefr fleiner Jgaten; Eenfer $10 \mathrm{~cm}$ wont

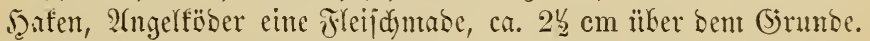
Man lyant jofort kei ber Yeicejten Berüfrung an, ba- ber Fijd

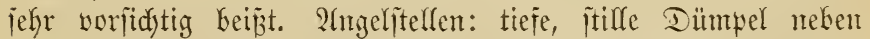
itarfen Ctrönungent. Der Bafen piffegt jefre jejt zu jüzen, mo 


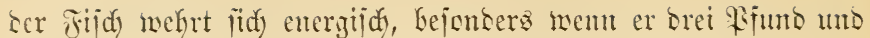
idwerer ijt. (sa werben oit jehr gute Jünge gemadyt.

\section{Die flunder. Platessa Flesus.}

(Édorre, The Flounder, La Plie Franche.)

Die Flunber Yebt in Meere, geht aber and weit bie Flülife

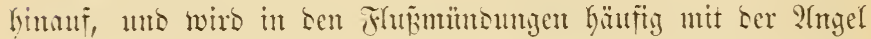

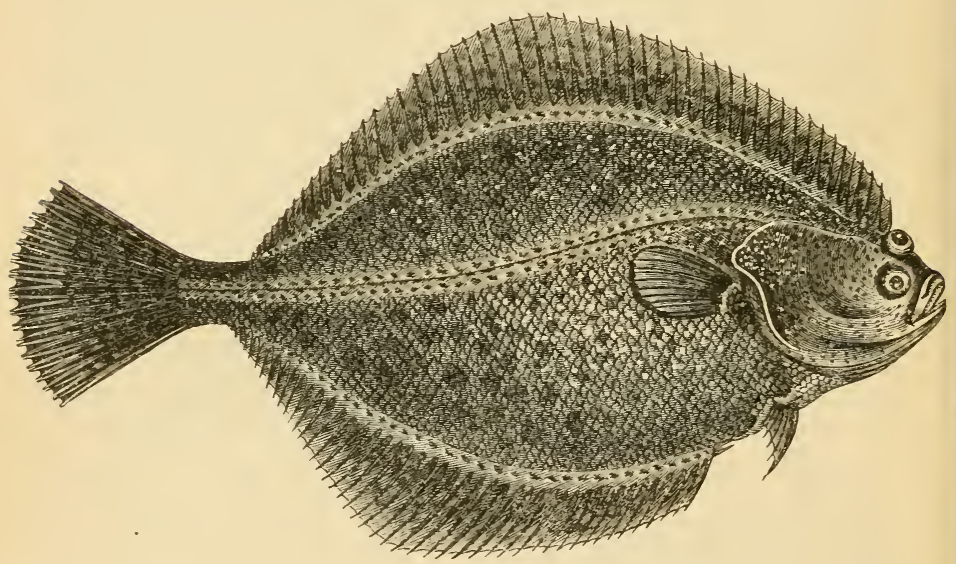

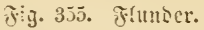

geimigen. Cie hält jith auj junsigem soer fiepigem Grunte, an

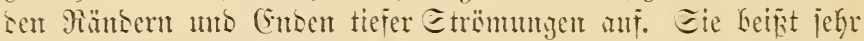

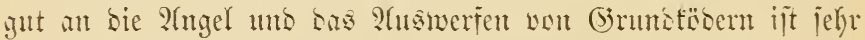
wirfiant. Die bejte Beit ijt, went Sab skrijer ebbt ofer flutet, bei warmem Setter und etwas sints.

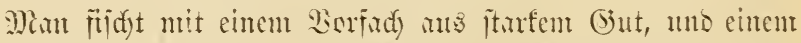
5-6 mm Greiten Dafen. Man fösert mit Megenwïrntern boer (irteven, bie am Grunse liegen milïen, und lǟt bem Jijue

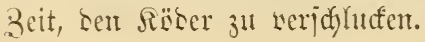




\section{Der 21al. Anguilla Vulgaris.}

(The Eel, L'Anguille.)

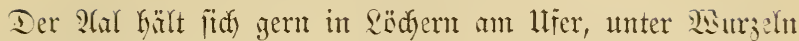
ıno Etringaufen, unter Mä̈glgerimten, an Brüdenffeilem umb

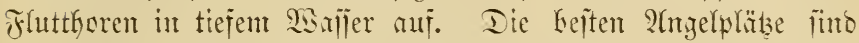
tiefe jitlle Dümpel ober idwadis Etrömungen, junbiger soer

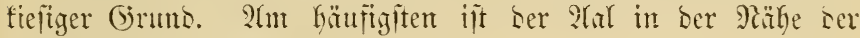
Münoung des Jutfes in Dir Meer.

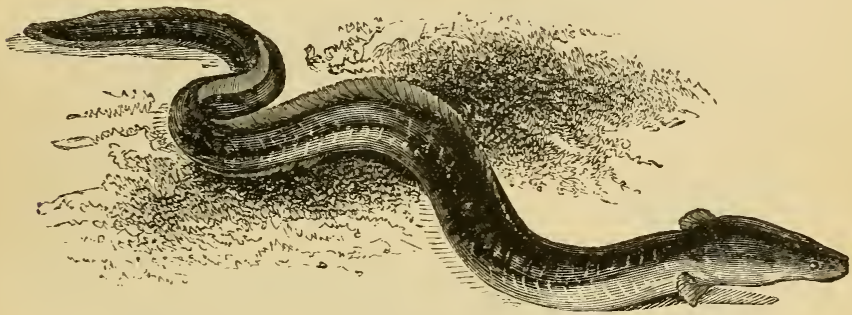

Fig. 356. 2al.

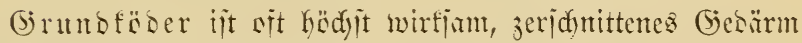
mit Cano gemijat ijt jefr baju geeignet.

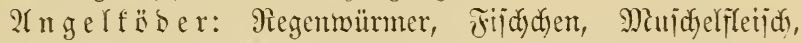

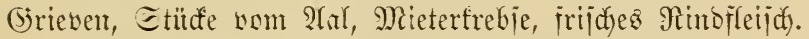

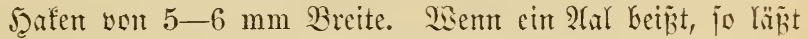
man ifm Beit, ben Röber geförig ju serfdhlucen, was er frit immer thut. Sisem er gelantoet ijt, fo tötet man ifnt burdy einen Ctich burd) bas (stenidf.

Ier Röber joll am Grumbe liegen.

Man haut erjt nad) 2-3 Eefunben an.

Fang mit ber Etopinabel. Man windet eine Etopinabel

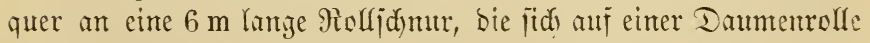
befinbet, Fig. 256. Tamn jofiebt man bie Stopinabel fo in 
cinen Inumurm, baj jie ganz Larin werkergen ijt, uno jtedt bie

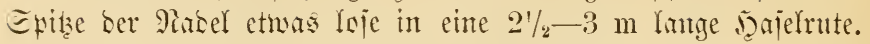
Sieje nimmt man in bie redyte, bie Dannenreffe in bie Yinfe

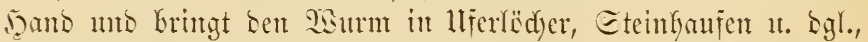

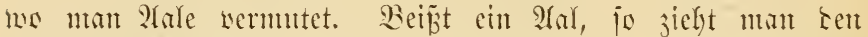

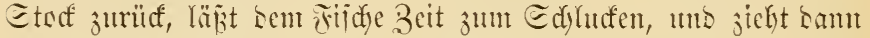

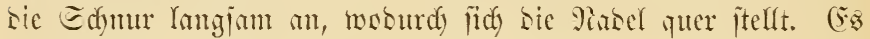

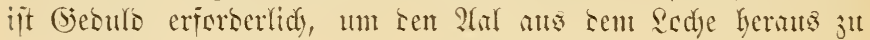
befommen.

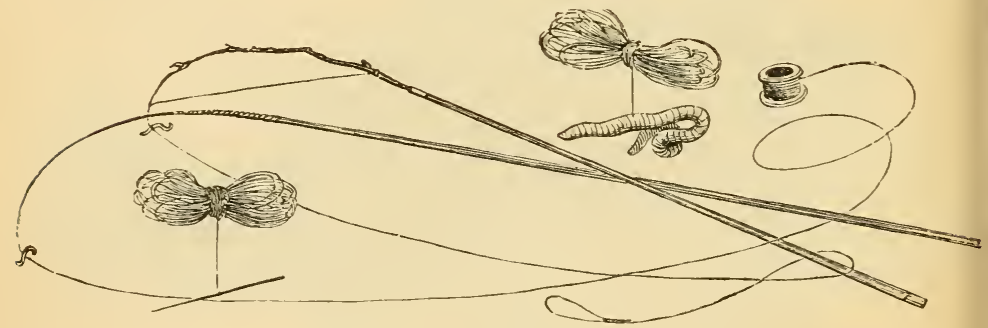

Fig. 357. Hafangel.

Fang mit sem moltenen Fasen. Man jielgt eine gröbere

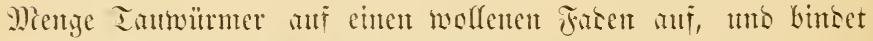
ite burdy eine Edyur ju cinem Bünod juiantmen, weldyes ntan

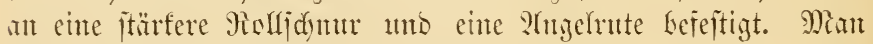

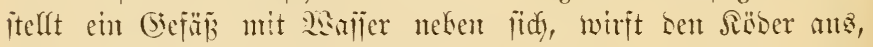

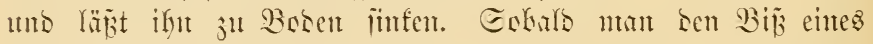

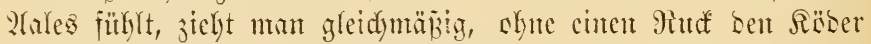

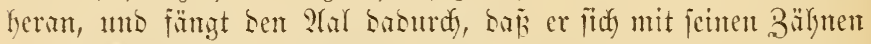

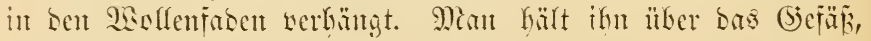
lä̈̆t ifn Gineinfallen, uns wirft ben Jäser wen neuem aus. Sin

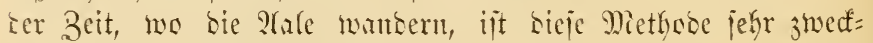
mäñig, uto weridyant sit cine reidye Beute. 
58. Die Elritze. Phoxinus Laevis.

(Pirille, Fiere, 阮mben, The Minnow, Le Veron.)

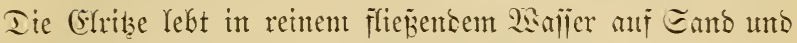

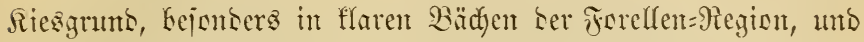
laidyt im Mai unt İuni.

Cie ijt cin vorjüglidyer Röberfijdy. Man finn lie mit ber

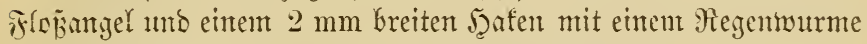
fangen, went man anf $2 / 3$ ber :

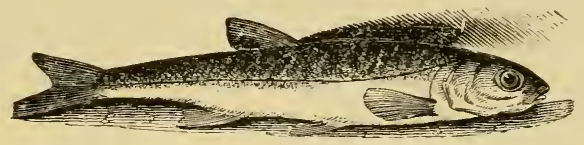

Fig. 3i8. Elritze.

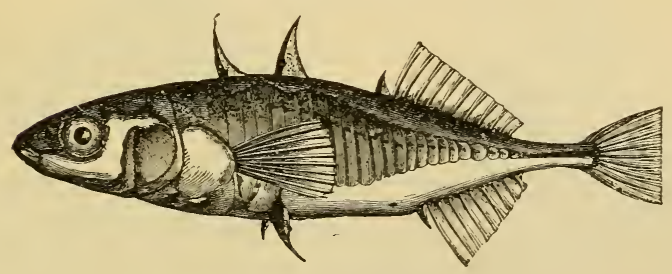

Fig. 359. Etidyling.

59. Der Stidgling. Gasterosteus.

Mant unterjucicet ben genteinen Etidyling (G. Aculeatus) unto ben fleinen Etidyling (G. Pungitius). (Etadyelinģu, Eteferling, Etrablbarid, Mob̧ärid, The Stickleback, L'Epinoche.)

Man faun ben Etidyling mit cinem $2 \mathrm{~mm}$ breiten $5 a$ afen unt

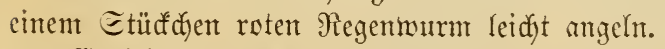

Gr lebt in ben fleimiten Säjlerchen mb Gräben unb ijt oft

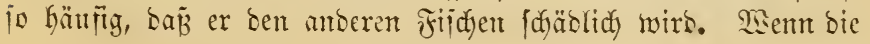




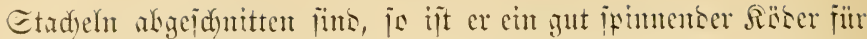
Barjd) und Farelle.

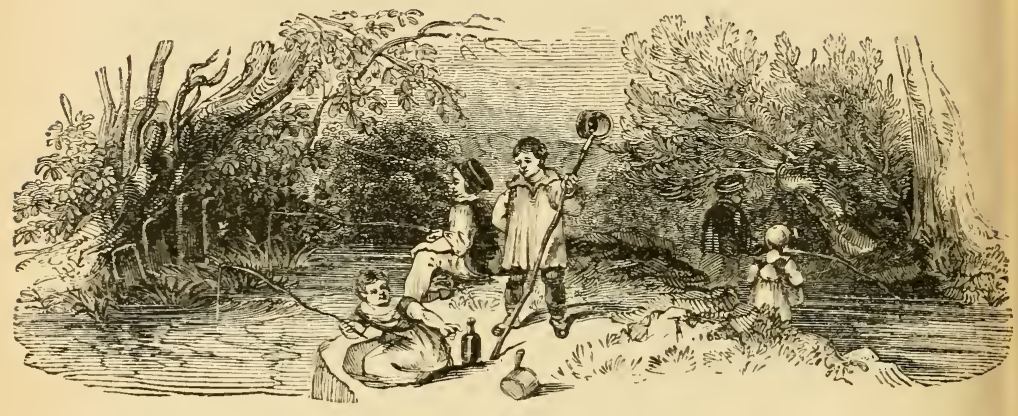

Fig. 360 Etidfingrang.

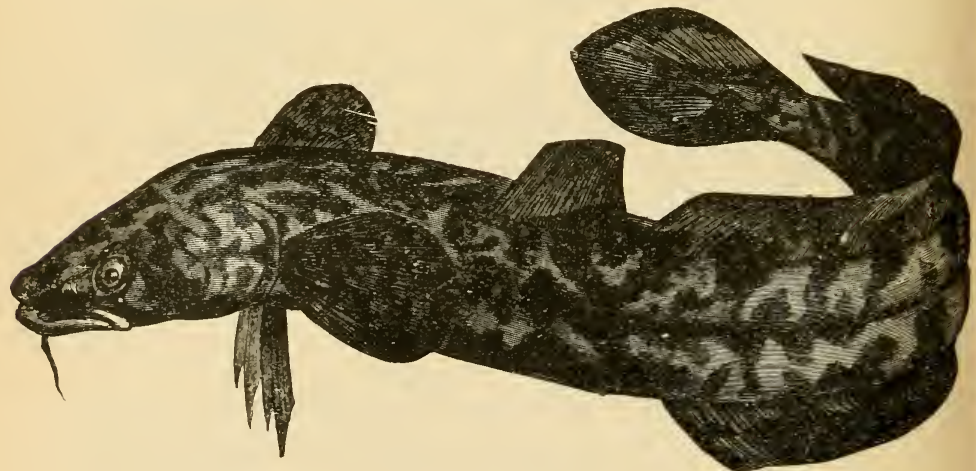

Fig. 361. Suappe.

40. Die Quappe. Lota Vulgaris.

(Palrute, Mutte, Ireijde, Iriije, Mufunfel, The Burbot, La Lotte.)

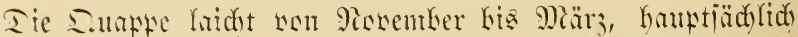

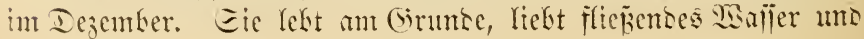


Lerfriedyt jidy unter Eteinen, 2Eurzeln unto in Ë̈djem. Cie irijt Injeften, sïumer unto jijde.

Sie füngt fïcf jelten an serगुatiangel, leidst an ber Pegenngel, nu= utentlid) int frïfyen

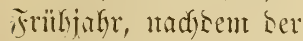
itrenge Frejt woriberift. Sïser: Fijddelt, Frëide, Inumümer,

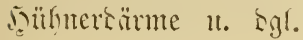

\section{Die อีärthe.} Abramis Vimba.

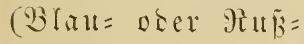
naije, Eimol.)

Eie jofent int Seseje uno Jigein= zebiet ju fefylen und fommet hautptiädylidy in

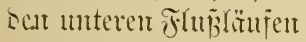
uns in ber Barben= Jegion nex. juti.

Raidyeit: Mari und

Eic wirb jerten ge= migelt. 2rugelföber: Erbjen, Teig, Ninbert,

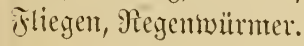

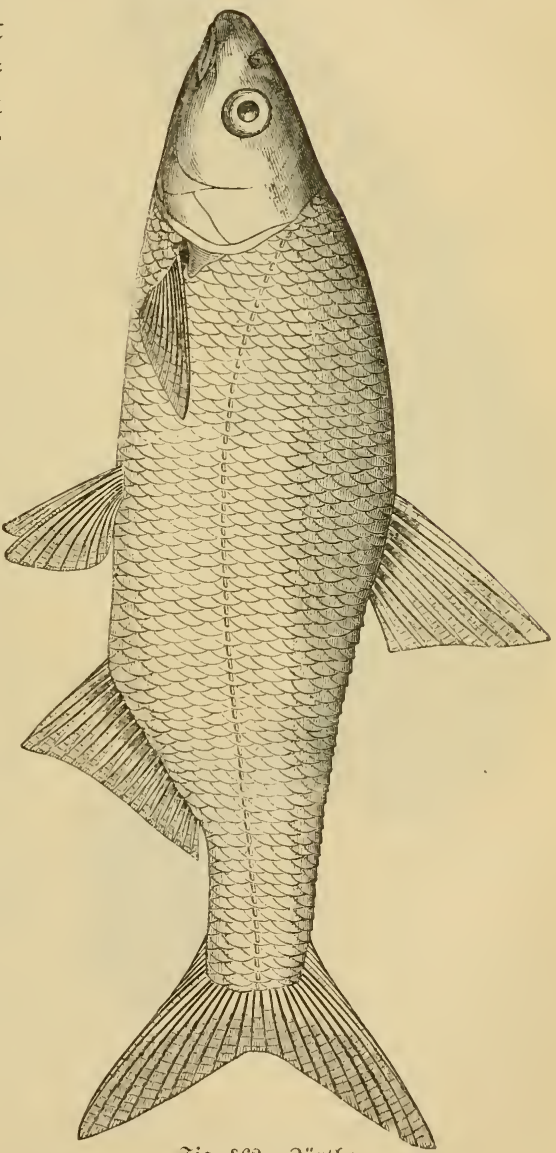

ริig. 562. 3ärthe.

v. ๖. Borne, 2Angeffiiderei. 3. Tuศt. 


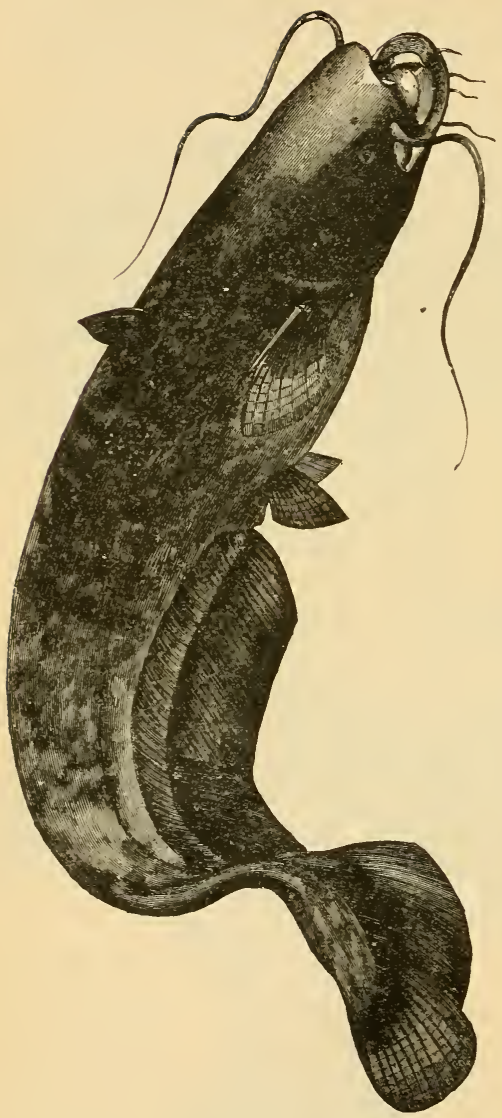

\section{Der Wels.}

Siluris Glanis.

(2Ealfer, Edyat,

Edaitu, Edascu,

$$
\text { (d)an.) }
$$

Ter Tiels ijt im ijit= lidyen Deutidylano nus int Donangebiet wer= breitet, feflet aber fait gant int 2escer= 1410 Migentughiet. (Ex lekt in Eeen Into Flüijen Des efrenen Santos añ ichlommigem Grumbe, wo er ruffing in ber Iiefi itebt. (E) raidyt im Juni IIIt Juli an sisajfer= ffilanjen. Mrit ber Santo= angel wirb er jeltener gejangen als mit sege= angeht. Röber fint: Jijuc, Frëldye, Rägel, Mäuје «.

Tig. 363. פBels. 


\section{Buveiter Teir.

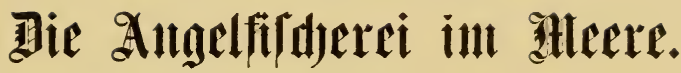

Das 2erfahren ift bem, weldyes im Citzmaijer angemantst

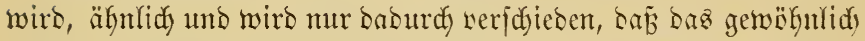

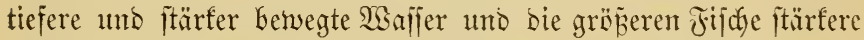
(S)eräte notwenbig madjen. Man angelt entweder vom Sanbe ober wou einem werauferten ober fahrenden Boote.

\section{Erfter 21bīhnitt. \\ Altgelmetfooen und Anngelgeräte.}

\section{Die Grundangel.}

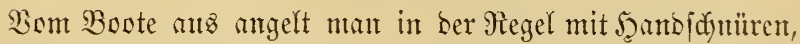
vom Hfer jowohl Giermit, wie mit ber Ingelmute.

Die Edyur ift entweder georeft ober geflodyten. Eritere joll nicht zu breff jein, bantit jie fidy nidyt verjedürzt. Man hat

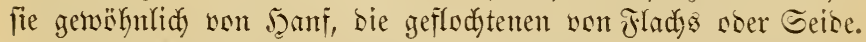

Willcod empfiehlt in jeinem Sea Fisherman folgende Etärfen für gedrehte Edfnüre:

Tir. 1. $5 \mathrm{~mm}$ Durdymejier. $1 \mathrm{~m}$ ijt $20 \mathrm{gr}$ jefwer. Wirb zil Regeangeln nady idweren Fijdyen verwentet. 


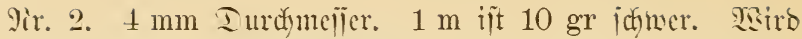
3u Grumbangeln mady Nittlimis uno Miecral gebrandst, wenn 3-6 \&ifs. jomere Eenter notwensig jüto.

Эir. 3. $2 \mathrm{~mm}$ Iurduntiler. $1 \mathrm{~m}$ ijt $4_{2}^{1} \mathrm{gr}$ juner. Tient

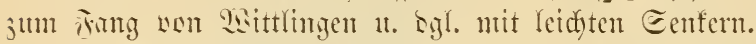

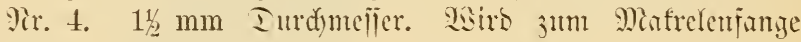
nerwentoct.

Pir. 5. $1 \mathrm{~mm}$ Durdymefler. SEird ju Barfüdtern bei ber LEittlingsifijdere vermendet.

Iie Eenfer, welde bei ber (Brunbangel angebradst werben,

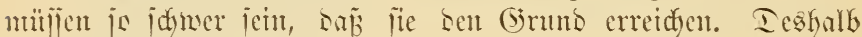
fïto bei grojer Siefe uno ftarfer Etrënumy ser Ebbe uno flut sit jefr idwere Gewidate erforberlid. WEenn man mit mebreren Edunuren jugleid) angelt, io nimut man bie Eenfer werjoficson

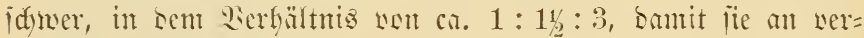
idfesenen Etellen ben (Grunt erreidyen nuto weridfeben meit son ber Etrümung fortgejüfrt merben. Bei 60-90 m Iiefe und itarter Etrömung find bie Eenter $21 / 2-4-7$ Pifut idner.

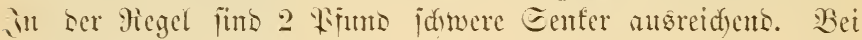

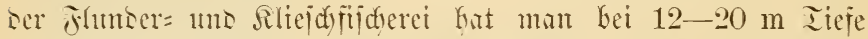

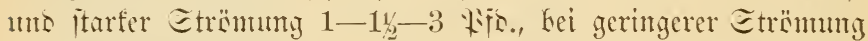

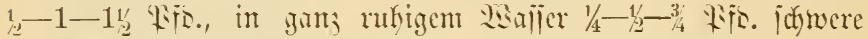

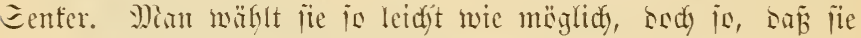
jidjer ben (Sirunts erreichen.

Ier Eenfer wiro ummittelbar an ber 2Angelidumer befejtigt; leime Form it weridsesen:

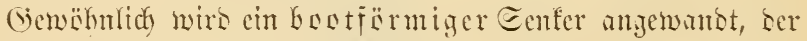
an jesem (Ente einten, mehrere Iezimeter langen, mit Sejen ver= jebenen itarten Iraht hat, um an ber einen Eeite bie ?tngelidnut, an ber anberen bas Borfind bejejtigen ju fünnen. Dearber

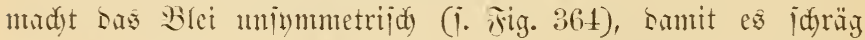

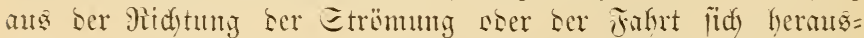

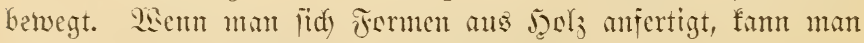

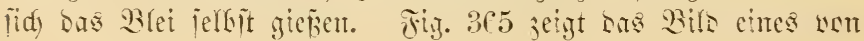




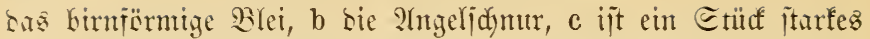

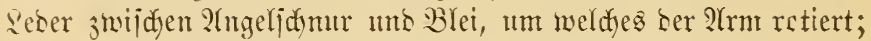
d ijt eine MetalfGülie, um weldye ber (Sinam (Boom) $f$ in Tig. 367, jomie Ler Ioffelarm (Chopstick) $f$ in Fig. 368 rotieren fam.

In Fig. 369 iit d Dearber's afmefmbarcr Inffelarm,

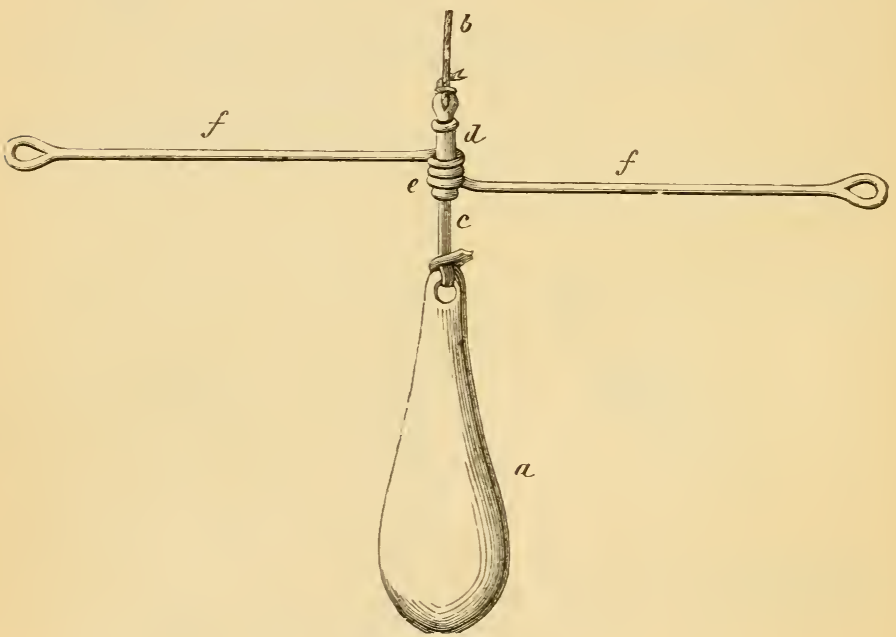

Fig. 368. Senter.

Ler jidf ganj leidjt uno juntell an einem anteren Eenfer an= bringen lä̈t.

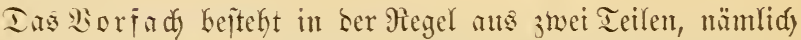

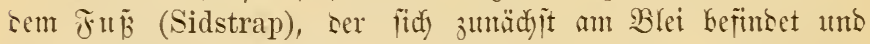

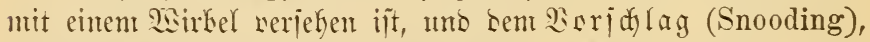

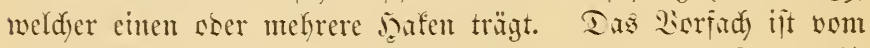
Blei bis jum erjten Dafen beim 2lngeln make am Sambe $1 \frac{1}{4}$ Gis $1 \frac{1}{2} \mathrm{~m}$, in sfifener Eee $3 \mathrm{~m}$ lang. Das 2 sorfact wirb aus

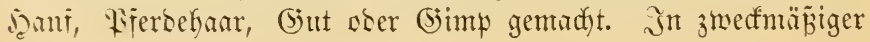




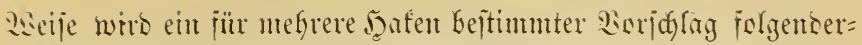
ntaben genudyt. Mian bringt an eintent ca. $125 \mathrm{~cm}$ langen Faben won Gimt an jerem (Ente eine Edyleife an, unt legt sanm won einem (snte $10 \mathrm{~cm}$ jo um, son eine sritte Edyleife entitefst, bie

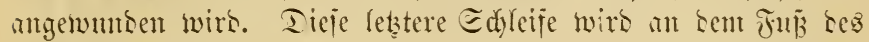

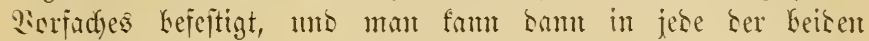

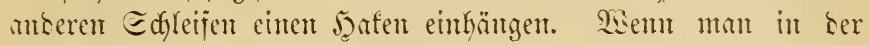

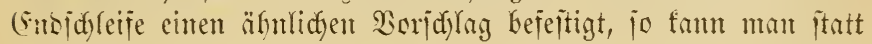

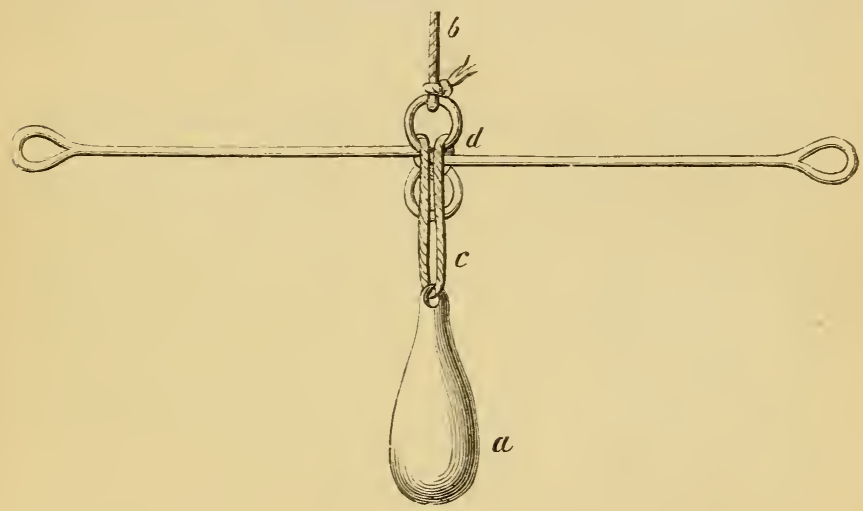

Tig. 369. ङenfer.

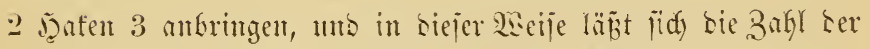
5aten beliebig wermehren. Jis. 270, E. 144.

Die jafen fint nerzimut, bantit fie nidyt rojten, uns mit Whättdyen ober Seje verjeken, unt jie jidjerer befejtigen zu fünmen.

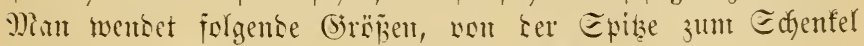
semeijen, für sen Frnty ber terịdiebenten Eeefijde an:

$38 \mathrm{~mm}$ für Thun= unt anbere grofie Fifdye.

26-28 mm Miceraal, Rabeljau.

23-26 $\mathrm{mm}$ Ecebaridy.

12-20 $\mathrm{mm}$ 厄teinbutte. 


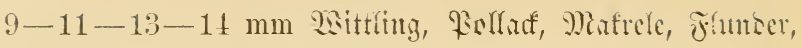
Rifiejde, ?hrl.

$8 \mathrm{~mm}$ Necrbarbe, Etint.

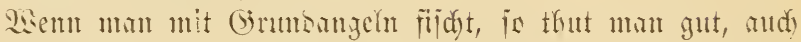

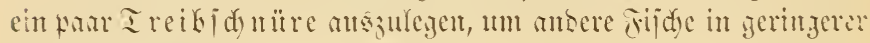
Iicfe z" intzu.

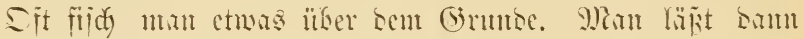

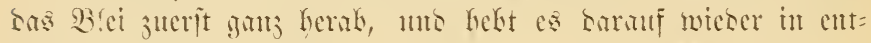
ipredenser secije. Hefer sem (intuse wirt ser Räser nidyt is reidyt wa Sirkben unb Eecitemen abgejeljen.

\section{Grunsff̆nur jum fang von flunbern uns Klielden.}

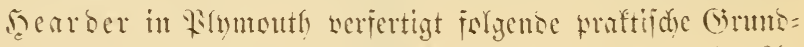

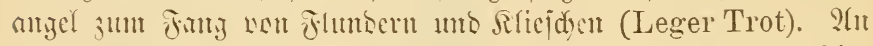

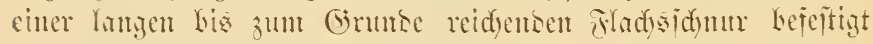

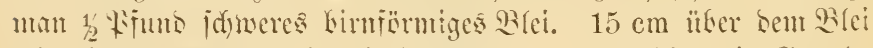

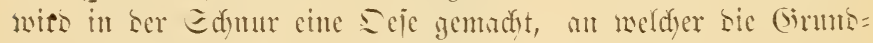

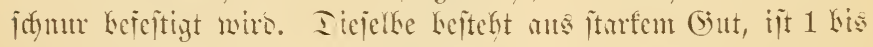
$6 \mathrm{~m}$ fong uns ijt an anseren (s)to mit cintent zweiten fleitreren

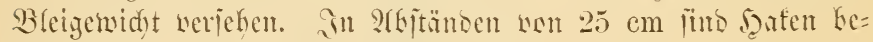

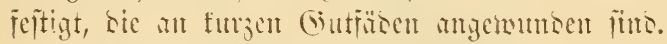

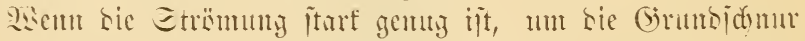

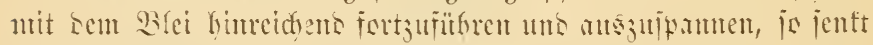

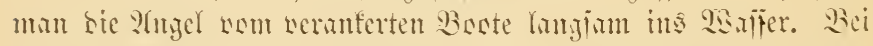

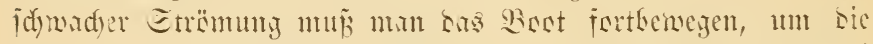

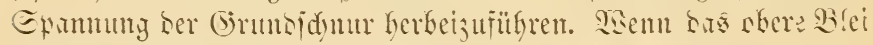

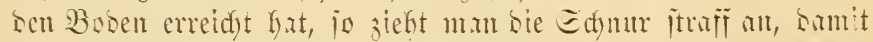

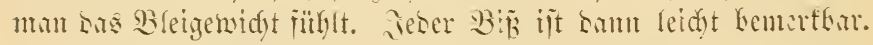

\section{Die fifheret mit ber Treibjąnur. \\ (Drift-Line Fishing.)}

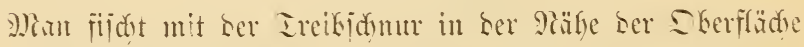

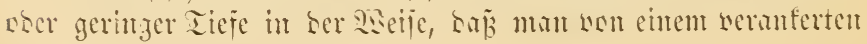


Baot, woer aud) woht wom llier, bie Edyur non ber Etrömung

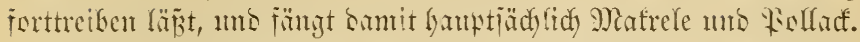

Die Treibjdnur ijt entweser in ?(bjtänoen won $4 \mathrm{~m}$ mit

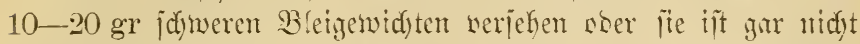
bejdyert, entipredyand ber Etürfe ber Etrümumb, aber bet gamb

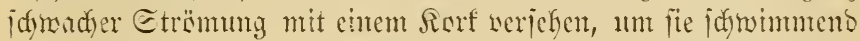

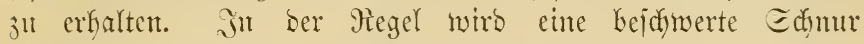
angewand. Bei fdyadjar Etrïmung giebt man etwas mefir

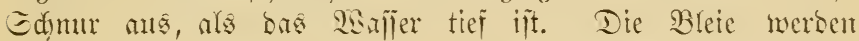

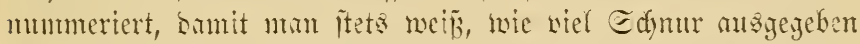
iit. Bei 6-8 m tiefem \$sajjer werden ber Etärfe ber Etrïmutg

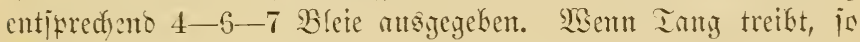
minifen alfe parar Minuten bie Bleie revibiert merben. Eteft

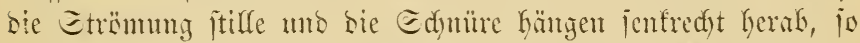
wiro gernbert and mit ber Edy?erfangel gefijdyt. jut ber Regel

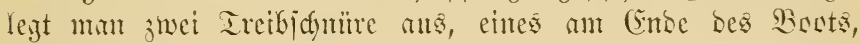

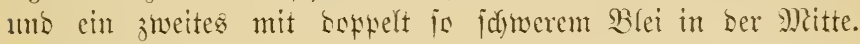

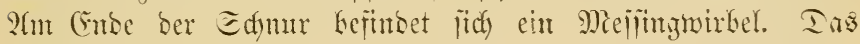

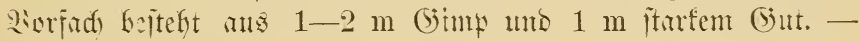
Bei jefyr jtarter Etrëmung ninmt man nux einen jofweren

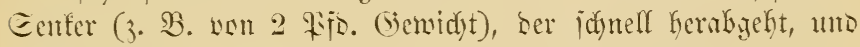

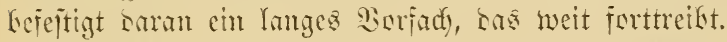

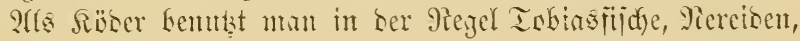

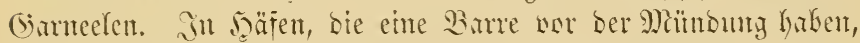
bie gewölynlidy reidy an Tobiasfitiden ift, angelt ntan an ber immenjeite bes tiefen (Ënganges, wo ber Etrom ber Gezeiten aus = uno eingeft, uno jefre tiejes senfier herworbringt. Jier

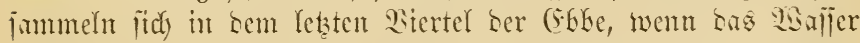

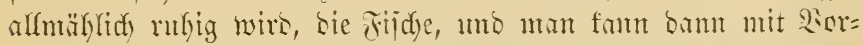

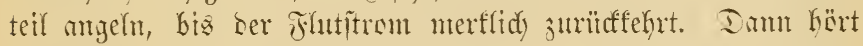

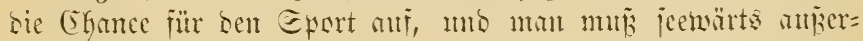
loulb ser Barreniptize angehr.

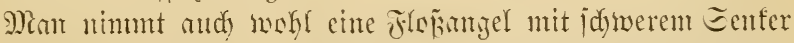

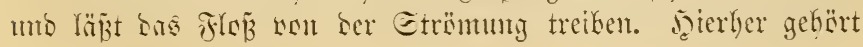




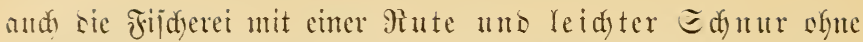

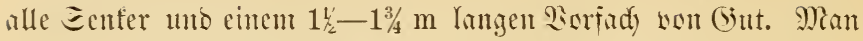
läßjt won einem Miolenfopi soer einem woripringenten Jeljen bie

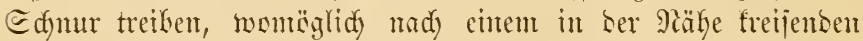

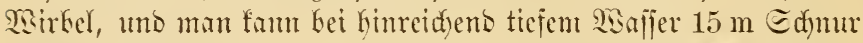

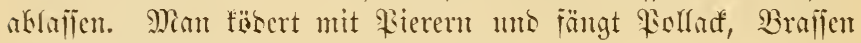
Etëder, Eeebaridye, Maftelen n. Igl.

\section{Das fifḩen mit ber Shlleppangel}

bejteft Larin, baj Ginter cintent fahrenton Boot eine lange mit Eenter und 2arfady werjekene 2lugelidnur nadjgeidyleppt wirs.

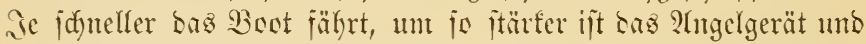
un io jumerere Eenfer finto erforberlich. Tas Boot wirb ent= weser gertibert ober es jegelt. in eriteren falle jint bie Eenfer $20-250 \mathrm{gr}$, in Yebterem 450-1500 gr id)wer. Man wäh)l Das

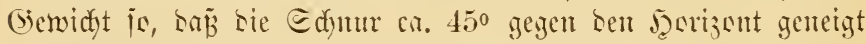

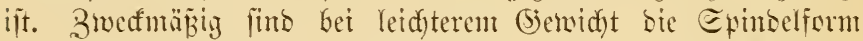
(Jig. 366), hei idwerem Eenfen tie Boctiorm (Jig. 364, 365), coer bas Girnförmige Blei mit retictenem (Einarm (Jig. 367).

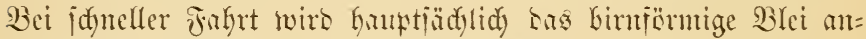
gewentet. F(ud) cine mit mehreren fleineren Bleigewidyten be= idwerte Edymur (i. Ireibjdyur E. 190) wirb bismeilen an=

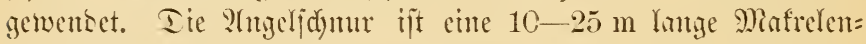

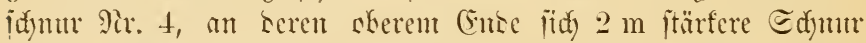
befintet, weldye nidyt leidyt an Borb surdyerteben wirt. Das

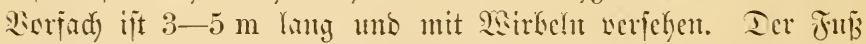
sellelken ijt (5imf, ser 2orjd)lag 1-2 m itürfiter (but. Man iiid)t entweser mit ciutem $13 \mathrm{~mm}$ breiten $5 a f e n$ mo natürlidjen Sösern, ober mit füntlidyen Epinnern soer glänzenben Fliegen.

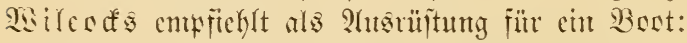

2 Edyüre, $10 \mathrm{~m}$ lang, Blet 3 Pifo., Sarfact) $3 \mathrm{~m}$ unit $1 \mathrm{~m}$ itärfittem Gut ant Ento.

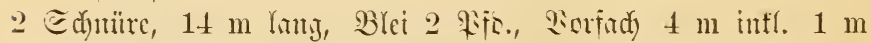
itärfitem (but an Entse. 


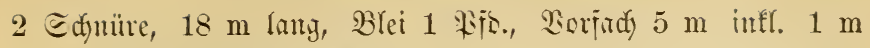
itürfitent Gut am Conde.

2 Edyüre, $25 \mathrm{~m}$ \ang, Błei y itärfitem (S)ut ant Conbe.

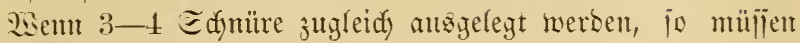
bie Eenter werjajesen jajwer jein, Damit jidy bie Edyüte nidyt begegnen. 2Hz Räber benubst man Yebende coer tote Tobias= fiid)e (Fig. 382-385), fleine 10-15 cm lange 2 fale (Jig. 386)

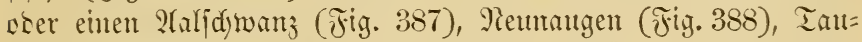
mürnter (Jig. 380), fleine tote Fiidge, Etïdfe rom Dornthedt, Miafrelen :c. 392, 393), \$ierer (Jig. 375), Mierëicen (Fiy. 377,

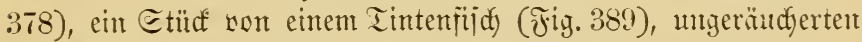

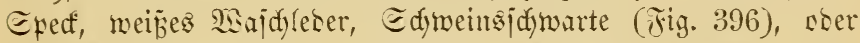
einen fümitlidjen Epinner (Jig. 235-242, 厄. 124-127), woer

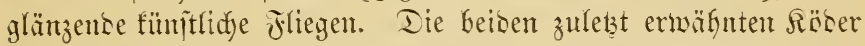
finto nad) Enmenuntergang bejenbers wirfiamt. Man fängt Gauptiädylidy Mafrele, Wollad, Eeebarid, Eeehabn. In ser Sitjee werben bei Etraljuto unb Burth im jeerbit mit fümptlidjen

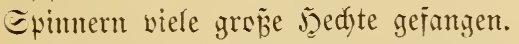

Man fafre quer gegen bie Midjtung Ler Etrïmung ber (be=

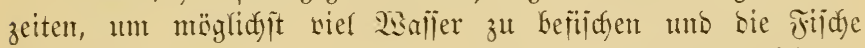
möglidjit menig ju beunrufigen, was bejonsers bei sem jdyenen Eeebarids widjtig ijt. Man fiid)t sam auperfalb bes Riel=

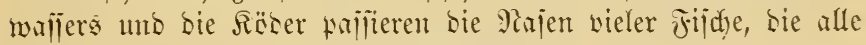
gegen sie Ridftumg ser Etrömung itefyen.

Ter $\mathfrak{A} u \mathfrak{B}$ leger (Tell-Tale; Cane-Outrigger) ift ein 1 bis

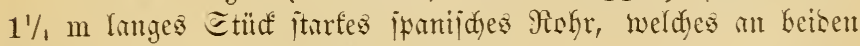

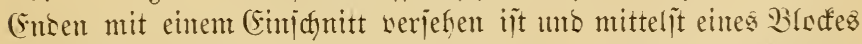
won zähem 5oolz, burd) ben es geitedft ift, an Berd bes Bootes

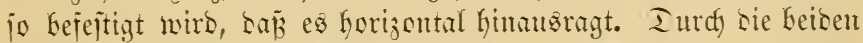
(Finjuntte wirb bie Edyun gelegt unto tam jeserzeit leidut

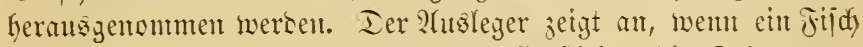

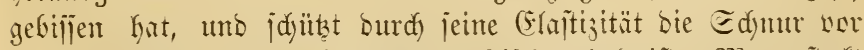
Lem Berreizen, mem ein idwerer Fiid) gefaft iit. Man itedft 


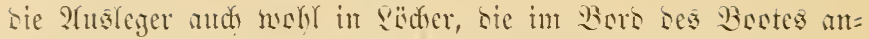

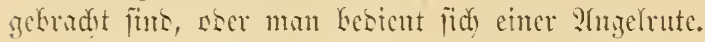

\section{Das fifhen mit Ser 2rngelrute.}

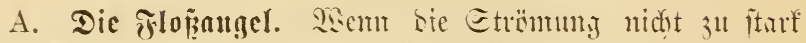
iit, is fant man won Mielenfüfien uns Pastigebirgen ans bic

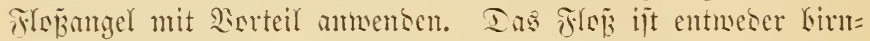

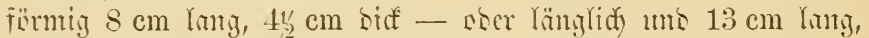

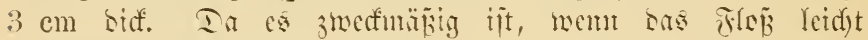

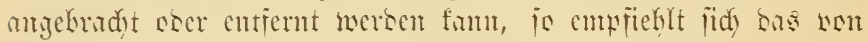

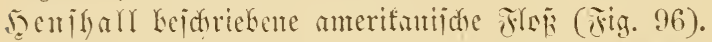

Mint angelt entweser mit curem idyeren Eenfer uno cunem

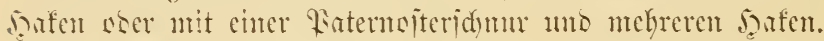

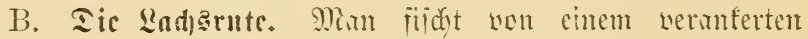

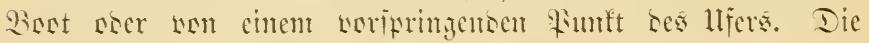

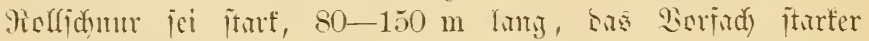

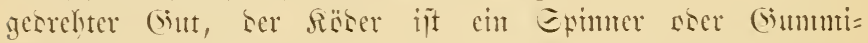

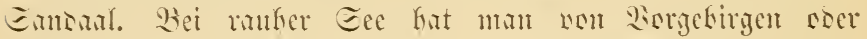

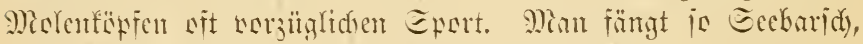
Forfacf uns mamentfich int Eeptemter Martelen. Mant wirft

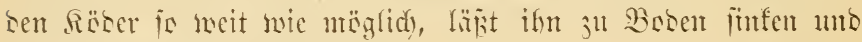

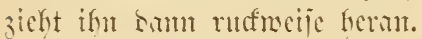

Mian fijdet mit ser Fliege som lifer, anf jteilen Feljen,

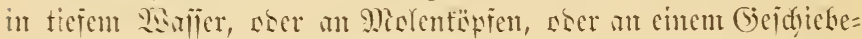

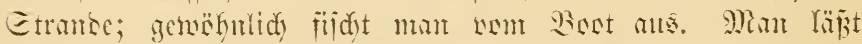
$40 \mathrm{~m}$ Edyur ans mo jieht fie ramginn umber.

\section{Das Bobenblei}

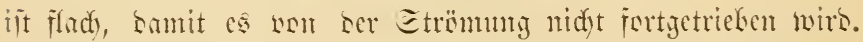

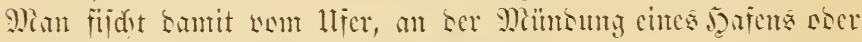

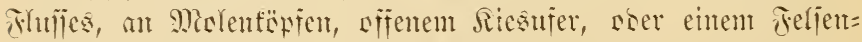

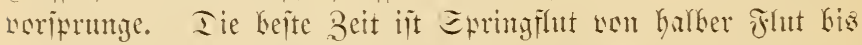

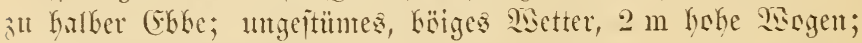

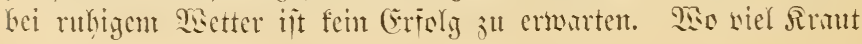




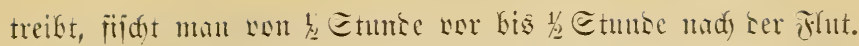

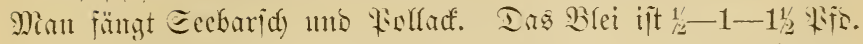

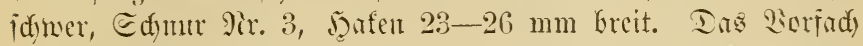

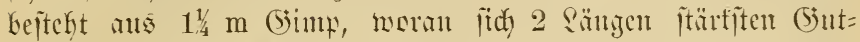

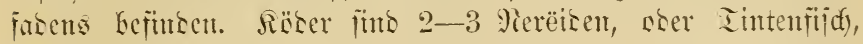

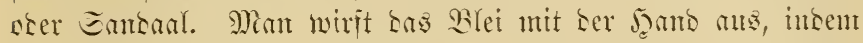

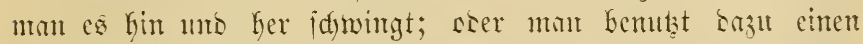
furzen, itarten, am (Ence gegabelten Etouf (Fig. 263, 264, હ. 142).

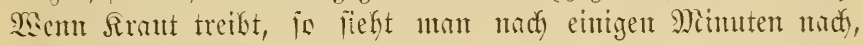
ob etwas saven an Łer Edunt büngt.

\section{Die Paternofter 2 2lngel,}

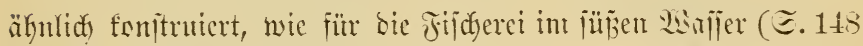
bis 150, Jig. 270-272), ijt jefr geeignet jum Jumig ser ser=

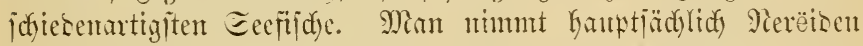

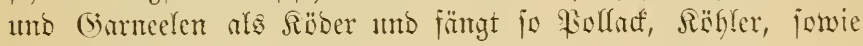

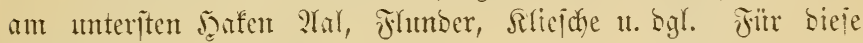

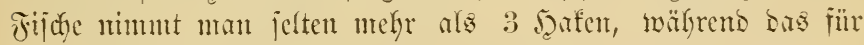

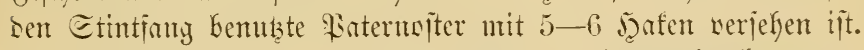

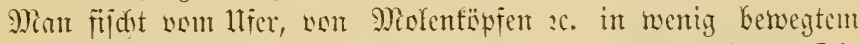
Sanjer, an Jafenmïnoungen, sber aud in sffener Eec. Die Jeafen Kaben $0,6 \mathrm{~m}$ 2(bitand von eintalber.

\section{Eegeangeltr.}

Zegeangefn bejtefen ats ciner Jäuptjoun, weldye burd? Eenfer unb Edwimuer an cinem bejtimuten Srte und in einer bejtinnten Tieje gefalten wiro uno ans eimer saran be=

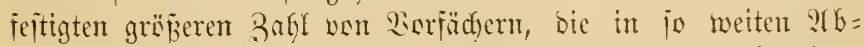

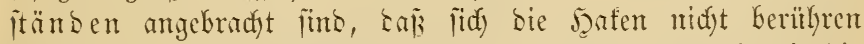

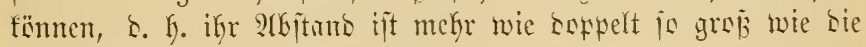
Sorfä̌der lang fiǹ.

A. Edjwimmende Regenngel. Tie Janutidume ijt $60 \mathrm{~m}$ lang uno mit Rerfen verjefen, bie fie fawimmento erbalten; fie 


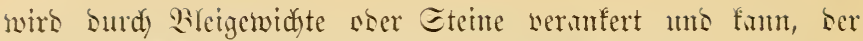

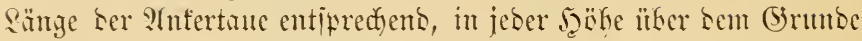
gebalten werben. Cine Brje ermïglidyt ans SBiebcrmifimoen bes Srts, wo bie 2(ngel Viegt. Sebes Sarjady ijt an einem Rorfen

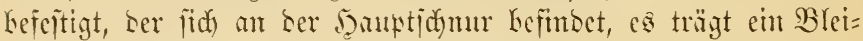

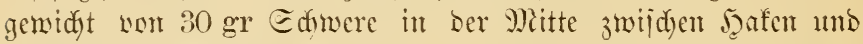

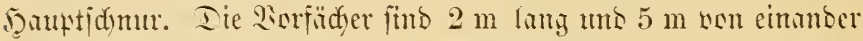
entfernt. Rëber: Yehenbe ober tote Tobiasfijde, Perëtisen, Fierer,

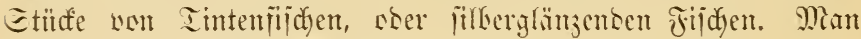

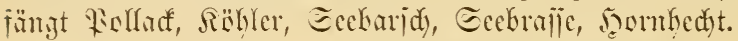

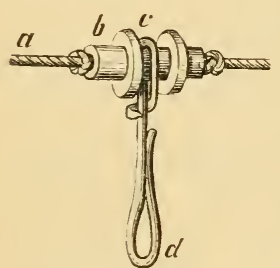

Fig. 370.

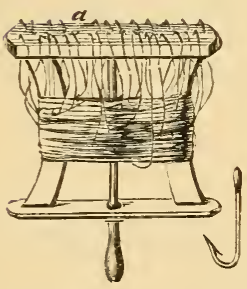

Fig. 371. Molle.

B. Brundegeangel, wirt surdy Eenfer am (Brmbe gekalten. Sänge ber Norfäd)er $1 \mathrm{~m}$, 2(kitanto berjelken $2,2 \mathrm{~m}$. Mian jängt Rabelin, Miceraal, Etcin= Gutte, Edyalle uno ambere, am (birumbe rebente Jijde. Rïber: Tokiasfijdy,

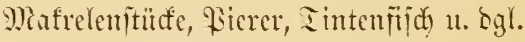

Dearbersegeangel. Santhtidyutr $80 \mathrm{~m}$ lang, 50 Siorfäd)er in $1 \frac{1}{4} \mathrm{~m} 216=$ itans; fino mit je einem sirthel ner= ieken, unb an ser Dauptidyut ourd) cin retierenses Ropfitïn (Jig. 370) (Jearber Brass Revolving HeadLink) Fefejtigt, weldyes es reidyt madjt, jebes Berfady abzutefmen ober anjubringen. a ift bie Soutptidnur, b eine Meifing= füllie, ed eine rotierente Iraftidyleife, in weldye sas Worfady eingefängt miro. Sgenrber madyt audy Rorlen fïr Qege=

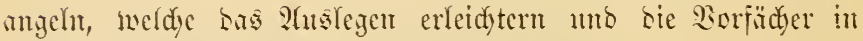

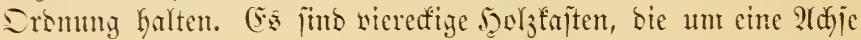
retieren, barm wirb bie Somptidyur getwidelt uto an sheren

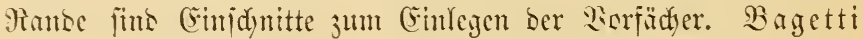

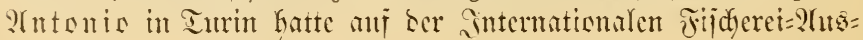




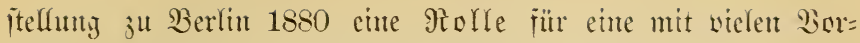

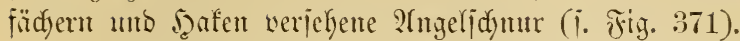

C. Ireiblegenngel. 2fn einer gewöbnlichen Edynur befindet iid) ein Eenter, ber eine lange I reibjdnum trïgt, bie mit 50 ñafen

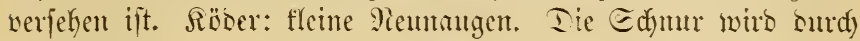
bie Ctrönung am Girunbe gejtreft. Mant fängt Iutbot 1 . Dgl.

D. Legenugel zum $\mathfrak{A}$ ifholen vou $\mathfrak{H}$ jer aus. Bei Ëble

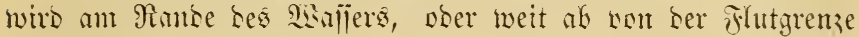

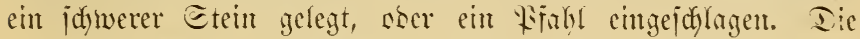

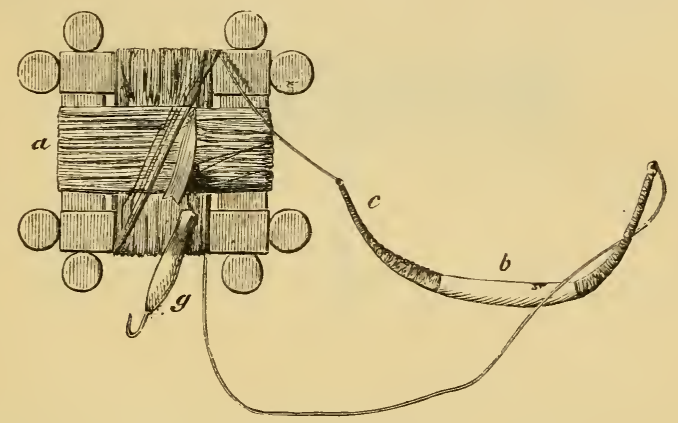

Fig. 372. Minte.

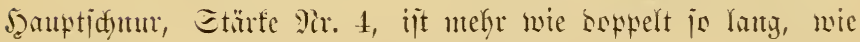

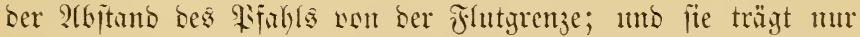
an einer Eeite Borfï̈dyer mit Saaten. Man legt jie um bent Fifafl mo bejejtigt beibe (Enten sberbalb ber Flutgrenze. Eie fant bamn bei flut herangezogen wersen. Janfen $40 \mathrm{~mm}$ breit.

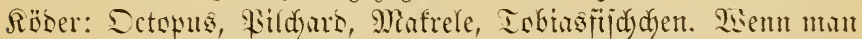

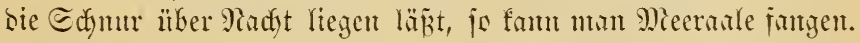
Dam milijen aber bie für ben Jang biejes jijdyes notwentigen

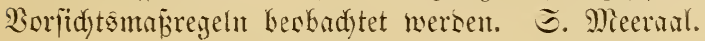

\section{Die Minde}

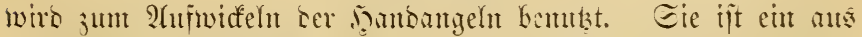

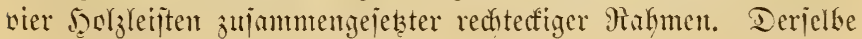




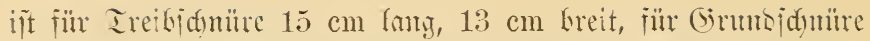
$21 \mathrm{~cm}$ fang, $15 \mathrm{~cm}$ freit, für jefr lange farfe $\Xi$ dyüre $48 \mathrm{~cm}$ long, $36 \mathrm{~cm}$ kreit. (ङ. Jig. 373.)

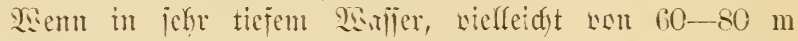
genugelt wirs, io iit ons büufige 2tuffeben uno Eenfen eines

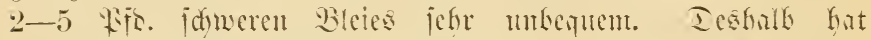

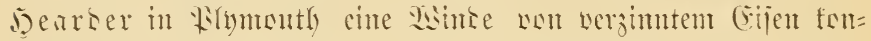

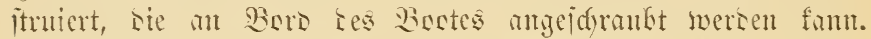
Eine Echleife, mu ben Jornogrifï gelegt, itellt bie se:nbe fejt.

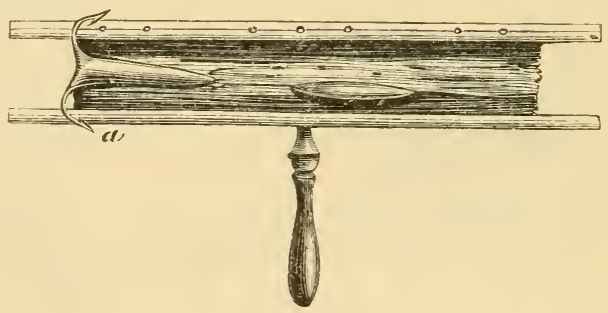

Fig. 373. Sambrolle.

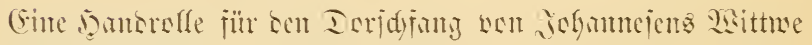
in (Efriptinia (jig. 373) mar 1880 aा ber intermationalen Fijderet=2(noitelfung in Berlin.

\section{כ̄weiter 21bfdunitt. \\ Altgefköder fïr Seefildjerei.}

Iic Mieŝmuidyel (Mytilus Edulis, The Mussel) ift ber an häufigiten gebraudyte Rëber fïr Gruncfilde.

I ie Narfmujdel (Patella Vulgaris, The Limpet). Ins Fleijch wirb eine Etunte in bie Eomme gelegt, smmit es zäbe mirs. (Sut fïr Eecfirruidue und Morrhua Lusca (Whiting Pout.) 


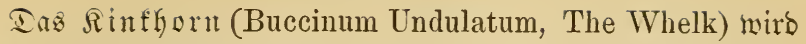
viel zum Rabeljanfang Kentz̧t. Man jerjalägt bie Canule mit einent Danmer und nimmt bas Tier heraus.

Die Majjeridneibe (Solen, The Razor Fish). Dieje Mujdel bohrt jidy int Eante ein, uno ijt ein guter Rïber für (Strumbangeltr.

Wenn Molfusfen am Salen befejtigt merben, fo füfrt man

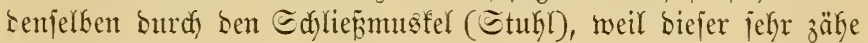
ift. Fig. 374a.

Der Rierer (Arenicula piscatorum, The Lug IVorm).

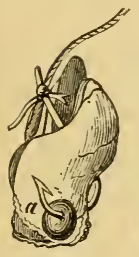

Fig. 374.

Milidiel.

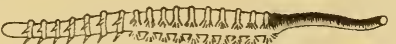

Fig. 375. \$ierer.

Man bemerft in Eanse ber Räjte fleine Grb̈hungen, worin 10 bis $15 \mathrm{~cm}$ lange siümer won ber Diffe eines Edyanentiels

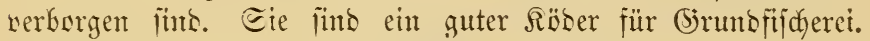
(Fig. 375.)

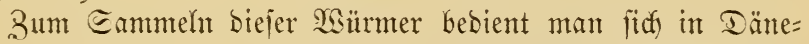
marf unb Jolitein ber jogenannten 2 urmitampfe (Jig. 376a). Diejelbe bejteft ants einent runben Brette von $25 \mathrm{~cm}$ Durdymejer, in befien Mitte ein Gïlzermer (briff von $1-4 \mathrm{~m}$ \&änge angejeţt ift. Der Canbbrien wirb im flachen Malfer vom Boot alb, ober beim Kanten mit ber Wurmitampje (2Surmpumpe) auf= gerïhrt, bie Wïrmer werben in Mienge blö̧gelegt, unb mit bem Ramme b Fig. 376, ober mit einer Gölsernen $0,7 \mathrm{~m}$ langen 3ange aufgensmmen.

Nerëiben (The Rag-, Rock-, Mud-Worm). Man unter= v. ๖. Borne, 2ngelfifiderei. 3. 2uff. 
fareibet jwei :raten: bie fleinere ijt $7-8 \mathrm{~cm}$ limg, uno benohnt

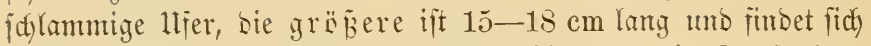
unter Etemen, anf Retten, Eand mo Ries, ober in Jelsipalten.

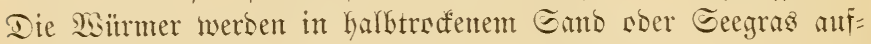

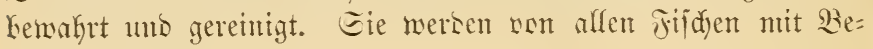
gietre gefrelien.

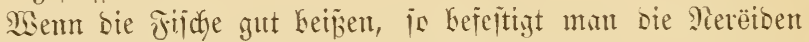

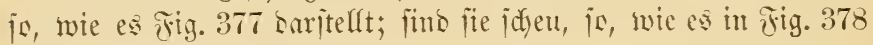

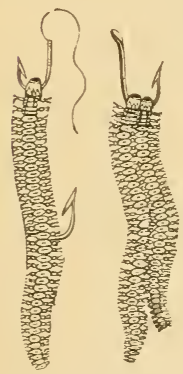

Æig. 377. ซ̀ig. 378. Nerëisen.

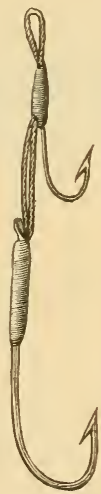

Jig. 379 . Saumurut.

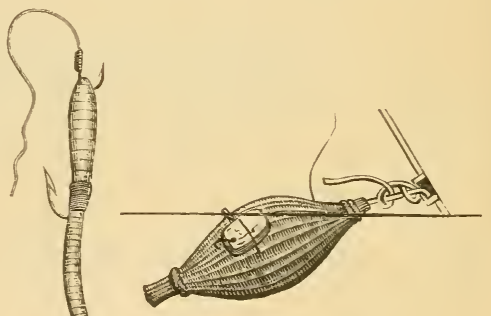

Æig. 381. Æiłdforb.

abgebiloet ijt. Man bemahrt bie \$ïtmer in einem fleinen Rajten, in $1 \frac{1}{2} \mathrm{~cm}$ tiefem Safjer anf.

Nereis versicolor (The White Sand Worm) fintet fith in fdhlammigent Gano ber Jeäfen uno Buchten uno wiro $15 \mathrm{biz}$ $18 \mathrm{~cm}$ Kang. Mkan fängt Samit hauptjädjlidy Etinte, aber aud) Wittling, Foffać, Meerbralle, Ratruts.

The Varm - Sea Tape Worm, finset fid) unter grojent Eteinen bei tiejer (56be, und wirb bis $45 \mathrm{~cm}$ lang und $1,3 \mathrm{~cm}$ bidf. (Es ift jehr gut für Wittling, Folfacf, Brafle.

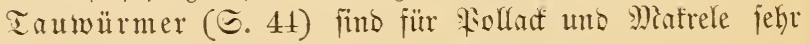


gut. Jig. 379 itellt bas Jateniyjtem, und Jig. 380 bie Be= feitigungen Des Taumums Dar.

Iobiaşija). Eanbaal (Sand-Eel) iit an fanbigen Riliten

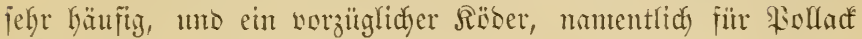

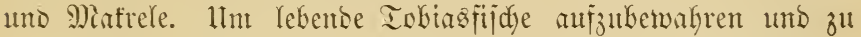
transportieren, bedient man fidf eines Sorbes von SGeidentuten, Der $0,6 \mathrm{~m}$ lang ift und $0,18-0,20 \mathrm{~m}$ Dutduntefier Gat. (5r wirb finten an Boot bejeitigt (i. Jig. 381). Qebente Tobiagfijde finto

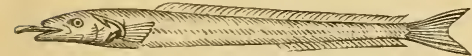

Jig. 382. Tobiasfijud.

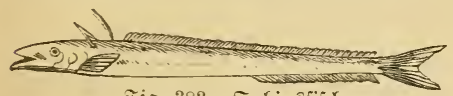

Fig. 383. Iobiasfitid.

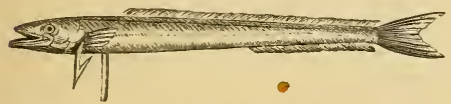

J゙ig. 381. Iobiasfiid).

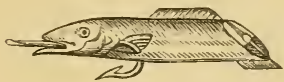

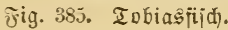

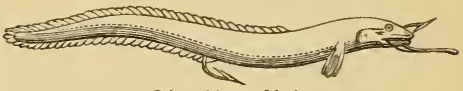

Fig. 386. 2aY.

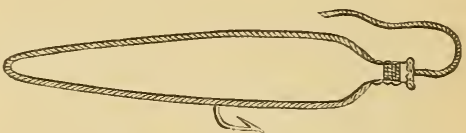

Fig. 387. शraliditwanz.

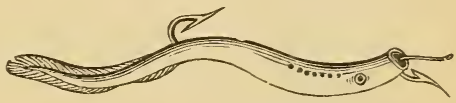

Fig. 388. Reunauge.

ein auŝgezeicyneter Röber. Fig. 382-385 jtellen bie Bejejtigungen

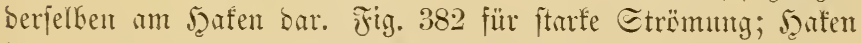
in Den Munt Kinein, aus ber Riemenöfinung Keraus, Dann georeht,

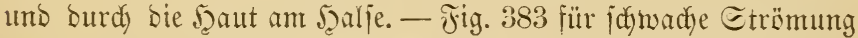

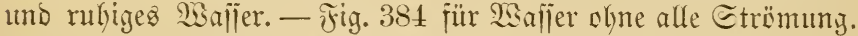
Fig. 385 ift ein Gurlber Eandaal, für bie Mafrelangel won eintent weranterten Boot. Der Jif ch wirb erit zeridynitten, wem er über Borb gemorfen werben joll, weil ber ansftr̈mende Rörperjaft bie Fijche anloct.

Sileine 2Aale von $12-15 \mathrm{~cm}$ \&änge jind fehr gute Sïber. Ulm jie zut fangen, legt man Bünbel von Meibenruten in einen idjlammigen Ditmpel eirtes Badjes, ber viele $\mathfrak{A} a$ le enthält, ober 


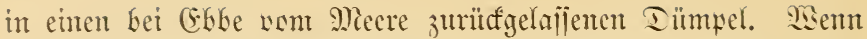
man nad einigen Iagen nadjiefyt, jo finbet man jmijdyen ben

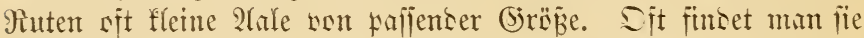
andy in fleinen Bädyen unter Eteinen unb fann pie mit einer Iifdgabel ipiē̄en. Eie jüno gut für bie Edyleppangel. (Fig. 386.)

Renn man is fleine Rale nidyt erlangen fam, jo fertigt man ans eincm $0,3-0,4 \mathrm{~m}$ langen $\mathfrak{T}$ al ben in irlant gebräudflidyen 2aljdmanjfïber (Fig. 387), ber jehr gut für bie Edjleppangel ijt.

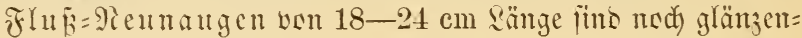
bor uno beffer als fleme 2fale, namentlich für Podfact. Mian finset jie, bejonters im Jrühjafr, in tleinen Bäden in Mienge an Eteinen, wo jie jidy angeingen kaben. Cie jint gut jür bie Edyleppangel. (Jig. 388.)

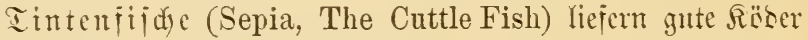
für Eeebarid), Rakelian, Mieeraal u. Egl. Sifr Fleijd ijt jehr zäbe, neshalb fie zum Fang von Ceebarjden bejonters zwedf=

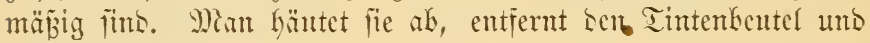
wäjd)t jie rein.

Rarmar (Loligo vulgaris, The Squid) ijt ned) Eelijer, als ber serige, er ift mentiger zäh und ift bejenters gut jür Mieeraal und Eeckarid.

Enmohl Sintenfiich wic Ralmar werben in Etüuc won $8 \mathrm{~cm}$ Eänge geidunitten, uno io an Daafen Kefejtigt, wie es Fig. 389 baritellt.

Octopus vulgaris (The Sucker, Poulpe) wirt jum Fang von Meeriar, Polfact unto Geebarid benutzt.

(3) arueclen (Crangon vulgaris, Palaemon serratus, Palaemon squilla, The Shrimp, The Prawn) wiro lekento für \&offacf, tat fïr Eecbarte uno (Sirefforf (Mugil cephalus), gefodyt uno

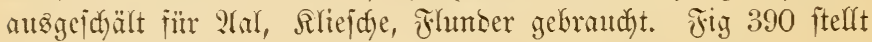
bar, wie cine lebende Gianeele am Jaaten bejejtigt wirb.

(5) meine Rrabben (Carcinus Maenas, The Common Green Crab) bembat man furz bewor lie bie Echale abmerfen, fo ba $\tilde{B}$ jie leidgt ansgejchält werben fömten (Soft Crab), nament= 
lich um \&abruß ober Eeebarjh ju fangen. Mim füngt bie Rrabben in biejen Buftande in Menge, wenn man bei Gbbe eine grop̉e

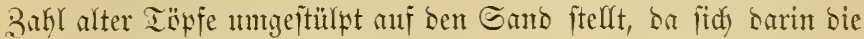
mieternoen Rrabben gern veritedfen. Rleine Rrabben fïnnen mit

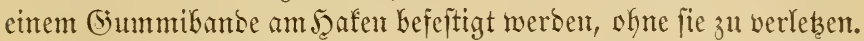
Ier Bernfardatebz (Pagurus Bernhardus, Soldier

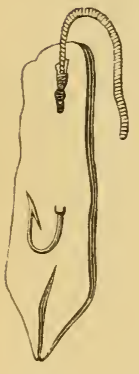

Fig. 389. Iintenfiid.

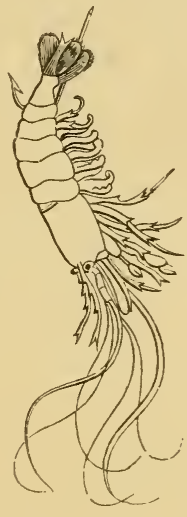

Fig. 390. Giarneele.

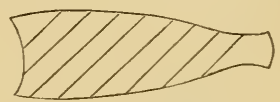

Fig. 391. Fifdifleifd.

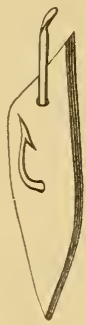

Fig. 392

अidfleifd.

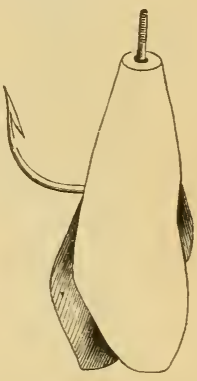

Fig. 393. Fijdidiwanz.

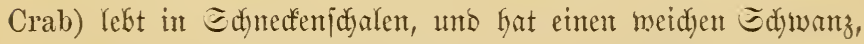
ber zum Fintg bon \$sittling, Brafjen u. Dgl. Gentust wirb.

Stücfe won Fifden, z. B. Mafrelen, Jeeringen, J̧ont=

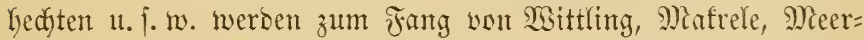

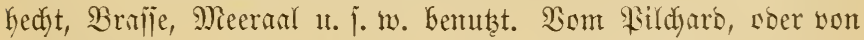
ber Sarbine, ober bem Jering werben in folgenter 2 seije $\Re$ öber für bie (Sruntongel gemadyt. Der Fifich wirb geiduppt, bann

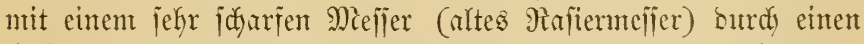

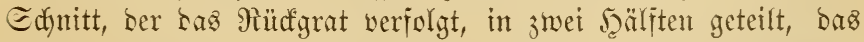
Ritufgrat entfernt, bie filberglünzenbe Eeite nad) unten gefehrt, auf Rorf gelegt, unb bann bas Fleijd in Etürfe geidnitten, bie 
$2 \mathrm{~cm}$ breit find (Fig. 391). Das Flcijed barf nidyt jut bict fein, bamit ser Röber an ber Edylespangel flattert, morauf jeine

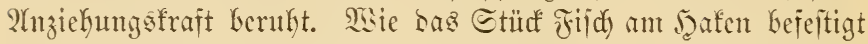
wirs, weranjdautlidt Fig. 392. Dic Fig. 393 cnolidy jeigt, wie ein Dafen mit bem Edywanz eines fleinen ober anderen fifdyes beföbert wirb. Die Edymanflofie ift ab= gejduitten, ser Rëser jur şälfte gejpalten, und io weit bies ge=

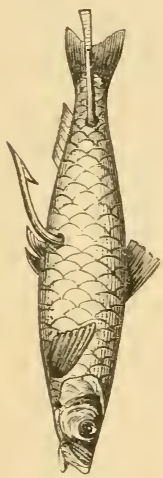

Fig. 391. gilduars.

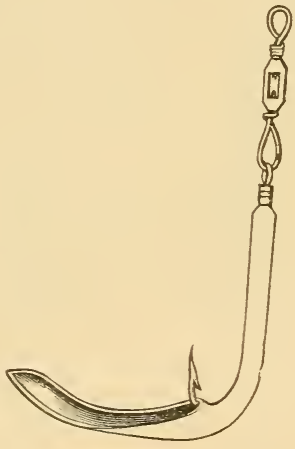

รig. 395.

(3)นmmi=Tobiaşitid).

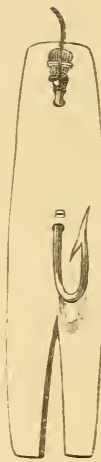

₹ig. 396.

๔a) weineidmarte.

jahah, bas Rïdfgrat entfernt. Man fijd)t Lamit nady Meeraal, Rabeljau, Mierlan.

Fig. 394 weranichatlidyt, wie ein Filldarb ober äfnllidyer fleincr Fijdy für sen Jang won Miecraal, Kateljan, Mierlan am Jafen befeftigt wirs.

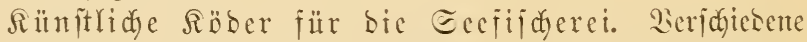
fünftlidye Epinner, ähnlidy senen, welde man im Sïjwaffer an=

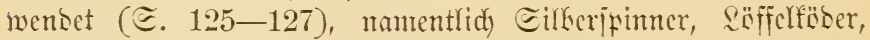
Błinter, (5)mmi=Tobiasfifde, Dicjelken fpimnent (i. Jig. 395); fünttlidye Fliegen, äbnlid) Icn Ead)Efliegen, aber einfadjer, jum Teil nur aus ein paar weiben ober roten Febern gemadt. 
Die beiten fünjtlichen Tobiasfijdye fann man nady SGilco of B feidyt jelbit in folgenoer Saeije madyen; man juneibet einen Gummiring won $4 \mathrm{~cm}$ Iurdymefïer, wie er zum Bufammenfalten won Brieffedaften benulzt wirb, an einer Etelle Dutrd). (5s giebt

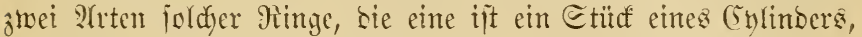
bie anbere ift ans einer (Summi=Tafel geidntitten. Tie Yetztere 2rut ift bie ridftige. Sienn man ein (Enbe bes burd)gejdynittenten Ringes an bem Edyenfel eine Ingelgafens antwinbet, io nimmt

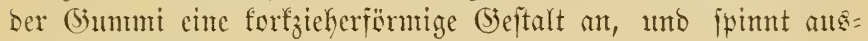

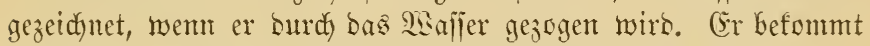

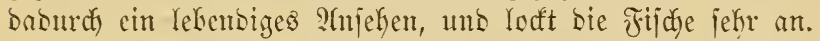

2uf bem Dzean wirb ein Röber gebraudyt, ber aus roher Sdymeinsidymarte geidnitten wirb (i. Jig. 396), ber Safen ift $12 \frac{1}{2} \mathrm{~cm}$, bie Edywarte $25 \mathrm{~cm}$ lang 4 mb wirb mit Iraht am Scafen befeptigt.

\section{Dritter 21bịnitt.}

\section{Die Seeftidje und deren Giang mit der ditgef.}

\section{Atugelfaleuder.}

Gamuar. Ratdzeit. Räbler.

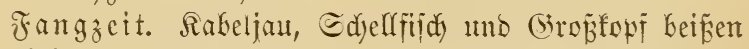
am beften. פgittling.

Jebruar. Raidzect. Rabeljau, Edyelfijidy, Räbler.

Fangzeit. Rabeljau, Edyelfijid und (Srofifopf beipen ant beften. Mafrelen erjocinen, uno ifr Fang begimnt.

Miär $\mathfrak{z}_{j}$. Iaidzzit. Edjelfifij, Mittling.

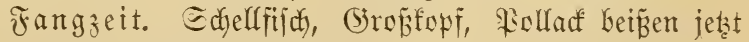
am bejten. Matrele, Meeranl. 
Mpril. Saidjeit. Flunder, Gilattbutte, Etint.

Fangzeit. Folfact uns nornkedf keizen jebst am beiten. Mafrele uno Meerar. - in ser Sitjee wiro Dorid ge= jangert.

Mai. Laidjeit. Finnoer, Ghattbutte, Etcinfutte, Ecehahn, Jemberit.

Fangzeit. Follad, Flumber umo Edyalle keipen jeb̧t ant bejten.

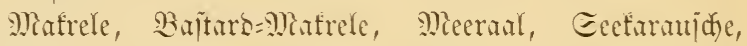
Rliejue.

In ser Sitjee wirb Iorid, bei Irieit merben \$ndafrelen gejangen.

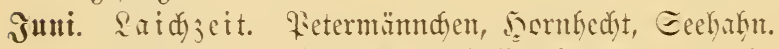

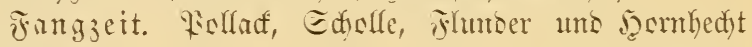
Geipen jek̨t ant beiten. Miafrele, Baitarsmaftele, Meeraal,

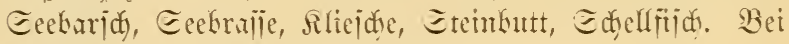
Trieit werben Martelen gefingen.

juli. Saidszeit. Fetermännden.

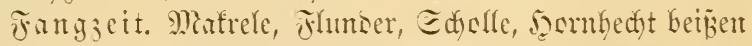
jeţa am beiten. Iie Miatrelen rerlailen (Ense Jult bie

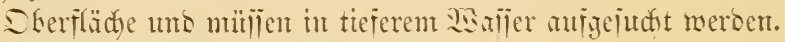
Sie werben foci Irieit gejungen.

Bajturs=-Matrele, Eeefaridy, Eeebralle, Meerbarbe, Micerfedt, Meerial, Rollat, Eteintutte.

Qugujt. Fangjeit. Wafrele, Finnoer, Edydle, Fetermüntuch Keipen jeķt am bejten. Maffelen merten bei Iriejt ge= jangen. Eie jins in Ler Acrsice in tejerent judten.

Bajtaromafrele, Ecebajid, Ecefraile, Grof̈topi, Folfact, 2.

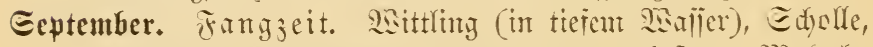

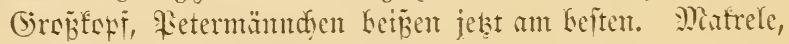
Bajtars= Mafrele, Meeraal, Eecbarj, Meerbarbe. Jutnge

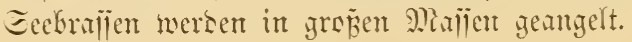


Dftober. Saidjeit. War? Die Miatrelen füllen fïb in Sftober mit Earidy.

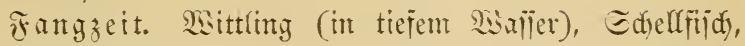

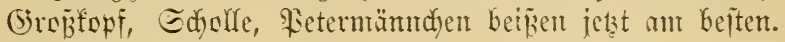
Meerbarbe, Doridy.

November. Raidzeit. Ral?

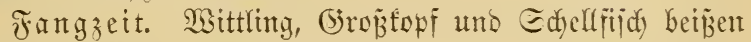

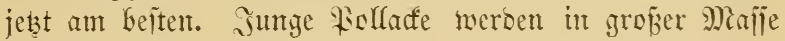
geangelt.

Meerbarbe, Modyen, Rëhler, Iorjw.

Dezember. Saidjeit. Nal? Rëhler.

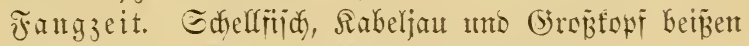

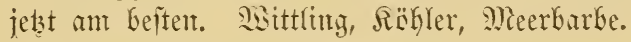

1. Der Kabeljau. Norrhua Vulgaris.

(Iorid), Etodfijd, The Cod, La Morue.)

Der Siakeljan ijt eit Bemobner ber noroijchen Meere, uno fommt an ber beuticten Piorbicefitite vor, wo or an ber Edyles=

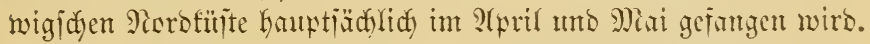

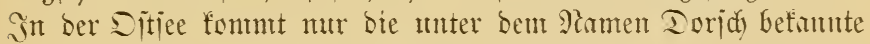
fleinere Barietät vor uno iīt iiberalf verbreitet, am fäufigiten bei

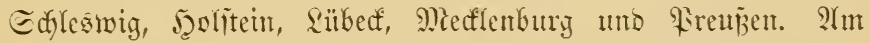

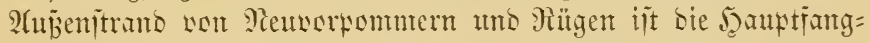
zeit ber Soerbit, sisuter und Friffling. (5r wirb mit Orumb= angeln gefangen, als Räser werben Iintenfitio, Jering, Matrele,

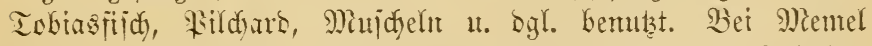
werben viele Doride ven ben Miclen mes genthelt. Der Sabeljau laidyt im Jebruar.

\section{Der Wittling. Merlangus Tulgaris.}

(2Beizling, The Whiting, Le Merlu.)

Der Mittling liebt weidyen, idslammigen Grunt uns foumt hauptiädlidy in 50-60 m Tiefe ror. Er fintet fide in ber Porb=

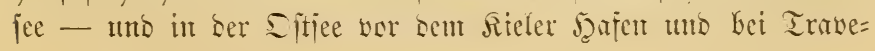




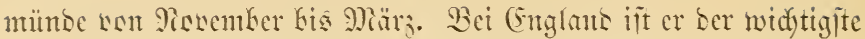

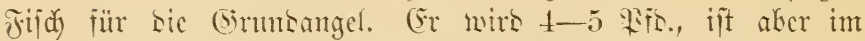
Iurdhidnitt mur ca. 2 Fifun jojmer. In geringerer Iieje fängt

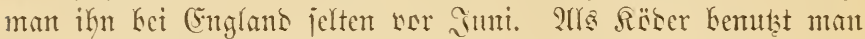

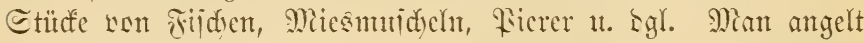
ca. $1 \mathrm{~m}$ ïfer bem (5rumbe. Ier 2 Sittling laidst im Fechruar und

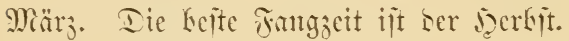

\section{Morrhua Lusca (The Thiting Pout, Le Tacaud)} fonmt in Ser Persiec sor uns wirs chenio wie ser Mittling gefangen.

Mian fiid)t mit Mierëisen uns Minidyeln sidut üfer bem Sirunte.

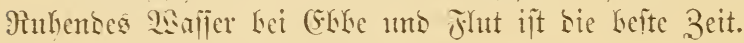

\section{Der ฮ̄weraborjø. Morrhua Minuta. (The Power, Poor Cod)}

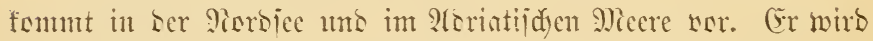
mit ser (sirutsangel funtptiäd)lich mit aierërsen gefangen.

\section{Der Sđellfifh. Gadus Aegliformis.}

(The Haddock, L'Égrefin.)

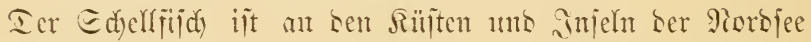

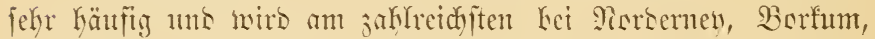

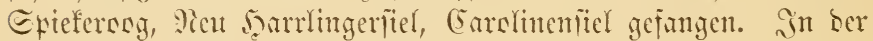
Sitjee ift er jelten. Man fängt ifn mit ber Strumbangel uno nimmt hauptiädylid Miujdyeln, Fijdye, Fierer als Röber. Der

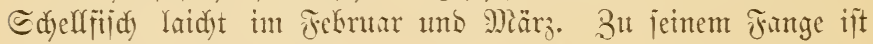
bie Beit won Märj Kis Juni unt won Sftoker bis Jaunar nm gecignetiten.

\section{Der 21Terlan. Merluccius Tulgaris.}

(Mecrfect, fleiner Etodfijd, The Hake, Le Merlu.)

Ier Merlan trmmt in ber Mirsjee uns sem aroriatijdyen

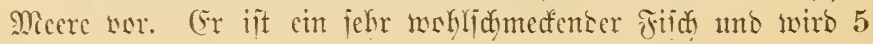




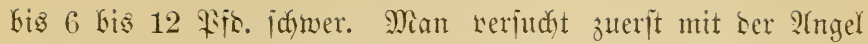
ant (binunte unb hebt biejelbe allntäblidy, bis man bie Tieje er= mittelt hat, in weldyer ber Fifd) fid) aufgält. Das Borfady mun (Simp jein, santit es ber Fijd) nidet surd)beipt. (5r muE tot= gejdylagen werben, fobals er gefangen ift, weil er fduwer zu bänsigen ift. (Sr keiñt nutr in ber ?adyt.

\section{Die Kolief̧e. Platessa Limanda.}

((5)lahrfe, The Dab, La Limande.)

Iie Rliejde ift in Ler Morbjee uns sem Mittelmeer bäufig, in ber Sitjee jeltert. Eie liebt janbigen und julammigen (Srumb

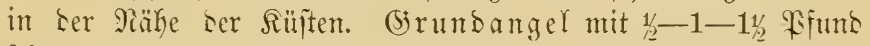

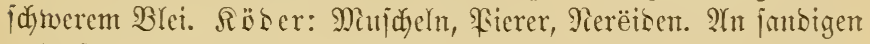
und fallammigen 1lfern ift bie bejte Beit, wenn bie Bjezeiten ftrëmen. - Raidzseit ift Februar uno Miärz.

\section{Die flunder. Platessa Flesus.}

(The Flounder, The Fluke, Le Flet, Le Flez.)

Die Flunber ift in ber 9iorb= ums Sitiee häufig unt geht weit bie Jiüfie Gunanf (ङ. 252). Man fängt fie ebenjo wie

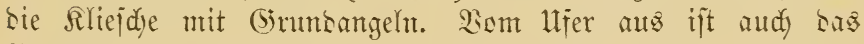
BrbenGlei mit Jafent von $10 \mathrm{~mm}$ Breite gut. Räser: ant beiten mieternte Rrabben (Soft Crab), ferner Pierëisen, \$iterer. Im

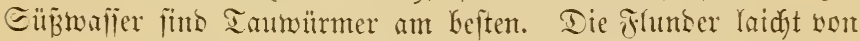
Februar bis Mari auf Eanbbobent. Mai fis 2uguit ijt bie bejte Fang3cit (Fig. 355).

\section{Die Scholle. Platessa Vulgaris.}

(The Plaice, La Plie Franche.)

Tie Echulfe ijt häufig in ber Parb= uno Sitjee, bleibt aber in Yetzterer tlemer. Eic Yiebt janbigen (simuns. Cis mirb mit Srunbangeln ebenjo mie bie Flunber gefangen, ber bejte Röber fino Fierer. Tie Edydle laidyt in Februar uts März und miro non Mai bis Sftwer am neiften gefangert. 
10. Die Geiligenbutte. Platessa Hippoglossus.

(The Halibut, Le Flétan.)

Die Seiligenbutte, ber Mitcje unter ben Plattriicten, woirs 100-150-400 \$ijo. jumer. Sie ijt in ber Porbjee nidjt jehr Günfig und fommt in ber Sitjee s̈itlid von Riel nidgt nor. Sie wirb jelten an ber Grunbangel gejangen. Bei Riel ijt fie von Staber bis Tezember nidjt jelten.

\section{Die Seejunge. Solea Vulgaris.}

(The Sole, La Sole).

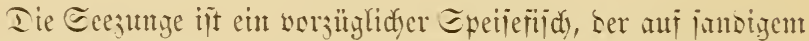
(Brutnde bäufig in ber Norbjee, ielten in ser Sitjee vorfomutt, uno jelten mit Sintusangeln, bäuftg mit Regenngeln gejangen wiro.

\section{Die Steinbutte, Rhombus Maximus.}

(The Turbot, Le Turbot).

Dic Etcinbutte ift in ber Norbjee Gänfiger und grëßer als in ber Sitiee. Die Steinbutte Yaidyt im Mai, und ifre Daupt= fangzeit ijt non Mat bis Eeptember. Sie wiro zifter an Sege=

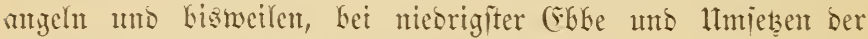
Etrëmung an ser Ginmongel gejangen.

15. Dic Glattbutte. Rhombus Tulgaris. (The Brill, Le Barbue).

Die Gilattbutte ijt in ber Porbjec hänfig, nidyt Gäufig in ber

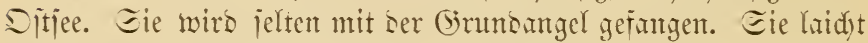
non März Giz Mai.

\section{Der Glattroḑer. Raja Batis.}

(The Skate, La Raie Bâtis).

Der Glattrodyen ijt in Der Porbjec auj janbigem unb

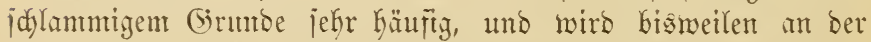
(S)unbangel, häufig mit Regeangeln gejangen. F(ujer siejer ver= 


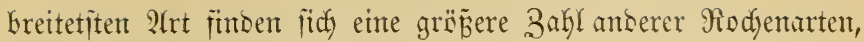
bie jumeilen sie 2 ngel nefymen.

\section{Der 2Teeraal. Conger Vulgaris. \\ (The Conger, Le Congre).}

Fer Meeraal ift in ber Porbjee häufig, 3. B. ant ber Slben= burger Rüjte unb ber SEejernünoung, ebenjo im Iforiatijdyen Meere. Er feglt in ber Sitjee. (Er wirb bis 100 \$ifs. fidwer; am häufigiten findet mau ifn auf felpigen Girunde. (Er wirb mit jefr iturfen (5runbangeln gefangen, bie ein Worfach von (Simtp

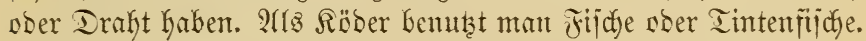
Rebztere werben worker geflopit, weil ber Meeraal meidje Röber liebt. (5r ijt jefr jtarf uno. unbünbig uno fant 2 rme uno Beinte ber Fijdjer zerbredjen, weşhalf er surd) einten Edylag betäubt unb surdy einen Etich ins (sentiff jojort getötet wirb.

\section{Der Seng. Lota Molva. \\ (The Ling, La Lotte de Mer).}

Der Sent ijt häufig in ber Morbjee, und fommt noch bei Ritel nor, idyeint aber meiter öftlidy in ber Sitjee ju fehlent. (5r

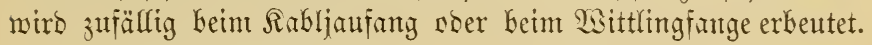
Er wirb bis $1 \frac{1}{2} \mathrm{~m}$ lang.

\section{Die Lippfifiche. Labrus.}

(The Wrasse, The Rock Fish, Le Labre).

Sippfiiche fängt man bismeilen beim (5rundangeln. Röber find Barm, Muideln, an beiten mieternbe Rrabben, ober fleitre Sirabber.

18. Der Stint. Osmerus Eperlanus.

(The Smelt, L'Éperlan).

Der Etint fommt in $\mathfrak{S} a ̈$ fen und Meerbujen oft in groß̈er Mienge vor, und gewährt bann eine jefre ergiebige Fijderet. Man fiidst mit Siaternojter, bas an eitem 2 m langen Borfach von (5ut 


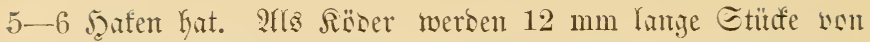

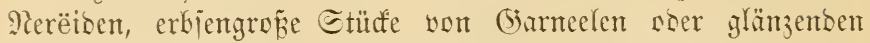

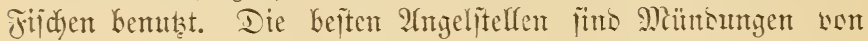
Bädjen, uno freine ferfige Budyten. Dr man ben Bí bes Etint faum füflt, fo muí man affe $5-6$ Eefunten anthaten unb bie Prngelrute heben (ङ. 221). Ier Etint laidjt int 2lprif.

\section{Der Grofłopf. Mugil Capito ober Cephalus.} Meer=2(ejde, The Grey Mullet, Le II ulet Capiton, Le Mulet Gris).

Diejer Fijd wirb meber an ben bentidjen 920 ro $=$ ned Ditjee= fiijten ermähnt, obgleid) er in ber Marejee käufig norfonment. Man

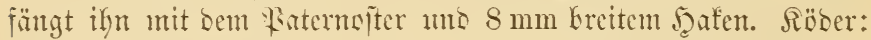
Fierer uns (5ameelen. Eebetere werben für biejen Fang ein=

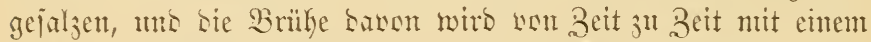

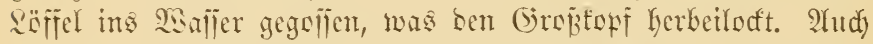
Fitid)rogen, Rartoffeln, jeritampite Garneelen jinto als Girumb=

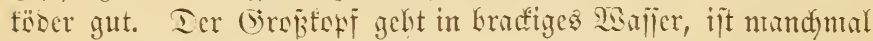
jehr zahleteidy in Inds, an Misten, Dajentanten in haffartigen Etrandjeen. (Ẽr wirb Gauptiäçlid) von Eeptember bis März gejungen.

\section{Der Pollact. Melangus Pollachius.}

(The Pollak, Whiting Coal, The Coal, Le Lieu).

Der Polfact it häufig in ber Porbjee, wereingelt in ber Ditfee.

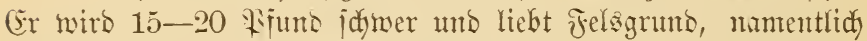
werjunfene Slippen vor einer Santipitze, bie weit ins Micer finaus= reidht. Man finbet ifn fier bas ganje Gafhr. Die bejte Fang=

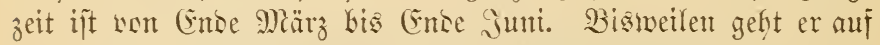
Eandgrunto, un Iobiasfijde ju frefien, sie er jebr liebt. Er frijt in alfen Iiejen, aber riel nefyr über wie am Grmnse, ijt rit in Menge an ber Sberiäläc.

Die Ireibjonur wirb jefr siel angewanot mit lebenden Iobiağiiden, Perëisen, lebensen Garneelen, $12-15 \mathrm{~cm}$ langen 
Aalen, fleinen Neunugen, Etüden non Donnhedten uno anberen Fijuden.

Sdyleppangel, jowohl bei idnelfer mie lamgiamer Jaht. Röber: Yebense uno tote Tobiasfijde, Meunaugen, Enumürmer,

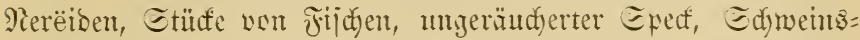

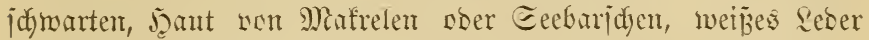

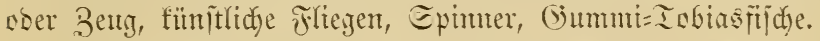

Mit Der PIngelrute uno fümitfidyen Fliegen, Epimern, Tobiasfitiden, won lljer oder einem feitgelegten Boot.

2tud bie Frozangel mo Faternoiterangel futs jum Fing biejer Fijdje geeigntet.

\section{Der Köh̨ler. Merlangus Carbonarius.}

(The Coal Fish, Le Colin).

Der Rögler ijt an Der nërolidjen Rüjte wen (Englano am Käufigiten, nimmt nad) Ëtsen ab unD iit an ben Eeutichen Rüpten

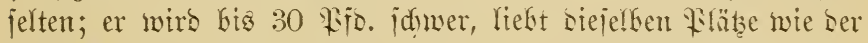

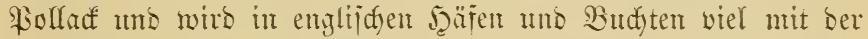
Rute gejunger. Der Röbler latht nom Iejember bis Februar.

\section{Die Miárele. Scomber Scombrus.}

(The Mackerel, Le Maquereau).

Die Miafrele ijt in Der Morojee, in sem Mittellänoijhen

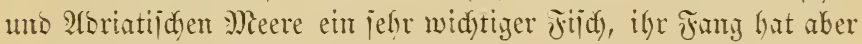
an ben beutichen Rïiten lange nidyt bie Bebentung mie bei (England. Sn ber sitice fommt ite ebenjalfs vor, veridymintet aber sijtlidy wou

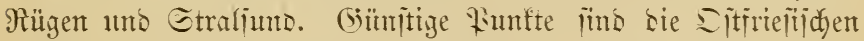
Injeln, bie gisejernünoung, நgelgoland, bic Travenünoung.

Die Mafrele genührt einten norzüglidyen Eport. Ein Fang von 500-600 Fijdyen in cinem Boot pro Tay ijt teine Eelten= Geit, uno ijt es worgefoumen, baj 1500 mo mebr Fijdye in einem Tage erbentet wurbert.

Man angelt mit ber Ėdeppangel bei juntller ober lang= famer Fafirt. 
Söber: Etiate nom Edimanj ber Mafrele, tote Tobiasfifiche, glänzente fïmitliche Fliegen, fünttldye Eximter.

Int Exätismmer uns Deerbit hält fïd tie Mafrele mełr ant (Srunoc auf unb wirb bann mit (5) runsangeln gefangen. Röber:

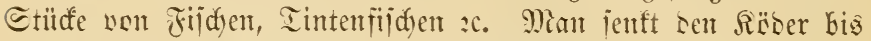
żm (irumbe unt hebt ifn bann langjam bis bahin, wo man bie

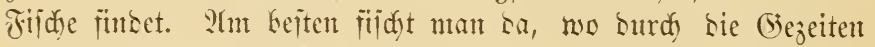
ganz wenig ober gar feine Etrëmung entiteft. Blei bootförmig, ober fpintelfürmig. Sorfad) $1 \frac{1}{2} \mathrm{~m}$ (Simf, wher bofpelter ftarfer

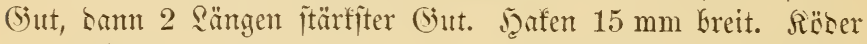
wom Rildyars, seffen Bitden orer Eingemeise, oser Ralmar. Sebente Tobiasfitide fint rorjüglich. Detben unt Eenfen bes Röbers ijt juedmä̈ig.

Ireibidnur. im 2luguit, Eeptentber unt Sftoker mirb

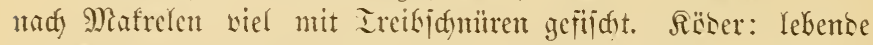
soer halbe tote Tobiasfifde (lebtere, wemn ber Röber länger als $15 \mathrm{~cm}$ iit), (Singeneise nom Fildurs, Merëiben. Defler Mont =

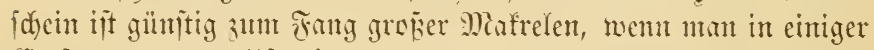

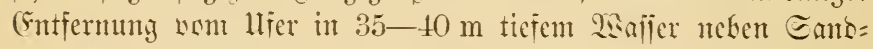

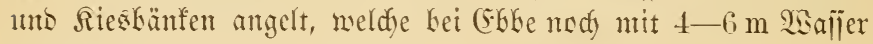
berecft jints.

Edyleptangel. Dat man cinen Edmarm non Mafrelen getroffen, fo fährt man io lange weiter, wie sic rifde beizen, io wie ïe anfGëren, mentet man bas Boot unb fährt benjelken Sutş zurüd, we man fie sann gemëhnlidy wicber trifft. Bei wolfigem Sietter in einter frijon Brieje jint sit 5-600 und mefr Diafrelen gefangen morben. Beim Fubern it 100 idjon

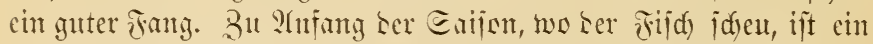
toter Iobiasfijd sit ein guter Räser. Zu idundle Fahtrt ijt nidyt gut.

Fïr sie Mafrele nührt bie Eaijon won Mfril bis Eeptemter,

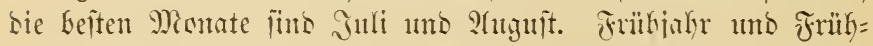
jonmer fint am keiten für bie Edyleffangel, Epätiommer uno Seerbit für bie Sinmoangel.

Bei Trięt iłt ber Mafrelenfang wom Mai bis ?huguît lohnent. 


\section{Die $\mathfrak{B}$ aftar $\delta=2$ Mafrele. Scomber Trachurus.} (Mïjefer, Etöffr, The Scad, The Horse-MackereI,

$$
\text { Le S a urel.) }
$$

Tie $\mathfrak{B}$ ajtarb= Mafrele ift weniger idnmadfaft als bie Matrele.

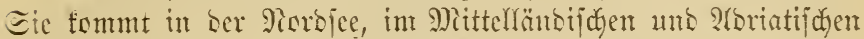
Meere und and in ber Sitjec frei Sitel nor. Eie wirb oft jufälliz gefangen, wem man nady Mafrelen uno Folfict angelt.

\section{Der Seebarjạ. Labrax Lupus.}

(2Bolfabridy, The Bass, Le Bar, Le Loup, Loubine.) Der Ecebrich ift im Mittelmeer amo Aloriatijchen Meer, fonie in ber Norsjee, an ber framzöpijidyen und englijdyen Ritite in grojen Edwoürnten anzutreffen. Eein \$orfommen an ben norb= beutjojen Rïiten wirb won sisittma cf nidyt erwähnt. Diejer

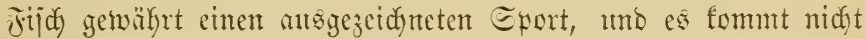
jelten vor, Daj in einigen frïhen Norgenitunben 70-80 Etït à 3 bis 15 㱠und mit ber Fliegenangel gefangen werben. Ier Seebarid liebt Flubmitnoungen, wo er mit ber (sbbe ans= und

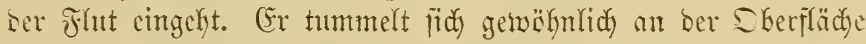
unther, wirb aber and oft tiejer und aud an (Sirumbe angetreffen. Er ift in 2lpril uno Mai gewöhnlicy an ber Sberfläche. Jat

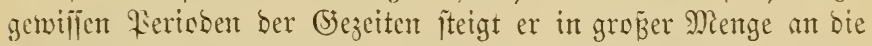

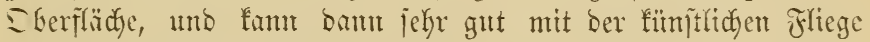
aber Treifidum gefangen werben.

Minn füngt ifn mit Der $\subseteq$ d) leppangel mit fümtltidyen Fliegen, Epintern, (5)tmmi=Tobiagfildyen. Man mähle einen Iag, wo bie

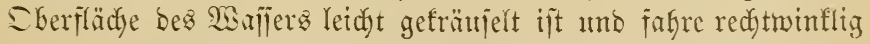
gegen bie Etrömung, mur bei lebhafter Brije, bie bent Flutitron entgegentweht.

Mit ber (5) randangel fijd) man bäufig yont llfer aus an Më̈toungen won Flïijen uno Jeäfen, Molenfüpfen uno felïgen 2orgebirgen. Mam bemutst bajt eine 2Angelrute unb ein Boben= blei und fübert mit Etïffen von Tintenfijdyen, Tobiasiijdyen, mieternden Srabben. Hurufiges SEetter iịt am beiten. Man bält v. ๖. $\mathfrak{B}$ orne, Angelp̈ifderei. 3. 2ufl. 


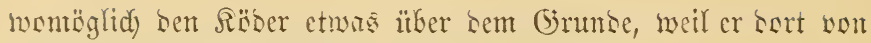
Rrabben u. Sgl. abgefreflen wirb.

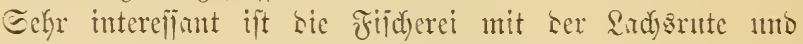
füntrliden Friegen, fünitliden Epinnern, Gummi= Iobiasfijden won einem fejtliegenten Boot noer wout llịer. Man twirft bieje Siöber fo meit wie mëglidy aus uno zieft fie

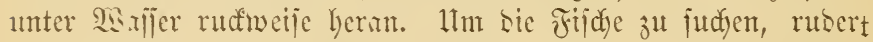

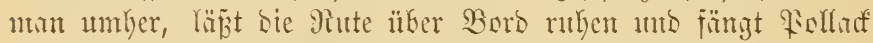

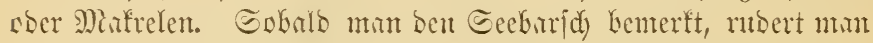
unter Sinto und wirft bann bie Fliege żwijden bic Fijdje. 11eber cinem Edywam won Eeekaridyen pflegen 2lïsen ju fumeben.

\section{Der Somtenfifid. Zeus Faber.}

(jeringofuntig, The Dory, La Dorée.)

Der Eonnenfija ijt cin mumberbar geitulteter, jehr wohl=

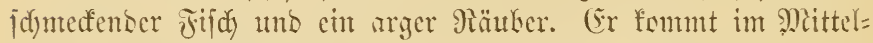
meer, im Mrortatijden Meer unb Ler Norbjee wereingelt ver. (Er wirb jü̈̈llig an ber Edjleptungel gefungen. Bejonters germ nimmt er lebende Tobiasifide unto anbere fleine Fijde.

\section{Der Gornheḑt. Belone Vulgaris.}

(The Gar-fish, The Long Nose, L'Orphie.)

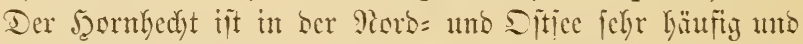
fommt aud im Mittelmeer wor. Ore wiro jumetlen an Treib= idjütren ober ber Edyleppangel gefungen uno jein Fleijd giebt einen guten Röber. Der Jeornfyedyt laidyt im Mat unb Suni uno

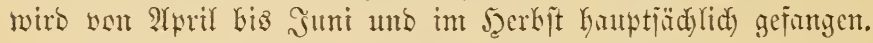

\section{Der graue Seehaḩn. Trigla Cuculus.}

(The Gurnard, The Gurnet, Le Trigle Grondin.)

Dor grame Eeebargn fonmt in ber Parbiee und an ter Medflenburgijugen Rüjte in ber Sitjee wor. (Er nimmt jeocn Röber, namentlid Epinnfüber, unto mirb s̈fter gefangen, went nadd Sittlingen boer Mafrelen geangelt wirb.

Der Fifid) Laidst int Mari uno Juni. 


\section{Katfifíne}

werben sit an ber Angel gebaft, aber jelten gefangen, ba fie bie

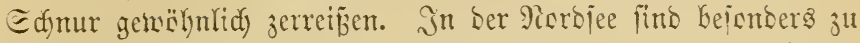
ermähnen: ber $\subseteq$ dy weinghai, Galeus Canis, ber mamentlidy an

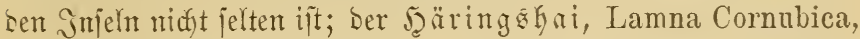

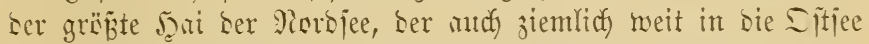
fineingeft; Der Iornhai, Spinax Acanthias, ber in ber Porbjee

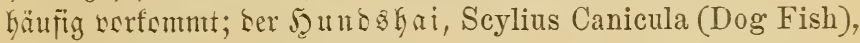

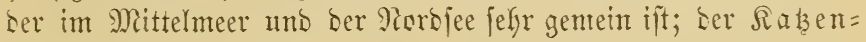
hai, Scylium Catulus, in ber Porsjee; ber blaue Jaai, Carcharias Glancus, in ber Norbjee.

\section{Die Seebraffen. Sparoidei. \\ (The Sea Bream', Le Pagre.)}

Sie fonmen in grof̈er Miannigfaltigfeit im Mittelmeer vor. Ter an ber englijdyen Rüijte jehr verbreitete Pagellus Centro-

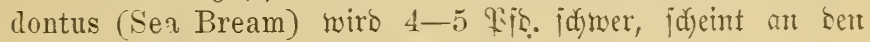
seutidyen Rüiten zu jeflen. Iie Eecbrallen werben in allent

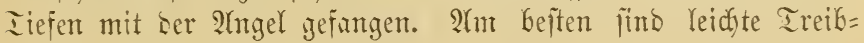

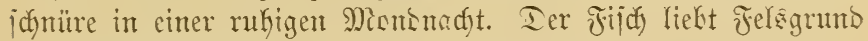

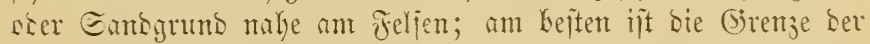

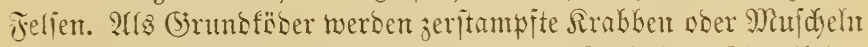

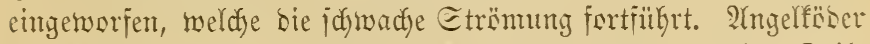

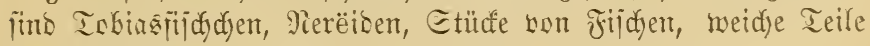
won Mirjeln.

\section{Die Mreerbarbe. Mullus surmuletus.}

(The Red Irullet, Le Surmulet, Le Rouget Mullet, Le Vrai Rouget.)

Die Nicerbarbe ijt cine brüfmte Ielifatelie ser Rünter.

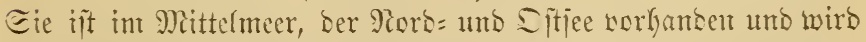
bisweilen nit ber 2lngel gefangen, bejonbers mit Gruntongeln

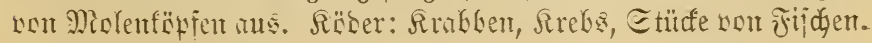


292 Die Seepijche und Deren Fang mit ber Alngel.

\section{Der Gering. Clupea Harengus.}

(The Herring, Le Hareng.)

Der Jering ijt in ungekeurer Mienge in ber Porb $=4$ to

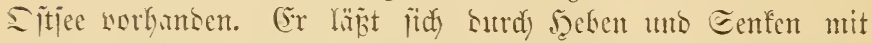
cinem unbetïberten blumfen 2fngelfafen fangen. (Ër lavidyt mäfrento bes gimjen Eonmer.

\section{Das Petermännׁ̆en. Trachinus Draco.}

(Iradyenfijd, S.teije. The Sting Bull.)

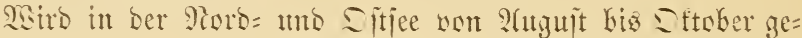

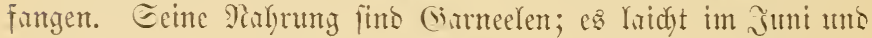
Sulti tno wirs won 2htguit bis Sftwber am meiften gefangen.

\section{5j. Der 2ral. Anguilla Tulgaris.}

(The Eel, L'Anguille.)

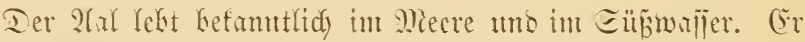

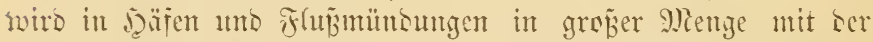

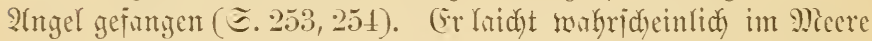
won Sftoker Kis Iejemficr. Im Sinter liegt er im Echlamm wergraben uns miro bant nidyt gejangen. 


\section{Sifferafutr.}

gachweiintng eintger Büther und Beitid)riften, welche ber Beachtung

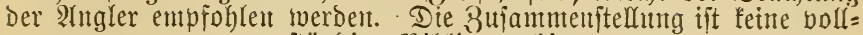
ftändige Bibliographie.

Aldam, W. H. A Quaint Treatise on Flees and The Art a Artyfichall Flee Making by An Old Man. London 1876. (Heber fiuntitliche Frorellentfiegen. (s'ntte: Butch, theuer.) Mit 2 (rbbildungen. D'Arlaten, Franz Rubrig Seerm. Sollftändigez Sattobuth Der fei= ttern 2̂ttgelfuttit. Retpzig 1862.

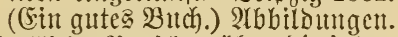
grutfid)e Seridhte über bie inter= nationale giticherei=22usitellutg 3u Berlit 1880 (Berlin 1881,

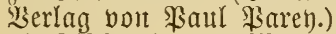

1. Fif Sornte, 5 . Saact, §. Michaelis. II. Seefiticherei vonDr.MI.Rintoe= man. Mit 162 Solzichnitten. III. Sitßwafierfif cherei von Dr. A.Meßger. Mittsolzidnutten.

IV. 2Baffertiere unt Fiticherei= produltte volt Dr. 57. Dohrut.

V. WBifientichaftliche Stbteilutg voit Э. 2famts, (5. Friedel, Dr. D. Serme, Dr. F. Solde= flein, Dr. \$․ Mragutu, Dr. (5. Ehorter, Dr. \& $\$$ sttmate. Mit 101 52olajchnitten.

Bailey, William. The Anglers Instructor. 3. Ed. Nottingham 1878. Der Lerfaffer gilt für einten Der getwansteitent 9lotting= ham $=$ tho Flokangler. (5r i itt Die withtigite Dutelle fitr Dieje Art von Jijecherei, namentlich für Den Fantg Der SBarbe.

Bainbridge, Geo. C. The Fly Fishers Guide. Liverpool 1816. lleber Fiticherei mit fïnftlichen Ffliegen, zahtreiche 2rbbilloungen.
Berners, Dame Juliana, The Book of Saint Albans; St. Albans 1486. A Treatyse of Fysshynge Wyth An Angle.

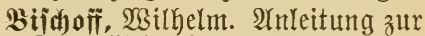
2Angelfiticheret. Münthen 1859.

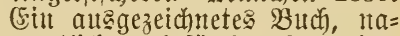
mentlich auch fiitr ben fang beg Szucthent. Sitele 2rbbildungent.

Blackers Art of Fly Making etc. London 1855. Witele fchöne 2 (b) biloungen. Berfafier war einer Der berithmteiten Frliegentwinder feinter 3eit. Sein $2 B$ ert bejdreibt bie 2Atfertigutg fünitlicher ơlie= gent ausfiuthrlich int eigentün= litcher 2 setie.

Blaine, Delabere P. An Encyclopaedia of Rural Sports. New Ed. London 1870 (sitt vortreffliches untentbeheliches Wert.) Siele 2rbbilbutngen.

Blanchère, H. de la. La pêche et les poissons. Nouveau dictionnaire génẻral des pêches. Paris 1868. (Unentbehrlich.) Litere âtbildoungent.

Borte, Max non Dem. Wegmeijer für 2Attgler Dutrch Dentichland, Deiterreich und Die Echweiz. Berlin 1877. (Sin Meijehandbutch für ŶAgler, mit Ylachbeijung von 2 Ingelgelegenheiten.

Borte, Mar von dem, Dr. $2 . B_{e}=$ necfe und (5. Dallnter. Sand= buth) Der Fitichzutcht und Fiticherei. Sherlin 1886.

Bowlker's Art of Angling, Containing Directions for Fly- 
Fishing,Trolling, Making Artificial Flies etc. New Ed. Ludlow. 1854. 2rbbildungen. Das Bud hat viele 2luflagen gehabt. Widhtig fül bie Jijuerei mit fünftlidien Fliegent. Der Der= fafier galt für ben gewantofeften Fliegenfitcher feiner Beit.

vary, Sir Humphry Bart. Salmonia, or Days of Fly-Fishing. 5. Ed. London 1869. (Fin inter= effantes Buch eines als giatur= forjecer beriibmten Berfajier.

Davy, Sir Humphry. Salmonia ober nem Ingeltage. Deutich von Vienbert. Berlin 1840.

Dentidje Fijucrei $=3$ Beitung. Etettin. Bringt Sufiäbe über Ungelfitcherei.

Ephemera (Edw. Fitzgibbon). A Handbook of Angling, Teaching Fly-Fishing, Trolling, Bottom-Fishing and SalmonFishing. 4. Ed. London 186 ó. Der Verfaffer war ein jehr ge= wanter IIngler; Daz Bud ijt bortrefflid and wird jebr vielge= lejent. Ubbildungen.

Ephemera. The Book of The Salmon. London 1850. Mit farbigen stbbildungen bon Rachoffliegen.

Fenuel, Greville. The Book of The Roach. London 1870. (Wichtig für Plökenfifcherei.)

Field, The Country Gentleman's Newspaper. London. Ias ge= lejenjte, in sngland ericheinentbe Sountal iiber alle 2rten bon Eport, namentlich aud) :rngel= fiicherei. Die 2 Mtriäbe jiber Fificherei rebigient ber belicbte \&ngelifuriftiteller Ma. Franciä Trancis.

Fishing Gazette. London. 3eit= forift, nur fïr angelfiticheret.
Forest and Stream. New-Iork. Beitichrift für @port, namentlich Wlitgelfifherci $2 c$. 2on bem be= fanntell Fifchzüicfter Mir. Fred yather bearbeitet.

Francis Francis. A Book on Angling. 5. Ed. London 1880. (5injehr gutes̃, bielgelejentes $\mathfrak{B} \|$ d) itber alle Irten bon 2lnaelfificherei in @ïfrwafjer. Bringt Das boll= itänbigite Qerzeichniß fünitlicher Trliegen zum Fang bon Sach, Meerforellen, Eceforellen, Jo= rellen 2c. Biele arbbiloungen.

Francis Francis. Angling. London, 2. Ausgabe 1883. (Ein jehr gutes Budb, lurae 2tutweijung fiir alle 2Irten Wnalfijchere int

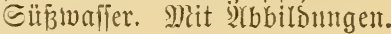

Francis Francis. By Lake and River. London 1574.

Fraucis Franci:. Hot Pot or Miscellaneons Paper. London 1880.

Francis Francis, Angling Reminiscenses. London 1887.

Hale, Captain, How to Tie Salmon Flies. London 1892. (5ine jehr gute $\mathfrak{A} u$ weijung zum Binden ber sachsfliegen.

Halford, Frederic II, Flouting Flies, and How to Dress Them. London 1886.

Halford, Frederic M., Dry-Fly Fishing in Theory and Practice. London 1889. (sinte aub= fïlorliche 2ntweijung zum 2Gerfent mit Der Fliegenrute.

Hallock.Ch. The FishingTourist. New-Tork 1873. Sntereffant.

llearder,J.N.\&Son. Sea-Fishing unto Bretslitte bon 2 Ingelgerätent.

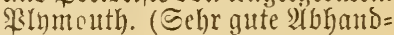
lung ïber 2lugelfijcherei im gleere.)

Heuderson, William. My life as 
an Angler. London 1879. F゙̈̈r Forellentang mit dem Jiegent=

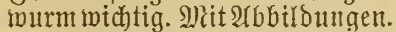
Henshall, James. Book of The Black Bass Cincinnati 1881. Epochemachend für bie ?otting= hant= Fijcheret und bie shagel= fitherei mit lebendent Jitichent. Mit rbbildungen.

Henshall, James. More About The Black Bass Being a Supplement to The Book of The Black Bass. Cincinnati 1839. Sorrode, John. Die Simt Der Tliegenfitherei auf Forellent nto Seichen in Deutichlauto und Dejterreich. Weimar 1874. Mrit

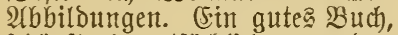
föpoft hauptiäclich aus ben Werten von Francis und $\Re D=$ nalos.

Jackson, John. The Practical Fly-Fisher. More Particularly for Grayling or Umbre. 2. Ed. London 1862. (5nthält eine lange Sifte limftricher Fliegen mit far= bigen 2ubbildungen derjelben, und Der lebenden Snieftent.

Jesse, Edward. An Anglers Rambles. London 1836. Das Butch ijt reich an guten Ratichlägen.

Land and Water, London. (sine Beitjcrift für Naturgejoichte und Sport. Das Drgan bes ver=

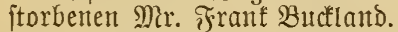

Nobbes, Rev. Robert. The Complete Troller. London 1682. Das $\mathfrak{B u d}$ hat viele $\mathfrak{A}$ trflagen erlebt, namentlich in unjerent Jahrhundert, es lehrt Den Jantg Des Sechts mit der Trollangel, fo, wie er noch beute auggentbt wirb.

Norris, Thaddeus. The American Anglers Book. Philadelphia 1865. Recht gut. Mit $\mathfrak{A b}=$ bildungen.
The North Country Angler. London 1817. Wichtig fütr Błuj( )= angelei tnd Tippficherei (poaching).

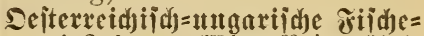
$\mathbf{r e}=3$ citung. Wien. $B$ rimgt $\mathfrak{A} u \hat{i}=$ fäke ïber antigelfijcherei.

Orvis, Charles F. and A. Nelson Cheney. Fishing with the Fly. Boston and New-Tork 18s6. (5nthält ein : Umerita bei dem Jang De马 Black Bass gebrauchten Jiliegen. Pennell, H. Cholmondeley. The Nodern Practical Angler. London 1870. Die?, wie die anderen Werfe von \$ennell jind jehr incrtooll.

Pennell, H. Cholmondeley. The Book of the Pike. 3. Ed. London.

Pennell, H. Cholm. Bottom or Float-Fishing. London.

Pennell, H. Cholm. Fly-Fishing and Worm-Fishing for Salmon, Trout and Grayling. London.

Pennell, H. Cholm. Trolling for Pike, Salmon and Trout.

Pennell, H. Cholm. Fishing Gossip. Edinburgh 1886.

Pennell, H. Cholm. Fishing in The Badminton Library. 1 Y ol. Salmon and Trout. 2 Vol. Pike and Other Coarse Fish. London 1889.

Pennell, H. Cholm. Modern Improvements in Fishing Tackle and Fish Hooks. London.

Poitevin, M. B. L'Ami du Pêcheur. Paris 1873. (5m= pFeblenswert für âtgelfijcherei mit natïrlichem sïber.

Pritt, T.E. North-Country Flies. London 1886.

Palman, G. P. R. The Tademecum ofFly-Fishing for trout. London 1851. (Enthält einte gute 


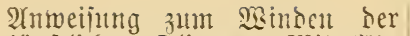
füntlidfen Jilegen. Mit $2 \mathrm{~b}=$ billoungen.

Roualds, Alfr. The Fly-Fishers Entomology. 7. Ed. London 1868. (5podhemachent für bie 2lngelfijcherei mit fünitlidbest Fliegen, und für bie ?achbildung lebender Injeften. Mlit farbigen

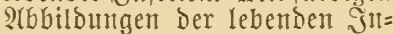
jeften und fünitliden Fliegen.

Salter, T. F. The Anglers Guide, Being a Plain and Complete Practical Treatise on The Art of Angling for Sea, River and Pond Fish. 8.Ed. London 1833.

Scott, Genio, C. Fishing in American Waters. New-York 1869. J̄nterejiant. 2rbbiloungen.

Shooting and Fishing, eine 3eit= idnrift, Deren Angling Editor Mr. A. N. Cheney itt; erjueint zu Boston, Mass.

Souti, Theophilus. The FlyFisher's Textbook. London 1841. (5nthält einige jehr gute Sngaben über das serfen mit Der Filiegentute.

stewart, IV. C. The Practical Angler, or The Art of Tront Fishing, more Particularly Applied to Clear Water. 6. Ed. London 1814. Der Berfaijer inal Der gemanteite Forellentangler jeiner Beit, und jein Budh gehört zu Den beften, bie e? giebt. Mlit Qtbbildungen.

Stoddart, Thomas'Tod. TheAnglers Companion to The Rivers and Lochs of Scotland. 2. Ed. Edinburgh and London 1853. (5in ganz vortreffliches zuch. Mrit $\mathfrak{2} b$ bildnngen.

Theakston, Michael. British Angling Flies. London 1883. Revised by F. W. Walbron.
Thomas,Henry Sullivan,Madras. The Rod in India, Being Hints How to Obtain Sport, With Remarks on The Natural History of Fish etc. 2.Ed. London 1881. Das wath enthält viele slit= tcilumaen über 2 Ingelfiicherei, bie auch für (5uropa von : Sert pind.

Walton, Izaak. The Complete Angler. Die erite 2 rngabe er= ichien 1653. Sein Buth erfrent fich einer gröвeren \$̧opularität in (England, wie diejes. (5. hat unzählige 2uf́lagest gehabt. SEid)tig für Den Jang von Döbcl mo 2 lei, Die Jitherei mit uatïr= lichen Sïbern.

Walton, Izaak and Charles Cotton by Ephemera.London 1853. Mit 2umerfungen Des 5eraus: gebers. Waalton behandelt haupt= iächlich bie silicherei mit natitr= liden Sïbern, (5otton Das 2Ugelı ntit fïmitlichen Fliegen. - Einte lleberjebung von ङ dumadber er= ichen in 52amburg 1859.

Wells, Henry P. Fly-Rod and Fly-Tackle. Suggestions as to Their Manufactury and Use. London.

Wheatley, Hewett. The Rod and Line. London 1849. Mit $21 b=$ bildungen. (5)auptquelle fïr das 5ebent und Eentent). Jang bon J̈prellen und शlejchen.

Wiicocks, J. C. The Sea-Fisherman. 3. Ed. London 1875. (Tas wid)tigite 23 erf fijcherei im Meere.) Mit $\mathfrak{U} b=$ bilbungent.

Willamson, Capt. J. The Complete Anglers Vade-Necum. London 1808. 11eber Eüīmaijer= und ๔eeficjerei 2oul Wert fitd Die פyitteilungen über 2 ngel= itellen. 


\section{UNTER GANZ NEUER LEITUNG

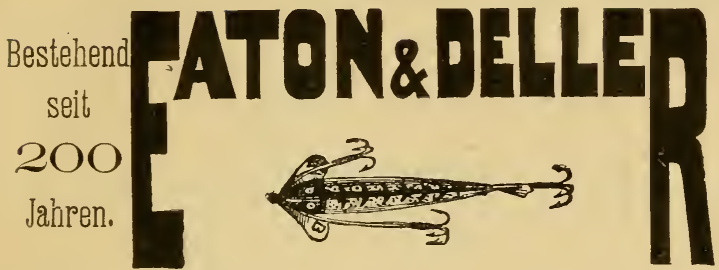 \\ Bestehend \\ seit \\ 200 \\ Jahren.}

haben das grösste Lager in der Welt von in London gemachten Angelgeräten.

FABRIKANTEN JEDER ART VON ANGELRUTEN, ROLLEN, SCHNÜREN, KÜNSTLICHEN FLIEGEN \& KÖDERN. SEIDENWURM-GUTFADEN FEINSTER QUALITÄTEN JEDERZEIT VORRÄTIG.

\section{SPECIALITÄTEN:}

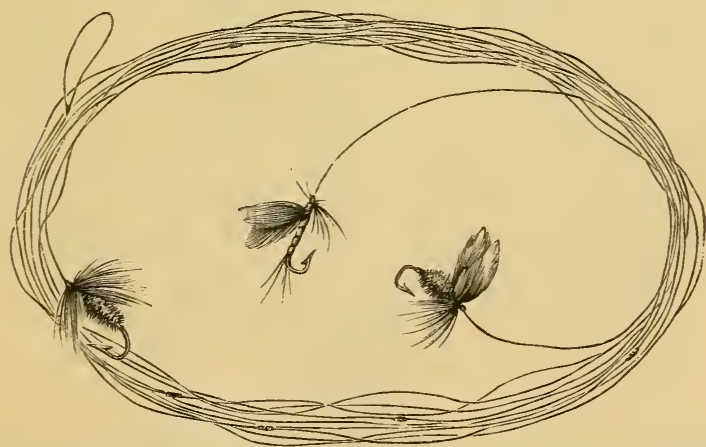

GESPLITZTE BAMBUS-RUTEN, RONALD'SCHE \& ANDERE FLIEGEN.

Preislisten auf Verlangen gratis übersandt.

BEANWORTUNG ALLER BRIEFE MIT POSTWENDUNG.

$\longrightarrow$ \& Zahlung durch Postanweisung. \}-

v. ๖. $\mathfrak{B} \circ$ rne, 2tngerfijderei. 3. 2tuๆ. 


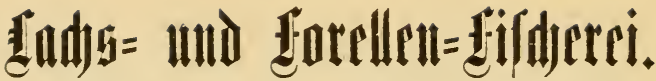

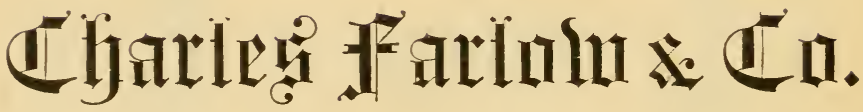

\section{Stranto}

\section{sondon, W. C.,}

berjertigen bie vorzïglidfiten angelruten, namentlich aud bie be=

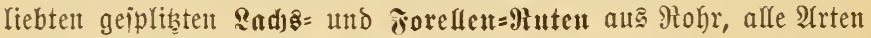
vou Mollen, Eduliren, fïujtliden Sadjs=, Foreflen= unD sed)t= Jliegen und fïultlidjen Epinuföberu in ber grȫ̄ten Mannigfaltigfeit; Snjten und čtuis für ?ngelgeräte, vollitändig ausgerüjtet für alle

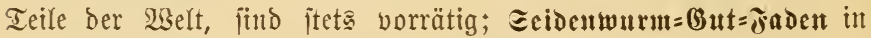
Der ausgezeichntiten Sualität, bou ben ftärfiten bis zu den feinjten Gortimenten it itets zu haben; Sharles Farlow \& (50. futb in \&ondon

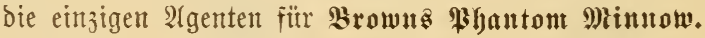

\section{Jreistoutrants werùen auf llerlannen nratis iiberfanot.}

Orbres werben mit Remiijen verjegen erbeten. 


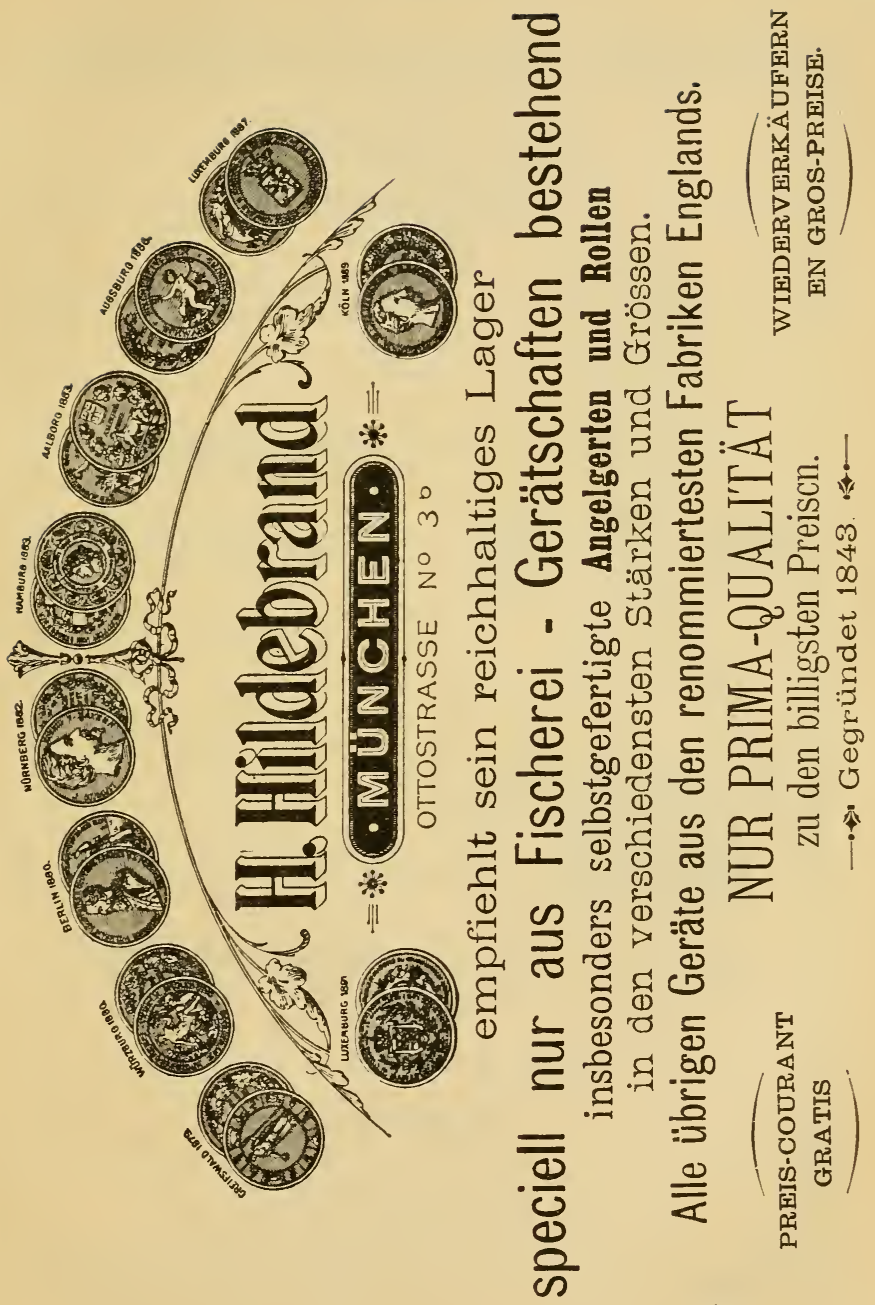




\section{Joseph Oszvald's Nachf. Josef Gerhard}

Wollzeile No. 1. WIEN. Wollzeile No. 1.

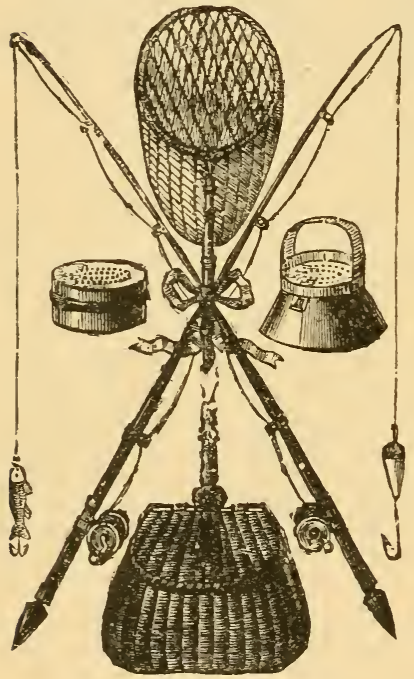

\section{Grösstes Lager von}

FischereiRequisiten englischer,

amerikanischer und

eigener Erzeugung.

Joseph Oszvald's Nachf. Josef Gerhard

Wollzeile No. 1. WIEN. Wollzeile No. 1.

$\rightarrow$ En gros et en detail $3-$

Illustrierte Preis-Courants gratis. 


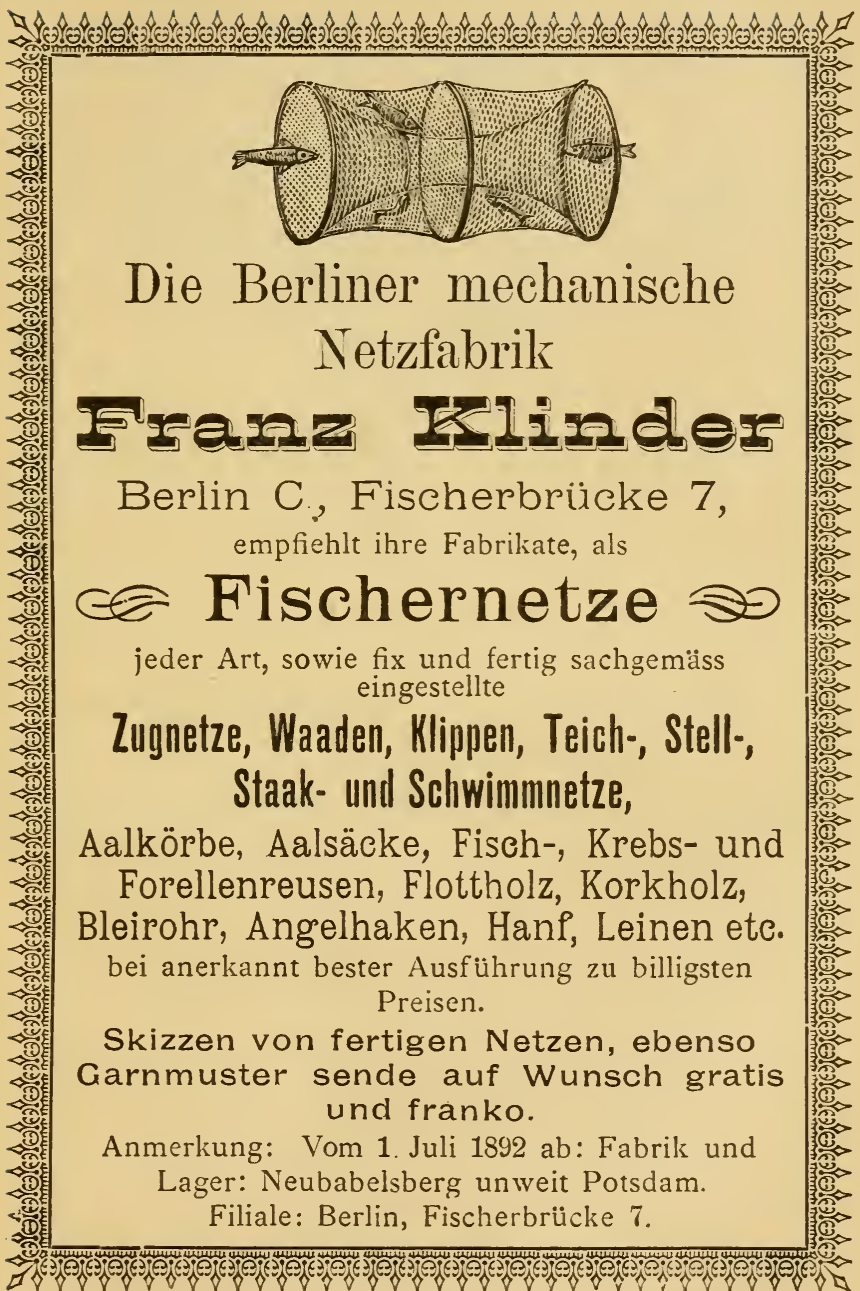




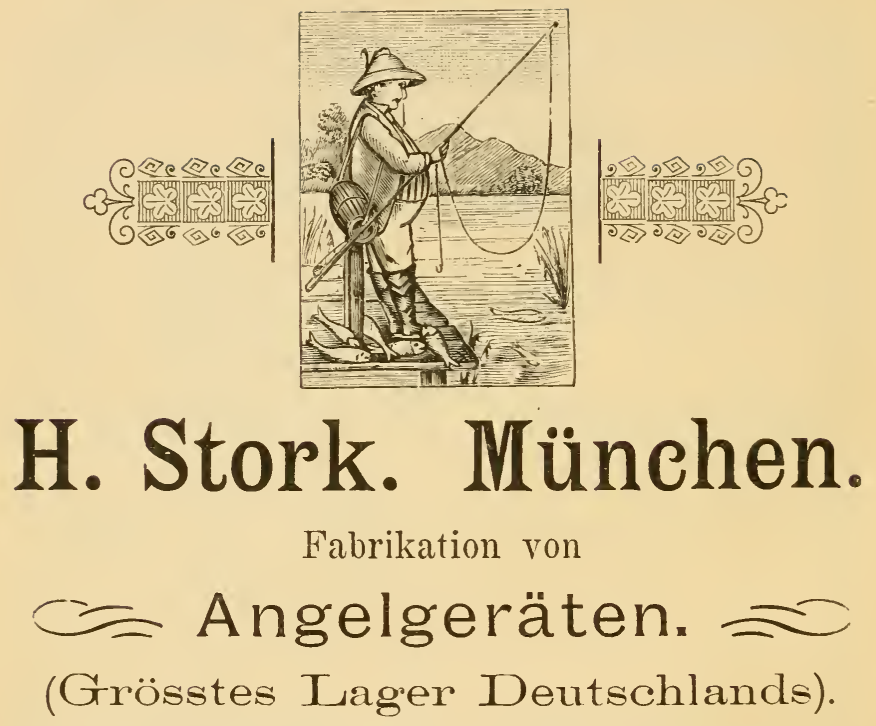

Eigene Specialfabrikate in Angelschnüren, Metallspinnern, montierten Fangzeugen, Ruten etc ;

Englische und amerikanische Fıscherei-Geräte.

Vertretung der Netzfabrik Itzehoe.

Import spanischen Seidenwurmdarmes.

\section{Medaille I ondon 1883.}

grosse Silb. Medai!le Berlin 1880
," Br.
",
Berlin ,

grosse Goldene Medaille aus Oesterreich 1887

Silb. Medaille Cöln 1889

Silb.

Würzburg 1880

Silb.

Linz

Hessen

Preussen

10 Diplome div. Länder. 


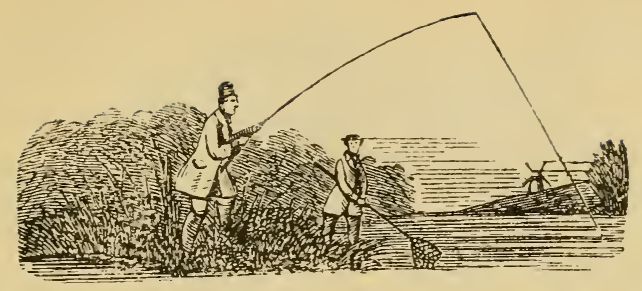

J. Gust. Grube J. E. R. Waitz Nachf.

Gänsemarkt 48 HAMBURG. Gänsemarkt 48.

Niederlage französischer, deutscher, sowie

englischer Fischerei - Geräte von S. Alleock \& Co. Redditeh.

$\propto$ Preislisten gratis. $=0$

Eisen- und Kurzwaaren. Spiel-Waren.

Haushaltungs-Gegenstände.

Gartenmöbel. Gartenwalzen.

Rasenmähmaschinen . 


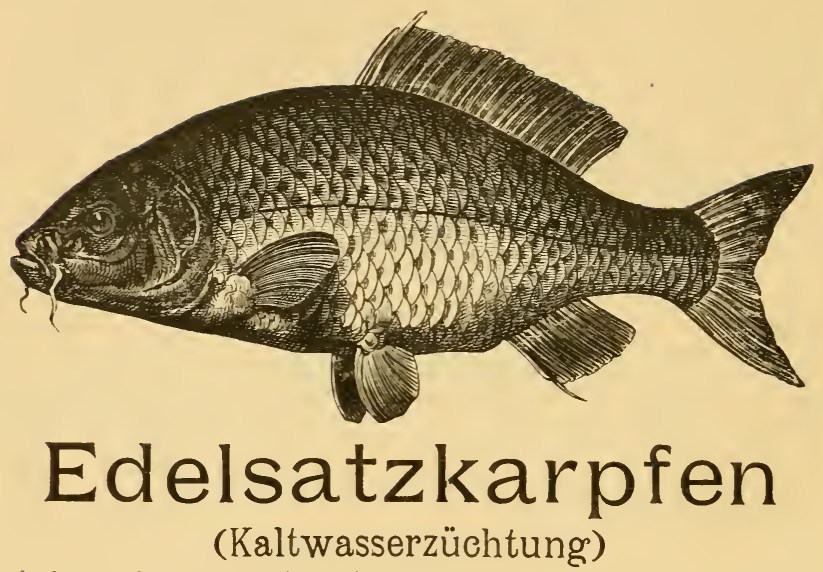

in jedem Wasser gedeihend, äusserst schnellwüchsig, offeriere wie folgt:

Edelsatzkarpfen, gemischt mit Edelspiegel- und Edellederkarpfen, pro 100 St. M. 6,00, 2-sömmerige desgl., pro 60 St. M. $20,00,3$ sömmerige pro 60 St. M. 30,00 .

Goldorfen, einjährig, pro St. M. 0,50, laichtähige pro St. M. 200. Goldkarpfen, 100 St M. 6,00, 2-sömmerige 60 St. M. 20,00, laichfähige pro St. M. 1,00.

Goldkarpfen-Karausche (delikater Speisefisch), 1-sömmerige pro 100 St. M. 6,00, 2-sömmerige 60 St. M. 20,00, laichfähige pro St. M. 1,00.

Goldkarausche, 1-sömmerige pro 100 St. M. 3,00, 2-sömmerige pro 100 St. M. 6.00.

Ferner empfehle:

\section{Goldfische, Schlei- und Aalsatz.}

Gefässe werden leihweise geliefert und sind innerhalh 3 Tagen franlio zu retournieren. Der Versand geschieht per Eilgut, doch wird nur gewöhnlicher Frachtgutsatz berechnet. Versandzeit im ganzen Jahre und fällt nur bei ganz hoher oder ganz niederer Temperatur aus.

Verunglückte Sendungen werden gratis nachgeliefert. Der Bestellung ist der Betrag beizufügen.

\section{Robert Friedrich,}

Fischzüchterei und Handlung. Zahna, Bezirli Halle. 

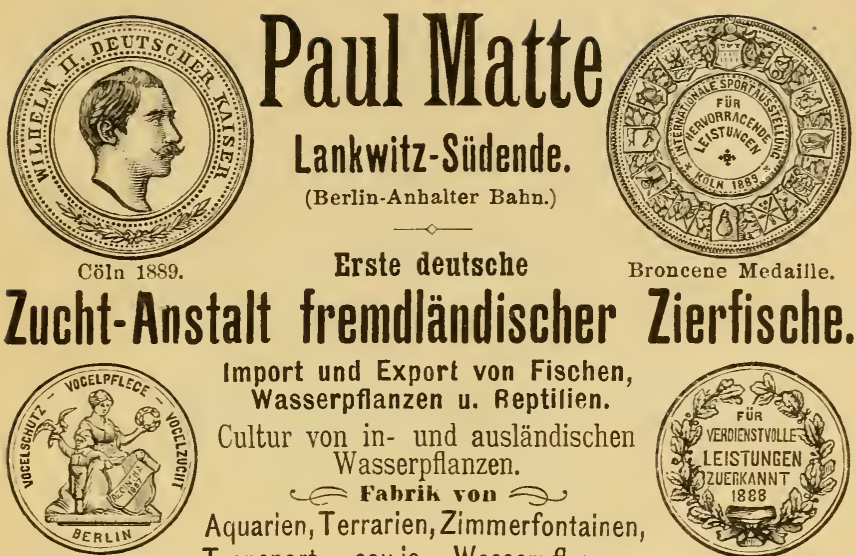

Import und Export von Fischen, Wasserpflanzen u. Reptilien.

Cultur von in- und ausländischen Wasserpflanzen.

$\Longleftarrow$ Fabrik von 20

Aquarien, Terrarien, Zimmerfontainen, Transport- sowie Wasserpflanzen-

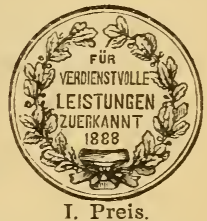

Berlin 1888 .

" 1892. Gefässen,Thermometern,Stechhebern,

Gr. silb. Medaille.

Durchlüftungs-Apparaten und den verschiedenen Hilfsmitteln zur

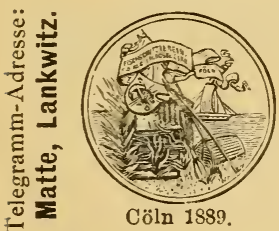

Instandhaltung von Aquarien.

Preis-Verzeichnis gratis und franko.

Gegründet 1876.

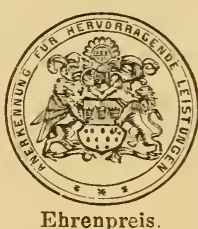

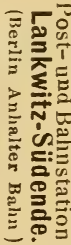

Prämilert auf allen beschickten Ausstellungen.

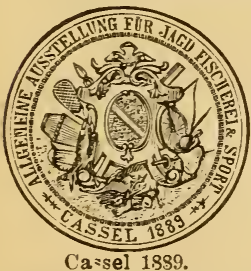

Prämilert

Internationale Fischerei-Ausstellung

Berlin 1880.

Berlin 1890.

1 Fhrenpreis,

7 gr. silb. Medaillen.

1 broncene Medaille.

Fremdländische Zierfische. Winke zur Beobachtung. PHege und Zucht der Makropoden, Guramis, Gold-, Telescop-, Hundsfische u. a. Nebst Anhang: Bemerkungen über die Axolot1. Nach Mitteilungen P. Matte's, Fischzüchter, sowie eigenen Beobachtungen und Erfahrungen von $B r u n o$ Düringen. Nit Abbildungen 1,50 Mark. 


\section{LUDWIG FREYHOFF}

Hoflieferant. - Krebsmästerei.

\section{Schwedt an der Oder,}

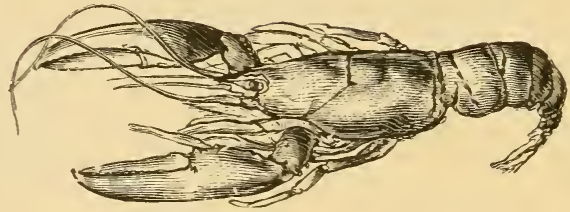

liefert während der Fangezeit von April bis Oktober

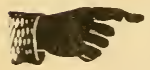

lebende Krebse

à Schock $=60$ Stuick von 3 bis 16 Mark in streng reeller Auswahl, sowie bei Vorhandensein

Satzkrebse mit Eiern 4 bis 8 Mark.

Aus Fichtenlıolz gefertigt:

Krebs-Reusen (Krebsfangekörbe) à 60 Pfge. Aal-Reusen (Aalfangekörbe) à 1 1/2, 3, $4^{1 / 2}$ U. $6^{1 / 2} \mathrm{H}$. Neunaugen-Reusen à 1 llark.

\section{Weidene Körbe in allen Grössen}

werden zu billigen Preisen nach Vorschrift angefertigt. 


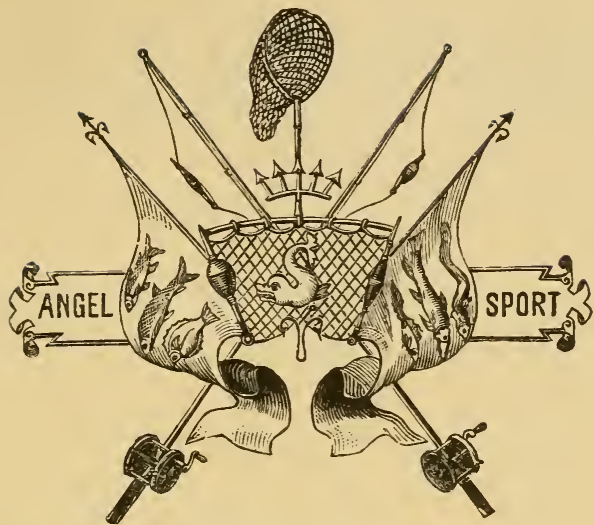

\section{Oscar Ziegenspeck} Berlin S., Kommandantenstr. 56.

Grösstes Spezial-Geschäft für

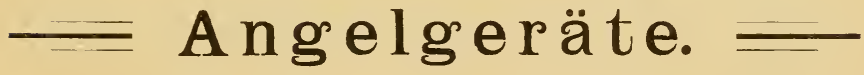

Eigene, sowie Fabrikate der

hervorragendsten Häuser des Auslandes.

Fischnetze, Reusen ete. jeder Art.

Beständiger Eingang aller auf dem Gebiete des Angelsports erscheinenden Neuheiten.

Export nach allen Ländern.

Reichhaltige, illustrierte Kataloge gratis und franko. 


\section{W.BARTLLEETI \& SONS ABBEY MILLS, REDDITCH \\ und}

53 GRESHAM STREET, LONDON (ENGLAND).
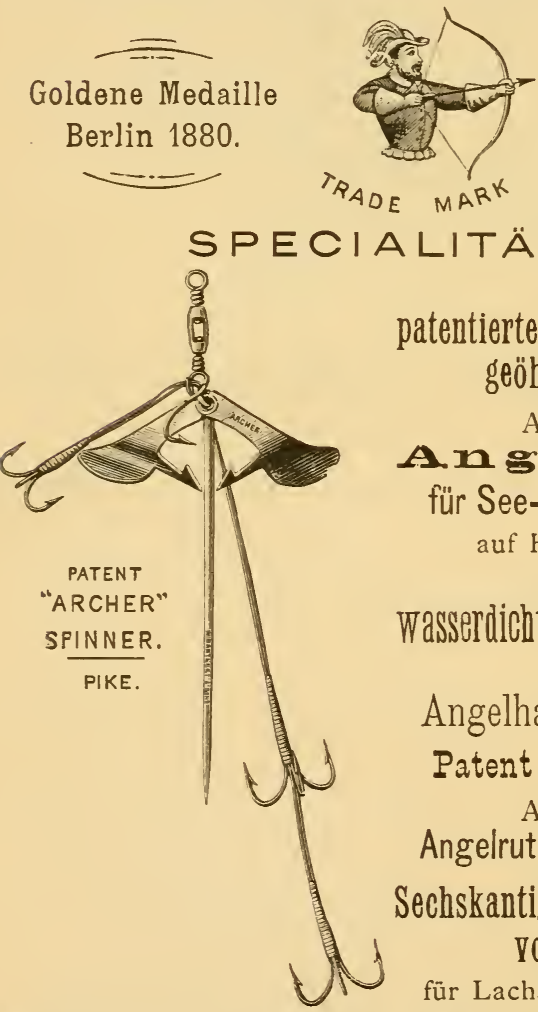

Erhielt 16 goldene und Preis-Medaillen.

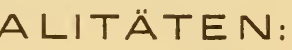

PENNELL's patentierte, einwärts gekrümmte, geöhrte Angelhaken.

Alle Arten von

An 901 halzon für See- und Binnenfischerei auf Haltbarkeit geprüft. , ARCHER", Wasserdichte seidene Angelschuntre. , , ARCHER', Angelhaken an Gutfäden. Patent „Archer" Spinner. Alle Arten von Angelruten und Angelgeräten.

Sechskantig gesplitzte Angelruten Neuheiten in künstlichen Ködern und Angelgeräten aller Art.

für Lachs- u. Forellenfischerei. 
NOTIZEN. 
NOTIZEN. 
NOTIZEN. 
NOTIZEN. 
Verlag von PAUL PAREY in Berlin SW., 10 Hedemannstrasse.

\section{DIE FISCHZUCHT. \\ Von}

Max von dem Borne.

Dritte, neubearbeitete Auflage.

Mit 111 Textabbildungen. Gebunden, Preis 2 M. 50 Pf.

\section{Wegweiser fiir Angler}

durch Deutschland, Österreich und die Schweiz.

von

Max von dem Borne.

Gebunden, Preis $4 \mathrm{M}$.

\section{Fischerei und Fischzucht im Harz}

mit besonderer Berticksichtung der

Forellen

und der

Centralfischzuchtanstalt zu Michaelstein in Braunschweig.

Von

Max von dem Borne.

Mit 9 Holzschnitten. Preis 1 M. 50 Pf.

\section{Die Teichwirtschaft.}

Praktische Anleitung zur Anlage von Teichen und deren Nutzung durch Fisch- und Krebszucht.

Von Dr. B. Benecke, w. Professor an der Universität Königsberg.

Zweite Auflage.

Nit 80 Abbildungen. Kartonniert, Preis 1 M. 75 Pt.

\section{Das Fischereigesetz für den Preuss. Staat} vom 3 . Mai 1874

nebst den für die sämtl. Provinzen erlassenen Ausführungs-Verordnungen sowic dem Vertrage wegen Regelung der Lachsfischerei im Stromgebiet des Rheins rom 30. Juni 1885 und dem Vertrage, betreffend die polizeiliche Regelung der Fischerei in der Nordsee ausserhalb der Küstengewässer rom 6. Mai 1885. Text-dusgabe mit Anmerk. Mit 33 Fischabbildungen. Preis $1 \mathrm{M}$.

$\mathrm{Zu}$ beziehen durch jede Buchhandlung. 


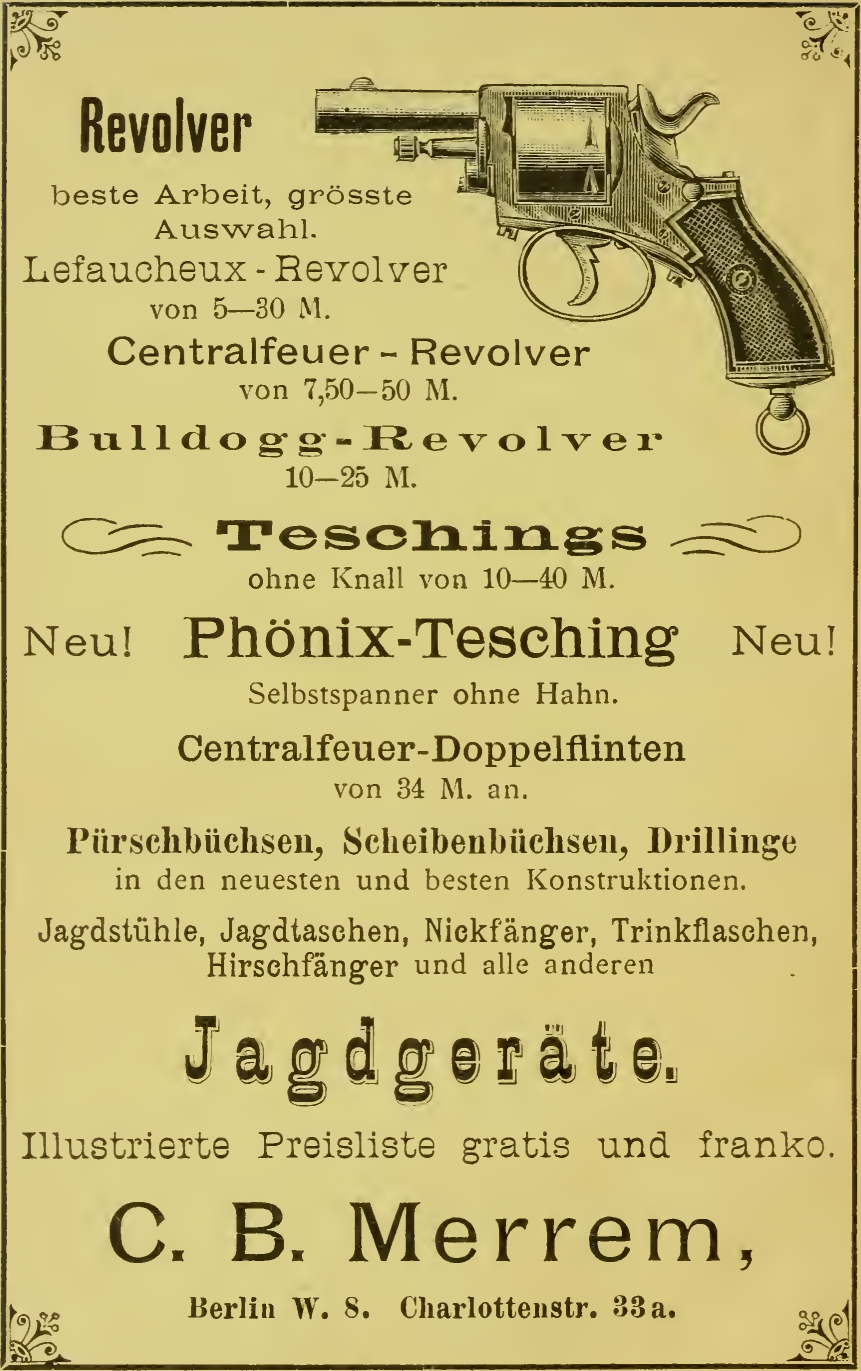




\section{Mechanische Netzfabrik n. Weberei}

Actiengesellschaft

\section{Itzehoe (Holstein).}

Cirösste und bestrenommimerte Fabsik des Continents.

\section{Prämiiert}

auf den Ausstellungen des In- und Auslandes mit den ersten Preisen.

Staatspreise der preuss. und österreich. Regierung.

\section{Fischernetze und Fischergarne} aller Art,

auch fertig montierte Netze als Zug. oderSchleppnetze, dreiwandige Staack- und Spiegelnetze, Aalreusen, Fischkörbe etc.

Prospekte und Beschreibungen werden auf briefliche Anfragen gern erteilt.

Agenten und Wiederverkäufer gesucht.

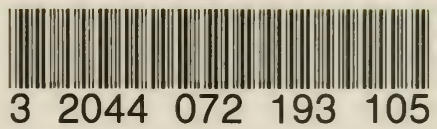


